\title{
Consolidation and coordination in urban freight transport
}

\author{
W.J.A. VAN HEESWIJK
}

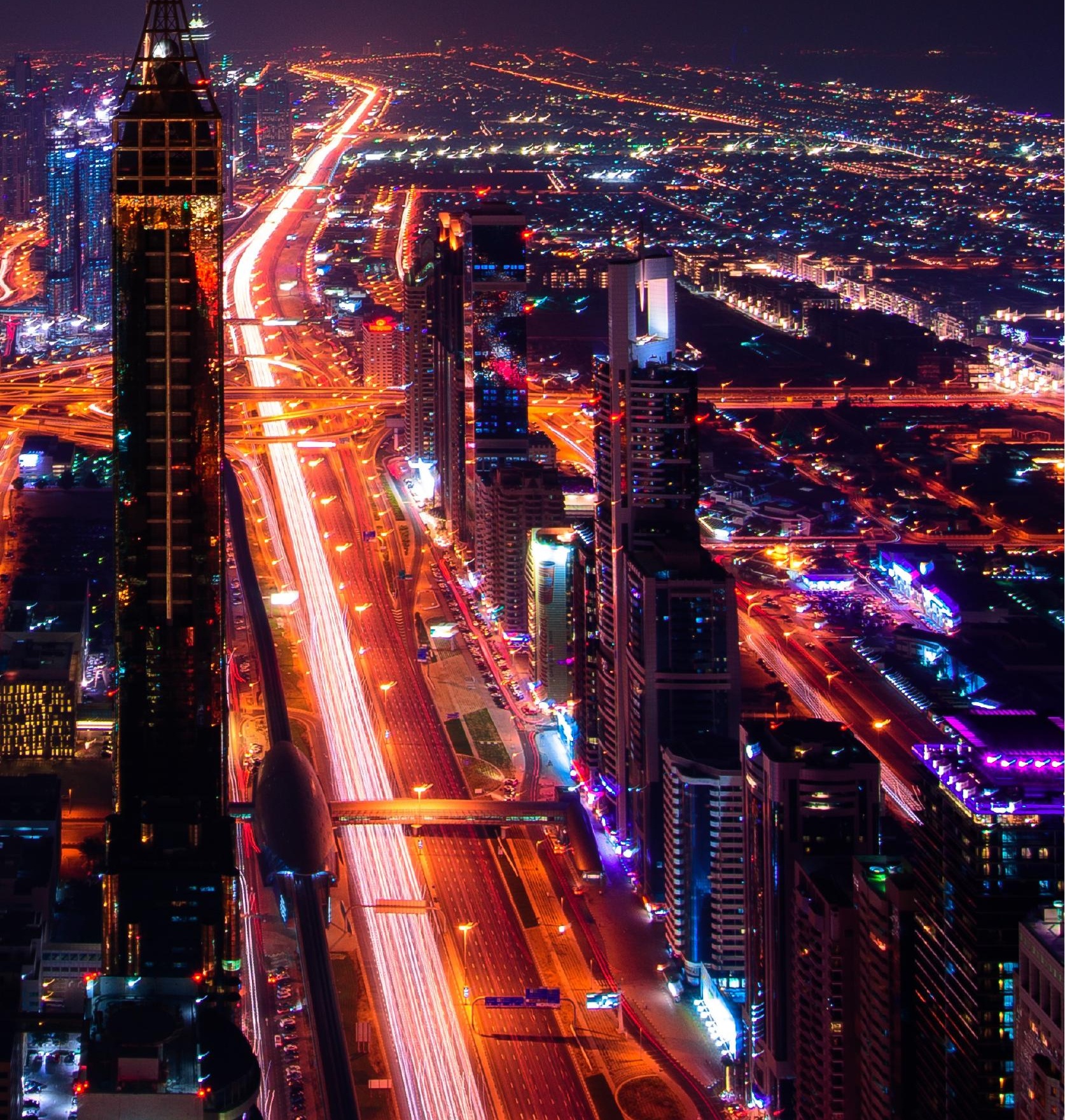




\title{
CONSOLIDATION AND COORDINATION IN URBAN FREIGHT TRANSPORT
}

\author{
W.J.A. van Heeswijk
}


This thesis is number D207 of the thesis series of the Beta Research School for Operations Management and Logistics. The Beta Research School is a joint effort of the departments of Technology Management and Mathematics and Computer Science at the Eindhoven University of Technology and the Centre for Telematics and Information Technology at the University of Twente. Beta is the largest research center in the Netherlands in the field of operations management in technology-intensive environments. The mission of Beta is to carry out fundamental and applied research on the analysis, design and control of operational processes.

\section{Graduation committee}

Chairman \& Secretary: Prof. dr. T.A.J. Toonen

Promotor: Prof. dr. W.H.M. Zijm

Assistant promotors: Dr. ir. M.R.K. Mes

Dr. ir. J.M.J. Schutten

Members: $\quad$ Prof. dr. J.van Hillegersberg

Prof. dr. ir. E.C. van Berkum

Prof. dr. T. van Woensel

Prof. dr. ir. L.A. Tavasszy

Dr. W. Ploos van Amstel

This research has been funded by Dinalog, the Dutch Institute for Advanced Logistics.

PhD thesis, University of Twente, Enschede, the Netherlands

Printed by Ipskamp Printing

○W.J.A. van Heeswijk, Enschede, 2017

All rights reserved. No part of this publication may be reproduced without the prior written permission of the author.

ISBN 978-90-365-4313-2 


\title{
CONSOLIDATION AND COORDINATION \\ IN URBAN FREIGHT TRANSPORT
}

\author{
PROEFSCHRIFT
}

ter verkrijging van

de graad van doctor aan de Universiteit Twente,

op gezag van de rector magnificus,

Prof. dr. T.T.M. Palstra,

volgens besluit van het College voor Promoties

in het openbaar te verdedigen

op vrijdag 19 mei 2017 om 14.45 uur

door

Wouter Johannes Albertus van Heeswijk

geboren op 14 september 1988

te Gendringen, Nederland 
Dit proefschrift is goedgekeurd door de promotor, Prof. dr. W.H.M. Zijm

en de co-promotoren, Dr. ir. M.R.K. Mes

Dr. ir. J.M.J. Schutten 


\section{Contents}

Preface ix

Part I. Introduction 1

Chapter 1. Introduction 3

1.1. Research motivation 3

$\begin{array}{lll}1.2 . & \text { Research setup } & 15\end{array}$

$\begin{array}{lll}1.3 . & \text { Literature overview } & 19\end{array}$

1.4. Structure of the thesis 26

$\begin{array}{ll}\text { Part II. Consolidated planning methods } & 31\end{array}$

Chapter 2. Planning orders in networks with reloads 33

2.1. Introduction 34

2.2. Literature on consolidation in intermodal transport 36

2.3. Problem formulation 38

2.4. Consolidation algorithm 40

2.5. Numerical experiments 56

2.6. Conclusions 67

Appendix 2A: Runtime and upper bound analysis $\quad 70$

Appendix 2B: Cost functions and savings 76

$\begin{array}{lll}\text { Chapter 3. Last-mile dispatch decision problem } & 79\end{array}$

$\begin{array}{ll}3.1 . & \text { Introduction } \\ 3.20\end{array}$

$\begin{array}{lll}\text { 3.2. Literature on dynamic and stochastic dispatching problems } & 81\end{array}$

3.3. Problem formulation 84

3.4. Solution method 92

3.5. Experimental setup 101

3.6. Numerical experiments 108

3.7. Conclusions 116 
Appendix 3A: ILP model for large action spaces 117

Appendix 3B: Proofs for the sizes of state, action, and outcome space 122

Appendix 3C: Recursive least squares method for nonstationary data 129

Part III. Evaluating urban logistics initiatives 131

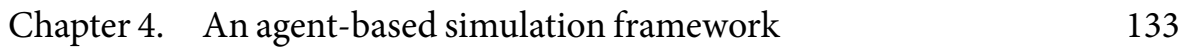

4.1. Introduction 134

4.2. Literature on evaluating urban logistics initiatives $\quad 135$

4.3. Framework design 139

4.4. Illustration of simulation framework 161

\begin{tabular}{lll}
\hline 4.5. Conclusions & 167
\end{tabular}

Chapter 5. Simulation of urban logistics schemes 169

5.1. Introduction 169

5.2. Literature on agent-based studies and urban data collection 171

5.3. Solution method 174

5.4. Experimental setup 193

5.5. Numerical experiments 210

5.6. Conclusions 232

Chapter 6. Consolidation in the city of Copenhagen: a simulation study 235

6.1. Introduction 236

$\begin{array}{lll}6.2 . & \text { Literature on business models for UCCS } & 237\end{array}$

6.3. Solution method 240

6.4. Experimental setup 251

6.5. Numerical experiments 261

6.6. Conclusions 278

Part IV. Conclusions 281

Chapter 7. Conclusions and further research 283

7.1. Reflection on research objective and research questions 283

7.2. Managerial insights 290

\begin{tabular}{lll}
\hline 7.3. & Discussion and further research & 292
\end{tabular}

$\begin{array}{ll}\text { Summary } 301 & 301\end{array}$

$\begin{array}{ll}\text { Samenvatting } & 307\end{array}$ 
\begin{tabular}{ll}
\hline Bibliography & 315
\end{tabular}

List of abbreviations 331

List of mathematical symbols 333

$\begin{array}{ll}\text { Chapter } 2 & 333\end{array}$

$\begin{array}{ll}\text { Chapter } 3 & 335\end{array}$

$\begin{array}{ll}\text { Chapter } 4 & 338\end{array}$

$\begin{array}{lr}\text { Chapter } 5 & 341\end{array}$

$\begin{array}{ll}\text { Chapter 6 } & 344\end{array}$

$\begin{array}{ll}\text { List of academic work } & 347\end{array}$

$\begin{array}{ll}\text { Journal papers } & 347\end{array}$

\begin{tabular}{ll}
\hline Conference papers & 347
\end{tabular}

\begin{tabular}{ll}
\hline Working papers & 347
\end{tabular}

\begin{tabular}{ll}
\hline Peer-reviewed conference proceedings & 348
\end{tabular}

\begin{tabular}{ll}
\hline Academic presentations at international conferences & 348
\end{tabular}

\begin{tabular}{ll}
\hline Theses & 349
\end{tabular}

Layout and design 351

Layout 351

Font 351

\begin{tabular}{ll}
\hline Figures & 351
\end{tabular}

Cover 351

About the author 353 



\section{Preface}

Before starting my master graduation project in 2012, the thought of pursuing a $\mathrm{PhD}$ degree had never even crossed my mind. Now, at the moment of writing this preface, the coveted title of doctor is within reach, marking the end of a long road that was sometimes jarring, but ultimately very rewarding. With this preface, I want to briefly reflect on the past four years and express my gratitude to the people who made the creation of this dissertation possible. Although I have met many smart and kind people during my $\mathrm{PhD}$ studies, I would like to address a number of people in particular.

While writing my master's thesis, I found that the challenge to tackle problems by collecting information, applying mathematical methods and attempting to find original solutions was considerably more enjoyable than trying to reproduce existing knowledge from text books. Simultaneously, the process made me aware of how much more there was yet to learn as a master's graduate, and I was eager to explore academic subjects into more depth. Still, without the right inspiration, I would probably never have made the step to apply for a $\mathrm{PhD}$ position. Therefore, I want to thank Reinoud Joosten for both sparking my interest in research and encouraging me to pursue $\mathrm{ahD}$. Despite having a background in finance, I ended up doing academic research in logistics. I am grateful to Henk Zijm for giving me the opportunity to join the IEBIS department as a $\mathrm{PhD}$ candidate.

During my research, I could always rely on my supervisors Martijn Mes and Marco Schutten, who helped me to familiarize myself with the field of logistics as well as various solution methods, and always were willing to provide helpful and detailed feedback on my work. Aside from the smooth cooperation on the work floor, I also enjoyed the home-cooked dinners and our memorable conference trips. Henk, no matter how many times my work had been reviewed and rewritten, your keen eye always found aspects to improve. I am glad to have had you as my promotor. 
My research was part of the CONCOORD project, which included partners from The Netherlands, Austria, Denmark and Turkey. I am happy to have exchanged ideas on urban logistics with you in our meetings all over Europe. In particular, I want to thank Birgit Hendriks from Binnenstadservice for challenging our sometimes rigid and abstract academic views and for providing indispensable insights into the practice of urban consolidation centers. Furthermore, I enjoyed collaborating with Bart Geelen from TU/e on the urban logistics game.

I was fortunate to spend the summer of 2016 in the peaceful green surroundings just north of Copenhagen. I thank Rune Larsen and Allan Larsen for graciously hosting me at Danmarks Tekniske Universitet. Our collaboration hasled to the paper on which Chapter 6 of this dissertation is based. My DTU colleagues - especially Cagatay, Evelien, Filipe, George, Ioulia, Jonas, Martin Philip, and Roberto - made me feel welcome and from the very beginning included me in their leisure activities. I enjoyed our weekly outings to Copenhagen and the occasional dinners and barbecues; your hospitality made my stay much more enjoyable. Ian, thank you for helping me to settle in Denmark and for accommodating me in your house with the stunning lake view.

The IEBIS department has always been a pleasant group to work in. In particular, I would like to thank my fellow $\mathrm{PhD}$ candidates (and the sporadic postdoc), with whom I spent much time both inside and outside of the university. My office mates Abhistha, Andrej, Floor, Gréanne, Lucas, Robin, Simon, and Sjoerd, thank you for the (highly frequent) coffee breaks and the interesting conversations, sometimes serious and sometimes amusing. Furthermore, I want to thank Arturo, Devrim, Dissa, Engin, Elisa, Fabian, Guido, Miha, Nardo, Nils, Rick, Sajjad, Sina, Sameh, Vahid, Wenyi, and Xavier for the games of hearts, lunch walks, dinners, nights out, and all other activities.

During my PhD, I served in the board of the PhD network P-NUT for a little over two years. This position allowed me to develop myself outside of academics and to organize events for the $\mathrm{PhD}$ community. More importantly, it allowed me to meet many interesting people from all sorts of backgrounds. Even mentioning just the board members that I have worked with yields a sizable list: Adithya, Anja, Bugra, Burcu, David, Elahe, Febriyani, Federica, Floris, Guido, Imke, Inês, Janne, Joana, Jonathan, Kasia, Koen, Marian, Mihaela, Mohammad, Nana, and Sarah, thank you for your efforts and the pleasant cooperation. I hope that P-NUT will continue to organize fun events and to bring together the PhD candidates of this university. 
Obtaining my $\mathrm{PhD}$ would not have been possible without the support of my parents, Gerard and Annemieke. Together with my sister Linda, you have always been there for me when I needed you. I could not have wished for a better family.

Agata, moj kochanie, this preface would not be complete without thanking you. The time-consuming process of writing this thesis and the odd working hours in academics have not always been easy for you, but you supported me nevertheless. I am looking forward to build up a future with you and our daughter on the way. 



\section{Part I}

\section{Introduction}





\section{CHAPTER 1}

\section{Introduction}

In the near future, urban areas around the world will deal with a vastly increasing number of freight transport movements (Transmodal, 2012). Handling these freight flows is becoming increasingly difficult, causing major social, ecological and economic problems. The main objective of this thesis is to identify viable solution concepts for freight consolidation and coordination between supply chain actors, contributing to an efficient and sustainable urban logistics system. To this end, we develop a number of models and apply a variety of analytical techniques from the field of operations research.

In Section 1.1, we provide the motivation for our work. We describe the setup of the research in Section 1.2. Section 1.3 gives a literature overview of the main techniques and concepts used, a high-level overview of related literature, and the literature gap that we address with this work. Finally, in Section 1.4 we outline the structure of the thesis.

\subsection{Research motivation}

In this section, we describe the motivation for this research. We first highlight several trends and statistics in urban freight transport. Subsequently, we explain how these trends reduce the efficiency of freight transport, and as a consequence have a negative impact on urban areas themselves. We proceed with a high-level overview of solution concepts in the context of urban freight transport, and address various implementation issues associated with these solutions. We conclude the section by delineating the scope of our thesis, our main research objective, and the research questions that we address.

1.1.1. Trends and statistics in urban freight transport. There are various trends that contribute to an expected increase in the number of urban transport movements. In this section, we highlight the most far-reaching trends, which are expected to significantly impact the way the urban freight transport system will be shaped in the future (Transmodal, 2012; Ploos Van Amstel, 2015). 
Trends in urban logistics are not equivalent everywhere around the world; the scope of our work will be on European cities. European cities are often similarly structured, in the sense that they have gradually been constructed around medieval city centers. Typically, commercial and residential premises are strongly intertwined (Ambrosini and Routhier, 2004). European cities seek to preserve this layout, as it provides synergy between agreeable living conditions and economic activity. However, a consequence is that many European cities suffer from spatial scarcity.

Worldwide, a strong population growth is projected over the next decades (United Nations, 2014). The European population is expected to grow as well, but at a relatively slow rate. Figure 1.1 shows the historical population growth within the 28 current European Union member states, as well as projections of demographic developments (Eurostat, 2016). From an urban logistics perspective, changes in demography are more influential than the absolute population growth. Europe is facing an aging population; the share of people aged 65 years or older is expected to grow from $18.9 \%$ in 2015 to $28.1 \%$ in 2050 (see Figure 1.2). Many of the older people are eventually expected to move back from the suburbs to city centers, with stores and services abundantly available (Transmodal, 2012). By the year 2025, over three-quarters of the European citizens are expected to live in urban areas; this percentage may even rise to $84 \%$ in the year 2050 . With a large share of the people residing in urban areas, the demand for goods in these areas will increase as well.

The role of the retailer has been changing over time, for an important part due to the emergence of e-commerce. Whereas consumers used to purchase the bulk of their goods at retailers located in the city center, these shops now have to compete with highly efficient online stores. As a result, profit margins are under pressure. Retailers increasingly adopt Just-In-Time principles, meaning that they keep inventory levels at a minimum (Dablanc, 2011). The (expensive) floor space allocated to storage can therefore be reduced, and subsequently dedicated to sales as much as possible. The result is that retailers tend to order more frequently and in smaller quantities. An exemplary illustration of this development are high-frequency stores, which have very small amounts of each good on sale, and replenish these goods on an ad hoc basis. Furthermore, a resurrected interest in small-format stores can be witnessed; the demand for such stores is expected to reverse the trend of ever-expanding chains of large retail stores (Dablanc, 2007; Transmodal, 2012). It is evident that these changing order patterns will impact the operations of shippers and carriers. 


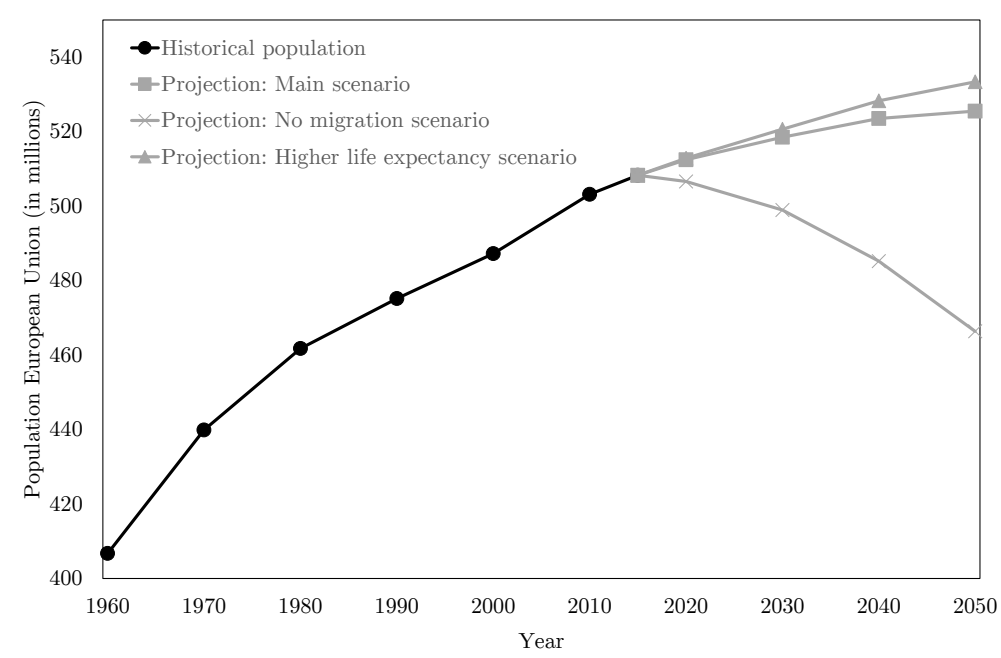

FIGURE 1.1. Eurostat projections of the population growth in European Union member states.

Another observable trend is the increase in service levels. Many customers expect delivery within 24 hours after placing an order. Companies like Amazon even started offering same-day delivery services (Hayashi et al., 2014). Furthermore, receivers - particularly end-consumers - have more freedom in selecting time slots and delivery locations. This makes it difficult to efficiently utilize vehicle capacity, as carriers need to deal simultaneously with increased shipment frequencies and decreased volumes (Boerkamps and van Binsbergen, 1999; Savelsbergh and Van Woensel, 2016).

A final development that we wish to stress is the diversification of freight flows. This trend is partially induced by the aforementioned rise of small-format stores. Besides the traditional flows from manufacturers to retailers, there are alternative freight flows that continue to grow. In the retail market, online purchases have displayed double-digit annual growth figures, with the market share of ecommerce rising from $7.2 \%$ in 2014 to $9.4 \%$ in 2016 (Centre for Retail Research, 2016). The presence of online sales platforms also enables customers to directly sell goods to each other; this form ofe-commerce keeps rising in popularity. Due 


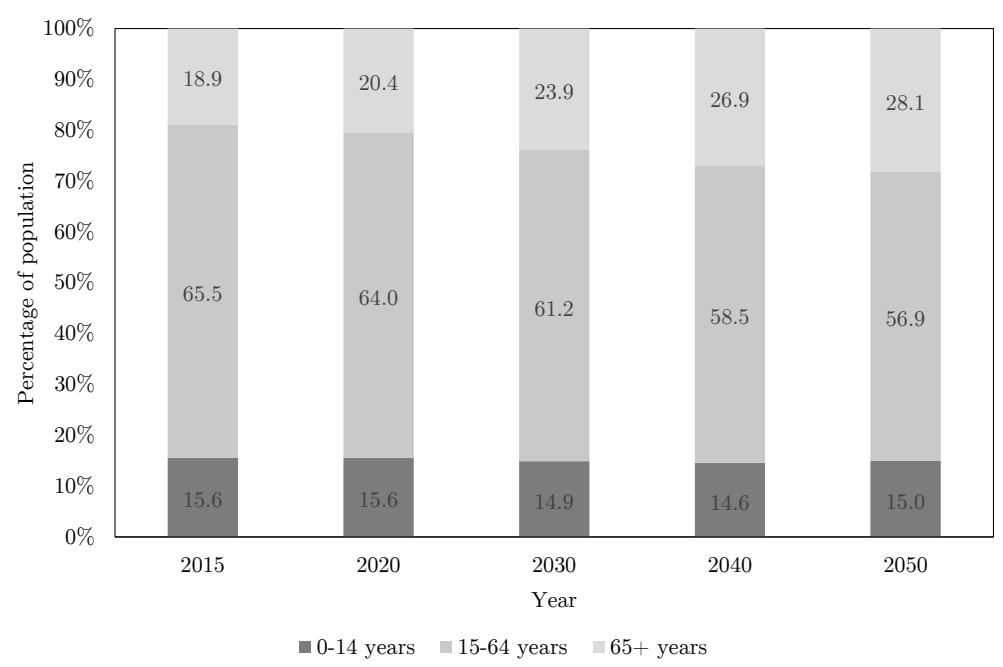

FIGURE 1.2. Eurostat projection of the age demography in European Union member states.

to these developments, many retailers nowadays feel the need to participate in e-commerce as well. Products may be delivered directly from the suppliers, but retailers may also sell goods held in their shop inventory via the internet (Hayashi et al., 2014). Furthermore, return streams are becoming more important. These streams entail, e.g., the returns of goods purchased online, but also services such as waste collection.

The aforementioned trends imply a drastic growth in the number of transport movements in urban areas, a development that could already be witnessed in recent years (Transmodal, 2012). The fragmentation of freight flows makes it increasingly difficult for carriers to efficiently perform last-mile distribution. Last-mile distribution is already recognized as the least efficient segment of supply chain logistics (Gevaers et al., 2011). Given the fact that $85 \%$ of the transport market is comprised by small freight carriers (Dablanc, 2011) - which often operate only a single truck - the inefficiency of last-mile distribution is only expected to worsen. Thus, major challenges are awaiting in the future of urban freight transport. 
1.1.2. Impact of freight transport in urban areas. Freight transport movements within urban areas contribute to a number of problems, which are only expected to increase in magnitude with the trends mentioned in the preceding section. These problems can be divided into three main categories (Quak, 2011; Macharis et al., 2014), which we discuss now.

First, excess freight transport has a negative impact from an economic perspective. Urban infrastructures often do not suffice to handle large amounts of freight transport. Congestion is a common problem in many cities. Furthermore, a lack of loading zones often forces trucks to double-park while supplying stores, thereby blocking the road for other vehicles. Congestion and blocked roads cause higher transport costs, which translates into lower profits. Also, an abundant number of trucks in the city centers reduces the attractiveness of cities, particularly in shopping areas (Ambrosini and Routhier, 2004). Finally, Europe has many historic city centers that are characterized by narrow streets and monumental structures. In these settings, the problems related to freight transport are amplified, as the system may easily be blocked by a single truck. Additionally, monumental structures may be damaged by the vibrations caused by heavy vehicles.

The second concern with urban freight transport is its environmental impact. Compared to cars, freight trucks generate disproportionally large amounts of hazardous particles. Partially, this is caused by the fact that the trucks used for urban distribution are generally older and more polluting than trucks operating over longer distances (Dablanc, 2007). Although freight transport comprises about $15 \%$ of the total traffic in urban areas, it is responsible for up to $50 \%$ of emissions (Dablanc, 2007). We particularly highlight the dangers of emission with local consequences, such as aerosols, volatile hydrocarbons, and smogcausing substances, which can affect both health and environment. Other environmental effects caused by freight transport are noise hindrance and vibrations. Also in this respect, trucks have a much larger impact than cars.

The last problem class that we distinguish is the social impact. In this category, we consider effects that reduce the quality of life for urban residents. One concern is the health impact of freight transport. The aforementioned emissions are known to have a hazardous impact on people's health. Also, a significant share of road accidents can be attributed to abundant amounts of freight transport (Van Essen et al., 2011). Furthermore, noise pollution negatively affects the daily lives of residents. In addition, the presence of trucks in itself may be perceived as a hindrance, e.g., due to blocking roads or hampering the accessibility of shopping areas. 
Many of the negative impacts of urban logistics are considered to be so-called 'external costs'. Although the impacts are known, they are typically difficult to accurately measure or to allocate to stakeholders. For example, it is clear that congestion has negative effects on the economy, the environment, and the society, but the translation into concrete financial metrics remains complicated.

It should be stressed that despite the negative effects, freight transport is a vital artery of the local economy. Goods that are consumed within urban areas are typically produced in other areas; transporting goods such that they can be consumed at the desired place and time is an essential need of a well-functioning economy. In addressing the negative effects of urban freight transport, one should therefore also keep in mind the tradeoff with the viability of the city as an economic entity.

1.1.3. Solution concepts. Both in academics and in practice, various solution concepts have been proposed and implemented to reduce the negative effects of urban freight transport. We provide a broad overview here, following the classification provided by Quak (2011) that distinguishes between four distinct classes of solution concepts. The first two classes require changing the existing infrastructure of the transport system, whereas the last two classes aim to reduce the impact of urban freight transport within the existing context of the transport system. Solution concepts may be implemented individually, but also in combination with each other, e.g., implementing regulation that supports company initiatives. A setting in which one or more concepts are combined is commonly known as an urban logistics scheme. Figure 1.3 gives an overview of these solution concepts, and show how they are linked to trends in urban freight transport and implementation challenges. In the next sections, we will discuss the elements of the figure.

1.1.3.1. Physical infrastructure. The first class of solution concepts is on the level of the physical infrastructure. This entails developing the proper infrastructure to reduce the impact of urban freight transport. For example, the local administrator might discourage the use of heavy trucks by placing obstacles on the road, or alternative dedicate special traffic lanes to environmental-friendly electric vehicles. A solution concept within the class of physical infrastructures that we pay special attention to is the urban consolidation center (UCC). An urban consolidation center is a transfer hub that is located in the proximity of an urban area, serving as a physical decoupling point in the transport flow (AIlen et al., 2012b). Inbound trucks no longer need to enter the city center, but can instead unload at the UCC. Subsequently, goods stemming from multiple 
carriers can be temporarily held and bundled at the UCC. This allows to construct more efficient delivery routes than the carriers could perform independently. Furthermore, the UCC can use dedicated delivery vehicles with a lower environmental impact. The potential benefits of UCCs are greatest when targeting independent carriers and receivers, which are typically associated with lowvolume, high-frequency deliveries. In addition, the UCC can provide a range of value-adding services for the receivers, such as waste collection and storage. For carriers, cost savings (the costs of last-mile distribution are disproportionally high (Gevaers et al., 2011)) and legislative restrictions are the main reasons to use a UCC. For receivers, poor accessibility by truck, lower receiving costs (due to bundled deliveries), and the offer of value-adding services are reasons to consider delivery via the UCC (Van Rooijen and Quak, 2010).

1.1.3.2. Reorganization of transport system. The second solution class entails the reorganization of the transport system, e.g., a switch from traditional truck transport to alternative modes such as electric bicycles or boats. Measures that require replacing existing resources are often capital-intensive, and require a long-term commitment of multiple parties. Alternative modes often have certain advantages over truck transport, but are unable to handle the complete distribution of urban freight. Hence, the operators of separate modes must work together in order to maintain an overall efficient distribution system. Often, a neutral party is introduced into the supply chain to coordinate the planning and financial exchanges between the actors.

1.1.3.3. Policy initiatives. We proceed to discuss the class of policy initiatives. On a high level, the legislation with respect to logistics is created by (supra)national governments. Within the boundaries set by legislation, local administrators apply specific measures for their own city or municipality. One option that they have is to impose hard restrictions to (i) prevent certain vehicle classes from entering the city, such as weight restrictions or engine requirements, or (ii) only allow certain vehicle classes in the city during designated access times (Russo and Comi, 2010). Implementations that dictate a minimum load factor exist as well. In addition to such restrictions, local administrators may implement measures that encourage carriers to adjust their behavior. Such measures include congestion charges, zone access fees, and parking costs. Although not enforcing carriers to alter their operations, these policies give them an incentive to operate more efficiently, entering the city at times during which traffic is limited, avoiding certain areas of the city, or outsourcing their last-mile distribution. On the other side of the spectrum, governments may financially support initiatives that aim to reduce the impact of urban freight transport, for 
example by providing subsidies to carriers that use electric vehicles. On an abstract level, the role of the administrator is to monetize external costs, and redistribute them in order to obtain better system-wide solutions.

1.1.3.4. Company-driven change. The final class of solution concepts that we discuss is company-driven change. The transportation sector is known to be highly competitive and subject to low profit margins (Dablanc, 2007). Companies are therefore interested in initiatives that improve efficiency, which in turn often reduces negative side-effects. This class is decomposed into technological advances, planning, and cooperation initiatives. We start with a description of technological advances. In general, trucks are becoming increasingly efficient in their fuel use, while emission filters also help to reduce the environmental impact of freight transport. Other improvements are continuously made with respect to noise reduction and safety concerns. Furthermore, electric vehicles and natural gas vehicles are becoming more and more technically viable alternatives to gas- and diesel powered trucks. Replacing the traditional fleet with such vehicles may significantly reduce the hazardous impact of freight transport, but currently such vehicles are considerably more expensive.

Companies can internally improve their performance by utilizing existing resources more efficiently. Novel planning algorithms, for example, can help to improve the efficiency of operations. Furthermore, companies may achieve higher efficiencies by cooperating with external parties, aiming to obtain synergies in a larger segment of the supply chain (Anderson et al., 2005). In this context, we distinguish between horizontal and vertical collaboration. In horizontal collaboration, companies that are active in the same stage of the supply chain cooperate. An example is a coalition formed by multiple carriers, such that they can bundle their freight and pool their vehicles to perform deliveries more efficiently. Vertical collaboration entails extensive coordination efforts with parties that are positioned upstream or downstream in the supply chain. An illustration is an improved information exchange between a carrier and the shippers, such that the carrier may anticipate future shipments in its schedule.

The improvements introduced by a company are often driven by developments in information technology. State-of-the art technology - such as GPS and RFID tags - present new opportunities to improve operational efficiency. It is now commonplace that companies possess detailed real-time information regarding vehicle locations, inventory levels, and capacity levels. Despite the availability of such information, there are still many business opportunities to put this information to good use. The capabilities to physically respond to changes in the system are currently not in line with the opportunities; companies might 
significantly improve their performance if they effectively use the abundance of available data.

While information technology can be used to improve the efficiency of individual business, it also opens doors for collaboration structures. This is perhaps best illustrated by the concept of the Physical Internet, which gives a potential preview of the logistics system of the future (Montreuil, 2011). In the Physical Internet, every transport order is accompanied with information specifying its transport requirements (e.g., origin, destination, time windows), yet within these requirements there is a high degree of flexibility. The progress of the transport order is monitored in real time. Similarly, the state of transportation units (e.g., vehicle allocation, departure time, remaining capacity) is dynamically updated. The transport orders are matched to available transport capacity as efficiently as possible, and allocations may be updated in real time based on the state of the system. Thus - similar to the digital internet - a parcel may be transported by multiple carriers and transshipped at intermediate hubs, as long as it arrives at the right destination at the desired time. Certain hubs function as entrance and exit nodes of the system, these hubs may correspond to the UCC (or smaller satellite facilities) in the context of urban logistics. The European Technology Platform ALICE has developed a road map, which is shown in Figure 1.4. It shows the required developments in separate areas (including urban logistics). The eventual objective is to have a seamlessly integrated logistics system by 2050 , in which urban logistics would no longer be considered as an isolated system. 


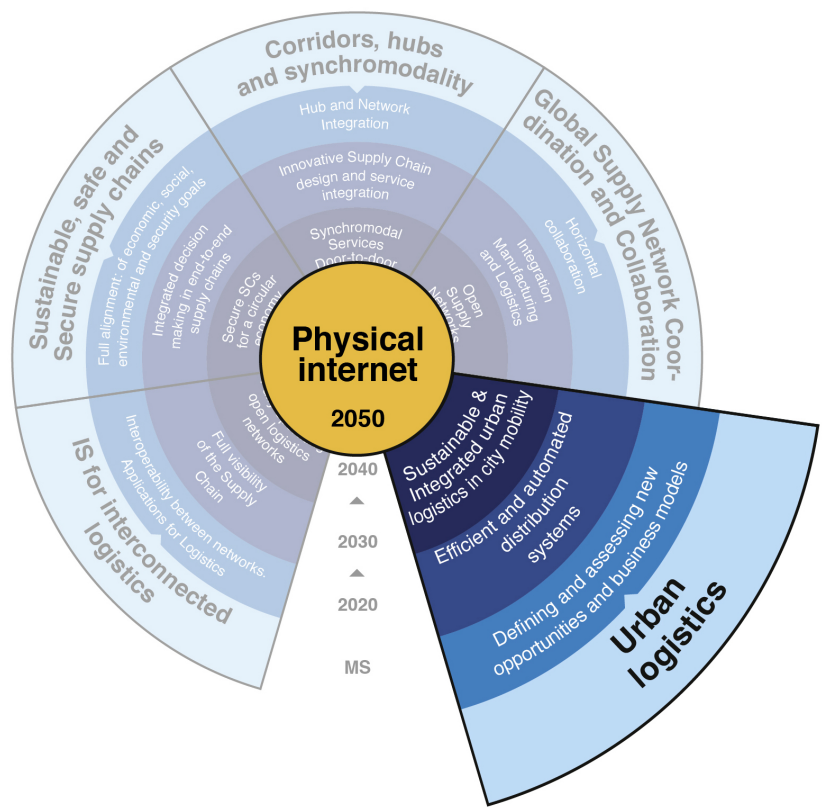

FIGURE 1.4. Roadmap for the development of the Physical Internet (source: European Technology Platform ALICE).

1.1.4. Implementation challenges. In Section 1.1, we described a number of solution concepts that have been proposed to improve the efficiency and combat the hazardous impacts of urban freight transport. Here, we discuss various challenges related to the implementation of such solutions.

On the policy side, governments and local administrators have to balance between the negative impacts of urban freight transport and the performance of the local economy Ambrosini and Routhier (2004). Particularly in periods of economic turmoil - such as the recent economic crisis - the economic objectives typically gain priority, leaving administrators with little room to address societal and environmental concerns. Budget cuts, legislative restrictions and lack of political will form major obstacles for local administrators to effectively address the problems caused by urban freight transport. Dablanc (2007) states that only very strict regulations may significantly impact the behavior of carriers, yet such regulations often violate constitutional principles. Finally, particularly 
for smaller cities, municipalities often lack the specific knowledge to properly analyze and implement measures.

Wiesenthal et al. (2011) list various barriers in the European transport sector that prevent transportation companies to successfully innovate and adopt new practices. A major barrier is that innovations in this sector typically require high capital expenditure, which is often problematic given the low profit margins in the industry. Also, the transportation sector is characterized by a relatively conservative mindset, with decision makers that are not known for quickly adopting innovative solutions. This mindset is reinforced by the complexity of the sector; innovations can seldom be accomplished without requiring extensive coordination efforts with external parties. Finally, due to the strong competition and high fragmentation in the sector, companies are reluctant to share any information that may benefit their competitors, thereby hampering the speed of development.

From the demand perspective, it is the receiver that normally initiates the transport flow by placing an order. However, after agreeing with the shipper on a delivery time, they are no longer involved in the logistics planning process (Verlinde et al., 2012). The shipper may subsequently deliver the order with its own distribution means, but usually a professional carrier is hired to conduct the transport. There is no direct contractual obligation between receiver and carrier. Although recent developments such as track-and-trace systems improve the communication between both parties, the contact between them is still largely restricted to the delivery moment. Inefficiencies on both sides are therefore often left unaddressed. In addition, transport costs are usually embedded in the order price, therefore receivers do not know how much an order delivery actually costs. As receivers have no insight in how their order patterns affect the transport flows - and hence have no financial incentive to alter these patterns it is challenging to make receivers change their behavior.

Urban consolidation centers are at the heart of many initiatives in city logistics, yet the concept did not make a breakthrough in practice. In an exhaustive overview of UCC initiatives, pilots, and feasibility studies, Browne et al. (2005) note that the success rate of UCCs is low. As an illustration, the authors report that out of 200 known UCCs in Germany, only 15 were still in operation at the time of the study. Furthermore, the economic viability of UCCs is being questioned (Muñuzuri et al., 2005; Verlinde et al., 2012; Janjevic et al., 2013). The required transshipment introduces additional costs. For a transshipment in a 
transport chain to be financially viable, these additional costs should be compensated by a reduction in the last-mile costs (Konings, 1996; Trip and Bontekoning, 2002). The high costs of the UCC are an obstacle that prevent its use for last-mile distribution, particularly because external costs are often not monetized. This obstacle is partially caused by the absence of generally established business models for UCCs (Allen et al., 2012b; Van Duin et al., 2016). Receivers may to some extent benefit from receiving bundled deliveries. However, as transport service costs are already embedded in the original order price (often implicitly) that is paid to the shipper, receivers often perceive the payment of additional transport costs to the UCC as an unnecessary expense. Also, shippers do not give a discount on transport costs when receivers list the UCC as their delivery address. Due to the unwillingness of receivers to pay additional transport costs, it is typically the carrier that incurs the full costs for the lastmile distribution. For carriers, the potential savings by outsourcing last-mile distribution are often outweighed by the costs of the UCC, making outsourcing an unappealing alternative (Janjevic et al., 2013). The UCC has the potential to reduce external costs, yet these benefits are primarily garnered by the residents. Thus, the imbalance in the allocation of (external) costs and gains is a key challenge for UCCs to overcome. Furthermore, both carriers and receivers often perceive a lack of added value from UCCs (Verlinde et al., 2012). Both parties are often satisfied with their existing transport arrangements, and do not see an urgent need to use the services of a UCC. It should also be noted that outsourcing the last-mile distribution rises issues such as contractual obligations, insurance, etc. (Gonzalez-Feliu, 2012). A final reason for failure is that UCCs tend to rely too much on subsidies (Browne et al., 2005; Kin et al., 2016). At the time when the UCC stops receiving financial support, they have often still failed to commit sufficient customers to reach the break-even point.

Arguably the biggest challenge in achieving sustainable solutions for urban freight transport is the fact that multiple stakeholders are involved. In most urban supply chains, one can distinguish between the roles of shippers, receivers, carriers, urban residents, and the local administrator (Macharis et al., 2014). It should be noted that a single entity can have multiple roles, e.g., a resident may also be a receiver and a shipper. The objectives corresponding to each role are typically divergent (Bektaș et al., 2015), which makes it challenging to find solutions that are beneficial to all actors involved.

We illustrate the difficulty to align objectives with an example. Night deliveries allow to distribute freight flows more evenly over time, alleviating the strain on the transport system during peak traffic hours. From the perspectives of the 
local administrator and carriers, this may be seen as beneficial. However, residents may complain about the noise levels at night, whereas the receivers may need to employ additional personnel to receive the goods outside opening hours. The conflicting arguments regarding night deliveries show how difficult it is to find solutions to which all parties are willing to commit.

Another discrepancy that we highlight is the definition of efficient routes (Verlinde et al., 2012). Suppose a truck visits customers in five different cities during a single route. From the perspective of the carrier, this may be an efficient route, being able to achieve a high load factor and minimizing travel distance. However, from the perspective of the city, it is undesirable that a heavy truck enters the city center to deliver only a relatively small volume. Again, we see how different objectives impact the perceived quality of a solution.

Diverging objectives may partially be overcome by re-allocating gains and costs over the actors; in particular local administrators can adopt the role of distributor. When finding a solution that yields system-wide gains, one might theoretically be able to allocate the gains and costs in such a way that all actors commit to a solution. This can for example be done by monetizing environmental and social costs, which can subsequently be divided over actors via freight transport pricing and subsidizing. However, it is not trivial to find a correct allocation that satisfies every actor.

To conclude, there are no clear-cut solutions to increase transport efficiency and tackle environmental and societal challenges. In particular, it is difficult to achieve environmental objectives without jeopardizing financial performance. Urban logistics takes place in a complex environment, in which multiple stakeholders pursue their own objectives. As local administrators only have limited ability and resources to address city logistics, implementing and enforcing strict regulation is typically not easily achievable. It is therefore imperative that solutions in urban freight transport are based on a solid business case that satisfies the objectives of all actors involved.

\subsection{Research setup}

In Section 1.1, we described the motivation of our research. In this section, we present the setup of our research. We demarcate our research scope, define the main objective of our thesis, and introduce the research questions that we address in this thesis.

1.2.1. Research scope and objective. This research is conducted as part of the CONCOORD project, which stands for Consolidation and Coordination. 
CONCOORD is a European research project, in which various academic institutes and industry partners collaborate. The overarching concept of the project is to stop considering separate transport flows in urban areas, and rather consider the transport system from a holistic point of view. Subsequently, we develop models and algorithms to optimize transport flows within the integrated system. This entails the pooling of orders, exchanging information, and sharing physical resources. The goals of the project are to identify ways to organize urban logistics in a more efficient manner and to reduce the negative societal and environmental impacts of urban freight transport.

Based on the objective of the project, we demarcate the research scope of our thesis. Various market sectors are distinguished within the domain of urban freight transport. On a high level, we may distinguish between retail, parcels and post, $\mathrm{HoReCa}$ (Hotels, Restaurants and Catering), construction, and waste collection (Transmodal, 2012). Each sector has vastly different logistic systems and faces unique business challenges. The contributions of this thesis link most strongly to the retail sector. The retail sector is suitable for our study on coordination and consolidation in urban logistics, as (i) retail orders often comprise small volumes, (ii) the relatively simple goods make sharing of resources more viable, and (iii) coordinating logistics structures can be designed for the long term. Parcel logistics revolves around small-volume deliveries, thus making this an interesting sector for consolidation studies as well. However, parcel logistics is often controlled by a small number of delivery services, which control dedicated hub-and-spoke networks that facilitate efficient transport. These networks function well due to economies of scale associated with the large total amounts of volume handled (Çetiner et al., 2010). Next, HoReCa logistics is for a large part composed of service logistics and food transport, therefore involving a lot of specific requirements that require tailored solutions (Verlinden et al., 2016). Construction logistics is often inefficiently organized, but typically revolves around temporary building projects, which makes this sector less suitable to study long-term solution concepts (Allen et al., 2012b). Finally, waste collection is inherently a pickup process, and often cannot be combined with the transport of other goods (Buhrkal et al., 2012).

Although we link to retail logistics in this thesis, the findings cannot be generalized to the entire retail sector. Many retail supply chains are already efficiently organized. Particularly larger retailers (e.g., retail chains) often have dedicated logistics systems; freight consolidation for these systems already takes place internally. We instead focus on the freight transport movements associated with small, independent receivers. Such receivers - which account for $30-40 \%$ of 
daily deliveries in urban areas (Transmodal, 2012) - are most vulnerable to the trends discussed earlier in this chapter. They typically have no direct control over their shipments, which is particularly challenging if they receive goods from multiple suppliers. Generally, they do not pay directly for transport, and do not interact with the carriers. The consequences are low vehicle fill rates and much time spent on receiving deliveries (Transmodal, 2012).

The optimization problem corresponding to the holistic view of the $\mathrm{CONCO}$ ORD project can be viewed as a resource-allocation problem, in which we seek to match the resources of the system as efficiently as possible to the jobs that need to be executed. Consequently, we focus on environments in which multiple actors participate that are willing to share their resources. In such an environment, there is a key role for high-level actors that facilitate and coordinate the collaboration between independent actors. This is often the task of a Logistics Service Provider (LSP). More specifically, we look at the role of a fourth party logistics service provider (4PL) as a central coordinator in the system. 4PLs do not own any transport resources or physical facilities themselves, but instead work together with a number of contracted carriers. This allows them to match orders and available vehicle capacity in an efficient manner, while maintaining a neutral position in the market. The second facilitating role that we highlight is that of the UCC; to efficiently bundle freight it should collaborate with both carriers and receivers.

Although the potential of pooling transport orders and resources can be readily seen from a system-wide perspective, such solutions are less obvious from the viewpoint of the individual actors. On a horizontal level, centralized solutions often require actors to cooperate with competitors. On a vertical level, actors typically have conflicting objectives, making it challenging to find solutions to which all actors in the supply chain are willing to commit. Furthermore, the coordinating parties (the 4PL and the UCC) require adequate solution methods to tackle the complex decision problems that they face in integrated logistics systems.

To address these problems, we define a research objective and a number of corresponding research questions. The main research objective of this thesis can be formulated as follows:

To develop mathematical models that support high-level actors in consolidated freight planning, and to obtain quantitative insights into the challenges and requirements to establish integrated logistics systems, in which independent actors coordinate their decisions to improve the efficiency of urban transport. 
1.2.2. Research questions. In the previous section, we defined the main research objective. In this thesis, we address this objective by answering a number of corresponding research questions. These research questions are formulated below.

1. How can we efficiently allocate dynamically arriving orders of fractional truckload size to transport resources that operate in a network with transfer hubs? We consider a 4PL that operates on a network with contracted carriers, and assume that orders of a known size arrive that are subject to pickup- and delivery times. Within the time constraints, we have the flexibility to schedule orders as efficiently as possible, using the opportunities to adjust departure times and to reload goods at transfer hubs. In Chapter 2, we develop a solution method to schedule orders of fractional truckload size, allowing to alter both the physical routes and the departure times of orders in response to consolidation opportunities that arise over time.

2. How can we efficiently plan the last-mile distribution of dynamically arriving orders at an urban consolidation center?

To answer this question, we assume that the urban consolidation center does not have perfect knowledge regarding future arrivals, but that there exists a stochastic model that represents the arrival process. Based on the existence of such a stochastic model, we develop a solution method which is described in Chapter 3. This solution method produces a consolidation policy that supports UCCs in their decision which subset of orders to dispatch at a decision moment.

3. How can we test the behavior and interaction of autonomous actors in urban supply chains under a variety of circumstances?

To analyze how autonomous actors make decisions under varying scenarios, and how this impacts the overall financial and environmental performance of the system, we develop an agent-based simulation framework in Chapter 4 . We define the KPIs, objective functions, and decisions for the different stakeholders that are active within urban supply chains.

4. What are abstract representations of urban supply chains for small retailers in Western European cities, which can be used to test the impact of interventions in urban freight transport?

We provide an abstract representation of a Western European city in Chapter 5. We obtain the properties of this abstract city based on a literature study, publicly 
available data sources, and expert interviews. In addition, we define various receiver profiles to reflect the large variety of retailers' order patterns in practice.

5. How is the urban freight system affected when combining various companydriven initiatives and government regulations?

To address this question, we make use of the simulation framework that we develop in Chapter 4 . Using the abstract representation of a Western European city as a test instance, in Chapter 5, we test scenarios that combine various companydriven initiatives and government regulations. In the same chapter, we measure their impacts on both the performance of the individual actors and the system as a whole, and evaluate which urban logistics schemes significantly reduce system-wide costs, while simultaneously generating commitment from the involved stakeholders.

6. What are the prospects for an urban consolidation center in a realistic setting? Again applying the simulation framework developed in Chapter 4, we perform a case study in Chapter 6 . We create a realistic test instance based on the city of Copenhagen. On this instance, we evaluate the prospects of a starting UCC under various subsidy schemes. Both the test instance and the experimental setup are validated by means of expert interviews.

In Chapter 7, we reflect on the individual research questions, and show how they together provide an answer to the main research objective. Furthermore, we provide various suggestions for future research that can help to expand this answer.

\subsection{Literature overview}

This section is divided into three parts. In the first part, we reflect on the literature related to our research questions. We restrict ourselves to a high-level overview in this chapter, while explicitly contrasting our work to existing literature on specific topics in the corresponding chapters. The second part describes the literature gaps that we address with this thesis. In the third part of this section, we provide a general overview of the operations research techniques and concepts that are used in this thesis.

1.3.1. Related literature. We discuss the main literature classes that are related to the work in this thesis. The first class is consolidation in networks with reloads, the second class is the delivery dispatching problem, and the third class is the evaluation of initiatives in urban freight transport. 
1.3.1.1. Consolidation in networks with reloads. The use of urban consolidation centers has a prominent role in this thesis. In a broader logistics perspective, a UCC can be viewed as a transfer hub. In essence, a transfer hub is a logistics facility where freight can be shifted to another modality (e.g., from a heavy truck to a delivery van), but it also facilitates the unloading and reloading of goods on the level of the individual vehicle, i.e., splitting or merging loads if this yields consolidation benefits (Crainic and Kim, 2006). The reloads apply, for example, when small loads delivered by several trucks are bundled into a single delivery van.

We can use networks with transfer hubs to transport Less-than-Truckload goods. We consider a setting in which the transfer hubs allow for the reload of goods and the transport means (which may be trucks, but also for example barges and trains) operating on this network may be subject to timetables. We classify the corresponding planning problem as a dynamic pickup and delivery problem with reloads and timetables. Literature on this problem is scarce. Bock (2010) describes a local search algorithm for the dynamic variant, focusing on real-time applications. Although providing a rich model, multiple modes operating on fixed routes and their corresponding timetables are not addressed. Ferrucci and Bock (2015) provide a tabu search heuristic that builds upon the framework provided by Bock (2010). Another heuristic solution for the dynamic problem is described by Goel (2010). His proposed solution evaluates routing and shipment decisions on a time-expanded network that includes multiple modalities and timetables.

1.3.1.2. Delivery dispatching problem. A key objective of a UCC is to dispatch freight as efficiently as possible, thereby minimizing both transport costs and environmental impact. Therefore, an important decision for UCCs is the timing of dispatching orders. In the literature, this decision problem is known as the Delivery Dispatching Problem (DDP). In the DDP, order arrivals follow a stochastic process and are dispatched in batches at a given decision moment (Minkoff, 1993). The aim of solving the DDP is to find a consolidation policy that returns the optimal dispatch time for an accumulated set of orders. With each new arrival, the decision maker assesses (i) the time elapsed since the first order in inventory arrived, and (ii) the volume of the accumulated orders. The consolidation policy is based on one or both of these measures. Dispatching the accumulated orders is subject to a cost function, in which route duration and route costs are pre-defined inputs (Minkoff, 1993). 
Relatively little work has been done on the optimization of consolidation policies in the DDP; most studies rather focus on evaluating existing consolidation policies (Çetinkaya, 2005; Mutlu et al., 2010; Bookbinder et al., 2011). Studies that do address optimization tend to consider only volume and arrival time as order properties, and present results that are valid only for a limited set of distributions. Çetinkaya and Bookbinder (2003) derive results on optimal policies that are either quantity-based or time-based, but do not combine both measures into a hybrid policy. Bookbinder et al. (2011) describe a generic solution method, which can handle several distributions. Finally, Cai et al. (2014) present an algorithm that returns an optimal consolidation policy for the DDP under a number of restrictions. Although some papers on optimal policies exist, they are generally valid only for a limited set of distributions, and run into computational difficulties when trying to solve large instances.

1.3.1.3. Evaluation of initiatives in urban freight transport. We explained that many urban logistics initiatives fail, in part due to a lack of analysis. Decision makers tend to implement measures without quantifying the results up front, and do not take into account how different types of stakeholders are affected (Bektass et al., 2015). Consequently, measures often do not have the impact that was intended.

Taniguchi et al. (2012) provide an overview of techniques that are used to evaluate initiatives in urban freight transport. Various manners exist to quantitatively analyze initiatives in urban freight transport. For now, we restrict ourselves to a brief assessment of agent-based simulation, which is considered the most suitable method to study the behavior of and interaction between the various stakeholders for urban logistics schemes (Taniguchi et al., 2014). Agent-based simulation is not fit to study detailed interactions (Bektaş et al., 2015), yet it is suitable to deduce generic insights into the system performance. A number of agent-based simulation studies in the context of urban logistics have been performed (Taniguchi and Van Der Heijden, 2000; Tamagawa et al., 2010; Van Duin et al., 2012; Wangapisit et al., 2014). They define various stakeholders in the urban areas, and test a number of measures, mainly governmental policies. The studies mentioned tend to focus on the simulation methodology, with the numerical component serving primarily as an illustration of the method. Furthermore, most of them run experiments on $5 \times 5$ grids, rather than more realistic urban networks.

1.3.2. Literature gap. With this thesis, we aim to address several gaps in the literature. These gaps, and the specific contributions that we make, are listed below. 
Chapter 2 contributes to the literature in two ways. First, we present an algorithm to schedule the transport of LTL freight via networks with reloads, thereby contributing to the limited number of dynamic planning studies in this field. We deviate from existing studies by explicitly focusing on heuristics that construct consolidated routes, rather than the more improvement-based heuristics typically encountered in literature. In addition, our problem description as a time-dependent intermodal network with reloads and timetables contributes to the formal definition of this problem class. Second, we present an efficient method to identify and plan consolidation opportunities in a delimited solution space, for which various parameters can be fixed to control the maximum computational effort.

In Chapter 3, we extend the Delivery Dispatching Problem (DDP) by embedding time windows into the problem formulation. The absence of time windows is a major shortcoming in the traditional DDP, as they are an integral component of real-life logistics. The inclusion of time windows significantly enriches the problem; the UCC operator must decide which subset of orders to dispatch at a given decision moment. Our extension to the DDP provides a better fit with real-life consolidation problems, allowing for applications to dispatching problems in general. We contribute to existing literature by (i) formulating a Markov decision model to capture the dynamic and stochastic nature of the DDP with time windows, and (ii) designing an algorithm that can handle large instances of this problem class.

Contrasted to existing agent-based simulation models in urban logistics, the contribution of the evaluation framework that we introduce in Chapter 4 is twofold. First, we take into account the transport process outside the city. As lastmile distribution accounts for only part of the transport process, a narrow perspective does not properly assess the decisions made by shippers and carriers. Second, we explicitly include various forms of cooperation between companies, while existing studies tend to have a strong focus on testing governmental policies. As practice shows that successful schemes require both policies and commitment from companies, a framework including both aspects is essential for proper evaluation of these schemes.

The main literature contribution of Chapter 5 is that a variety of schemes is discussed. We referred to a number of studies that tested various measures, yet these studies are applied to specific instances and test only a small number of measures. For these reasons, it is difficult to derive general conclusions from these studies. We overcome this difficulty by (i) designing abstract representations of urban supply chains based on the literature and expert interviews, (ii) 
testing a large number of scenarios which combine various measures, and (iii) performing sensitivity analysis on the results.

Finally, Chapter 6 contributes to the literature with quantitative insights into urban logistics schemes. In contrast to Chapter 5, this chapter has a more narrow scope, and focuses explicitly on the viability of a UCC. To obtain results that are credible to real-life UCCs, we strive for a high degree of realism in our case. The test instance is inspired by the city of Copenhagen; we use an actual UCC location and real retailer locations, and work with the corresponding street network. Data from the literature, as well as real UCC data, are used to reflect the properties of the urban supply chains. Again, we conduct expert interviews to validate both the setup of the study and the case data.

1.3.3. Operations research techniques and concepts. This thesis draws upon various techniques from the field of operations research to solve a number of optimization problems. Here, we give a brief overview of the main concepts and techniques that are applied.

1.3.3.1. Graph theory. Commonly, transport problems are represented by means of a graph. In such a graph, the nodes represent physical locations, such as pickup- and delivery locations or urban consolidation centers. The nodes are connected by a set of arcs. A basic graph contains only travel durations or travel distances between the nodes, yet much more properties can be attached to both the nodes and arcs. In the context of urban logistics, such properties could be, e.g., transshipment costs, zone access fees to the city center, or emission functions. In dynamic applications, the properties of a graph may change over time, for example when considering multiple scheduled vehicle departures on the same line-haul service. Such dynamics can be represented by either timedependent graphs or time-expanded graphs (West et al., 2001; Köhler et al., 2002; Ding et al., 2008).

1.3.3.2. Mathematical programming. Mathematical programming is an optimization technique widely applied in the field of logistics. Particularly common in the field of transportation are linear programs (LPs) and integer linear programs (ILPs). In an (I)LP, we formulate an objective function with a number of decision variables and a set of constraints that must be satisfied. Such a model yields a solution space in the form of a polyhedron. With advanced solvers, LPs can be solved in a highly efficient manner; other variants of mathematical programs pose more computational challenges (Dantzig, 1998; Williams, 2013).

1.3.3.3. Markov decision models. Markov decision models are well-versed to represent transportation problems with dynamic and stochastic properties. In a Markov decision model, a set of decision moments is defined in discrete 
time; these moments are separated by time intervals. At each decision moment, the system is in a certain problem state, in which a number of actions can be taken. Each action yields a direct reward (or cost). Furthermore, new random information arrives at the decision moments. A prerequisite for the application of Markov decision models is that the memoryless property is satisfied. If this is the case, then the transition from the current to the future problem state does not depend on events that occurred in the past. If this property holds, the future state depends on the current state, the action that is taken, and the random information process. This implies that future rewards are subject to randomness, however - if we know the transition probabilities - we can compute expected future values. By taking into account all possible actions and outcomes corresponding to a problem state, the expected value of a given state at a given point in time can be computed. Consequently, it is possible to calculate the optimal policy by backwards induction. Using this notion, Markov decision models can be solved to optimality with mathematical programming or dynamic programming (Bertsekas, 2005; Puterman, 2014).

1.3.3.4. Approximate dynamic programming. When considering problem instances of realistic sizes, Markov decision models often suffer from computational intractability. In fact, the size of the decision problem typically grows exponentially with respect to the instance size in three areas: the state space, the outcome space, and the action space. Approximate dynamic programming is a modeling framework that enables to solve stochastic optimization problems of realistic sizes, combining techniques from dynamic programming, mathematical programming, simulation, and statistics. It does not guarantee finding optimal policies, but instead aims to return close-to-optimal policies for problem instances that cannot be solved exactly within reasonable time (Si, 2004; Powell, 2011).

1.3.3.5. Heuristics. A heuristic is a systematic method to create and/or improve a solution for an optimization problem. As we generally deal with problems that are too large to solve to optimality, heuristics are an integral part of our solution concepts. Heuristics do not guarantee an optimal solution, but often provide good or close-to-optimal solutions within limited computational time (Lenstra, 1997; Winston and Goldberg, 2004; Campbell and Savelsbergh, 2004; Bräysy and Gendreau, 2005).

1.3.3.6. Discrete-event simulation. Discrete-event simulation is a framework used to model the functioning of a system as a discrete sequence of events in time. The model contains a finite number of decision moments, which are separated by discrete time steps. At every decision moment the system is in a given 
problem state, based upon which a number of actions can be taken. Discreteevent simulation generates a sequence of random events; the combination of the current state, the action that is taken, and the event that occurs, the transition from the current state to the state at the next decision moment is determined. In discrete-event models, the system does not change in between decision moments. Under this assumption, after making a decision we can instantaneously jump to the next decision moment, rather than performing a simulation in continuous time. To ensure statistic validity of the model, events are typically generated randomly. The performance of decision-making policies is evaluated by perform a sufficiently high number of simulation runs. (Jerry et al., 2005; Law, 2007).

1.3.3.7. Agent-based simulation. A special form of discrete-event simulation that we highlight is agent-based simulation. Rather than assuming the role of a single decision maker, we explicitly model multiple decision makers. Each decision maker is represented by a so-called 'agent', which is an artificial representation of a real-life actor. Every agent aims to optimize the value of its own objective function, which depends on both its own actions and the current and future states of the system. The latter at least partially depends on the decisions of other agents. Hence, we can observe how the system functions under a variety of scenarios, and how agents interact with each other. Agents may differ in their degree of intelligence in decision making. Reactive agents simply apply the same fixed rule on the prevailing state of the system, whereas learning agents may alter their decisions based on their past and forecasted results. Agent-based simulation can be used either to analyze the behavior of actors in existing systems, or to optimize a problem via the agent intelligence (Gilbert, 2008; Niazi and Hussain, 2011).

1.3.3.8. Solution methods for the Vehicle Routing Problem. After representing the transportation problem in the form of a graph, routing algorithms can be applied to construct routes that serve all customers. In order to minimize costs, the carrier wants to construct routes that are as efficient as possible. The classical example of this problem is the Traveling Salesman Problem (TSP), which is a restricted version of the Vehicle Routing Problem (VRP). In the TSP, a single truck is dispatched from a depot, and must visit every customer before returning to the depot. The objective is to find a solution that visits all customers and travels the minimum distance or completes the route in minimum time.

When generalizing the TSP to a problem in which multiple vehicles may be dispatched, we obtain the VRP. This introduces an additional dimension to the problem, as it also requires a decision on which vehicle visits which subset of 
customers. The VRP is arguably the best studied problem in the transport literature, and many different variants have been studied. Variants that are typical for urban settings include, e.g., time-based and weight-based access restrictions, delivery time windows, emission objectives, and congestion effects.

We distinguish between two branches of research within the VRP community. One branch studies exact solution methods. Exact solutions generally rely on mathematical programming or dynamic programming. Nowadays, fairly large instances can be solved to optimality within reasonable computational time. The other branch of VRP research is dedicated to heuristics. Heuristic solutions allocate customers to vehicles based on a ourset of rules. We can distinguish between constructive heuristics and improvement heuristics. Constructive heuristics generate an initial solution, whereas improvement heuristics seek to transform existing solutions to improve their quality. Despite the advances made in the development of exact solution methods, heuristic solutions are still commonplace in practice, particularly in real-time applications (Laporte, 1992; Crainic and Laporte, 1997; Golden et al., 2008).

\subsection{Structure of the thesis}

This thesis is divided into four parts. The outline of the thesis is presented in Figure 1.5. This chapter belongs to Part I, and provides the motivation, context and scope of our research.

Part II contains two chapters in which we address challenges faced in the context of an integrated logistics system. These chapters mainly focus on the operations research methodology, describing and solving optimization problems that are faced by the coordinating parties in the supply chain. The decision processes of carriers and shippers start well outside the city boundaries, and the upstream decisions may have a significant impact on the last-mile delivery. It is therefore essential to gain insights into the decisions made on the line-haul as well; carriers typically make the bulk of their costs and profit on the line-haul. We start by analyzing consolidation of less-than-truckload orders on theline-haul in Chapter 2. We consider the decision process of a 4PL, which assigns dynamically arriving orders to a specific set of modes and corresponding departure times. Transport units can be transshipped at transfer hubs that connect the arcs. Also, the hubs facilitate reloading freight from one transport unit to the other. For this setting, we develop a planning algorithm, which we test for a variety of network configurations. Next, in Chapter 3, we study the last-mile dispatch decision from the perspective of the UCC. We consider a UCC at which orders arrive according 
to a random arrival process. The UCC faces the decisions which orders to dispatch and which orders to hold to consolidate more efficiently at a later point in time. We formally define this problem as a Markov decision model, and solve realistic-sized instances by introducing a solution method based on Approximate Dynamic Programming.

In Part III, we introduce an agent-based simulation framework, which we subsequently use to perform numerical experiments. Chapter 4 introduces the framework itself, in which we integrate the decision problems of multiple actors. We introduce the various agent types that interact in the context of urban logistics, and define their objective functions and KPIs. Also, we discuss the techniques that can be applied to solve the optimization problems faced by the actors. In our assessment, we distinguish between the physical streams, the information streams, and the monetary streams that occur between the actors. Subsequently, we apply this simulation framework in Chapter 5. We design representative virtual cities based on data obtained from the literature, and test a variety of both government measures and company-driven initiatives on these instances. We also apply the simulation framework to a setting with an additional layer of realism, based on the city of Copenhagen. This study is described in Chapter 6. We use real network data obtained from OpenStreetMap. We study a starting UCC, for which both receivers and carriers can periodically decide to opt-in or opt-out for its services. The local administrator facilitates the UCC. The goal is to find a subsidy scheme that allows the UCC to operate in a self-sustained way after a limited period of time.

Finally, in Part IV, Chapter 7, we present our main conclusions. First, we reflect on the individual research objectives. Subsequently, based on the results obtained from the simulation studies, we formulate generic recommendations for practice. Finally, we provide some directions for future research, as well as a brief outlook on the urban logistics system of the future. 
Developments in urban freight transport

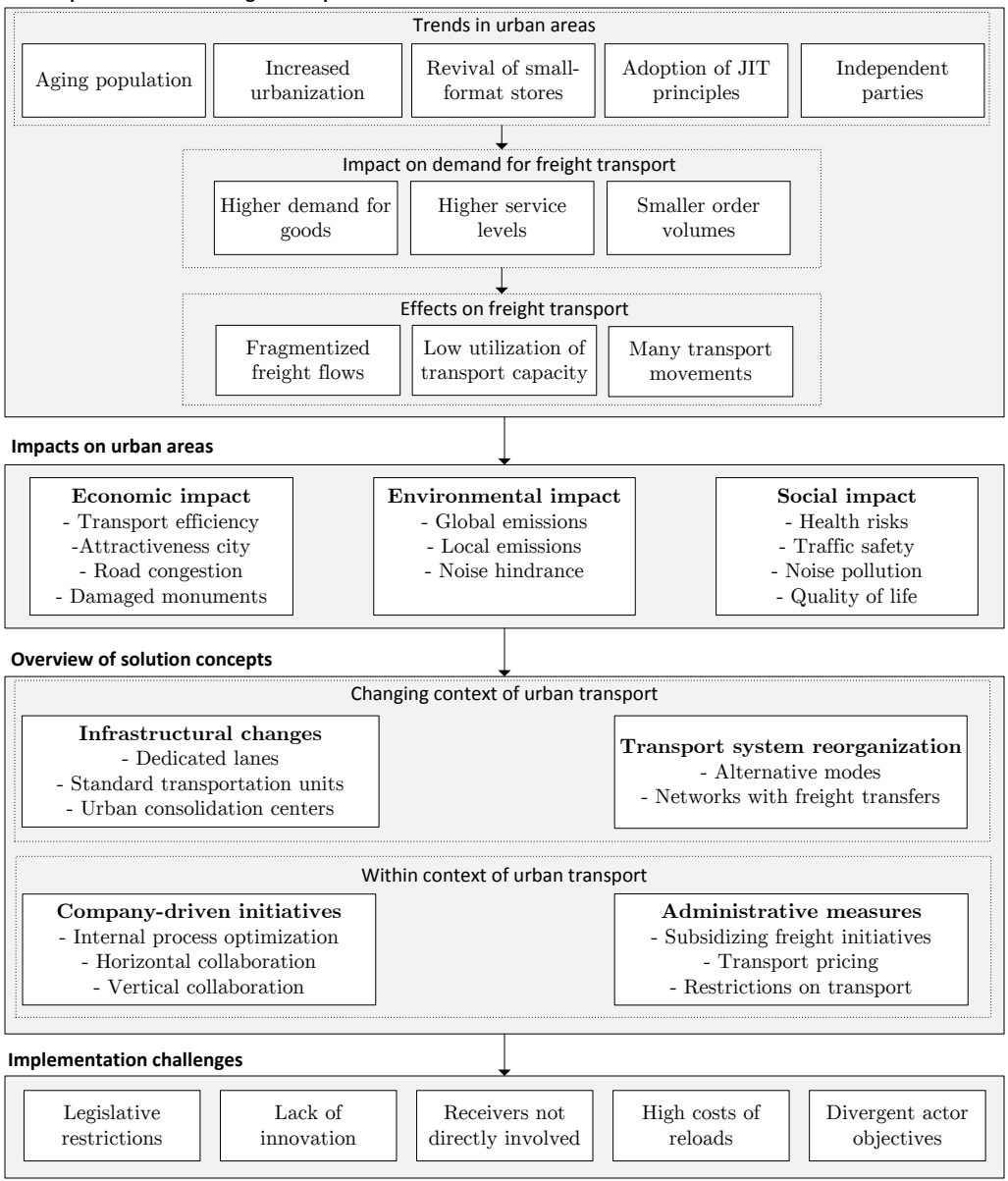

FIGURE 1.3. Summary of developments, impacts, solution concepts and implementation challenges in the context of urban freight transport. 


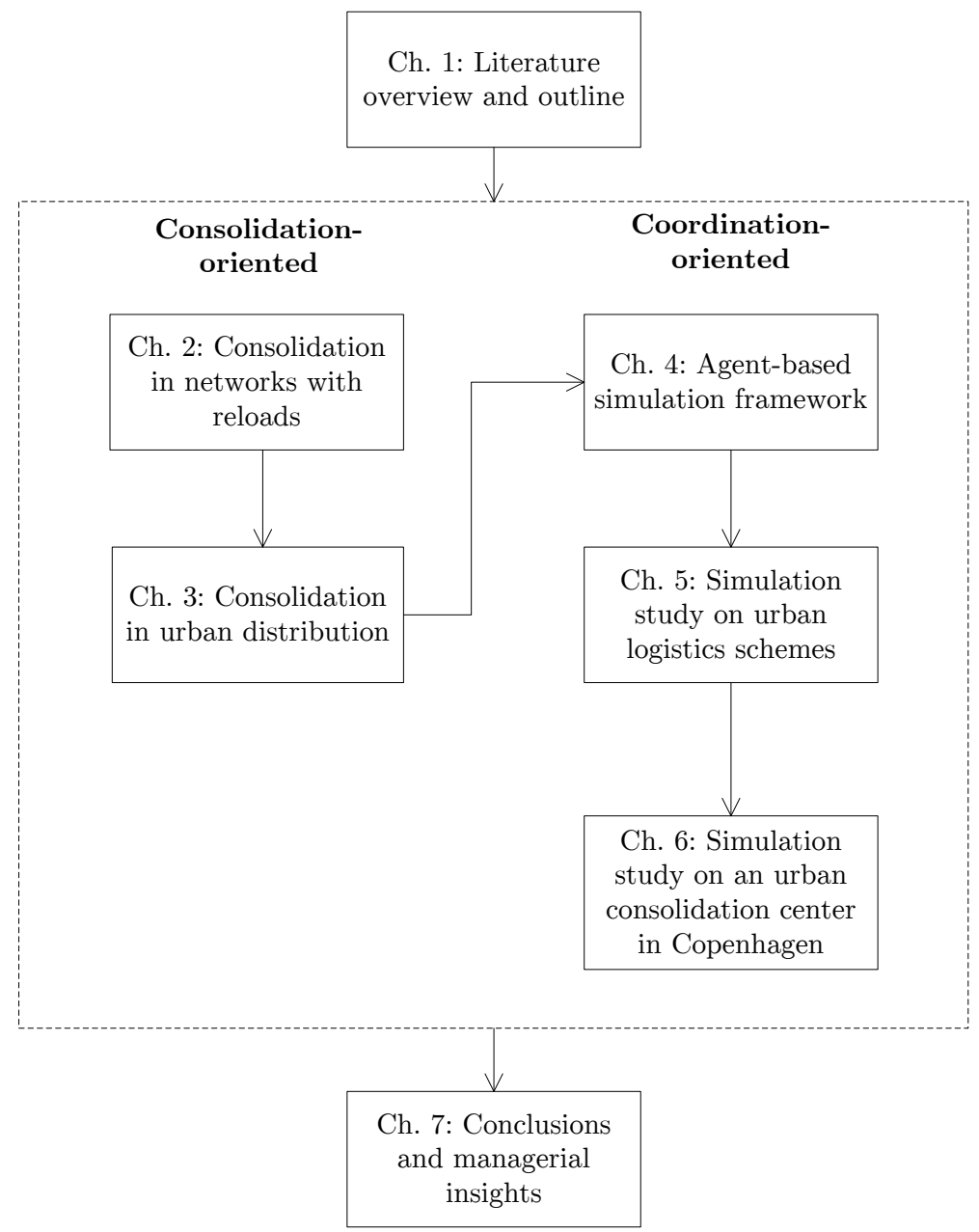

FIGURE 1.5. Thesis outline. 



\section{Part II}

\section{Consolidated planning methods}





\section{CHAPTER 2}

\section{Planning orders in networks with reloads}

In this chapter! we provide insights in the consolidation of orders in networks with reloads. Studies on urban logistics often tend to focus solely on the last-mile distribution of goods, yet orders are generally transported to the city from a remote location. The efficiency of transport within urban areas is our primary research interest, but it should be viewed within the context of a larger logistics system. Although the costs for last-mile distribution per kilometer are higher than those on the line-haul (Gevaers et al., 2011), the cost proportion for carriers on the last-mile is often modest in comparison with the line-haul costs, due to the much larger distance covered on the latter. Transport planners therefore primarily aim to achieve high capacity utilization on the line-haul. In this chapter, we therefore study the decision process on the line-haul, given a dynamic order arrival process. We present a solution method for a coordinating $4 \mathrm{PL}$; this agent aims to transport orders as efficiently as possible, using a network of contracted carriers. This chapter discusses how coordination can be used to efficiently consolidate small freight flows, and embeds the last-mile distribution in a supply chain perspective.

The chapter is structured as follows. First, we present a problem introduction and demarcation of our scope in Section 2.1. We provide a literature review in Section 2.2, assessing studies on several topics that are relevant to our work. Section 2.3 outlines the problem setting, followed by a description of the consolidation algorithm in Section 2.4. We conduct numerical experiments on several networks, measuring the performance of the algorithm - both with respect to costs and emissions - under a variety of circumstances (Section 2.5). Section2.6 closes the chapter with the main conclusions.

\footnotetext{
${ }^{1}$ This chapter is based on the paper W.J.A. van Heeswijk, M.R.K. Mes, J.M.J. Schutten \& W.H.M. Zijm (2016). Freight consolidation in intermodal networks with reloads. Flexible Services and Manufacturing. (Van Heeswijk et al., 2016b)
} 


\subsection{Introduction}

Various forms of networks with reloads exist. As mentioned, a network with reloads can link the urban consolidation centers of a variety of cities, using the reload opportunities at the centers to ensure efficiency both on the line-haul and within the urban areas. Furthermore, on the scale of an individual city, one might envision a one-tier or two-tier network, linking large distribution centers at the city limits to smaller satellite facilities in close proximity to or within the city center. Especially for large cities or metropolitan areas, such a structure is relevant. Also, networks with reloads are applicable to intermodal transport, where route segments are operated by various transport modes such as trucks, barges, and trains. Reload operations are performed at the hubs connecting these modes.

In this chapter, we will use the case of intermodal transport as an illustration for our solution method. First, the concepts of timetabling and reloads in this form of transport are more advanced than in road transport, thus resulting in a closer fit with current practice. Second, the transport modes significantly vary in terms of travel speeds and emission, thereby yielding a more interesting analysis of the impacts of consolidation decisions. Finally, we have a real data set available for intermodal transport, which allows us to better validate the solution method.

Intermodal transport is becoming an increasingly important alternative to road transport. Developments in real-time information provisioning, planning systems, governmental regulation, and more consideration of external costs (e.g., emissions, congestion, noise hindrance), make intermodal transport both increasingly relevant and competitive. In a typical intermodal setting, a load is placed in a container (or another transportation unit, such as a trailer), picked up by a truck at the customer, then transported via one or more railroad/waterway connections that are linked by transfer hubs, and finally distributed by truck towards its destination location. The transfer hubs allow for transshipments of containers from one modality to another, but also facilitate the unloading and reloading of goods on the level of the individual container, i.e., splitting or merging loads if this yields consolidation benefits (Crainic and Kim, 2006). Although intermodal networks are traditionally associated with transporting full containers, the use of transfer hubs that allow for reload operations could be regarded as a step towards the Physical Internet (Montreuil, 2011). Reload operations enable to create a dynamic transport network, via which we can transport small volumes by allocating them to the large load capacities of existing transport flows. 
From an environmental perspective, the potential benefits of increasing the share of intermodal transport is twofold. First, the additional flexibility offered by such networks allows for more efficient transport, thus requiring less transport movements. Second, barges and trains have distinct advantages over trucks in terms of emissions. A shift from road transport to other transport modes may significantly reduce emissions and other external costs (Janic, 2007). However, for fractional container loads, it is challenging to integrate these modes with road transport while maintaining high container fill rates. To be a financially viable alternative for direct road transport, the detour- and transshipment costs of intermodal transport have to be compensated by a reduction in transport costs (Konings, 1996; Trip and Bontekoning, 2002). For networks with reloads, consolidating orders in a container results in lower transport costs and allows to divide the transshipment costs per container over multiple loads, but at the expense of additional reload costs.

We consider the case in which the logistics coordination in an intermodal setting is in the hands of a $4 \mathrm{PL}$ service provider, i.e., a logistics service provider that matches orders to the transport services of external carriers. The 4PL matches incoming orders - specified by a volume, a pickup location, a delivery location, and a corresponding time window - to contracted carriers. In an intermodal setting, the 4PL can construct routes between a pickup location and a delivery location by combining various route segments (arcs) that connect transfer hubs, each arc being operated by a separate vehicle. We distinguish between two sorts of arcs. Main arcs are fixed connections between two hubs, and are operated by a specific type of modality (in this chapter either barge, train, or truck). These modalities may operate according to a timetable. Free arcs are connections between any two points in the network, and are operated by trucks that can be commissioned at any desired time. The key distinction between both types is that there exist regular transport flows on main arcs, while transport on free arcs must be rented for the individual order.

Although intermodal networks have the potential to significantly improve transport efficiency, attention for consolidation in networks with reloads remains limited. This chapter aims to contribute to this knowledge domain, more specifically, to the operational planning of consolidated routes in a dynamic environment. We present a scheduling algorithm that is able to efficiently identify and plan consolidated routes for networks with reloads, and might serve as a decision support system for $4 \mathrm{PL}$ planners. The $4 \mathrm{PL}$ can take into account multiple criteria to evaluate routes, e.g., costs, $\mathrm{CO}_{2}$ emissions, and duration. As the transport market is characterized by strong competition and low profit margins, cost 
minimization remains the leading objective for most 4PLs. However, the resulting efficiency gains themselves significantly contribute to a reduction in $\mathrm{CO}_{2}$ emissions; we measure this reduction in the numerical experiments. We illustrate the algorithm using a case study at a leading 4PL based in the Netherlands, which is active in the European transport market. In this chapter, we apply the algorithm on intermodal networks in which containers are placed on and lifted from the transport modes at transfer hubs. For the sake of completeness, we note that many transport modes are designed to carry wheeled cargo. Therefore, lifting operations are often not required in waterway transport and railroad transport. As the associated transshipment costs are lower and transshipment times are shorter, intermodal transport becomes a more attractive alternative in this case. We therefore see applications of the consolidation algorithm in this context as well.

\subsection{Literature on consolidation in intermodal transport}

In this chapter, we consider the consolidation of goods in an intermodal transport setting. Intermodal transport is formally defined as the transport of goods via at least two arcs that are operated by distinct transport means, with transshipments taking place at transfer hubs (Dewitt and Clinger, 2000; Crainic and Kim, 2006). Examples of such hubs are rail yards, harbors, and inland terminals (Groothedde et al., 2005; Bektaş and Crainic, 2008). A common feature in intermodal transport is the use of fixed timetables, particularly in railroad- and waterway transport (Macharis and Bontekoning, 2004). Despite the increasing number of applications of intermodal networks, serious knowledge deficits exist in the research fields of multi-modality, network design, and operational planning (Veenstra and Zuidwijk, 2010).

Macharis and Bontekoning (2004), Caris et al. (2008), and SteadieSeifi et al. (2014) provide overviews of intermodal transport research, and all indicate that only few studies have been performed on operational planning. Additional insights can be obtained from the conceptually related ride-sharing problem. Some problems in this field also consider the fixed schedules of public transport and transfers between different transport means. An overview on the dynamic ride-sharing problem can be found in Agatz et al. (2012).

Before assessing the studies that relate to our problem, we refer to a number of studies that provide building blocks to construct routes in an intermodal setting. In a full truckload setting, Boardman et al. (1997) use a $k$-shortest path algorithm to perform intermodal planning, evaluating multiple routes before selecting the route that best fits the established criteria. By coupling a vector 
of path length estimates to each vertex and using these estimates as a criterion on whether to evaluate a route, the authors keep computational time limited. Ziliaskopoulos and Wardell (2000) explicitly take into account timetables and transfer times, making use of Bellman's optimality principle while recursively updating time windows for every route segment. Horn (2004) provides an arcexpansion approach for the problem with timetables. He starts by generating routes consisting of only a single arc, and gradually increases the number of arcs in a route while simultaneously establishing upper bounds. He states that this procedure generally leads to an optimal schedule quickly: good routes tend to contain only few arcs, as this keeps detour distance and transshipment costs limited. Mes and Iacob (2016) use a comparable arc-extension approach, generating a large number of routes, and storing the $k$ best routes as suitable planning options.

To incorporate timetables in operational planning problems on a graph, we distinguish between two approaches. The first is to create a time-expanded graph, where each arc is characterized by a time component (Ford Jr and Fulkerson, 1958; Köhler et al., 2002). A separate arc is generated for each departure time in the timetable, allowing to include specific properties such as the realized fill rate on the vehicle. The major drawback of a time-expanded graph is that it can become very large, although this disadvantage is partially mitigated by the need to consider the graph only over a limited time interval. Furthermore, potentially many geographically identical routes are generated, differing only in their departure times. The alternative approach is to consider a time-dependent graph (Ding et al., 2008; Delling and Wagner, 2009). The graph itself is then defined only in space; time-dependent arc characteristics are taken into account by applying a function with the departure time as input.

We classify our problem as a dynamic pickup and delivery problem with reloads and timetables. Amongst others, Oertel (2000), Chang (2008) and Masson et al. (2013) address the static variant of this problem. Due to the complexity of the problem, heuristics are used to solve it, mainly utilizing local search principles. Literature on the dynamic variant of the problem is scarce. Bock (2010) describes a local search algorithm for the dynamic variant, focusing on realtime applications. Although providing a rich model, different modes operating on fixed routes and their corresponding timetables are not addressed. Ferrucci and Bock (2015) provide a tabu search heuristic that builds upon the framework provided by Bock (2010). Another heuristic solution for the dynamic problem is described by Goel (2010). The proposed solution evaluates routing and shipment decisions on a time-expanded network that includes multiple modalities 
and timetables. However, the consolidation role of transfer hubs is explicitly left out of consideration.

Our contribution to the intermodal planning literature is twofold. First, we present an algorithm to schedule the transport of LTL freight via intermodal networks, thereby contributing to the limited number of dynamic planning studies in this field. In particular, we deviate from existing studies by explicitly focusing on constructing consolidated routes, rather than the more improvement-based heuristics typically encountered in literature. In addition, we believe that our problem description as a time-dependent intermodal network with reloads and timetables contributes to the formal definition of this problem class. Second, we present an efficient method to identify and plan consolidation opportunities in a delimited solution space, for which various parameters can be fixed to control the computational effort.

\subsection{Problem formulation}

We study a dynamic planning problem with reloads and timetables. Orders are tentatively planned once they become known. Our choice to consider dynamic planning rather than static optimization (over a rolling horizon) is motivated by two arguments encountered in practice. First, the time between pickup and delivery in an intermodal setting spans several days, and volumes with the same order-destination pairs are low. When modalities with low travel speed or low departure frequencies are considered, a delay of the actual planning moment might result in exclusion of routes with longer duration. This would eliminate certain opportunities for consolidation. Second, we design the algorithm to be used as a decision support tool for planners at a $4 \mathrm{PL}$, allowing to directly reply to customers requests while retaining flexibility to consolidate with future orders. For these reasons, we directly plan orders when the shipper poses a request, while retaining the opportunity to alter the plan at a later stage.

Our representation of the intermodal network is as follows. Let $\mathcal{G}=\{\mathcal{V}, \mathcal{A}\}$ be a directed graph with $\mathcal{V}$ the set of vertices and $\mathcal{A}$ the set of arcs. $\mathcal{V}^{H} \subset \mathcal{V}$ represents the set of transfer hubs in the network. The remaining vertices signify the subset of order origins, $\mathcal{V}^{O} \subset \mathcal{V} \backslash \mathcal{V}^{H}$, and the subset of order destinations, $\mathcal{V}^{D} \subset \mathcal{V} \backslash \mathcal{V}^{H}$. The set of arcs can be divided into a set of free $\operatorname{arcs} \mathcal{A}^{F} \subset \mathcal{A}$ and a set of main $\operatorname{arcs} \mathcal{A}^{M}=\mathcal{A} \backslash \mathcal{A}^{F}$. Every vertex pair in the graph is connected by a free arc, and operated by vehicles without time schedules. Free arcs can therefore be used to link origins and destinations either directly to each other or to transfer hubs, but may also facilitate transport between hubs. The main arcs connect fixed vertex pairs of the subset $\mathcal{V}^{H}$, and possibly operate on a timetable. 
A single hub pair can be connected by multiple arcs, representing the availability of various modalities or carriers.

Each $\operatorname{arc} a \in \mathcal{A}$ is described by a tuple $\left(v_{a}, v_{a}^{\prime}, \tau_{a}^{t r}\right)$, where $v_{a} \in \mathcal{V}$ is the starting vertex of the arc, $v_{a}^{\prime} \in \mathcal{V}$ is the end vertex of the arc, and $\tau_{a}^{t r} \in \mathbb{R}^{+}$is the deterministic transport time (based on the transport means operating the arc). An arc connects exactly one vertex pair, meaning that containers using this arc are not reloaded or transshipped at intermediate hubs. Every arc has a corresponding volume-dependent transport cost function $C_{a}^{t r}: l \mapsto \mathbb{R}^{+}$, with $l$ being the order volume. We define a transshipment as moving a container from one mode to another. We use $c_{v}^{t m}$ to denote the costs of placing a container on a mode at hub $v$ at the start of the arc, and $c_{v^{\prime}}^{t m}$ to denote the costs of lifting the container off the mode at the end of the arc. We split the transshipment costs in two components to ease the cost allocation to individual orders, since a reload may take place between lifting and placing the container. We define a reload as unloading orders from one container and subsequently loading them into another container; the corresponding reload costs at hub $v$ are given by $c_{v}^{r l}$. Finally, $\tau_{v}^{t m}$ denotes the transshipment time at hub $v \in \mathcal{V}^{H}$.

Let $i \in \mathbb{N}$ and $j \in \mathbb{N}$ be order indicators, with order $i$ representing an order that just became known, and order $j$ an open order (i.e., an order that has not yet started the last main arc of its route). Each order $i$ can be described by a tuple $\left(v_{i}^{O}, v_{i}^{D}, t_{i}^{\min }, t_{i}^{\max }, l_{i}\right)$. Here, $v_{i}^{O} \in \mathcal{V}^{O}$ is the origin vertex and $v_{i}^{D} \in \mathcal{V}^{D}$ the destination vertex. The order has an earliest pickup time $t_{i}^{\text {min }}$; from this time onwards the order can be retrieved from the origin $v_{i}^{O}$. Furthermore, it has a strict deadline $t_{i}^{\max }$; the order must arrive at its destination $v_{i}^{D}$ at or before this time. Finally, we define the set of feasible order sizes - expressed in terms of fractional container capacity - as $\mathcal{L}=\left\{\frac{1}{y}, \frac{2}{y}, \ldots, 1\right\}$, with integer $y \geq 1$. The size of order $i$ is $l_{i} \in \mathcal{L}$.

We make a number of key assumptions in the definition of our problem. First, we assume that consolidation is only possible on the main arcs. Free arcs generally correspond to first-mile and last-mile transport; typically a 4PL will outsource the task of constructing detailed routes to the carrier. Our second assumption is that carriers utilize a concave and monotonically increasing cost function with respect to volume, reflecting economies of scale. This assumption is in line with general practice, and retains a large degree of flexibility. Third, we assume that all travel times and transshipment times are deterministic. Transshipment times are independent of whether reloads take place; in practice the time required for a reload is often minor compared to the full transshipment time. A fourth assumption is that reload costs are independent of the volume 
moved; a fixed fee is charged for every reload operation. Also, we assume that reload costs are independent of the time the orders are held at the transfer hub, i.e., no holding costs are charged. Fifth, as we consider hard time windows, we assume that for every order a time-feasible route exists. Sixth, we ignore capacity constraints on the main arcs. We do this because we adopt the perspective of an individual 4PL; the volumes handled by such actors typically only marginally contribute to the total container capacity on trains and barges. However, we note that this assumption can be relaxed in our problem, by simply removing the departure of a completely filled mode from the timetable.

\subsection{Consolidation algorithm}

In this section, we describe the consolidation algorithm. First, we briefly describe our solution method in Section 2.4.1. Subsequently, we outline the three key steps of the algorithm: planning $k$ individual routes for an incoming order (Section 2.4.2), constructing a decision tree with all consolidation actions (Section 2.4.3), and finally evaluating and implementing consolidation actions (Section 2.4.4).

2.4.1. Solution method. We briefly explain the general idea behind the consolidation algorithm. The algorithm is triggered by every new order arrival. First, a large preset number of unconsolidated routes is generated for the new order, of which the $k$ best routes are stored. To generate the initial routes, we use an arc expansion approach comparable to Horn (2004) and Mes and Iacob (2016). The key benefits of this method are that it is efficient, takes into account the cost structure of routes (small numbers of arcs generally yield the cheapest routes), and its computational effort can be controlled. To incorporate timetables in the model, we make use of a time-dependent graph, considering the case in which the departure time is the only varying component (hence, travel times and costs remain constant). Time-expanded graphs may require a large value for $k$ in order to maintain sufficient diversity in the routes, although the unique arcs would allow for quicker evaluation. In a time-expanded graph, consolidation opportunities could already be assessed during route generation, i.e., the costs on an arc may be conditioned on its current fill rate. However, the time-dependent graph allows for a more generic approach towards the planning problem. Particularly when departures are frequent, the time-dependent graph provides a more compact representation than the time-expanded graph. The main reason for choosing time-depending graphs is that the departure windows allow for flexibility in the departure time even after consolidating. To realize this, we apply a mapping function which generates a set of feasible departure times based 
on the corresponding timetable, if such a table exists. The mapping function only needs to be applied when mutual arcs with overlapping departure windows are identified. Such an evaluation is somewhat more complex than for a timeexpanded graph, but requires to evaluate each physical arc only once.

After obtaining the $k$ best routes, we construct an evaluation set for every main arc in each of these routes that include all consolidation opportunities on the arc. A consolidation opportunity for a given order exists if a route corresponding to an open order (or set of orders) has (i) the same main arc incorporated in its route as the route of the currentorder, (ii) a mutually feasible departure time on the arc, and (iii) sufficient capacity in the container to add the new order. To assess the consolidation opportunities on the various main arcs in conjunction, we construct a decision tree to compute the achievable savings and check whether the consolidation action yields a time-feasible schedule for all orders involved. Note that the opportunity refers to consolidation on a single arc, whereas the action refers to a unique combination of opportunities. Consolidation divides the fixed transshipment costs per container over the consolidated orders and typically reduces transport costs, but also introduces reload costs. The consolidation savings should compensate for using routes that are more expensive from the perspective of the individual order.

After consolidating, we fix the routes of orders that are consolidated on at least one arc of their respective routes, and update the departure schedules and container fill rates. We fix the routes because (i) if not, replanning routes may undo existing consolidation actions, therefore potentially requiring to re-solve the static problem at every order arrival, (ii) it significantly reduces the solution space, and (iii) it requires to change the selection of modes at most once, which is preferable in most practical settings. Although we geographically fix routes after consolidation, further consolidation and the adjustment of departure windows remains possible on these routes. The main structure of the algorithm is shown in the flowchart in Figure 2.1. Throughout this section, we use pseudo-code to describe the algorithm. We use a running example to illustrate its working.

2.4.2. Generating $k$ routes for an incoming order. For each incoming order $i$, we start by generating routes using a $k$-shortest path procedure similar to Mes and lacob (2016), based on iteratively expanding the number of main arcs in a route. Free arcs are added to ensure the route connects the order origin to its destination. As good routes generally contain only a few main arcs, we expect to identify these routes and their costs early in the process. Storing alternative routes with higher initial costs than the best individual route provides more consolidation opportunities. However, for these routes to be economically feasible, 


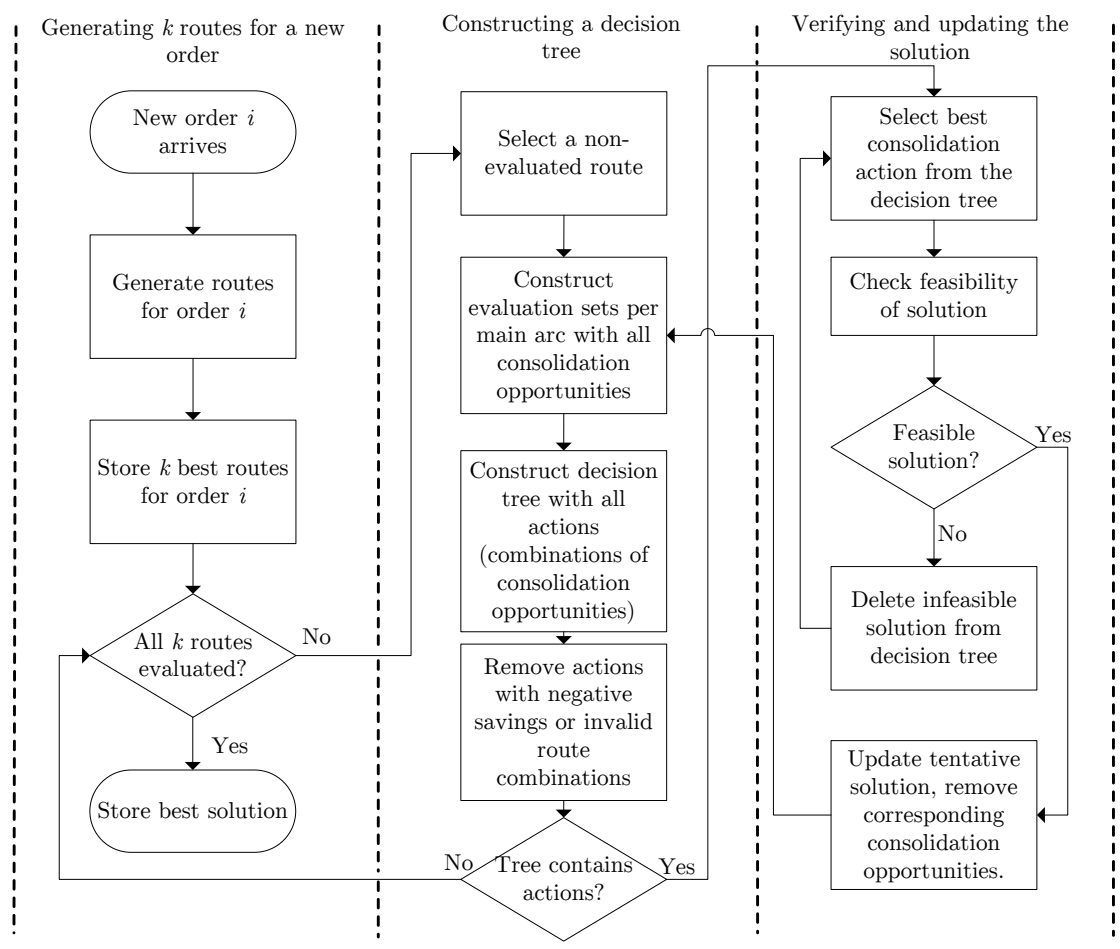

FIGURE 2.1. Flowchart with the main steps of the consolidation algorithm. The algorithmic procedure for each column is outlined in the subsequent sections.

their higher costs should be compensated by the consolidation savings. Thus, we want to exclude routes that are so expensive that the savings are unlikely to outweigh the additional costs. For this purpose, we introduce a factor $\beta>1$. To set a dynamic threshold, we multiply $\beta$ with the cost of the cheapest route found so far; we do not store a route if its costs exceed the threshold. Hence, we should set $\beta$ such that we can reasonably expect a route to contribute to improving solutions (e.g., $\beta=1.2$ implies no route is allowed to be over $20 \%$ more expensive than the best route). Also for partially constructed routes, we can use $\beta$ to check whether we can discard them, thereby considerably speeding up the procedure. In Theorem 2.1 (Appendix 2A), we provide a theoretical upper bound on $\beta$ that 
guarantees that the optimal solution can be found within the routes having costs lower than the threshold resulting from $\beta$. Finally, we also discard routes that are not time-feasible.

After generating a large number of routes, we store the $k$ least expensive routes for the individual order. The order is tentatively scheduled to follow the least expensive route. Timetables may result in slack in the schedule; departure policies - setting a tentative departure time - determine where in the schedule this slack will be allocated. The chosen departure policy impacts the consolidation opportunities on the route, given that we continue to seek for consolidation opportunities while the route is being executed (Powell, 1987).

To formally describe the properties of a route, we introduce some mathematical notation. Let $R_{i}$ denote the set of all $k$ stored routes for order $i$. A route $r_{i, n} \in R_{i}$, with $n \in\{1, \ldots, k\}$, is an ordered set of arcs - both main arcs and free $\operatorname{arcs}$ - connecting $v_{i}^{O}$ to $v_{i}^{D}$. We define an indicator $z \in\left\{1,2, \ldots,\left|r_{i, n}\right|\right\}$ to specify the arc sequence of a route. We refer to the $z^{\text {th }}$ arc incorporated in $r_{i, n}$ as $a_{i, n, z} \in r_{i, n}$; a route is formally defined by the ordered set $r_{i, n}=\bigcup_{z=1}^{\left|r_{i, n}\right|} a_{i, n, z}$. Each arc $a_{i, n, z} \in r_{i, n}$ has an associated departure window $\left[t_{i, n, z}^{e d}, t_{i, n, z}^{l d}\right]$; departing at any point in time within this window ensures that the destination can be reached before the delivery deadline. Let $\mathcal{T}_{i, n, z}$ be the set of feasible departure times on $\operatorname{arc} a_{i, n, z}$. We define a function $T_{a}$ that maps the departure window to a set of feasible departure times, i.e., $T_{a}:\left(t_{i, n, z}^{e d}, t_{i, n, z}^{l d}\right) \mapsto \mathcal{T}_{i, n, z}$. If no timetable exists for the arc (e.g., a free arc), every departure time within the window is feasible, otherwise $\mathcal{T}_{i, n, z}$ contains a finite number of departure times. Finally, we introduce a number of counters used in the algorithm: $M$ is the number of main arcs in a route, $M^{\max }$ the maximum number of main arcs that we allow in a route, \#Routes $M$ a loop variable for the number of routes containing $M$ main arcs, and \#Routes ${ }_{M}^{\max }$ the maximum number of routes that we generate containing $M$ main arcs. We describe the route-generating procedure in Algorithm 2.1. For each number of main arcs, we generate the specified number of routes. We select the required number of main arcs; the connections from the origin to the first main arc and from the last main arc to the destination are obtained by adding free legs. If the main arcs do not connect to each other, free arcs are added to connect these as well. We keep track of the cheapest route found so far; routes with costs that exceed the costs of the cheapest route by more than a factor $\beta$ are discarded. From all the routes generated, we store the $k$ routes with the lowest costs. In Algorithm 2.2, we calculate the departure windows for all arcs; the same algorithm is also used to update the departure windows after 
consolidation. In the latter case, we can input a mutually feasible departure window $\left[\hat{t}_{i, n, z}^{e d}, \hat{t}_{i, n, z}^{l d}\right]$ of two orders to synchronize their departure windows. The algorithm recursively computes the feasible departure times based on the departure times, travel times, and handling times at the adjacent arcs.

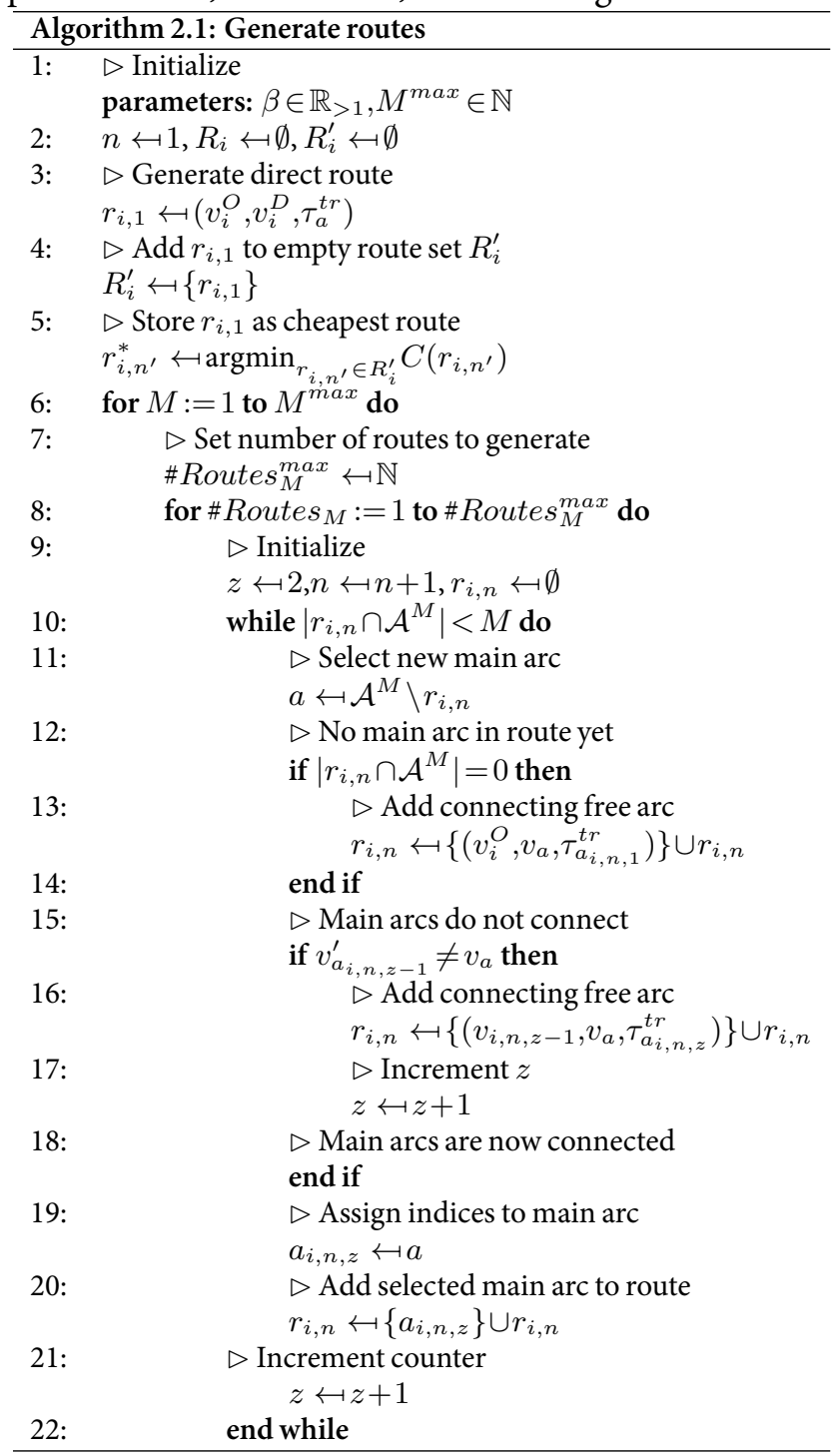



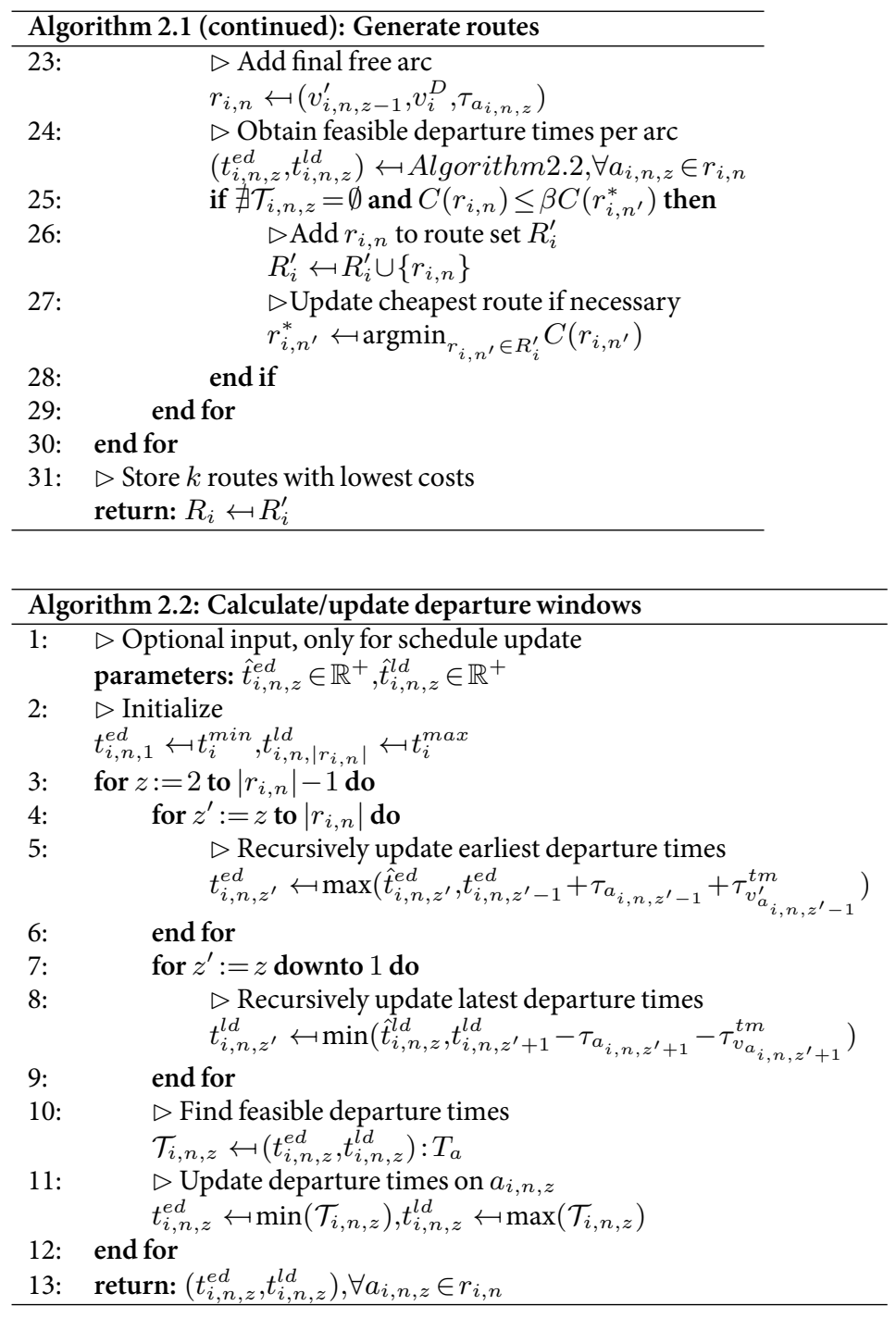

\section{Example 1: Route structure for incoming orders}

Consider the network as described in Figure 2.2. where order 1 has a load size $f_{1}=0.3$, pickup location 1 , and delivery location $1^{\prime}$. Suppose $k=4$. We denote each route $r_{1, n} \in R_{i}$ as an ordered set of arcs; every free arc is referred to as 0 . In 
this example, suppose that route $r_{1,2}$ is the least expensive solution, such that this route is set as the initial best solution.

TABLE 2.1. Characteristics and $k$ routes for order 1.

\begin{tabular}{cc}
\hline Order & 1 \\
\hline$f_{1}$ & 0.3 \\
\hline$r_{1,1}$ & 0 \\
$r_{1,2}$ & $\mathbf{0 - 1 - 0}$ \\
$r_{1,3}$ & $0-1-2-0$ \\
$r_{1,4}$ & $0-1-3-0$ \\
\hline
\end{tabular}

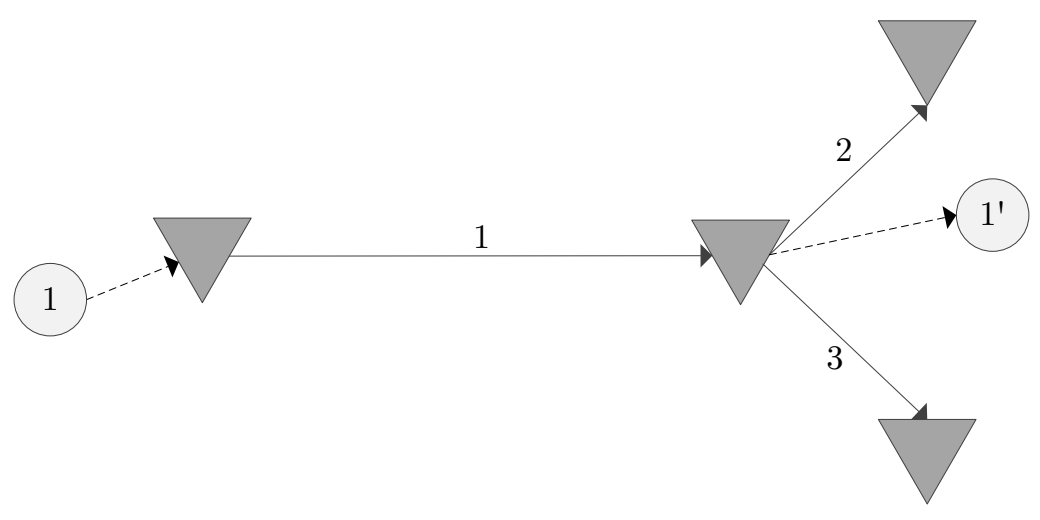

FIGURE 2.2. Initial best solution for order 1, utilizing only main arc 1.

Order 1 has an earliest pickup time of 11.40 and a latest delivery time of 20.00 on the same day. Assume that vehicles traverse main arc 1 with an hourly departure, that traversing this arc takes two hours, and that the reload time is always half an hour. In Figure 2.3, the corresponding time schedule for the route is provided, given a latest departure policy. In this figure, window 1 indicates the time interval in which departure on main arc 1 yields a feasible schedule. 


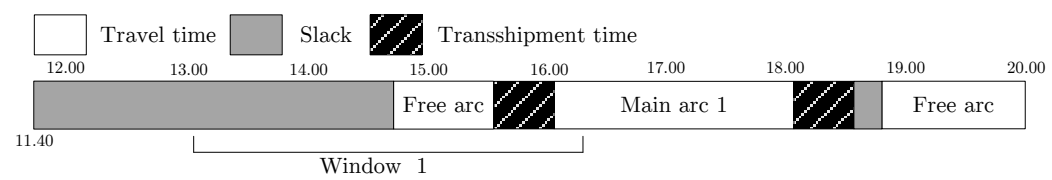

FIGURE 2.3. Gantt chart for route $r_{1,2}$ under a latest departure policy; window 1 indicates the feasible departure window.

2.4.3. Construction of a decision tree. After we have generated and stored $k$ routes for the new order $i$, we look for consolidation opportunities to reduce the system-wide costs. Let $j \in \mathbb{N}$ refer to an open order, i.e., an order that has not reached its destination yet. Let $\mathcal{J}$ be the set of all open orders, excluding the current order $i$. For an open order $j$, we use the route index $m \in\{1, \ldots, k\}$. Consolidation opportunities are arcs found in both $r_{i, n} \in R_{i}$ and $r_{j, m} \in R_{j}, \forall j \in \mathcal{J}$, for which (i) a mutually feasible departure time exists and (ii) container capacity is sufficient to consolidate the orders. We note that order $j$ may already have been consolidated with other orders at a preceding decision moment.

To retain flexibility and account for possible future consolidation opportunities, we assign orders to a container to be transported via the main arc, rather than to a transport means departing at a fixed time. Let $q, q^{\prime} \in \mathbb{N}, q \neq q^{\prime}$ be container indices. We define a consolidation opportunity $\Omega_{q, a}$ as the set of orderand route indices representing the orders (and their corresponding routes) currently assigned to container $q$ on arc $a$. We may always decide not to consolidate on an arc; we define the corresponding opportunity as $\Omega_{q, a}=\{(0,0)\}$. The remaining feasible consolidation opportunities with order $j$ on arc $a$ in route $m$ are denoted by $\Omega_{q, a} \ni(j, m)$. We also keep track of the orders that are consolidated with order $i$ on arc $a$ in route $n$ during the consolidation process; we describe the corresponding set as $\hat{\Omega}_{q^{\prime}, a}$. Before consolidating on route $n$, we have $\hat{\Omega}_{q^{\prime}, a}=\{(i, n)\}$. After a consolidation action $x$ takes place - described in more detail later on - we update the set of consolidated orders and obtain $\hat{\Omega}_{q, a}^{x}=\hat{\Omega}_{q^{\prime}, a} \cup \Omega_{q, a} ;$ subsequently we update $\hat{\Omega}_{q^{\prime}, a}$ and $\Omega_{q, a}$ as well. Note that we move goods from container $q^{\prime}$ to $q$, requiring a reload.

After we confirm that consolidating order sets $\hat{\Omega}_{q^{\prime}, a}$ and $\Omega_{q, a_{i, n, z}}$ is feasible, we can store $\Omega_{q, a_{i, n, z}}$ in the evaluation set $\mathcal{S}_{i, n, z}$. As the opportunity to not consolidate always exists, it is guaranteed that $\left|\mathcal{S}_{i, n, z}\right| \geq 1$. To keep the evaluation 
set as small as possible, we can use the upper bound on attainable savings as described in Theorem 2.2. A set $\Omega_{q, a} \ni(j, m)$ is not included in $\mathcal{S}_{i, n, z}$ if this bound is negative. When route $r_{i, n}$ contains multiple main arcs, we might consolidate with distinct order sets on the various main arcs. We define the action space for route $r_{i, n}$ as $\mathcal{X}_{i, n}=\prod_{z=1}^{\left|r_{i, n}\right|} \mathcal{S}_{i, n, z}$.

A consolidation action $x \in \mathcal{X}_{i, n}$ represents a unique combination of consolidation opportunities, i.e., $x=\bigcup_{z=1}^{\left|r_{i, n}\right|} \Omega_{q, a_{i, n, z}}$. We assess all actions to (i) compute their corresponding savings (reload costs can only be computed when assessing consolidation on all main arcs simultaneously) and (ii) check whether actions are time-feasible (as consolidation actions alter the departure windows of the entire schedule). To this end, we construct a decision tree for every $r_{i, n} \in R_{i}$, with every path representing an action. As we show in Theorem 2.3, the number of actions may increase exponentially with the number of main arcs in the route. In a practical setting, routes rarely contain more than two main arcs, keeping the decision tree at a well-manageable size. We assess consolidation opportunities per main arc with one order set $\Omega_{q, a}$ at a time; simultaneously evaluating all possible combinations of orders may result in an unfeasibly large decision tree. Instead, after selecting the best action from the tree, we update the order sets and departure windows, and use them as input to construct a new decision tree. We repeat this procedure until no more feasible cost-saving actions can be found. In Algorithm 2.3, we outline the procedure to create evaluation sets and decision trees.

The costs of a route $r_{i, n} \in R_{i}$ of order $i$ - taking into account possible consolidation actions - consist of three components, (i) the transport costs $C^{t r}(i, n)$, (ii) the transshipment costs $C^{t m}(i, n)$, and (iii) the reload costs $C^{r l}(i, n)$. We give detailed descriptions of these functions in Appendix 2B; for a general understanding of the algorithm we only need the aggregate cost function $C(i, n)=$ $C^{t r}(i, n)+C^{t m}(i, n)+C^{r l}(i, n)$. In the experiments, we also demonstrate a multi-objective cost function, in which we monetize emissions, delays, and hub risk (representing, e.g., missed connections and damage during handling). This extension shows how planners may embed non-financial objectives into the cost function.

The savings $S(x)$ for an action $x \in \mathcal{X}_{i, n}$ are given by the difference between the total costs before and after consolidation. Savings are composed of (i) the reduction in transport cost, plus (ii) the reduction in transshipment costs, minus (iii) the additional reload costs, minus (iv) the cost differences between the best routes and the evaluated routes of the orders involved. We provide the formal definition of the savings $S(x)$ in Appendix 2B. 


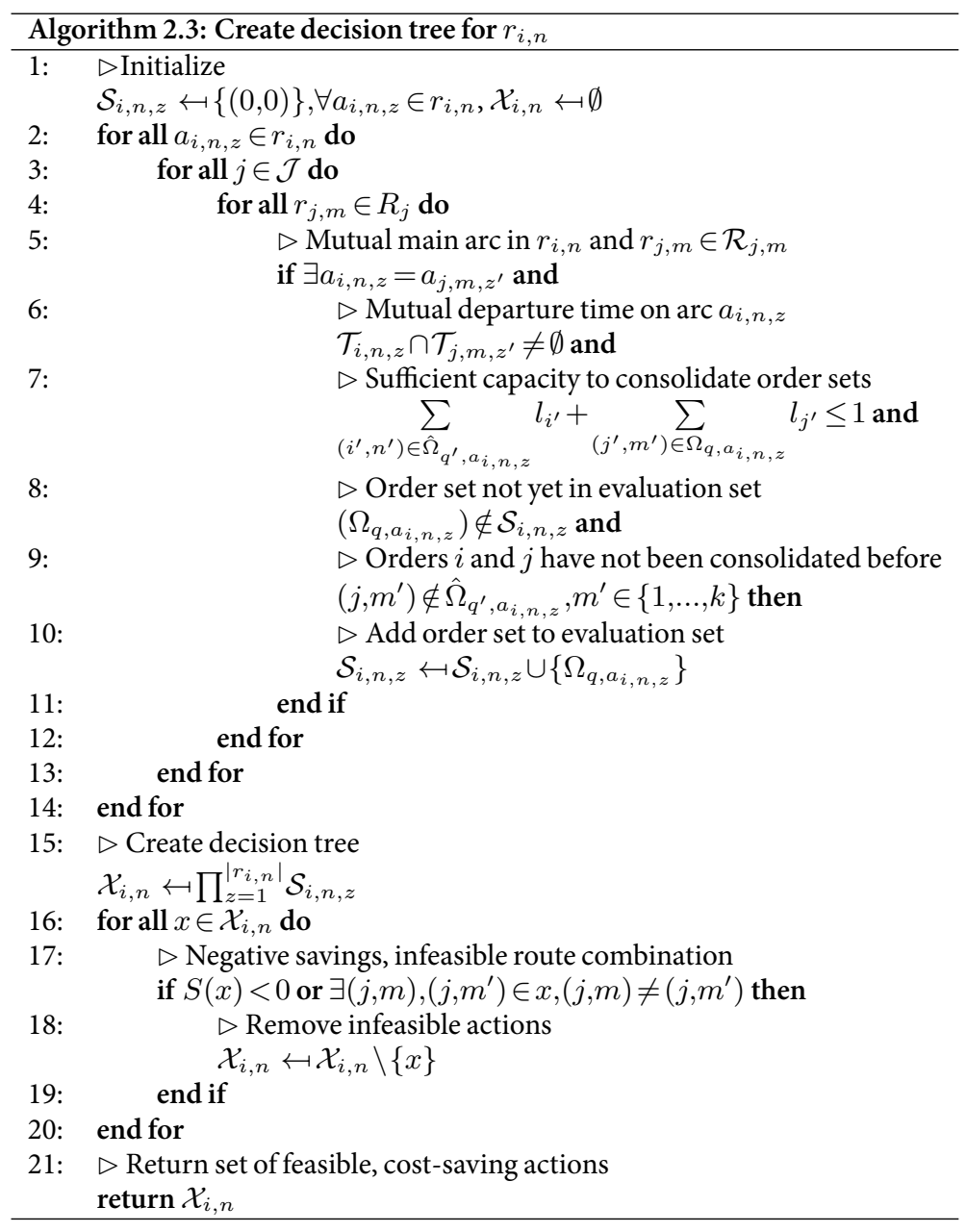

\section{Example 1 [Continued]: Construction of a decision tree}

Cycling through all $k$ routes for order 1 , assume we now arrived at route $r_{1,3}$, with trajectory 0-1-2-0. Suppose three open orders (2,3 and 4) have main arcs in common with this route, that are feasible in departure time and capacity, and therefore included in the evaluation set. Orders 2 and 3 were already consolidated in an earlier stage, hence only one route remains for both of these orders. Order 4 still 
has four available routes, Figure 2.4 shows the current schedule, Table 2.2 shows all available routes for the orders.

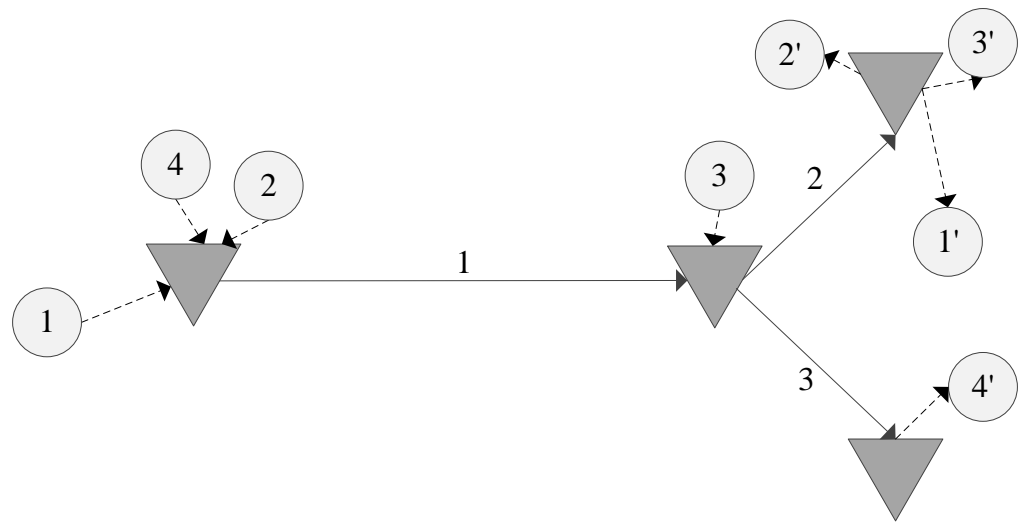

FIGURE 2.4. Current routes for orders 1,2, 3, and 4.

TABLE 2.2. Order properties for orders 1, 2, 3, and 4 .

\begin{tabular}{ccccc}
\hline Order & 1 & 2 & 3 & 4 \\
\hline Fill quantity & 0.3 & 0.4 & 0.3 & 0.5 \\
\hline Route(s) & $r_{1,1}: 0$ & $r_{2,1}: 0-1-2-0$ & $r_{3,1}: 0-2-0$ & $r_{4,1}: 0$ \\
& $r_{1,2}: 0-1-0$ & & & $r_{4,2}: 0-1-0$ \\
& $r_{1,3}: 0-1-2-0$ & & & $r_{4,3}: 0-1-2-0$ \\
& $r_{1,4}: 0-1-3-0$ & & & $r_{4,4}: 0-1-3-0$ \\
\hline
\end{tabular}

In Table 2.3, the timetable corresponding to the relevant main arcs is presented, giving the departure times with the routes that are eligible for leaving at each departure time between parentheses. The transshipment times at the transfer hubs are 30 minutes.

When constructing the evaluation set, we see that consolidation at main arc 1 is feasible for three routes of order 4, corresponding to two nodes in the decision tree. On main arc 2, consolidation is possible with $r_{2,1}, r_{3,1}$, and $r_{4,3}$. Observe that since orders 2 and 3 utilize the same container on main arc 2, this consolidation opportunity needs to be assessed only once. Finally, on both arcs we can also choose not to consolidate, leaving the original time window intact. With five opportunities on 
TABLE 2.3. Timetable on main arcs 1 and 2, showing the feasible departure times for orders 1, 2, 3 and 4.

\begin{tabular}{clc}
\hline Main arc & Feasible departure time with corresponding route sets & Travel time \\
\hline 1 & $13.00\left\{r_{1,3}\right\},\left\{r_{4,2}\right\},\left\{r_{4,3}\right\},\left\{r_{4,4}\right\}$ & 2 hours \\
& $14.00\left\{r_{1,3}\right\},\left\{r_{4,2}\right\},\left\{r_{4,4}\right\}$ & \\
\hline 2 & $15.00\left\{r_{1,3}\right\},\left\{r_{2,1}\right\},\left\{r_{4,2}\right\},\left\{r_{4,4}\right\}$ & 1 hour \\
& $15.30\left\{r_{1,3}\right\},\left\{r_{4,3}\right\}$ & \\
& $16.30\left\{r_{1,3}\right\},\left\{r_{2,1}, r_{3,1}\right\}$ & \\
& $17.30\left\{r_{1,3}\right\}$ &
\end{tabular}

main arc 1 and three on main arc 2 (including not consolidating), we have fifteen combinations to assess. However, not all of them are feasible. It is not possible to combine different routes for order 4 in the same schedule, while it is not possible either to consolidate both on main arc 1 with $r_{2,1}$ and on main arc 2 with $r_{4,3}$ due to time constraints. As shown in Algorithm 2.3, such infeasible combinations are identified and discarded. The decision tree in Figure 2.5 shows all combinations. By calculating the savings corresponding to each path, the decision maker can determine the consolidation opportunity with the largest feasible saving.

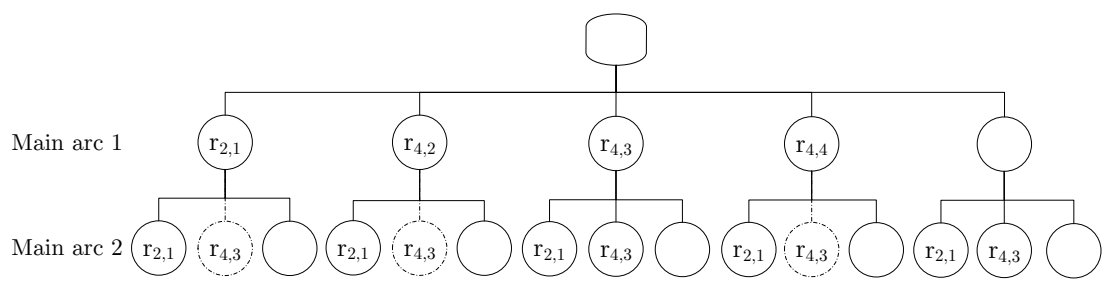

FIGURE 2.5. Decision tree containing all feasible combinations of consolidation opportunities for route $r_{1,3}$. The empty nodes represent the action where we do not consolidate. The dotted nodes represent infeasible actions.

2.4.4. Verifying actions and updating the solution. In the previous sections, we showed how to generate routes and how to construct the corresponding decision trees. The next step is to identify the consolidation actions that yield the largest savings. We evaluate the consolidation actions for every $r_{i, n} \in R_{i}$. The procedure to evaluate these actions consists of (i) generating a decision tree corresponding to $r_{i, n}$, (ii) selecting the feasible action that yields the largest savings, 
(iii) updating the schedules. Attempting to fill up the remaining container capacity, we recursively execute these three steps until no cost-saving actions can be identified, and store the obtained savings $S_{i, n}$. After performing this evaluation for all $r_{i, n} \in R_{i}$, we select the solution (e.g., the set of actions for a certain route) that yields the largest savings, thereby also taking into account the cost differences between routes. In Algorithm 2.4, we describe the evaluation procedure.

After identifying and fixing the best set of actions $x_{i, n}^{*}$, we must update the schedules accordingly. As we may consolidate with distinct order sets on the various main arcs in a route, this could also affect the departure schedules of orders that we do not consolidate with. As an example, suppose that we consolidate orders $i$ and $j$ on a given main arc. Further suppose that on a subsequent main arc in its route, order $j$ was already consolidated with order $j^{\prime}$. Then the departure time of order $j^{\prime}$ may be affected by consolidating order $i$, even though orders $i$ and $j^{\prime}$ are not consolidated. We therefore distinguish between consolidated and interrelated orders. The latter refers to every order for which departure times may need to be adjusted due to a consolidation action. Formally, we denote the set of orders interrelated to order $j$ according to Equation (2.4.1), where we combine all existing sets of consolidated orders corresponding to the current action. To ensure that the planning is updated consistently after a consolidation action, we must update the schedules for all order-route pairs $\left(j^{\prime}, m^{\prime}\right) \in \tilde{\Omega}_{j}$. We outline this procedure in Algorithm 2.5.

$$
\tilde{\Omega}_{j}=\bigcup_{z=1}^{\left|r_{j, m}\right|} \bigcup_{\left(j^{\prime}, m^{\prime}\right) \in \Omega_{q, a_{j^{\prime}, m^{\prime}, z}}} \bigcup_{z^{\prime}=1}^{\left|r_{j^{\prime}, m^{\prime}}\right|} \Omega_{q, a_{j^{\prime}, m^{\prime}, z^{\prime}}}
$$

Example 1 [Continued]: Updating the departure windows after consolidation Based on the decision tree as constructed in the preceding section, we decide to consolidate $r_{1,3}$ on main arc 1 with $r_{4,4}$ and on main arc 2 with $\left\{r_{2,1}, r_{3,1}\right\}$. In this example, we describe how the departure windows are updated for consolidation, and how updating affects the rest of the schedule. We again assume that departures are tentatively scheduled as late as possible; however, earlier departure times can be selected to facilitate consolidation. From Figure 2.6, the mutually feasible departure times on both arcs can be observed.

It can be seen that 13.00, 14.00 and 15.00 are feasible departure times for both order 1 and 4 . However, when selecting 15.00, consolidation at main arc 2 would no longer be possible. Hence, 13.00 and 14.00 are the only feasible departure times for 


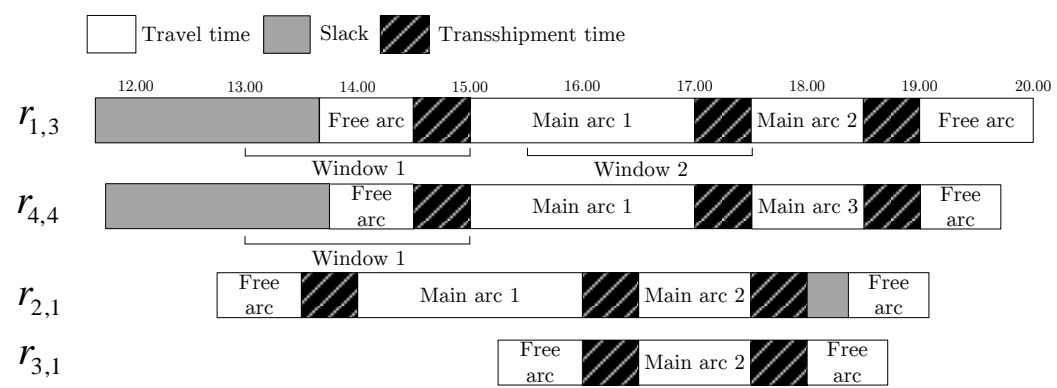

FIGURE 2.6. Gantt charts of routes before consolidation. A mutually feasible departure time must exist to consolidate on any given arc.

this particular combination. The departure window is therefore narrowed, causing scheduled slack after the second main arc. The schedule after consolidation for order 1 is shown in Figure 2.7.

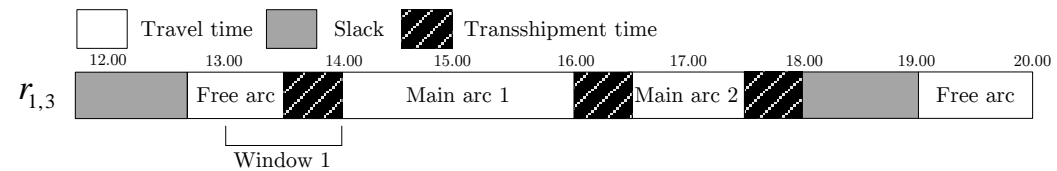

FIGURE 2.7. Gantt chart of route $r_{1,3}$ after consolidation on both main arcs. 


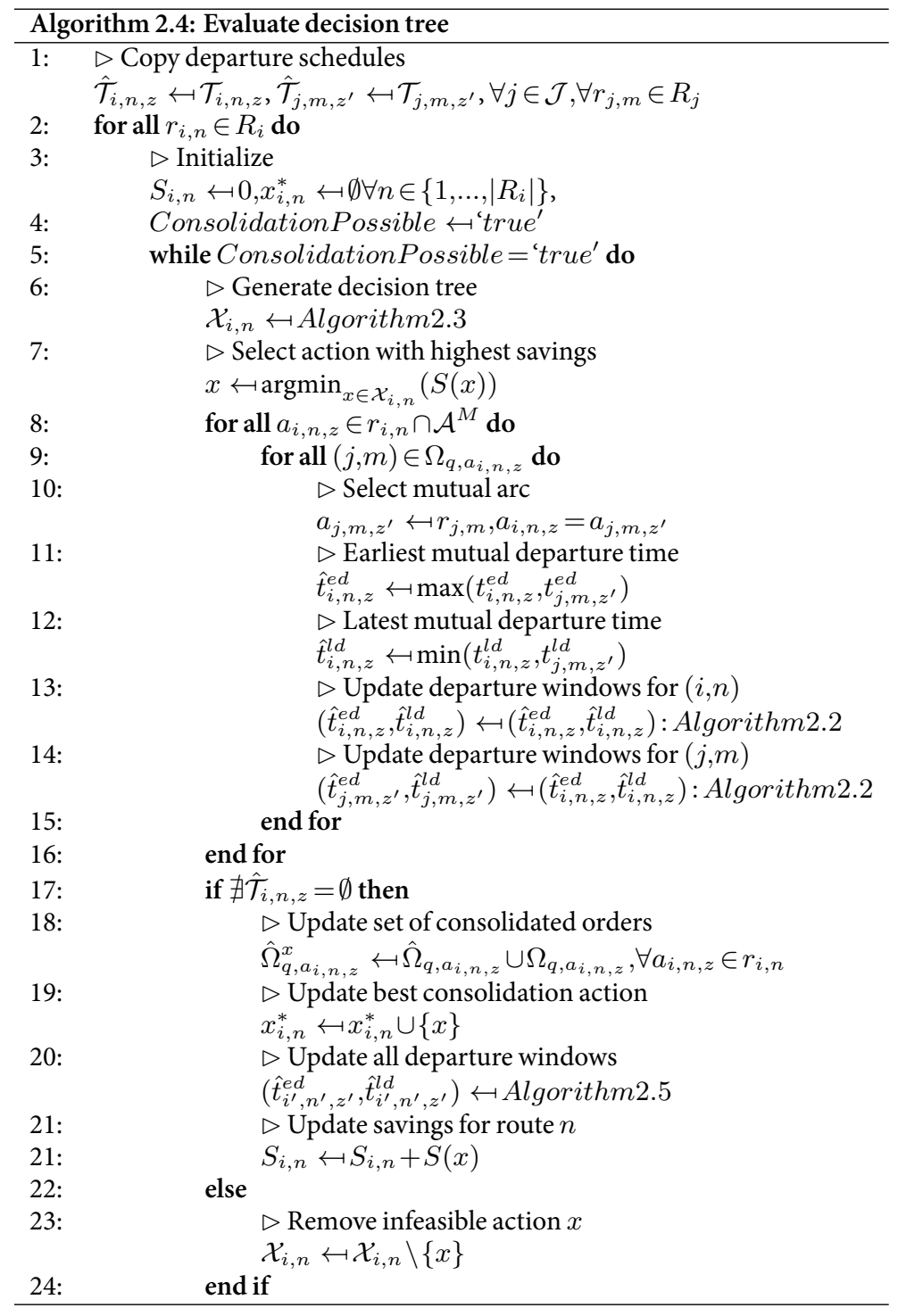



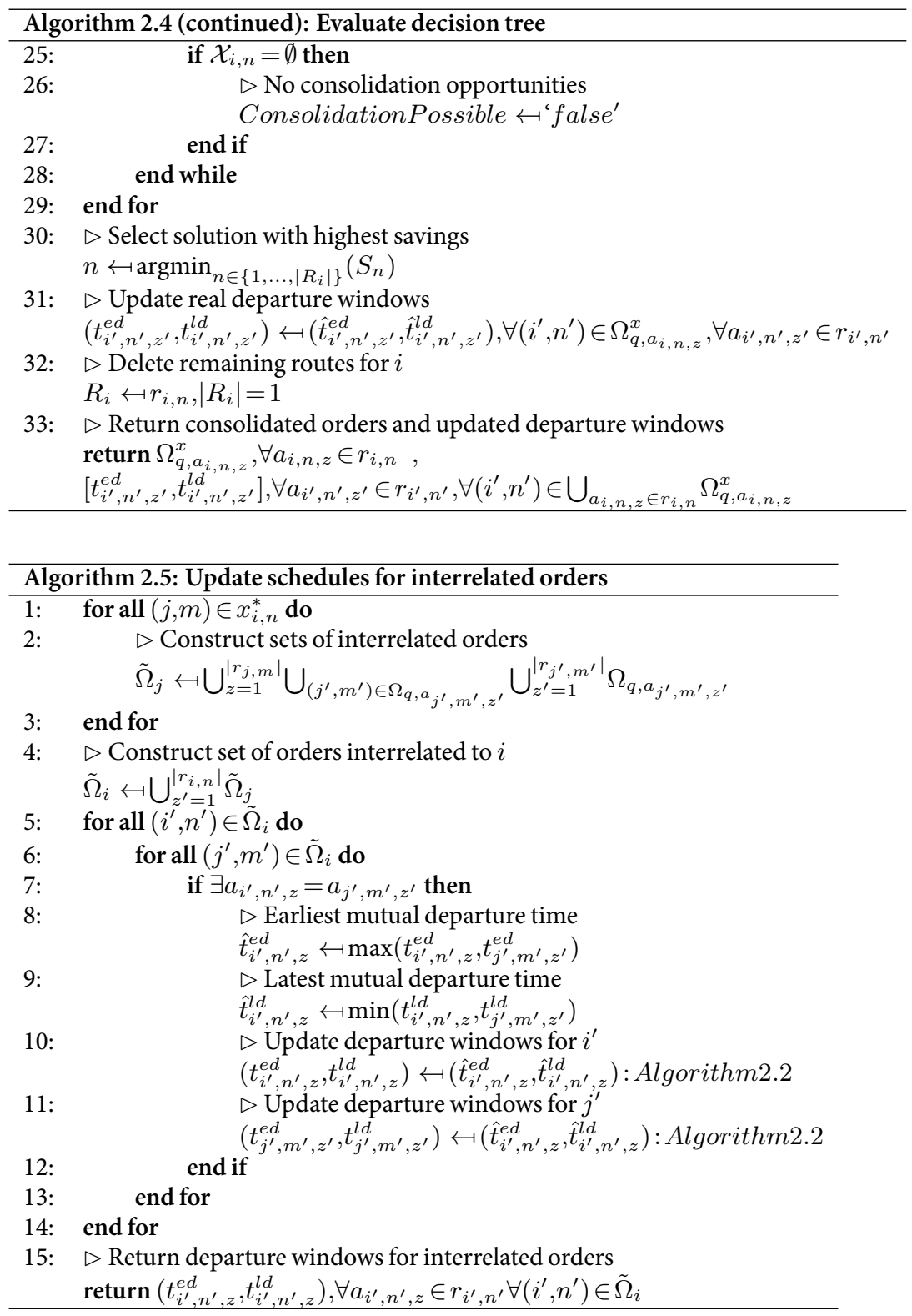


\subsection{Numerical experiments}

In this section, we describe the experiments to test the performance of the algorithm. In Section 2.5.1, we identify three key design choices of the algorithm and describe alternative solution methods as a benchmark to test the impact of these choices. Section 2.5.2 describes our experimental settings and instances, consisting of both virtual networks and a real-life case of a Dutch 4PL. In Section 2.5.3 we provide the numerical results of the simulation study, providing insights in the cost performance, impact on $\mathrm{CO}_{2}$ emissions, and computational times.

2.5.1. Solution methods for benchmarks. Threekey design choices for the algorithm are that a) the search space for consolidation opportunities is limited to $k$ routes per order, $\mathrm{b}$ ) routes are geographically fixed after consolidating, and c) orders are planned dynamically. Although these choices greatly reduce the computational effort, they may also prematurely eliminate fruitful consolidation opportunities. In Table 2.4, we propose a number of alternative solution methods (denoted by $\pi$ ) as benchmarks to evaluate the impact of these design choices.

To assess the impact of a), we use various settings for $k$ to evaluate the marginal contributions of storing an extra route $\left(\pi^{k=k^{\prime}}\right)$; this solution method is the consolidation algorithm that we introduced in this chapter. The rationale is that as routes become more expensive, they have lower potential for savings, hence their marginal contribution to the solution quality will decrease. To quantify the impact, we compute multiple comparison metrics, using the basic solution methods $\pi^{d i r}$ and $\pi^{i n t}$. Here, $\pi^{d i r}$ always directly transports orders from origin to destination by road transport, and $\pi^{i n t}$ uses the cheapest (possibly intermodal) route for each individual order. The solution method $\pi^{i n t}$ is equivalent to $\pi^{k=1}$, absent the possibility to consolidate.

To test the effect of design choice b), we use a solution method where we completely replan all orders that are not yet shipped. We do this by replanning all open orders at each new order arrival, thereby essentially solving the static problem at every arrival. The orders are planned one-by-one according to a given sequence. We consider two forms of sequences, (i) sorted based on the latest delivery time $\left(\pi^{t i m}\right)$, and (ii) random planning sequence $\pi^{r n d}$. The first sequence form gives priority to urgent orders, granting them more opportunities to be consolidated. With the random sequence benchmark, we test multiple random sequences and select the cheapest solution. Evaluating all possible sequences 
would solve the static variant of the problem to optimality, but this is computationally unmanageable for larger instances.

Finally, we define the benchmark for design choice c). The optimal solution can only be achieved by having perfect foresight for all orders to come and solving the associated static scheduling problem (with all future orders included) to optimality with $\pi^{\text {opt }}$. We define 'optimality' here within the constraint that a finite number of routes is available; as before, we take into account only $k$ routes for each order. The acquired solution is always at least as good as the optimal solution to the dynamic problem (the same is not true without perfect foresight, as solutions eliminate certain future consolidation opportunities once they are fixed). Due to the high computational effort, this solution method can only be applied to instances containing a small number of orders.

TABLE 2.4. Solution methods used as benchmarks.

\begin{tabular}{ll}
\hline Solution method & Description \\
\hline$\pi^{d i r}$ & $\begin{array}{l}\text { Shortest route (single free arc), direct transport from origin } \\
\text { to destination }\end{array}$ \\
$\pi^{i n t}$ & $\begin{array}{l}\text { Cheapest (possibly intermodal) route, not allowing } \\
\text { for consolidation }\end{array}$ \\
$\pi^{k=k^{\prime}}$ & Consolidation algorithm with various settings $k \in \mathbb{N}$ \\
$\pi^{t i m}$ & $\begin{array}{l}\text { Re-solve static problem at order arrival, orders sorted } \\
\text { on latest delivery time }\end{array}$ \\
$\pi^{r n d}$ & $\begin{array}{l}\text { Re-solve static problem at order arrival } r \text { times, } \\
\text { using } r \in \mathbb{N} \text { random sequences }\end{array}$ \\
$\pi^{\text {opt }}$ & Solve static problem with perfect foresight to optimality \\
\hline
\end{tabular}

2.5.2. Experimental settings. We perform numerical experiments on four virtual networks, each one representing generalizations of common consolidation scenarios. In Figure 2.8, panel a) shows a cluster with pickup locations, a cluster with delivery locations, transfer hubs in the center of both clusters, and a single main arc connecting these two hubs (STR). Panel b) shows a split network, consisting of a single origin cluster and two distant destination clusters (SPL). Panel c) shows a triangular network (TRI), where making a detour could yield additional consolidation opportunities. Finally, panel d) shows a scaleddown version of a semi-random network (RND). The pickup points and distributions are uniformly generated within a distance of $\frac{1000 \mathrm{~km}}{\# h u b s}$ from randomly selected hubs, based on the notion that hubs tend to be positioned within areas of high demand and vice versa. Orders generated have a preset minimum distance of $250 \mathrm{~km}$ between origin and destination, and always move to the right. 
The random networks vary in the number of hubs and the number of main arcs denoted as RND(\# hubs/\# main arcs) - namely RND(15/30), RND(20/40), and $\mathrm{RND}(20 / 100)$. For all virtual networks, we consider variants where all main arcs are operated by either trucks, electric trains, diesel trains, or barges.

Besides the virtual networks, we also perform experiments on the 4PL case. We study their operations on the Rhine-Alpine corridor, which connects a variety of economic regions in the Netherlands, Germany, Austria, Switzerland and Italy. The $4 \mathrm{PL}$ ships orders (with an average volume of 0.33 container) from several locations in the Netherlands to a variety of locations within this corridor. To facilitate this transport, the $4 \mathrm{PL}$ utilizes a network operated by various contracted carriers. This dedicated network consists of 37 hubs, which are connected by 110 waterway and railroad arcs. We use the real timetables and travel times for these arcs. 
a) Straight network

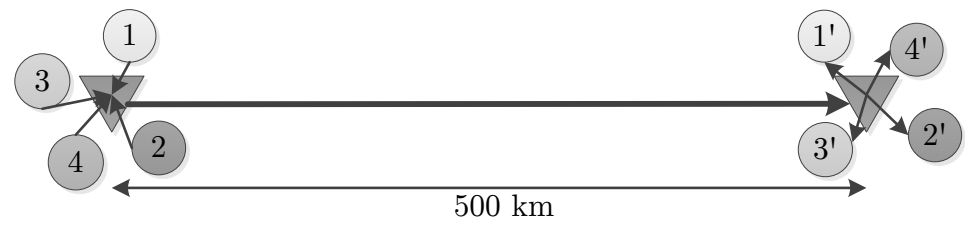

b) Split network

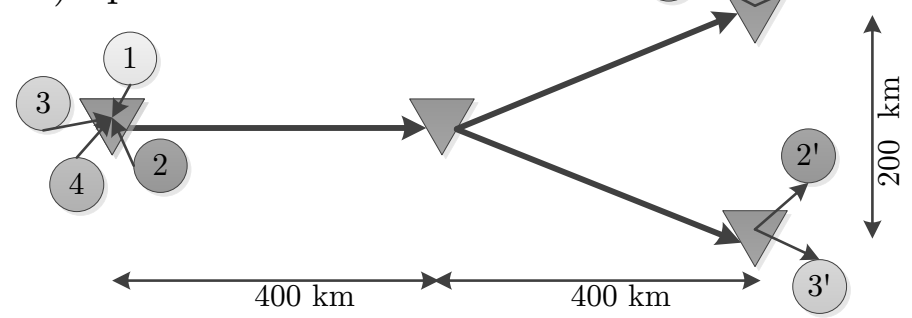

c) Triangular network

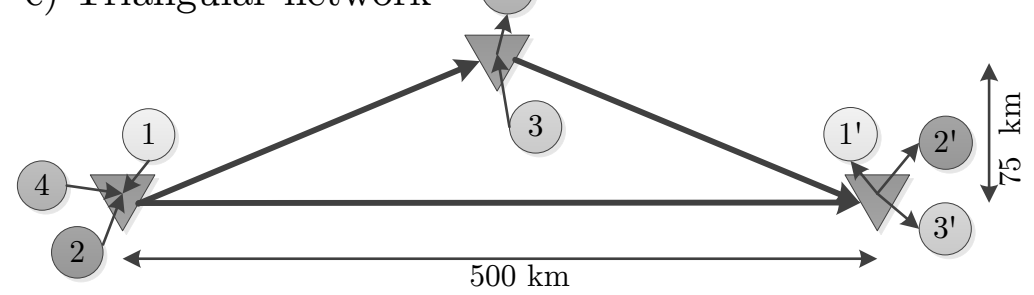

d) Semi-random network

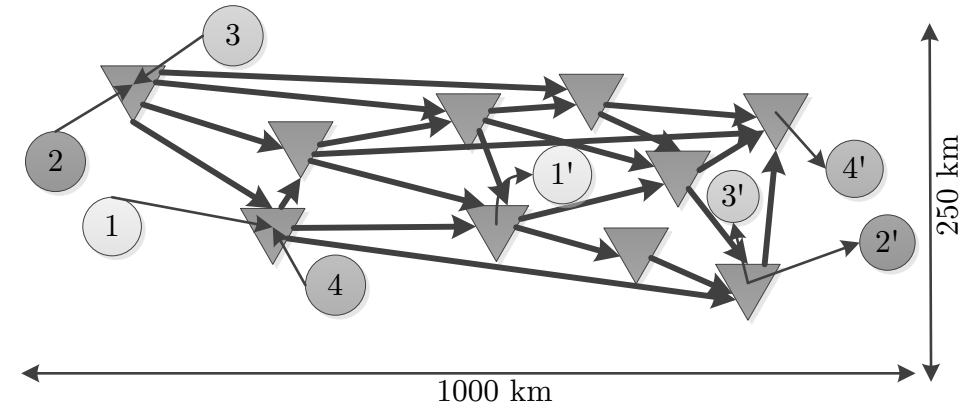

FIGURE 2.8. Graphical representation of the four virtual networks used in the experiments. Four order-destination pairs are shown for each network; in the actual experiments much larger numbers of orders are available at the same time. 
The mode characteristics used in the experiments are shown in Table 2.5. The travel speeds per mode are acquired from the Dutch 4PL, averaging over oftenused train connections and barge connections. We obtain container volume data and the $\mathrm{CO}_{2}$ emission per modality (calculated by 2020 engine standards) from Boer et al. (2011). Emissions are computed on a well-to-wheel basis, including both exhaust emission and upstream emission (i.e., electricity production and refinement). We consider containerized average density goods; for these goods container volume is more restrictive than weight. Extrapolating the figures for twenty-feet containers to forty-feet containers (Boer et al., 2011), we set a full container load equal to 21 ton.

For trucks, we take into account the load factor when computing $\mathrm{CO}_{2}$ emissions. Emissions strongly depends on whether the truck drives in an urban area, non-urban area, or on a motorway; we take the average emissions over the road shares corresponding to a truck trailer. The obtained bounds on $\mathrm{CO}_{2}$ emission are $589 \mathrm{gram} / \mathrm{km}$ (empty truck) and $897 \mathrm{gram} / \mathrm{km}$ (full truck); Boer et al. (2011) assume that between these bounds, emission increases linearly with the transported weight. For trains and barges, we use the average container weight to compute $\mathrm{CO}_{2}$ emissions, as container consolidation by the $4 \mathrm{PL}$ only marginally affects the total weights of these modes.

For a 4PL to successfully consolidate freight, it is essential that the prices charged by carriers give an incentive to consolidate. By consolidating, carriers themselves are able to reduce their costs by economies of scale, i.e., lower costs due to higher utilization of assets (Berwick and Dooley, 1997; Krajewska and Kopfer, 2006). This is because fixed asset costs can then be divided over more orders. If this decreasing cost function for the carrier is properly reflected in the prices they charge, the $4 \mathrm{PL}$ might be able to benefit from higher utilization as well. However, prices agreed upon between shippers and carriers do not necessarily reflect the actual costs that are made to provide the service. Precise cost allocation may be difficult, as operational transportation costs depend on many factors (Yan et al., 1995). A cost function may, among other things, depend on average fill rate, haul length, shipment size, percentage of LTL traffic, and composition of output (Spady and Friedlaender, 1978). Such factors are often only partially reflected in the prices charged by the carrier. In container transport, for example, it is common to charge a flat rate per container rented, regardless of its fill rate. Clearly, such price functions give the $4 \mathrm{PL}$ a strong incentive to attain a high fill rate. At the other end of the spectrum, the rates charged by parcel services usually do not offer incentives to consolidate (Klausner and Hendrickson, 2000). The associated price function, which is linear in the number of packages, 
would make a 4PL almost indifferent about consolidating. Clearly, the larger the cost reduction when combining loads into the same container, the more beneficial it becomes for the 4PL to actively consider consolidation opportunities.

Our standard cost functions, shown in Table 2.5, are based on the case of the Dutch 4PL; costs are normalized for confidentiality reasons. For the experiments on virtual networks, all modes have a fixed rate per container. For the real-life case, the barges and trains on the main arcs use the same fixed rates. However, to more accurately reflect the costs that they make for road transport, the trucks on the free arcs have a capped linear cost function of the form $\min (1,0.31+0.78 \cdot l)$. Finally, we set the costs for a single transshipment operation (container lift or placement) at 22 (hence 44 for a full transshipment) and for a reload (per order, including both unloading and loading) at 35.

To demonstrate how the algorithm is able to handle multiple criteria, we test an extension in which we monetize various additional objectives. We add the following costs to the original objective function: 40 per ton $\mathrm{CO}_{2}$ emission (see Maibach et al. (2008), based on 2020 standards), 0.10 per hour of additional transport time (compared to direct transport), and 20 per order transfer to represent hub risk.

For the virtual networks, orders are randomly generated, with their origin, destination and volume - selected from the set of order sizes $\left\{\frac{1}{25}, \ldots, \frac{25}{25}\right\}$ - being drawn from uniform distributions. The times between order arrivals follow a Poisson distribution with a mean of 3 hours. We make use of common random numbers, thus excluding the influence of stochastic fluctuation when comparing the performances. Orders can be picked up directly after becoming known; the latest delivery time is 5 days ahead. Modes operating on the main arcs depart every 6 hours. For our case study, we use an order set of the Dutch 4PL, consisting of 1006 orders transported over a quartile in 2013. Their actual locations, order volumes, pickup- and delivery times are used. The operating modes are subject to the real timetables and travel speeds. The case study shows to some extent how the algorithm performs under non-uniform distributions. Future research may further explore the impact of such distributions.

We conclude this section with the key simulation settings. Unless specified otherwise, for the virtual instances we use 6 replications with 650 order arrivals - setting both the warm-up and cool-down periods equal to 25 order arrivals to achieve a relative cost error of at most $5 \%$ for the $95 \%$ confidence intervals. Based on preliminary tests that determine the marginal contributions for various levels for $k$, we use $k=2$ for the straight and split networks, $k=3$ for the 
triangular network, $k=31$ for the random networks, and $k=40$ for the 4 PL network. Furthermore, we set $\beta=2$, meaning that we store routes being up to twice as expensive as the cheapest route found. Settings for $\beta>2$ did not show any performance improvement. Some other values $1<\beta<2$ yielded comparable results, while requiring less computational effort. However, as $\beta=2$ already yields reasonable computational times for the simulation study, we did not attempt to fine-tune this setting. Finally, preliminary tests indicate that a latest departure policy clearly and consistently outperforms an earliest departure policy due to offering more flexibility and consolidation opportunities. We stress that we assume deterministic travel times in the experiments; in practical applications we might add some safety margin to account for disturbances. In the experiments, every order therefore leaves its route vertices as late as the corresponding departure windows allow. The algorithm was coded in Plant Simulation 11, and ran on a computer with $8 \mathrm{~GB}$ RAM and a $2.90 \mathrm{GHz}$ Intel Core i7 processor.

TABLE 2.5. Mode characteristics as used in the numerical experiments.

\begin{tabular}{lllll}
\hline Mode & $\begin{array}{l}\text { Costs per km } \\
\text { (normalized) }\end{array}$ & $\begin{array}{l}\text { Speed } \\
\text { km/hour }\end{array}$ & $\begin{array}{l}\mathrm{CO}_{2} \\
\text { gram/ton/km }\end{array}$ & $\begin{array}{l}\mathrm{CO}_{2} \text { gram/ } \\
\text { container/km }\end{array}$ \\
\hline Truck & 1.00 & 68 & - & $589-897$ \\
Train (electric) & 0.51 & 55 & 12 & 252 \\
Train (diesel) & 0.51 & 55 & 31 & 651 \\
Barge & 0.33 & 15 & 31 & 651 \\
\hline
\end{tabular}

2.5.3. Numerical results. We divide the presentation of the numerical experiments in two parts: (i) the performance of the algorithm under various network settings and (ii) the performance of the algorithm compared to several alternative solution methods. We start by presenting the performance for a variety of networks. First, we describe the results for the experiments on the virtual networks, using the standard settings. In Table 2.6, we provide key insights on the performance of the algorithm. We show the reduction percentages in costs and $\mathrm{CO}_{2}$ emissions, as well as the increase in container fill rate, compared to results obtained with the benchmarks $\pi^{d i r}$, $\pi^{i n t}$, and $\pi^{k=1}$. The first two policies (which do not consider consolidation) are clearly outperformed both in terms of costs and emissions. Compared to road transport, electric train networks yield the greatest reduction in $\mathrm{CO}_{2}$ emissions, as the $\mathrm{CO}_{2}$ emission of electric trains is significantly lower than the emission associated with trucks. Similarly, the low costs of barges translate in the greatest reduction in terms of costs. Diesel train networks perform worst in terms of emission. For the semi-random networks, direct road transport even outperforms diesel train transport. For the 
first three network configurations, comparison to $\pi^{k=1}$ indicates that storing $k>1$ routes has virtually no effect. Due to the small diversity in routes for these networks, the algorithm is generally able to use the best routes of orders when consolidating, such that storing more than one route has a negligible impact. However, for the random networks we see that the added flexibility of storing more routes can yield 3-17\% reduction in $\mathrm{CO}_{2}$ emissions, $3-12 \%$ cost savings, and a $14-50 \%$ improvement in fill rates. Furthermore, we show the results for the multi-objective extension in Table 2.6 as well; the tests are performed on the semi-random truck networks. We see that while average savings decrease, intermodal transport is still financially attractive. The impact on emissions and fill rates is negligible. Hence, the solutions appear to be fairly robust when taking into account non-financial objectives of the planner. We conclude our analysis with some notes on how typical solutions look like. The use of two or more main arcs within one route is exceptional. Compared to direct transport, the average increase in travel time is $22 \%$. Of the consolidated solutions, the average drayage distance comprises $32 \%$ of the route. The average volume of consolidated orders is 0.41 container load; orders with a volume over 0.60 are notably more difficult to consolidate. 
TABLE 2.6. Key results from the numerical experiments. The percentages show the improvement in performance of the algorithm, relative to the results obtained with the benchmark policies. For the transport modes, we use the abbreviations $R$ (road transport by truck), $T \_e$ (electric train), T_d (diesel train), and B (barge). The symbol * indicates that the instance was solved with a multi-objective function.

\begin{tabular}{|c|c|c|c|c|c|c|c|c|c|}
\hline \multirow[b]{2}{*}{ Network } & \multicolumn{3}{|c|}{ Cost reduction } & \multicolumn{3}{|c|}{$\mathrm{CO}_{2}$ reduction } & \multicolumn{3}{|c|}{ Increase fill rate } \\
\hline & $\pi^{d i r}$ & $\pi^{i n t}$ & $\pi^{k=1}$ & $\pi^{d i r}$ & $\pi^{i n t}$ & $\pi^{k=1}$ & $\pi^{d i r}$ & $\pi^{i n t}$ & $\pi^{k=1}$ \\
\hline STR-R & $28 \%$ & $28 \%$ & $0 \%$ & $28 \%$ & $28 \%$ & $0 \%$ & $73 \%$ & $73 \%$ & $0 \%$ \\
\hline STR-T_e & $52 \%$ & $28 \%$ & $0 \%$ & $74 \%$ & $36 \%$ & $0 \%$ & $74 \%$ & $74 \%$ & $0 \%$ \\
\hline STR-T_d & $52 \%$ & $28 \%$ & $0 \%$ & $32 \%$ & $92 \%$ & $0 \%$ & $74 \%$ & $74 \%$ & $0 \%$ \\
\hline STR-B & $62 \%$ & $23 \%$ & $0 \%$ & $43 \%$ & $40 \%$ & $0 \%$ & $73 \%$ & $73 \%$ & $0 \%$ \\
\hline SPL-R & $13 \%$ & $13 \%$ & $13 \%$ & $15 \%$ & $15 \%$ & $15 \%$ & $36 \%$ & $36 \%$ & $36 \%$ \\
\hline SPL-T_e & $52 \%$ & $30 \%$ & $0 \%$ & $74 \%$ & $35 \%$ & $0 \%$ & $72 \%$ & $72 \%$ & $0 \%$ \\
\hline SPL-T_d & $52 \%$ & $30 \%$ & $0 \%$ & $33 \%$ & $92 \%$ & $0 \%$ & $72 \%$ & $72 \%$ & $0 \%$ \\
\hline SPL-B & $62 \%$ & $25 \%$ & $0 \%$ & $42 \%$ & $38 \%$ & $0 \%$ & $68 \%$ & $68 \%$ & $0 \%$ \\
\hline TRI-R & $20 \%$ & $20 \%$ & $20 \%$ & $22 \%$ & $22 \%$ & $22 \%$ & $62 \%$ & $62 \%$ & $62 \%$ \\
\hline TRI-T_e & $49 \%$ & $26 \%$ & $0 \%$ & $71 \%$ & $33 \%$ & $1 \%$ & $71 \%$ & $71 \%$ & $1 \%$ \\
\hline TRI-T_d & $49 \%$ & $26 \%$ & $0 \%$ & $25 \%$ & $86 \%$ & $3 \%$ & $71 \%$ & $71 \%$ & $1 \%$ \\
\hline TRI-B & $59 \%$ & $20 \%$ & $0 \%$ & $40 \%$ & $38 \%$ & $2 \%$ & $69 \%$ & $69 \%$ & $2 \%$ \\
\hline RND(15/30)-R & $12 \%$ & $12 \%$ & $12 \%$ & $17 \%$ & $17 \%$ & $17 \%$ & $50 \%$ & $50 \%$ & $50 \%$ \\
\hline RND(15/30)-T_e & $31 \%$ & $14 \%$ & $5 \%$ & $54 \%$ & $18 \%$ & $5 \%$ & $54 \%$ & $54 \%$ & $17 \%$ \\
\hline RND(15/30)-T_d & $31 \%$ & $14 \%$ & $5 \%$ & $-20 \%$ & $46 \%$ & $12 \%$ & $54 \%$ & $54 \%$ & $17 \%$ \\
\hline RND(15/30)-B & $42 \%$ & $9 \%$ & $3 \%$ & $26 \%$ & $27 \%$ & $8 \%$ & $51 \%$ & $51 \%$ & $14 \%$ \\
\hline $\mathrm{RND}(20 / 40)-\mathrm{R}$ & $12 \%$ & $12 \%$ & $12 \%$ & $17 \%$ & $17 \%$ & $17 \%$ & $49 \%$ & $49 \%$ & $49 \%$ \\
\hline RND(20/40)-T_e & $32 \%$ & $13 \%$ & $5 \%$ & $54 \%$ & $17 \%$ & $4 \%$ & $54 \%$ & $54 \%$ & $18 \%$ \\
\hline RND(20/40)-T_d & $32 \%$ & $13 \%$ & $5 \%$ & $-19 \%$ & $43 \%$ & $9 \%$ & $54 \%$ & $54 \%$ & $18 \%$ \\
\hline RND(20/40)-B & $43 \%$ & $9 \%$ & $3 \%$ & $27 \%$ & $27 \%$ & $9 \%$ & $52 \%$ & $52 \%$ & $16 \%$ \\
\hline $\operatorname{RND}(20 / 100)-\mathrm{R}$ & $12 \%$ & $12 \%$ & $12 \%$ & $17 \%$ & $17 \%$ & $17 \%$ & $47 \%$ & $47 \%$ & $47 \%$ \\
\hline RND(20/100)-T_e & $35 \%$ & $13 \%$ & $8 \%$ & $57 \%$ & $15 \%$ & $3 \%$ & $54 \%$ & $54 \%$ & $24 \%$ \\
\hline RND(20/100)-T_d & $35 \%$ & $13 \%$ & $8 \%$ & $-10 \%$ & $39 \%$ & $9 \%$ & $54 \%$ & $54 \%$ & $24 \%$ \\
\hline RND(20/100)-B & $46 \%$ & $8 \%$ & $5 \%$ & $29 \%$ & $28 \%$ & $16 \%$ & $50 \%$ & $50 \%$ & $25 \%$ \\
\hline $\operatorname{RND}(15 / 30)-\mathrm{R}^{*}$ & $8 \%$ & $8 \%$ & $8 \%$ & $17 \%$ & $17 \%$ & $17 \%$ & $46 \%$ & $46 \%$ & $46 \%$ \\
\hline $\mathrm{RND}(20 / 40)-\mathrm{R}^{*}$ & $9 \%$ & $9 \%$ & $9 \%$ & $17 \%$ & $17 \%$ & $17 \%$ & $45 \%$ & $45 \%$ & $45 \%$ \\
\hline $\mathrm{RND}(20 / 100)-\mathrm{R}^{*}$ & $7 \%$ & $7 \%$ & $7 \%$ & $17 \%$ & $17 \%$ & $17 \%$ & $43 \%$ & $43 \%$ & $43 \%$ \\
\hline Real-life 4PL case & $15 \%$ & $6 \%$ & $6 \%$ & $31 \%$ & $20 \%$ & $20 \%$ & $28 \%$ & $28 \%$ & $28 \%$ \\
\hline Average & $34 \%$ & $17 \%$ & $5 \%$ & $30 \%$ & $33 \%$ & $9 \%$ & $57 \%$ & $57 \%$ & $21 \%$ \\
\hline
\end{tabular}

Next, we discuss the results of the experiment with the real-life 4PL case. Orders transported from the Netherlands to Germany do not favor the use of the 
intermodal network; for distances below $200 \mathrm{~km}$ intermodal transport is not beneficial. For distances over $500 \mathrm{~km}$ we see the most use of the intermodal network. Therefore, without considering consolidation, only $21 \%$ of orders is transported via the intermodal network. When considering only the best routes, the 215 intermodal routes use 28 distinct main arcs. Consequently, when we only consider the best routes $(k=1)$, only few consolidation opportunities exist. As indicated by the results in Table 2.6, increasing planning flexibility by storing multiple routes notably increases the performance, illustrating the added value of the algorithm. Compared to $\pi^{k=1}$, costs are reduced with $6 \%, \mathrm{CO}_{2}$ emissions drops by $20 \%$, and container fill rates are increased by $28 \%$.

We continue with the results regarding our evaluation of the design choices by comparing to the benchmark methods. First, we assess the impact of storing $k$ routes. Figure 2.9 shows the outcomes for applying $k=\{1,2, \ldots, 40\}$ on three semi-random truck networks; 15 hubs with 30 main arcs, 20 hubs with 40 main arcs, and 20 hubs with 100 main arcs. The same procedure is applied to the real-life network. The added value of storing more alternative routes is significant, yielding savings up to $12 \%$ compared to $\pi^{k=1}$. Note that the saving potential for the 4PL case is lower due to the larger number of short-distance orders. Marginal savings decrease with increasing settings of $k$; the lower quality of the stored routes decrease the savings potential. As illustrated in Figure 2.9, the marginal savings decrease, such that at a certain point storing additional routes no longer significantly impacts the solution quality. This implies that we can fix $k$ at some level, such that higher values for $k$ would not yield additional benefits. Furthermore, we note that a higher setting for $k$ does not guarantee a higher saving. This may seem counter-intuitive at first. However, a higher $k$ results in a higher probability to identify and fix opportunities with minor savings shortly after the order becomes known, thus reducing consolidation opportunities (potentially with a greater savings potential) later on.

We proceed with the benchmark results of fixing routes after consolidating. Our instances are the 20/40 semi-random networks for truck, train, and barge. To keep the experiment computationally manageable, we use the relatively small setting of $k=5$; recall that the benchmarks policies re-solve the static problem at every order arrival. Whereas the algorithm takes less than 1 second to plan an order, $\pi^{r n d}$ takes close to 10 second per order. Also, it is not scalable to larger instances, making this benchmark method unfit as an online solution method. The policy $\pi^{t i m}$ outperforms our algorithm $0.4 \%$ on average. In Figure 2.10, we show the performance gap between the algorithm and $\pi^{r n d}$. Testing up to 50 
random sequences, we see that the performance gap remains below 3\%, seemingly converging to a stable performance gap.

The last design choice that we test is that of planning orders dynamically by comparing the performance to $\pi^{o p t}$. As the benchmark policy is computationally intensive, we require a small and simplistic instance for this experiment. We run 1,000 instances on the straight truck network (STR-R), each consisting of 7 orders. Solving these toy-sized instances on average takes 15 seconds per instance, compared to a fraction of a second for the $k$-path algorithm. We emphasize that $\pi^{\text {opt }}$ yields a high upper bound due to perfect foresight, as the dynamic planning problem does not entail future information. In Figure 2.11, we show the average deviation. In $38 \%$ of the instances, we obtain the optimal solution with our algorithm; the average deviation in terms of costs is $4.7 \%$.

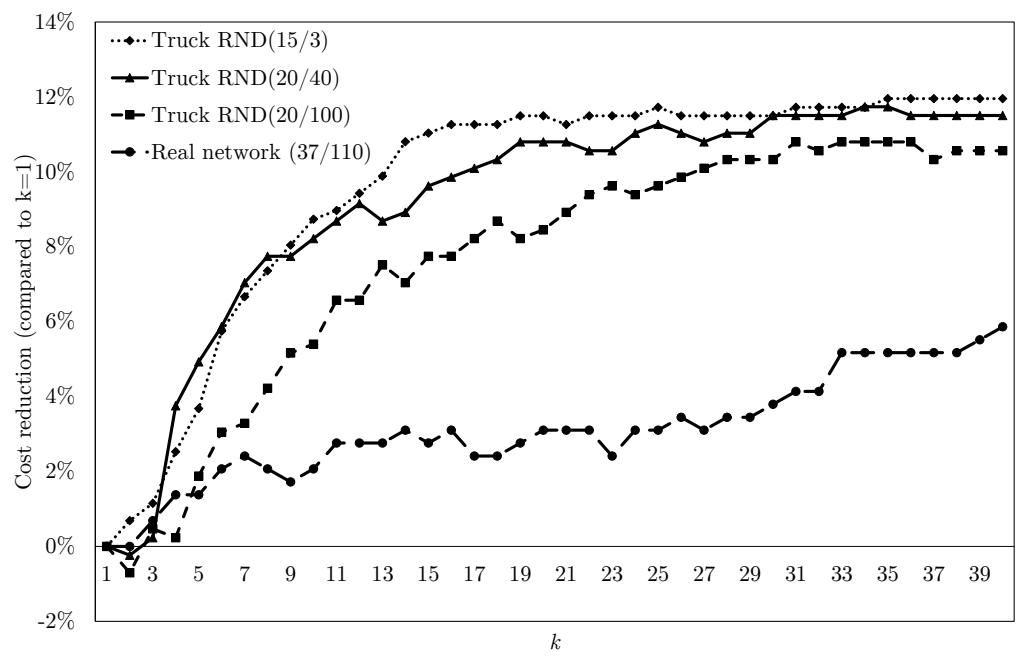

FIGURE 2.9. Average cost reduction compared to $k=1$ for three semi-random networks and the real-life 4PL network, measured for $k=\{1,2, \ldots, 40\}$. 


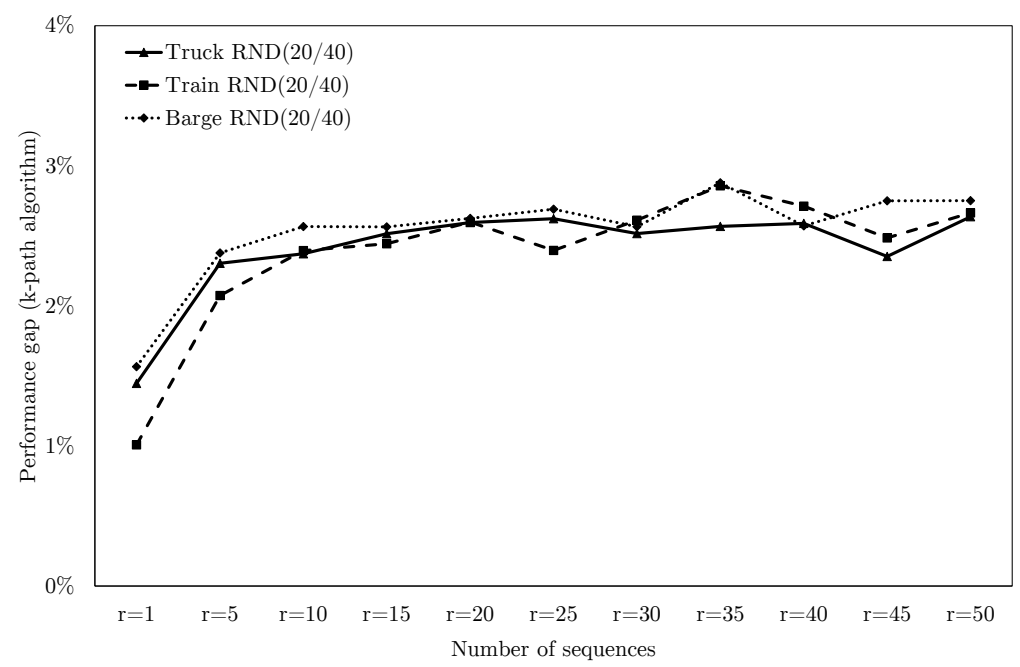

FIGURE 2.10. Comparison between the algorithm and $\pi^{\text {rnd }}$ for varying numbers of planning sequences $r$, applied on truck, train and barge networks.

\subsection{Conclusions}

We have demonstrated that networks with reloads have the potential for significant improvements, both in terms of transport costs and environmental impact. However, the required transshipments introduce a financial barrier for this shift. For intermodal transport to compete with direct transport of LTL goods, it is essential that freight is consolidated as much as possible. We therefore have proposed an algorithm to dynamically plan routes in intermodal networks with transshipments and reloads. Timetables of the modes are captured in a time-dependent graph. We use an intuitive arc-expansion approach to efficiently construct multi-arc routes. The best $k$ routes for every order are stored, yielding a controlled solution space to search for consolidation opportunities. To facilitate consolidation, we create flexibility in both space and time, by (i) storing multiple geographically distinct routes for every order, and (ii) using departure windows rather than fixed departure times. The algorithm is designed 


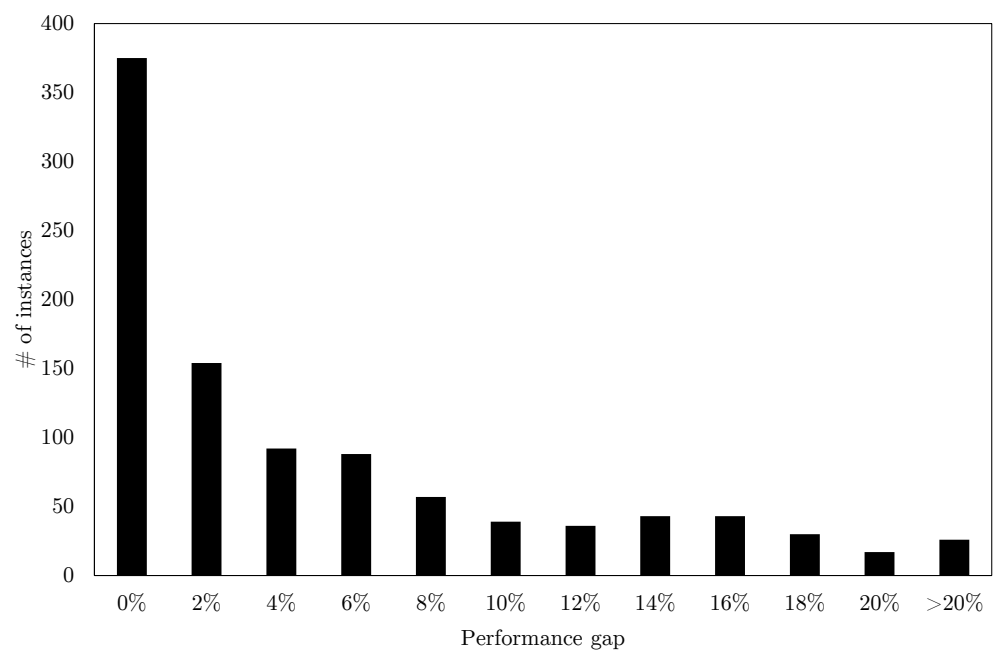

FIGURE 2.11. Histogram showing the deviations between the algorithm and $\pi^{o p t}$, applied on a straight truck network with instances containing 7 orders.

as a decision support tool for human planners at 4PLs, allowing to plan orders using multiple modes and to provide a direct reply to their customers.

To make the algorithm suitable for online planning, we have introduced three key design choices to restrict the search space. We experimentally tested the impact of these design choices by introducing alternative solution methods as benchmarks. First, we assessed the effect of storing only $k$ routes. We have shown that due to decreasing marginal savings, a relatively small number of stored routes already captures the savings potential. Second, we assessed the impact of fixing routes once they contain consolidated orders. We compared our algorithm to a policy in which we solve the static problem at every order arrival. The cost reduction compared to the algorithm remains below $3 \%$. These results indicate which improvements can be achieved by, e.g., extending the algorithm with local search techniques. Finally, we have tested the impact of considering dynamic planning rather than static planning, e.g., the effect of directly planning incoming orders rather than periodically re-optimizing the schedule. For 
this purpose, we solved small instances to optimality, yielding an average performance gap of $4.7 \%$. As we assumed perfect foresight for this benchmark, this provides an upper bound for the results.

For multiple networks, we demonstrated the potential of our solution method to significantly and consistently reduce costs and $\mathrm{CO}_{2}$ emissions, while improving vehicle fill rates. First, we performed numerical experiments on a variety of virtual networks. Compared to direct road transport, on average we save $34 \%$ in costs, reduce $\mathrm{CO}_{2}$ emissions by $30 \%$, and improve the container fill rate by $57 \%$. Compared to the case in which we consolidate on the best routes only, the additional flexibility yields $5 \%$ cost savings, $9 \% \mathrm{CO}_{2}$ reduction, and a $21 \%$ increase in fill rate. Furthermore, we performed a numerical experiment on a real-life 4PL network. For orders transported from the Netherlands to Austria and Italy, consolidated planning notably improves the performance. Compared to consolidation on the best route only (i.e., $k=1$ ), our solution method saves $6 \%$ in costs, $20 \%$ in $\mathrm{CO}_{2}$ emissions, and $28 \%$ in container fill rate. The results indicate that the flexibility introduced by the solution method has the potential to significantly reduce both costs and $\mathrm{CO}_{2}$ emissions, thus being a promising planning tool for 4PLs active in intermodal transport.

The numerical results show that consolidated trips typically contain only one main arc. Detours of over $20 \%$ are common, implying that networks with reloads not necessarily need to offer perfect connections between origin and destination to facilitate consolidated trips. The orders that are most viable for consolidated trips require no more than $40 \%$ transportation unit volume, and have an origin-destination distance of at least $250 \mathrm{~km}$.

In this chapter, we studied a coordinated method to consolidate LTL freight on line-haul networks with reloads. We have shown that for the 4PL, the flexibility to replan routes and alter dispatch times significantly improves transport efficiency. In the next chapter, we will focus on last-mile deliveries within urban areas via an urban consolidation center (UCC). The two chapters in Part II link in various ways. Whereas this chapter addresses consolidation in the line-haul operations, Chapter 3 studies consolidation in the last-mile distribution. When adopting a broad perspective, transport operations take place within a network of cities that have their own UCCs. Clearly, the consolidation decisions made on the line-haul segments will impact the operations of the UCCs. In particular, the decision whether to directly transport orders to their destination or instead use the UCC is important from the perspective of the urban area. Furthermore, replanning orders on the line-haul affects the arrival process at the UCC. Given the flexibility that the $4 \mathrm{PL}$ has to adjust its transportation decisions, one can 
imagine that the arrival process at the UCC is prone to uncertainty. This is particularly the case when the 4PL and UCC operator do not exchange real-time arrival information. Under this uncertainty, the challenge for the UCC is to correctly time the dispatch of orders, such that they can carry out their last-mile deliveries as efficiently as possible. This problem will be addressed in Chapter 3 .

\section{Appendix 2A: Runtime and upper bound analysis}

We provide three theorems and their corresponding proofs. Theorem 2.1 gives an upper bound on the savings for a given route; this result can be used to set $\beta$ such that optimal solutions are guaranteed to be in the search space. Theorem 2.2 provides an upper bound on the savings achievable by consolidating with a given order, which allows to exclude certain orders from the decision tree up front. Finally, Theorem 2.3 gives the computational complexity of evaluating the decision tree (Algorithm 2.4).

Theorem 2.1: Let $C_{a}^{\text {tr }}$ be a concave monotonically increasing cost function of the fill rate $l \in \mathcal{L}$ on arc a. Let $r_{i, n}$ be a route in the tentative routeset $\mathcal{R}_{i}^{\prime}$ andlet $r_{i, n^{\prime}}^{*}$ be the cheapest route in $\mathcal{R}_{i}^{\prime}$. Let $l^{\text {min }}=\min (l \mid l \in \mathcal{L})$ be the smallest possible order size and define $\bar{S}^{*}=M^{\text {max }} \cdot \frac{1}{l^{\text {min }}} \cdot C_{a}^{\text {tr }}\left(l^{\text {min }}\right)-C_{a}^{t r}\left(\left(\frac{1}{l^{\text {min }}}\right) \cdot l^{\text {min }}\right)+\left(\frac{1}{l^{\text {min }}}-\right.$ $1) \cdot\left(c_{v}^{t m}+c_{v^{\prime}}^{t m}\right)$ as the maximum attainable savings for every route. Setting $\beta=$ $\frac{C\left(r_{i, n^{\prime}}^{*}\right)+\bar{S}^{*}}{C\left(r_{i, n^{\prime}}^{*}\right)}$ guarantees that the optimal solution will never be discarded a priori.

Proof: Suppose that $\sum_{\left(i^{\prime}, n^{\prime}\right) \in \hat{\Omega}_{q^{\prime}, a}} l_{i^{\prime}}<1$, such that consolidation remains possible. From the inequality posed in Equation (2.6.1), we see that $\exists \hat{\Omega}_{q, a}^{x}=\hat{\Omega}_{q^{\prime}, a} \cup$ $\Omega_{q, a}$ such that

$$
\begin{array}{r}
C_{a}^{t r}\left(\sum_{\left(i^{\prime}, n^{\prime}\right) \in \hat{\Omega}_{q^{\prime}, a}} l_{i^{\prime}}+\sum_{\left(j^{\prime}, m^{\prime}\right) \in \Omega_{q, a}} l_{j^{\prime}}\right) \leq \\
C_{a}^{t r}\left(\sum_{\left(i^{\prime}, n^{\prime}\right) \in \hat{\Omega}_{q^{\prime}, a}} l_{i^{\prime}}\right)+C_{a}^{t r}\left(\sum_{\left(j^{\prime}, m^{\prime}\right) \in \Omega_{q, a}} l_{j^{\prime}}\right) .
\end{array}
$$


Hence, maximum savings on transport costs are obtained by consolidating to a fill rate of 1 . Recall that with every action $x \in \mathcal{X}_{i, n}$, we consolidate with at most one order set per arc. It follows that before Algorithm 2.4 terminates, we can consolidate with up to $\frac{1}{l^{\text {min }}} \in \mathbb{N}$ orders per arc. By means of deduction, the following inequality holds

$$
\begin{array}{r}
C_{a}^{t r}\left(\frac{1}{l^{\text {min }}} \cdot l^{\text {min }}\right) \leq \\
C_{a}^{t r}\left(\left(\frac{1}{l^{\text {min }}}-1\right) \cdot l^{\text {min }}\right)+C_{a}^{t r}\left(l^{\text {min }}\right) \leq \\
C_{a}^{t r}\left(\left(\frac{1}{l^{\text {min }}}-2\right) \cdot l^{\text {min }}\right)+2 \cdot C_{a}^{t r}\left(l^{\text {min }}\right) \leq \\
\ldots \leq \\
\frac{1}{l^{\text {min }}} \cdot C_{a}^{\text {tr }}\left(l^{\text {min }}\right) .
\end{array}
$$

As every $l \in \mathcal{L}$ may be expressed by $l^{\text {min }} \cdot y$, with $y \in \mathbb{N}$, the upper bound for the savings is $\frac{1}{l^{m i n}} \cdot C_{a}^{t r}\left(l^{\text {min }}\right)-C_{a}^{t r}\left(\frac{1}{l^{m i n}} \cdot l^{\text {min }}\right)$, achieved by consolidating with $\frac{1}{l^{\text {min }}}$ orders of size $l^{\text {min }}$.

Next, we show that for any consolidation action $x$ and any $a \in \mathcal{A}^{M}$, the savings on transshipment costs are $c_{v}^{t m}+c_{v^{\prime}}^{t m}$ :

$$
\begin{array}{r}
\sum_{\left(i^{\prime}, n^{\prime}\right) \in \Omega_{q, a}^{x}}\left(\frac{c_{v}^{t m}}{\left|\hat{\Omega}_{q, a}^{x}\right|}+\frac{c_{v^{\prime}}^{t m}}{\left|\hat{\Omega}_{q, a}^{x}\right|}\right)= \\
c_{v}^{t m}+c_{v^{\prime}}^{t m} \leq \\
2 \cdot\left(c_{v}^{t m}+c_{v^{\prime}}^{t m}\right)= \\
\sum_{\left(i^{\prime}, n^{\prime}\right) \in \hat{\Omega}_{q^{\prime}, a}}\left(\frac{c_{v}^{t m}}{\left|\hat{\Omega}_{q^{\prime}, a}\right|}+\frac{c_{v^{\prime}}^{t m}}{\left|\hat{\Omega}_{q^{\prime}, a}\right|}\right)+\sum_{\left(j^{\prime}, m^{\prime}\right) \in \Omega_{q, a}}\left(\frac{c_{v}^{t m}}{\left|\Omega_{q, a}\right|}+\frac{c_{v^{\prime}}^{t m}}{\left|\Omega_{q, a}\right|}\right) .
\end{array}
$$

Hence, the savings on transhipment costs on any arc when consoidating two route sets are $c_{v}^{t m}+c_{v^{\prime}}^{t m}=2 \cdot\left(c_{v}^{t m}+c_{v^{\prime}}^{t m}\right)-\left(c_{v}^{t m}+c_{v^{\prime}}^{t m}\right)$. It readily follows that we maximize savings on transshipment costs by consolidating with as many orders as possible, i.e., we again only consolidate with orders of size $l^{\text {min }}$, thereby saving $\left(\frac{1}{l^{m i n}}-1\right) \cdot\left(c_{v}^{t m}+c_{v^{\prime}}^{t m}\right)$. 
Theorem 2.2 states that an upper bound on savings is found by consolidation on all main arcs in a route. Let $M^{\max } \leftrightarrow \mathbb{N}$ be the maximum number of main arcs we allow in a route, and let $a^{*} \in \mathcal{A}^{M}$ be the arc that yields the highest upper bound on savings, obtained by solving

$$
\begin{gathered}
a^{*} \underset{a \in \mathcal{A}^{M}}{\operatorname{argmax}}\left(\left(\frac{1}{l^{\text {min }}}\right) \cdot C_{a}^{t r}\left(l^{\text {min }}\right)-C_{a}^{t r}\left(\frac{1}{l^{\text {min }}} \cdot l^{\text {min }}\right)\right. \\
\left.+\left(\frac{1}{l^{\text {min }}}-1\right) \cdot\left(c_{v}^{t m}+c_{v^{\prime}}^{t m}\right)\right) .
\end{gathered}
$$

We denote the corresponding savings (representing the upper bound on savings for any route) as

$$
\begin{aligned}
\bar{S}^{*}= & M^{\max } \cdot \frac{1}{l^{\min }} \cdot C_{a}^{t r}\left(l^{\text {min }}\right)-C_{a}^{t r}\left(\left(\frac{1}{l^{\text {min }}}\right) \cdot l^{\text {min }}\right) \\
& +\left(\frac{1}{l^{\text {min }}}-1\right) \cdot\left(c_{v}^{t m}+c_{v^{\prime}}^{t m}\right) .
\end{aligned}
$$

It follows that if $C\left(r_{i, n}\right) \geq C\left(r_{i, n^{\prime}}^{*}\right)+\bar{S}^{*}$, the potential consolidation gains for $r_{i, n}$ will never suffice to obtain a solution with lower costs than $r_{i, n^{\prime}}^{*}$. Thus, the value for $\beta$ that never excludes improving solutions is given by

$$
\beta=\frac{C\left(r_{i, n^{\prime}}^{*}\right)+\bar{S}^{*}}{C\left(r_{i, n^{\prime}}^{*}\right)}
$$

Theorem 2.2: For all $a \in \mathcal{A}$, let $C_{a}^{\text {tr }}$ be a concave, monotonically increasing cost function of the fill rate $l \in\left\{\frac{1}{y}, \ldots, 1\right\}$, with $y \in \mathbb{N}$. Let $i$ be an order which we seek to consolidate with $j \in \mathcal{J}$. Let $x$ be the action that consolidates on all $a \in r_{i, n} \cap r_{j, m}$, and let $r_{j^{\prime}, m^{\prime \prime}}^{*}$ be the cheapest route for order $j$ before consolidation. The upper bound on savings by consolidating on the routes $r_{i, n}$ and $r_{j, m}$ is given by 


$$
\begin{aligned}
& \bar{S}_{j, m}=\sum_{a \in r_{i, n} \cap r_{j, m}}\left(C_{a}^{t r}\left(\sum_{\left(i^{\prime}, n^{\prime}\right) \in \hat{\Omega}_{q^{\prime}, a}} l_{i^{\prime}}\right)+\right. \\
& \left.C_{a}^{t r}\left(\sum_{\left(j^{\prime}, m^{\prime}\right) \in \Omega_{q, a}} l_{j^{\prime}}\right)-C_{a}^{t r}\left(\sum_{\left(i^{\prime}, n^{\prime}\right) \in \hat{\Omega}_{q, a}^{x}} l_{i^{\prime}}\right)\right)+ \\
& \sum_{a \in r_{i, n}}\left(\frac{c_{v}^{t m}}{\left|\hat{\Omega}_{q, a}\right|}+\frac{c_{v^{\prime}}^{t m}}{\left|\hat{\Omega}_{q, a}\right|}+\frac{c_{v}^{t m}}{\left|\Omega_{q, a}\right|}+\frac{c_{v^{\prime}}^{t m}}{\left|\Omega_{q, a}\right|}-\frac{c_{v}^{t m}}{\left|\hat{\Omega}_{q, a}^{x}\right|}-\frac{c_{v^{\prime}}^{t m}}{\left|\hat{\Omega}_{q, a}^{x}\right|}\right)- \\
& \sum_{\left(j^{\prime}, m^{\prime}\right) \in x}\left(C\left(r_{j^{\prime}, m^{\prime}}\right)-C\left(r_{j^{\prime}, m^{\prime \prime}}^{*}\right)\right)- \\
& |x| \cdot \frac{1}{\left|\Omega_{q, a}\right| \cdot\left|r_{i, n} \cap \mathcal{A}^{M}\right|}\left(C\left(r_{i, n}\right)-C\left(r_{i, n^{\prime}}^{*}\right)\right)- \\
& \min _{a \in r_{i, n} \cap r_{j, m}} 2 \cdot C^{r l}(a) \text {. }
\end{aligned}
$$

Proof: We first show that maximum savings in transshipment costs are attained when consolidating on all mutual arcs $a \in r_{i, n} \cap r_{j, m^{\prime}}$. Depending on possible previous consolidations of order $j$, for all mutual arcs we have a set of consolidated orders $\Omega_{q, a}$, with $(j, m) \in \Omega_{q, a}$. To define the set of orders currently consolidated with order $i$ on $\operatorname{arc} a$, we have $\hat{\Omega}_{q^{\prime}, a}$. Finally, we denote the set of orders after consolidating orders $i$ and $j$ as $\hat{\Omega}_{q, a}^{x}=\hat{\Omega}_{q^{\prime}, a} \cup \Omega_{q, a}$. Clearly, $\left|\hat{\Omega}_{q^{\prime}, a}\right| \leq\left|\hat{\Omega}_{q, a}^{x}\right|$. Given that $c_{v}^{t m} \geq 0, \forall v \in \mathcal{V}^{H}$, the following inequality holds $\forall a \in r_{i, n} \cap r_{j, m}$, such that overall transshipment costs are minimized by consolidating on all mutual arcs: 


$$
\begin{array}{r}
\frac{c_{v}^{t m}}{\left|\hat{\Omega}_{q, a}^{x}\right|}+\frac{c_{v^{\prime}}^{t m}}{\left|\hat{\Omega}_{q, a}^{x}\right|} \leq \\
\frac{c_{v}^{t m}}{\left|\hat{\Omega}_{q, a}\right|}+\frac{c_{v^{\prime}}^{t m}}{\left|\hat{\Omega}_{q, a}\right|} \leq \\
\frac{c_{v}^{t m}}{\left|\hat{\Omega}_{q^{\prime}, a}\right|}+\frac{c_{v^{\prime}}^{t m}}{\left|\hat{\Omega}_{q^{\prime}, a}\right|}+\frac{c_{v}^{t m}}{\left|\Omega_{q, a}\right|}+\frac{c_{v^{\prime}}^{t m}}{\left|\Omega_{q, a}\right|} .
\end{array}
$$

We show that the lowest transport costs are also achieved by consolidating on all mutual arcs. To show this, we make use of the fact that the function $C_{a}^{t r}(l), \forall a \in \mathcal{A}$ is concave and monotonically increasing. Given these properties, the following inequality is satisfied when substituting the fill rates of all orders involved (before and after consolidation) into Equation (2.6.2):

$$
\begin{aligned}
& \sum_{\left(i^{\prime}, n^{\prime}\right) \in \hat{\Omega}_{q, a}^{x}, a} \frac{l_{i^{\prime}}}{\sum_{\left(i^{\prime \prime}, n^{\prime \prime}\right) \in \hat{\Omega}_{q, a}^{x}} l_{i^{\prime \prime}}} C_{a}^{t r}\left(\sum_{\left(i^{\prime \prime}, n^{\prime \prime}\right) \in \hat{\Omega}_{q, a}^{x}} l_{i^{\prime \prime}}\right)= \\
& 1 \cdot C_{a}^{t r}\left(\sum_{\left(i^{\prime \prime}, n^{\prime \prime}\right) \in \hat{\Omega}_{q, a}^{x}} l_{i^{\prime \prime}}\right) \leq \\
& 1 \cdot C_{a}^{t r}\left(\sum_{\left(i^{\prime \prime}, n^{\prime \prime}\right) \in \hat{\Omega}_{q^{\prime}, a}} l_{i^{\prime \prime}}\right)+1 \cdot C_{a}^{t r}\left(\sum_{\left(j^{\prime \prime}, m^{\prime \prime}\right) \in \Omega_{q, a}} l_{j^{\prime \prime}}\right)= \\
& \sum_{\left(i^{\prime}, n^{\prime}\right) \in \hat{\Omega}_{q^{\prime}, a}} \frac{l_{i^{\prime}}}{\sum_{\left(i^{\prime \prime}, n^{\prime \prime}\right) \in \hat{\Omega}_{q^{\prime}, a}} l_{i^{\prime \prime}}} C_{a}^{t r}\left(\sum_{\left(i^{\prime \prime}, n^{\prime \prime}\right) \in \hat{\Omega}_{q^{\prime}, a}} l_{i^{\prime \prime}}\right)+ \\
& \sum_{\left(j^{\prime}, m^{\prime}\right) \in \Omega_{q, a}} \frac{l_{j^{\prime}}}{\sum_{\left(i^{\prime \prime}, n^{\prime \prime}\right) \in \Omega_{q, a}} l_{j^{\prime \prime}}} C_{a}^{t r}\left(\sum_{\left(j^{\prime \prime}, m^{\prime \prime}\right) \in \Omega_{q, a}} l_{j^{\prime \prime}}\right) .
\end{aligned}
$$


We showed that both transport costs and transshipment costs are minimized by consolidating on all $a \in r_{i, n} \cap r_{j, m}$. By substituting the corresponding action into Equation (2.6.6), we would get an upper bound that deducts the cost differences $C\left(r_{j, m}\right)-C\left(r_{j, m^{\prime}}^{*}\right)$ and $C\left(r_{i, n}\right)-C\left(r_{i, n^{\prime}}^{*}\right)$ from the maximum savings. However, the latter term cannot be fully allocated to $\bar{S}_{j, m}$. Let $w_{j^{\prime}, m^{\prime}} \in$ $[0,1], \forall\left(j^{\prime}, m^{\prime}\right) \in x$ be an allocation weight, subject to $\sum_{\left(j^{\prime}, m^{\prime}\right) \in x} w_{j^{\prime}, m^{\prime}} \leq 1$. We show that this allocation constraint is necessary to guarantee that $S(x) \leq$ $\sum_{\left(j^{\prime}, m^{\prime}\right) \in x} \bar{S}_{j^{\prime}, m^{\prime}}$ for any action $x$. For the sake of our proof by contradiction, suppose that $\sum_{\left(j^{\prime}, m^{\prime}\right) \in x} w_{j^{\prime}, m^{\prime}}>1$, and that $\Omega_{q, a} \cap \Omega_{q^{\prime}, a^{\prime}}=\emptyset, \forall a, a^{\prime} \in r_{i, n}, a \neq a^{\prime}$ (i.e., we consolidate with a unique order set on every main $\operatorname{arc}$ in $r_{i, n}$ ). The following inequality then holds - implying that the sum of upper bounds on savings are lower than the actual savings obtained by solving Equation (2.6.6) - which is a contradiction.

$$
\begin{aligned}
& \sum_{\left(j^{\prime}, m^{\prime}\right) \in x} \bar{S}_{j^{\prime}, m^{\prime}}= \\
& \sum_{a \in r_{i, n}}\left(C_{a}^{t r}\left(\sum_{\left(i^{\prime}, n^{\prime}\right) \in \hat{\Omega}_{q^{\prime}, a}} l_{i^{\prime}}\right)+\right. \\
& C_{a}^{t r}\left(\sum_{\left(j^{\prime}, m^{\prime}\right) \in \Omega_{q, a}} l_{j^{\prime}}\right)-C_{a}^{t r}\left(\sum_{\left(i^{\prime}, n^{\prime}\right) \in \hat{\Omega}_{q, a}^{x}} l_{i^{\prime}}\right)+ \\
& \sum_{a \in r_{i, n}}\left(\frac{c_{v}^{t m}}{\left|\hat{\Omega}_{q^{\prime}, a}\right|}+\frac{c_{v^{\prime}}^{t m}}{\left|\hat{\Omega}_{q^{\prime}, a}\right|}+\frac{c_{v}^{t m}}{\left|\Omega_{q, a}\right|}+\frac{c_{v^{\prime}}^{t m}}{\left|\Omega_{q, a}\right|}-\frac{c_{v}^{t m}}{\left|\hat{\Omega}_{q, a}^{x}\right|}-\frac{c_{v^{\prime}}^{t m}}{\left|\hat{\Omega}_{q, a}^{x}\right|}\right)- \\
& \sum_{\left(j^{\prime}, m^{\prime}\right) \in x}\left(C\left(r_{j^{\prime}, m^{\prime}}\right)-C\left(r_{j^{\prime}, m^{\prime \prime}}^{*}\right)\right)-\sum_{\left(j^{\prime}, m^{\prime}\right) \in x} w_{j^{\prime}, m^{\prime}}\left(C\left(r_{i, n}\right)-C\left(r_{i, n^{\prime}}^{*}\right)\right) \leq \\
& S(x) .
\end{aligned}
$$

We have established that $\sum_{\left(j^{\prime}, m^{\prime}\right) \in x} w_{j^{\prime}, m^{\prime}} \leq 1$ must hold to establish a feasible upper bound. In theory, we could freely allocate the weights $w_{j^{\prime}, m^{\prime}}$, as long as this property is satisfied. However, to guarantee that the sum of $w_{j^{\prime}, m^{\prime}}$ for 
independently established upper bounds never exceeds 1, without loss of generality we set $w_{j, m}=\frac{1}{\left|\Omega_{q, a}\right| \cdot\left|r_{i, n} \cap \mathcal{A}^{M}\right|}$. From this definition, it follows that for any $x$, the inequality $S(x) \leq \sum_{\left(j^{\prime}, m^{\prime}\right) \in x} \bar{S}_{j^{\prime}, m^{\prime}}$ holds.

Finally, we relax the assumption that reload costs are 0 . If we have $C^{r l}\left(r_{i, n}\right) \geq$ 0 , it is no longer guaranteed that maximum savings are attained by consolidating on all mutual main arcs. However, we know that for any $x$ with $(j, m) \in x$, at least two reloads are required, such that the minimum reload costs are given by $\operatorname{argmin}_{a \in r_{i, n} \cap r_{j, m}} 2 \cdot C^{r l}(a)$. Hence, when including reload costs, we obtain the upper bound. With this proof, we establish an upper bound on the maximum savings that can be attained by consolidating orders $i$ and $j$ using routes $r_{i, n} \in R_{i}$ and $r_{j, m} \in R_{j}$.

Theorem 2.3 Let $R_{i}$ be the route set corresponding to order $i$ which we seek to consolidate, $\mathcal{J}$ be the set of open orders, $k$ be the number of routes stored per order, and $l^{\text {min }} \in(0,1]$ be the smallest possible load size. Take $r_{i, n} \in R_{i}$ such that $\left|r_{i, n}\right| \geq\left|r_{i, n^{\prime}}\right|, \forall r_{i, n^{\prime}} \in R_{i}$. Algorithm 2.4 has a worst-case complexity of $\mathcal{O}\left(k \cdot \frac{1}{l^{m i n}} \cdot(|\mathcal{J}| \cdot k)^{\left|r_{i, n}\right|}\right)$.

Proof: Recall that the set of actions is defined as $\mathcal{X}_{i, n}=\prod_{z=1}^{\left|r_{i, n}\right|} \mathcal{S}_{i, n, z}$, with $\mathcal{S}_{i, n, z}$ being the evaluation set for arc $a_{i, n, z}$. Clearly an order cannot be allocated to more than one container on an arc, such that for every order-route combination per arc, there exists at most one consolidation opportunity $\Omega_{q, a} \ni(j, m)$. As we have an open order set of size $|\mathcal{J}|$ and up to $k$ routes per order, it follows that there are at most $|\mathcal{J}| \cdot k \geq \mathcal{S}_{i, n, z}$ opportunities per arc $a_{i, n, z} \in r_{i, n}$ to consolidate order $i$ with some order $j \in \mathcal{J}$. As route $r_{i, n}$ contains $\left|r_{i, n}\right|$ arcs, a decision tree contains up to $(|\mathcal{J}| \cdot k)^{\left|r_{i, n}\right|}$ actions. For every decision tree, we consolidate with at most one order set per arc, hence we must evaluate up to $\frac{1}{l^{\text {min }}}$ decision trees per route $r_{i, n^{\prime}} \in R_{i}$. The same procedure is repeated for all $r_{i, n^{\prime}} \in R_{i}$, hence computational effort increases linearly with the number of routes $\left|R_{i}\right|=k$. Hence, the worst-case complexity for Algorithm 2.4 is given by $\mathcal{O}\left(k \cdot \frac{1}{l^{\text {min }}} \cdot(|\mathcal{J}| \cdot k)^{\left|r_{i, n}\right|}\right)$.

\section{Appendix 2B: Cost functions and savings}

In this appendix, we provide detailed cost functions for a route $r_{i, n}$, adopting a generic notation that handles both consolidated and unconsolidated orders. The transport costs are given by 


$$
C^{t r}(i, n)=\sum_{a_{i, n, z} \in r_{i, n}} \frac{l_{i}}{\sum_{\left(i^{\prime}, n^{\prime}\right) \in \hat{\Omega}_{q, a_{i, n, z}^{x}}} l_{i^{\prime}}} C_{a}^{t r}\left(\sum_{\left(i^{\prime}, n^{\prime}\right) \in \hat{\Omega}_{q, a_{i, n, z}}^{x}} l_{i^{\prime}}\right) .
$$

Transshipment costs are shared by all orders in the same container. From a system point of view, transshipment costs can be arbitrarily allocated to orders transported in the same container. We therefore equally distribute them over all orders in a container:

$$
C^{t m}(i, n)=\sum_{z=1}^{\left|r_{i, n}\right|} \frac{c_{v}^{t m}}{\left|\hat{\Omega}_{q, a_{i, n, z}}^{x}\right|}+\frac{c_{v^{\prime}}^{t m}}{\left|\hat{\Omega}_{q, a_{i, n, z}}^{x}\right|} .
$$

To ease the notation of the reload costs, we first provide the cost formula per $a_{i, n, z} \in r_{i, n}, z<\left|r_{i, n}\right|:$

$$
C_{a}^{r l}\left(a_{i, n, z}\right)= \begin{cases}c_{v^{\prime}}^{r l} & \text { if }\left|\hat{\Omega}_{q, a_{i, n, z}}^{x} \cap \hat{\Omega}_{q, a_{i, n} z+1}^{x}\right|=1 \\ & \max \left(\left|\hat{\Omega}_{q, a_{i, n, z+1}}^{x}\right|,\left|\hat{\Omega}_{q, a_{i, n, z+1}}^{x}\right|\right)>1 \\ 0 & \text { otherwise } \\ . & \end{cases}
$$

The total reload costs for order $i$ are given by

$$
C^{r l}(i, n)=\sum_{a_{i, n, z} \in r_{i, n}, z<\left|r_{i, n}\right|} C_{a}^{r l}\left(a_{i, n, z}\right) .
$$

Finally, we define $S(x)$ as the savings of an action $x \in \mathcal{X}_{i, n}$. To account for the cost differences between routes for the same order, let $r_{i, n^{\prime}}^{*} \in R_{i}, r_{j, m^{\prime}}^{*} \in R_{j}$, be such that $C\left(r_{i, n^{\prime}}^{*}\right) \leq C\left(r_{i, n}\right), \forall r_{i, n} \in R_{i}$ and $C\left(r_{j, m^{\prime}}^{*}\right) \leq C\left(r_{j, m}\right), \forall r_{j, m} \in R_{j}$. The savings for an action $x \in \mathcal{X}_{i, n}$ are defined as 


$$
\begin{aligned}
& S(x)= \\
& \sum_{a_{i, n, z} \in r_{i, n}}\left(C_{a}^{t r}\left(\sum_{\left(i^{\prime}, n\right) \in \hat{\Omega}_{q^{\prime}, a_{i, n, z}}} l_{i^{\prime}}\right)+C_{a}^{t r}\left(\sum_{\left(j^{\prime}, m\right) \in \Omega_{q, a_{i, n, z}}} l_{j^{\prime}}\right)\right. \\
& \left.-C_{a}^{t r}\left(\sum_{\left(i^{\prime}, n\right) \in \hat{\Omega}_{q, a_{i, n, z}^{x}}^{x}} l_{i^{\prime}}\right)\right)+ \\
& \sum_{a_{i, n, z} \in r_{i, n}}\left(\frac{c_{v}^{t m}}{\left|\hat{\Omega}_{q^{\prime}, a_{i, n, z}}\right|}+\frac{c_{v^{\prime}}^{t m}}{\left|\hat{\Omega}_{q^{\prime}, a_{i, n, z}}\right|}+\frac{c_{v}^{t m}}{\left|\Omega_{q, a_{i, n, z}}\right|}+\frac{c_{v^{\prime}}^{t m}}{\left|\Omega_{q, a_{i, n, z}}\right|}\right. \\
& \left.-\frac{c_{v}^{t m}}{\left|\hat{\Omega}_{q, a_{i, n, z}}^{x}\right|}-\frac{c_{v^{\prime}}^{t m}}{\left|\hat{\Omega}_{q, a_{i, n, z}}^{x}\right|}\right)- \\
& C^{r l}\left(r_{i, n}\right)-\left(C\left(r_{i, n}\right)-C\left(r_{i, n^{\prime}}^{*}\right)\right)-\sum_{(j, m) \in x}\left(C\left(r_{j, m}\right)-C\left(r_{j, m^{\prime}}^{*}\right)\right)
\end{aligned}
$$




\section{CHAPTER 3}

\section{Last-mile dispatch decision problem}

In this chapter ${ }^{\llbracket}$, we address the last-mile dispatch decision problem that the operator of a UCC faces. As shown in the previous chapter, the flexibility to dynamically adjust the departure times can significantly improve the transport efficiency on the line-haul. With the development of the physical internet, the degree of flexibility in transport is expected to further increase. However, the freedom to re-plan poses challenges for the last-mile distribution. If the exact arrival times of orders are unknown, the UCC operator needs to deal with orders that arrive according to a random process. To efficiently execute the lastmile distribution, the UCC operator should consider vehicle availability, current inventory, and expected order arrivals to schedule the urban transport as efficiently as possible over time. We represent this optimization problem over time as a Markov decision model. A solution model based on approximate dynamic programming is proposed to solve realistic-sized instances of this problem.

This chapter is structured as follows. Section 3.1 gives an introduction and motivation to the problem. Section 3.2 provides a literature review on the DDP and other related works. We specifically focus on transportation problems dealing with both dynamic and stochastic properties. Section 3.3 gives a formal definition of the DDP-TW and the corresponding decision problem, which is modeled as a Markov Decision Problem. Section 3.4 elaborates on our solution method. The setup of the numerical experiments is given in Section 3.5. The corresponding results - including managerial implications - are discussed in Section 3.6. Finally, we present the conclusions of our work in Section 3.7.

\footnotetext{
${ }^{1}$ This chapter is based on the papers: W.J.A. van Heeswijk, M.R.K. Mes, \& J.M.J. Schutten (2015). An approximate dynamic programming approach to urban freight distribution with batch arrivals Lecture Notes in Computer Science 9335, 61-75 (Van Heeswijk et al., 2015) and W.J.A. van Heeswijk, M.R.K. Mes, \& J.M.J. Schutten (2017). The delivery dispatching problem with time windows for urban consolidation centers. Transportation Science, 1-37 (Van Heeswijk et al., 2017b)
} 


\subsection{Introduction}

This chapter studies the dispatch decision that a UCC operator faces, when dispatching orders at discrete decision moments. At each decision moment, the operator must decide on the composition of order batches to dispatch. Cost minimization requires that vehicles are used as efficiently as possible. Therefore, the operator has an incentive to wait for future orders to arrive, which may result in better consolidation opportunities and more efficient tours. However, in real-life settings, the order arrival process is subject to replanning and disruptions within the supply chain. The degree of communication between the actors within the supply chain dictates how accurate the information on future arrivals is. Furthermore, dispatch decisions are generally bound by time windows, as well as vehicle availability and storage capacity. The operator faces complete or partial uncertainty in the arrival process, and wants to determine the optimal consolidation policy that minimizes the costs under this uncertainty.

We consider a setting in which arriving orders are dynamically revealed to the operator. Orders either arrive at the consolidation center without advance notice, or are pre-announced to arrive at a future point in time. Once orders arrive at the consolidation center, they can be dispatched to the customers in the city. Arriving orders may have a large variety in destinations, dispatch windows, load sizes, etc. With the dispatch decisions, the operator aims to minimize costs by consolidating orders based on these properties, striving for high utilization of vehicle capacity and minimal travel distance. Based on both the available knowledge regarding current orders and the anticipation of new orders, the operator is able to make informed consolidation decisions.

To formalize the problem, we extend the Delivery Dispatching Problem (commonly abbreviated to DDP) by including time windows. We will refer to this extension as the DDP with time windows, or DDP-TW. The absence of time windows is a major shortcoming in the traditional DDP; time windows are an integral component of logistics in general, and perhaps even more so in an urban logistics context. Shipment consolidation policies stemming from the traditional DDP indicate when the full accumulated set of orders should be dispatched. However, when orders are subject to time windows, one tends to dispatch orders with distinct time windows at different times, holding on to some orders for a later dispatch time. Hence, in this setting the decision maker wants to know which subset of orders to dispatch. Therefore, our extension to the DDP provides a better fit with real-life consolidation problems, allowing for applications to dispatching problems in general. 
The dynamic and stochastic nature of our DDP is modeled by means of a Markov decision model. Solving instances of this model provides an optimal consolidation policy. In line with the DDP definition as provided by Minkoff (1993), we explicitly separate the dispatch decision from the routing decision. Although the focus of this chapter is on the dispatch decision, we also demonstrate an integrated method that embeds the routing decision. We apply a cost function that estimates the transportation costs for dispatching a given set of orders. After determining which orders to dispatch, a VRP algorithm may subsequently be applied to obtain detailed delivery tours. The objectives of this chapter are to formally define the DDP with dispatch windows, to design an algorithm that can handle large instances for this problem class, and to apply it to an urban logistics context.

\subsection{Literature on dynamic and stochastic dispatching problems}

We present a literature overview that includes studies on the DDP and several related problems. We refer to recent literature studies for these related problems, and highlight a number of studies that address characteristics comparable to our problem. We discuss various solution approaches to transport problems with dynamic and stochastic properties, and evaluate their suitability to solve the DDP-TW. We conclude with the literature gap that we address.

Optimization problems that embed both stochastic and dynamic properties are notoriously hard to solve (Powell et al., 2012). Even though stochastic information is recognized as an integral aspect of optimization in transport problems, the majority of papers in the transport literature focus on deterministic problems. Traditionally, mathematical programming and (meta)heuristics are used to handle high-dimensional transport problems. However, these methods generally do not cope well with stochastic information being revealed over time (Powell et al., 2012). Successful incorporation of stochastic information in solution methods is still an ongoing development (Powell et al., 2012; SteadieSeifi et al., 2014). Suitable solution methods are usually based on either (i) approximating a value function that uses analytical expressions to define the stochastic process or (ii) sampling scenarios and solving the decision problems for the corresponding outcomes (Lium et al., 2009; Pillac et al., 2013). The latter method is generally applied to extend heuristics and mathematical programs designed for deterministic problems towards stochastic problems. A key challenge with scenario sampling is to correctly represent the stochastic process; incorrect sampling may yield poor results (Lium et al., 2009). When approximating a value function, the stochastic process is fully defined, but the corresponding 
expected values may be hard to compute. These expected values therefore tend to be computed offline; the resulting policies can subsequently be used in online decision-making. As these policies return decisions as a function of the input state, they tend to be faster than online sampling methods.

In the DDP, order arrivals follow a stochastic process and are dispatched in batches at a given decision moment (Minkoff, 1993). The aim of solving the DDP is to find a consolidation policy that returns the optimal dispatch time for an accumulated set of orders. With every new arrival, the decision maker assesses (i) the time elapsed since the first order in inventory arrived (representing the maximum service delay, as experienced by the customer), and (ii) the volume of the accumulated orders. The consolidation policy is based on one or both of these measures. Dispatching the accumulated orders is subject to a cost function, in which route duration and route costs are pre-defined input (Minkoff, 1993). For our DDP-TW, we study recurrent consolidation policies, meaning that the dispatching decision depends on the state of the problem (Higginson and Bookbinder, 1995; Powell, 2011). The stochastic and dynamic elements embedded in such DDPs give rise to the use of Markov decision models (Minkoff, 1993). Although Markov decision models are useful to define decision problems, practical implementations generally suffer from intractably large state spaces and expected values that cannot be calculated exactly (Minkoff, 1993; Powell, 2011; Pillac et al., 2013). Çetinkaya (2005) provides an overview on DDP literature, although describing the problem class as inventory and shipment consolidation decisions'. Relatively few studies address the optimization of consolidation policies; instead most studies focus on evaluating existing consolidation policies (Çetinkaya, 2005; Mutlu et al., 2010; Bookbinder et al., 2011). Studies that do address optimization tend to consider only volume and arrival time as order properties, and present results that are valid only for a limited set of distributions. Çetinkaya and Bookbinder (2003) derive results on optimal policies that are either quantity-based or time-based, but do not combine both measures into a hybrid policy. Bookbinder et al. (2011) describe a generic solution method based on a batch Markovian arrival process, which is able to cope with a multitude of distributions. However, all transition probabilities must be computed to describe the arrival process. This is computationally challenging when considering orders with multiple stochastic properties. Finally, Cai et al. (2014) present an algorithm that - under the assumption of a specific cost structure returns an optimal consolidation policy. The drawback of this algorithm is that every state must be visited, thus it requires the state space to be sufficiently small to fully enumerate. 
We now look at various problem classes related to the DDP-TW. The Vehicle Routing Problem (VRP) is concerned with routing rather than dispatching decisions. Despite this key distinction, it has some overlap with the DDP-TW. A common characteristic is that both problems are customer-driven. Assigning time windows to orders is common in VRPs. However, in a VRP the time windows usually only affect the routing decision, not the dispatching decision as in the DDP-TW. Ritzinger et al. (2016) provide a literature overview of the dynamic and stochastic VRP, which generally considers re-optimization during the execution of routes. The VRP variant that has the strongest relation to our DDP-TW combines dynamic order requests with stochastic customer information. Examples of works that address this variant are Simão et al. (2009) and Ulmer et al. (2015).

Also the Inventory Routing Problem (IRP) has ties to the DDP (Minkoff, 1993). The IRP addresses repeated stock replenishment from a facility to a fixed set of customer locations, the product quantities to deliver, and the decision when to visit a location. The facility optimizes these decisions, given the - possibly stochastic - depletion rates at the customers. The dispatching decision is an integral part of the IRP. A key distinction between the DDP and the IRP is that the latter is generally not order-based. This implies that the facility is not subject to an inbound arrival process, and goods are typically not coupled to individual customers. Furthermore, only one or several types of goods have to be distributed along the customers. For the solution of stochastic IRPs, generally either mathematical programming is applied, or Markov decision models are heuristically solved. We mention a few IRP studies that handle both dynamic and stochastic properties. An example is the study of Coelho et al. (2012), who propose a heuristic solution with scenario sampling for this IRP class. Bertazzi et al. (2013) formulate the stochastic IRP as a Markov decision model, and solve the associated decision problem with a hybrid rollout algorithm. An overview of the IRP literature can be found in Coelho et al. (2014).

The next related problem class that we address is Service Network Design (SND). SND entails the selection and timing of transport services. Known solution methods mostly use mathematical programming, (meta)heuristics, and graph theory. The majority of SND studies focus on deterministic instances. SteadieSeifi et al. (2014) mention a number of SND studies that do incorporate stochastic demand, e.g, Lium et al. (2009) and Meng et al. (2012). Solutions to the stochastic SND generally are multi-stage mathematical programs, in which scenarios are added to reflect uncertainty in future demand. Lium et al. (2009) 
state that generating a compact yet representative scenario tree is one of the key challenges in this solution method.

Finally, we discuss the emerging problem class of same-day deliveries. In same-day delivery problems, the decision maker dynamically receives order requests during the day, and must decide when to dispatch a vehicle that delivers (a subset of) the accumulated orders. If a request is not fulfilled during the day, a penalty is incurred. Voccia et al. (2015) define a corresponding Markov decision model and propose a solution method based on scenario-sampling. Arslan et al. (2016) solve a ride-sharing variant of the problem using a rolling horizon approach. Klapp et al. (2016) study a single-vehicle variant of this problem, where the customers are located on a line (as a consequence, route duration depends only on the customer located farthest away from the depot). They propose both a rollout algorithm and a solution based on approximate linear programming to address the stochasticity embedded in the problem.

The contribution of this chapter is threefold. First, we formulate a Markov decision model for the DDP-TW, in this way formally defining the time-window extension of the DDP.Second, we develop a solution method to solve large instances of this problem class. Based on the stochastic modeling frameworks of Topaloglu and Powell (2006) and Powell (2011) for dynamic resource-allocation problems, we develop an algorithm based on Approximate Dynamic Programming (ADP) to solve the DDP-TW. The main contribution is the design of our value function approximation, while also reflecting on several implementation issues that are encountered in the design phase. In particular, we contribute to literature by studying a variety of basis functions that can be used to estimate the downstream costs of decisions. Third, we formulate an Integer Linear Program (ILP) to solve the decision problem within the ADP algorithm. With the ILP, we find approximate solutions when the embedded decision problem cannot be enumerated within reasonable time.

\subsection{Problem formulation}

This section introduces the decision problem, which we model as a Markov decision model. We aim to provide a generic formulation, such that it can be applied to a variety of instances. We assume that the characteristics of arriving orders are stochastic and have a probability distribution that is known to the UCC operator. When an order is pre-announced or arrives at the center, its exact properties are revealed. Certain variables that are treated as stochastic in our description may be known in practice. In that case, the random variable can simply be replaced by the actual information regarding future orders, creating 
a restricted instance of the problem. We optimize over a finite set of decision moments $\mathcal{T}=\{0,1, \ldots, T\}$, which are separated by equidistant time intervals. The dispatching decisions are made at decision moments $t \in \mathcal{T}$. The equidistant time intervals that separate the decision moments correspond to a setting in which the consolidation center dispatches orders a few times a day. This choice is motivated by the common practice that consolidation centers often make two delivery rounds per day (Bohne et al., 2015; Nesterova and Quak, 2015). The practice of dispatching vehicles at fixed times may be reinforced by access time restrictions set by local governments, only allowing trucks within the city center during one or two time intervals per day (Nuzzolo et al., 2012). We stress that the aforementioned motivation applies to modeling decision moments at pre-defined times, not to the actual practice of commencing delivery tours at each decision moment. The model that we define does not require to dispatch orders at every decision moment. Furthermore, our model does not place any restrictions on the length of time intervals between decision moments.

The following properties are modeled as random variables in the problem: the number of orders arriving per decision moment, order volumes, order destinations, and the dispatch window. Every order must be shipped within its dispatch window. We choose to use dispatch windows at the consolidation center, rather than the more common delivery windows at the receiver. The main reason for this choice is that it simplifies the presentation of the problem. In a typical urban logistics setting, adopting dispatch windows rather than delivery windows will not pose problems. Urban deliveries are characterized by relatively short travel times; when time intervals between dispatching moments are set relatively large, a feasible delivery plan can generally be constructed within this interval. In addition, Cherrett et al. (2012) state that the majority of independent retailers (the typical target customers for an urban consolidation center) have no influence on the exact delivery times. Nevertheless, we stress that delivery windows can be handled within the solution method; in Section 3.3.5 we provide suggestions for this extension.

At every decision moment $t \in \mathcal{T}$, we must choose which subset of the accumulated orders to dispatch. To take into account the impact of this decision on the future, we evaluate the effects of postponing the dispatch of orders. It may be possible to combine postponed orders into a dispatch batch with future orders, thereby decreasing overall costs. As such, we optimize over a planning horizon rather than at a single decision moment; we measure the impact of current decisions on the downstream costs, i.e., the expected costs over the remainder of the horizon. 
We consider an urban area with a fixed set of customer locations (i.e., order destinations). Last-mile distribution takes place via a consolidation center at the edge of the area. Our representation of the urban distribution network is as follows. Let $\mathcal{G}=\{\mathcal{V} \cup 0, \mathcal{A}\}$ be a directed graph, with $\mathcal{V} \cup 0$ being the set of vertices and $\mathcal{A}$ being the set of arcs. Vertex 0 represents the consolidation center in the network. The remaining vertices signify the set of order destinations $\mathcal{V}=\{1,2, \ldots,|\mathcal{V}|\}$.

3.3.1. Fleet description. For the orders transport we restrict ourselves to vehicles with capacity 1 , although the method allows to handle heterogeneous fleets as well. We distinguish between primary and secondary vehicles. The set of primary vehicles - denoted by $\mathcal{Q}^{p r}$ - is finite, and either represents a fleet owned by the consolidation center or a rented fleet. To ensure feasible solutions at all times, we assume that there are always enough secondary vehicles in the set $\mathcal{Q}^{s e}$ to dispatch all accumulated orders, and that secondary vehicles are more expensive than primary vehicles. We refer to an individual vehicle as $q \in \mathcal{Q}^{p r} \cup \mathcal{Q}^{s e}$. Secondary vehicles may represent an actual backup option (e.g., renting additional vehicles in case of shortage) or a dummy fleet with infinitely high costs. Such a dummy fleet effectively serves as a bound on vehicle capacity, and eases the formulation of the model. We only dispatch secondary vehicles if all primary vehicles are in use.

A vehicle $q$ dispatched at decision moment $t \in \mathcal{T}$ has a finite and deterministic route duration $r_{t, q} \in\left\{1, \ldots, \tau^{\text {Max Route }}\right\}$. The dispatched vehicle is available for dispatch again at $t+r_{t, q}$, where $r_{t, q}$ is determined by the deployed routing function $\Delta: \mathcal{V}_{t, q} \mapsto\left\{1, \ldots, \tau^{\text {MaxRoute }}\right\}, \forall q \in \mathcal{Q}^{p r} \cup \mathcal{Q}^{s e}$, with $\mathcal{V}_{t, q} \subseteq \mathcal{V}$ being the subset of locations visited by vehicle $q$. After making a dispatching decision, the route durations $r_{t, q}$ determine the next dispatch availability of each vehicle. As we take into account downstream costs in the decision, we must keep track of the dispatch availability of primary vehicles both now and in the future; the return time of secondary vehicles is not relevant for our decisions. The earliest availability of vehicle $q$, relative to the current time $t$, is denoted by $\tau_{t, q} \in\left\{0, \ldots, \tau^{\text {MaxRoute }}\right\}$; vehicle $q$ can only be dispatched at decision moment $t$ if $\tau_{t, q}=0$. When dispatching a vehicle $q$ at time $t$, we set $\tau_{t, q}=r_{t, q}$. As the index is relative to the decision moment, it is updated. We store the dispatch availability of each primary vehicle - based on preceding dispatching decisions - in the vector $Q_{t}=\left[\tau_{t, 1}, \tau_{t, 2}, \ldots, \tau_{t,\left|\mathcal{Q}^{p r}\right|}\right]$.

3.3.2. Order types. An order is characterized by four properties: destination $v$, load size $l$, earliest dispatch time $t^{e}$, and latest dispatch time $t^{l}$. We refer to 
every unique combination of these four properties as the order type $\left(v, l, t^{e}, t^{l}\right)$. The order destination is represented by a vertex $v \in \mathcal{V}$. The size of an order is an element $l \in \mathcal{L}$, with $\mathcal{L}=\left\{\frac{1}{y}, \frac{2}{y}, \ldots, 1\right\}$ being the discretized set of feasibleload sizes, and $k \in \mathbb{N}$. The element $l=1$ represents a full load for an urban vehicle. Next, we formulate the dispatch windows. As all time indices are relative to the decision moment $t$, dispatch windows for accumulated orders must be updated over time; we introduce this procedure in Section 3.3.6. Every order has a dispatch window, within which the order must be dispatched from the depot. The dispatch window is defined by an earliest dispatch time $t^{e} \in \mathcal{T}^{e}$ and a latest dispatch time $t^{l} \in$ $\mathcal{T}^{l}$. Order types with $t^{e}>0$ describe pre-announced orders for which all properties are known and deterministic, but that have not yet arrived at the consolidation center. We impose that $t^{e} \geq 0$, as knowledge regarding dispatch times in the past does not impact the decision, while $t^{e}$ for a pre-announced order is bounded from above by $\tau^{\text {Max Ahead }}$. Hence, the set of feasible earliest dispatch times is

$$
\mathcal{T}^{e}=\left\{0, \ldots, \tau^{\text {MaxAhead }}\right\} .
$$

The latest dispatch time cannot be a moment in time before the earliest dispatch time, we therefore impose that $t^{l} \geq t^{e}$. If $t^{l}=0$, it is mandatory that the order is dispatched. Furthermore, we set a maximum length $\tau^{\text {MaxWindow }} \in \mathbb{N}$ on the dispatch window, such that $t^{e} \leq t^{l} \leq t^{e}+\tau^{\text {MaxWindow }}$. Hence, we have

$$
\mathcal{T}^{l}\left(t^{e}\right)=\left\{t^{e}, \ldots, t^{e}+\tau^{\text {MaxWindow }}\right\} .
$$

3.3.3. State description. We have introduced all properties that define an order type. Let $I_{t, v, l, t^{e}, t^{l}} \in \mathbb{N}$ be the available number of orders of a given type at decision moment $t$. We capture the deterministic knowledge regarding both pre-announced orders and accumulated orders at $t$ as

$$
I_{t}=\left[I_{t, v, l, t^{e}, t^{l}}\right]_{v \in \mathcal{V}, l \in \mathcal{L}, t^{e} \in \mathcal{T}^{e}, t^{l} \in \mathcal{T}^{l}} .
$$

For the sake of readability, we omit from here on the set notation when referring to these elements. We continue to define the state of the problem. The state at decision moment $t$ is denoted as $S_{t}$ in state space $\mathcal{S}$; its definition combines the earliest possible dispatch time of each primary vehicle as embedded in vector $Q_{t}$ and the deterministic order knowledge stored in vector $I_{t}$ :

$$
S_{t}=\left[Q_{t}, I_{t}\right] .
$$


3.3.4. Action description. We now describe the actions we can take in a state. Let $l^{\text {max }} \in \mathbb{N}$ be the maximum number of orders that can be held in the consolidation center, i.e., the maximum inventory remaining after a decision. This maximum should take into account some safety margin to anticipate the orders that may arrive within a time interval. At each decision moment $t$, we decide how many orders of every order type in inventory to dispatch. Orders that are not dispatched remain in inventory, and are available at the next decision moment. Let the integer variable $x_{t, v, l, t^{e}, t^{l}, q}$ describe the number of orders of a specific type to be dispatched at $t$, served by vehicle $q$. As a consequence of assigning orders to individual vehicles, the route durations of dispatched vehicles may vary. A feasible action at decision moment $t$ is given by

$$
x_{t}\left(S_{t}\right)=\left[x_{t, v, l, t^{e}, t^{l}, q}\right]_{\forall v, l, t^{e}, t^{l}, q},
$$

where

$$
\begin{array}{rlrl}
\sum_{v, l, t^{l}}\left(I_{t, v, l, 0, t^{l}}-\sum_{q \in \mathcal{Q}^{p r} \cup \mathcal{Q}^{s e}} x_{t, v, l, 0, t^{l}, q}\right) & \leq l^{\text {max }}, & \\
\sum_{q \in \mathcal{Q}^{p r} \cup \mathcal{Q}^{s e}} x_{t, v, l, t^{e}, t^{l}, q} \leq I_{t, v, l, t^{e}, t^{l}} & & \forall v, l, t^{e}, t^{l} \\
\sum_{q \in \mathcal{Q}^{p r} \cup \mathcal{Q}^{s e}} x_{t, v, l, 0,0, q} & =I_{t, v, l, 0,0} & & \forall v, l, \\
x_{t, v, l, t^{e}, t^{l}, q} & =0 & & t^{e}>0, \forall v, l, t^{l}, q \\
\sum_{v, l, t^{l}} x_{t, v, l, 0, t^{l}, q^{\prime}} \cdot l \leq 1 & & \forall q, \\
r_{t, q} \leq \tau^{\text {MaxRoute }} & & \forall q, \\
x_{t, v, l, t^{e}, t^{l}, q} \in \mathbb{N} & \forall v, l, t^{e}, t^{l}, q
\end{array}
$$

Constraint (3.3.2) ensures that at most the maximum inventory remains after dispatching. Observe that only orders with $t^{e}=0$ are part of inventory. We note that one might instead place a bound on the maximum volume with a similar constraint. With Constraint (3.3.3), we ensure that we cannot dispatch more orders of a certain type than are available at the decision moment $t$. Constraint (3.3.4) stipulates that all orders with a latest dispatch time equal to the current 
time need to be dispatched. Constraint (3.3.5) prevents the dispatch of preannounced orders that are not yet in inventory. Constraint (3.3.6) ensures that the order volume assigned to a vehicle does not exceed its capacity, whereas Constraint (3.3.7) prevents violations of the maximum route duration. Recall from Section 3.3.1 that the route duration is the outcome of routing function $\Delta$. Finally, Constraint (3.3.8) ensures that the number of orders dispatched of any type is nonnegative. The set of feasible actions in a given state is described by $\mathcal{X}_{t}\left(S_{t}\right)$.

3.3.5. Cost function. When evaluating a state, we must compute the direct costs $C\left(S_{t}, x_{t}\right)$ for all actions in $\mathcal{X}_{t}\left(S_{t}\right)$. We reiterate that we consider a twophase solution method, where we first make the dispatching decision based on approximate routing costs, and subsequently optimize the route for the selected dispatch decision. Although we will perform some numerical experiments in which we integrate the routing problem and heuristically solve it, our main focus is on the dispatching decision. The approximate routing costs must be computed for every possible action for a large number of states. Therefore, an efficient cost function is required. For this study, we use the approximation formula of Daganzo (1984) to estimate the routing costs.

Daganzo's formula is known to provide good estimates of VRP distances (Robusté Antón et al., 2004). The formula is based on the notion that when increasing the number of locations in an area, the length of the tour visiting these locations asymptotically converges to a constant multiplied by the square root of the number of points and the service area (Daganzo, 1984). After determining the total tour length with Daganzo's formula, we determine the number of vehicles required. We assume that the total distance to cover is equally distributed over the dispatched vehicles, thus resulting into identical route durations for all vehicles. We emphasize that these identical route durations only apply to the particular cost function that we define here, not to the model itself. After determining the required travel distance, we incrementally increase the number of vehicles, until it satisfies the maximum route duration constraint and there is enough capacity to carry the total volume of dispatched orders. The costs are composed of three elements: a fixed cost per dispatched truck, distantdependent route costs, and handling costs per location visited. To demonstrate that route durations may be different, we will also perform experiments in Section 3.6 that allow vehicles dispatched at the same time to have different route durations.

The cost function deployed in this chapter is subject to several major simplifications. For the sake of completeness, we stress that more advanced expressions 
exist as well, which provide accurate estimates for more realistic VRPs. Figliozzi (2009) presents expressions for multiple VRP variants, for example taking into account capacity constraints and time windows at the customer. The parameters for such expressions can be determined a priori by first generating a large number of routes (preferably with the routing algorithm that is eventually used) and then apply regression to estimate the parameters in the expression.

3.3.6. Transition function. Based on the order arrivals that may occur during the planning horizon, we can compute a consolidation policy $\pi_{t} \in \Pi_{t}$, with $\pi_{t}: S_{t} \mapsto x_{t}$, and $\Pi_{t}$ being the set of possible policies. To model the uncertainties with respect to the properties of arriving orders, we introduce five random variables. These are (i) the number of orders $O_{t}$ arriving in the time interval $(t-1, t]$, (ii) the order destination $V$, (iii) the order size $L$, (iv) the earliest dispatch time $T^{e}$, and (v) the width of the dispatch window $T^{\text {window }}$. Based on the realizations of these random variables (given by the lower case representation of the variables), we can deterministically compute the latest dispatch time with $T^{l}=T^{e}+T^{\text {window }}$. The corresponding probability distributions are discrete and finite. To represent all probability distributions with a single variable, we define the exogenous information variable $\tilde{I}_{t, v, l, t^{e}, t^{l}} \in \mathbb{N}$, which indicates the number of arrivals of order type $\left(v, l, t^{e}, t^{l}\right)$ in the interval just before $t \geq 1$. The generic variable $W_{t}$ captures all exogenous information, i.e., all orders that arrive between $t-1$ and $t$ (i.e., before making decision $x_{t}$ ):

$$
W_{t}=\left[\tilde{I}_{t, v, l, t^{e}, t^{l}}\right]_{\forall v, l, t^{e}, t^{l}} \quad t \geq 1 .
$$

We now proceed to describe the transition from $S_{t}$ to the next state $S_{t+1}$. The transition is affected by the action $x_{t}$ and the new arrivals $W_{t+1}$. Orders that are not dispatched remain in inventory, hence must be included in $S_{t+1}$. Furthermore, we adjust the dispatch windows, as it is always relative to the current time. Actions determine the change in vehicle availability from $Q_{t}$ to $Q_{t+1}$, and update the remaining orders in inventory. For a given action, we have $r_{t, q}$ as the route duration for a dispatched vehicle $q \in \mathcal{Q}^{p r}$. By combining the route durations for dispatched vehicles with the earliest dispatch times stored in $Q_{t}$, we can compute $Q_{t+1}$. This gives us the transition function $S^{M}$ :

$$
S_{t+1}=\left[Q_{t+1}, I_{t+1}\right]=S^{M}\left(S_{t}, x_{t}, W_{t+1}\right),
$$

where 


$$
\begin{aligned}
I_{t+1, v, l, 0, t^{l}}= & \sum_{\tilde{t}^{e}=0}^{1}\left(I_{t, v, l, \tilde{t}^{e}, t^{l}+1}\right)-\sum_{q \in \mathcal{Q}^{p r} \cup \mathcal{Q}^{s e}} x_{t, v, l, 0, t^{l}+1, q}+\tilde{I}_{t+1, v, l, 0, t^{l}} \\
& \forall v, l, t^{l} \\
I_{t+1, v, l, t^{e}, t^{l}=} & I_{t, v, l, t^{e}+1, t^{l}+1}+\tilde{I}_{t+1, v, l, t^{e}, t^{l}} \\
& t^{e}>0, \forall v, l, t^{l}, \\
\tau_{t+1, q}= & \max \left(0, \tau_{t, q}-1, r_{t, q}-1\right) \quad \forall q
\end{aligned}
$$

Constraints (3.3.10) states that the number of an order type at $t+1$ is the number of the order type that we have in $S_{t}$, minus the number of the order type that is dispatched at $t$, plus the number of the order type that arrives at $t+1$. Constraint (3.3.11) is similar, but takes into account that orders with $t^{e}>0$ cannot be shipped. Constraint (3.3.12) ensures that $Q_{t+1}$ is consistently updated.

3.3.7. Objective function. The objective when solving the problem is to minimize the total dispatching costs over the full planning horizon, denoted by $\sum_{t \in \mathcal{T}} C\left(S_{t}, x_{t}\right)$. The costs corresponding to $t>0$ cannot be computed deterministically beforehand, as we do not know which orders will arrive within the planning horizon. Instead, we minimize the expected future costs by summing over all possible future outcomes, which is classical backwards dynamic programming. Let $\Omega_{t}$ be the set of all possible order arrivals, and let $\omega_{t} \in \Omega_{t}$ be a particular realization of order arrivals, hence of the random variable $W_{t}$. We can now introduce the optimality equation

$$
\begin{aligned}
V_{t}\left(S_{t}\right)= & \min _{x_{t} \in \mathcal{X}_{t}\left(S_{t}\right)}\left(C\left(S_{t}, x_{t}\right)+\right. \\
& \left.\sum_{\omega_{t+1} \in \Omega_{t+1}} \mathbb{P}\left(W_{t+1}=\omega_{t+1}\right) V_{t+1}\left(S_{t+1} \mid S_{t}, x_{t}, \omega_{t+1}\right)\right) .
\end{aligned}
$$

We describe the expression for $\mathbb{P}\left(W_{t}\right)$. For $t \geq 1$, let $o_{t}$ be the number of orders arriving in the interval $(t-1, t]$, with $o_{t} \in\left\{0,1, \ldots, o_{t}^{\max }\right\}$. The probability of $o_{t}$ orders arriving is given by $\mathbb{P}\left(O_{t}=o_{t}\right)$. The probability of an arriving order being of a certain order type (i.e., the unique combination of order properties) follows from the multivariate distribution $\mathbb{P}\left(V, L, T^{e}, T^{l}\right)$. The properties of 
these random variables have been outlined at the beginning of this section. The probability function for new arrivals $\mathbb{P}\left(W_{t}=\omega_{t}\right)$, with $t \geq 1$, is given by

$$
\begin{aligned}
\mathbb{P}\left(W_{t}=\omega_{t}\right)= & \mathbb{P}\left(O_{t}=o_{t}\right) \frac{o_{t} !}{\prod_{\tilde{I}_{t, v, l, t^{e}, t^{l}} !}^{\tilde{I}_{t, v, l, t^{e}, t^{l}} \in \omega_{t}}} \\
& \cdot \prod_{v, l, t^{e}, t^{l}} \mathbb{P}\left(V=v, L=l, T^{e}=t^{e}, T^{l}=t^{l}\right)^{\tilde{I}_{t, v, l, t^{e}, t^{l}}} .
\end{aligned}
$$

\subsection{Solution method}

For realistic instance sizes, the decision problem becomes intractably large in terms of state space, action space, and outcome space. Appendix 3B shows the sizes of these spaces. Powell (2011) refers to this phenomenon as the 'three curses of dimensionality', and provides an Approximate Dynamic Programming (ADP) framework that addresses these problems. Based on this framework, we develop an ADP algorithm that we use to solve the DDP-TW. ADP is a solution method that uses simulation to solve instances of problems represented by a Markov decision model. It consists of an offline and an online phase. In the offline phase, we aim to find a good policy by learning from repeated observations. The computational effort to learn a good policy may be considerable, but the offline phase only needs to be completed once. After learning the policy, it can be applied in the online phase. In this phase, the policy is no longer altered based on observations. The online application of the policy requires that decisions can be generated sufficiently fast for decision-making. During the offline learning phase, we learn estimates for the downstream costs corresponding to state-action pairs. This is done by performing a Monte Carlo simulation, in which we (i) step forward in time, (ii) sample a random order arrival scenario, and (iii) learn the value of being in a given state by observing its associated costs. By repeatedly doing so, we become able to recognize good actions. The goal is to obtain the optimal consolidation policy, which can subsequently be used for online decision making. In this section, we separately discuss the three curses of dimensionality that we address. Subsequently, we give an overview of the ADP algorithm. We conclude the section with the design of the basis functions. 
3.4.1. Dimensionality of the outcome space. We start by addressing the size of the outcome space. In traditional dynamic programming, when evaluating a given state-action pair, we compute its downstream costs by multiplying the value for every possible outcome state $S_{t+1}$, given $\left(S_{t}, x_{t}, \omega_{t+1}\right)$, with the probability that arrival scenario $\omega_{t+1}$ occurs (see Equation 3.3.13). In our problem, the number of order arrival scenarios increases exponentially with the maximum number of arrivals. For realistic numbers of arrivals, this results in very large outcome spaces. Therefore, rather than evaluating the full outcome space for every action, we replace the expression for the downstream costs $\sum_{\omega_{t+1} \in \Omega_{t+1}} \mathbb{P}\left(W_{t+1}=\omega_{t+1}\right) V_{t+1}\left(S_{t+1} \mid S_{t}, x_{t}, \omega_{t+1}\right)$ with a single cost estimate. For this purpose, we introduce the concept of the post-decision state (Powell, 2011). The post-decision state $S_{t}^{x}$ is the state immediately after action $x_{t}$, but before the order arrivals $\omega_{t+1}$. Applying action $x_{t}$ in state $S_{t}$ results in a deterministic transition to the post-decision state $S_{t}^{x}$. We express this transition in the function

$$
S_{t}^{x}=S^{M, x}\left(S_{t}, x_{t}\right)
$$

where

$$
\begin{aligned}
I_{t, v, l, t^{e}, t^{l}}^{x} & =I_{t, v, l, t^{e}, t^{l}}-\sum_{q \in \mathcal{Q}^{p r} \cup \mathcal{Q}^{s e}} x_{t, v, l, t^{e}, t^{l}, q} & & \forall v, l, t^{e}, t^{l}, \\
\tau_{t, q}^{x} & =\max \left(r_{t, q}, \tau_{t, q}\right) & & \forall q \in \mathcal{Q}^{p r} .
\end{aligned}
$$

For the problem, the post-decision state comprises (i) the number of orders per type remaining at the UCC and (ii) the updated vehicle availability after the dispatching decision. In the offline learning phase, we operate on the postdecision state as follows. For a given state $S_{t}$, we make a decision $x_{t}$ to arrive at the post-decision state $S_{t}^{x}$. Then, we randomly sample an order arrival $\omega_{t+1} \in \Omega_{t+1}$ and observe the costs of the next state $S_{t+1}$. We use these observed costs to improve the estimate for the downstream cost $\mathbb{E}\left\{V_{t+1}\left(S_{t+1}\right) \mid S_{t}^{x}\right\}$ for post-decision state $S_{t}^{x}$. Thus, by using the estimated value of the post-decision state rather than the values of all possible pre-decision states at $t+1$, we need to evaluate only a single outcome per action.

3.4.2. Dimensionality of the state space. We proceed to address the problem of the large state space. As mentioned before, we learn values by observing states in the offline learning phase, which we encounter by randomly sampling order arrivals. Suppose that we would use a lookup table containing estimates of the downstream costs for each post-decision state. Filling this table implies that 
we should visit every post-decision state at least once to estimate its associated downstream costs. As the size of the state space grows exponentially with the number of order types, for realistic-sized instances we would need to perform many simulation iterations to learn all these values. Rather than explicitly learning the expected downstream cost $\mathbb{E}\left\{V_{t+1}\left(S_{t+1}\right) \mid S_{t}^{x}\right\}$ for every post-decision state, we replace this term with a single function that returns estimates for the downstream costs of all post-decision states, regardless of whether we visited a state before. This function, known as the value function approximation (VFA) for downstream costs (Powell, 2011), is denoted by $\bar{V}_{t}^{n}: S_{t}^{x} \mapsto \mathbb{R}$. The key benefit of the VFA is that we no longer need to fill a lookup table with all the expected downstream costs.

Before applying the learned policy in an online setting, the parameters of $\bar{V}_{t}^{n}$ must be learned offline via observations. In the solution method, the VFA is a function that contains a number of variables that explain the cost structure of the problem, using so-called basis functions (Powell, 2011). Adopting the ADP terminology, we refer to an explanatory variable as a 'feature. Let $\mathcal{F}$ be a set of features (which we can compute based on the post-decision state), with $f \in \mathcal{F}$ representing an explanatory variable for the true value function, for example the number of primary trucks available or the volume per receiver. Furthermore, let $\phi_{f}: S_{t}^{x} \mapsto \mathbb{R}$ be a basis function of feature $f$. Such a basis function is often equal to the feature itself, but may also be a polynomial or another mathematical operation performed on the feature, e.g., the number of primary vehicles squared. A set of basis functions is denoted as $\phi \supset \phi_{f}\left(S_{t}\right), \forall S_{t} \in \mathcal{S}$. Such a set may be viewed as an aggregate state description, retaining sufficient detail to capture the cost structure, but requiring less variables to do so. For example, the number of primary vehicles available at $t+1$ and the volume per receiver held in the UCC may be good indicators to estimate the downstream costs, while requiring only $1+|\mathcal{V}|$ variables instead of the full state description. In Section 3.4.5, we will discuss the basis function design for the model. Finally, let $\theta_{t, f}^{n}$ be a weight corresponding to $\phi_{f}$ at decision moment $t$. As we consider a finite horizon problem, at each decision moment $t \in \mathcal{T}$ the downstream costs are computed over the time interval $t+1, \ldots, T$, i.e., downstream costs are time-dependent. Therefore, we learn weights separately for each decision moment $t \in \mathcal{T}$. We use the iteration counter $n$ to track the number of iterations performed, and we use $\bar{V}_{t}^{n}\left(S_{t}^{x}\right)$ to denote the VFA during the offline learning phase. We obtain the value function 
approximation

$$
\bar{V}_{t}^{n}\left(S_{t}^{x}\right)=\sum_{f \in \mathcal{F}} \theta_{t, f}^{n} \phi_{f}\left(S_{t}^{x}\right) \quad \forall t \in \mathcal{T} .
$$

To improve the estimate of the downstream costs, after every iteration $n$ we must update the weights $\theta_{t, f}^{n}, \forall f \in \mathcal{F}, t \in \mathcal{T}$. For the weight updates, we use a regression procedure, which we discuss in Section 3.4.5.5. As we use a single set of weights to estimate all downstream costs, new observations adjust the cost estimates for all states that we may encounter. To exemplify, suppose that the number of primary vehicles available at $t+1$ is the only basis function, implying that we have one estimated weight at $t$. By multiplying this weight with the number of primary vehicles in the post-decision state corresponding to a certain action, we obtain the downstream cost estimate corresponding to that post-decision state, even if we never visited that state before.

3.4.3. Dimensionality of the action space. The final curse of dimensionality that we address relates to the action space. By now, we have replaced the original objective function with a function in which the VFA (operating on the post-decision state) reflects the estimated downstream costs. As a consequence, the decision problem reduces to a deterministic minimization problem. By solving this problem at decision moment $t$ for iteration $n$, the best action - given the estimate $\bar{V}_{t}^{n-1}\left(S_{t}^{x}\right)$ - is found:

$$
\tilde{x}_{t}^{n}=\underset{x_{t} \in \mathcal{X}_{t}\left(S_{t}\right)}{\operatorname{argmin}}\left(C_{t}\left(S_{t}, x_{t}\right)+\bar{V}_{t}^{n-1}\left(S_{t}^{x}\right)\right) .
$$

Although the computational effort required to solve the decision problem sharply decreases by operating on the post-decision state rather than the full outcome space, we are still required to evaluate every action $x_{t} \in \mathcal{X}_{t}\left(S_{t}\right)$. For our problem, the action space increases exponentially with the number of orders. Therefore, complete enumeration of all decisions is not feasible for large instances. Generally, either mathematical programming or metaheuristics are used to solve large decision problems in ADP (Powell, 2009). In this chapter, we describe a mathematical program to handle large action spaces. We formulate the decision problem - which is solved within the ADP - as an Integer Linear Program (ILP). This ILP solves Equation (3.4.5), and is subject to the set of action constraints (3.3.2) - (3.3.6) and post-decision constraints (3.4.2) - (3.4.3). The post-decision variables are required to compute the basis functions. As ILPs can be solved relatively fast with the solvers available nowadays, we can handle much larger decision problems than enumeration would allow. However, the 
decision problem remains computationally challenging due to the need to precompute $2^{|\mathcal{V}|}$ route durations to cover all subsets of destinations that might be visited, which would all require a corresponding decision variable in the ILP. We therefore propose an additional simplification. Instead of explicitly computing all possible route distances, we define a set of approximate travel distance classes as input for the ILP. The formulation of the ILP enforces that the approximate distance used to compute the routing costs in the ILP is at least equal to the distance computed with the Daganzo formula. The more distance classes that we pre-define, the closer we can approximate the actual travel distance. To set the number of distance classes such that both accuracy and computational speed are satisfactory, we measure the performance gap with preliminary numerical experiments. With the ILP, we can solve the large instances within reasonable time, and closely approximate the solutions from the Daganzo formula. The full ILP model is shown in Appendix 3A.

We conclude our discussion of the actions with a note on incorporating exploration while learning the dispatching policy. Solving the deterministic minimization problem in Equation (3.4.5) yields the best action given the current VFA to estimate the downstream costs. Especially in the early iterations, the estimates for the downstream costs may be poor. As a consequence, the algorithm might continuously visit the same suboptimal post-decision states, while never learning the values of better states. To avoid this form of cycling, we do not always select the 'best' action. Instead, with probability $\epsilon \in(0,1)$ we select a random action $x_{t} \in \mathcal{X}_{t}\left(S_{t}\right)$. This so-called $\epsilon$-greedy strategy allows us to explore new states (Powell, 2011). However, too much exploration will decrease the quality of the estimates for the (near-)optimal states that we are interested in. We therefore test various settings for $\epsilon$, such that we find the right balance between visiting new states and accurately learning the values of good states.

3.4.4. Algorithmic outline. We have explained how we handle the dimensionality of the outcome space, state space, and action space of the problem. Algorithm 3.1 provides the outline of the ADP algorithm; a detailed description of the full procedure can be found in Powell (2011). To update the value functions, we make use of a backward pass procedure (Sutton and Barto, 1998), in which the VFAs are updated only after completing a full forward iteration. With every iteration $n$, we generate a random order arrival path $\omega^{n}=\left\{\omega_{1}^{n}, \ldots, \omega_{T}^{n}\right\}$. At every $t \in\{0, \ldots, T-1\}$, we solve Equation (3.4.5) to obtain the action that minimizes the sum of direct costs and estimated downstream costs. To facilitate exploration of the state space, we choose a random action with probability $\epsilon$. After selecting an action, we generate a random order arrival $W_{t+1}\left(\omega^{n}\right)$, and 
find the next state $S_{t+1}$ by solving Equation (3.3.9). This procedure is repeated until reaching $t=T$. After completing the forward iteration, we move backwards to recursively update $\bar{V}_{t}^{n-1}\left(S_{t}^{x}\right)$, based on the observed downstream costs $\hat{v}_{t}^{n}=C_{t}\left(S_{t}, x_{t}\right)+\hat{v}_{t+1}^{n}$. We perform this update with a regression procedure that we discuss in Section 3.4.5.5, the updating function is defined as follows:

$$
\bar{V}_{t-1}^{n}\left(S_{t-1}^{x}\right) \leftrightarrow U^{V}\left(\bar{V}_{t-1}^{n-1}\left(S_{t-1}^{x}\right), S_{t-1}^{x}, \hat{v}_{t}^{n}\right) .
$$




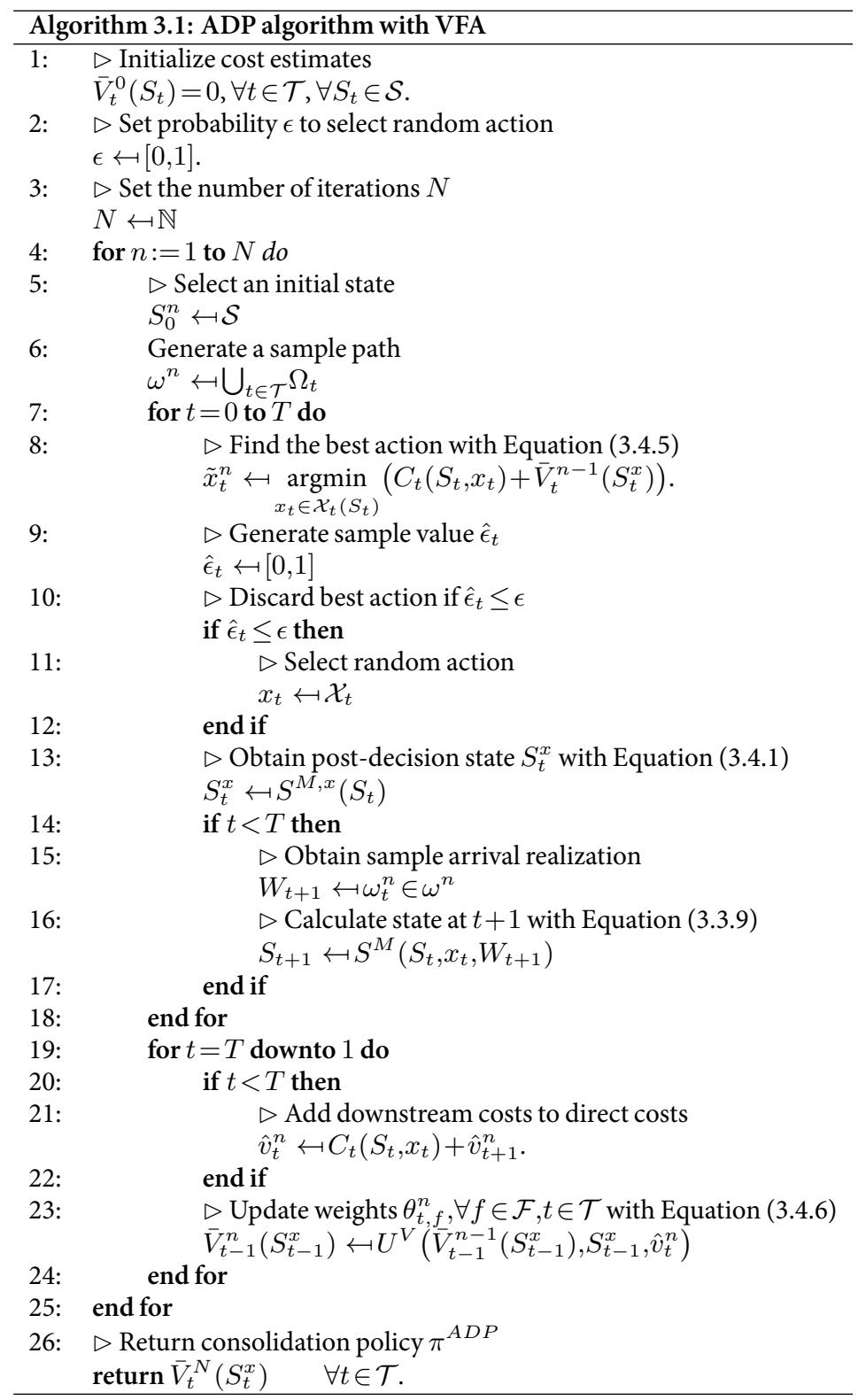


3.4.5. Basis function design. We conclude this section with the design of the basis functions. We discuss the following design-related topics: (i) a categorization of the basis functions for the DDP-TW, (ii) the selection of a set of basis functions, (iii) the pitfalls of non-segregated basis functions, and (iv) the updating procedure to learn the weights corresponding to basis functions. Finally, we present the selected sets of basis functions that we test in the numerical experiments.

3.4.5.1. Categorization of basisfunctions. The selected set of basis functions should accurately represent the downstream costs. Hence, the basis functions need to capture the cost structure of the problem. In Section 3.5, we introduce the sets of basis functions that we will test. For now, we restrict ourselves to presenting a categorization of the basis functions. The cost structure of our problem mainly depends on (i) the locations visited, (ii) the volume dispatched, (iii) the number of primary vehicles available, and (iv) the flexibility to postpone orders. The basis functions should reflect one or more of these properties. It is important that basis functions within a set are independent of each other; as we learn the weights by means of regression, we must avoid collinearity. We divide the basis functions into three categories that are assumed to be independent: vehicle-based, location-based, and volume-based. A time component may be added to each category, e.g., computing vehicle availability up until the maximum route duration. By carefully selecting a set of basis functions that incorporates these factors, we are able to estimate the values of post-decision states, even those we did not visit before. As such, the VFA enables us to cope with large state spaces.

3.4.5.2. Exploration of the state space. Solving the deterministic minimization problem in Equation (3.4.5) yields the best action given the current VFA to estimate the downstream costs. Especially in the early iterations, the estimate for the downstream costs may be poor. As a consequence, the algorithm might continuously visit the same suboptimal post-decision states, while never learning the values of better states. To avoid this form of cycling, we do not always select the 'best' action. Instead, with probability $\epsilon \in(0,1)$ we select a random action $x_{t} \in \mathcal{X}_{t}\left(S_{t}\right)$. This so-called $\epsilon$-greedy strategy allows us to explore new states. However, too much exploration will decrease the quality of the estimates for the (near-)optimal states that we are interested in. We therefore test various settings for $\epsilon$, such that we find the right balance between visiting new states and accurately learning the values of good states.

3.4.5.3. Design of basis functions. As explained in Section 3.4.2, our solution method requires the design of a set of basis functions. A key difficulty here 
is to define a set that enables us to accurately approximate the downstream costs. We obtain a first sense of good basis functions by performing regression analysis on the exact values of states; these values are obtained by solving small instances to optimality. We compute the $R^{2}$ values (i.e., the coefficient of determination that describes the fit of the model) of various sets of basis functions to evaluate how well they explain the costs.

Although the $R^{2}$ values give an initial insight into the quality of basis functions, a high explanatory value does not guarantee that the corresponding policy that we learn also performs well (Pérez Rivera and Mes, 2016). There are three key reasons for this. First, as we only observe a sample of the state space, the weights that we learn with ADP may differ from those obtained with regression analysis on the values of all states. Second, we can only compute exact values for small instances; these instances do not capture all the dynamics embedded in the problem. Third, correctly estimating the downstream costs is important, yet good policies may be obtained without accurate estimates of the downstream costs (Chang et al., 2013). Essentially, we require estimates that allow us to rank actions correctly, such that we are able to consistently select good actions. For these reasons, we also compare the performance resulting from our basis function designs.

3.4.5.4. Use of non-segregated basis functions. Non-segregated basis functions (e.g., the ratio of vehicle capacity and volume per dispatch time) combine several features and therefore may have a higher explanatory value. However, they are difficult to combine with other basis functions due to the high probability of introducing collinearity. Another drawback is that the associated weights may be more difficult to learn, as we try to simultaneously learn the impact of multiple explanatory variables. Furthermore, we also incorporate the basis functions within the ILP formulation; the use of non-linear basis functions potentially requires a large number of artificial variables. In the design of the basis functions, we therefore focus on basis functions that are both linear and segregated.

3.4.5.5. Regression procedure to update the weights. After every simulation iteration, we must update the weights corresponding to the basis functions to improve the VFA. We do this by regressing the VFA on the observed data. The regression procedure that we use is known as recursive least squares for nonstationary data (Powell, 2011); this procedure is briefly described in Appendix 3C. A shortcoming of this procedure is that it is sensitive to outliers. Unstable behavior of the regression is common for large sets of basis functions in particular. This instability follows from observing deviating values, to which the regression 
procedure responds by assigning a very large weight to a basis function of small magnitude. Subsequent observations may then yield extreme estimates. Burger and Repisky (2012) discuss these problems, as well as various other pitfalls in least squares regression. In the design of the basis functions, we aim to keep the number of basis functions relatively low; as a rule of thumb, we consider sets that contain 10 or less basis functions as sufficiently small. Furthermore, by using only additive basis functions - rather than, e.g., polynomials, multiplications, and divisions - we prevent basis functions from returning extremely small values; the smallest non-zero value that we may obtain relates to volume-based basis functions and is equal to $\min (l \mid l \in \mathcal{L})$. All other basis functions use integer counters, such that their smallest non-zero value is 1 .

3.4.5.6. Selected basis functions. Having discussed the categories of basis functions for the problem and the design requirements, we now introduce the sets of basis functions that we will test in the numerical experiments. The selected basis functions reflect one or more of these categories; we first test sets containing separate basis functions, and then sets that combine multiple aspects. We now describe the 13 sets of basis functions that we test in the numerical experiments, with the aim of finding the basis functions that yield the best ADP policy. The sets are shown in Table 3.1. Every set of basis functions $\phi$ contains a constant to capture the average costs of a state. The set $\phi^{1}$ contains only such a constant; note that this set assigns the same value to every post-decision state, as such yielding the direct cost-minimization policy $\pi^{m i n}$. In set $\phi^{2}$, we consider the total number of destinations that we may visit at the current decision moment $t$. Set $\phi^{3}$ describes the number of primary vehicles available from $t$ to $t+\tau^{\text {MaxRoute }}$. Set $\phi^{4}$ gives the volume per latest dispatch moment, e.g., the urgent volume at various decision moments. In $\phi^{5}$, we keep track of the volume per destination. Set $\phi^{6}$ contains the number of orders per order type. As this set does not aggregate state data, it is not a well-designed set of basis functions; this set is added only for illustrative purposes. The remainder of the sets $\left(\phi^{7}-\phi^{13}\right)$ are either linear combinations or non-segregated combinations of the aforementioned sets. Again, we stress that non-segregated basis functions do not meet all our design requirements and are included for the sake of illustration.

\subsection{Experimental setup}

In this section, we describe the setup of the numerical experiments. The main goal of the experiments is to identify the set of basis functions that enables us to most accurately capture the cost structure of the problem and show how the resulting policy that we learn performs relative to a variety of benchmark policies. 
TABLE 3.1. Selected sets of basis functions.

\begin{tabular}{lll}
\hline Set & Basis functions & \# basis functions \\
\hline$\phi^{1}$ & Const. & 1 \\
$\phi^{2}$ & Const.+\# available destinations at $t^{e}=0$ & 2 \\
$\phi^{3}$ & Const.+\# vehicles & $1+\tau^{\text {MaxRoute }}$ \\
$\phi^{4}$ & Const.+Total vol. per $t^{l}$ & $1+\tau^{\text {LatestDispatch }}$ \\
$\phi^{5}$ & Const.+Total vol. per $v$ & $1+|\mathcal{V}|$ \\
$\phi^{6}$ & Const.+\# orders per type & $1+|\mathcal{I}|$ \\
$\phi^{7}$ & Const.+\# Vehicles/\# available destinations & 2 \\
$\phi^{8}$ & Const.+\# Vehicles/Total vol. per $t^{l}$ & $1+\min \left(\tau^{\text {MaxRoute }}, \tau^{\text {LatestDispatch }}\right)$ \\
$\phi^{9}$ & Const.+\# destinations per $t^{l}$ & $1+\tau^{\text {LatestDispatch }}$ \\
$\phi^{10}$ & Const.+Vol. per destination per $t^{l}$ & $1+\left(\tau^{\text {LatestDispatch }} \cdot|\mathcal{V}|\right)$ \\
$\phi^{11}$ & Const.+\# available destinations+ & $2+\tau^{\text {LatestDispatch }}$ \\
& Total vol. per $t^{l}$ & \\
$\phi^{12}$ & Const.+\# vehicles+Total vol. per $t^{l}$ & $1+\tau^{\text {MaxRoute }}+\tau^{\text {LatestDispatch }}$ \\
$\phi^{13}$ & Const.+\# available destinations+ & $2+\tau^{\text {MaxRoute }}+\tau^{\text {LatestDispatch }}$ \\
& \# vehicles per $\tau_{t}+$ Total vol. per $t^{l}$ & \\
\hline
\end{tabular}

We distinguish between experiments in the offline learning phase and the online phase. In the offline learning phase, we evaluate the sets of basis functions as defined in Section 3.4.5.6. For each set of basis functions we learn the corresponding dispatching policy; subsequently, we compare the performances of these policies with each other. In the online phase, we test the performance of the ADP policy obtained with the best set of basis functions only, relative to a number of benchmark policies. For large instances, we also report the computational times required to solve the decision problem.

We divide the experiments in three classes - small (S), medium (M), large (L) - each class having its own purpose. Preliminary tests on medium-sized instances indicate that 5,000 iterations suffice to learn a policy; further observations do not significantly increase the quality of policies. We test the online performance of policies using 1,000 iterations. In the offline learning phase, we select a random action with probability $\epsilon=0.05$. Preliminary tests on a representative instance indicated this as the best-performing setting, having tested $\epsilon \in\{0,0.05, \ldots, 0.25\}$. The policy obtained under $\epsilon=0$ performed the worst, with a difference in solution quality of $3.3 \%$ compared to $\epsilon=0.05$. Our algorithm is coded in Delphi XE6 and we use a computer with $8 \mathrm{~GB}$ RAM and a $2.90 \mathrm{GHz}$ Intel Core i7 processor. The ILP is solved with CPLEX 12.2.

The outline of the remainder of this section is as follows. In Section 3.5.1, we define the properties of all the test instances. Section 3.5.2 describes the benchmark policies that we use to evaluate the performance of the policies that we 
learn with ADP. In Section 3.5.3, we describe the procedure that we use to generate a set of representative initial states, from which we draw at the start of every iteration.

3.5.1. Instance properties. In this subsection, we describe the properties of the instances that we perform experiments on. We define 2 small instances, 20 medium-sized instances, and 2 large instances. With the experiments on small instances, we primarily evaluate the behavior of ADP in the offline learning phase, comparing the results with the exact results. We perform tests on two instances, named S1 and S2. For S1, we consider three customers located at equal distances from the depot, while for $\mathrm{S} 2$ the depot-destination distances vary. We consider a dispatch window of width 1 , and no pre-announced orders. The instances are sufficiently small (93,000 states) to be solved exactly with dynamic programming. With the obtained values, we compute the explanatory power of the basis functions by computing the $R^{2}$ value and unveil possible dependencies between them. We do this for multiple sets of basis functions and then test how closely we approximate the exact values with the corresponding policies.

Our experiments on the medium-sized instances (\# states $\gg 10^{22}$ ) provide insights into both the offline learning phase and the online phase. Table 3.2 shows the main properties of these instances. We consider up to 15 arrivals per decision moment and up to 30 orders in inventory. We are primarily interested in the performance of ADP in relation to two properties: (i) the degree of dynamism (DoD) and (ii) the flexibility in dispatching times. The degree of dynamism is the ratio between the number of orders with the property $t^{e}=0$ (i.e., not announced in advance) and the total number of orders (Larsen et al., 2002). A DoD of 1 indicates that all orders become known dynamically, whereas $\mathrm{DoD}=0 \mathrm{im}$ plies that all orders are pre-announced. With the flexibility in dispatching times, we refer to the maximum width of the dispatch windows of orders. The first six instances (M1-6) vary both the DoD and the flexibility in dispatching times. We take the first 10 customers of the 25-customer Solomon instance R101, scaled to a $10 \mathrm{~km} \times 10 \mathrm{~km}$ area. We consider instances with maximum dispatch windows of 1 and 2 respectively, providing us with two levels of flexibility. Furthermore, we vary the $\mathrm{DoD}$, testing ratios of $0,0.5$, and 1 . The remaining instances are variants of M2 and M5, in which we test the effects of skewed demand (M7-8), different customer locations (M9-10), variations in fleet sizes (M11-14), distances scaled to a $50 \mathrm{~km} \times 50 \mathrm{~km}$ area (M15-16), and tests in which we use a routing algorithm within the ADP method (Clarke-Wright savings algorithm with 2-opt) instead of using Daganzo's formula (M17-18). Finally, in M19 and M20 we test more realistic instances inspired by the city of Copenhagen. We take the location of an 


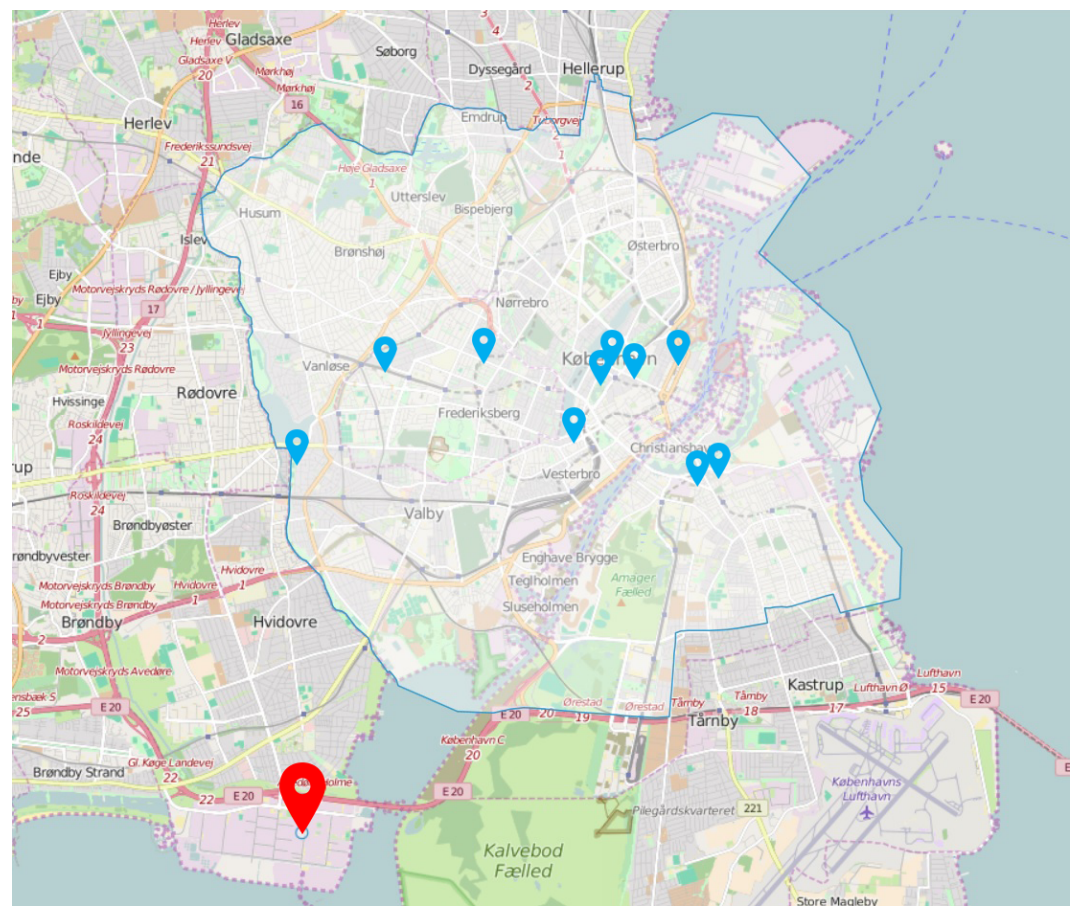

FIGURE 3.1. Copenhagen test instance, showing the low-emission zone (shaded area), the UCC location (large red marker), and customer locations (blue markers). (o) OpenStreetMap contributors

existing urban consolidation center, 10 real customer locations within the lowemission zone of Copenhagen (see Figure 3.1), and compute the corresponding distance matrix using the OpenStreetMap implementation of Luxen and Vetter (2011). We again solve these instances with the routing algorithm as used in M17-18.

Finally, we perform simulation experiments on two large instances (\# states $\gg 10^{178}$ ). For these instances, we consider up to 40 arrivals per decision moment and inventories up to 60 orders. We test two instances: the 25-customer Solomon instance R101 (L1) and the 50-customer Solomon instance R101 (L2), again scaled to a $10 \mathrm{~km}^{2}$ area. The results in the online phase are compared to those of the benchmark policies that we describe in Section 3.5.2. The contribution of these experiments is to demonstrate that realistic-sized instances can 
TABLE 3.2. Settings for the medium-sized instances.

\begin{tabular}{ll}
\hline Instance & Description \\
\hline M1 & DoD 1, $\tau^{\text {MaxWindow }}=1$ \\
M3 & DoD $=0.5, \tau^{\text {MaxWindow }}=1$ \\
M4 & DoD $=0, \tau^{\text {MaxWindow }}=1$ \\
M5 & DoD $=1, \tau^{\text {MaxWindow }}=2$ \\
M6 & DoD $=0, \tau^{\text {MaxWindow }}=2$ \\
M7 & As M2, 2 customers $50 \%$ demand \\
M8 & As M5, 2 customers $50 \%$ demand \\
M9 & As M2, but with last 10 customers R101 \\
M10 & As M5, but with last 10 customers R101 \\
M11 & As M2, but with 4 vehicles \\
M12 & As M5, but with 4 vehicles \\
M13 & As M2, but with unlimited fleet size \\
M14 & As M5, but with unlimited fleet size \\
M15 & As M2, but with $50 \mathrm{~km}^{2}$ network \\
M16 & As M5, but with $50 \mathrm{~km}^{2}$ network \\
M17 & As M2, but with routing algorithm \\
M18 & As M5, but with routing algorithm \\
M19 & As M17, but with Copenhagen network \\
M20 & As M18, but with Copenhagen network \\
\hline
\end{tabular}

be handled by applying the ILP for the decision problems. Finally, we provide insights into the computation times required to solve the ILP.

3.5.2. Benchmark policies. In this subsection, we define the benchmark policies that are used to evaluate the performance of the ADP policies. We introduce three myopic policies and one lookahead policy.

The small instances of the problem can be solved to optimality, giving us an exact benchmark for the ADP method. However, these instances do not capture all the dynamics embedded in the problem, whereas medium-sized instances cannot be solved exactly. To evaluate the online performance of ADP, we compare with three consolidation policies without lookahead, as well as a rollout policy that incorporates a downstream cost estimate based on randomly generated future orders (Goodson et al., 2017). Under the first myopic policy $\pi^{\text {direct }}$, we always ship orders as soon as possible, as long as primary vehicle capacity is available. With the second myopic policy $\pi^{\text {post }}$, we postpone as many orders as possible, until an order reaches its latest dispatch time. For both policies, we fill up a vehicle that is to be dispatched with orders that are sorted and assigned as described in Algorithm 3.2; the sorting mechanism gives priority to orders that 
are more urgent, and for equally urgent orders prioritizes smaller volumes. The third myopic policy is $\pi^{\text {min }}$, which enumerates the full set of actions and minimizes the direct costs without estimating downstream costs. As no extra costs are charged to transport additional orders to locations that are already visited, we dispatch as much volume as possible if the costs are equal. Thus, the three myopic policies make use of consolidation opportunities by utilizing the remaining capacity of dispatched vehicles, but do not take into account the stochastic arrival process.

We proceed to explain the lookahead policy $\pi^{\text {roll }}$. We apply this benchmark policy to the medium-sized instances; later on we will explain why we do not use this lookahead policy for the large instances L1 and L2. The idea behind $\pi^{r o l l}$ is that we generate a number of sample arrival paths and heuristically solve the decision problems for these paths, thereby obtaining an estimate for the downstream costs. This benchmark policy is comparable to the rollout procedures as presented in, e.g., Goodson et al. (2013) and Goodson et al. (2017). More precisely, our benchmark policy may be viewed as a version of the postdecision rollout that is described in Goodson et al. (2017), in which we look ahead for multiple time steps. To apply $\pi^{\text {roll }}$ at a given decision moment, we first generate a set $\Omega^{m}$, which contains $m$ random arrival paths of length $\tau^{\text {roll }}$, i.e., $\omega^{m}=\left\{\omega_{t+1}^{m}, \ldots, \omega_{t+\tau^{r o l l}}^{m}\right\}$. We then enumerate all actions $x_{t}$ - meaning that the action space should be sufficiently small - to obtain the direct costs $C\left(S_{t}, x_{t}\right), \forall x_{t} \in \mathcal{X}_{t}$. Next, we select a path $\omega^{m} \in \Omega^{m}$, and obtain $S_{t+1}=$ $S^{M}\left(S_{t}, x_{t}, W_{t+1}\left(\omega_{t+1}^{m}\right)\right)$. After arriving in $S_{t+1}$, we use the heuristic-based policy $\pi^{\text {direct }}$ to obtain decisions for the remainder of the horizon, and denote the resulting costs as $\tilde{V}_{t}\left(S_{t}, \omega^{m}\right)$. These costs are calculated recursively for $t^{\prime}=t+1, \ldots, t+\tau^{r o l l}$ using

$$
\tilde{V}_{t^{\prime}}\left(S_{t}^{\prime}, \omega^{m}\right)=C\left(S_{t^{\prime}}, x_{t^{\prime}}\right)+\tilde{V}_{t^{\prime}+1}\left(S_{t^{\prime}+1}, \omega^{m}\right),
$$

with $S_{t^{\prime}+1}=S^{M}\left(S_{t^{\prime}}, x_{t^{\prime}}, W_{t^{\prime}+1}\left(\omega_{t^{\prime}+1}^{m}\right)\right)$ and $x_{t^{\prime}} \leftrightarrow S_{t^{\prime}}: \pi^{\text {direct }}$. We repeat this procedure for every post-decision state and for all arrival paths $\omega^{m} \in \Omega^{m}$. Thus, the estimated post-decision values are obtained as follows:

$$
\hat{V}_{t}\left(S_{t}^{x}\right)=\frac{\sum_{\omega^{m} \in \Omega^{m}} \tilde{V}_{t+1}\left(S_{t+1}, \omega^{m}\right)}{\left|\Omega^{m}\right|} .
$$


After estimating the post-decision values, we obtain the best decision by solving

$$
\underset{x_{t} \in \mathcal{X}_{t}\left(S_{t}\right)}{\operatorname{argmin}}=C\left(S_{t}, x_{t}\right)+\hat{V}_{t}\left(S_{t}^{x}\right) .
$$

We do not compare the performance of ADP with post-decision rollout in $\mathrm{L} 1$ and $\mathrm{L} 2$, as the procedure is not directly scalable to large action spaces. Specifically, our rollout procedure requires to calculate downstream costs for every post-decision state in advance. As the ILP is designed to handle outcome spaces that are too large to enumerate, we also are unable to pre-generate the downstream costs for all post-decision states. This problem might be overcome by either assigning downstream costs to aggregated post-decision states or by reducing the decision space. However, the design of such benchmark policies would justify a separate study, which is beyond the scope of this chapter.

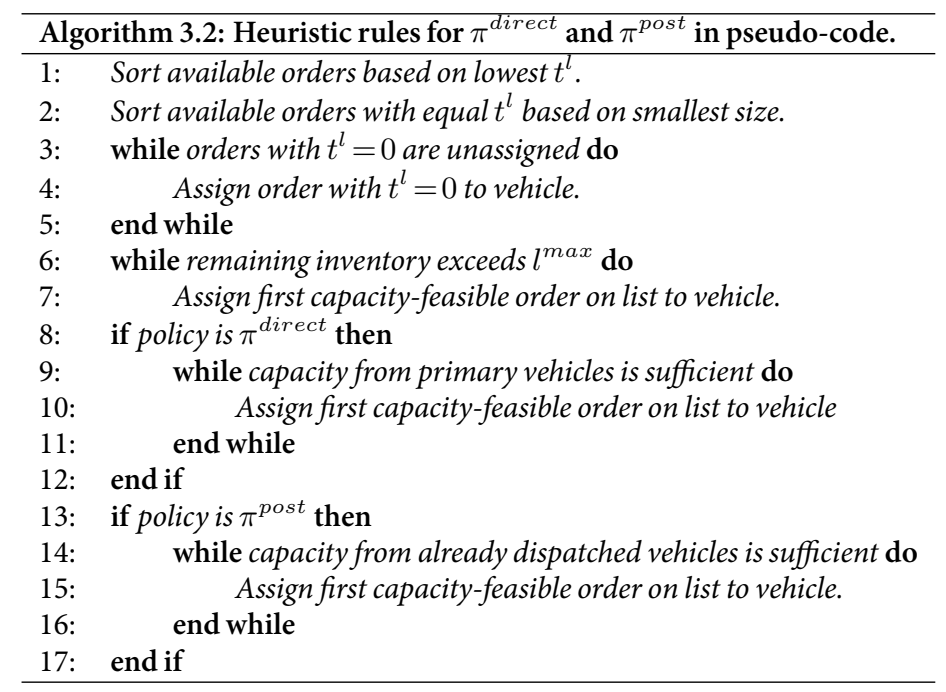

3.5.3. Generating sets of initial states. In this subsection, we describe the procedure to generate sets of initial states from which we draw at the start of each iteration. To learn a policy with ADP based on a given initial state, we might start every iteration with the same initial state and obtain a policy tailored to that specific state. While this is useful for testing purposes, in practical settings we might not repeat the entire offline learning phase for every state we encounter. Instead, we want to perform the offline learning phase only once, obtaining a policy that 
is applicable to all states over a longer period of time. However, randomly generating an initial state at every iteration may result in policies based on states that we rarely encounter in practice. To obtain a set of realistic initial states $\mathcal{S}^{\prime} \subset \mathcal{S}$, we perform a predefined number of simulation iterations with $\pi^{\text {direct }}$ - starting every iteration with a randomly generated initial state - and store the state encountered at $T / 2$ (avoiding possible warmup and cool-down effects) in $\mathcal{S}^{\prime}$. Next, at the start of each iteration $n$, we select $S_{0}^{n} \in \mathcal{S}^{\prime}$ with a probability proportional to the frequency it was observed. As order arrivals are random, we still visit states $S_{t} \notin \mathcal{S}^{\prime}$. However, by learning based on commonly encountered states, the results provide a better fit in practical settings.

\subsection{Numerical experiments}

This section presents the numerical results of the experiments. First, we show the results for small instances, then for medium-sized instances, and finally for large instances. When presenting results for the offline learning phases, we focus on the identification of suitable sets of basis functions. When discussing the results for the online phases, we compare the performance of the best ADP policy (using the most suitable set of basis functions) to various benchmark policies. For each class of instances, we will provide a separate outline of the results that we discuss.

3.6.1. Experiments on small instances. We separately present the results of the offline learning phase and the online phase. In the offline learning phase, we test various sets of basis functions, for which we assess how well they approximate the optimal outcomes obtained with dynamic programming, while also discussing the properties of good sets of basis functions. In the online phase, we apply the dispatching policy that we learned for the best set of basis functions and compare the results obtained with this policy to the optimal outcomes.

3.6.1.1. Results offline learning phase. To assess the explanatory value for various sets of basis functions, we compute their $R^{2}$ values and test how these sets perform in the offline learning phase. Using the procedure described in Section 3.5.3, we obtain a state set $\mathcal{S}^{\prime}$. For each simulation run, we use a state in $\mathcal{S}^{\prime}$ as the initial state and learn the corresponding policy. We consider a set of basis functions as a candidate set to apply on larger instances if it satisfies the following criteria: (i) the basis functions in the set are independent of each other, (ii) every basis function in the set contributes significantly to the $R^{2}$ value, (iii) regression can be performed within reasonable time, (iv) the number of basis functions is scalable to larger instances, (v) the numerical outcomes are robust 
TABLE 3.3. $R^{2}$ values and value gaps for various sets of basis functions.

\begin{tabular}{llllll}
\hline Set & Basis functions & \multicolumn{3}{c}{ S1 } & \multicolumn{2}{c}{ S2 } \\
& & $R^{2}$ & Avg. gap & $R^{2}$ & Avg. gap \\
\hline$\phi^{1}$ & Const. & - & $6.76 \%$ & - & $6.14 \%$ \\
$\phi^{2}$ & Const.+\# available destinations at $t^{e}=0$ & 0.16 & $2.80 \%$ & 0.14 & $3.57 \%$ \\
$\phi^{3}$ & Const.+\# vehicles & 0.34 & $6.77 \%$ & 0.31 & $6.14 \%$ \\
$\phi^{4}$ & Const.+Total vol. per $t^{l}$ & 0.35 & $2.11 \%$ & 0.32 & $2.78 \%$ \\
$\phi^{5}$ & Const.+Total vol. per $v$ & 0.27 & $2.97 \%$ & 0.31 & $3.34 \%$ \\
$\phi^{6}$ & Const.+\# orders per type & 0.35 & $5.27 \%$ & 0.40 & $5.36 \%$ \\
$\phi^{7}$ & Const.+\# Vehicles/\# available destinations & 0.41 & $5.63 \%$ & 0.37 & $6.21 \%$ \\
$\phi^{8}$ & Const.+\# Vehicles/Total vol. per $t^{l}$ & 0.23 & $6.48 \%$ & 0.20 & $6.70 \%$ \\
$\phi^{9}$ & Const.+\# destinations per $t^{l}$ & 0.14 & $2.92 \%$ & 0.12 & $3.50 \%$ \\
$\phi^{10}$ & Const.+Vol. per destination per $t^{l}$ & 0.35 & $2.97 \%$ & 0.39 & $3.34 \%$ \\
$\phi^{11}$ & Const.+\# available destinations+ & 0.48 & $2.05 \%$ & 0.43 & $2.76 \%$ \\
& Total vol. per $t^{l}$ & & & & \\
$\phi^{12}$ & Const.+\# vehicles+Total vol. per $t^{l}$ & 0.69 & $2.31 \%$ & 0.62 & $2.92 \%$ \\
$\phi^{13}$ & Const.+\# available destinations+ & 0.82 & $2.03 \%$ & 0.74 & $2.75 \%$ \\
& \# vehicles per $\tau_{t}+$ Total vol. per $t^{l}$ & & & & \\
\hline
\end{tabular}

for all states in $S^{\prime}$, and (vi) the average value gap (difference between optimal value and estimated value) is small.

The results for the sets of basis functions are shown in Table 3.3. We observe some discrepancies between the $R^{2}$ values and the actual performance of the corresponding policies that we learned. In general, we see that sets including the volume per $t^{l}$ yield estimates of good quality, which indicates that this property well explains the ranking of values of states. During the experiments, we observed that combining more than three types of basis functions often results in unstable behavior. Similar instabilities are found when combining multiple basis functions of the same type, e.g., two volume-based basis functions. Nonsegregated, nonlinear functions do not yield favorable results in terms of accuracy, and are notably difficult to combine with other basis functions. The set $\phi^{13}$ yields the best results of all tested sets. This set consists of the following basis functions: a constant, the number of available destinations, the number of vehicles available per dispatch time $t+1, \ldots, \tau^{\text {Max Route }}$, and the total volumes sorted per latest delivery time. We note that the set captures the key properties of the cost structure as listed in Section 3.4.5, while only requiring a total of 4 basis functions for the small instances to estimate the downstream costs with an error of less than $3 \%$. In comparison, a set such as $\phi^{6}$ contains many more basis functions, but yields considerably worse estimates. 


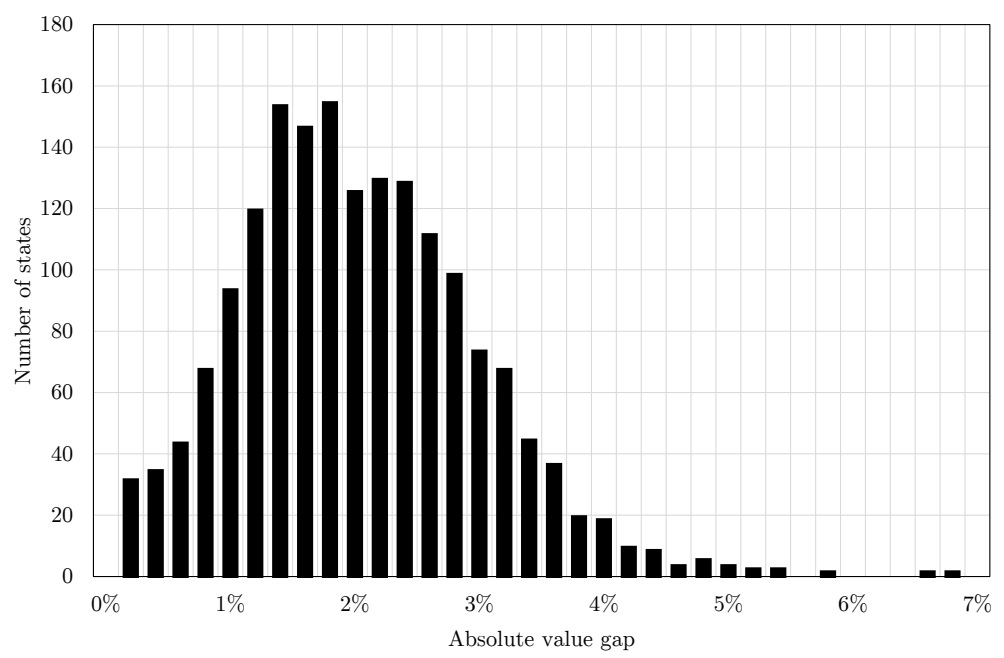

FIGURE 3.2. Histogram of absolute differences between exact values and ADP estimates for all states in $\mathcal{S}^{\prime}$.

3.6.1.2. Results online phase. We illustrate the ADP performance using the policy learned with set $\Phi^{13}$ on S1, comparing ADP estimates to exact values for the states in $\mathcal{S}^{\prime}$. Figure 3.2 shows the distribution of absolute deviations from the optimal value. For virtually all states, ADP yields estimates that are somewhat higher than the optimal value, with an average absolute deviation of $1.9 \%$. The tendency to overestimate costs follows from using suboptimal policies to estimate the downstream costs (Powell, 2011). For $0.24 \%$ of the states, the estimated and optimal values differ by more than $5 \%$; these deviations cause the tail on the right. In Figure 3.3, we show the ADP estimates for all $S_{0} \in \mathcal{S}^{\prime}$, plotted against the optimal values. This plot provides insight into the ranking of ADP estimates compared to the true ranking. We see that the ranking is rather well-preserved; ADP will not select actions that deviate much from the optimum.

3.6.2. Experiments on medium-sized instances. We separately present the results of the offline learning phase and the online phase. In the offline learning phase, we again test the performance for various sets of basis functions to 


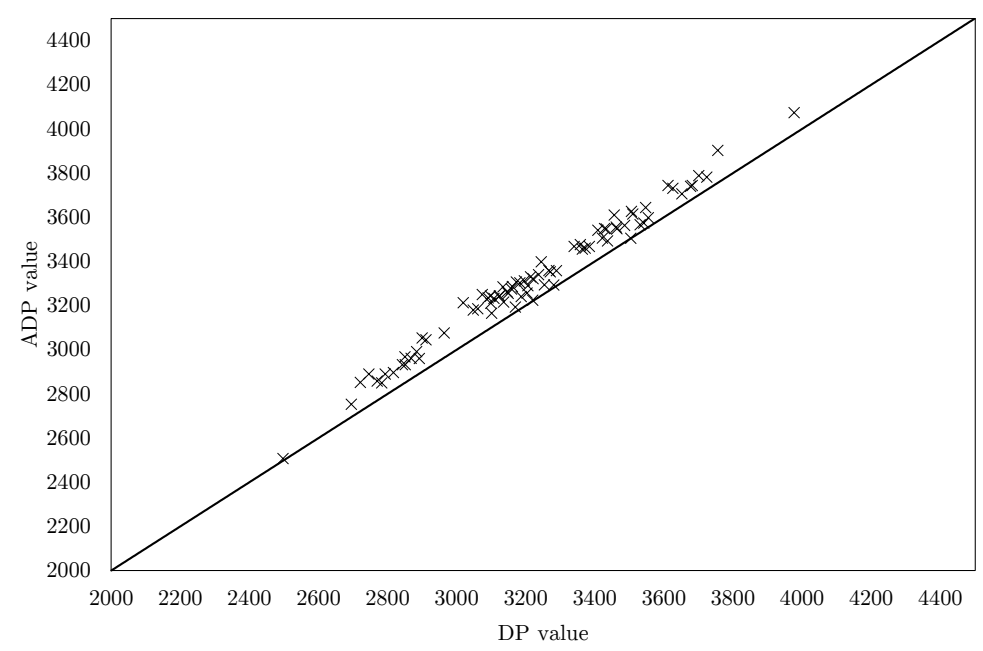

FIGURE 3.3. ADP estimates plotted against exact values for all states in $\mathcal{S}^{\prime}$.

evaluate how they perform under increased complexity, this time comparing to the direct cost minimization policy. In the online phase, we take the best set of basis functions to obtain the ADP policy; we compare this policy to a variety of benchmark policies on 20 different instances. Based on the results, we describe the instance properties under which ADP performs best. Furthermore, we discuss how the performance of ADP relates to both the performance of myopic policies and the rollout policy.

3.6.2.1. Results in offline learning phase. In the medium-sized instances, the number of order types is much larger than in the small instances. Therefore, we test how the sets of basis functions perform under this increased complexity. From Table 3.3, we take the sets of basis functions for which the corresponding policies yielded a value gap smaller than $5 \%$ on both S1 and S2. We repeat the offline learning phase for each set of basis functions meeting this criterion, yielding us $8 \mathrm{ADP}$ policies. We re-evaluate the performance of these sets on M2 and M5, as these may be considered as hybrids of the other medium-sized instances. To learn $\pi^{A D P}$, we randomly draw an initial state from $\mathcal{S}^{\prime}$ at every iteration. Subsequently, we measure the performance of all policies relative to 
TABLE 3.4. Performance of various $\phi$ on $M 2$ and M5, relative to $\phi^{1}$.

\begin{tabular}{llll}
\hline Set & Basis functions & Avg. gap (M2) & Avg. gap (M5) \\
\hline$\phi^{1}$ & Const. & $0 \%$ & $0 \%$ \\
$\phi^{2}$ & Const.+\# available destinations at $t^{e}=0$ & $-0.6 \%$ & $0.2 \%$ \\
$\phi^{4}$ & Const.+Total vol. per $t^{l}$ & $4.2 \%$ & $10.1 \%$ \\
$\phi^{5}$ & Const.+Total vol. per $v$ & $5.0 \%$ & $9.9 \%$ \\
$\phi^{9}$ & Const.+\# destinations per $t^{l}$ & $3.4 \%$ & $7.5 \%$ \\
$\phi^{10}$ & Const.+Vol. per location per $t^{l}$ & $3.7 \%$ & $7.8 \%$ \\
$\phi^{11}$ & Const.+\# available destinations+Total vol. per $t^{l}$ & $2.4 \%$ & $8.9 \%$ \\
$\phi^{12}$ & Const.+\# vehicles+Total vol. per $t^{l}$ & $7.7 \%$ & $13.3 \%$ \\
$\phi^{13}$ & Const.+\# available destinations+ & $7.9 \%$ & $14.0 \%$ \\
& \# vehicles per $\tau_{t}+$ Total vol. per $t^{l}$ & & \\
\hline
\end{tabular}

the performance using set $\phi^{1}$. The results are shown in Table 3.4. Similar to the results for the small instances, for both M2 and M5 the best results are obtained with $\phi^{13}$. Observe that the relative improvement in M5 is notably stronger than with M2; it appears that ADP performs better when there is more flexibility in the dispatching decision, i.e., when the dispatch windows of orders are wider.

3.6.2.2. Results in online phase. In this section, we test the performance of ADP policies on the medium-sized instances M1-M20, using the policies obtained with the set of basis functions $\phi^{13}$. Again, we randomly draw an initial state from $\mathcal{S}^{\prime}$ at every iteration. We learn $\pi^{A D P}$ individually for each mediumsized instance and evaluate the performance to the benchmark policies. We run the rollout policy $\pi^{\text {roll }}$ for various numbers of sample paths and lengths of the sample path, e.g., $\pi_{2,5}^{\text {sample }}$ denotes a policy with 2 sample paths of 5 time units each.

The computational results of the simulation phase are shown in Table 3.5. We highlight the main takeaways from the results. First, we see that ADP consistently and significantly outperforms all myopic benchmark policies. The values obtained by the rollout procedure are closer, although ADP typically outperforms the post-decision rollout as well. These results indicate that looking into the future pays off for the DDP-TW. Second, we observe that ADP outperforms rollout more strongly when considering wider dispatch windows. Voccia et al. (2015) suggest that - due to the larger number of possible arrival paths - a longer lookahead horizon requires more sample paths. This hypothesis appears to be confirmed by our results: when using only 2 or 5 sample paths, the rollout policy performs on average better with a lookahead interval of 1 . When comparing ADP to the rollout procedures, the results seem to indicate that ADP with VFA 
is more useful when considering decisions that have an impact on multiple future decision moments, taking into account the large number of paths required for the rollout procedure. Third, ADP performs best when the problem instance offers sufficient flexibility in terms of dispatch times, while vehicle capacity is scarce (M11-14). Fourth, we see that for larger networks (M15-16), ADP performs relatively strong. For these instances, travel costs have a relatively high weight compared to visiting costs, whereas restrictions on maximum route duration become more stringent. Finally, we note that the performance of ADP decreases when the basis functions do not properly address problem-specific characteristics (e.g., in the case of uneven demand distributions, see M7-8). A similar effect is observed for more realistic instances (M17-20), which are solved with a routing algorithm. Comparing to the results of $\pi^{\mathrm{min}}$, the outcomes suggest that lookahead has less added value for these instances. Interestingly, this effect is also observed for the rollout benchmarks; increasing the number of sample paths does not necessarily improve performance for these instances. As the outcomes of the routing algorithm are more difficult to learn than those of the Daganzo formula, additional basis functions might be necessary to improve the performance of $\pi^{A D P}$ for these instances. 


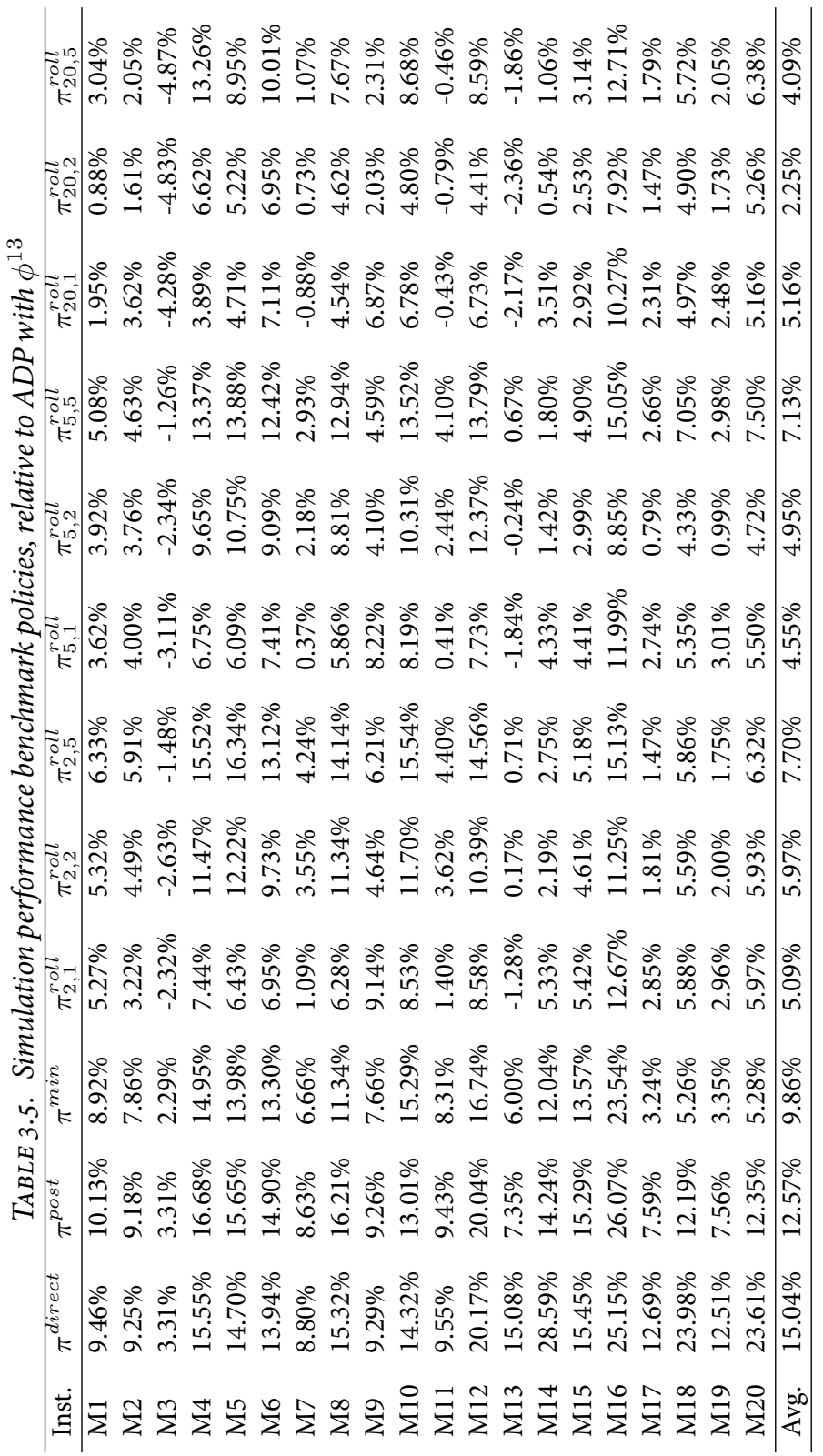


TABLE 3.6. Simulation performance of ADP policies against benchmark policies, relative to ADP with $\phi^{13}$.

\begin{tabular}{lcccccc}
\hline Instance & $\pi^{\text {direct }}$ & $\pi^{\text {post }}$ & $\pi^{\text {min }}$ & $\begin{array}{c}\text { Avg. solving } \\
\text { time ILP }\end{array}$ & $\begin{array}{c}\text { \% solved } \\
\text { within 60s }\end{array}$ & $\begin{array}{c}\text { Diff. with } \\
\text { cost function }\end{array}$ \\
\hline L1 & $7.9 \%$ & $6.7 \%$ & $20.8 \%$ & $3.08 \mathrm{~s}$ & $100.00 \%$ & $1.44 \%$ \\
L2 & $6.9 \%$ & $6.0 \%$ & $18.9 \%$ & $5.96 \mathrm{~s}$ & $99.31 \%$ & $1.81 \%$ \\
\hline Average: & $7.40 \%$ & $6.35 \%$ & $19.85 \%$ & $4.52 \mathrm{~s}$ & $99.66 \%$ & $1.63 \%$ \\
\hline
\end{tabular}

3.6.3. Experiments on large instances. This subsection reflects on the suitability of our solution method to handle large action spaces. First, we compare the results of the ADP policy (learned using the best set of basis functions) to those obtained with the myopic benchmark policies. Second, we provide statistics on the computational time required to solve the ILP.

For the large instances, we deal with state spaces of size $\gg 10^{178}$ and action spaces with sizes up to $2^{120}$. Enumerating all actions within reasonable time is not possible for these instances. In the experiments, we solve the decision problem at each decision moment with the ILP. In Section 3.4.3, we explained that the ILP requires to define a set of pre-defined input distances. The smaller this set is, the faster we can solve the ILP, but at the cost of reduced accuracy. Based on preliminary testing on medium-sized instances, we define 30 distinct distanceto-depot sums to estimate the actual travel distance of a decision. On average, this setting yields an average absolute cost difference between the ILP (using the approximate distances) and the Daganzo function of less than $2 \%$. The results of the experiments on L1 and L2 are shown in Table 3.6. As stated before, we compare the performance of ADP to the myopic benchmark policies. The benchmark policies are clearly outperformed, although the performance gaps are not as large as for the medium-sized experiments. A possible explanation for the smaller performance differences is that it might be more challenging to learn a good ADP policy due to the larger state space (more iterations may be required to properly learn values) and the introduction of the distance approximation error (as the approximation to some degree overestimates the direct costs). In addition to the performance gaps, we provide statistics on the time it takes to solve the ILP with CPLEX. Although significant computational effort is required in the offline learning phase (as we need to solve the decision problem for $N$ iterations times $T+1$ time periods), the ILP is shown to be fast enough to solve the decision problem in the online application. 


\subsection{Conclusions}

We addressed the dispatching problem faced by operators of urban consolidation centers. These centers have a key role in many urban logistics initiatives, as they allow bundling of fragmented freight flows to facilitate efficient distribution within urban areas. The operator of the center receives less-than-truckload orders that need to be distributed as efficiently as possible, but does not have perfect foresight into the arrival process. The operator periodically dispatches batches of accumulated orders, taking into account both current and future consolidation opportunities. To provide a formal definition of this problem, we extended the Delivery Dispatching Problem by including dispatch windows. We formulated this problem as a Markov decision model. Like many stochastic models, this Markov decision model becomes computationally intractable for larger instances of the problem. To be able to solve larger instances, we provided an ADP algorithm based on value function approximation, with which we are able to overcome the computational problems associated with large state spaces and outcome spaces. The consolidation policy is learned offline and might be periodically updated, e.g., if the stochastic arrival process changes. The learned policy can be applied to online decision problems. To address computational problems with large action spaces, we formulated an ILP model that can be used to solve the decision problem within the ADP algorithm.

In the numerical experiments, we demonstrated a three-step methodology to find suitable VFAs for realistic-sized problems. First, we showed how to use small instances to evaluate basis functions, using the exact values obtained by dynamic programming as a benchmark. We tested various sets of basis functions. The set of basis functions yielding the best approximations contains the following explanatory variables: (i) the number of possible destinations to visit (ii) the number of vehicles available per dispatch time, and (iii) the total volumes per latest delivery time. The resulting policy returns values that on average deviate $2.39 \%$ from the optimal values. Second, we evaluated the performance of the ADP policies on various medium-sized instances, testing the effects of various network settings and flexibility in dispatch windows. To evaluate the quality of the ADP policies, we compared their performance to two policies based on heuristic rules, to a direct cost minimization policy, and to a post-decision rollout policy. Using the best set of basis functions, we obtained a ADP policy that consistently outperformed the benchmark policies. The myopic benchmark policies are typically outperformed by $10-15 \%$, the rollout benchmark policies are outperformed by $2-8 \%$. Third, we solved two large instances - having state spaces of sizes $\gg 10^{178}$ and action spaces of sizes up to $2^{120}-$ using the ILP to 
solve the decision problems within ADP. The results illustrate the applicability of the algorithm for realistic-sized instances. Again, the resulting ADP policy outperformed the benchmark policies, for these instances the myopic benchmark policies are outperformed by $6 \%$ to $20 \%$. On average, we solved the ILP in 4.52 seconds in the large instances, which is fast enough for online decision making. However, due to the large number of iterations needed in ADP, the required computational effort to learn the consolidation policy remains significant. Therefore, heuristic reduction of the action space might be beneficial before implementing our approach in practice.

This chapter considered the dispatch procedure from the perspective of an independent UCC. The model included pre-announced orders, which reflects the possibility of communication between the line-haul carrier that supplies the city and the UCC itself. Nevertheless, in Chapter 2 and Chapter 3 we focused on freight consolidation from the perspective of isolated decision makers. The discrepancy between their perspectives already became clear to some extent; the LSP prefer the flexibility to replan orders (implying variation in the arrival process for the UCC), whereas planning for the UCC would be notably easier when all order arrivals are known up front. This illustrates the difficulties in aligning the objectives of various actors. In Chapter 4, we introduce an agent-based simulation framework, in which we explicitly model the various actors involved in urban supply chains. This framework allows us to evaluate the system-wide impact of the decisions of individual actors. Furthermore, it enables us to test a variety of governmental interventions, collaboration structures, and information exchange protocols.

\section{Appendix 3A: ILP model for large action spaces}

As stated in Section 3.4.3, we use an ILP to solve decision problems that cannot be solved in reasonable time by full enumeration. We present the ILP model in this appendix, starting with introducing the notation required for the objective function. As noted in the main text, we replace the original cost function (which allows all travel distances) with a cost function based on a limited set of distance classes to keep the size of the input manageable; only the number of locations visited and the numbers of primary and secondary vehicles dispatched determine the costs. Compared to the action formulation defined earlier, we therefore omit the subscript $q$, as we no longer assign orders to individual vehicles. The parameters $c^{p r, f i x}$ and $c^{s e, f i x}$ indicate the fixed costs per primary and secondary 
vehicle dispatched. The parameters $q^{p r, \max }$ and $q^{s e, \max }$ are the maximum numbers of primary and secondary vehicles that can be dispatched, we define corresponding sets $\hat{\mathcal{Q}}^{p r}=\left\{0, \ldots, q^{p r, \max }\right\}$ and $\hat{\mathcal{Q}}^{s e}=\left\{0, \ldots, q^{\text {se, } \max }\right\}$. The binary variables $z_{q^{p r}}^{p r}$ and $z_{q^{s e}}^{s e}$ indicate whether $q^{p r} \in \hat{\mathcal{Q}}^{p r}$ primary vehicles and $q^{s e} \in \hat{\mathcal{Q}}^{s e}$ secondary vehicles are dispatched. Let $d \in \mathcal{D}=\left\{0,1, \ldots, d^{\max }\right\}$ be an index corresponding to a pre-defined sum of distances-to-depot $\delta_{d} \in \mathbb{R}^{+}$, such that we have a set of distances $\left\{\delta_{0}, \delta_{1}, \ldots, \delta_{d^{\max }}\right\}$ with $\delta_{d}<\delta_{d+1}, \forall d \in \mathcal{D} \backslash d^{\max }$. The average distance-to-depot is approximated by $\delta_{d} /\left|\mathcal{V}^{\prime}\right|$. We set $\delta_{d^{\text {max }}}=\sum_{v \in \mathcal{V}} d_{0, v}$; although the theoretical value that guarantees feasible solutions at all times would be double this number, the selected maximum value suffices for all our solutions. Furthermore, we define $\mathcal{N}=\{0, \ldots,|\mathcal{V}|\}$ as the set containing the number of locations we may visit. The variable travel costs depend on the number of locations visited $n$, the distance class $d$ for the approximate sum of distances-to-depot $\delta_{d}$, and the dispatched number of primary vehicles $q^{p r}$ and secondary vehicles $q^{s e}$. These costs are given by parameter $c_{n, d, q^{p r}, q^{s e}}^{v a r}$ the binary variable $z_{n, d, q^{p r}, q^{s e}}$ indicates whether the model chooses the corresponding combination of number of locations, distance class, and vehicles dispatched. When customer $v \in \mathcal{V}$ is visited, handling costs $c_{v}^{h d l}$ are incurred. A visit to a location is indicated by the binary variable $z_{v}^{v i s}$. The objective function for the decision problem is

$$
\begin{aligned}
\min _{x \in \mathcal{X}_{t}\left(S_{t}\right)} & \left(\sum_{q^{p r} \in \hat{\mathcal{Q}}^{p r}} z_{q^{p r}}^{p r} \cdot q^{p r} \cdot c^{p r, f i x}+\sum_{q^{s e} \in \hat{\mathcal{Q}}^{s e}} z_{q^{s e}}^{s e} \cdot q^{s e} \cdot c^{s e, f i x}+\right. \\
& \sum_{n \in \mathcal{N}} \sum_{d \in \mathcal{D}_{q^{p r} \in \hat{\mathcal{Q}}^{p r}} \sum^{s e} \in \hat{\mathcal{Q}}^{s e}} z_{n, d, q^{p r}, q^{s e}} \cdot c_{n, d, q^{p r}, q^{s e}}^{v a r}+ \\
& \left.\sum_{v \in \mathcal{V}} z_{v}^{v i s} \cdot c_{v}^{h d l}+\sum_{f \in \mathcal{F}} \theta_{t, f} \cdot \phi_{f}\left(S_{t}^{x}\right)\right) .
\end{aligned}
$$

The objective function is subject to a number of constraints. First, we introduce several inventory-based constraints. Constraint (3.7.1) warrants that after an action, at most $l^{\max }$ orders remain in inventory. Constraint (3.7.2) ensures that no more orders of a certain type can be shipped than the number available of that type. Constraint (3.7.3) stipulates that all orders with $t^{l}=0$ must be dispatched, while Constraint (3.7.4) prevents orders with $t^{e}>0$ from being dispatched. Finally, Constraint (3.7.5) ensures that the post-decision inventory is 
calculated correctly.

$$
\begin{gathered}
\sum_{v \in \mathcal{V}} \sum_{l \in \mathcal{L}} \sum_{t^{l} \in \mathcal{T}^{l}}\left(I_{t, v, l, 0, t^{l}}-x_{t, v, l, 0, t^{l}}\right) \leq l^{\text {max }}, \\
x_{t, v, l, t^{e}, t^{l}} \leq I_{t, v, l, t^{e}, t^{l}} \\
\forall\left(v, l, t^{e}, t^{l}\right) \in \mathcal{V} \times \mathcal{L} \times \mathcal{T}^{e} \times \mathcal{T}^{l}, \\
x_{t, v, l, 0,0}=I_{t, v, l, 0,0} \\
\forall(v, l) \in \mathcal{V} \times \mathcal{L}, \\
x_{t, v, l, t^{e}, t^{l}}=0 \\
t^{e}>0, \forall\left(v, l, t^{l}\right) \in \mathcal{V} \times \mathcal{L} \times \mathcal{T}^{l}, \\
I_{t, v, l, t^{e}, t^{l}}^{x}=I_{t, v, l, t^{e}, t^{l}-x_{t, v, l, t^{e}, t^{l}}} \\
\forall\left(v, l, t^{e}, t^{l}\right) \in \mathcal{V} \times \mathcal{L} \times \mathcal{T}^{e} \times \mathcal{T}^{l} .
\end{gathered}
$$

Constraints (3.7.6) and (3.7.7) are included to compute the artificial binary variable $z_{\tau_{r}}^{\text {away }}$ for all route durations. A value $z_{\tau_{r}}^{\text {away }}=1$ indicates that dispatched vehicles are still away at $t+\tau_{r}^{\text {away }}$, whereas $z_{\tau_{r}}^{\text {away }}=0$ indicates that the dispatched vehicles are available again. Travel times depend on the number of locations $n$ visited, the approximate sum of distances-to-depot $\delta_{d}$, and the total number of dispatched vehicles $q^{p r}+q^{s e}$. Feasible travel times are given by the parameter $s_{n, d, q^{p r}+q^{s e}} \leq \tau^{\text {MaxRoute }}$. The value of $z_{\tau_{r}}^{\text {away }}$ is 1 for all $\tau_{r}$ at which vehicles are executing a route and 0 when they are available for dispatch again.

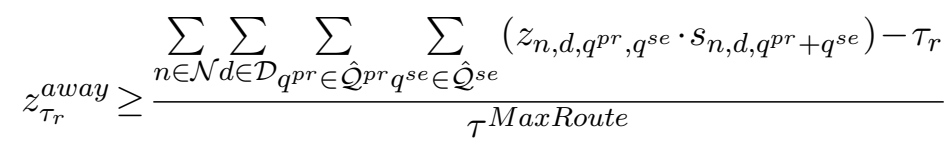

$$
\sum_{\tau_{r} \in\left\{0, \ldots, \tau^{\text {Max Route }\}}\right.} z_{\tau_{r}}^{\text {away }}=\sum_{n \in \mathcal{N}} \sum_{d \in \mathcal{D}_{q^{p r} \in \hat{\mathcal{Q}}^{p r}} \sum_{q^{s e} \in \hat{\mathcal{Q}}^{s e}} z_{n, d, q^{p r}, q^{s e}} \cdot s_{n, d, q^{p r}+q^{s e}}}^{\forall \tau_{r} \in\left\{0, \ldots, \tau^{\text {MaxRoute }}\right\}},
$$

The variable $q_{t, \tau_{r}}^{p r}$ indicates the number of vehicles available for dispatch at $t+\tau_{r}$. Variable $q_{t, \tau_{r}}^{x, p r}$ indicates the same for the post-decision state. With constraints (3.7.8), (3.7.9), and (3.7.10), we consistently update the post-decision 
availability of primary vehicles based on the average dispatch time:

$$
\begin{aligned}
& q_{t, \tau_{r}}^{x, p r} \leq q_{t, \tau_{r}}^{p r} \\
& \forall \tau_{r} \in\left\{0,1, \ldots, \tau^{\text {MaxRoute }\}}\right. \\
& q_{t, \tau_{r}}^{x, p r} \leq q_{t, \tau_{r}}^{p r}-\sum_{q^{p r} \in \hat{\mathcal{Q}}^{p r}} z_{q^{p r}}^{p r} \cdot q^{p r}+\left(1-z_{\tau_{r}}^{\text {away }}\right) q^{p r, \text { max }} \\
& \forall \tau_{r} \in\left\{0,1, \ldots, \tau^{\text {MaxRoute }}\right\} \\
& q_{t, \tau_{r}}^{x, p r} \geq q_{t, \tau_{r}}^{p r}-\sum_{q^{p r} \in \hat{\mathcal{Q}}^{p r}} z_{q^{p r}}^{p r} \cdot q^{p r} \\
& \forall \tau_{r} \in\left\{0,1, \ldots, \tau^{\text {MaxRoute }}\right\}
\end{aligned}
$$

The binary variable $z_{d}^{d i s}$ corresponds to the distance parameter $\delta_{d}$. Constraint (3.7.11) states that the capacity of the dispatched vehicles should be sufficient to carry all dispatched orders. Constraints (3.7.12) and (3.7.13) ensure that the approximate sum of depot-to-customer distances is at least equal to the actual sum. Constraint (3.7.14) ensures that no secondary vehicles are dispatched unless all primary vehicles have been dispatched. Finally, constraints (3.7.15) and (3.7.16) state that only one integer number of vehicles may be dispatched, both for primary and for secondary vehicles.

$$
\begin{aligned}
\sum_{q^{p r} \in \hat{\mathcal{Q}}^{p r}} z_{q^{p r}}^{p r} \cdot q^{p r}+\sum_{q^{s e} \in \hat{\mathcal{Q}}^{s e}} z_{q^{s e}}^{s e} \cdot q^{s e} & \geq \sum_{l \in \mathcal{L}} \sum_{v \in \mathcal{V}} \sum_{t^{e} \in \mathcal{T}^{e}} \sum_{t^{l} \in \mathcal{T}^{l}} x_{t, v, l, t^{e}, t^{l}} \cdot l \\
\sum_{v \in \mathcal{V}} z_{v}^{v i s} \cdot d_{0, v} & \leq z_{d}^{d i s} \cdot \delta_{d} \quad \forall d \in \mathcal{D}, \\
\sum_{d \in \mathcal{D}} z_{d}^{d i s} & =1, \\
z_{q^{p r, m a x}}^{p r} & \geq z_{q^{s e}}^{s e} \quad \forall q^{s e} \in \hat{\mathcal{Q}}^{s e} \backslash 0 \\
\sum_{q^{p r} \in \hat{\mathcal{Q}}^{p r}} z_{q^{p r}}^{p r} & =1 \\
\sum_{q^{s e} \in \hat{\mathcal{Q}}^{s e}} z_{q^{s e}}^{s e} & =1 .
\end{aligned}
$$


Constraints (3.7.17) and (3.7.18) ensure that $z_{v}^{v i s}=1$ if and only if location $v$ is visited. Constraint (3.7.19) states that the total number of locations visited is equal to the sum of unique locations visited; its correct working is asserted by Constraint (3.7.20).

$$
\begin{array}{rlrl}
z_{v}^{v i s} & \geq \frac{\sum_{l \in \mathcal{L} t^{e} \in \mathcal{T}^{e}} \sum_{l^{l} \in \mathcal{T}^{l}} x_{t, v, l, t^{e}, t^{l}}}{I^{\text {total }}} & & \forall v \in \mathcal{V}, \\
z_{v}^{v i s} & \leq \sum_{l \in \mathcal{L} t^{e} \in \mathcal{T}^{e}} \sum_{t^{l} \in \mathcal{T}^{l}} x_{t, v, l, t^{e}, t^{l}} & & \forall v \in \mathcal{V}, \\
\sum_{n \in \mathcal{N}} n z_{n}^{l o c} & =\sum_{v \in \mathcal{V}} z_{v}^{v i s}, & \\
\sum_{n \in \mathcal{N}} z_{n}^{l o c} & =1 .
\end{array}
$$

The set of constraints (3.7.21) $-(\overline{3.7 .25})$ enforce that $z_{n, d, q^{p r}, q^{s e}}=1$ for exactly one combination of the number of locations visited, total tour distance, primary vehicles dispatched, and secondary vehicles dispatched.

$$
\begin{aligned}
& z_{n, d, q^{p r}, q^{s e}} \leq z_{n}^{l o c} \\
& \forall\left(n, d, q^{p r}, q^{s e}\right) \in \mathcal{N} \times \mathcal{D} \times \hat{\mathcal{Q}}^{p r} \times \hat{\mathcal{Q}}^{s e}, \\
& z_{n, d, q^{p r}, q^{s e}} \leq z_{q^{p r}}^{p r} \\
& \forall\left(n, d, q^{p r}, q^{s e}\right) \in \mathcal{N} \times \mathcal{D} \times \hat{\mathcal{Q}}^{p r} \times \hat{\mathcal{Q}}^{s e}, \\
& z_{n, d, q^{p r}, q^{s e}} \leq z_{q^{s e}}^{s e} \\
& \forall\left(n, d, q^{p r}, q^{s e}\right) \in \mathcal{N} \times \mathcal{D} \times \hat{\mathcal{Q}}^{p r} \times \hat{\mathcal{Q}}^{s e}, \\
& z_{n, d, q^{p r}, q^{s e}} \leq z_{d}^{d i s} \\
& \forall\left(n, d, q^{p r}, q^{s e}\right) \in \mathcal{N} \times \mathcal{D} \times \hat{\mathcal{Q}}^{p r} \times \hat{\mathcal{Q}}^{s e},
\end{aligned}
$$

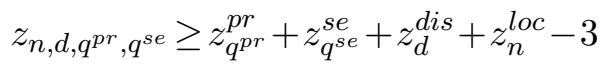

$$
\begin{aligned}
& \forall\left(n, d, q^{p r}, q^{s e}\right) \in \mathcal{N} \times \mathcal{D} \times \hat{\mathcal{Q}}^{p r} \times \hat{\mathcal{Q}}^{s e} .
\end{aligned}
$$


Finally, the values of the decision variables are subject to:

$$
\begin{array}{rlrl}
z_{\tau_{r}}^{\text {away }} \in\{0,1\} & & \forall \tau_{r} \in\left\{0, \ldots, \tau^{\text {MaxRoute }}\right\}, \\
z_{q^{p r}}^{p r} & \in\{0,1\} & & \forall q^{p r} \in \hat{\mathcal{Q}}^{p r}, \\
z_{q^{s e}}^{s e} & \in\{0,1\} & & \forall q^{s e} \in \hat{\mathcal{Q}}^{s e}, \\
z_{n}^{l o c} & \in\{0,1\} & & \forall n \in \mathcal{N}, \\
z_{v}^{v i s} & \in\{0,1\} & & \forall v \in \mathcal{V}, \\
z_{d}^{d i s} & \in\{0,1\} & & \forall d \in \mathcal{D}, \\
z_{n, d, q^{p r}, q^{s e}} & \in\{0,1\} & & \forall\left(n, d, q^{p r}, q^{s e}\right) \in \mathcal{N} \times \mathcal{D} \times \hat{\mathcal{Q}}^{p r} \times \hat{\mathcal{Q}}^{s e}, \\
I_{t, v, l, t^{e}, t^{l}}^{x} & \in \mathbb{N} & & \forall\left(v, l, t^{e}, t^{l}\right) \in \mathcal{V} \times \mathcal{L} \times \mathcal{T}^{e} \times \mathcal{T}^{l}, \\
q_{t, \tau_{r}}^{x, p r} & \in \mathbb{N} & & \forall \tau_{r} \in\left\{0,1, \ldots, \tau^{M a x R o u t e}\right\}, \\
x_{t, v, l, t^{e}, t^{l}} & \in \mathbb{N} & & \forall\left(v, l, t^{e}, t^{l}\right) \in \mathcal{V} \times \mathcal{L} \times \mathcal{T}^{e} \times \mathcal{T}^{l} .
\end{array}
$$

Appendix 3B: Proofs for the sizes of state, action, and outcome space

We first provide two lemmas regarding the number of order types and the number of vectors that denote vehicle dispatch availability. These results are later used in proving the sizes of the state space, action space, and outcome space. For the proofs we rely on various standard results from the field of combinatorics, such as the binomial coefficients to compute the numbers of multiset combinations and multiset permutations.

Lemma 3.1 Let $I_{t, v, l, t^{e}, t^{l}}$ with $v \in \mathcal{V}, l \in \mathcal{L}, t^{e} \in \mathcal{T}^{e}, t^{l} \in \mathcal{T}^{l}$ represent a unique order type. We define $\tau^{\text {Max Ahead }} \in \mathbb{N}$ as the maximum number of time units an order can be known in advance, and $\tau^{M a x W i n d o w} \in \mathbb{N}$ as the maximum number of time units between the earliest and latest dispatch time. Let $\mathcal{T}^{e}=\left\{0, \ldots, \tau^{\text {MaxAhead }}\right\}$ be the set of allowed earliest dispatch times, and $\mathcal{T}^{l}=\left\{t^{e}, \ldots, t^{e}+\tau^{\text {MaxWindow }}\right\}$ be the sets of corresponding latest dispatch times. Then, the number of unique order types is given by

$$
\left(\tau^{\text {MaxAhead }}+1\right) \cdot\left(\tau^{\text {MaxWindow }}+1\right) \cdot|\mathcal{V}| \cdot|\mathcal{L}|
$$

Proof: The set $\mathcal{T}^{l}$ is dependent on $\mathcal{T}^{e}$, as we impose that by definition $t^{e} \leq t^{l}$ for every order type $\left(v, l, t^{e}, t^{l}\right)$. As we have $t^{e} \in \mathcal{T}^{e}=\left\{0, \ldots, \tau^{\text {MaxWindow }}\right\}$, it 
follows that there are $\left|\mathcal{T}^{e}\right|=1+\tau^{\text {MaxWindow }}$ earliest dispatch times. Furthermore, we have $t^{l} \in \mathcal{T}^{l}=\left\{t^{e}, \ldots, t^{e}+\tau^{\text {MaxWindow }}\right\}$, such that we have $\left|\mathcal{T}^{l}\right|=$ $\tau^{\text {MaxWindow }}+1, \forall t^{e} \in \mathcal{T}^{e}$ latest dispatch times. It follows that the number of unique dispatch windows is given by $\left(\tau^{\text {MaxAhead }}+1\right) \cdot\left(\tau^{\text {MaxWindow }}+1\right)$. The remainder of the order-defining variables are independent of the dispatch windows. Any combination of a valid time window with an arbitrary destination $v \in \mathcal{V}$ and an arbitrary load size $l \in \mathcal{L}$ represents a unique order type. Hence, $\left(\tau^{\text {MaxAhead }}+1\right) \cdot\left(\tau^{\text {MaxWindow }}+1\right) \cdot|\mathcal{V}| \cdot|\mathcal{L}|$ unique order types exist.

Lemma 3.2 Let $\mathcal{Q}^{p r}$ be the set containing the primary vehicles, with $q \in \mathcal{Q}^{p r}$ representing an individual vehicle. The vector $Q_{t}=\left[\tau_{t, 1}, \ldots, \tau_{\left.t, \mid \mathcal{Q}^{p r}\right]}\right]$ represents the earliest availability per vehicle, with $\tau_{t, q} \in\left\{0, \ldots, \tau^{\text {MaxRoute }}\right\}$. Let $\tau^{\text {cum }} \in\left\{0, \ldots,\left|Q^{p r}\right| \cdot \tau^{\text {Max Route }}\right\}$ represent the cumulative route duration from all primary vehicles that are dispatched. Let $\xi_{a}^{\tau^{\text {cum }}}-$ with $a \in \mathbb{N}$ - be a vector described by $\left[n_{1}^{a}, n_{2}^{a}, \ldots, n_{\left|\mathcal{Q}^{p r}\right|}^{a}\right]$ with $n_{j}^{a} \in\left\{0, \ldots, \tau^{\text {MaxRoute }}\right\}, \forall j \in\left\{1, \ldots,\left|\mathcal{Q}^{p r}\right|\right\}$ that satisfies $\sum_{j=1}^{\left|\mathcal{Q}^{p r}\right|} n_{j}^{a}=\tau^{\text {cum }}$ and $n_{j}^{a} \leq n_{j+1}^{a}, \forall j \in\left\{1, \ldots,\left|\mathcal{Q}^{\text {pr }}\right|\right\}$. Let $\Xi_{\tau^{\text {cum }}}$ be the set containing all these vectors. The number of possible vectors $Q_{t}$ is given by

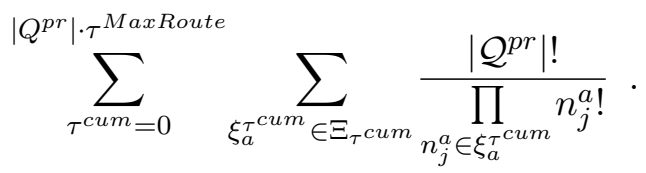

Proof: By definition, the vector $Q_{t}$ has a length $\left|\mathcal{Q}^{p r}\right|$. For every vehicle $q \in \mathcal{Q}^{p r}$, the set from which we draw $\tau_{t, q}$ contains $\tau^{\text {MaxRoute }}+1$ elements. The dispatch decisions per vehicle are independent of each other, meaning that we can obtain the expressions for $Q_{t}$ by computing all multiset permutations.

Take an arbitrary $\tau^{\text {cum }} \leftarrow\left\{0, \ldots,\left|Q^{p r}\right| \cdot \tau^{\text {MaxRoute }}\right\}$ and a corresponding vector $\xi_{a}^{\tau^{\text {cum }}} \leftrightarrow \Xi_{\tau^{\text {cum }}}$. Every permutation of $\xi_{a}^{\tau^{c u m}}$ represents a valid vehicle vector $Q_{t}$. The number of multiset permutations are computed by the multinomial coefficient

$$
\left(\begin{array}{c}
\left|\mathcal{Q}^{p r}\right| \\
n_{1}^{a}, \ldots, n_{\left|\mathcal{Q}^{p r}\right|}^{a}
\end{array}\right)
$$


For $0<\tau^{\text {cum }}<Q^{p r} \mid \cdot \tau^{\text {MaxRoute }}$ and $\left|\mathcal{Q}^{\text {pr }}\right|>1$, the set $\Xi_{\tau^{c u m}}$ contains multiple vectors $\xi_{a}^{\tau^{c u m}}$ that satisfy the restrictions. As every vector $\xi_{a}^{\tau^{\text {cum }}} \in$ $\Xi_{\tau^{\text {cum }}}$ by definition contains a unique combination of numbers, the corresponding permutations are unique as well. Hence, we sum the permutations of all $\xi_{a}^{\tau^{c u m}} \in \Xi_{\tau^{\text {cum }}}$. It is eminent that the vectors $\xi_{a}^{\tau^{c u m}}$ are unique for every distinct value $\tau^{\text {cum }}$. Therefore, we also sum the number of permutations over all $\tau^{\text {cum }} \in\left\{0, \ldots,\left|Q^{p r}\right| \cdot \tau^{\text {MaxRoute }}\right\}$. It follows that the number of unique vehicle vectors $Q_{t}$ is given by

$$
\begin{gathered}
\sum_{\tau^{\text {cum }}=0}^{\left|Q^{p r}\right| \cdot \tau^{\text {MaxRoute }}} \sum_{\xi_{a}^{\text {cum }} \in \Xi_{\tau^{c u m}}}\left(\begin{array}{c}
\left|\mathcal{Q}^{p r}\right| \\
n_{1}^{a}, \ldots, n_{\left|\mathcal{Q}^{p r}\right|}^{a}
\end{array}\right) \\
=\sum_{\tau^{\text {cum }}=0}^{\left|Q^{p r}\right| \cdot \tau^{\text {Max Route }}} \sum_{\xi_{a}^{\text {cum }} \in \Xi_{\tau^{\text {cum }}}} \frac{\left|\mathcal{Q}^{\text {pr }}\right| !}{\prod_{n_{j}^{a} \in \xi_{a}^{\text {cum }}} n_{j}^{a !}} .
\end{gathered}
$$

Proposition 3.1 Let $\mathcal{Q}^{\text {pr }}$ be the set containing the primary vehicles, with $q \in \mathcal{Q}^{\text {pr }}$ representing an individual vehicle. The vector $Q_{t}=\left[\tau_{t, 1}, \tau_{t, 2}, \ldots, \tau_{t,\left|\mathcal{Q}^{p r}\right|}\right]$ represents the earliest availability per vehicle, with $\tau_{t, q} \in\left\{0, \ldots, \tau^{\text {MaxRoute }}\right\}$. Let $\tau^{\text {cum }} \in\left\{0, \ldots,\left|Q^{p r}\right| \cdot \tau^{\text {MaxRoute }}\right\}$ represent the cumulative route duration from all primary vehicles that are dispatched. The number of multiset combinations $\bigcup_{\tau^{\text {cum }}=0}^{\left|Q^{\text {pr }}\right| \cdot \tau^{\text {Max Route }}} \Xi_{\tau^{\text {cum }}}$ is given by

$$
\frac{\left(\tau^{\text {MaxRoute }}+\left|\mathcal{Q}^{\text {pr }}\right|\right) !}{\left(\left|\mathcal{Q}^{\text {pr }}\right|\right) ! \cdot\left(\tau^{\text {MaxRoute }}\right) !} .
$$

Proof: Recall that we enforce that the elements in $\xi_{\tau}$ cum are in ascending order. Therefore, $\xi_{\tau \text { cum }}$ represents a combination rather than a permutation. Every set $\xi_{\text {cum }}$ is a multicombination of $\left|\mathcal{Q}^{p r}\right|$ elements obtained from the set $\left\{0, \ldots, \tau^{\text {MaxRoute }}\right\}$. As vehicle route durations are independent, repetition within the combinations is allowed. Thus, we compute the number of corresponding multicombinations with the multiset coefficient. By rewriting the multiset coefficient we obtain 


$$
\begin{gathered}
\left(\left(\begin{array}{c}
\tau^{\text {MaxRoute }}+1 \\
\left|\mathcal{Q}^{p r}\right|
\end{array}\right)\right)= \\
\left(\begin{array}{c}
\tau^{\text {MaxRoute }}+\left|\mathcal{Q}^{p r}\right| \\
\left|\mathcal{Q}^{p r}\right|
\end{array}\right)= \\
\frac{\left(\tau^{\text {MaxRoute }}+\left|\mathcal{Q}^{p r}\right|\right) !}{\left(\left|\mathcal{Q}^{\text {pr }}\right|\right) ! \cdot\left(\tau^{\text {MaxRoute }}\right) !} .
\end{gathered}
$$

Theorem 3.1 Let $S_{t}=\left[I_{t}, Q_{t}\right]$ be a problem state. Let $o_{t}^{\max , \text { tot }} \in \mathbb{N}$ be the maximum number of orders that can accumulate in a state. Let $o_{t}^{\text {tot }} \in\left\{0, \ldots, o_{t}^{\text {max }}\right.$ tot $\}$ be the number oforders in a state. We define $m_{i}^{b}=\mathbb{N}, i \in\left\{1, \ldots, o_{t}^{\text {max }}\right.$,tot $\}$ (with $b \in$ $\mathbb{N})$ as the number of orders of a specific type. Let $\gamma_{b}^{o_{t}}$ be a vector $\left[m_{1}^{b}, m_{2}^{b}, \ldots, m_{o_{t}^{t o t}}^{b}\right]$ that satisfies $\sum_{i=1}^{o_{t}^{\text {tot }}} m_{i}^{b}=o_{t}^{\text {tot }}$ and $m_{i}^{b} \leq m_{i+1}^{b}, \forall i \in\left\{1, \ldots, o_{t}^{\text {tot }}-1\right\}$. Let $\Gamma_{o_{t}^{\text {tot }}}$ be the set containing all vectors $\gamma_{b}^{t_{t}^{t o t}}$ for a given number of order arrivals $o_{t}^{\text {tot }}$. The size of the state space $\mathcal{S}_{t}$ is given by

$$
\begin{aligned}
& \left|\mathcal{S}_{t}\right|=\sum_{\tau^{\text {cum }}=0}^{\left|Q^{\text {pr }}\right| \cdot \tau^{\text {Max Route }}} \sum_{\xi_{a}^{\tau^{\text {cum }}} \in \Xi_{\tau^{\text {cum }}}} \frac{\left|\mathcal{Q}^{\text {pr }}\right| !}{\prod_{n_{j}^{a} \in \xi_{a}^{\text {cum }}} n_{j}^{a !}} .
\end{aligned}
$$

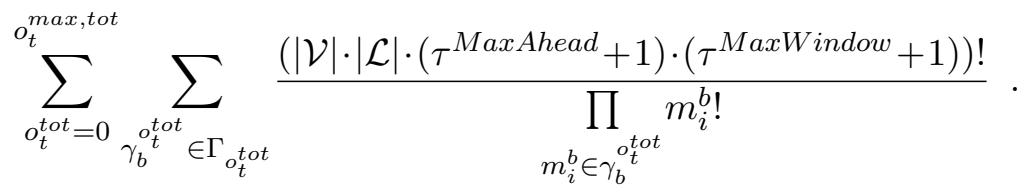

Proof: In Lemma 3.1, we established the number of unique order types. At each stage, we can have 0 to $o_{t}^{\max }$ new orders arriving. Orders can accumulate over time. The maximum latest dispatch time is given by $t^{l}=\tau^{\text {MaxAhead }}+$

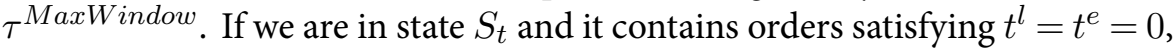
by Constraint (3.3.4) these orders must be shipped, such that they are no longer part of $S_{t+1}$. Presume that all arriving orders satisfy $t_{e}=\tau^{\text {MaxAhead }}$ and $t_{l}=$ $\tau^{\text {MaxAhead }}+\tau^{\text {MaxWindow }}+1$. It is eminent that orders with the latest possible 
dispatch time can be accumulated over the maximum period of time, hence, a state can contain at most $\left(\tau^{\text {MaxAhead }}+\tau^{\text {MaxWindow }}+1\right) \cdot o_{t}^{\text {max }}$ orders.

Every order that arrives can have any of the unique order types. We defined $m_{i}^{b}=\mathbb{N}, i \in\left\{1, \ldots, o_{t}^{t o t}\right\}$ as the number of orders of a specific type, and $\gamma_{b}^{o_{t}^{t o t}}$ as a vector $\left[m_{1}^{b}, m_{2}^{b}, \ldots, m_{o_{t}^{\text {tot }}}^{b}\right]$ satisfying the condition $\sum_{i=1}^{o_{t}^{\text {tot }}} m_{i}^{b}=o_{t}^{\text {tot }}$. For any given vector $\gamma_{b}^{o_{t}^{t o t}}$, we can compute the number of corresponding multiset permutations by solving

$$
\left(\begin{array}{c}
|\mathcal{V}| \cdot|\mathcal{L}| \cdot\left(\tau^{\text {MaxAhead }}+1\right) \cdot\left(\tau^{\text {MaxWindow }}+1\right) \\
m_{1}^{b}, \ldots, m_{o_{t}^{\text {tot }}}^{b}
\end{array}\right) .
$$

Every permutation represents a unique vector $I_{t}$. The permutations can be computed for every $\gamma_{b}^{o_{t}^{\text {tot }}} \in \Gamma_{o_{t}^{\text {tot }}}$ and for every $o_{t}^{\text {tot }} \in\left\{0, \ldots, o_{t}^{\text {max,tot }}\right\}$. Hence, the maximum number of unique vectors $I_{t}$ is given by

$$
\sum_{o_{t}^{t o t}=0}^{o_{t}^{\text {max }, \text { tot }}} \sum_{\gamma_{b}^{o t} \in \Gamma_{o_{t}^{\text {tot }}}}\left(\begin{array}{c}
\left(|\mathcal{V}| \cdot|\mathcal{L}| \cdot\left(\tau^{\text {MaxAhead }}+1\right) \cdot\left(\tau^{\text {MaxWindow }}+1\right)\right) \\
m_{1}^{b}, \ldots, m_{o_{t}^{t o t}}^{b}
\end{array}\right)
$$

In Lemma 3.2 we showed the number of distinct vehicle availability vectors that exist. A state combines a unique inventory position $I_{t}$ with a unique vehicle availability vector $Q_{t}$. The state space is therefore given by the sum of all possible multiset permutations of all allowed numbers of order arrivals, multiplied by the number of distinct vehicle availability vectors. It follows that the size of the state space is given by the binomial coefficients 


$$
\begin{aligned}
& \left|\mathcal{S}_{t}\right|=\sum_{\tau^{\text {cum }}=0}^{\left|Q^{p r}\right| \cdot \tau^{\text {MaxRoute }}} \sum_{\xi_{a}^{\tau^{\text {cum }}} \in \Xi_{\tau^{c u m}}}\left(\begin{array}{c}
\left|\mathcal{Q}^{\text {pr }}\right| \\
n_{1}^{a}, \ldots, n_{\left|\mathcal{Q}^{\text {pr }}\right|}^{a}
\end{array}\right) . \\
& \sum_{o_{t}^{\text {tot }}=0}^{o_{t}^{\text {max }, \text { tot }}} \sum_{\substack{\gamma_{b}^{\text {tot }} \in \Gamma_{o_{t}^{\text {tot }}} \\
\gamma_{t}}}\left(\begin{array}{c}
|\mathcal{V}| \cdot|\mathcal{L}| \cdot\left(\tau^{\text {Max Ahead }}+1\right) \cdot\left(\tau^{\text {MaxWindow }}+1\right) \\
m_{1}^{b}, \ldots, m_{o_{t}^{b t}}^{b}
\end{array}\right) \\
& =\sum_{\tau^{\text {cum }}=0}^{\left|Q^{\text {pr }}\right| \cdot \tau^{\text {Max Route }}} \sum_{\xi_{a}^{\tau^{\text {cum }}} \in \Xi_{\tau^{\text {cum }}}} \frac{\left|\mathcal{Q}^{\text {pr }}\right| !}{\prod_{n_{j}^{a} \in \xi_{a}^{\tau^{\text {cum }}}} n_{j}^{a !}} .
\end{aligned}
$$

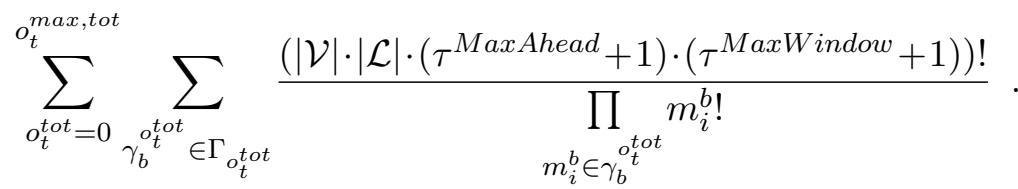

Theorem 3.2: Let $I_{t}=\left[I_{t, v, l, t^{e}, t^{l}}\right]_{\forall v \in \mathcal{V}, l \in \mathcal{L}, t^{e} \in \mathcal{T}^{e}, t^{l} \in \mathcal{T}^{l}}$ be a vector of order types, with $I_{t, v, l, t^{e}, t^{l}} \in \mathbb{N}, \forall v \in \mathcal{V}, l \in \mathcal{L}, t^{e} \in \mathcal{T}^{e}, t^{l} \in \mathcal{T}^{l}$. Furthermore, let $x_{t}=\left[x_{t, v, l, t^{e}, t^{l}}\right]_{\forall v \in \mathcal{V}, l \in \mathcal{L}, t^{e} \in \mathcal{T}^{e}, t^{l} \in \mathcal{T}^{l}}$ be an action on $I_{t}$ as defined in Equation (3.3.1), subject to the constraints (3.3.2)-(3.3.8). Let $\mathcal{X}_{t}$ be the set containing all actions. The size of the action space $\mathcal{X}_{t}$ is given by

$$
\begin{aligned}
\left|\mathcal{X}_{t}\right|= & \left(\sum_{v \in \mathcal{V}} \sum_{l \in \mathcal{L}} \sum_{t^{e} \in \mathcal{T}^{e}} \sum_{t^{l} \in \mathcal{T}^{l}} I_{t, v, l, t^{e}, t^{l}}\right) . \\
& \left|\mathcal{Q}^{p r} \cup \mathcal{Q}^{s e}\right| \cdot \prod_{v \in \mathcal{V} l \in \mathcal{L}_{t^{l}} \in \mathcal{T}^{l} \backslash\{0\}}\left(I_{t, v, l, 0, t^{l}}+1\right) .
\end{aligned}
$$

Proof: From Constraint (3.3.5) we obtain $x_{t, v, l, t^{e}, t^{l}}=I_{t, v, l, t^{e}, t^{l}} \mid t^{l}=0$. Hence, we have exactly 1 shipping option for order types that satisfy this $t^{l}=0$. Simil-

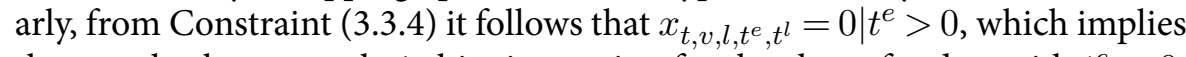
that we also have exactly 1 shipping option for the class of orders with $t^{e}>0$. For the remaining order types, Constraint (3.3.3) is valid, meaning that we can 
ship between 0 and $I_{t, v, l, t^{e}, t^{l}}$ units of these order types. For every order type that satisfies $t^{e}=0$ and $t^{l}>0$, we can ship $x_{t, v, l, t^{e}, t^{l}} \in\left\{0, \ldots, I_{t, v, l, t^{e}, t^{l}}\right\}$, such that we have $I_{t, v, l, t^{e}, t^{l}}+1$ options per order type. As in this case the maximum inventory constraint is not restrictive, the dispatch decisions for all order types are independent. It follows that the number of dispatch options is given by the number of permutations with repetitions allowed:

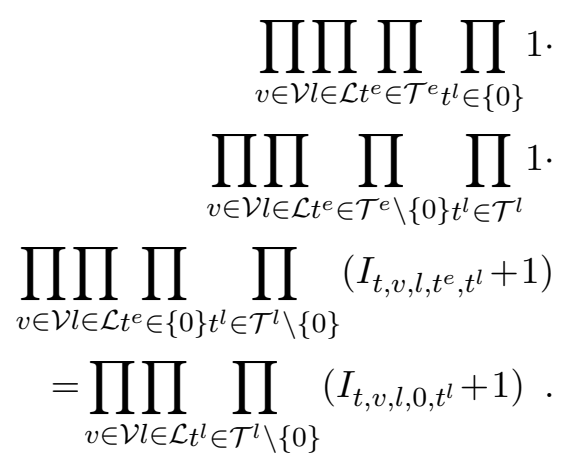

Each order can be assigned to any of the individual vehicles from either the primary fleet or the secondary fleet. Hence, every order that we ship can be assigned to one of $\left|\mathcal{Q}^{p r}\right|+\left|\mathcal{Q}^{s e}\right|$ unique vehicles. Thus, the number of unique ways to assign orders to vehicles is given by

$$
\sum_{v \in \mathcal{V}} \sum_{l \in \mathcal{L} t^{e} \in \mathcal{T}^{e}} \sum_{t^{l} \in \mathcal{T}^{l}} I_{t, v, l, t^{e}, t^{l}} \cdot\left|\mathcal{Q}^{p r} \cup \mathcal{Q}^{s e}\right|
$$

Multiplying this term with the number of possible dispatch decisions gives the size of the action space:

$$
\begin{aligned}
\left|\mathcal{X}_{t}\right|= & \left(\sum_{v \in \mathcal{V}} \sum_{l \in \mathcal{L}} \sum_{t^{e} \in \mathcal{T}^{e}} \sum_{t^{l} \in \mathcal{T}^{l}} I_{t, v, l, t^{e}, t^{l}}\right) . \\
& \left.\left|\mathcal{Q}^{p r} \cup \mathcal{Q}^{s e}\right| \cdot \prod_{v \in \mathcal{V} l \in \mathcal{L} t^{l} \in \mathcal{T} l \backslash\{0\}} \prod_{t, v, l, 0, t^{l}}+1\right) .
\end{aligned}
$$


Theorem 3.3 Let $\Omega_{t}$ be the outcome space corresponding to a state-action pair $\left[S_{t-1}, x_{t-1}\right]$. Let $o_{t}^{\max }$ be the maximum number of orders that can arrive at decision moment $t$. We define $m_{i}^{b}=\mathbb{N}, i \in\left\{1, \ldots, o_{t}\right\}$ as the number of orders of a specific type. Let $\gamma_{o_{t}}$ be a vector $\left[m_{1}^{b}, m_{2}^{b}, \ldots, m_{o_{t}}^{b}\right]$ that satisfies $\sum_{i=1}^{o_{t}} m_{i}^{b}=o_{t}$. Let $\Gamma_{o_{t}}$ be the set containing all these vectors for a given number of order arrivals $o_{t}$. Then, the size of the outcome space $\Omega_{t}$ is given by:

$$
\left|\Omega_{t}\right|=\sum_{o_{t}=0}^{o_{t}^{\max }} \sum_{\gamma_{b}^{o t} \in \Gamma_{o_{t}}} \frac{|\mathcal{V}| \cdot|\mathcal{L}| \cdot\left(\tau^{\text {MaxAhead }}+1\right) \cdot\left(\tau^{\text {MaxWindow }}+1\right) !}{\prod_{m_{i}^{b} \in \gamma_{b}^{o_{t}}} m_{i}^{b} !}
$$

Proof: In accordance with the transition function (3.3.9), the orders already held in inventory and the dispatch of vehicles do not influence the size of the outcome space, as these yield deterministic outcomes. Hence, only the realization of new order arrivals $\omega_{t}$ determines the size of the outcome space. The maximum number of arrivals is $o_{t}^{\max }$. Thus, for every order type, $\tilde{I}_{t+1, v, l, t^{e}, t^{l}} \in$ $\left\{0, \ldots, o_{t}^{\max }\right\}$ may arrive. Hence, we need to compute all permutations of orders arrivals. Every permutation represents a unique vector $\omega_{t}$. The permutations can be computed for every $\gamma_{b}^{o_{t}^{t o t}} \in \Gamma_{o_{t}^{t o t}}$ and for every $o_{t} \in\left\{0, \ldots, o_{t}^{\max }\right\}$. Hence, the maximum number of unique vectors $\omega_{t}$ is given by

$$
\begin{aligned}
\left|\Omega_{t}\right|= & \sum_{o_{t}=0}^{o_{t}^{\max }} \sum_{\gamma_{b}^{o_{t}} \in \Gamma_{o_{t}}}\left(\begin{array}{c}
|\mathcal{V}| \cdot|\mathcal{L}| \cdot\left(\tau^{\text {MaxAhead }}+1\right) \cdot\left(\tau^{\text {MaxWindow }}+1\right) \\
m_{1}^{b}, \ldots, m_{o_{t}}^{b}
\end{array}\right) \\
& \sum_{o_{t}=0}^{o_{t}^{\max }} \sum_{\gamma_{b}^{o t} \in \Gamma_{o_{t}}} \frac{|\mathcal{V}| \cdot|\mathcal{L}| \cdot\left(\tau^{\text {Max Ahead }}+1\right) \cdot\left(\tau^{\text {MaxWindow }}+1\right) !}{\prod_{m_{i}^{b} \in \gamma_{b}^{o t}} m_{i}^{b !}}
\end{aligned}
$$




\section{Appendix 3C: Recursive least squares method for nonstationary data}

In this appendix, we provide a brief discussion of the regression method that we apply in this chapter. As already noted in the main text, a complete description of this method can be found in Powell (2011). Recall that after every iteration $n$, we update the weights $\theta_{t, f}^{n}, \forall f \in \mathcal{F}, t \in \mathcal{T}$ using recursive least squares for nonstationary data. This procedure requires storing only a small amount of data, and can be swiftly executed during the ADP learning phase.

We proceed with an outline of the regression method. Let $B^{n}$ be a matrix of size $|\mathcal{F}| \cdot|\mathcal{F}|$. We recursively update this matrix with $B^{n}=\frac{1}{\lambda^{n}}\left(B^{n-1}-\right.$ $\left.\frac{1}{\gamma^{n}}\left(B^{n-1} \phi\left(S_{t}^{x}\right)\left(\phi\left(S_{t}^{x}\right)\right)^{T} B^{n-1}\right)\right)$, with discount factor $\lambda^{n}$ and $\gamma^{n}=\lambda^{n}+$ $\left(\phi\left(S_{t}^{x}\right)\right)^{T} B^{n-1} \phi\left(S_{t}^{x}\right)$. Next, we define a matrix $H^{n}=\frac{1}{\lambda^{n}} B^{n-1}$. The set of weights is now updated with

$$
\theta_{t, f}^{n}=\theta_{t, f}^{n-1}-H_{n} \phi_{f}\left(\bar{V}_{t-1}^{n-1}\left(S_{t-1}^{x}\right)-\hat{v}_{t}^{n}\right) \quad \forall f \in \mathcal{F}, t \in \mathcal{T} .
$$

To initialize the recursive least squares method, we set $B^{0}=\psi I$, with $\psi=$ 0.000001 and $I$ being the identity matrix. Furthermore, we set $\theta_{t, f}^{0}=1, \forall f \in$ $\mathcal{F}, t \in \mathcal{T}$. For the discount factor $\lambda^{n}$, we use $\lambda^{n}=1-\bar{\lambda} / n$. A value $\lambda^{n}<1$ indicates that we put more weight on recent observations, whereas $\lambda^{n}=1$ implies that equal weight is placed on all the observations. The latter would be the optimal choice for stationary data, but the observations obtained with ADP are typically obtained from a nonstationary process. Based on preliminary experiments, we fix $\bar{\lambda}=0.99$, meaning that we put relatively high weights on recent observations during the first few iterations. The reason for this is that the weights are initialized at 1 , such that the first estimates are far off from the actual values. Therefore, we do not want these early outcomes to strongly affect the estimates later on. 


\section{Part III}

\section{Evaluating urban logistics initiatives}





\section{CHAPTER 4}

\section{An agent-based simulation framework}

This chapter ${ }^{1}$ introduces an agent-based simulation framework that can be used to evaluate a variety of government policies and company-driven initiatives in the context of urban logistics. In particular, the framework enables to simultaneously analyze multiple measures, i.e., urban logistics schemes that contain more than one measure.

Part II of this thesis describes optimization techniques related to the decision problems of individual actors. In Part III, we adopt a more holistic view on urban supply chains, studying the behavior of autonomous actors that aim to optimally solve their own decision problems. Whereas Part II addresses the optimization of decision problems, Part III focuses on the evaluation of existing systems. We do not attempt to optimize the system itself, but instead analyze how the system behaves under varying circumstances. In this chapter, we design an agentbased simulation framework to evaluate urban logistics schemes. We provide a formal description of the agent types, discuss the decisions that they can make, and define the KPIs that we use to measure their performances. Although we provide an illustration of how the framework works by means of a small number of numerical experiments, full numerical studies based on the framework are saved for the remaining chapters of Part III.

The chapter is structured as follows. Section 4.1 introduces the generic problem and the contribution of this chapter. In Section 4.2, we provide a literature review on applications of operations research in the field of urban logistics. Section 4.3 introduces the agent-based simulation framework. We briefly illustrate the working of the framework in Section 4.4; Chapter 5 and Chapter 6 apply the framework to perform comprehensive simulation studies. Finally, Section 4.5 concludes the chapter and reiterates the contributions of the framework.

\footnotetext{
${ }^{1}$ This chapter is based on the paper: W.J.A. van Heeswijk, M.R.K. Mes, \& J.M.J. Schutten (2016). An agent-based simulation framework to evaluate urban logistics schemes. Lecture Notes in Computer Science 9855, 369-383 (Van Heeswijk et al., 2016a)
} 


\subsection{Introduction}

In Chapter 1, we discussed how both governments and companies try to find solutions in response to trends that negatively impact the efficiency of urban freight transport. Examples of such solutions are vehicle access restrictions or zone access fees that are set by local administrators. Companies may adopt new methods and resources to improve the efficiency of their operations, but also collaboration initiatives - both horizontal and vertical - are set up to reduce costs. There is a large variety of such initiatives in urban logistics; initiatives can be viewed separately, but also in combination with each other (e.g., the impact of vehicle access restrictions combined with carrier collaboration, often called freight consolidation). An urban logistics scheme consists of one or more forms of company-driven change and governmental policies, with the aim to improve efficiency and/or reduce external costs, i.e., intangible environmental and societal costs (Anderson et al., 2005). Urban consolidation centers (UCCs) have a central role in many schemes, facilitating order bundling and efficient last-mile distribution. Since actors that handle large volumes often already benefit from economies of scale and have the expertise to optimize their transport processes, our focus is on actors that handle small volumes, which are affected most by the increasing fragmentation in urban logistics.

Despite the eminent need for better organizing urban logistics, the vast majority of schemes fails after a short life-span (Browne et al., 2005). A major reason for this is that the involved actors typically have divergent objectives, making it difficult to find solutions to which all actors are willing to commit (Bektass et al., 2015). Administrators often attempt to generate commitment by providing financial incentives in the form of subsidies (Van Duin et al., 2012). However, such solutions are often not sustainable once the subsidies are halted (Ploos Van Amstel, 2015). Another problem for many schemes is that they are implemented after only little preliminary analysis, thereby not adequately evaluating their system-wide impact (Quak, 2011). A last shortcoming is that studies often focus solely on the processes within the city boundaries, while last-mile distribution accounts for only a small part of the supply chain. As such, they ignore the impact of upstream decisions. Of particular importance is the allocation of slack in the chain; holding freight early in the supply chain may improve vehicle fill rates, but reduces flexibility for the UCC in last-mile distribution. In our framework, we explicitly address these aspects.

A key success factor for urban logistics schemes is the right combination of company involvement and governmental policies (Browne et al., 2005). Actors 
must be willing to permanently change their behavior, without requiring perpetual external cash flows. Traditional optimization techniques may be used to find viable system-wide solutions, yet these are not guaranteed to be stable when their success depends on multiple decision makers. Furthermore, it is difficult to evaluate the impact of schemes that are comprised of multiple measures. Agentbased simulation is suitable to evaluate such schemes, as it is capable to monitor and alter the behavior of autonomous actors under conditions that can be flexibly adjusted (Taniguchi et al., 2014). In this chapter, we provide an agent-based simulation framework to evaluate the effectiveness of urban logistics schemes that include both governmental policies and company-driven initiatives.

\subsection{Literature on evaluating urban logistics initiatives}

As mentioned in Section 4.1, both companies and administrators actively take measures to improve the cost efficiency and environmental impact of urban freight transport. We briefly repeat the four classes of initiatives in urban logistics by Quak (2011), which have been introduced in Chapter 1. First, he considers improvements that require changing the context of urban logistics, which he divides in (i) physical infrastructure initiatives (e.g., UCCs, special lanes for electric vehicles) and (ii) transport-reorganizing initiatives (e.g., using alternative transport modes). Second, he considers improvements within the context of existing operations, in which he distinguishes between (iii) governmental policies (e.g., vehicle access restrictions, zone access fees, subsidization) and (iv) company-driven initiatives (e.g., improved routing algorithms, better use of real-time data, collaboration structures). To quantitatively evaluate the impact of schemes in one or more of the described initiative classes, a variety of operations research methods can be applied. For literature reviews on modeling efforts in urban logistics, we refer to Anand et al. (2012) and Bektaş et al. (2015). According to these reviews, most studies focus on describing and evaluating existing initiatives. Relatively few papers in the context of urban logistics adopt an operations research perspective (Crainic et al., 2009). In this section, we give a non-exhaustive overview.

The VRP is at the base of many transportation problems studied in literature. Also in the context of urban logistics, a number of VRP variants exist. Cattaruzza et al. (2015) and Kim et al. (2015) provide overviews of VRP solution methods in urban logistics; we highlight a few applications in various problem classes. A first class of applications relates to the incorporation of time-dependent travel times. Urban areas are often subject to congestion, such that the time of the day may considerably impact the travel times and the structures of optimal routes. 
Real-time extensions of the VRP with time-dependent travel times also take into account factors such as blocked roads and accidents, allowing to react instantly to changes in travel times. To solve the VRP with time-dependent travel times, Van Woensel et al. (2008) propose a solution method based on queueing theory. They show that accounting for time-dependent travel times considerably improves the quality of solutions for instances with congested traffic. Kok et al. (2010) study a variant of the problem that also takes into account the legally required driver breaks. They propose a restricted dynamic programming heuristic to solve this problem.

A second problem class that we discuss is the VRP with time access constraints. Many cities impose time access constraints that only allow trucks to drive within the city at designated time intervals. The rationale behind this measure is that the city is kept free of trucks for a large part of the day. Muñuzuri et al. (2012) study the VRP when time access constraints are imposed by the local administrator. They find that carriers require more trucks to serve the same set of customers, resulting in higher operating costs.

A final class that we discuss is the emission routing problem; Bektaş et al. (2016) provide a contemporary review on this topic. In this problem, the goal is to minimize emissions (within certain constraints) by adjusting driving speeds (Psaraftis et al., 2016). One of the early studies in emission routing is presented by Suzuki (2011), who applies a metaheuristic based on simulated annealing. His approach is based on the notion that if heavy loads are delivered first, the fuel requirements for the remainder of the route are reduced. Ehmke et al. (2016) study the emission routing problem in cities, in which vehicles must adapt to the prevailing traffic speeds. They propose a tabu search algorithm to solve this problem. Cheng et al. (2016) study a multi-period inventory routing problem, in which the carrier is subject to emission regulation. To solve this problem, they apply a genetic algorithm.

This thesis extensively addresses the role of distribution systems with consolidation centers to facilitate distribution in urban areas. We typically assume the presence of a single UCC in a city. A generalized version of this distribution system is the multi-echelon distribution system. Whereas a single UCC may suffice for many small to medium-size cities, larger cities might require more facilities. For such cities, a two-echelon distribution system may be more appropriate. Such a system is composed of large distribution centers in the outskirts of the city, with smaller satellite facilities located at the edge of urban regions. Goods can then either be delivered directly, via one of the distribution centers, or via 
one of the satellites. Cuda et al. (2015) provide a literature review on this problem class; we highlight a number of notable works. Crainic et al. (2009) provide a modeling framework for vehicle routing in multi-echelon systems, proposing a decomposition method to solve the two-echelon problem. Hemmelmayr et al. (2012) introduce an adaptive large neighborhood search heuristic for twoechelon vehicle routing problems arising in a city logistics context. Their main contributions are the design of functional operators (e.g., destroy and insert operators) and the exploitation of a problem structure that only allows VRP tours from the satellite facilities. An exact solution method for the problem is provided by Baldacci et al. (2013); they introduce a decomposition approach that allows to solve a set of subproblems within reasonable time. Dellaert et al. (2016) present a branch-and-price algorithm to solve the two-echelon vehicle routing problem with delivery windows being imposed by the customers.

Although the works mentioned before take into account various aspects that are relevant in urban logistics, the solution methods are designed from the perspective of isolated decision makers, typically carriers. Evaluating how a single actor would be affected by a scheme does not capture the system-wide effects of measures. Furthermore, such solution methods do not allow to study collaboration initiatives. A final aspect that is not addressed by single-actor decision analysis is that the objectives of actors in urban logistics are often divergent. A measure that yields beneficial results for one actor may be detrimental for others, thus raising opposition to the solution. As the inability to generate sufficient commitment is often problematic in practice, this is something that should be addressed in the analysis. To address this shortcoming in traditional analysis, Macharis (2007) describes the use of multi-criteria, multi-actor evaluation in the field of logistics. This method includes defining an objective function for every actor involved - the function may comprise both financial and non-financial components - and computing the impact of implementing a scheme for each actor involved. A number of applications of multi-criteria, multi-actor evaluation can be found in Macharis et al. (2012), Stathopoulos et al. (2012), and Verlinde et al. (2014). Although multi-actor decision analysis helps to evaluate the system-wide impact of schemes and illuminate discrepancies, it does not specify how the decision-making processes of the involved actors would be affected. As such, it is less suitable to analyze settings in which actors can take multiple actions and interact with each other.

A well-known solution method that takes into account the strategic interactions between various actors is game theory. We elaborate further upon the research opportunities related to game theory in Chapter 7; some applications 
in transport are briefly discussed here. One example of game theory applied in the context of logistics is the so-called flow game. In such a game, each arc in a network represents a transport service offered by an independent carrier. By cooperating, the carriers can increase the system-wide payoff. A prerequisite for the carriers to cooperate is that the system-wide gains are divided in a fair manner. Examples of studies on flow games in logistics are Reyes (2005) and Hafezalkotob and Makui (2015). Other game variants explicitly study the urban context, including the role of the UCC. Such games are studied by, e.g., Yang and Odani (2007) and Dahlberg (2015). Although game theory provides a framework to model and evaluate the interaction between multiple actors, it does have its flaws when the aim is to analyze urban logistics schemes. For one, classic game theory assumes that every actor has perfect knowledge regarding the other players' payoffs. In reality, it is common that actors do not know how their actions impact others, as we already noted in Chapter 1. Games with imperfect information do exist, yet these are typically very difficult to solve (Koller and Pfeffer, 1997). Furthermore, when analyzing the interactions between many actors - as is common in an urban logistics setting - the corresponding payoff matrices become very large, making it cumbersome to calculate the solutions. Finally, correctly modeling a complex environment can be difficult and timeconsuming, and needs to be redone at least partially for every scheme. This is particularly problematic when a large number of schemes must be evaluated.

Taniguchi et al. (2014) and Anand et al. (2016) state that agent-based simulation is the most applicable method to study the behavior of and interaction between the various actors for urban logistics schemes. Agent-based simulation is not capable to study detailed interactions (Bektaş et al., 2015), yet it is suitable to deduce generic insights on system performance. We mention some notable agent-based simulation studies in the field of urban logistics. Taniguchi et al. (2007) present an agent-based system in which carriers use a routing algorithm that dynamically adjusts to the prevailing travel times. They show that by adopting this more advanced routing algorithm, both carriers and shippers are able to benefit from the resulting cost reductions, while emissions are also reduced. A prerequisite for success is that a gain-sharing mechanism is installed, such that both carriers and shippers profit from the efficiency gains. Tamagawa et al. (2010) perform an agent-based simulation study in which they heuristically solve a VRP and iteratively update the actions of five agent types. They compare two learning mechanisms that the agents use to update their decisions, namely Monte Carlo simulation and Q-learning, which is a model-free reinforcement learning technique. The numerical experiments revolve around the effects of 
road pricing and truck bans. The authors state that despite the implementation of such measures, the carrier remains able to operate at transport costs comparable to those before. Boussier et al. (2011) apply an agent-based traffic simulation to study the use of electric delivery vans, with a reservation system for parking places being implemented to support the use of these vans. They claim that parking policies can be effective to improve the performance of distribution systems in urban areas. Van Duin et al. (2012) investigate the financial model and the environmental impact of UCCs, taking into account UCC service fees, road pricing, and subsidizing. Based on numerical experiments, they conclude that for a UCC to be financially viable, additional revenues from permanent subsidies or value-adding services are required. Wangapisit et al. (2014) evaluate the use of consolidation centers by imposing parking constraints and providing subsidies to carriers. They state that these measures significantly contribute to the reduction of emissions. Teo et al. (2014) study the effects of road pricing and a load factor control system, using customer complaints on late deliveries as a driver to alter the selection of carriers. The authors claim that the studied measures have a positive environmental effect.

The contribution of this chapter is twofold. First, in contrast to the agentbased simulation studies mentioned in this literature overview, we in addition take into account the transport process outside the city. As last-mile distribution accounts for only part of the transport process, a narrow perspective does not properly assess the decisions made by shippers and carriers. Second, we explicitly include various forms of cooperation between companies, while existing studies tend to have a strong focus on testing governmental policies. As practice shows that successful schemes require both policies and commitment from companies, a framework that explicitly includes both aspects is essential for proper evaluation of these schemes.

\subsection{Framework design}

In this section, we outline the design of our agent-based simulation framework. By presenting a mathematical formulation of the framework, we provide concrete guidelines for implementation as a discrete-event simulation. Figure 4.1 shows a high-level overview of the framework. To not overly complicate the mathematical presentation, at various points we introduce simplifications that may easily be omitted in actual implementations of the framework. The main simplifications that we make are (i) all routes are completed within a single time interval in the model, (ii) pickup routes outside the city are not specifically 
described, (iii) the network contains only a single UCC location, and (iv) price and cost functions are linear.

The outline of this section is as follows. We start by describing the roles of the agent types in Section 4.3.1. In Section 4.3.2, we formally define the state of the system. Section 4.3.3 introduces the dispatching decisions per agent type that can be performed based on the state of the system. The transition function that describes the state change over time is given in Section 4.3.4. We define the cost functions for each agent type and the environmental performance indicators in Section 4.3.5. In Section 4.3.6, we discuss various solution methods that can be applied within the framework on the levels of strategic, tactical, and operational decision-making.

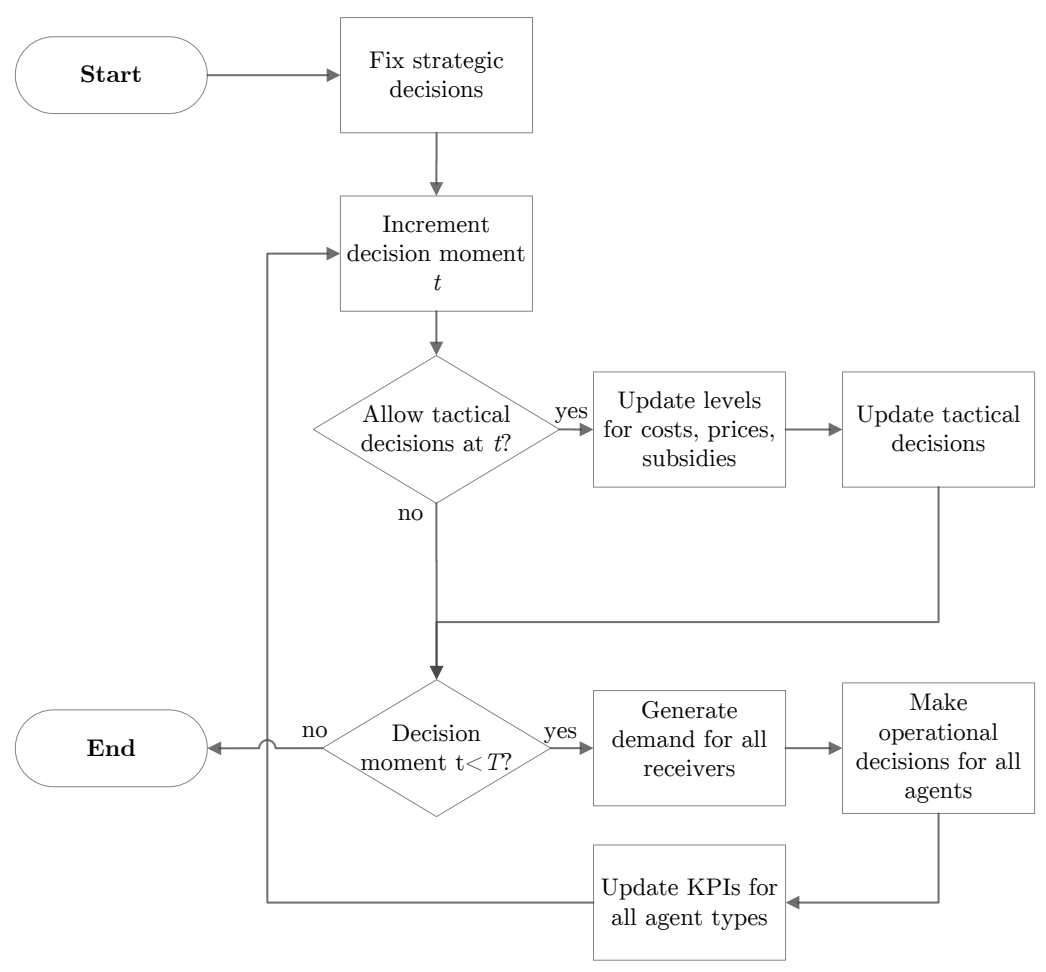

FIGURE 4.1. High-level flowchart of the simulation framework. 
4.3.1. Agent types. We design the agent-based simulation framework in a manner that allows to simultaneously evaluate company-driven initiatives and administrative policies. We focus on supply chains for a single city; the extension of the framework to multiple cities is relatively straightforward. In a context with multiple cities, harmonization of local policies and consolidation on the line haul are noteworthy challenges (Quak, 2011). In the simulation framework, we allow decisions to be made at discrete moments in time. Five types of agents are distinguished: receivers, shippers, line-haul carriers, the UCC operator, and the administrator. We proceed to briefly describe their roles; Figure 4.2 summarizes the actions, monetary flows, and information flows between the agents. The actions of and information exchanges between agents are defined in detail in Section 4.3.3 and Section 4.3.4; the cost functions corresponding to the monetary flows are defined in Section 4.3.5. We note that the real-life counterparts of the agents are not necessarily rational decision-makers, particularly when solutions require drastic changes in behavior. However, the simulation results yield insights into the effects on real-life agents if they would change their behavior, as such providing directions for change.

The receivers (denoted by the set $\mathcal{R}$ ) generate an order demand that is subject to some (stochastic) process. Receivers may order from multiple suppliers at a single decision moment. Fixed ordering moments may be defined for each receiver (e.g., twice per week); we assume that its ordering pattern already takes into account factors such as internal consolidation, storage costs, and stockouts. This implies that no measures can be taken to improve internal consolidation at the receiver level. Although this may seem a strong assumption, modeling order optimization at the receiver level would require many assumptions with respect to their operations as well. Both to prevent indefinite postponement of order shipping and to accurately model practice, receivers specify a delivery window for each order. In the standard situation, orders are directly delivered by the carrier to the address of the receiver, but a receiver can also opt to select the UCC as its delivery address. In that case, the UCC is responsible for the last-mile distribution. Finally, the receiver can decide to outsource value-adding services to the UCC as well.

The shippers in the framework are denoted by the set $\mathcal{S}$. They act on incoming orders from the receivers and hire carriers to transport orders. As carriers charge relatively lower prices for higher volumes (i.e., a lower price per volume unit), shippers have an incentive to bundle multiple orders before shipping. However, the shippers should dispatch the orders in time, such that the carrier is able to meet the delivery windows. For the sake of generality, we have separated the 
roles of the carrier and the shipper. In practice, it is also possible that the shipper and carrier are the same party. In that case, the shipper has a similar incentive, as consolidation allows a more efficient utilization of transport resources.

Line-haul carriers, denoted by the set $\mathcal{C}$, pick up goods at the shippers, and transport them either directly to the receivers or to the UCC. They may outsource the last-mile distribution to the UCC when this yields a financial benefit or is enforced by regulation. Also if the receiver sets the UCC as its delivery address, the carrier must use the UCC, but in that case it is the receiver who pays for the last-mile delivery service. The carrier uses a price function based on volume and line-haul distance (i.e., the distance between cities, ignoring distance variations due to routing) that reflects economies of scale. In the typical setting that we study, a dispatched truck will visit multiple cities during a single tour, yet we focus on a single city only. Consequently, the load destined for the city is generally much less than the truck's capacity.

The UCC receives goods from the line-haul and is responsible for their lastmile distribution. We use a generic set notation $\mathcal{H}$ for UCCs, but for simplicity only consider a single UCC for the outline of the framework. Goods are transported via the UCC if either the carrier outsources its last-mile delivery or the receiver selects the UCC as its delivery address. At the UCC, orders stemming from multiple carriers can be bundled and may be temporarily held to account for future consolidation opportunities. For modeling purposes we assume that the UCC uses its own vehicles to perform the last-mile distribution; in practice the carrier may also hire a neutral carrier, i.e., a carrier other than the agents representing the line-haul carriers and operating solely within the city, thereby not causing competitive frictions. Ideally, the efficiency gains on the last-mile compensate for the necessary reload costs, such that the UCC can offer competitive prices to carriers and receivers. To obtain additional revenues, the UCC can also perform value-adding services for the receivers; these should otherwise be performed in-house by the receiver itself.

Finally, the administrator can implement governmental policies to influence the behavior of agents. Since such policies are typically implemented for a longer time, we do this on a scenario basis. The financial gains stemming from these policies may be redistributed to subsidize agents. As mentioned, many schemes fail due to being overly dependent on subsidies. We therefore look for schemes that are financially sustainable without requiring permanent external cash flows. One way to achieve this involves temporary subsidies, paid until a new equilibrium state of the system is reached. Another sustainable solution can be achieved when the additional income due to specific policies implementation suffices to 
cover the subsidy expenses. In the latter case, the administrator essentially monetizes and redistributes external costs.

4.3.2. System state. In this section, we provide the notation required to define the state of the system and the actions of agents. We use discrete-event simulation to model the behavior of the system over time, more specifically, over a sequence of equidistant time intervals. Let $\mathcal{T}=\{0,1, \ldots, T\}$ be the set of discrete decision moments upon which agents can make decisions. We distinguish between three levels of decision making. First, we have a class of strategic decisions that are fixed at $t=0$. This class describes decisions of agents that are typically implemented for multiple years. Examples are the implementation of a new infrastructure or new regulations. Second, we have the class of tactical decisions. These decisions are made at designated tactical decision moments $t \in \mathcal{T}^{t a c} \subset \mathcal{T}$. The interval between two adjacent tactical decision moments corresponds to a commitment of an agent for a time period of medium length, e.g., a month up to a year. An example of a tactical decision is a receiver that subscribes to the UCC. Third, we have a class of operational decisions, these decisions are made at every $t \in \mathcal{T}$. Typically, the time between two adjacent decision moments will correspond to several hours up to a day. For example, we might have one decision moment in the morning and one in the afternoon, such that two rounds of deliveries can be performed during a day. 

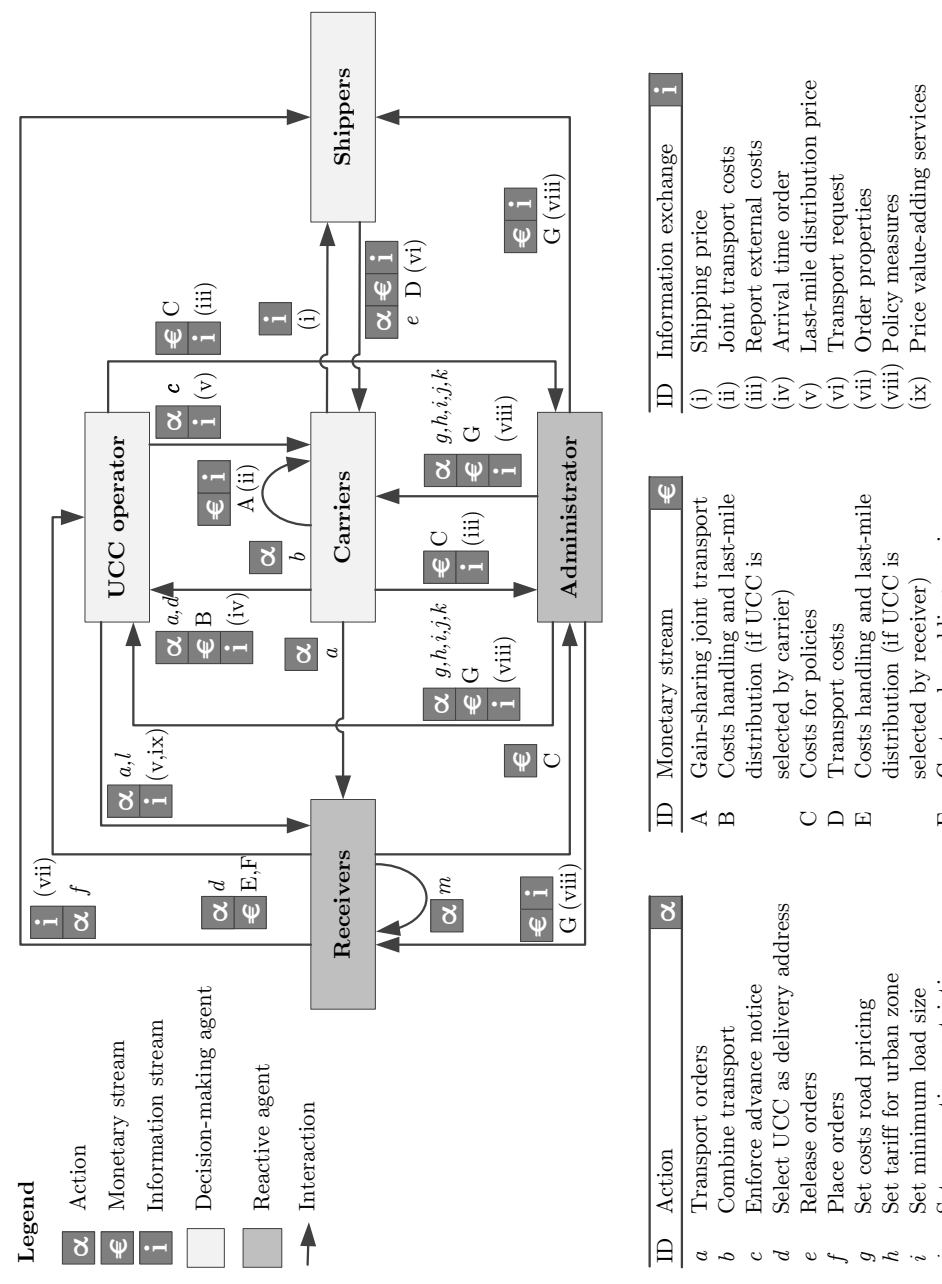

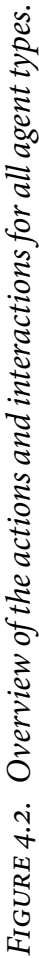

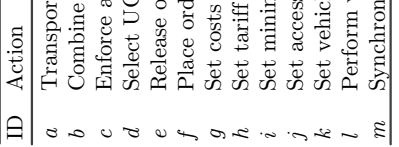
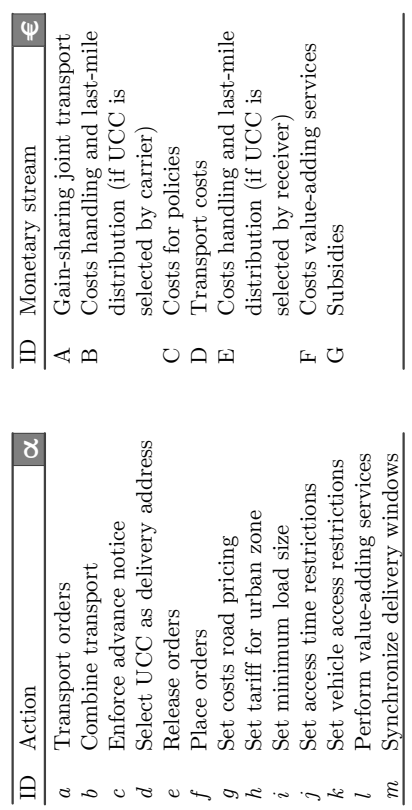
We define an abstract network consisting of a pickup area with shipper locations and the home depots of carriers, a line-haul distance, and the urban area with receiver locations and a single UCC. In Figure 4.3, we show the typical network setting that we consider. The network is comprised by a vertex set $\mathcal{V}$ and a set of $\operatorname{arcs} \mathcal{A}$. Vertex set $\mathcal{V}$ is defined as $\mathcal{V}=\mathcal{V}^{R} \cup \mathcal{V}^{S} \cup \mathcal{V}^{C} \cup \mathcal{V}^{H}$, i.e., the set consists of subsets of locations of receivers $\mathcal{V}^{R}$, shippers $\mathcal{V}^{S}$, carriers $\mathcal{V}^{C}$, and the UCC $\mathcal{V}^{H}$. As we consider a single UCC location for the sake of illustration, we define $\mathcal{V}^{H}=\left\{v^{u c c}\right\}$, with $v^{u c c}$ denoting the UCC location in the network. Every arc $a \in \mathcal{A}$ connects a vertex pair $\left(v_{a}, v_{a}^{\prime}\right)$, with $v_{a}, v_{a}^{\prime} \in \mathcal{V}$ and $v_{a} \neq v_{a}^{\prime}$. Let $\mathcal{L}=\left\{\frac{1}{y}, \frac{2}{y}, \ldots, 1\right\}$ (with integer $y>1$ ) be the set of possible order volumes, with a volume of 1 being equal to the smallest vehicle in the model (e.g., a delivery van). If necessary, volumes $>1$ may be defined as well, yet such volumes typically do not coincide with the type of freight flows that are analyzed with this framework. We define an order as a request to ship a certain load, with an order type being a unique combination of the delivery window $\left[t+t^{e}, t+t^{l}\right]$ (note that the delivery window is defined relative to $t$ ), the current position of the order $v \in \mathcal{V}$ (which indicates the agent responsible for handling the order at the current decision moment $t$ ), the receiver $r \in \mathcal{R}$, the carrier $c \in \mathcal{C}$, the shipper $s \in \mathcal{S}$, the order volume $l \in \mathcal{L}$, and an indicator $\gamma \in\{0,1\}$ that specifies whether delivery takes place via the UCC ( $\gamma=1$ sets the UCC as the delivery address for the carrier). Note that in Chapter 3 the window $\left[t+t^{e}, t+t^{l}\right]$ was related to the dispatch times from the perspective of the UCC, whereas here it relates to the delivery window at the receiver. The indicator $\gamma$ can be specified either by the receiver or by the carrier. The order type of an order may change during the simulation. As the delivery window is defined relative to the decision moment $t$, it must be updated after each time step. Furthermore, decisions regarding the use of the UCC may alter the specifications of an order. The transition functions that describe how orders change type are defined in Section 4.3.4. During the simulation, we keep track of the agents that utilize the UCC. The variable $\gamma_{t, r}^{r e c} \in\{0,1\}$ indicates whether receiver $r$ sets the UCC as its delivery address at decision moment $t$; the variable $\gamma_{t, c}^{c a r} \in\{0,1\}$ has the same purpose for carriers. Usually agents have a contractual agreement with the UCC, such that these variables are fixed for longer periods of time; we discuss strategic and tactical decision making in Section 4.3.6. In total there are $|\mathcal{R}|$ receivers and $|\mathcal{C}|$ line-haul carriers, the vectors $\gamma_{t}^{r e c}=\left[\gamma_{t, 1}^{r e c}, \ldots, \gamma_{t,|\mathcal{R}|}^{r e c}\right]$ and $\gamma_{t}^{c a r}=\left[\gamma_{t, 1}^{c a r}, \ldots, \gamma_{t,|\mathcal{C}|}^{c a r}\right]$ describe which agents are committed to the UCC at time $t$. The number of orders of a specific type in the 


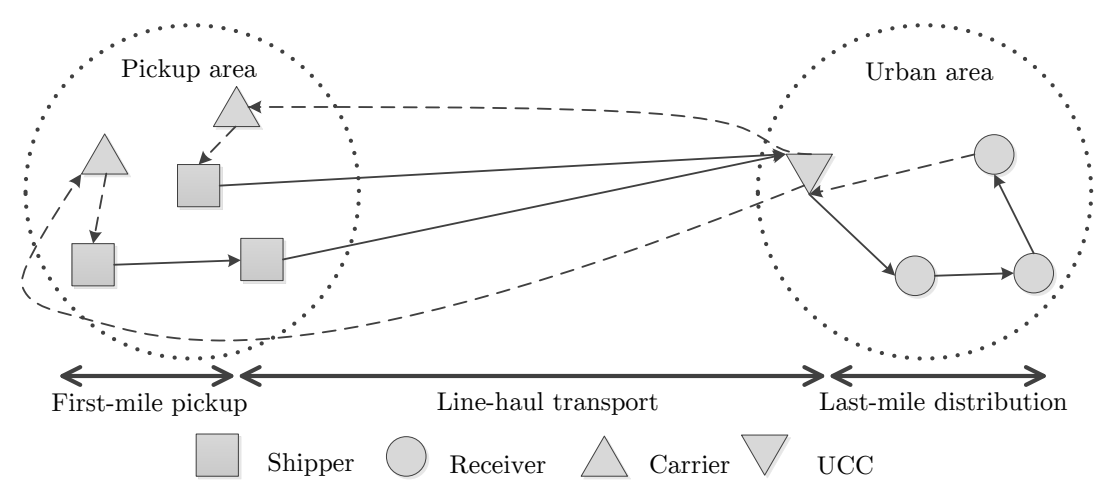

FIGURE 4.3. Example of a network with a UCC facilitating last-mile distribution. The dashed lines indicate empty vehicle movements, the solid lines indicate loaded vehicle movements.

system at time $t$ is denoted by $I_{t, t^{e}, t^{l}, v, r, c, s, l, \gamma} \in \mathbb{N}$. The vector $I_{t}$ describes the number of every order type in the system at time $t$, and is defined as

$$
I_{t}=\left[I_{t, t^{e}, t^{l}, v, r, c, s, l, \gamma}\right]_{\forall t^{e}, t^{l}, v, r, c, s, l, \gamma} .
$$

As order types are defined by a sequential decision-making process that spans multiple agent types, several indices of the order type are left undefined during the process. For instance, the receiver will not specify which carrier delivers the order; this index is left blank in the specification of the order type or the action which we denote by a dot - in place of the unspecified index - until the moment it is specified by the shipper. The decision-making process that defines the order types is described in Section 4.3.3.

The state of the system at $t$ is defined by $S_{t}=\left[I_{t}, \gamma_{t}^{r e c}, \gamma_{t}^{c a r}\right]$; this description provides all information required for decision-making and computing the performance indicators.

4.3.3. Definition of dispatching decisions. In this section, we introduce the definitions of the dispatching decisions of the agents. In some form, every agent is faced with a dispatching decision based on $S_{t}$. The dispatching decision of one agent transfers the responsibility for the dispatched orders to other agents; the dispatching decisions are made sequentially by the various agent types. Depending on the context, a dispatching decision may either be a physical transfer or an information transfer. Physically, orders move downstream from shipper 
to receiver via the carrier (and possibly via the UCC). In the basic version of the framework, information transfers only take place between receivers and shippers, i.e., the placement of orders. Both types of actions have in common that they update the (physical or virtual) order positions of other agents.

The dispatching decision for the receiver $r \in \mathcal{R}$ is the information transfer of its demand arising between time $t-1$ and time $t$, i.e., it places an order at the shippers that is equal to its demand. The carrier is undefined at this point. When placing the order, the receiver specifies a delivery window $\left[t+t^{e}, t+t^{l}\right]$, a value $t^{e}>0$ implies that the order must be held either at the shipper location or at the UCC before the earliest delivery time. The vertex $v_{r}^{r e c}$ in the subscript notation of decisions and order positions follows from the index $r$; the same holds for the other vertex indices used in the notation. The dispatching by receiver $r \in \mathcal{R}$ is defined as:

$$
x_{t, t^{e}, t^{l}, v_{r}^{r e c}, r, \cdot, s, l, \gamma}=I_{t, t^{e}, t^{l}, v_{r}^{r e c}, r, \cdot, s, l, \gamma} \quad \forall t^{e}, t^{l}, s, l, \gamma .
$$

The dispatching decisions for the shipper is a physical dispatching decision. Although Figure 4.1 also describes the possibility to pre-announce order arrivals to the UCC (i.e., an information transfer), we ignore this action to simplify the mathematical formulation of actions and transitions. By accumulating orders, the shipper can achieve lower average transport costs, yet the delivery windows imposed by the receivers must be respected. At every decision moment $t$, the shipper $s$ must select a carrier $c \in \mathcal{C}$ to perform the transport for the dispatched orders (i.e., one carrier for the complete shipment at $t$ ), we introduce the binary variable $z_{t, c, s} \in\{0,1\}$ to describe this choice. The carrier selection may be modeled as a cost minimization problem. Another modeling approach is to fix shipper-carrier pairs, for example when a contract between both parties exists. The dispatching decision of shipper $s \in \mathcal{S}$ is given by

$$
\left[x_{t, t^{e}, t^{l}, v_{s}^{s h p}, r, c, s, l, \gamma}\right]_{\forall t^{e}, t^{l}, r, c, l, \gamma},
$$

s.t. 


$$
\begin{array}{rlrl}
\sum_{c \in \mathcal{C}} z_{t, c, s} \cdot x_{t, t^{e}, t^{l}, v_{s}^{s h p}, r, c, s, l, \gamma} & \leq I_{t, t^{e}, t^{l}, v_{s}^{s h p}, r, c, s, l, \gamma} & & t^{l}>1, \forall t^{e}, r, l, \gamma \\
\sum_{c \in \mathcal{C}} z_{t, c, s} \cdot x_{t, 0,1, v_{s}^{s h p}, r, c, s, l, \gamma} & =I_{t, 0,1, v_{s}^{s h p}, r, c, s, l, \gamma} & & \forall r, l, \gamma, \\
x_{t, t^{e}, t^{l}, v_{s}^{s h p}, r, c, s, l, 0} & =0 & & t^{e}>0, \forall t^{l}, r, c, l, \gamma, \\
\sum_{c \in \mathcal{C}} z_{t, c, s} & =1, & \\
x_{t, t^{e}, t^{l}, v_{s}^{s h p}, r, c, s, l, \gamma} & \in \mathbb{N} & \\
z_{t, c, s} & \in\{0,1\} & \forall t^{e}, t^{l}, r, c, l, \gamma,
\end{array}
$$

Constraint (4.3.4) states that no more orders of any order type are shipped than that are placed by the receiver. Constraint (4.3.5) ensures that orders are shipped in time, such that the carrier or UCC can make the delivery to the receiver before the last allowed delivery time (given our assumption that all routes, including the line-haul component, are completed within a single time interval). Constraint (4.3.6) states that - if the order does not have the UCC as its delivery address - the shipper cannot ship orders before the earliest delivery date; this is because the carrier does not offer intermediate storage capacity. With Constraint (4.3.7), we enforce that exactly 1 carrier is selected to conduct the transport of the shipment, i.e., all orders that are dispatched by the shipper at time $t$. Constraint (4.3.8) and Constraint (4.3.9) are domain constraints.

The dispatching decision of the carrier is equivalent to transporting all orders that are physically released by the shippers to carrier $c$. Orders that are released by the shipper at $t$ are picked up and delivered by the carrier between $t$ and $t+1$. Recall that orders with $t^{e}>0$ can only be delivered to the UCC; unlike the UCC and the shipper, the carrier does not offer a temporary storage service. We define the dispatching decision of a carrier $c \in \mathcal{C}$ as follows:

$$
x_{t, t^{e}, t^{l}, v_{c}^{c a r}, r, c, s, l, \gamma}=I_{t, t^{e}, t^{l}, v_{c}^{c a r}, r, c, s, l, \gamma} \quad \forall t^{e}, t^{l}, r, s, l, \gamma .
$$

Finally, the definition of the dispatching decision for the UCC is similar to that of the shipper, although without the constraints relating to the carrier selection:

$$
\left[x_{t, t^{e}, t^{l}, v^{u c c}, r, c, s, l, 1}\right]_{\forall t^{e}, t^{l}, r, c, s, l, 1},
$$

s.t. 


$$
\begin{array}{rlrl}
x_{t, 0, t^{l}, v^{u c c}, r, c, s, l, 1} \leq I_{t, 0, t^{l}, v^{u c c}, r, c, s, l, 1} & & \forall t^{l}, r, c, s, l, \\
x_{t, 0,1, v^{u c c}, r, c, s, l, 1} & =I_{t, 0,1, v^{u c c}, r, c, s, l, 1} & & \forall r, c, s, l, \\
x_{t, t^{e}, t^{l}, v^{u c c}, r, c, s, l, 1} & =0 & & t^{e}>0, \forall t^{l}, r, c, s, l, \\
x_{t, t^{e}, t^{l}, v^{u c c}, r, c, s, l, 1} \in \mathbb{N} & & \forall t^{e}, t^{l}, r, c, s, l .
\end{array}
$$

4.3.4. Transition function. Having defined the state of the system and the corresponding actions, we proceed to describe the transition function. We start

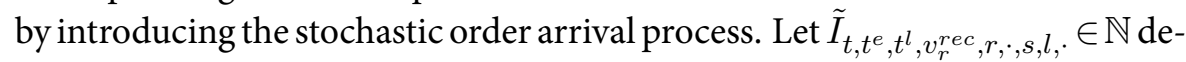
note the number of arrivals of a given order type during the time interval $(t-1, t]$. We denote arrivals by $\omega_{t}=\left[\tilde{I}_{t, t^{e}, t^{l}, v_{r}^{r e c}, r, \cdot, s, l,},\right]_{\forall t^{e}, t^{l}, r, s, l}$ and the set of all arrival scenarios by $\Omega_{t}$. Let $W_{t}$ be a random variable with $\omega_{t} \in \Omega_{t}$ being a realization of $W_{t}$. The random generation of orders implicitly reflects the ordering policies of the receivers.

As we consider a system with interacting agents, the actions that we discussed in Section 4.3.3 affect the states of other agents. The supply chain is structured as follows: (i) receivers communicate their order placements to the shippers, (ii) shippers dispatch a subset of the orders requested by receivers, (iii) carriers transport the orders that are dispatched by shippers, (iv) the UCC receives a subset of the orders transported by the carriers, and (v) the UCC dispatches a subset of orders in inventory for delivery. Dispatching decisions may also transform order types, e.g., when the carrier decides to deliver via the UCC. To correctly describe the transition function, we require a sequential updating procedure. The influence that the actions of one agent has on the order position of another agent is represented by the vertex subscripts. For example, in the dispatching decision of a receiver $r$ (communicating an order placement to the shipper), the subscript $v_{r}^{r e c}$ is specified. Processing this decision updates the order position of the shipper, the subscript $v_{s}^{s h p}$ is used to denote that the decision of the receiver affects the order position of the shipper.

To ease the presentation of the transition, we split the transition function into two components: the transition from the pre-decision state $S_{t}$ to the postdecision state $S_{t}^{x}$ (i.e., the state after applying action $x_{t}$ on state $S_{t}$, but before the arrival of new information $W_{t+1}$ ), and the transition from $S_{t}^{x}$ to $S_{t+1}$. Notations that relate to the post-decision state are defined with a superscript $x$.

We start with an initial state $S_{t}=\left[I_{t}, \gamma_{t}^{r e c}, \gamma_{t}^{c a r}\right]$. In this initial state, positive numbers of order types may exist for the receivers (newly generated orders according to the realization of $W_{t}$ ), the shippers (existing order positions), and the 
UCC (existing order position). From this initial position, we proceed to move to the post-decision state $S_{t}^{x}$. This state is reached via a sequential updating procedure, which we outline below.

The demand of receiver $r \in \mathcal{R}$ for a given order type is defined by the variable $I_{t, t^{e}, t^{l}, v_{r}^{r e c}, r, \cdot, s, l, .} \in \mathbb{N}$; the carrier and the use of the UCC are initially undefined, hence the dots - in the notation. If $\gamma_{t, r}^{r e c}=1$ (which may be fixed at the tactical or strategic level), this means that the receiver will use the UCC as its delivery address, thus we must set $\gamma=1$ for all its orders. If $\gamma_{t, r}^{r e c}=0$, the receiver is indifferent as to whether it will be delivered directly or via the UCC, meaning that this choice remains open to the carrier. The index for $\gamma$ is left blank in that case. Thus, iff $\gamma_{t, r}^{r e c}=1$, we perform the following update

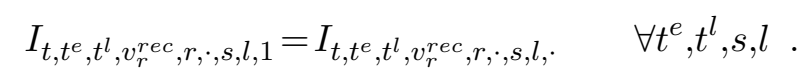

If we set $\gamma=1$, we must reset all order positions of the receiver without a specified value for $\gamma$ to 0 . Again, this update is only performed when $\gamma_{t, r}^{r e c}=1$ :

$$
I_{t, t^{e}, t^{l}, v_{r}^{r e c}, r, \cdot, s, l, \cdot}=0 \quad \forall t^{e}, t^{l}, s, l .
$$

Subsequently, the orders placed by the receiver are converted into an action according to Equation (4.3.2). After obtaining the action of the receiver, it is communicated to the shipper, which prompts an update of the order position of the shipper. At this stage, the carrier $c$ is still undefined. To obtain the order position of a shipper $s \in \mathcal{S}$ at $t$, the order requests by the receivers are added to the already existing order positions of the shipper (i.e., the post-decision position at $t-1)$. We first show how to compute the post-decision order position. The shipper selects an action as defined in Equation (4.3.3). By dispatching the orders, we can compute the post-decision order position for the shipper $s$ :

$$
\begin{aligned}
& I_{t-1, t^{e}, t^{l}, v_{s}^{s h p}, r, \cdot, s, l, \gamma}^{x}=I_{t-1, t^{e}, t^{l}, v_{s}^{s h p}, r, \cdot, s, l, \gamma}-\sum_{c \in \mathcal{C}} x_{t-1, t^{e}, t^{l}, v_{s}^{s h p}, r, c, s, l, \gamma} \\
& \forall t^{e}, t^{l}, r, l, \gamma .
\end{aligned}
$$

The order position of the shipper at $t$ follows from the post-decision order position at $t-1$ and the orders posed by the receivers at $t$. To take into account the time update of delivery windows relative to the current decision moment, we must update the order types of existing orders in the order position. Most 
time indices $t^{e}$ and $t^{l}$ are simply reduced by 1 , as they are relative to $t$. However, orders with an earliest delivery time $t^{e}=0$ or $t^{e}=1$ relative to $t-1$ both correspond to $t^{e}=0$ at the current decision moment $t$. We incorporate this update with the help variable $\tilde{t}^{e}$. The update of the order position is defined as follows:

$$
\begin{aligned}
& I_{t, 0, t^{l}, v_{s}^{s h p}, r, \cdot, s, l, \gamma}=\sum_{\tilde{t}^{e}=0}^{1}\left(I_{t-1, \tilde{t}^{e}, t^{l}+1, v_{s}^{s h p}, r, \cdot, s, l, \gamma}^{x}\right)+x_{t, 0, t^{l}, v_{r}^{r e c}, r, \cdot, s, l, \gamma} \\
& \forall t^{l}, r, l, \gamma \\
& I_{t, t^{e}, t^{l}, v_{s}^{s h p}, r, \cdot, s, l, \gamma} I_{t-1, t^{e}+1, t^{l}+1, v_{s}^{s h p}, r, \cdot, s, l, \gamma}^{x}+x_{t, t^{e}, t^{l}, v_{r}^{r e c}, r, \cdot, s, l, \gamma} \\
& t^{e}>0, \forall t^{l}, r, l, \gamma .
\end{aligned}
$$

We proceed with the update at the carrier level based on its decision whether to use the UCC. Based on the decision variables $\gamma_{t, c}^{c a r}$ and $\gamma_{t, r}^{r e c}$, the value for $\gamma$ may be updated. If $\gamma=1$ due to the preceding decision of the receiver, the carrier cannot set $\gamma=0$. However, if $\gamma_{t, r}^{r e c}=0$, the index $\gamma$ has not been defined and will be determined by the carrier. The update of the order types for the carrier is defined by

$$
\begin{array}{ll}
I_{t, t^{e}, t^{l}, v_{c}^{c a r}, r, c, s, l, 0}=I_{t, t^{e}, t^{l}, v_{c}^{c a r}, r, c, s, l, .} & \forall t^{e}, t^{l}, r, s, l \text { if } \gamma_{t, c}^{c a r}=0 \\
I_{t, t^{e}, t^{l}, v_{c}^{c a r}, r, c, s, l, 1}=I_{t, t^{e}, t^{l}, v_{c}^{c a r}, r, c, s, l, .} & \forall t^{e}, t^{l}, r, s, l \text { if } \gamma_{t, c}^{c a r}=1
\end{array}
$$

After completing the update for $\gamma$, the number of orders for types characterized by $\gamma=$ - are set to 0 :

$$
I_{t, t^{e}, t^{l}, v_{c}^{c a r}, r, c, s, l, \cdot}=0 \quad \forall t^{e}, t^{l}, r, s, l .
$$

We proceed with the inventory update of the UCC. The inventory at time $t$ depends on the post-decision inventory at $t-1$ and the dispatching decisions by the carriers taken at $t$, as defined in Equation $(4.3 .10)$. We first describe how we compute the post-decision inventory position of the UCC. To be consistent with the subsequent notation, we define the post-decision inventory at $t-1$ . For a given (pre-decision) inventory position, the UCC makes its dispatching decision as defined in Equation (4.3.11), which is used to compute its postdecision inventory position: 


$$
\begin{aligned}
I_{t-1, t^{e}, t^{l}, v^{u c c}, r, c, s, l, 1}^{x} & =I_{t-1, t^{e}, t^{l}, v^{u c c}, r, c, s, l, 1}-x_{t-1, t^{e}, t^{l}, v^{u c c}, r, c, s, l, 1} \\
\forall t^{e}, t^{l}, r, c, s, l & .
\end{aligned}
$$

The inventory position of the UCC at $t$ depends on the existing inventory from $t-1$ and the dispatching decisions by the carriers taken at $t$, as defined in Equation (4.3.10). Based on this information, we update the inventory position of the UCC as follows:

$$
\begin{gathered}
I_{t, 0, t^{l}, v^{u c c}, r, c, s, l, 1}=\sum_{\tilde{t}^{e}=0}^{1}\left(I_{t-1, \tilde{t}^{e}, t^{l}+1, v^{u c c}, r, c, s, l, 1}^{x}\right)+x_{t, 0, t^{l}, v_{c}^{c a r}, r, c, s, l, 1} \\
\forall t^{l}, r, c, s, l \\
I_{t, t^{e}, t^{l}, v^{u c c}, r, c, s, l, 1}=I_{t-1, t^{e}+1, t^{l}+1, v^{u c c}, r, c, s, l, 1}^{x}+x_{t, t^{e}, t^{l}, v_{c}^{c a r}, r, c, s, l, 1} \\
t^{e}>0, \forall t^{l}, r, c, s, l .
\end{gathered}
$$

Having defined the sequential procedure to obtain the post-decision state of the system, we proceed to describe the transition from post-decision state $S_{t}^{x}$ to the next pre-decision state $S_{t+1}$. First, the receiver orders are generated based on the realization of the random variable $W_{t+1}$. At this point, the carrier $c$ and the indicator $\gamma$ are still undefined. For receiver $r$, its order position at $t+1$ is given by

$$
I_{t+1, t^{e}, t^{l}, v_{r}^{r e c}, r, \cdot, s, l, \cdot}=\tilde{I}_{t+1, t^{e}, t^{l}, v_{r}^{r e c}, r, \cdot, s, l, \cdot \quad \forall t^{e}, t^{l}, s, l .} .
$$

For each shipper $s$, we need to update the time indices of its inventory, i.e., the post-decision inventory at decision moment $t$. This time update goes as follows:

$$
\begin{aligned}
I_{t+1,0, t^{l}, v_{s}^{s h p}, r, \cdot, s, l, \gamma} & =\sum_{\tilde{t}^{e}=0}^{1} I_{t, \tilde{t}^{e}, t^{l}+1, v_{s}^{s h p}, r, \cdot, s, l, \gamma}^{x} & \forall t^{l}, r, l, \\
I_{t+1, t^{e}, t^{l}, v_{s}^{s h p}, r, \cdot, s, l, \gamma} & =I_{t, t^{e}+1, t^{l}+1, v_{s}^{s h p}, r, \cdot, s, l, \gamma}^{x} & t^{e}>0, \forall t^{l}, r, l .
\end{aligned}
$$

The time update for the UCC is comparable: 


$$
\begin{array}{rlrl}
I_{t+1,0, t^{l}, v^{u c c}, r, c, s, l, 1} & =\sum_{\tilde{t}^{e}=0}^{1} I_{t, \tilde{t}^{e}, t^{l}+1, v^{u c c}, r, c, s, l, 1}^{x} & & \forall t^{l}, r, c, s, l, \\
I_{t+1, t^{e}, t^{l}, v^{u c c}, r, c, s, l, 1}=I_{t, t^{e}+1, t^{l}+1, v^{u c c}, r, c, s, l, 1}^{x} & t^{e}>0 \forall t^{l}, r, c, s, l .
\end{array}
$$

4.3.5. Cost functions and key performance indicators. In this section, we provide the cost (profit) functions and Key Performance Indicators (KPIs) of the agents. We start by introducing some notation required to describe the price functions and cost functions. For a variety of parameters and variables, we use the superscript $h d$ to refer to costs for handling operations (e.g., (un)loading by the driver), $r c$ for receiving (e.g., working hours spent on receiving, control of goods, and allocation to shelves), $s p$ for shipping (e.g., loading operations), and $s b$ for income from subsidies. In the description, we restrict ourselves to subsidies for using the UCC; schemes that subsidize other initiatives may be modeled in a comparable manner. Price functions $P$ describe the income of agents and cost functions $C$ describe their expenses. To reflect economies of scale, we define most price functions and cost functions based on a fixed component and a volume-dependent variable component. These functions serve as an illustration, and may be replaced by functions operating on alternative variables in implementations of the framework. The used order volumes depend on the context of the function. For example, the volume transported by carrier $c$ at time $t$ to the UCC is given by

$$
l_{t, \cdot, \cdot, v_{c}^{c a r},,, c, \cdot,, 1}=\sum_{t^{e}, t^{l}, r, s, l} x_{t, t^{e}, t^{l}, v_{c}^{c a r}, r, c, s, l, 1} \cdot l .
$$

We proceed with the notation required to denote routes. Let $\mathcal{Q}_{c}$ denote the set of vehicles operated by carrier (or UCC) $c \in \mathcal{C} \cup \mathcal{H}$. A vehicle $q \in \mathcal{Q}_{c}$ has a vehicle capacity $\geq 1$. The smallest vehicle defined in the simulation model has a capacity of 1 , order sizes and the capacities of other vehicles are defined relative to this volume. To ease the notation, we assume that all routes starting at $t$ are completed at $t+1$. For the same reason, we do not explicitly include the pickup tour. We denote a route started by vehicle $q$ of carrier $c$ at time $t$ as $\delta_{t, c, q}^{c a r}=\left\{\delta_{t, c, q}^{c a r, l h}, \delta_{t, c, q}^{c a r, l m}\right\}$, with the components referring to line-haul transport and last-mile distribution respectively. This distinction is used to assign distinct properties (e.g., fuel usage, road pricing, driver wage) to the associated 
travel distances $\eta^{l h}$ and $\eta^{l m}$. We let $\Delta_{t, c}^{c a r}$ denote the set of routes for carrier $c$ at decision moment $t$, and use $\Delta_{t}^{u c c}$ to describe the set of routes for the UCC. The UCC only has to deal with last-mile distribution, such that $\delta_{t, q}^{u c c}=\left\{\delta_{t, q}^{u c c, l m}\right\}$. We use $\Delta_{t}=\left[\left[\Delta_{t, c}^{c a r}\right]_{\forall c \in \mathcal{C}}, \Delta_{t}^{u c c}\right]$ to denote all routes starting at time $t$.

For the carriers and UCCs, handling costs depend on the subsets of locations visited. The carrier incurs handling costs at each shipper of the subset $\mathcal{S}^{\prime} \subseteq \mathcal{S}$ that it visits to collect shipments at a given decision moment. Both carriers and receivers incur handling costs at each location in the subset of receivers visited $\mathcal{R}^{\prime} \subseteq \mathcal{R} \cup \mathcal{H}$ (note that the UCC is a receiver from the perspective of the carrier). For each agent that conducts transport, the subsets $\mathcal{S}^{\prime}$ and $\mathcal{R}^{\prime}$, as well as the distances $\eta^{l h}$ and $\eta^{l m}$, can be derived from the route sets $\Delta_{t, c}^{c a r}$ and $\Delta_{t}^{u c c}$. The symbol $\alpha$ - with the appropriate subscripts and superscripts - refers to a fixed price or cost component; similarly, the symbol $\beta$ refers to a variable price or cost component. In Table 4.1, we provide price and cost components of the price and cost functions corresponding to the agent's actions. For notational convenience, we formulate all components linearly. For the same purpose, we assume that carriers use homogeneous fleets. Definitions are kept at a generic level; we define the objective functions of the actors and illustrate the main cost and price components, without introducing excessive notational complexity. For this reason, the detailed costs structures required to describe measures such as road pricing and coalitions are not shown. 


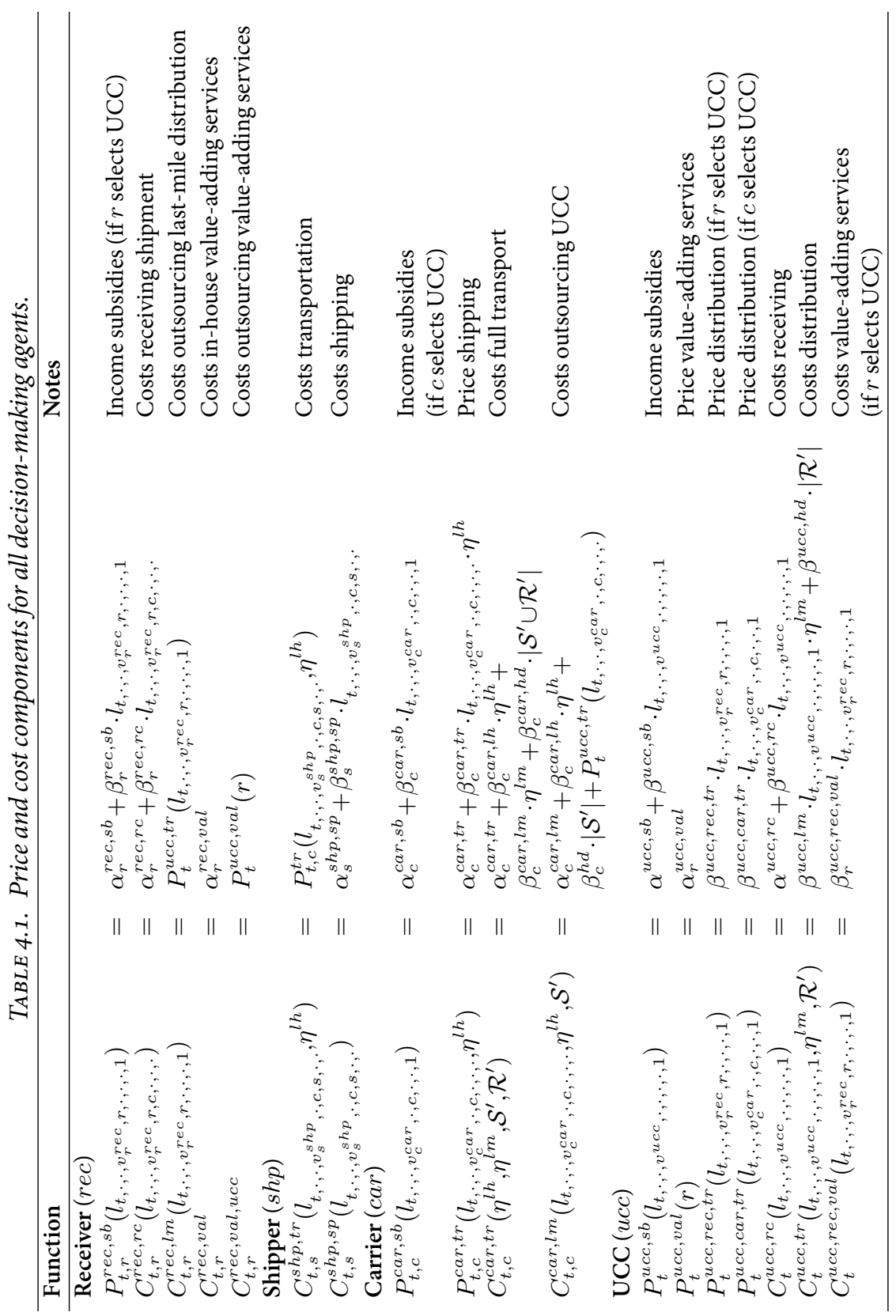


Having defined the price and cost components, we now introduce the cost functions for the agents. The outcomes of these cost functions over the full time horizon $\mathcal{T}$ serve as KPIs for the agents. To be consistent in our notation, we express the performance of each agent in terms of costs; price components are included as negative costs. Although agents aim to minimize their costs over the full planning horizon, they make periodic decisions based on incomplete information.

The objective of the shipper is to minimize the sum of transport costs and shipping costs. Shippers can influence these costs by selecting the set of orders to ship at every decision moment and select the cheapest carrier to conduct the transport of this order set, see Equation (4.3.3). The costs for shipper $s$ at time $t$ are given by

$$
\begin{aligned}
C_{t, s}^{s h p}\left(l_{t, \cdot,, v_{s}^{s h}, \cdot, c, s, \cdot, \cdot,}, \eta^{l h}\right)= & C_{t, s}^{s h p, t r}\left(l_{t, \cdot, \cdot, v_{s}^{s h p}, \cdot, c, s, \cdot, \cdot}, \eta^{l h}\right)+ \\
& C_{t, s}^{s h p, s p}\left(l_{t, \cdot, \cdot, v_{s}^{s h p}, \cdot, c, s, \cdot, \cdot}\right) .
\end{aligned}
$$

Carriers attempt to maximize profit (determined by the transport price, subsidy income, transport costs, and outsourcing costs) by selecting the route set $\Delta_{t, c}^{c a r, x}$ that minimizes costs at every decision moment. In addition, carriers can choose whether they perform the full transport themselves or outsource the last-mile transport to the UCC. In case of outsourcing, the carrier only pays for outsourcing volume that is not already outsourced by the receiver, i.e., only the volume characterized by $\gamma=\cdot$. The carrier also receives subsidies for this volume only. The cost function of a carrier $c$ at time $t$ is given by

$$
\begin{aligned}
& C_{t, c}^{c a r}\left(l_{t, \cdot, \cdot, v_{c}^{c a r}, \cdot, c, \cdot, \cdot, \cdot} l_{t, \cdot, \cdot, v_{c}^{c a r}, \cdot, c, \cdot, \cdot, 1}, \eta^{l h}, \eta^{l m}, \gamma_{t, c}^{c a r}, \mathcal{S}^{\prime}, \mathcal{R}^{\prime}\right)=
\end{aligned}
$$

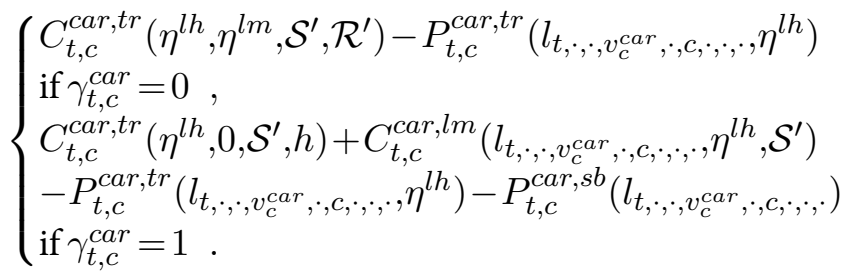

The objective of the UCC is to maximize profit, which is determined by the prices it charges, subsidy incomes, receiving costs, costs for performing valueadding services, and transport costs. If the receiver decides to select the UCC as its delivery address, it is the receiver that pays the UCC for the last-mile distribution costs. If the carrier decides to outsource its last-mile distribution, it 
only pays the UCC for the volume that has not already been outsourced by the receiver. To influence its profit, at every decision moment it selects a subset of orders to dispatch as defined in Equation (4.3.11), which yields a corresponding route set $\Delta_{t}^{u c c}$. The cost function for the UCC at time $t$ is defined by

$$
\begin{aligned}
& C_{t}^{u c c}\left(l_{t, \cdot, \cdot, v^{u c c}, \cdot, \cdot, \cdot, \cdot,}, \eta^{l m}, \mathcal{R}^{\prime}\right)= \\
& C_{t}^{u c c, r c}\left(l_{t, \cdot, \cdot, v^{u c c}, \cdot, \cdot, \cdot, \cdot 1}\right)+C_{t}^{u c c, t r}\left(l_{t, \cdot, \cdot, v e c}{ }^{u c c, \cdot, \cdot, \cdot, 1}, \eta^{l m}, \mathcal{R}^{\prime}\right)+ \\
& \sum_{r \in \mathcal{R}^{\prime}} C_{t}^{u c c, r e c, v a l}\left(l_{t, \cdot, \cdot, v_{r}^{r e c}, r, \cdot, \cdot, \cdot 1}\right)- \\
& P_{t}^{u c c, s b}\left(l_{t, \cdot, \cdot, v e c,, \cdot, \cdot, \cdot, 1}\right)-\sum_{r \in R^{\prime}} \gamma_{t, r}^{r e c} \cdot P_{t}^{u c c, r e c, t r}\left(l_{t, \cdot, \cdot, v^{u c c}, r, \cdot, \cdot, \cdot, 1}\right)- \\
& \sum_{c \in C^{\prime}} \gamma_{t, c}^{c a r} P_{t}^{u c c, c a r, t r}\left(l_{t, \cdot, \cdot, v_{c}^{c a r}, \cdot, c, \cdot, \cdot, 1}\right)-\sum_{r \in \mathcal{R}^{\prime}} P_{t}^{u c c, v a l}(r) .
\end{aligned}
$$

The performance of the receiver is measured as the sum of receiving costs. These depend on the tactical decision whether delivery takes place via the UCC. If the receiver does not mandate delivery via the UCC, it incurs costs for every carrier (possibly including the UCC) that delivers goods at the receiver. Furthermore, the receiver must perform its value-adding services in-house and incurs a cost for that. If the receiver mandates deliveries via the UCC, the receiver pays the UCC for last-mile delivery, but may incur lower receiving costs due to receiving bundled orders from only one carrier (i.e., the UCC). Furthermore, the receiver incurs costs when it decides to outsource the value-adding services. The costs for a receiver $r$ at time $t$ are given by:

$$
\begin{array}{r}
C_{t, r}^{r e c}\left(l_{t, \cdot, \cdot, v_{r}^{r e c}, r, \cdot, \cdot, \cdot,} l_{t, \cdot, \cdot, v_{r}^{r e c}, r, \cdot, \cdot, \cdot,}, \gamma_{t, r}^{r e c}\right)= \\
\left\{\begin{array}{l}
C_{t, r}^{r e c, r c}\left(l_{t, \cdot, \cdot, v_{r}^{r e c}, r, \cdot, \cdot, \cdot,}\right)+C_{t, r}^{r e c}, v a l \\
\text { if } \gamma_{t, r}^{r e c}=0 \\
C_{t, r}^{r e c, r c}\left(l_{t, \cdot, \cdot, v_{r}^{r e c}, r, \cdot, \cdot, \cdot 1}\right)+C_{t, r}^{r e c, l m}\left(l_{t, \cdot, \cdot, v_{r}^{r e c}, r, \cdot, \cdot, \cdot, 1}\right)+C_{t, r}^{r e c, v a l, u c c}- \\
P_{t, r}^{r e c, s b}\left(l_{t, \cdot, \cdot, v_{r}^{r e c}, r, \cdot, \cdot,, 1}\right) \text { if } \gamma_{t, r}^{r e c}=1 .
\end{array}\right.
\end{array}
$$

The performance of the administrator is measured with the following KPIs: (i) the number of vehicles per type that enter the urban area, (ii) the total distance covered within the urban area per vehicle type, (iii) the income from policies minus the provided subsidies, and (iv) the emission levels for $\mathrm{CO}_{2}, \mathrm{SO}_{2}, \mathrm{NO}_{\mathrm{x}}$, and particulate matter (PM). The first two KPIs indirectly capture effects such 
as noise hindrance and the contribution to road congestion. The third KPI is an indicator for the financial feasibility of a scheme.

4.3.6. Solution methods for decision-making. In this section, we describe various solutions methods that can be applied in the framework. We distinguish between solution methods on the strategic level (Section 4.3.6.1), the tactical level (Section 4.3.6.2), and the operational level (Section 4.3.6.3). The descriptions of the solution methods are deliberately kept at an abstract level for this framework; the specific methods to be used depend on the experimental setup that is applied.

4.3.6.1. Strategic decisions. Strategic decisions are embedded in the framework as pre-defined scenario input; they are fixed at $t=0$, thereby committing the agent to a strategic decision for the full duration of the simulation run. We discuss implementation guidelines for two classes of strategic decisions, namely governmental policies and company-driven initiatives.

Governmental policies are typically described by forms of access pricing or access restrictions. Such policies are incorporated in the framework by (i) defining restrictions and pricing functions as arc properties, (ii) defining the structure for cost allocations and conditions for transport via urban network arcs, and (iii) specifying allocation rules for possible redistribution in the form of subsidies. Although the subsidy measures themselves are fixed, allocation rules and time thresholds can be defined in such a way that they allow for a dynamic distribution of subsidies.

We proceed to discuss company-driven initiatives. To evaluate measures that permanently affect agents - such as adjusting the type of vehicles used or a mandatory pre-announcement of shipments - the characteristics of the agents can be adjusted in the model to reflect their strategic decisions. Finally, initiatives that physically reorganize infrastructure or transport are incorporated by modifying the network and may entail, e.g., positioning the UCC or including special transport lanes for licensed vehicles. It is important that the applied routing algorithms properly take into account such restrictions.

We conclude with a brief discussion on setting up coalition structures in the framework. Examples of coalitions in urban logistics are receivers that synchronize their delivery windows and carriers that pool their orders and transport resources. We describe the setup of a coalition structure in four steps. First, it should be specified which members can join or leave the coalition. Second, the action space of the coalition needs to be defined, including rules for the allocation of tasks to agents. Third, a single objective function must be specified for the coalition. Stable solutions require that the profit of the coalition is at 
least equal to the sum of individual profits of the coalition members. Fourth as coalitions require rational agents that are willing to cooperate - an appropriate gain-sharing mechanism should be incorporated. Such mechanisms may be drawn from the field of cooperative game theory.

4.3.6.2. Tactical decisions. In the simulation framework, tactical decisions are made only at a pre-defined subset of decision moments $\mathcal{T}^{t a c} \subset \mathcal{T}$. Hence, these decisions commit agents for time periods of medium length. Examples of tactical decisions are adjusting price levels, committing to transport via the UCC, and joining a coalition. In this subsection, we discuss a sampling procedure to make decisions on the tactical level.

Although the tactical decisions themselves may be diverse, they have in common that they affect the performance of agents over a longer period of time. Therefore, each agent should evaluate how its performance in the longer run is affected by its tactical decisions. One approach to evaluate the impact of tactical decisions is to measure performance over some past time interval (e.g., the time elapsed since the preceding tactical decision moment) and compare it to the performance that could have been achieved under other tactical decisions. However, the state of the system may have been updated over time, e.g., due to altered price levels or subsidies. In that case, it is more sensible to incorporate some lookahead estimate.

To provide an example of a lookahead estimate, consider a receiver that must decide whether to select the UCC as its delivery address. The estimated costs for this option are computed with a sampling procedure. For this purpose, we generate $N$ sample paths of order arrivals of length $\tau^{\text {sample }}$, with $n \in\{1, \ldots, N\}$ being the index for the sample path and $t^{n} \in\left\{0, \ldots, \tau^{\text {sample }}\right\}$ being the time index for the sample states of path $n$. We obtain sets of sample arrivals that are denoted by $\left\{\tilde{\omega}_{0}^{n}, \ldots, \tilde{\omega}_{\tau_{\text {sample }}^{n}}^{n}\right\}, \forall n \in\{1, \ldots, N\}$. From these samples, we

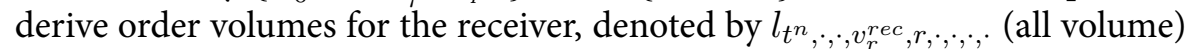
and $l_{t^{n}, \cdot, \cdot, v_{r}^{r e c}, r, \cdot, \cdot,, 1}$ (volume delivered via the UCC). Let $\tilde{\gamma}_{r}^{r e c} \in\{0,1\}$ be a binary variable that indicates whether the receiver uses the UCC in the sampling scenario; to compare both options we compute the costs for $\tilde{\gamma}_{r}^{r e c}=0$ as well as for $\tilde{\gamma}_{r}^{r e c}=1$. This way, every receiver samples its expected costs $\tilde{C}^{r e c}$ for both $\gamma_{t, r}^{r e c}=0$ and $\gamma_{t, r}^{r e c}=1$. In mathematical form, the following minimization problem is solved to update the tactical decision:

$$
\gamma_{t, r}^{r e c}=\underset{\tilde{\gamma}_{r}^{r e c} \in\{0,1\}}{\operatorname{argmin}} \tilde{C}^{r e c}\left(\tilde{\gamma}_{r}^{r e c}, l_{t^{\prime}, \cdot, \cdot, v_{r}^{r e c}, r, \cdot, \cdot, \cdot, \cdot,} l_{t^{\prime}, \cdot, \cdot, v_{r}^{r e c}, r, \cdot, \cdot,, 1}\right)
$$


The sample costs $\tilde{C}^{r e c}\left(\tilde{\gamma}_{r}^{r e c}, l_{t^{n},,,,, v_{r}^{r e c}, r, \cdot, \cdot,,,}\right)$ are computed as follows:

$$
\begin{aligned}
& \tilde{C}^{r e c}\left(\tilde{\gamma}_{r}^{r e c}, l_{t^{n}, \cdot, \cdot, v_{r}^{r e c}, r, \cdot, \cdot, \cdot, \cdot}\right)=
\end{aligned}
$$

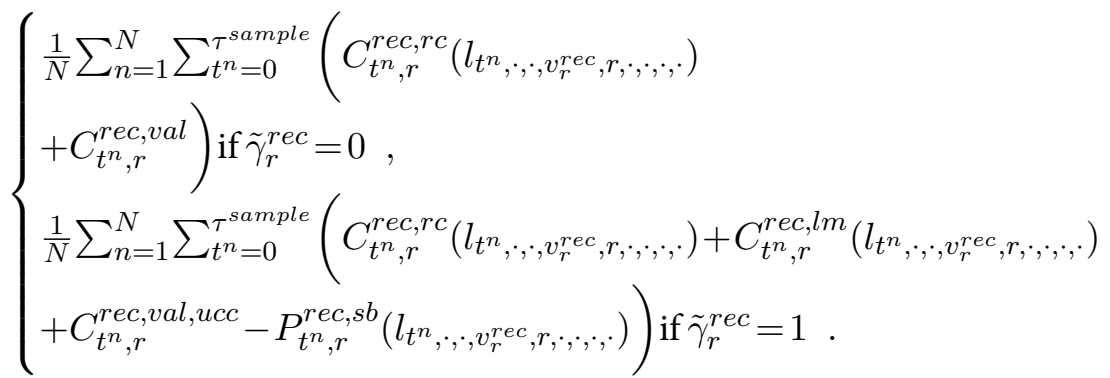

In the sampling function that we describe, it is assumed that the agent responds instantly to changes in the system such as new price levels. The price and cost levels are updated at the beginning at each tactical decision moment, i.e., before updating the tactical decisions. In practice, agents may display a more gradual approach in altering their tactical decisions. Such behavior can be reflected by incorporating learning mechanisms such as Q-learning, which place weight on both past observations and future expectations of state-action values. This results in a smoother transition of the state of the system. Although learning mechanisms may reflect the behavior of real-life actors more accurately, a downside is that more computational effort is likely required to identify the steady state (if such a state exists) of the system.

4.3.6.3. Operational decisions. All agents aim to optimize their own objective functions. In our dynamic environment, the corresponding operational decision problems are subject to incomplete information. Exact solution methods for stochastic problems usually require a large computational effort, which is why we typically resort to heuristic solutions in agent-based simulation. Various policies can be used to tackle the operational decision problems. The specific policies to be used in the simulation are selected based on the instance, and are part of the simulation settings.

Both the shippers and the UCC face a variant of the Delivery Dispatching Problem, which was discussed extensively in Chapter 3. As we consider a single line-haul arc, shippers face the basic version of this problem, in which they only need to decide on the time at which they dispatch orders. For them, a basic threshold policy suffices to capture their decision-making process: as the orders are subject to delivery windows, there is no incentive to dispatch orders as fast as possible. Hence, they can be expected to accumulate volume until (i) at 
least one of the orders becomes urgent, prompting the shipper to dispatch the accumulated volume or (ii) the accumulated volume hits some threshold volume that yields an acceptable carrier price. The UCC faces the DDP-TW, implying that at each decision moment they must decide which subset of orders to dispatch. Possible solution methods are learning a dispatching policy with ADP, a lookahead policy based on sampling, or some heuristic policy.

Carriers, as well as the UCC, are required to solve a vehicle routing problem (VRP). Orders arrive via the line-haul arc, implying a single entry point to the city that functions as the starting point of the route. For orders delivered via the UCC, the UCC location is the starting point of the route. In Section 4.2 we gave an overview of VRP methods specifically designed to deal with the context of urban logistics. As we split the decision problems into a periodic dispatch decision and a vehicle routing problem, solution methods for the static VRP can be applied. Which routing algorithm to apply depends on the level of detail of the model, the learning objectives of the simulation study, and the computational budget. If the purpose of the simulation study is to establish credibility of the simulation model with the stakeholders involved, it may be sensible to incorporate algorithms similar to the ones used in practice.

\subsection{Illustration of simulation framework}

In Chapter 5 and Chapter 6, we apply the presented framework using two different experimental designs. To give a brief illustration of the working of the framework, we already test a few urban logistics schemes here. Although we use approximate data and apply limited experimental designs, the experiments presented in this section already give an indication of the possibilities of the framework. Also, the results are helpful in interpreting the outcomes of the later simulation studies that we perform.

We have implemented the framework as a discrete-event simulation model in Delphi XE6. We represent the city by a virtual $10 \mathrm{~km}^{2}$ area, with 1 UCC located at the middle of the edge of the area, 3 carriers, 10 shippers, and 20 receivers. We assume that all agents of the same type are identical. The properties of the network are chosen such that they sufficiently reflect flexibility and diversity. The line-haul distance between shippers and receivers is set at $100 \mathrm{~km}$. We consider a planning horizon with 500 decision moments and perform 5 replications per scenario. In this illustration, we include decision making on the operational and on the strategic levels; the tactical decision level is omitted here. Based on preliminary experiments in which we apply Welch's graphical method (Law, 2007), 
we set the warmup period equal to 10 decision moments. We represent the uncertainty in the system by randomly generating the information variables $W_{t}$ according to uniform distributions. At every decision moment, receivers have a demand between 0 and 5 orders, with order sizes ranging between 0.05 and 0.20 of the capacity of a delivery van. The earliest delivery time varies between 0 and 2 time units; the length of the delivery window varies between 1 and 4 time units.

In Table 4.2, we show the vehicle properties that we use. Vehicle capacities and average emission values are obtained from Boer et al. (2011), using capacities for voluminous goods and 2020 engine standards. The UCC uses large vans ( $>2$ ton), the line-haul carriers deploy medium-sized trucks (10 - 20 ton). For the delivery van, we deduce costs per hour and vehicle speeds from Roca-Riu et al. (2016). We multiply these costs with 1.5 for medium-sized trucks. We set the transport price charged by the carrier to a fixed shipment fee of $€ 35$ and a variable cost of $€ 1.5$ per $\mathrm{km}$; the transport price of the carrier is assumed to be independent of the volume shipped. In contrast, the UCC charges a volumebased price of $€ 100$ per van-load, and it incurs receiving costs of $€ 20$ for every incoming truck. For receivers, we set the receiving costs at $€ 5$ per vehicle that delivers a shipment. Similarly, for shippers we assume shipping costs of $€ 5$ per vehicle that visits its premises to collect a shipment.

\begin{tabular}{ccc} 
TABLE 4.2. Vehicle properties for & \multicolumn{3}{c}{ UCC (delivery van) and carriers (truck). } \\
\hline Vehicle type & Large van $>2$ ton & Truck $10-20$ ton \\
\hline Loading capacity (ton) & 1.2 & 8 \\
Speed line-haul (km/hour) & 50 & 50 \\
Speed urban area (km/hour) & 25 & 25 \\
Handling costs $(€ /$ receiver) & 7.9 & 7.9 \\
Costs line-haul $(€ / \mathrm{km})$ & - & 1.24 \\
Costs urban $(€ / \mathrm{km})$ & 1.35 & 2.03 \\
$\mathrm{CO}_{2}(\mathrm{~g} / \mathrm{km})$ & 299.5 & 943 \\
$\mathrm{SO}_{2}(\mathrm{mg} / \mathrm{km})$ & 2.3 & 7.2 \\
$\mathrm{NO}_{\mathrm{x}}(\mathrm{g} / \mathrm{km})$ & 0.55 & 3.1 \\
$\mathrm{PM}_{2.5}(\mathrm{mg} / \mathrm{km})$ & 42 & 56 \\
\hline
\end{tabular}

We now describe the applied decision methods. For the shippers, we define a simple policy that dispatches all accumulated orders when it contains an urgent order and holds the shipment otherwise. For the UCC, we use a onestep lookahead sampling policy. Although an online ADP policy as introduced in Chapter 3 could have been executed sufficiently fast, repeating the offline 
learning phase for every scenario is computationally intense. In the simulation framework, we therefore opt for the sampling approach as discussed in Section 3.5.2. Even though its online application is slower than a policy learned with ADP, it provides good results - compared to myopic policies - and eliminates the need to perform the computationally expensive learning phase. In the sampling procedure, we sample 5 realizations of order arrivals; for each realization we compute the expected future costs per action. We select the dispatch decision that minimizes the sum of direct costs and expected downstream costs. The routing problem in the simulation model can be defined as a VRP with deterministic travel times and delivery windows. For both the carriers and the UCC, we use the cheapest insertion algorithm to solve their routing problems. We define the following strategic decisions: selection of the UCC by the receiver, forming a carrier coalition, setting parking costs, and subsidization of the UCC by the administrator. As already mentioned, we omit the tactical decision level in this illustration.

For the allocation of the gains obtained by a carrier coalition, we make use of the Shapley value as applied in cooperative game theory. We briefly explain the concept of the Shapley value here, for further detail we refer to Osborne and Rubinstein (1994). The Shapley value is an allocation concept that takes into account the marginal contribution of each player to the coalition. For any coalition $\mathcal{C}^{\prime} \subseteq \mathcal{C}$, transporting orders as described by the joint dispatching de-

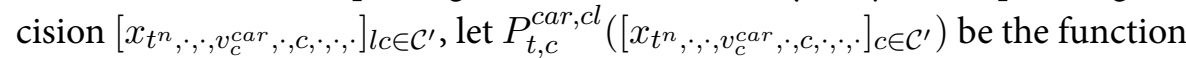
that yields the net income for $\mathcal{C}^{\prime}$. For each carrier $c \in \mathcal{C}$, for a given dispatching decision the gains are allocated as follows:

$$
\begin{array}{r}
\phi_{c}\left(P_{t, c}^{c a r, c l}\right)=\sum_{\mathcal{C}^{\prime} \subseteq \mathcal{C} \backslash\{c\}} \frac{\left|\mathcal{C}^{\prime}\right| !\left(|\mathcal{C}|-\left|\mathcal{C}^{\prime}\right|-1\right) !}{|\mathcal{C}| !}\left(v\left(\mathcal{C}^{\prime} \cup\{c\}\right)-\right. \\
\left.P_{t, c}^{c a r, c l}\left(\left[x_{t^{n}, \cdot, \cdot, v_{c}^{c a r},,, c, \cdot, \cdot,}\right]_{c \in \mathcal{C}^{\prime}}\right)\right) .
\end{array}
$$

We test the following measures: (A) carriers may deliver via the UCC (operational decision), (B) receivers mandate delivery via the UCC (strategic decision), (C) carriers form a coalition (strategic decision), using the Shapley value as a gain-sharing mechanism, (D) parking costs of $€ 3$ per stop, applicable only to trucks (strategic decision), and (E) volume-based subsidy to the UCC, set at $€ 70$ per full van-load (strategic decision). Based on these measures, we compose and test eight urban logistics schemes. With these schemes, we aim to show how both individual and combined measures affect system performance. For each 
agent type, we compare the impact of a scheme based on their performance under Scheme 1, which is the default scheme in which none of the five described measures are implemented. In Table 4.3, we show the results of the agent types for all schemes, stating the financial performance and an indicator for the external costs relative to Scheme 1 . The latter indicator is selected from the set of indicators $\{--,-, \mathrm{o},+,++\}$, with -- describing external costs that are much worse than under the default scheme and ++ describing a external costs that are much better than under the default scheme. Agents that are negatively affected by a scheme are likely to resist the new measures, we mark their costs in bold to indicate that these agents will not favor the scheme. 


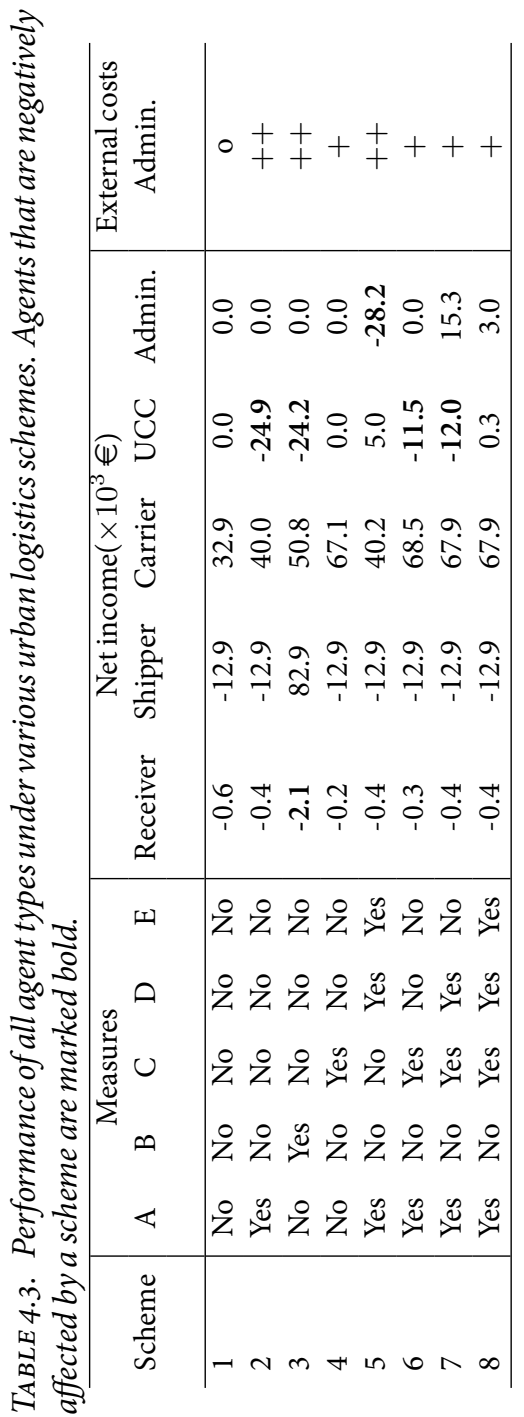


We proceed to reflect on the outcomes of the individual schemes. We stress that the analysis of the results is merely illustrative to the framework, rather than aiming to provide insights that are applicable to real-world settings. Furthermore, we note that we do not test any measures that impact the shippers; their performance is therefore equal for every scheme.

Schemes 2 to 4 each contain only a single measure. In Scheme 2, carriers have the option to deliver via the UCC. This option yields considerable gains in terms of environmental performance, but the UCC suffers substantial financial losses. In Scheme 3, the receivers mandate that all orders are delivered via the UCC. In this case, the receivers pay for the last-mile distribution, such that they lose money compared to the default scheme. Furthermore, the income from the receivers only does not suffice to cover the costs of the UCC. Scheme 4 tests the effect of carriers forming a coalition. Although this scheme does not have the same impact as the UCC, it generates additional profits for the carriers and is financially viable for all agent types.

Schemes 5 to 8 all combine multiple measures. With Scheme 5, we seek to improve the performance of the UCC by imposing parking costs on carriers on the one hand (given them a stronger incentive to use the UCC) and subsidizing the UCC on the other hand. When comparing to Scheme 2 and 3, we observe that the scheme is effective in generating a positive net income for the UCC. However, for the administrator the income stemming from the parking policy does not cover its subsidy expenses to the UCC. Thus, the administrator needs to provide permanent funding from external sources to the UCC, which is typically not a desirable situation. Scheme 6 combines both the presence of the UCC and the carrier coalition. Although the scheme reduces the deficit of the UCC mainly because the carriers make less use of the UCC 00 the net income for the UCC remains negative. Scheme 7 is similar to Scheme 6, but also imposes parking costs on the carriers. Although this measure seemed effective in Scheme 5, it has a relatively weaker effect when carriers cooperate - thus entering the city with less vehicles - and does not provide sufficient incentive for the collaborating carriers to utilize the UCC. Finally, Scheme 8 is similar to Scheme 7, but with the administrator providing subsidies to UCC. Although the subsidy does not increase the use of the UCC, it suffices to generate a positive net income for the UCC; the parking income is sufficient to cover the original deficit of the UCC. This combination of measures does not negatively impact any of the involved agents, while having a positive environmental impact as well.

Scheme 8 is the best performing scheme that is also financially sustainable. It significantly cuts emissions $\left(\mathrm{CO}_{2}\right.$ by $47.5 \%, \mathrm{SO}_{2}$ by $47.4 \%, \mathrm{NO}_{\mathrm{x}}$ by $53.1 \%$, 
and $\mathrm{PM}_{2.5}$ by $30.2 \%$ ), reduces the number of trucks in the city center by $60 \%$, and reduce the overall urban transport distance (by both trucks and vans) by $20 \%$. Although many measures have the potential to reduce external costs, the challenge remains to combine them into a economically viable scheme.

The results underline the difficulty to find feasible schemes. Individual measures often fail to generate the required commitment from all agents. The funding of the UCC is particularly complex; it is not financially sustainable without any supporting measures, yet it is not always clear how agents change their behavior when such supporting measures are implemented. Furthermore, we see that carrier coalitions are effective from a financial perspective, but do not have the same environmental benefits as the UCC, although still yielding an improvement compared to the base scheme. For a proper analysis of urban logistics schemes, both the environmental impact and the (financial) effects on each of the involved stakeholders should be assessed.

\subsection{Conclusions}

Although the need for improving urban freight transport is widely recognized, existing initiatives often fail due to a lack of commitment by the actors involved. In this chapter, we have designed an agent-based simulation framework that enables to evaluate a wide array of urban logistics schemes. We define the roles of five agent types, namely receivers, shippers, carriers, the UCC, and a local administrator. For each agent type, we describe its actions, monetary streams, and information streams. Furthermore, we specify KPIs to measure its performance. To reflect the practice of urban logistics - where we attempt to align the interests of multiple actors - every agent is modeled as an autonomous decision maker. As such, we assess the behavior and performance of every agent type. We divide the decision-making processes of the agents into three levels: operational, tactical, and strategic. Operational decisions such as routing and dispatching are solved at every decision moment; we discuss a number of solution methods to address these decisions. To solve tactical decisions (such as outsourcing last-mile distribution to the UCC), we propose a sampling procedure at a pre-determined subset of decision moments. Finally, we suggest that strategic decisions (e.g., administrative policies) are embedded into the analysis as scenario input.

As practice indicates that schemes combining both governmental policies and company-driven initiatives yield the best results, we have designed the framework such that both aspects are well represented. The urban consolidation center (UCC) has a key role in the framework, as it facilitates both consolidation 
and the deployment of dedicated delivery vehicles with lower environmental impacts. Furthermore, we include governmental policies such as road pricing, zone access fees, and parking costs; gains stemming from these measures can be used to subsidize agents in a closed-loop scheme. To define company-driven initiatives, we describe collaboration between carriers, as well as various interactions between the companies and the UCC. Another distinctive feature of our framework is that we explicitly take into account the effect of line-haul transport on the last-mile distribution, rather than focusing only on the last mile. With our simulation framework, we are able to measure the impact of schemes on the financial performance and the external costs and verify whether autonomous actors might be tempted to commit to such a scheme in the long run.

The agent-based simulation framework presented in this chapter has only been illustrated through a small set of numerical experiments. These experiments show that most measures have the potential to improve environmental performance, but that it is challenging to identify schemes that do not decrease the financial performance of any of the agents. In line with literature, we find that the combination of regulation, subsidies, and company-driven initiatives are the most viable schemes. In Chapter 5, we will perform a full simulation study, applying this framework to capture the dynamics of urban supply chains. We collect data from technical reports, various case studies, and expert interviews, enabling us to represent urban supply chains in a credible manner. Subsequently, we test a variety of urban logistics schemes. As indicated in literature and illustrated with the numerical results presented in this chapter, a combination of company-driven initiatives and government support is typically required to create sustainable long-term solutions. The focus is therefore on schemes that combine initiatives from both classes. 


\section{CHAPTER 5}

\section{Simulation of urban logistics schemes}

In Chapter 4, we developed an agent-based simulation framework to evaluate urban logistics schemes. We provided a brief numerical example to illustrate the working of the framework. In this chapter we perform a full simulation study, with the goal to obtain generic insights into the effects of a variety of measures in urban logistics. We assess the impact of individual measures, but are particularly interested in the combination of company-driven initiatives and administrative policies, as such combinations are generally most successful in practice. Therefore, we test over 2,000 schemes - applied on 6 distinct instances - that combine subsidy allocations, pricing policies, regulatory measures, and company-driven initiatives. By applying a fractional factorial experimental design, we obtain insights into both the main effects and the interaction effects of the tested measures. To reflect the diversity of urban supply chains in Western European cities, we collect data from a variety of urban logistics studies and case studies. By means of aggregation, we create a data set that enables us to construct abstract representations of urban supply chains.

The setup of this chapter is as follows. In Section 5.1, we introduce the study. Section 5.2 provides an overview of the literature that we use to extract our data from. Furthermore, it briefly contrasts the contribution of our simulation study to existing agent-based simulation studies in urban logistics. In Section 5.3, we discuss our methodology, which is an application of the framework defined in Chapter 4. In Section 5.4 we introduce the experimental setup. Numerical results are presented in Section 5.5; Section 5.6 concludes the chapter and summarizes the main findings.

\subsection{Introduction}

Although many urban logistics initiatives have been implemented in practice, a lack of insight remains regarding the degree to which measures actually contribute to the desired effects. The majority of studies in urban logistics is of a 
qualitative nature (Crainic et al., 2009). They are mostly case studies that evaluate either past or existing schemes, whereas some others are theoretical studies that hypothesize the impact of schemes. Although they may provide detailed reasons for the success or failure of a scheme, these reasons are often only applicable for that specific context and the manner in which the scheme has been implemented. Although existing quantitative studies provide some insights into the impact of schemes under a variety of conditions, these studies tend to focus on specific cases and a limited number of measures. Furthermore, they are often applied on overly simplified instances, without providing insights into the extent to which the findings are applicable to more realistic instances. Thus, it is challenging to deduce generic insights from the current body of literature and to translate them into well-performing logistics schemes. Given the high rate of urban logistics schemes that do not live up to their intended benefits, it would be valuable to gain insights into which measures are most effective and in which combination they should be applied.

As already mentioned in Chapter 4, it is typically the combination between administrative measures and company-driven initiatives that yields the best results. This symbiosis is often not explicitly studied, with papers usually focusing only on one of the two classes. As individual measures do not fully capture the potential for improvement, we are also interested in the interaction effects between various measures, particularly the interaction between companydriven initiatives and administrative policies. These interaction effects also yield valuable insights to practitioners. For example, if companies start a promising initiative, the administrator would like to know which measures to implement in order to support this initiative and strive for a high probability of success.

The contribution of this chapter is twofold. First, we provide the characteristics of abstract representations of urban supply chains. To obtain credible results, it is essential that (i) the instances use data that accurately reflect practice and (ii) the experiments are sufficiently generic to be representative for a variety of instances, rather than being tailored towards a particular case. For this purpose, we collect data from a variety of sources and aggregate these into a data set that is representative for Western European cities. Part of this representation comprises the development of retailer profiles. Second, we numerically test a large variety of urban logistics schemes. By testing these schemes on instances that are based on aggregate data, we aim to obtain quantitative insights that can be generalized to the wider context of urban logistics in Western Europe. 


\subsection{Literature on agent-based studies and urban data collection}

This literature overview is comprised of two parts. Section 5.2.1 reflects on the managerial insights provided by existing agent-based studies in the context of urban logistics. Section 5.2.2 discusses the main data sources that we use for the simulation study that we perform in this chapter.

5.2.1. Agent-based simulation studies. We have discussed a number of agent-based studies in the context of urban logistics in Section 4.2. In that section, we focused on their contributions on the methodological level; in the current section we instead reflect on their contributions to managerial insights in urban logistics. First, the current body of agent-based studies in urban logistics is tested on relatively small grid networks (as we show later on), whereas realistic instances of urban areas contain vastly larger numbers of agents. It is unclear to what extent the results obtained on small grid networks are scalable to larger and more realistic instances. The grid networks also have the limitation that they assume that all agents - including the shippers and carriers - are located within the city; in practice this often is not the case. Second, most studies consider the effects of the measures in isolation. Although these studies provide insights into a number of isolated measures, it remains difficult to deduct generic insights from these works. Third, the existing papers strongly focus on governmental policies. As indicated by Browne et al. (2005), it is usually the combination of company-driven initiatives and administrative policies that yields the best results in practice.

Taniguchi et al. (2007) perform a simulation study on two instances: a $2 \times 2$ grid network and a $7 \times 7$ grid network. The first contains 2 carriers and 2 shippers; receivers are not explicitly modeled. The latter represents a $28 \mathrm{~km}^{2}$ area, in which 10 carriers (all located at the corner vertices, i.e., multiple carriers use the same depot locations) and 45 shippers (located at the remaining vertices) are operating. Carriers dynamically adjust their prices, and pick up orders at the shipper vertices. The administrator may impose road pricing and provide subsidies to shippers that select environment-friendly carriers. Carriers are able to cooperate with other carriers, thereby increasing vehicle fill rates. Tamagawa et al. (2010) study a $5 \times 5$ grid (representing a $25 \mathrm{~km}^{2}$ area), with a setup similar to the one presented in Taniguchi et al. (2007). They test the effects of truck bans and road pricing, both as isolated measures and in combination with each other. Van Duin et al. (2012) perform numerical experiments on a network containing 3 carriers and 240 retailers; every day, a subset of 10 randomly selected retailers places orders. The authors test the impacts of dynamic UCC fees 
depending on the time of the day, congestion levels, subsidies for the UCC, and road pricing. They conclude that it is unlikely that UCCs are sustainable without permanent subsidies or revenues from value-adding services such as collecting waste or labeling goods. Wangapisit et al. (2014) represent the city with a $5 \times 5$ grid, containing 1 UCC location, 4 carrier depots, 3 receivers, and 17 vertices representing residents; the role of the shipper is not incorporated in the model. Subsidies may be provided to the UCC; furthermore parking constraints may be imposed on the carriers. Teo et al. (2014) study two measures, namely road pricing and restrictions on the minimum load factors. They perform experiments on a network that is based on the city of Osaka in Japan and consider 3 shippers, 3 carriers and 30 receivers within this network. The authors conclude that when implementing both measures, the financial performance of shippers and receivers is better than when only implementing road pricing. Finally, the work of Boussier et al. (2011) differs from the aforementioned studies as it uses agents to represent individual vehicles in a traffic simulation; vehicles can choose a variety of actions when parking places are occupied.

5.2.2. Literature on urban freight transport data. We proceed to discuss the data sources on urban logistics. The first source we discuss is the report of Browne et al. (2005), who provide an exhaustive overview of UCCs, taking into account operational schemes, trial projects, and feasibility studies. Although several hundred UCC initiatives have been started, for many of them no public data has been disclosed. The report contains descriptions of 67 UCC schemes for which sufficient data was available to perform a meaningful analysis. The data availability varies per instance and is often presented in different formats, yet the report provides data on a wide variety of UCCs. Although the analysis in this chapter is not limited to UCCs, the report provides valuable information that aid us to represent urban freight flows.

We highlight two reports that resulted from the BESTUFS project. The report 'Quantification of Urban Freight Transport Effects I' (Schoemaker et al., 2006) focuses on quantifying urban freight transport. They obtain data from a variety of sources, namely statistical offices, a variety of research projects, urban freight platforms, case study reports, traffic studies, and expert interviews. The report provides figures regarding, for instance, the characteristics of urban freight demand, delivery trips, and vehicle properties. The complementary report 'Quantification of Urban Freight Transport Effects II' (Dasburg and Schoemaker, 2006) quantifies the effects of measures aimed to improve external cost performance. 
The report reflects on both government policies and company-driven initiatives, providing insights into the environmental impacts (and to a lesser extent the financial performance) stemming from a variety of measures.

As part of the Green Logistics project, Allen et al. (2008) provide an overview of the results collected in a large number of urban freight studies that were performed in the UK. Allen et al. (2012a) perform an international literature review, in which 114 UCC schemes from 17 countries are identified. They reflect on these schemes and provide quantitative insights. Finally, the book by Ambrosino et al. (2005) documents the properties and impacts of various urban logistics schemes implemented throughout Europe.

In addition to these main sources, we mention a variety of case studies that provide data within the scope of our research. Ambrosino et al. (2007) reflect on the experiences with a UCC in Lucca (Italy), contrasting the results to those of a number of other UCCs. Quak and De Koster (2007) study how retailers in Western Europe respond to time access restrictions in cities, and how this in turn affects financial and environmental performances. They use the figures provided by 14 Dutch retailers from different sectors and cities. In a comparable setting, Quak and de Koster (2009) investigate the effects of both time access restrictions and vehicle access restrictions for two Dutch retail chains. Van Rooijen and Quak (2010) perform a case study on a UCC in Nijmegen (the Netherlands), providing a variety of characteristics and quantitative results. Van Duin et al. (2010) perform a feasibility study on a fictive UCC in The Hague (the Netherlands). Based on literature, expert interviews, and collaboration with the municipality of The Hague, they estimate a variety of parameters to perform a quantitative analysis. They also provide summaries of various other UCC initiatives. Dablanc (2011) provides an overview of freight transport in European cities, describing a number of representative common properties and developments. Finally, in the two related papers Roca-Riu and Estrada (2012) and Roca-Riu et al. (2016), the feasibility of an urban consolidation center in an abstract setting is studied. Various figures that are representative for a variety of European cities are provided.

With this chapter, we address two gaps in the literature. First, we contribute with a simulation study on larger and more realistic instances than has been done before. Existing agent-based studies often operate on relatively small and simplified grid networks, study only the decisions made within the urban area, and tend to focus strongly on administrative measures. In this chapter, we consider realistically sized instances that also embed the roles of shippers and carriers outside the city boundaries, whereas existing studies assume that all agents are 
located within the city. Furthermore, we explicitly address the combination of administrative measures and company-driven initiatives. The second literature gap that we address is the current lack of an representative data set to model urban supply chains. Although a fairly large amount of data is available, data sources are fragmented and often incomplete. By aggregating data from many data sources, we create a data set that is representative for Western European cities in particular. This data set may aid in generating test instances for quantitative studies in urban logistics.

\subsection{Solution method}

In Chapter 4, we presented a general framework to evaluate urban logistics schemes. Here, we describe the specific implementation of this framework that we use for this simulation study. Because we aim to analyze a large number of schemes, we omit the layer of tactical decisions. Although this design choice removes some of the richness and realism of the framework, it significantly reduces the required simulation run time, because (i) the computationally expensive procedure to evaluate tactical decisions does not need to be performed and (ii) the system reaches a steady state much faster. Therefore, we assume the decisions made at a strategic and tactical level as one-time decisions fixed at the beginning of each simulation run, and we focus on the operational decisions made at each decision moment.

The structure of the remainder of this section is as follows. In Section 5.3.1, we present an outline of the simulation model, describing the sequential flow of decisions and introducing the generic notation used to define the model. Section 5.3.2 provides the cost functions per agent type for the default settings; the effects of measures such as outsourcing to the UCC and receiving subsidies are explained textually. Finally, Section 5.3.3 describes the agent intelligence for each agent type. We distinguish between agent intelligence at the strategic or tactical level (fixed at the beginning of each simulation run) and agent intelligence on the operational level.

5.3.1. Outline of the simulation model. In this subsection, we outline the simulation model that we use in this chapter. Many of the properties are comparable to those presented in Chapter 4 . Therefore, we only discuss the notation and definitions required for a general understanding of the simulation model here, and refer to Section 4.3 .3 for the detailed definitions of the dispatching decisions and to Section 4.3 .4 for the corresponding transition functions.

Similar to the framework introduced in Chapter 4 , let $\mathcal{V}$ be a set of vertices. Furthermore, let $\mathcal{T}=\{0, \ldots, T\}$ be a set of decision moments separated by 
equidistant time intervals, and let $t \in \mathcal{T}$ refer to a specific discrete decision moment. We characterize each order by a number of properties; a unique combination of order properties is called an order type. Each order type is characterized by six properties: the earliest delivery time $t^{e} \in \mathcal{T}^{e}$ (relative to $t$ ), the latest delivery time $t^{l} \in \mathcal{T}^{l}$ (relative to $t$ ), the vertex $v \in \mathcal{V}$, the receiver $r \in \mathcal{R}$, the carrier $c \in \mathcal{C}$, the shipper $s \in \mathcal{S}$, the order volume $l \in \mathcal{L}$, and a binary variable $\gamma \in\{0,1\}$ that indicates whether the transport takes place via the UCC. An order type is denoted by $\left[t^{e}, t^{l}, v, r, c, s, l, \gamma\right]$, whereas the number of orders of a specific type in the system at time $t$ is denoted by $I_{t, t^{e}, t^{l}, v, r, c, s, l, \gamma} \in \mathbb{N}$. The vector $I_{t}=\left[I_{t, t^{e}, t^{l}, v, r, c, s, l, \gamma}\right]_{\forall t^{e}, t^{l}, v, r, c, s, l, \gamma}$ describes the number of orders of each type in the system at decision moment $t$.

We provide some explanation on how to interpret the notation of order types, specifically the meaning of the index $v$ and the connotation of blank indices. The set $\mathcal{V}$ is composed of the subsets of receiver locations $\mathcal{V}^{R}$, shipper locations $\mathcal{V}^{S}$, carrier locations $\mathcal{V}^{C}$, and the UCC location $v^{u c c}$. A carrier location $v_{c}^{c a r} \in \mathcal{V}^{C}$ refers to the home depot of carrier $c$, which is the location from which carrier $c$ starts its routes. The locations $v_{r}^{r e c} \in \mathcal{V}^{R}$ denote the delivery locations and the shipper locations $v_{s}^{s h p} \in \mathcal{V}^{S}$ are the pickup locations. In the order type, the vertex indicates which decision maker is able to perform an action on the order type. For example, if an order type is described by a receiver vertex $v_{r}^{r e c}$ and the number of this order type is positive, this means that receiver $r$ can communicate the demand for this order type to the shipper. This example also illustrates that the vertex does not necessarily indicate the physical location of the order, but may also indicate the agent that holds information regarding the order, see Section 4.3.3 for the discussion of the distinction between physical transfers and information transfers. Another important aspect in the definition of order types is that when we use a dot · instead of a sub-index, this indicates that the index has not yet been defined. Recall that the sub-indices are defined during a sequential transition function. For example, a receiver may leave open the decision whether to be delivered via the UCC $(\gamma=\cdot)$; in that case the carrier eventually defines the setting for $\gamma$.

We briefly describe the vehicle-related notation here; we will reflect on the specific vehicle properties used in the simulation study in Section 5.4. We assume that all carriers use identical trucks. The UCC has a homogenous fleet, but uses smaller trucks than the carriers. Furthermore, we assume that both the carriers and the UCC always have sufficient vehicles available to perform the requested order transports. Let $\psi_{c}^{c a r} \in \mathbb{R}_{\geq 1}$ be the load capacity of the vehicles 
operated by carrier $c$ and $\psi^{u c c} \in \mathbb{R}_{\geq 1}$ be the load capacity of the vehicles operated by the UCC, with $\psi^{u c c}<\psi_{c}^{c a r}, \forall c \in \mathcal{C}$.

We proceed to describe the dispatching decisions. In generic notation, the dispatching decisions made in the system at time $t$ are denoted by the vector $x_{t}=\left[x_{t, t^{e}, t^{l}, v, r, c, s, l, \gamma}\right]_{\forall t^{e}, t^{l}, v, r, c, s, l, \gamma}$, with the variable $v \in \mathcal{V}$ indicating which agent is taking the decision. As mentioned, the dispatching decisions used in this simulation model are comparable to those described in Section 4.3.3; we therefore do not discuss them in detail here. Figure 5.1 gives a high-level overview of the simulation model as applied in this chapter. The sequence of the decisions made at each decision moment $t \in \mathcal{T}$ is as follows: (i) the receiver generates orders, (ii) the receiver decides whether it wants to use the UCC as its delivery address for the set of placed orders (choosing not to use the UCC leaves the ultimate decision to the carrier), (iii) the shipper decides whether to ship its batch of accumulated orders, (iv) the carrier decides whether to outsource the delivery of its non-urgent deliveries to the UCC, (v) the carrier executes the route, (vi) the UCC decides which subset of orders to dispatch, and (vii), the UCC executes the route for the subset of dispatched orders. Actions (ii) and (iv) imply that both the receivers and the carriers may decide whether to use the UCC at each decision moment. Although the use of a UCC will typically be less flexible in practice, treating the selection of the UCC as an operational decision yields insights in the circumstances under which customers are inclined to use the UCC.

The time intervals corresponding to transport times deviate from the ones described in Chapter 4; here we describe how they are modeled in this chapter. Direct transport by the carrier to the receiver is completed within 1 time unit, i.e., if the shipper dispatches orders at $t$, they are delivered to the receiver in the interval $(t, t+1]$. Carriers do not offer storage capacity, therefore, if the receiver did not set the UCC as its delivery address, shippers may only dispatch orders for which $t^{e}=0$ to ensure that orders are not delivered before the earliest arrival time. In this chapter, we model the UCC such that it needs 1 time interval to handle incoming goods. Thus, an order dispatched by the shipper at time $t$ is ready for dispatch from the UCC at time $t+1$, such that it can be delivered to the customer within the interval $(t+1, t+2]$. This implies that when the shipper dispatches orders characterized by $t^{l}=1$, the carrier must directly transport these orders to the city to deliver at the receivers on time; it cannot outsource the delivery of these orders to the UCC. Orders may only be delivered via the $\mathrm{UCC}$ when $t^{l}>1$. All orders are generated with $t^{l} \geq 2$, such that theoretically it is always possible to deliver via the UCC; it depends on the dispatch times of the shippers whether the carrier may outsource to the UCC. The implication of this 
design is that carriers may only use the UCC if the shipper is willing to allocate slack. When the receiver selects the UCC as its delivery address, an order with $t^{l}=2$ must already be dispatched by the shipper to reach the receiver on time.

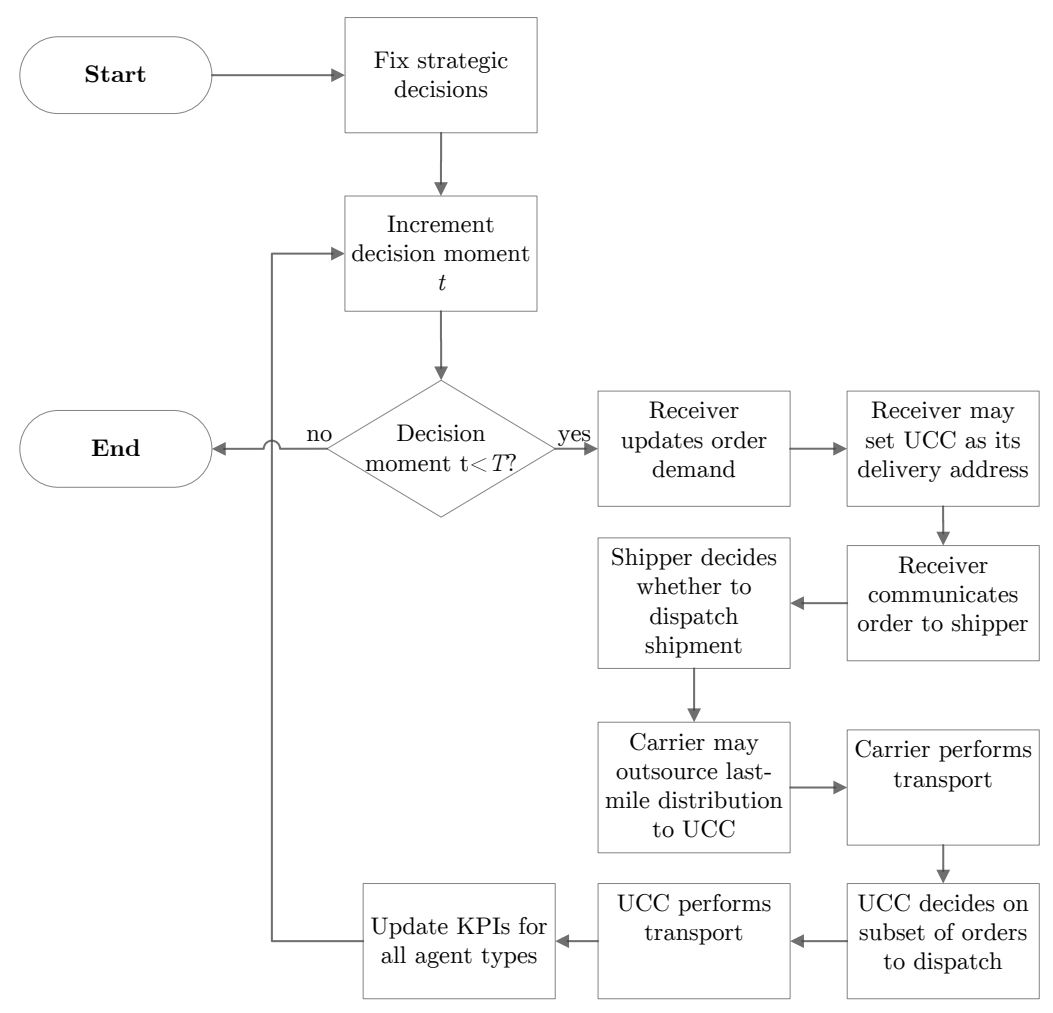

FIGURE 5.1. Flowchart of the simulation model.

The last topic of this outline is the transition of the system state over time. The demand for order types arising in the time interval $(t-1, t]$ is denoted by the information variable $\tilde{I}_{t, t^{e}, t^{l}, v, r, \cdot, s, l, \cdot} \in \mathbb{N}$; note that the exogenous variables $\gamma$ (indicating whether to ship via the UCC) and the carrier selection $c$ are not specified initially. To describe the new demand for all order types, we use the notation

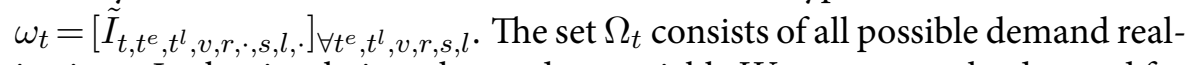
izations. In the simulation, the random variable $W_{t}$ represents the demand for 
orders arriving between $t-1$ and $t$, with $\omega_{t} \in \Omega_{t}$ being a particular realization of $W_{t}$. Together with the order position $I_{t-1}$ and the dispatching decisions that are made at time $t-1$, this information enables us to model the transition of the system from $t-1$ to $t$. The transition functions have been given in Section 4.3.4.

5.3.2. Cost functions. In this section, we describe the cost function for shippers, carriers, receivers and the UCC at a decision moment $t$. Detailed representations of the cost and price elements have already been introduced in Section 4.3.5; here we only provide a generic overview of the specific cost structures for each agent type as modeled in this chapter. To keep the functions comprehensible, in the formal mathematical definitions we restrict ourselves to describing the agents' costs and incomes under standard operations, i.e., not including the costs and incomes related to specific measures and initiatives such as outsourcing, collaborating, or subsidizing. Although we incorporate these measures and initiatives in the model, to prevent the formulation from becoming unnecessarily complicated we will instead textually describe their financial consequences for each agent type. The incorporation of the additional measures into the standard cost functions is self-explanatory.

To denote volumes corresponding to a certain set of orders, we use the same notational style as introduced in Chapter 4 . We briefly recap the essential notation, starting with the notation of routes. For a carrier $c$, we describe the set of routes performed at $t$ by $\Delta_{t, c}^{c a r}=\left[\Delta_{t, c}^{c a r, p u}, \Delta_{t, c}^{c a r, l h}, \Delta_{t, c}^{c a r, l m}\right]$, with each route set being divided in a pickup component $\Delta_{t, c}^{c a r, p u}$, a line-haul component $\Delta_{t, c}^{c a r, l h}$, and a last-mile component $\Delta_{t, c}^{c a r, l m}$; a single carrier may simultaneously dispatch multiple vehicles. All pickups must be completed before transport on the line-haul segment commences. The UCC only needs to perform transport on the last-mile segment; its route set is denoted by $\Delta_{t}^{u c c}=\left[\Delta_{t}^{u c c, l m}\right]$. The manner in which we describe volumes is also the same as introduced in Chapter 4 . We provide an example; other volumes are computed in a similar way. Suppose that for a receiver $r$, we want to denote the volume that is ordered at decision moment $t$ with a given earliest delivery time $t+t^{e}$. For this purpose, we use the following notation:

$$
l_{t, t^{e},,, v_{r}^{r e c}, r, \cdot, \cdot, \cdot,}=\sum_{t^{l}, s, l} x_{t, t^{e}, t^{l}, v_{r}^{r e c}, r, \cdot, s, l, \cdot} \cdot l .
$$

The costs incurred by the shipper is given by the sum of two cost functions and is based on the volume that it dispatches at $t$. As we will further discuss 
in Section 5.3.3, each shipper uses a single carrier $c \in \mathcal{C}$ that conducts all its transport for the full simulation. The first cost function is the transport price $P_{c}^{c a r, t r}\left(l_{t, \cdot, \cdot, v_{s}^{s h p},,, c, s, \cdot,}\right)$ that is charged by the selected carrier $c$. The transport price consists of a fixed component per truckload dispatched and a volumedependent component. Due to this structure, the shipper has a financial incentive to accumulate orders; this decision is reflected in its shipping policy. The second cost function, $C_{s}^{s h p, s p}$, describes the handling costs that are incurred when the carrier visits the premises of the shipper to collect a shipment. These costs are composed of a fixed component only. The costs for shipper $s \in \mathcal{S}-$ again given that the selected carrier $c$ is known - at time $t$ are given by

$$
C_{s}^{s h p}\left(l_{t,,, \cdot, v_{s}^{s h p},, c, s, \cdot, \cdot}\right)=P_{c}^{c a r, t r}\left(l_{t, \cdot, \cdot, v_{s}^{s h p},,, c, s, \cdot, \cdot}\right)+C_{s}^{s h p, s p} .
$$

We proceed with the cost function for the carriers. The goal of each carrier is to maximize its profit; to have a consistent notation for each agent type we formulate it as a cost function nevertheless. Here, we only present the basic cost function of the carrier, in which the carrier executes the entire route - composed of the pick-up of shipments, the line-haul transport, and the last-mile deliveryby itself. The costs may deviate from this standard function in three ways. One way to influence the cost function is by collaborating with other carriers. In that case, the costs and incomes are divided over all carriers; we do not address the question what this allocation looks like in this chapter. The second way to influence the cost function is to outsource the last-mile distribution to the UCC; the corresponding cost function has been described in Section 4.3.5. We note that when operating in a coalition, the carriers may still outsource their last-mile delivery to the UCC. The third way to influence the costs is through a volume-based subsidy that may be received for outsourcing to the UCC. To keep the function comprehensible, we do not introduce the additional notation required for these extensions.

For the standard function, we have the routing costs $C_{c}^{c a r, t r}\left(\Delta_{t, c}^{c a r}\right)$ - which include the costs for driving, (un)loading, and policy-related expenses such as road pricing - and a volume-based price $P_{c}^{c a r, t r}\left(l_{\left.t,,,, v_{s}^{s h p},, c, s, \cdot, \cdot\right)}\right)$ that is paid to the carrier by shipper $s$; recall that this price consists of a fixed and a volumedependent component. The cost function of carrier $c \in \mathcal{C}$ at time $t$ is given by 


$$
\begin{array}{r}
C_{c}^{c a r}\left(\Delta_{t, c}^{c a r},\left[l_{t, \cdot, \cdot, v_{s}^{s h p},,, c, s, \cdot,}\right]_{s \in \mathcal{S}}\right)=C_{c}^{c a r, t r}\left(\Delta_{t, c}^{c a r}\right)- \\
\sum_{s \in \mathcal{S}} P_{c}^{c a r, t r}\left(l_{t, \cdot, \cdot, v_{s}^{s h p}, \cdot, c, s, \cdot, \cdot}\right)
\end{array}
$$

The objective of the UCC is to maximize profit, which - in its standard form is determined by one price function and two cost functions. The UCC charges a fixed fee per volume unit outsourced by carriers or receivers; the corresponding price function is denoted by $P^{u c c, t r}\left(l_{t, \cdot, \cdot, v^{u c c},,, \cdot, \cdot, 1}\right)$. The UCC can only acquire an income from an order once; i.e., if the receiver sets the UCC as its delivery address, the UCC does not charge carriers for orders destined to that receiver; instead the receiver pays the UCC for these orders. The UCC incurs costs for all the volume that it handles; these costs are denoted by $C^{u c c, h d}\left(l_{t, \cdot, \cdot, v^{u c c}, \cdot, \cdot, \cdot, 1}\right)$. Finally, the UCC dispatches a subset of orders - as has been described in Equation (4.3.11) - and incurs costs for the corresponding route $\Delta_{t}^{u c c}$ that it executes; these transport costs are denoted by $C^{u c c, t r}\left(\Delta_{t}^{u c c}\right)$. Subsidies allocated to the UCC are a fixed percentage of the volume-based price it charges to the carriers and receivers (we motivate this design choice in Section 5.3.3); this income source is not included in the standard cost function. The cost function for the $\mathrm{UCC}$ at time $t$ is defined by

$$
\begin{aligned}
& C^{u c c}\left(\Delta_{t}^{u c c}, l_{t, \cdot, \cdot, v^{u c c}, \cdot, \cdot, \cdot, \cdot, 1}\right)= \\
& C^{u c c, h d}\left(l_{t, \cdot, \cdot, v^{u c c}, \cdot, \cdot, \cdot, \cdot 1}\right)+C^{u c c, t r}\left(\Delta_{t}^{u c c}\right)-P^{u c c, t r}\left(l_{t, \cdot, \cdot, v^{u c c}, \cdot, \cdot, \cdot, \cdot 1}\right) .
\end{aligned}
$$

The receiver incurs a receiver-specific cost per truck that visits its premises. This cost component is based on the average time it spends on receiving goods, as this time is highly specific and not necessarily related to volume. We denote the receiving costs by $C_{r}^{r e c, r c}\left(\Delta_{t}^{u c c} \cup \bigcup_{c \in \mathcal{C}} \Delta_{t, c}^{c a r}\right)$. This cost function takes into account the effects of access time windows. If the administrator offsets the access time windows such that shops cannot be visited at their regular opening times, the receiver must pay for additional personnel hours to receive the goods, which results in an extra cost. We note that the smaller trucks that are operated by the UCC are exempted from this restriction, such that the receiver does not need to pay the extra receiving costs when outsourcing to the UCC. An extension that is not included in the standard cost function is the possibility to obtain a subsidy for outsourcing to the UCC. This subsidy is a fixed percentage of the outsourcing price that is equal for all receivers. The basic cost function for receiver $r \in \mathcal{R}$ at time $t$ is given by: 


$$
C_{r}^{r e c}\left(\Delta_{t}^{u c c} \cup \bigcup_{c \in \mathcal{C}} \Delta_{t, c}^{c a r}\right)=C_{r}^{r e c, r c}\left(\Delta_{t}^{u c c} \cup \bigcup_{c \in \mathcal{C}} \Delta_{t, c}^{c a r}\right)
$$

5.3.3. Agent intelligence. In this section, we discuss the decisions that each agent can make in the model. We distinguish between decisions made on two levels: the one-time decision level and the operational level. The one-time decisions are fixed at $t=0$ for the duration of the simulation run. Operational decisions are made at each decision moment $t \in \mathcal{T}$. The dispatching decisions that are made by each agent have already been discussed in Section 5.3.1; in this section we explain the remainder of the operational decisions that are made by the agents.

In Table 5.1, we give an overview of the agent intelligence that is embedded in the model. This section first describes the decisions per agent type that are made on the one-time decision level. Then, we discuss the operational decisions that are made at every decision moment, again itemized per agent type. Before providing detailed descriptions of the decisions, we already discuss the main design choices of each agent type here.

Both the carrier and the receiver decide whether to outsource to the UCC at the operational level. In practice, it is likely that users will commit to the UCC for longer periods of time, as we will study in Chapter 6. However, in this chapter we are primarily interested in the properties of orders for which outsourcing is financially appealing; operational decisions serve this purpose better. The remaining operational decisions are routing decisions and dispatching decisions, these are performed at the operational level to accurately compute the cost functions.

At $t=0$, receivers may synchronize their delivery windows for the entire horizon. This decision implies that all receivers that use a centralized ordering system synchronize their delivery windows. Although in practice such a measure may be difficult to conceive, testing it does provide us with insights into the potential effects of synchronizing delivery windows on the efficiency of urban freight transport. For the carriers, we model the one-time decision to form a coalition. If the carriers opt for this decision, they pool all their resources and orders. Again, in practice such a collaboration is unlikely to be so strong and permanent, yet our goal here is to determine an upper bound on the attainable savings and the system-wide effects. For the shipper, setting the threshold volume until which it accumulates orders before shipping them and selecting the carrier to conducts all its transport are one-time decisions. As the ordering process of receivers is stationary, having a fixed threshold volume is a natural design choice. Selecting a single carrier to conduct all transports for the shipper 
(possibly excluding the last mile) results in distinct profiles for the carriers in terms of customer locations to visit and volumes transported. Furthermore, as we model all carriers as homogenous, selecting carriers on the operational level would yield no meaningful insights.

TABLE 5.1. Overview of agent intelligence per agent type, itemized for decisions on the operational level and on the one-time decision level.

\begin{tabular}{|c|c|c|}
\hline Agent type & Operational level & One-time decision level \\
\hline Receiver & Outsource to UCC & Synchronize delivery windows \\
\hline Carrier & $\begin{array}{l}\text { Outsource to UCC } \\
\text { Routing decision }\end{array}$ & Form coalition \\
\hline Shipper & Dispatching decision & $\begin{array}{l}\text { Set dispatching threshold } \\
\text { Select carrier }\end{array}$ \\
\hline UCC & Routing decision & Open UCC \\
\hline Administrator & Dispatching decision & $\begin{array}{l}\text { Mandate pre-announcement } \\
\text { Cluster receiver locations } \\
\text { Set subsidy levels } \\
\text { Set pricing policies } \\
\text { Impose restrictions }\end{array}$ \\
\hline
\end{tabular}

The description of the decisions per agent type is fairly detailed and aims to explain the rationale behind our model choices. As an aid to the reader, we provide an itemized summary of the decisions per agent type for both one-time decisions and operational decisions at the end of each subsection. These summaries suffice for a general understanding of the decisions.

5.3.3.1. One-time decisions shipper. The first agent type that we assess is the shipper. Shippers select a threshold value for the volume dispatched, expressed in terms of the capacity of the UCC vehicles (i.e., $\psi^{u c c}$ ) (as $l$ is defined in terms of this capacity as well):

$$
\zeta_{s} \in\left[\min (l \mid l \in \mathcal{L}), \max _{c \in \mathcal{C}} \psi_{c}^{c a r}\right] .
$$

A threshold of $\min (l \mid l \in \mathcal{L})$ implies that the shipper always directly ships any order with $t^{e}=0$ or $\gamma=1$ when it is revealed; a value of $\left\{\max _{c \in \mathcal{C}} \psi_{c}^{c a r}\right\}$ implies that the shipper waits until the volume suffices to completely fill the largest carrier vehicle in the system, under the condition that none of the accumulated orders is urgent. When at least one of the orders is urgent and must be dispatched, the shipper dispatches all orders that have been accumulated until that point. Furthermore, the shipper must take into account that the carrier cannot collect orders for which $t^{e}>0$ and $\gamma=0$, as the carrier does not offer intermediate 
storage capacity. When $\gamma=1$, orders with $t^{e}>0$ may also be shipped, as these orders can be stored at the UCC. The threshold $\zeta_{s}$ is set on the one-time decision level; we will describe the operational dispatching policy itself in Section 5.3.3.1.

The second one-time decision that the shipper takes is the selection of a carrier to conduct all its transport. The shipper selects a carrier with a probability that is inversely proportional to the distance between the shipper location and the carrier depot, i.e., it is most likely to select the carrier that is most closely located. With this design choice, we want to reflect that the shipper has a preference for nearby carriers, but may have particular reasons to select another carrier. The decision is made at the one-time decision level to diversify the routing properties of the carriers; depending on the supply chains corresponding to the shippers, some carriers will mainly handle large volumes and few receivers, whereas others will deliver many small loads. To be able to compare the results between different schemes, the selection of carriers is equivalent for all simulation runs.

\begin{tabular}{ll}
\hline One-time decisions shipper & Description \\
\hline Set threshold value $\zeta_{s}$ & $\begin{array}{l}\text { If accumulated volume (i) is higher than } \zeta_{s} \text { or (ii) contains } \\
\text { at least one urgent order, then shipper } s \text { dispatches all } \\
\text { accumulated orders. }\end{array}$ \\
& $\begin{array}{l}\text { Select carrier to conduct all transports of shipper } s \text { with } \\
\text { probability that is inverse to the distance between shipper } \\
\text { location and carrier depot. }\end{array}$ \\
\hline
\end{tabular}

5.3.3.2. One-time decisions carrier. We proceed with the one-time decision for the carrier by describing the policy for order allocation when a carrier coalition is formed. When carriers form a coalition, all orders to be transported are placed in a joint pool. Subsequently, at each decision moment $t \in \mathcal{T}$, one of the carriers is randomly selected to transport all orders dispatched by the shippers; the underlying rationale is to divide the workload between carriers in a fair manner. Recall that we do not assume restrictions on the fleet size of carriers; for the coalition we rationalize this choice by presuming that carriers share their vehicles. By bundling orders, the carriers can attain higher fill rates than would be possible when operating individually. The focus of this simulation study is to investigate whether system-wide gains might be obtained by carriers by sharing their orders and transport resources, and if so, under which circumstances. We do not explicitly look into the gain-sharing mechanism here, but instead measure the total attainable gains and the environmental impact of carrier coalitions. In reality, achieving gains in itself is not sufficient for a coalition to be acceptable to all participants; advantages and disadvantages should be distributed in a fair manner between the carriers. A further complication that we ignore is that 
shippers select a specific carrier, and might not accept other carriers executing the order transport.

\begin{tabular}{ll}
\hline One-time decisions carrier & Description \\
\hline Form carrier coalition & $\begin{array}{l}\text { Bundle all orders to be transported. At every decision } \\
\text { moment } t \in \mathcal{T} \text {, a randomly selected carrier } c \in \mathcal{C} \\
\text { executes the transport for the bundled orders. }\end{array}$ \\
\hline
\end{tabular}

5.3.3.3. One-time decisions receiver. We discuss the one-time decision that can be made by receivers. As we will discuss in Section 5.4, receivers may place orders either ad hoc or according to a planned ordering system. If the latter is the case, we assume that the receiver has a pre-specified order frequency, e.g., it places orders every Tuesday and Friday of the week. If receivers have planned ordering systems, they are able to synchronize their delivery windows with other receivers. When the receivers in the target group synchronize their windows, it becomes easier for carriers to attain higher fill rates. As we do not address gainsharing mechanisms, the receiver will not directly benefit from the associated efficiency gains. However, it remains useful to verify whether synchronization of delivery windows indeed yields a positive effect on the system; this might lead to providing some financial incentive for synchronization.

\begin{tabular}{ll}
\hline One-time decisions receiver & Description \\
\hline Synchronize delivery windows & $\begin{array}{l}\text { All receivers with planned ordering systems synchro- } \\
\text { nize their delivery windows; receivers with the same } \\
\text { order frequency receive their deliveries at the } \\
\text { same weekdays. }\end{array}$ \\
\hline
\end{tabular}

5.3.3.4. One-time decisions UCC. For the UCC, we model three one-time decisions. The first decision is whether to open a UCC. If no UCC is opened, it is eminently not possible for carriers or receivers to select the UCC to perform the last-mile deliveries. The second decision is that the UCC may dictate a mandatory pre-announcement of orders. In that case, carriers that decide to use the UCC must communicate the orders that they transport to the UCC already one time period in advance. This way, the UCC always has insight into arrivals one time period in advance. The third decision involves assigning receivers to a number of clusters. As mentioned in the assessment of the operational decisions, the UCC faces a delivery dispatching problem as we discussed in Chapter 3. In that chapter, we proposed an ADP algorithm that learns a dispatching policy based on the stochastic arrival process. As we test a large number of scenarios in this chapter - each of them possibly resulting in a unique arrival 
process at the UCC - we would have to learn many policies, which is computationally challenging. To reduce the computational effort, we instead opt for a lookahead policy based on sampling, as described in Section 3.5.2. However, in that section we also noted that this approach is not scalable to larger numbers of receivers. We therefore introduce an additional simplification, in which the UCC decides whether or not to visit certain clusters of receivers. For this purpose, the UCC clusters the receiver locations (all locations in $\mathcal{V}^{R}$ ) into $k$ different zones; we use a basic $k$-means clustering algorithm for this, namely Lloyd's algorithm (Kanungo et al., 2002). This algorithm allocates $k$ centroids (which may be seen as virtual vertices) in the area and assigns each receiver location to the closest centroid. Subsequently, the algorithm recursively performs the following two steps: (i) move the positions of the centroids to minimize the total distance to its set of assigned receiver locations and (ii) if possible, re-assign receiver locations to the closest (updated) centroid position. The algorithm terminates when it is no longer possible to re-assign receivers and yields a local optimum. For a detailed description of Lloyd's algorithm, we refer to Kanungo et al. (2002).

\begin{tabular}{ll}
\hline One-time decisions UCC & Description \\
\hline Open UCC & $\begin{array}{l}\text { UCC may be (permanently) opened or closed; out- } \\
\text { sourcing is only possible if the UCC has been opened. }\end{array}$ \\
Mandatory pre-announcement & $\begin{array}{l}\text { Carriers must notify the UCC one decision moment in } \\
\text { advance, allowing the UCC to better match current and } \\
\text { future dispatching decisions. }\end{array}$ \\
& $\begin{array}{l}\text { All receivers are assigned to clusters based on their } \\
\text { proximity to each other. At each decision } \\
\text { mossign receivers to clusters } \\
\text { moment } t \in \mathcal{T} \text {, the UCC decides whether to } \\
\text { visit the receivers in a given cluster. }\end{array}$ \\
\hline
\end{tabular}

5.3.3.5. One-time decisions administrator. The final class of one-time decisions relates to the administrator. First, the administrator is able to set subsidy levels for using the UCC. These subsidy levels are expressed as fixed percentages of the price charged by the UCC and are equal for all agents of a specific type. Subsidies may be awarded to the UCC, the carriers, and the receivers. For receivers and carriers, subsidizing makes outsourcing to the UCC a more attractive alternative compared to direct transport, whereas for the UCC it may help to overcome financial deficits.

Second, the administrator determines its pricing policies. These policies are intended to discourage the use of heavy trucks, therefore they are not applicable to the small trucks that are used by the UCC. We distinguish between three pricing policies, (i) road pricing (a fee charged per kilometer driven within the 
city), (ii) parking costs (a fixed fee per delivery stop), and (iii) a fixed zone-access fee that must be paid when a truck enters the city.

Third, the administrator may impose restrictions on the vehicles that enter the city. Similar to the pricing policies, these restrictions have the purpose to discourage the use of heavy trucks in the city center. As such, the restrictions are not applicable to the small trucks that are operated by the UCC. We test four restrictions that may be implemented by the administrator: (i) an access time window during which heavy trucks may operate within the city, (ii) offsetting the access time window before the opening times of shops, such that receivers must be present early to receive their goods, (iii) setting a minimum load factor (e.g., $60 \%$ of the vehicle capacity should be used at the moment of entering the city), and (iv) a strict ban of heavy trucks, such that all last-mile deliveries must be performed by the UCC (as the carriers in our simulation model do not operate small trucks).

\begin{tabular}{ll}
\hline One-time decisions administrator & Description \\
\hline Set subsidy levels & $\begin{array}{l}\text { Subsidy levels for carriers and receivers } \\
\text { (as a \% of UCC price) for using the UCC and for the }\end{array}$ \\
& UCC (as a \% of its income). At most one subsidy \\
& measure may be in effect in any scheme. \\
& Price levels for heavy trucks, based either on \\
access, distance, or \# stops. At most one pricing & policy may be in effect in any scheme. \\
Access restrictions on heavy trucks: there are two \\
forms of access time windows, a minimum load \\
factor, and a strict ban on heavy trucks. Multiple \\
access restrictions may be in effect simultaneously.
\end{tabular}

5.3.3.6. Operational decisions shipper. In Section 5.3.3.1, we have discussed the dispatching decision faced by the shippers. On the one-time decision level, the shipper sets the threshold value $\zeta_{s}$; if the accumulated volume exceeds this threshold all orders are dispatched. Alternatively, the shipper ships the accumulated orders when the set of orders contains at least one urgent order. On the operational level, the dispatching decisions of the shipper are made based on this policy.

Let $l_{t, s}^{r e a d y}=l_{t, 0, \cdot, v_{s}^{s h p},,,,,,,, \cdot}+\sum_{t^{e} \in \mathcal{T}^{e}} l_{t, t^{e},, v_{s}^{s h p}, \cdot, \cdot,,,, 1}$ be the total volume that may be dispatched by the shipper at time $t$ and let $l_{t, s}^{u r g e n t}=l_{t, 0,1, v_{s}^{s h p}, \cdot,,,, \cdot,}+$ $\sum_{t^{e}=0}^{1} l_{t, t^{e}, 2, v_{s}^{s h p}, \cdot, \cdot, s, \cdot, 1}$ be the volume that must be dispatched at $t$ to meet the 
imposed delivery windows. Recall that transport via the UCC requires an additional time interval, hence when the receiver sets the UCC as its delivery address, the order is classified as urgent when $t^{l}=2$. We define two sets of orders: let $\mathcal{J}_{t, s}^{\text {urgent }}$ be the set of urgent orders at time $t$ and let $\mathcal{J}_{t, s}^{\text {nonurgent }}$ be the set of non-urgent orders that are ready for dispatch. The variable $l_{t, s}^{\text {shipped }} \in \mathbb{R}^{+}$denotes the volume that is shipped at time $t$ by shipper $s$. In Algorithm 5.1, we show the dispatching policy $\pi_{s}^{s h p}$ of a shipper $s \in \mathcal{S}$ in pseudo-code.

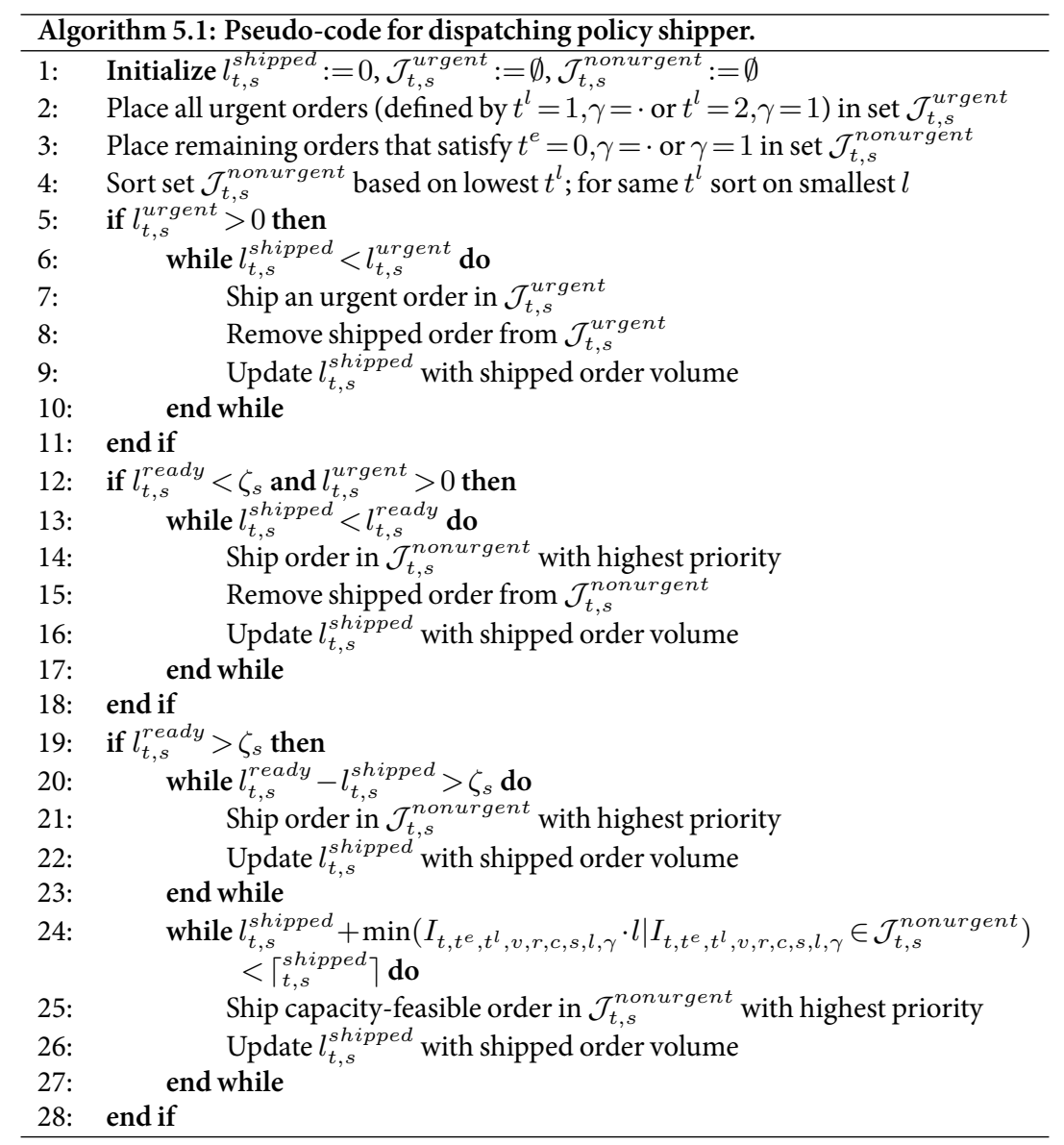




\begin{tabular}{ll}
\hline Operational decisions shipper & Description \\
\hline Dispatching decision & $\begin{array}{l}\text { Dispatch batch of orders when the accumulated volume } \\
\text { exceeds the volume threshold } \zeta_{s} \text { or when } \\
\text { urgent orders must be shipped. }\end{array}$ \\
\hline
\end{tabular}

5.3.3.7. Operational decisions carrier. For the carriers, we model two operational decisions: (i) determining a route to deliver its orders and (ii) outsourcing the last-mile delivery to the UCC. During the simulation, each carrier faces routing decisions. A truck dispatched by a carrier commences routes at its home depot, picks up orders at the shipper locations, drives the line-haul distance, and finally makes a delivery tour in the urban area. The return trip is beyond the scope of the model, as in practice a truck may visit multiple cities before returning to its home depot. To solve the routing problems, we first use the Clarke-Wright savings algorithm to construct routes and subsequently apply a 2-opt improvement heuristic. Although this solution method is relatively simple, it allows us to solve the routing problems embedded in the model relatively quickly; furthermore, heuristic approaches usually yield solutions within 2-3\% of the optimum (Golden et al., 1980). Quak and de Koster (2009) state that similar approaches are used in practice.

Each carrier may decide to outsource its last-mile delivery to the UCC. We explain the procedure that determines whether a carrier will outsource to the UCC at a given time $t$. Let $\tilde{\gamma}_{t, c}^{c a r} \in\{0,1\}$ be a help variable that describes the use of the UCC. We use $\Delta_{t, c} \mid \tilde{\gamma}_{t, c}^{c a r}=0$ to describe the route performed by carrier $c$ at time $t$, assuming that it would not outsource its last-mile delivery to the UCC (as indicated by the help variable $\tilde{\gamma}_{t, c}^{c a r}$ ). Similarly, $\Delta_{t, c} \mid \tilde{\gamma}_{t, c}^{c a r}=1$ denotes the route of carrier $c$ at time $t$ if it would outsource its last-mile distribution to the UCC; in this case the last-mile component consists of visiting the UCC to unload its non-urgent orders and directly delivering any urgent orders it may carry to the receivers. In the cost function that we defined in Section 5.3.2, we may substitute $\Delta_{t, c}$ with these alternative route notations.

For the decision whether to outsource, the carrier aims to minimize the sum of costs incurred on the last mile. First, if the carrier does not outsource to the UCC, it must visit all receivers for which it transports a load and incurs corresponding routing costs for the last-mile component $C^{c a r, t r}\left(\Delta_{t, c}^{c a r, l m} \mid \tilde{\gamma}_{t, c}^{c a r}=0\right)$; recall that these costs also include policy-related costs such as a zone-access fee. If the carrier outsources to the UCC, it must still deliver all orders with $t^{l}=1$ directly to the receivers; the UCC is not able to deliver these orders in time; all orders with $t^{l}>1$ are outsourced to the UCC. We denote the routing costs incurred 
by the carrier in this case by $C^{c a r, t r}\left(\Delta_{t, c}^{c a r, l m} \mid \tilde{\gamma}_{t, c}^{c a r}=1\right)$. Second, the carrier must pay the volume-based price of the UCC, $P^{u c c, t r}=\beta^{u c c} \cdot l_{t, t^{e}, \cdot, v_{c}^{c a r}, c, \cdot, \cdot, \cdot, \text { (with }}$ $\beta^{\text {ucc }} \in \mathbb{R}^{+}$), when it outsources its last-mile distribution. We note that the UCC does not charge outsourcing costs for orders for which the receiver has selected the UCC as delivery address; the sub index $\gamma=\cdot$ implies that the receiver has not selected the UCC as its delivery address for its orders. If $\gamma=1$ at this point in the transition, this implies that the receiver has selected the UCC and pays for the last-mile delivery of its orders. Finally, the carrier might receive a subsidy $P_{c}^{c a r, s b}\left(l_{\left.t, t^{e},,, v_{c}^{c a r}, c,,, \cdot, \cdot,\right)}\right)$ when outsourcing, again only for the orders that are not already supposed to be delivered at the UCC (i.e., set by the receiver). The outsourcing decision for carrier $c$ at time $t$ is formulated as follows:

$$
\begin{aligned}
& \gamma_{t, c}^{c a r}=\underset{\tilde{\gamma}_{t, c}^{c a r} \in\{0,1\}}{\operatorname{argmin}}\left(1-\tilde{\gamma}_{t, c}^{c a r}\right) \cdot C^{c a r, t r}\left(\Delta_{t, c}^{c a r, l m} \mid \tilde{\gamma}_{t, c}^{c a r}=0\right)+ \\
& \tilde{\gamma}_{t, c}^{c a r} \cdot C^{c a r, t r}\left(\Delta_{t, c}^{c a r}, l m \mid \tilde{\gamma}_{t, c}^{c a r}=1\right)+\tilde{\gamma}_{t, c}^{c a r} \cdot\left(\beta^{u c c} \cdot l_{t, t^{e}, \cdot, v_{c}^{c a r}, c, \cdot, \cdot, \cdot, \cdot}\right)- \\
& \tilde{\gamma}_{t, c}^{c a r} \cdot P_{c}^{c a r, s b}\left(l_{\left.t, t^{e}, \cdot, v_{c}^{c a r}, c, \cdot, \cdot, \cdot, \cdot\right)}\right) \text {. }
\end{aligned}
$$

\begin{tabular}{ll}
\hline Operational decisions carrier & Description \\
\hline Routing decision & Generate set of routes $\Delta_{t, c}^{c a r}$ with \\
& $\begin{array}{l}\text { Clarke-Wright savings algorithm, improve with } \\
\text { 2-opt improvement heuristic. } \\
\text { Decide whether to outsource last-mile distribution } \\
\text { of orders transported at decision moment } t \in \mathcal{T} \\
\text { to the UCC, selecting the option with the lowest costs. }\end{array}$ \\
\hline
\end{tabular}

5.3.3.8. Operational decisions receiver. For receivers, we model one operational decision, namely whether to select the UCC as its delivery address for the set of orders placed at $t$. Let $\tilde{\gamma}_{t, r}^{r e c} \in\{0,1\}$ be a variable that is used to compute the expected costs for receiver $r$ for both outsourcing and not outsourcing the last-mile distribution to the UCC, with $\tilde{\gamma}_{t, r}^{r e c}=1$ corresponding to the decision in which the receiver selects the UCC as its delivery address at decision moment $t$. We use $Q_{t, r}$ for the number of trucks that visits the premises of receiver $r$ at time $t$; the receiver incurs a fixed cost $\beta_{r}^{r e c, r c} \in \mathbb{R}^{+}$per visiting truck. Thus, we can specify the cost function that we introduced in Section 5.3.2 as follows: $C_{r}^{r e c, r c}\left(\Delta_{t}^{u c c} \cup \bigcup_{c \in \mathcal{C}} \Delta_{t, c}^{c a r}\right)=\beta_{r}^{r e c, r c} \cdot Q_{t, r}$. To decide whether to outsource to the UCC, the receiver substitutes $Q_{t, r}$ with both the expected number of visiting trucks when outsourcing and when not outsourcing. We start by describing 
how the receivers compute the expected number of visiting trucks under direct transport. Let $\mathbb{E}\left(Q_{t, r} \mid \tilde{\gamma}_{t, r}^{r e c}=0\right)$ be the expected number of vehicles visiting the receiver for the orders placed at $t$ when neither the receiver nor any of the carriers utilizes the UCC, i.e., all orders are transported directly to the receivers by the carriers. Orders placed at a single decision moment may have distinct earliest delivery dates, which implies multiple visits. When estimating the costs, the receiver assumes that neither the shipper nor the UCC bundles deliveries with distinct earliest delivery times; thus, the actual receiving costs might be lower. As we have explained, each shipper uses a fixed carrier for all its transport. Therefore, the receiver knows which carriers will visit its premises and particularly whether distinct shippers use the same carrier. With this information, the receiver estimates the number of trucks that visit its premises for the orders placed at $t$. We compute the expected number of trucks visiting the receiver as follows:

$$
\mathbb{E}\left(Q_{t, r} \mid \tilde{\gamma}_{t, r}^{r e c}=0\right)=\sum_{s \in \mathcal{S}} \sum_{c \in \mathcal{C}} \sum_{t^{e} \in \mathcal{T}^{e}}\left\lceil\frac{l_{t, t^{e}, \cdot, v_{r}^{r e c}, r, s, c, \cdot, \cdot}}{\psi_{c}^{c a r}}\right\rceil .
$$

Similarly, let $\mathbb{E}\left(Q_{t, r} \mid \tilde{\gamma}_{t, r}^{r e c}=1\right)$ be the expected number of trucks visiting the premises of the receiver when outsourcing to the UCC. The expected number of trucks that would visit the receiver's premises if the receiver selects the UCC as its delivery address is given by the following definition:

$$
\mathbb{E}\left(Q_{t, r} \mid \tilde{\gamma}_{t, r}^{r e c}=1\right)=\sum_{t^{e} \in \mathcal{T}^{e}}\left\lceil\frac{l_{t, t^{e}, \cdot, v_{r}^{r e c}, r, \cdot, \cdot, \cdot,}}{\psi^{u c c}}\right\rceil .
$$

Let $\beta^{u c c}$ be the price charged to the receiver by the UCC for outsourcing a full truckload. We have now introduced all notation required to determine $\gamma_{t, r}^{r e c}$. In the cost function, we substitute the real number of trucks with both the expected number of visiting trucks for direct transport and the expected number of trucks when outsourcing. The receiver selects the value for $\gamma_{t, r}^{r e c}$ that minimizes the expected costs:

$$
\begin{array}{r}
\gamma_{t, r}^{r e c}=\underset{\tilde{\gamma}_{t, r}^{r e c} \in\{0,1\}}{\operatorname{argmin}} \tilde{\gamma}_{t, r}^{r e c} \cdot\left(\mathbb{E}\left(Q_{t, r} \mid \tilde{\gamma}_{t, r}^{r e c}=1\right) \cdot \beta^{r e c, r c}+l_{t, t^{e},, v_{r}^{r e c}, r, \cdot, \cdot, \cdot, 1} \cdot \beta^{u c c}\right) \\
+\left(1-\tilde{\gamma}_{t, r}^{r e c}\right) \cdot \mathbb{E}\left(Q_{t, r} \mid \tilde{\gamma}_{t, r}^{r e c}=0\right) \cdot \beta^{r e c, r c} .
\end{array}
$$




\begin{tabular}{ll}
\hline Operational decisions receiver & Description \\
\hline Outsourcing decision & $\begin{array}{l}\text { Decide whether to select the UCC as the delivery } \\
\text { address for the orders placed at decision moment } t \in \mathcal{T}, \\
\text { selecting the option with the lowest expected costs. The } \\
\text { cost difference is in the number of visiting trucks and } \\
\text { - when access time windows are set before the opening } \\
\text { time of the receiver - additional personnel costs. }\end{array}$ \\
\hline
\end{tabular}

5.3.3.9. Operational decisions UCC. Similar to the shippers, the UCC must solve a dispatching problem as well, yet this problem is more complex. For the shipper, costs are always computed based on the same line-haul distance, therefore only the total accumulated volume is relevant for the dispatching decisions. For the UCC however, the transport costs within the city depend on how efficiently it executes its delivery routes. Thus, the UCC has an incentive to visit receivers that are located close to one another in the same route. Rather than deciding when to dispatch the complete set of accumulated orders, the UCC therefore decides on which subset of orders to dispatch at a given decision moment. In this decision, the UCC also takes into account possible future order arrivals that yield better consolidation opportunities. In Section 3.4.3, we already indicated that full enumeration of the dispatching problem per order type is computationally intractable for large problems, as it requires computing all possible permutations. The ILP that we proposed in Chapter 3 is able to solve large dispatching problems within several seconds, yet even this speed is insufficient given the large number of experiments that we are going to perform in this chapter. Furthermore, we pointed out that integration with sampling procedures is computationally challenging.

To reduce the computational effort required to solve the dispatching problem for the UCC, we reduce the size of the action space. Aggregation to the level of individual receivers seems reasonable; if the UCC already visits a receiver location, it makes sense that it delivers all orders available for this receiver. However, also in this case the need to compute all permutations quickly becomes prohibitive for larger numbers of receiver locations. For this reason, we divide the receiver locations in a number of clusters that is sufficiently small to repeatedly compute its corresponding permutations during the simulation. The corresponding one-time decision for allocating receivers to clusters has been described in Section 5.3.3.4. For each cluster $\mathcal{V}_{i}^{c l u}$, we decide whether to visit it at time $t$. Let $z_{i} \in\{0,1\}, \forall i \in\{0, \ldots, k\}$ (with $k$ being the maximum number of clusters) be a variable indicating whether the receivers in $\mathcal{V}_{i}^{c l u}$ are visited at time $t$. A value $z_{i}=1$ indicates that cluster $\mathcal{V}_{i}^{c l u}$ is visited, if $z_{i}=0$ the cluster is not visited. If a 
cluster $\mathcal{V}_{i}^{c l u}$ contains a receiver that has an urgent demand for an order, i.e, $t^{l}=1$, then the UCC delivers all orders for the receivers located in this cluster. We may compute the costs for any route that visits a given set of clusters - indicated by the vector of binary variables $\left[z_{1}, \ldots, z_{k}\right]$ - by substituting $\Delta_{t}^{u c c}$ by $\Delta_{t}^{u c c} \mid\left[z_{1}, \ldots, z_{k}\right]$ in the cost function $C^{u c c, t r}\left(\Delta_{t}^{u c c}\right)$ that we defined in Section 5.3.2. By varying the composition of $\left[z_{1}, \ldots, z_{k}\right]$, we can compute the direct routing costs corresponding to multiple dispatching decisions.

To take into account possible future consolidation opportunities, we use a one-step lookahead policy, as we found in Chapter 3 that considerable benefits are obtained when taking into account downstream costs for the delivery dispatching problem. We distinguish between two forms of lookahead; lookahead for pre-announced orders and lookahead for orders that are not pre-announced. If orders are not pre-announced, the UCC generates a sample of order arrivals denoted by $\hat{\omega}_{t+1}$ - based on its knowledge regarding the stochastic arrival process. Given the current order position $I_{t}$ and the sample arrivals $\hat{\omega}_{t+1}$, the UCC can compute the expected costs at $t+1$ for each decision $\left[z_{1}, \ldots, z_{k}\right]$ made at $t$; note that the decision $\left[z_{1}, \ldots, z_{k}\right]$ influences the remaining UCC inventory at $t+1$. The set of UCC routes at $t+1$ is denoted by $\hat{\Delta}_{t+1}^{u c c} \mid I_{t}, \hat{\omega}_{t+1},\left[z_{1}, \ldots, z_{k}\right]$. The corresponding costs are described by again substituting the route notation in the cost function, such that we obtain $C^{u c c, t r}\left(\hat{\Delta}_{t+1}^{u c c} \mid I_{t}, \hat{\omega}_{t+1},\left[z_{1}, \ldots, z_{k}\right]\right)$. At each decision moment $t$, the UCC aims to minimize the sum of the costs incurred at $t$ and the expected costs incurred at $t+1$ according to the following formula:

$$
\begin{array}{r}
\min _{z_{1} \in\{0,1\}, \ldots, z_{k} \in\{0,1\}} C^{u c c, t r}\left(\hat{\Delta}_{t}^{u c c} \mid\left[z_{1}, \ldots, z_{k}\right]\right)+ \\
C^{u c c, t r}\left(\hat{\Delta}_{t+1}^{u c c} \mid I_{t}, \hat{\omega}_{t+1},\left[z_{1}, \ldots, z_{k}\right]\right),
\end{array}
$$

s.t

$$
\begin{array}{lll}
z_{i}=0 & \text { if } & \mathcal{V}_{i}^{\text {clu }}=\emptyset \\
z_{i}=1 & \text { if } & \sum_{r \in \mathcal{R} \mid v_{r}^{r e c} \in \mathcal{V}_{i}^{c l u}}\left(I_{t, 0,1, v^{u c c}, r, \cdot, \cdot, 1}\right)>0 .
\end{array}
$$

Constraint (5.3.2) ensures that the UCC does not visit $\mathcal{V}_{i}^{c l u}$ if there are no orders available for this cluster, whereas Constraint (5.3.3) states that cluster $\mathcal{V}_{i}^{\text {clu }}$ must be visited if urgent orders are available for this cluster.

Equation (5.3.1) describes the minimization problem for the UCC in case orders are not pre-announced. For the case in which orders are pre-announced 
before arriving at the UCC, we use a similar procedure to compute the costs at $t+1$. However, instead of using samples, we then use the actual orders that are announced for arrival at $t+1$ to compute $\hat{\Delta}_{t+1}^{u c c}$; we do not introduce additional notation to describe this case.

The final operational decision of the UCC is the routing decision. For each dispatching decision, the UCC must solve the associated routing problem. Unlike the carrier, the UCC only makes delivery tours within the urban area; it does not operate in the pickup area or on the line-haul segment. As for the carriers, we use a combination of the Clarke-Wright savings algorithm and a 2-opt improvement heuristic to solve the routing problem of the UCC.

\begin{tabular}{ll}
\hline Operational decisions UCC & Description \\
\hline Dispatching decision & Decide which subset of orders to dispatch at decision \\
& moment $t \in \mathcal{T}$, selecting the option with the lowest \\
& expected costs. The cost difference is in the number of \\
& visiting trucks and possibly - if access time windows are \\
& set before opening time of the receiver - additional \\
& personnel costs. \\
& Generate set of routes $\Delta_{t}^{u c c}$ for the set of \\
& dispatched orders with the Clarke-Wright savings \\
Routing decision & algorithm, improve with 2-opt improvement heuristic. \\
\hline
\end{tabular}

\subsection{Experimental setup}

This section is divided into two subsections. In Section 5.4.1, we present the data that we collected. Some of the data will be directly used as a metric in the model, whereas others are implicitly imbedded in, e.g., the cost settings of agents. Section 5.4.2 provides the experimental design.

5.4.1. Data collection. The objective of the data collection is to accurately model urban supply chains in Western European cities, such that we can construct representative instances that yield generic insights. This requires a substantial amount of data on both agent properties and the structure of geographical networks. Unfortunately, no single centralized data sources exist that contain all the information that we require. Instead, we use the data sources that we discussed in Section 5.2.2. Although the data sources are fragmented, by aggregating their data we may obtain representative parameter ranges. In Table 5.2, we provide an overview of the aggregated data; we discuss the presented data in more detail when we define the agent properties. For each parameter, we describe the mean (the average obtained by aggregating the reported mean values 
of all data sources), the range of the means (the highest and lowest mean values obtained from individual data sources), and if sufficient data is available, the full range of low and high values (the lowest value and the highest value obtained from individual data sources).

The outline of this section is as follows. In Section 5.4.1.1, we discuss the design of our test networks. Subsequently, we describe the properties of the carriers (Section 5.4.1.2), receivers (Section 5.4.1.3), shippers (Section 5.4.1.4), the UCC (Section 5.4.1.5), and the administrator (Section 5.4.1.6). 


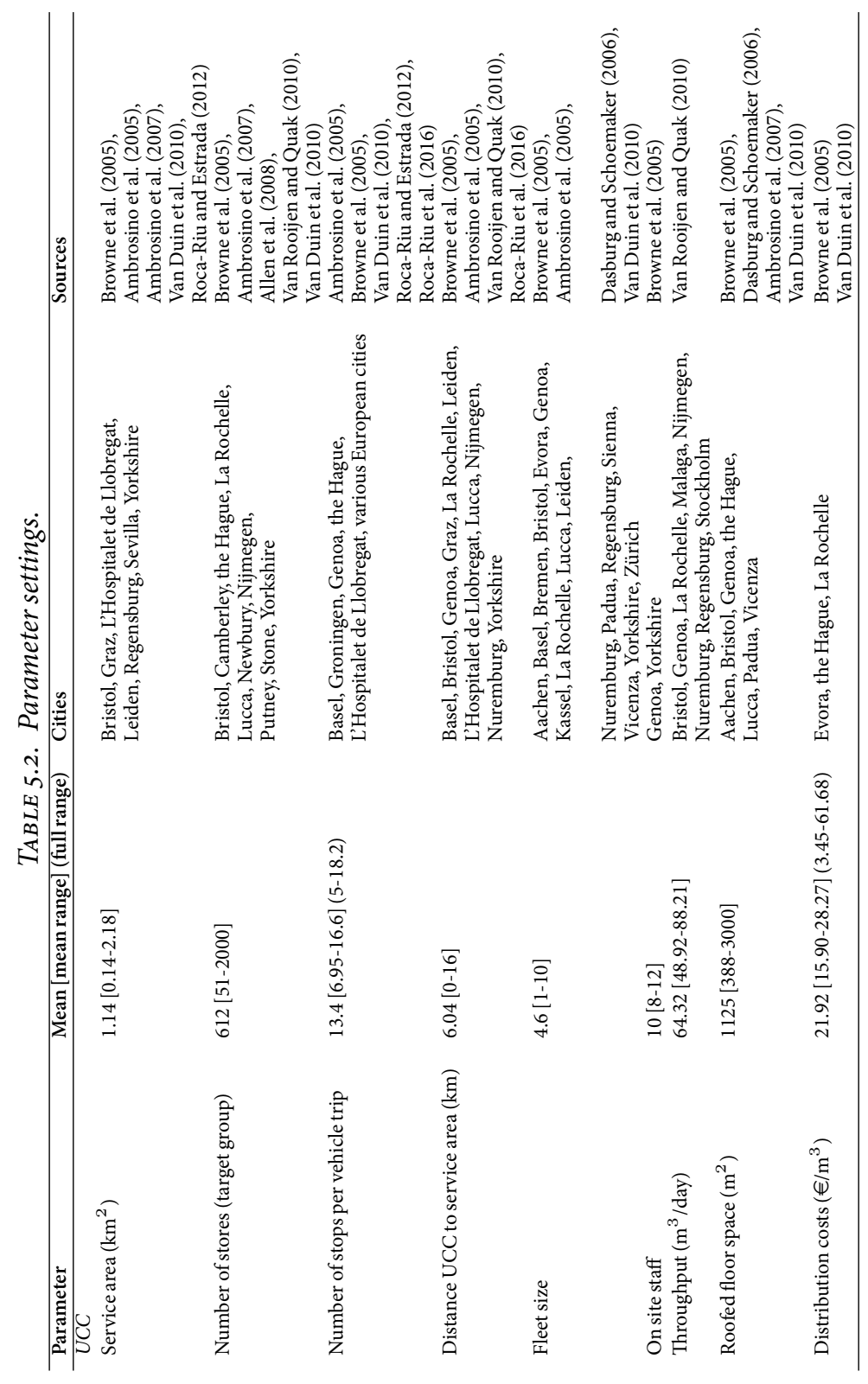




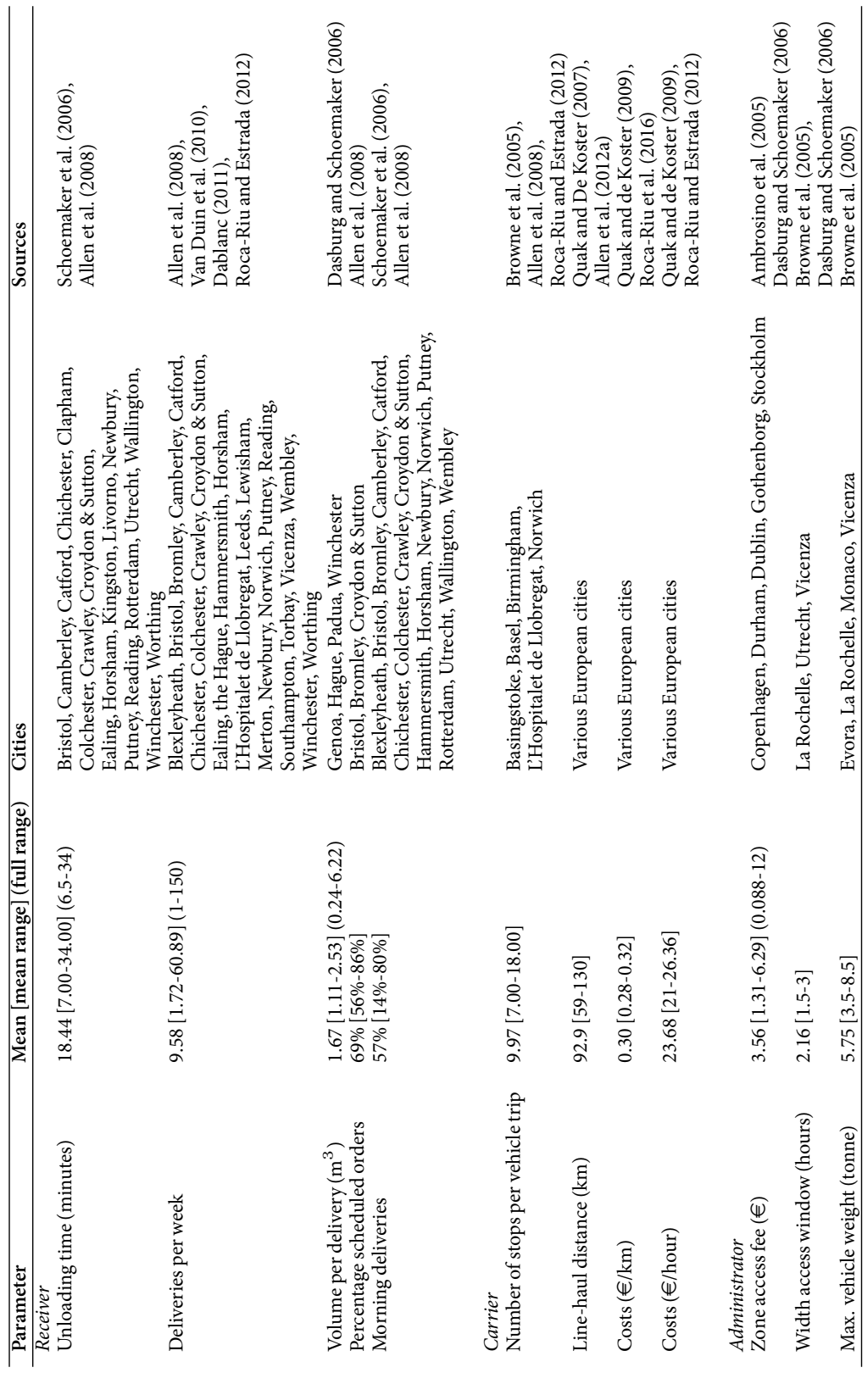


5.4.1.1. Network design. In this section, we present the design of the networks that we perform experiments on. In Section 5.2.1, we stated that existing agent-based simulation studies tend to represent urban distribution systems as grid networks with small numbers of locations and agents. To obtain more generic insights, we design networks that contain substantially more receivers. Furthermore, we test three distinct manners in which the receiver locations are distributed within the service area. Nevertheless, we do not claim to reflect the freight transport process of a complete city; our aim is to design the networks such that they sufficiently represent the diversity of urban supply chains. For the purpose of generality, in this chapter we perform experiments on abstract networks only. To complement the insights obtained in this chapter, Chapter 6 will present numerical experiments based on a real city.

In accordance with the collected data, we set the size of the urban area - in which the receivers are located - equal to $1.14 \mathrm{~km}^{2}$, assuming a square area. The UCC is located on a distance of $6.04 \mathrm{~km}$ away from the middle of the upper edge of the service area. This distance implies that the UCC is located relatively far from the urban area in which we seek to improve the environmental performance, such that we may assume that any restriction on freight transport does not hold for trucks that only visit the UCC. In line with the shop density stated by Roca-Riu et al. (2016), we assume the presence of 100 receiver locations within the urban area. Although this number is on the low end of the spectrum of the aggregated data - with 614 being the average - it is sufficiently large for the purpose of the experiments, as it allows modeling a diverse range of urban supply chains. Furthermore, higher densities of receivers would likely require aggregation of locations to solve the model within reasonable computational time. To represent a variety of service areas, we use three functions for the distribution of the receiver locations; Figure 5.2 shows the resulting three sets of receiver locations. The first is a uniform distribution of the receiver locations. The second is a truncated bivariate normal distribution of receiver locations and shows a high density of receivers in the center and an increasingly lower density on the outskirts. The third is a clustered distribution, which corresponds to a city where stores are clustered in multiple areas, e.g., various shopping centers. For this instance, we scale the 100-customer Solomon instance C101 - which defines customer locations grouped in clusters within a $90 \times 85$ area - to a $1.14 \mathrm{~km}^{2}$ area.

We proceed to describe the design of the pickup area. The attractiveness of collaboration mainly depends on the size of the pickup area. Thus, we test two different sizes for the pickup area; based on preliminary experiments, we set one 
pickup area to $20 \mathrm{~km} \times 20 \mathrm{~km}$ and one pickup area to $100 \mathrm{~km} \times 100 \mathrm{~km}$. Combining the two pickup areas with the three delivery areas gives a total of six geographically distinct instances. We set the line-haul distance - i.e., the distance between the pickup area and the urban area - to $92.9 \mathrm{~km}$, as defined in Table 5.2 .

To conclude the discussion of the network design, we reflect on its travel properties. We apply the Manhattan distance metric to compute the distance between each pair of vertices in the graph. In accordance with Roca-Riu et al. (2016), we set the travel speed on the line-haul segment to $50 \mathrm{~km} /$ hour and the travel speed in both the pickup area and in the urban area to $25 \mathrm{~km} /$ hour.

5.4.1.2. Carrier properties. In this subsection, we determine the number of carriers that we use in the simulation, discuss the prices that the carriers charge to shippers, and provide the vehicle properties for the carriers.

First, we must determine the number of carriers in the simulation. In Table 5.2, we indicate that the average number of delivery stops per tour is 9.97. Based on preliminary experiments, we choose the number of carriers such that the average number of stops per carrier per day is approximately 10. For individual carriers, the exact number of stops and the volumes that they transport may vary considerably based on the shippers that they work with. Carriers may dispatch more than one truck at a single decision moment. To achieve the average number of 10 stops per carrier, we need some 12 carriers. The locations of the 12 carrier depots - from which the carriers commence their routes - are generated in the pickup zone according to a uniform distribution.

We base the transport prices as charged by the carriers to the shippers on the price structure published by a Dutch transport company (De Transporters, 2016). For each shipment, this carrier charges a fixed fee of $€ 45$ and a distance fee of $€ 1.05$ per kilometer. The distance fee applies only to the line-haul distance, thus ignoring the length of the pickup and delivery tours. Furthermore, we note that the transport price does not depend on volume; this provides the shipper with an incentive to consolidate shipments as much as possible.

We continue to describe the vehicles used by the carrier. The transport market for carriers is known to be highly competitive and the offered transport services are subject to high substitutability. Therefore, we assume that all carriers use the same trucks and offer the same prices to shippers. For the carriers we use trucks with a capacity of $28 \mathrm{~m}^{3}$; the properties of these medium-sized trucks are shown in Table 5.3. As the weight of such trucks exceeds 3.5 tonnes, they typically fall in the vehicle category for which city access restrictions apply. In accordance with Quak and de Koster (2009), we set the driver's wage of the carriers to $€ 21$ per hour. The reason that we separate these costs from the vehicle costs is that 


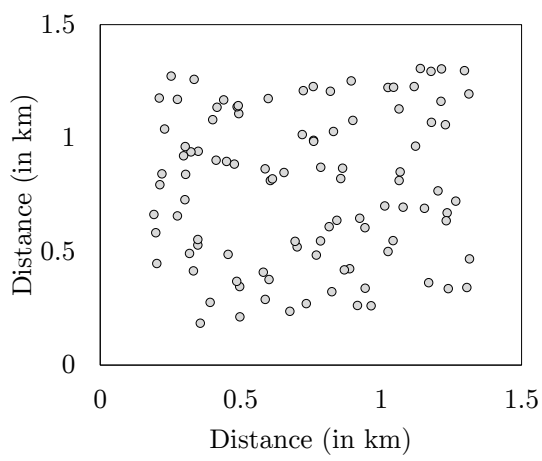

Uniform distribution

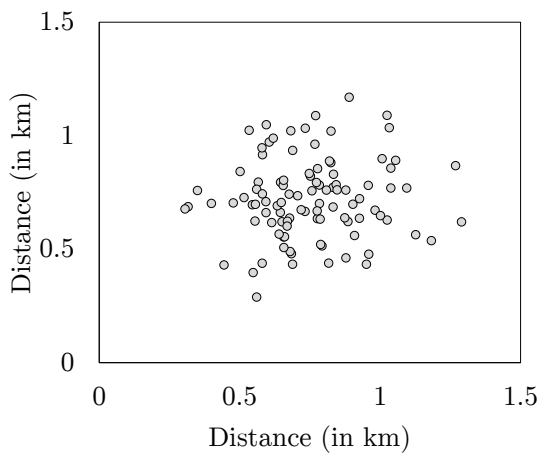

Bivariate normal distribution

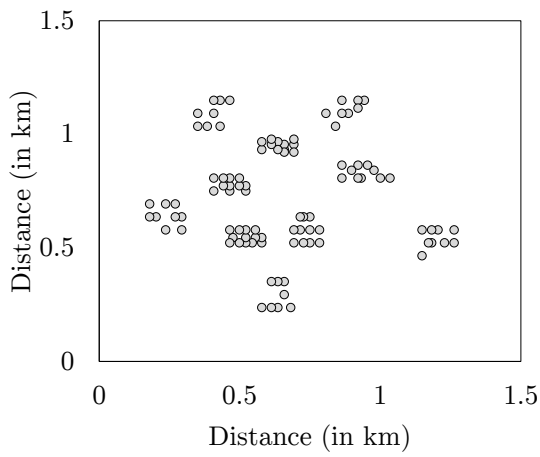

Clustered distribution

FIGURE 5.2. Sets of receiver locations generated according to 3 distributions. 
the driver also spends a considerable amount of time to load and unload goods; during this time no vehicle costs are incurred. The vehicle costs are expressed in euros per kilometer and are based on Quak and de Koster (2009) and Roca-Riu et al. (2016). Finally, we use the emission values for $\mathrm{CO}_{2}, \mathrm{SO}_{2}, \mathrm{NO}_{\mathrm{x}}$ and $\mathrm{PM}_{2.5}$ as reported in Boer et al. (2011).

TABLE 5.3. Vehicle properties for the UCC (light truck) and carriers (medium-sized truck).

\begin{tabular}{lcc}
\hline Vehicle type & Light truck & Medium-sized truck \\
\hline Load capacity $\left(\mathrm{m}^{3}\right)$ & 18 & 28 \\
Driver's wage $(€ /$ hour$)$ & 21 & 21 \\
Costs urban transport $(€ / \mathrm{km})$ & 0.72 & 0.86 \\
(excluding driver’s wage) & & \\
$\mathrm{CO}_{2}(\mathrm{~g} / \mathrm{km})$ & $455-553$ & $821-1,065$ \\
$\mathrm{SO}_{2}(\mathrm{mg} / \mathrm{km})$ & $3.5-4.2$ & $6.3-8.1$ \\
$\mathrm{NO}_{\mathrm{x}}(\mathrm{g} / \mathrm{km})$ & $1.5-1.8$ & $2.7-3.5$ \\
$\mathrm{PM}_{2.5}(\mathrm{mg} / \mathrm{km})$ & $35-37$ & $53-59$ \\
\hline
\end{tabular}

5.4.1.3. Receiver properties. We describe the receiver profiles that define the following properties: the stochastic order process of receivers, the unloading times at receiver premises, and the personnel costs of receivers.

In Table 5.2, we show several ranges with respect to the order properties of receivers. Although we might draw order properties directly from these ranges, this would ignore certain relationships between the properties. Specifically, an important distinction can be made between centralized and decentralized ordering systems (Cherrett et al., 2012). In centralized systems, the receiver places all its orders with a single shipper or hires an LSP that already consolidates its orders upstream. In a decentralized system, the receiver places orders separately with multiple shippers. From the described order systems, some relationships between certain order properties may be inferred. For example, a receiver that has a centralized ordering system is more likely to order higher volumes when placing a single order and to place orders less frequently.

To reflect the high diversity in ordering patterns that exists in practice, we define 10 distinct receiver profiles, with a profile consisting of four ordering properties. The properties of each receiver profile are shown in Table 5.4. We randomly assign profiles to receivers; all order profiles are equally likely to occur and are identical for each simulation run. The first profile property is the order frequency, which is the number of moments per week that the receiver places orders. At each order moment, the receiver may place one or more orders; the receiver may also decide not to place any orders. The second property is the 
number of orders placed per order moment. Receivers with a centralized ordering system always place orders with a single shipper, whereas receivers with decentralized supply chains may place orders with up to 12 distinct shippers at a single order moment. Each receiver has a fixed set of shippers from which it may order; the maximum number of distinct shippers with which a single receiver may place orders is given by the order frequency multiplied with the maximum number of orders. For example, a receiver that may order 3 times per week and may order up to 5 orders at each ordering moment may have $3 \cdot 5=15$ distinct shippers. Shippers are selected randomly for each receiver and are the same for each simulation run. The third property is the volume of orders. Based on the volume range listed in Table 5.2, we distinguish between receivers with low-volume demand (0.18-2.88 $\mathrm{m}^{3}$ per order) and high-volume demand (3.24$6.30 \mathrm{~m}^{3}$ per order); order volumes are uniformly drawn from these ranges for each generated order. We assume that $60 \%$ of the receivers with decentralized ordering systems have order volumes that fall within the small volume range, whereas $60 \%$ of the receivers with centralized ordering systems draw from the large volume range. The fourth property of a receiver profile is whether its ordering system is planned; a planned ordering system implies that the earliest delivery times of all orders are set in the future at the moment the order is placed (i.e., $t^{e}>0$ ), whereas an ad hoc ordering system indicates that all orders may be delivered directly (i.e., $t^{e}=0$ when the order is placed). To reflect that centralized ordering systems are typically more structured than decentralized ordering systems, we assume that $60 \%$ of the centralized ordering systems are planned, compared to $40 \%$ of the decentralized ordering systems.

TABLE 5.4. Receiver profiles.

\begin{tabular}{llll}
\hline $\begin{array}{l}\text { Order frequency } \\
\text { per week }\end{array}$ & $\begin{array}{l}\text { \# orders per } \\
\text { order moment }\end{array}$ & $\begin{array}{l}\text { Average volume } \\
\text { per order }\end{array}$ & $\begin{array}{l}\text { Planned ordering } \\
\text { system }\end{array}$ \\
\hline 1 & 1 & $0.18-2.88 \mathrm{~m}^{3}$ & Yes \\
1 & 1 & $3.24-6.30 \mathrm{~m}^{3}$ & Yes \\
$3-5$ & 1 & $0.18-2.88 \mathrm{~m}^{3}$ & No \\
$3-5$ & 1 & $3.24-6.30 \mathrm{~m}^{3}$ & No \\
$3-5$ & 1 & $3.24-6.30 \mathrm{~m}^{3}$ & Yes \\
1 & $2-12$ & $0.18-2.88 \mathrm{~m}^{3}$ & Yes \\
1 & $2-12$ & $3.24-6.30 \mathrm{~m}^{3}$ & Yes \\
1 & $2-12$ & $0.18-2.88 \mathrm{~m}^{3}$ & No \\
$3-5$ & $2-12$ & $3.24-6.30 \mathrm{~m}^{3}$ & No \\
$3-5$ & $2-12$ & $0.18-2.88 \mathrm{~m}^{3}$ & No \\
\hline
\end{tabular}


Next, we discuss the settings for the receiving times. Table 5.2 states that unloading times at the receivers lie in the range from 6.5 to 34 minutes. However, this is the time that is spent by the carrier; the time that the receiver itself spends on receiving the goods may vary greatly. Some receivers might only need to sign for the receiving the goods (requiring a minimal time spent on receiving goods), whereas others might be involved in the complete unloading process. Factors such as accessibility of the premises, the availability of handling equipment, and the need for quality checks may strongly impact the time that a receiver spends on receiving goods. Cherrett et al. (2012) state that the receiving time is typically independent of the volume received. Therefore, for each receiver, after generating the unloading time at its premises for the carrier, we randomly generate the time spent on receiving for a given receiver between 2 minutes and the unloading time, using a uniform distribution.

Finally, we determine the personnel costs for the receiver. We assume that one staff member is required for receiving goods. Following Van Duin et al. (2010), we set personnel costs for all receivers at $€ 15.3$ per hour. Thus, when a given receiver for example spends 12 minutes on receiving goods, the receiver incurs costs of $\frac{12}{60} \cdot 15.3=€ 3.06$ per truck that visits its premises.

5.4.1.4. Shipper properties. In this section, we describe two shipper properties, namely the number of shippers and the loading times at shipper locations. The shippers are included in the model mainly to represent the impact of dispatching decisions that are made upstream. However, for our analysis we are primarily interested in the effects of transport within the city. Although we seek to realistically reflect the decision-making process of shippers, we are concerned more with the mechanics that drive their decisions rather than accurately quantifying their performance. Thus, unlike for the other agent types, we do not base the representation of shippers on data obtained from literature.

First, we explain how we decide on the number of shippers. The number of shippers should be sufficiently small such that it is likely that multiple receivers order from the same shipper - allowing to test shipping policies - whereas the number of shippers must also be considerably larger than the number of carriers to create diversity in the transport operations. We select a number of 50 shippers; with this number, shippers on average serve 18 distinct receivers as customers and on average 4 shippers make use of each carrier. The shipper locations are generated in the pickup area according to a uniform distribution.

We assume that the loading times of shippers (i.e., the time that they spend when a carrier picks up goods) are generated in the same way as for the receivers and are subject to the same time range. Thus, we assume that the pickup time for 
the carrier at the shipper is between 6 and 34 minutes. The time expenditure of the shipper on the loading procedure ranges between 2 minutes and the number of minutes that the carrier spends on picking up the shipment. Both the pickup time for the carrier and the loading time for the shipper are generated according to uniform distributions. We also set the personnel costs per hour equal to 15.3 $€ /$ hour; this hourly wage is multiplied with the time that the shipper spends on the pickup process to obtain the handling costs of the shipper.

5.4.1.5. UCC properties. This section describes the vehicle properties, the handling costs, and the price structure of the UCC.

We start by describing the properties of the vehicles that are used by the UCC. Van Duin et al. (2010) argue that heavy trucks (i.e., trucks with a capacity of $28 \mathrm{~m}^{3}$ or higher), have a detrimental impact on the city and should therefore not be used by a UCC. In addition, such trucks also weigh over 3.5 ton and are therefore often subject to access restrictions. On the other end of the spectrum, UCCs might opt for small transport means such as delivery vans and cargo bikes. Although having a low environmental impact, the inability of such transport modes to transport larger loads - such as pallets and rolling cages - has been identified as a hampering factor in the success of UCCs. Another effect is that considerably more vehicles are required to transport the same volume as a single truck, which may nullify the better environmental performance of such transport means. In line with Van Duin et al. (2010), we assume that the UCC uses trucks with a capacity of $18 \mathrm{~m}^{3}$. The properties of these light trucks are shown in Table 5.3. Such trucks are often small enough to avoid being subject to access restrictions - giving them an advantage over carriers that use large trucks - while being sufficiently large to accommodate most potential users.

Table 5.2 establishes ranges for the UCC on the number of trucks, number of staff members, and the floor space. Although these figures are useful to estimate the costs of a specific UCC, we do not define a UCC of a pre-specified size for the numerical experiments. This is because in practice, the operator of the UCC will make an effort to align the size and resources with the demand. As the throughput of the UCC greatly depends on the specific scheme that we study, we do not place restrictions on the operational capabilities of the UCC.

We compute the handling costs per $\mathrm{m}^{3}$ (including cost such as the rent of the property, staff wages, and handling equipment, but excluding transport costs) of the UCC as follows. In Table 5.2, we estimate the distribution costs (comprising both handling and transport) of the UCC at $€ 21.92$ per $^{3}$. However, preliminary experiments have shown that, if we set the price charged to users at this level (implying a break-even level), the UCC is far too expensive for its users 
and will only be used when extreme measures are taken. To perform a meaningful analysis, we instead adopt the cost estimate of Van Duin et al. (2010), which is the most detailed cost breakdown that we encountered in the literature review. Van Duin et al. (2010) estimate that - for a high-throughput scenario - the costs per $\mathrm{m}^{3}$ are $€ 11$. This amount includes the transport costs, which we compute separately. To estimate the transport cost per $\mathrm{m}^{3}$ for the UCC, we performed a preliminary test with high utilization of the UCC and computed the corresponding transport costs with the routing heuristic. This test yielded average transport costs of $€ 3$ per $\mathrm{m}^{3}$. Subtracting the average transport costs of $€ 3$ yields handling costs of $€ 8$ per $\mathrm{m}^{3}$. When interpreting the results in this chapter, one should take into account that a realistic but favorable cost structure for the UCC is assumed; for UCCs in practice the handling costs may be higher.

Despite the relatively low cost settings, the UCC is typically still expensive when compared to the receiving costs of the receiver and the last-mile transport costs of the carrier, particularly when considering larger volumes. To approximately achieve a break-even level - assuming sufficiently high throughput to efficiently conduct the last mile transport - the price that the UCC charges to both carriers and receivers is set at $€ 11 \mathrm{per}^{3}$. Thus, the UCC charges $11 \cdot 18=$ $€ 198$ to outsource a full truckload. Consequently, with our simulation settings outsourcing is typically only financially appealing for small loads; such loads are indeed the target of the UCC. Recall that in practice, many UCCs indeed struggle to survive on the long term partially due to their high operational cost.

As we described in Section 5.3.2, we base the prices that the UCC charges to the receivers and carriers on volume only. The rationale behind this design choice is that - from an environmental perspective - we mainly would like to bundle small and inefficiently transported loads. However, other price functions might also have been adopted. For example, we might also use prices based on the number of stops, use a fixed fee, use negotiation to derive agent-specific prices, or combine multiple aspects in the price function.

5.4.1.6. Administrator properties. This section discusses the properties regarding the regulations, pricing policies and subsidizing measures that may be implemented by the local administrator.

For all measures that are implemented, the administrator must operate within the boundaries of the (supra)national legal framework. Therefore, the administrator is legally prohibited from discriminating among agents. Thus, for example, the administrator cannot simply dictate that all carriers should use the UCC. For this reason, the restrictions modeled in this chapter are vehicle-based. 
Extending our example, the administrator may impose a ban on vehicles weighing over 3.5 tonnes, such that carriers using large trucks must use the UCC. In the model this would result in a situation in which all carriers must outsource to the UCC, but in a real-life situation carriers that operate small trucks could still enter the city. In formulating the administrator restrictions and pricing policies, the legal basis is always a truck-related property. Also in defining subsidy measures, the administrator should not discriminate among agents. We express subsidies as a percentage of the price paid to the UCC, or if the UCC is the recipient of the subsidies, a percentage of its income. This allocation does not discriminate among agents, as each agent proportionally receives the same amount of subsidy for using the UCC. A note with respect to subsidizing the UCC is that such a measure might be considered as unfair competition, yet the UCC may also be organized as a public service rather than a purely commercial party.

We proceed to describe the rationale behind the pricing measures of the administrator. Each pricing measure aims to discourage the use of large trucks in the city and each subsidy measure aims to stimulate the use of the UCC. Essentially, pricing policies quantify environmental costs and re-allocate them to the carrier. Similarly, subsidies monetize the environmental benefits of the UCC and allocate them either to the UCC or to its users. Furthermore, combining multiple subsidy measures or pricing policies may result in agent behavior that is inconsistent with the intended effects of the measures. For these reasons, we impose that the administrator may implement at most one subsidy measure and at most one pricing policy per scheme. The rationale for this design choice is not strictly influenced by concerns with realism; in Section 5.4.2 we will explain how reducing the number of measures helps us to create a reliable experimental design. The administrative measures that we test are based on measures that have been implemented in practice or at least have been considered for implementation. However, some measures have severe drawbacks in practice, particularly with respect to validating whether the imposed regulations are actually respected. For example, the administrator may impose a minimum load factor on the trucks that enter the city, yet it is hard to efficiently check the load factors of trucks that enter the city. Another measure that we test is a complete ban of heavy trucks. Although this measure has been implemented in a few cities, in general such a measure is politically impractible. Thus, in interpreting the simulation results, one should not only consider the environmental impact, but also how strongly the measures affect the stakeholders when translated to practice. 
5.4.2. Design of the numerical experiments. In this section, we describe the design of the numerical experiments. We provide a stepwise explanation of the design, applying the following structured approach:

1. Create overview of measures to analyze.

2. Categorize measures as a means to support the construction of schemes.

3. Define a default scheme without active measures for the purpose of comparison.

4. Perform a sensitivity analysis to determine default and non-default values per measure to be used in a fractional factorial design.

a. Evaluate the impact of various parameter levels for each measure, with other measure levels kept to their default levels.

b. Select sensible levels for each measure.

5. Perform experiments according to the fractional factorial design.

a. Obtain insights into main effects of measures.

b. Obtain insights into two-way interaction effects between measures.

6. Perform a scenario analysis to evaluate and provide insights into effective schemes.

a. Compare performance of schemes to default scheme.

b. Identify schemes with good environmental performance and acceptable financial impact.

We first provide an overview of the measures (Step 1) and the corresponding categorization (Step 2). We study a total of 15 measures; an overview of these measures is shown in Table 5.5. We divide the measures in 4 categories: (i) restrictions, (ii) pricing policies, (iii) subsidies, and (iv) company-driven initiatives. We proceed with a definition of the default scheme (Step 3); this is the scheme in which measures are not effective or set equal to 0 , e.g., we have access time windows of $24 \mathrm{~h}$ and subsidy levels of $0 \%$. 

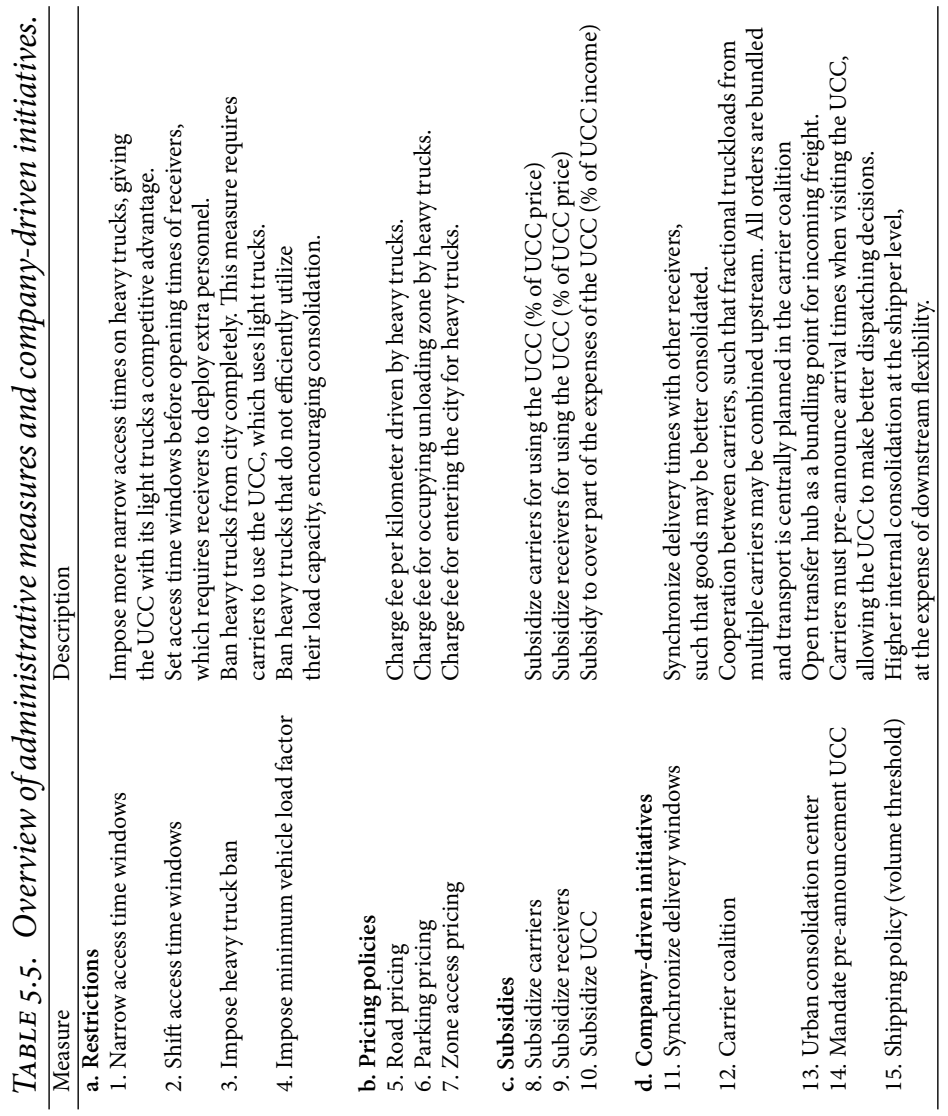
We proceed to explain the application of the sensitivity analysis (Step 4) to determine the values for all 15 measures. The fractional factorial design that we use requires the definition of a 'low' and a 'high' value for each measure. In our case, we let the 'low' values correspond to the default settings and the 'high' values to the non-default settings, i.e., the measure is active when at its non-default level. To determine the non-default levels, we run experiments separately for each measure. We test various levels for the measure of interest, while maintaining all other variables at their default levels (with the exception that the UCC needs to be open to perform a meaningful analysis for the measures). The purpose of this step is twofold: we (i) obtain insights into the sensitivity regarding the measure and (ii) determine reasonable settings for the non-default levels for the measures in the fractional factorial design. The sensitivity analysis is only performed for measures that require to set a numerical value; binary measures (i.e., the UCC is either open or closed) are excluded from the analysis.

After determining the default and non-default values for each measure, we perform a simulation study according to a $2^{k-p}$ fractional factorial design. We only explain the key concepts of this experimental design here and refer to Law (2007) and Montgomery (2008) for in-depth descriptions. First, we discuss the idea behind a $2^{k}$ factorial design (also called a full factorial design). We consider a set of $k$ measures; we assume that the effects of changing the level of a measure is - within reasonable bounds - approximately linear. As stated before, for each measure the design requires a 'low' value and a 'high' value (in our case the default and non-default values). The two values should be sufficiently distinct such that we might expect different responses for both levels. However, they should not be separated too far, such that they yield unrealistic configurations without practical applicability. Subsequently, we test all possible combinations of these levels. The observations yield insights into both the main effects of measures and the interaction effects between measures. We note that the effects are computed pairwise for all combinations of variables, thus, we compute effects over all experiments rather than comparing to the default scheme.

As stated before, in this chapter we test 15 distinct measures. A $2^{k}$ factorial design would thus yield $2^{15}$ different schemes, which we need to test on 6 distinct instances, yielding a computationally expensive total of $6 \cdot 2^{15}=196,608$ simulation runs. Instead, we opt to apply a $2^{k-p}$ factorial factorial design, in which we consider only a subset of all combinations. A fractional factorial design is an experimental design in which we carefully select a subset of simulation runs that allows us to expose the most important effects of the tested measures. Such a design relies on the notion that the state of a system is typically dominated by 
main effects and low-level interaction effects (i.e., the interactions between small numbers of measures), whereas high-level interaction effects (i.e., the interactions between high numbers of measures) are generally statistically insignificant; this notion is also known as the sparsity-of-effects principle. Standardized design tables exist to implement a fractional factorial design in a reliable way. The so-called resolution of a design (denoted in Roman numbers) describes its ability to distinguish main effects and low-order interactions from one another. A resolution of $V$ or higher ensures that two-factor interactions are not confounded with each other. For up to 11 measures, there are various useful fractional factorial design tables available; for more than 11 measures the designs become increasingly less reliable. By selecting at most one subsidy measure and one pricing measure per scheme, we bring the number of measures for the fractional factorial design back from 15 to 9; we have explained the practical motivation for this design choice in the last paragraph of Section 5.4.1.6. By selecting at most one subsidy measure and at most one pricing policy in a scheme (i.e., for both categories at most one active measure, or all measures are at their default levels), we obtain $4 \cdot 4=16$ combinations. Thus, we repeat the fractional factorial design 16 times to incorporate all combinations in our design. Omitting subsidizing and pricing from the design implies that for these categories we do not obtain interaction effects between measures of the same category, but we still obtain insights in their relations with the other measures.

Having brought back the selection of measures for the simulation design to 9 measures, we opt for a design with resolution VI. This design ensures that main effects are not confounded with two-factor interaction effects and that two-factor interaction effects are not confounded with each other or with threefactor interaction effects. For the design with 9 measures and resolution VI, we must perform 128 experiments for the 16 combinations of price- and subsidy measures and for 6 distinct instances. Thus, the total number of simulation runs that we perform is $16 \cdot 6 \cdot 128=12,288$. For two combinations of measures, we overrule measures to ensure feasibility of the last-mile distribution: if the UCC is not open (measure 13), then the heavy truck ban (measure 3) and the minimum load factor (measure 4 ) have no impact. Furthermore, the subsidy measures (8-11) are ineffective when the UCC is not opened, whereas shifting access windows (measure 2) has no impact when the access window is set at 24 hours (measure 1); these situations require no adjustments to the model.

By applying the $2^{k-p}$ design, we obtain insights into the main effects and the low-level interaction effects of the measures. We aim to reveal which measures are effective and in which combination they should be applied. To compute 
the effects for the subsidy- and price measures, we separately assess the relevant combinations of measures (out of the 16 different $2^{k-p}$ designs), i.e., we do not directly compare between distinct pricing measures and between distinct subsidy measures. Also for the 6 distinct instances, we provide a separate analysis. The effect of the remainder of the measures is computed over all results, i.e., by treating them as if a single $2^{k-p}$ experimental design. Based on the obtained insights, we use scenario analysis to further investigate the most promising schemes, i.e., schemes that yield substantial environmental improvements while simultaneously yielding good financial results for the agents involved. Ideally, no agent is worse off than under the default scheme, as this implies that real-life stakeholders would likely not object to the implementation of the scheme. To a certain extent, monetizing and re-allocating environmental cost may be justified in practice. However, it is crucial that if agents of a given type should change their behavior, they should be offered a positive financial incentive to do so.

\subsection{Numerical experiments}

In this section, we discuss the results of the numerical experiments. The outline of this section is as follows. In Section 5.5.1, we describe how we set up the experiments. In Section 5.5.2, we determine the default and non-default variable levels to be used in the $2^{k-p}$ design, testing various parameter settings for each measure. Section 5.5.3 discusses the main effects and the most important two-way interaction effects obtained with the $2^{k-p}$ design. In Section 5.5.4, we perform a sensitivity analysis to further investigate the most promising schemes. Section 5.5.5 reflects on the insights obtained with the numerical study.

5.5.1. Experimental setup. In this section we describe how we set up the parameters for the experiments. We briefly describe the batch means method that we use, as well as the selection procedure for the warmup period, the number of batches, and the batch length.

Each simulation run starts with an empty system, i.e., a system without any orders. As the model does not contain any time-varying processes, the system may be described by a stationary process after some warmup period. To efficiently perform the experiments we apply the batch means method, which means that we perform one simulation run for each scheme, with the run being divided into a number of batches of length $\tau^{\text {batch }}$. This method has the benefit that it only requires a single warmup period. The disadvantage is that the results of batches 
might be correlated; we therefore must set batch lengths sufficiently large such that correlation between batches is negligible.

Using Welch' graphical procedure (Law, 2007), we set the length of the warmup period equal to 20 . The corresponding graph is not shown here, yet we provide the underlying rationale. Given that orders may be pre-announced 2 time intervals before the earliest delivery time and given a maximum delivery window width of $4, t=6$ is the latest possible delivery date for any order placed at $t=0$, the system stabilizes relatively quickly. Adding some margin of safety, a warmup period of 20 time intervals is more than sufficient to reach the steady state.

We proceed to describe how we determine the number of batches and the length of each batch, under the condition that we want a relative error of at most $5 \%$ for a $95 \%$ confidence interval of the financial performances of each agent type. We start by determining the batch length. As orders remain within the system for a rather limited time, correlations between observations that are separated by multiple time intervals may be expected to be relatively weak; therefore it is implied that the batch lengths may be chosen fairly short. Our second criterion for determining the batch length is how much variance the results of the batches show.

To validate whether the batch observations can be treated as independent replications, we perform a lag-1 test, in which we calculate the correlation between each batch and its adjacent batch (Steiger and Wilson, 2001). For a batch length of $\tau^{\text {batch }}=20$ time intervals, taking the financial performance of each agent type as the batch results, we obtain a correlation of at -0.079 between the batches, implying that the batches are approximately independent. Lengths shorter than 20 intervals tend to yield stronger correlations and are therefore less reliable.

Given that the batch observations are approximately uncorrelated, we use the sequential batch replication procedure of Law and Carson (1979) to determine the number of batches. Using this procedure, we find that 4 batches suffice to yield a relative error of at most $5 \%$ for the $95 \%$ confidence interval of the financial performances. However, Law (2007) and Rossetti (2015) state that the ideal number of batches is between 10 and 30; batch numbers smaller than 10 may yield less reliable outcomes. Therefore, we set the number of batches in each simulation run equal to 10 .

5.5.2. Determining the levels of measures. In this section, we perform a sensitivity analysis to determine the default and non-default levels for the measures with non-binary values that we list in Table 5.6. During the analysis, all measures are set to their default levels, with the exception of the UCC - which 
is set to always open as other measures may depend on it - and the measure of interest, for which we test various levels. This analysis allows to find sensible levels for each measure, taking into account both the financial impact per agent type and the environmental impact. No sensitivity analysis is performed for measures of a binary nature. We discuss our main findings from the sensitivity analysis; note that these findings only apply to the measures in isolation; the analysis cannot detect possible interaction effects.

We assess the financial impacts per agent type and two proxies to indicate the environmental performance, namely (i) the changes with respect to distances covered by heavy trucks and (ii) the $\mathrm{PM}_{2.5}$ emissions. For all indicators, an impact higher than $0 \%$ (as shown on they-axis of the figures in this section) signifies a performance improvement. We briefly describe how we determine the levels for each variable based on the sensitivity analysis; the discussion of main effects of measures takes place in Section 5.5.3.

We first discuss the analysis of 3 restrictions: imposing access time windows, offsetting the access time window, and setting a minimum load factor. Figure 5.3 shows the results of the sensitivity analysis for these measures. First, for the access time window, we consider window widths varying between 0 hours (equivalent to a strict ban on heavy trucks) and 10 hours. Windows of 0 and 0.5 hour have a positive effect on the UCC, but result in large losses for the carriers (which in practice likely translates to carriers charging higher transport prices). A window of 0 has a positive environmental effect due to the mandatory use of the UCC. However, very narrow windows (but wider than 0, e.g., 0.5 hours) actually decrease environmental performance; carriers need to dispatch more trucks to deliver the same set of receivers while complying with the window. When the width of the window is set at 2 hours or more, it is ineffective in altering the behavior of the agents. For the experimental design, we set the non-default value for access time windows at 1 hour, which balances between environmental performance and utilization of the UCC. Second, for offsetting the access time window, we test values from 0 up to 2 hours. The effects of shifting the window are felt by the receivers, as they must dedicate additional personnel hours when waiting for a carrier that visits before opening hours. We see that even small offsets have positive effects for the UCC, yet at the expense of the receiver and only modest environmental benefits. We set the non-default value for the offset of the access time window to 1 hour. The third restriction that we evaluate is the minimum load factor. We see that increasing this factor has generally a positive impact on the UCC and the environmental performance, but a negative impact on the performance of carriers. We set the non-default level for the load factor 
at 0.7 ; this value appears to have a moderate impact on the carrier performance while achieving considerable environmental benefits.

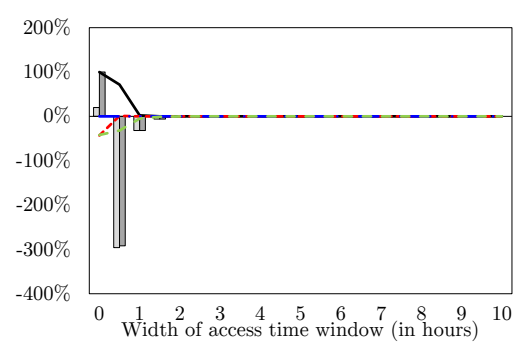

Access time windows

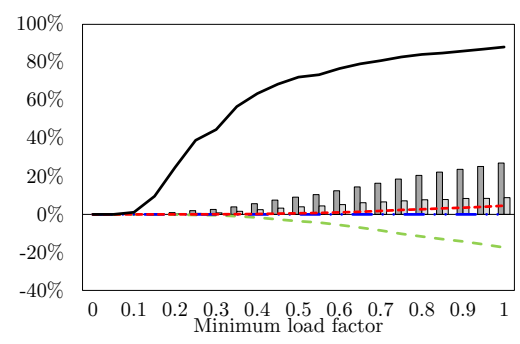

Load factor

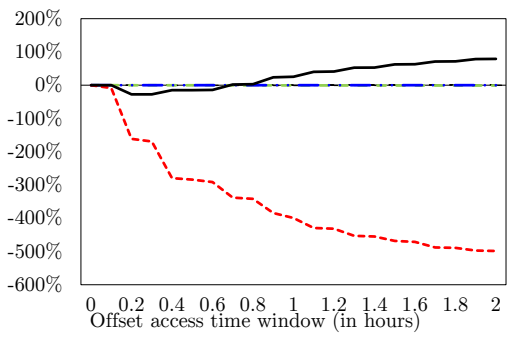

Offset time windows $\square$ Distance heavy trucks $\square$ PM emissions

- Fin. impact carriers -Fin. impact shippers

- -Fin. impact receivers -Fin. Impact UCC

FIGURE 5.3. Sensitivity analysis of non-binary access restrictions.

We proceed with the settings for the 3 pricing policies, see Figure 5.4 for the visual illustrations of the corresponding sensitivity analysis. We find that the pricing policies primarily have a negative impact on carriers and yield only modest environmental improvements for reasonable price levels. In the selection of the non-default pricing settings, the impact on the profit of the carrier is therefore leading, rather than the environmental impacts. We choose the settings such that the carrier remains able to generate a profit; the average carrier profit under the default settings is about $8 \%$. We set the zone-access fee at $€ 20$, the pricing for parking at $€ 4$ per delivery stop, and for road pricing we adopt a price of $€ 1$ per $\mathrm{km}$. Although these settings only marginally influence the environmental 
performance when the measures are independently implemented, in combination with other measures these price settings might contribute to significant improvements.

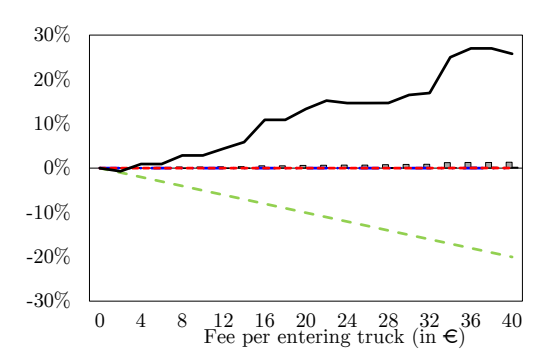

Zone-access fee

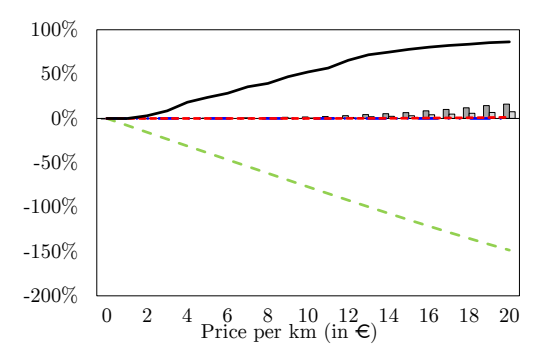

Road pricing

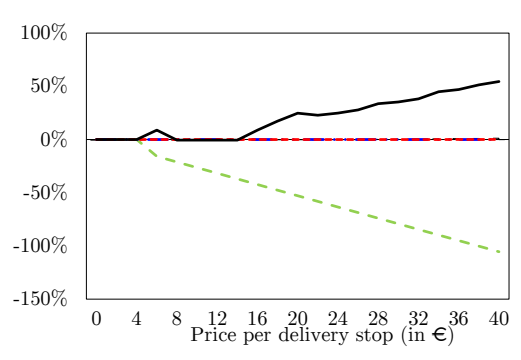

Parking costs $\square$ Distance heavy trucks $\square$ PM emissions

- Fin. impact carriers -Fin. impact shippers

- -Fin. impact receivers -Fin. Impact UCC

FIGURE 5.4. Sensitivity analysis of pricing measures.

Now, we describe the levels for the 3 subsidy measures; the sensitivity results are shown in Figure 5.5. With respect to subsidy levels, we see that potential UCC users require substantial subsidies to make the UCC a financially attractive alternative. Particularly for receivers, subsidies should be very high to convince them to use the UCC, as their receiving costs are typically much lower than the price charged by the UCC and potential savings by using the UCC are modest. Especially for large volumes, the receiver is virtually never inclined to outsource to the UCC. To have a substantial impact on the environmental performance, we test a subsidy level of $90 \%$ for the receivers. For carriers, lower subsidies 
appear to suffice, mainly when carriers transport relatively small loads. However, to realize a substantial shift from direct transport to transport via the UCC, high subsidy levels are still required; the setting of $70 \%$ of the UCC price aims to achieve a significant increase in outsourcing by the carriers. The UCC makes a substantial loss without supporting measures, as the use of the UCC is very limited, it is unable to efficiently conduct its transport. A subsidy level of $50 \%$ is used for the UCC; for scenarios with higher utilization of the UCC this might aid in attaining the breakeven level or even generate a profit. We remark that these settings are selected only based on their expected effects, not on how efficient they are in comparison to one another. Clearly, the administrator would like to allocate subsidies as efficiently as possible, yet it is very difficult to predict the expenditures for a large variety of schemes a priori.

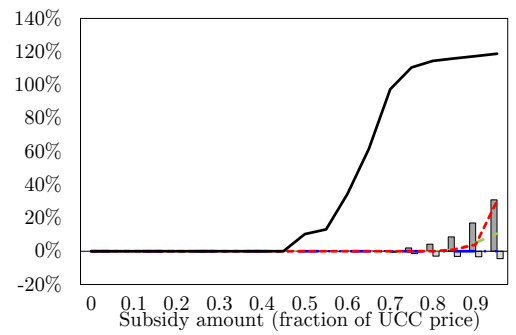

Subsidy receivers

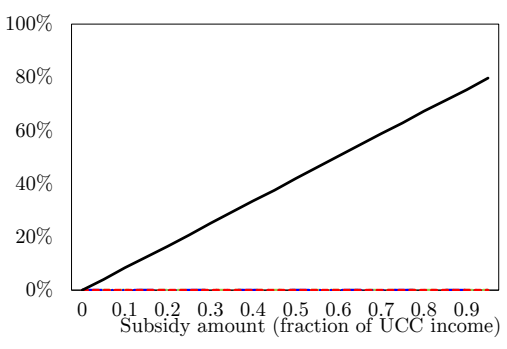

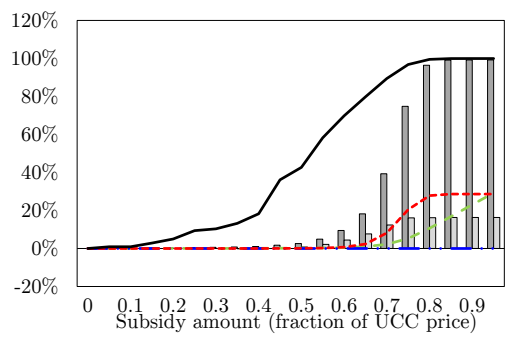

Subsidy carriers $\square$ Distance heavy trucks $\square$ PM emissions

- Fin. impact carriers -Fin. impact shippers

- -Fin. impact receivers -Fin. Impact UCC

Subsidy UCC

FIGURE 5.5. Sensitivity analysis of subsidy measures. 
Finally, we discuss the threshold setting for the shippers (Figure 5.6). The price structure of the carriers encourages shippers to delay shipments as much as possible. Indeed, the higher the threshold, the better the financial performance of the shipper. We set the non-default level for the threshold at 1 (i.e., the shipper always strive to accumulate a full truckload, expressed in terms of capacity of the light trucks operated by the UCC), yet we also note that this threshold is detrimental for the performance of the UCC.

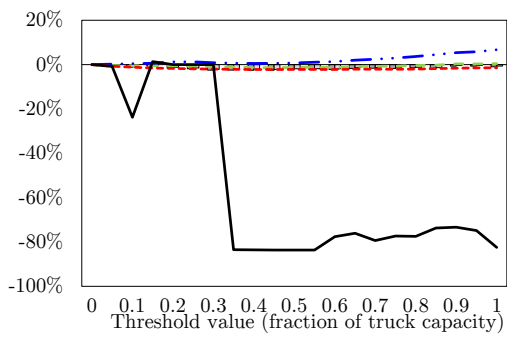

Threshold shipper $\square$ Distance heavy trucks $\square$ PM emissions

- Fin. impact carriers —Fin. impact shippers

- -Fin. impact receivers —Fin. Impact UCC

FIGURE 5.6. Sensitivity analysis of shipper threshold policy.

Based on the sensitivity analysis, we set the default and non-default values for each measure as stated in Table 5.6; these values are used in the $2^{k-p}$ design.

5.5.3. Main effects and interaction effects. In this section, we present and discuss the main effects and the most important two-way interaction effects that we obtain by applying the $2^{k-p}$ factorial design. As stated before, we compute the effects based on the aggregated results for the measures other than price-and subsidy measures. For the latter measures, we only consider the design corresponding to the particular combination of measures. The effects of varying the test instance are discussed separately.

5.5.3.1. Main effects. We first discuss the main effects; Figure 5.7 graphically shows the main effects on the financial performance and Figure 5.8 shows the main effects on the environmental performance. For the financial performance, we show the effects on the (i) costs of the receivers, (ii) costs of the shippers, (iii) profit margin of the carriers, and (iv) profit margin of the UCC. Where necessary, we separately reflect on the financial performance of the administrator. As proxies for the environmental performance, we show the effects on heavy 
TABLE 5.6. Overview of administrative measures and company-driven initiatives and their corresponding default and non-default values.

\begin{tabular}{lll}
\hline Measure & Default value & Non-default value \\
\hline 1. Narrow access time windows & 24h per day & 1 h per day \\
2. Shift access time windows & 0h before opening & 1 h before opening \\
3. Impose heavy truck ban & No & Yes \\
4. Impose minimum vehicle load factor & $0 \%$ of vehicle capacity & $70 \%$ of vehicle capacity \\
5. Road pricing & $€ 0$ per km & $€ 1$ per km \\
6. Parking pricing & $€ 0$ per delivery stop & $€ 4$ per delivery stop \\
7. Zone access pricing & $€ 0$ per truck & $€ 20$ per truck \\
8. Subsidize carriers & $0 \%$ of UCC price & $70 \%$ of UCC price \\
9. Subsidize receivers & $0 \%$ of UCC price & $90 \%$ of UCC price \\
10. Subsidize UCC & $0 \%$ of income & $50 \%$ of income \\
11. Synchronize delivery windows & No & Yes \\
12. Carrier coalition & No & Yes \\
13. Urban consolidation center & No & Yes \\
14. Mandate pre-announcement & No & Yes \\
15. Shipping policy (volume threshold) & $\tau_{s}=\min (l \mid l \in \mathcal{L})$ & $\tau_{s}=\psi^{u c c}$ \\
\hline
\end{tabular}

truck distance and $\mathrm{PM}_{2.5}$ emissions. All KPIs are normalized to aid the visual presentation and, later, to better compute the interaction effects; positive effects $(>0 \%)$ indicate an improvement. For all effects, we show the corresponding 95\% confidence intervals. Particularly for shippers and the UCC, the variance in the observations may be very small, resulting in very narrow confidence intervals. If the error bar of an effect crosses the $0 \% \mathrm{y}$-axis, the result is statistically insignificant. 


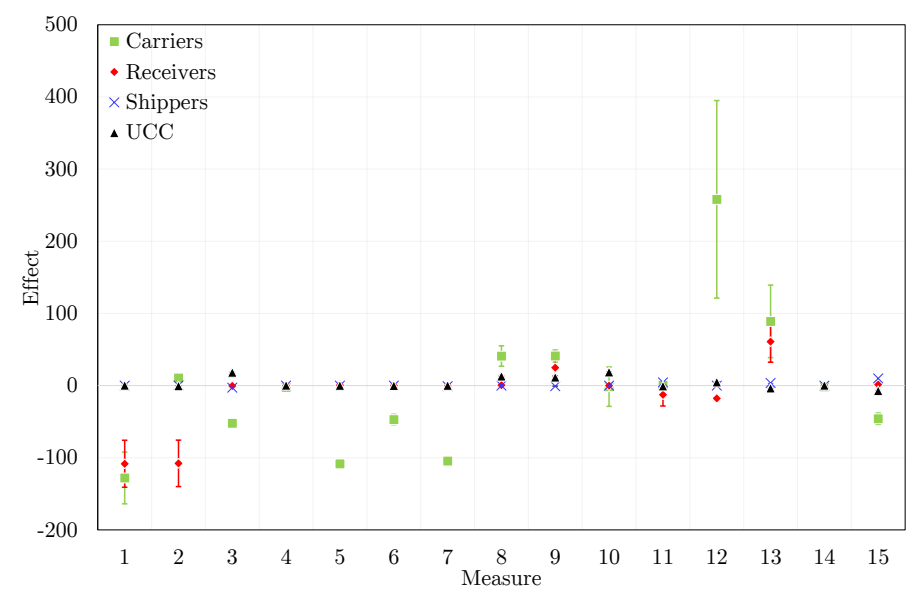

FIGURE 5.7. Main effects on the financial performance indicators.

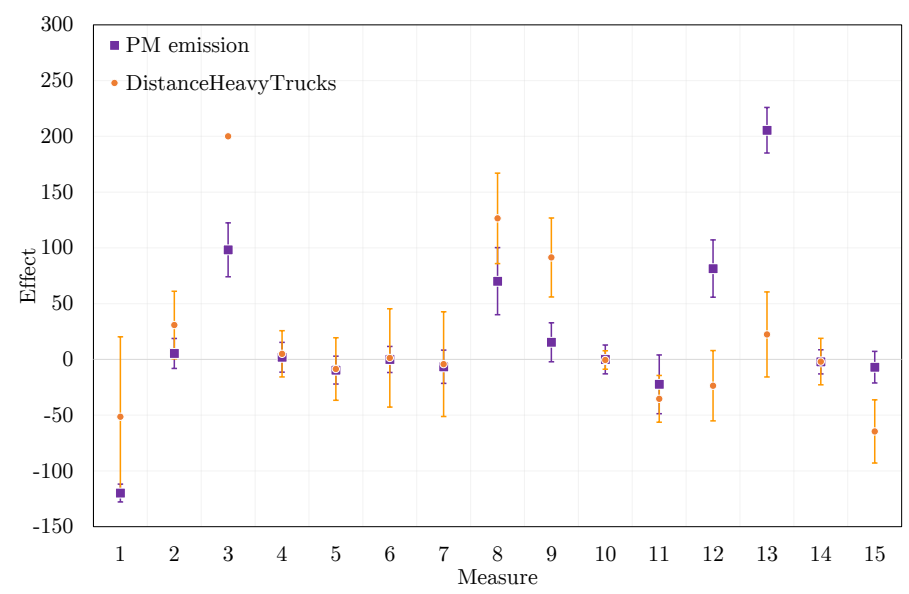

FIGURE 5.8. Main effects on the environmental performance indicators. 
We now provide insight into the significance of the main effects and provide explanations for the obtained results. During the discussion of the effects, we refer to individual measures as, e.g., M4 for measure 4, using the same ordering as in Table 5.6.

First, we assess the main effects of imposing narrow access time windows (M1) and shifting the access time windows (M2). We see that access windows have a detrimental effect on the financial performances of both carriers and receivers. Carriers are affected by the windows as they need to dispatch more trucks to deliver the same sets of receivers, while complying with the time restrictions. Receivers are not directly affected by access windows themselves, but are strongly affected by offsetting them. As M2 only exists in combination with M1, we find negative main effects on both measures. The fact that carriers must dispatch more trucks to deliver the same number of receivers is also seen in the main effects on environmental performance: the main effects of M1 on both distance of heavy trucks and on $\mathrm{PM}_{2.5}$ emissions are negative, although only the latter effect is significant. The environmental effects stemming from M2 are insignificant.

We proceed to discuss the main effects of the remaining access restrictions on heavy trucks. The effect of banning heavy trucks (M3) is significant for carriers, shippers, and the UCC. For the UCC the main effect is positive, although not as strong as might be expected. The reason for the relatively weak improvement is that the profit margin, unlike the profit in an absolute sense, is not strongly related to the total handled volume. The benefit of larger throughput is that the UCC may conduct its transport more efficiently, thus reducing the average costs per $\mathrm{m}^{3}$ of orders handled. Compared to their total operational costs, these savings are relatively small. For carriers and shippers, the main effect of banning heavy trucks is negative. Carriers are forced to pay the UCC for the last-mile distribution, whereas they are typically able to conduct this transport less expensively with their own vehicles. Shippers perform worse due to having less flexibility in their dispatching decisions; recall that they must dispatch orders one time interval earlier when delivering via the UCC. Although significant, the impact on the financial performance of shippers is minor. We proceed to discuss the load-dependent access restriction on heavy trucks (M4). Imposing a minimum load factor on heavy trucks has a significant negative impact on carriers and a significant positive impact on the UCC, although both effects are of small magnitudes. The main effects on the environmental performance are not significant.

The three pricing policies - road pricing (M5), parking pricing (M6), and zone-access pricing (M7) - negatively and significantly impact the profitability 
of the carriers; the financial impact on the other agent types is negligible. The measures might have some positive effect on the environmental KPIs - due to a somewhat higher use of the UCC by the carriers - yet these effects are not statistically significant. The administrator obtains the lowest income with the parking policy; it obtains 17\% more income with the zone-access fee and 23\% more income with road pricing.

Next, we discuss the main effects of the subsidies, which may be allocated to carriers (M8), receivers (M9), or the UCC (M10). In all three cases, the recipient of the subsidy significantly benefits from the subsidies; in terms of magnitude the carrier benefits the most. When subsidies are allocated to the receivers, the carriers also significantly benefit, as they are more often able to outsource their last-mile distribution for free (i.e., when the receiver selects the UCC as its delivery address). In terms of environmental performance, M8 yields significant improvements both in terms of emission and heavy truck distance; M9 only yields significant reductions of heavy truck distance. Subsidies allocated to the UCC do not affect the system other than the profitability of the UCC and therefore have no environmental effect. In terms of expenses by the administrator, subsidizing carriers is the most efficient subsidy measure: subsidizing receivers costs $368 \%$ more and subsidizing the UCC costs $275 \%$ more, while yielding lower environmental benefits.

We continue to discuss the main effects of the company-driven measures. Synchronizing delivery windows (M11) has a negative and significant impact on the heavy truck distance. The measure has a significant and positive main effect on the shippers, as they are able to dispatch higher volumes at a time. The main effect on the UCC is significant and negative; the higher volumes on given dispatch moments may result in a lower use of the UCC. The effect on receivers themselves is insignificant. The main effect on the heavy truck distance is negative and significant; a possible explanation for this is that the synchronization results in week days with many deliveries and week days with relatively few deliveries; transport on the latter type of days might be organized inefficiently.

The formation of a carrier coalition (M12) strongly improves the financial performance of the carriers. Sharing trucks and bundling order considerably improves the efficiency of their transport operations, as a result, the effect on the $\mathrm{PM}_{2.5}$ emissions is positive and significant. The use of the UCC is very low in schemes that include a carrier coalition.

Opening the UCC (M13) has significant and positive main effects on both carriers and receivers. Although these agents do not always make use of the UCC, its presence offers an alternative for the delivery of small volumes and 
when operating under strict regulations. The financial effect on the UCC itself is negative, implying that the UCC on average loses money on its operations. The main effect on emissions is positive and significant.

Dictating that carriers should pre-announce the arrival of outsourced orders to the UCC (M14) has a minor but significant positive effect on the UCC, implying that the UCC is able to make slightly better dispatching decisions than when working with expected future costs. For the other agents and for the environmental KPIs, the main effects are insignificant.

The last measure that we discuss is the shipping policy (M15). Setting the threshold volume equal to 1 significantly improves the financial performance of shippers. As the shippers pay lower transport costs, it follows that the carriers generate less income; indeed, the main effect on the carriers is negative and significant. The effect on the UCC is also negative, as carriers are not able to outsource urgent shipments when the shipper dispatches orders only one time interval before the latest delivery time. Furthermore, the policy has a significant negative effect on the distance driven by heavy trucks; again, this effect likely relates to the lower use of the UCC.

Although not part of the $2^{k-p}$ designs, we provide some insights into the effects of the 6 geographical instances. Both the effects of various customer distributions and varying the size of the pickup area are insignificant for all KPIs; almost all effects approximate 0 . Only for clustered instances, we observe a slightly positive (but also insignificant) effect on $\mathrm{PM}_{2.5}$ emission, possibly due to offering opportunities for more efficient bundling. The only substantial effect that we measure is when distinguishing the performance of carrier coalitions for pickup areas of size $20 \mathrm{~km}^{2}$ and size $100 \mathrm{~km}^{2}$; the latter yields $6 \%$ lower profits for the carriers. This may be explained by the longer pickup tours that the executing carrier has to perform. However, the overall financial effect on carriers is insignificant, as shippers tend to select carriers that are located nearby. Due to the insignificance of the impact of the instance, we ignore their effects for the remainder of this analysis.

We conclude this section with the key insights. We see that the following measures yield the most substantial improvements to the environmental performance: forming a carrier coalition, opening the UCC, imposing a ban on heavy trucks, and subsidizing carriers to use the UCC. As the latter three measures all relate to the UCC, it appears that considerable bundling efforts are required to achieve a significant impact on environmental performance, either 
performed upstream by the carriers or downstream by the UCC. The main effects of the truck ban and subsidies reconfirm that the impact of the UCC may substantially be improved by implementing supporting measures.

5.5.3.2. Interaction effects. In this section we discuss the most important two-way interaction effects. The experiments yield over 100 two-way interactions, of which a great deal are statistically significant for at least one agent type or environmental KPI. To focus the analysis, we only show the 10 interactions that yield the highest improvements for $\mathrm{PM}_{2.5}$ emissions; this indicator is relevant for determining good schemes, correlated to the transport costs of both carriers and the UCC, and challenging to improve upon. The financial interaction effects are shown in Figure 5.9 and the environmental interaction effects are shown in Figure 5.10. We refer to interactions between measures by their numbers, e.g., M1xM8 signifies the two-way interaction between M1 and M8.

The two-way interaction effects indicate the differing effect of one measure depending on the level of another measure, i.e., the effect of two measures that interact with each other. To compute these effects, we compare the average performance of schemes in which two measures are either both set at their default levels or both set at their non-default levels to schemes in which only one of the two measures is set at its default level. A positive two-way interaction effect implies that implementing the measures in conjunction on average yields better results than implementing only one of them, e.g., their joint effect exceeds the sum of their main effects. 


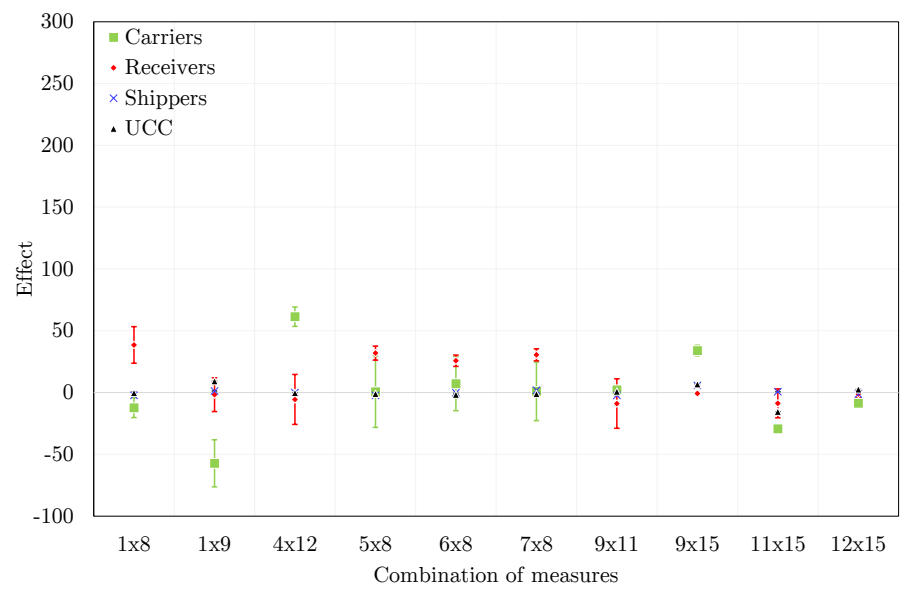

FIGURE 5.9. Interaction effects on the financial performance indicators.

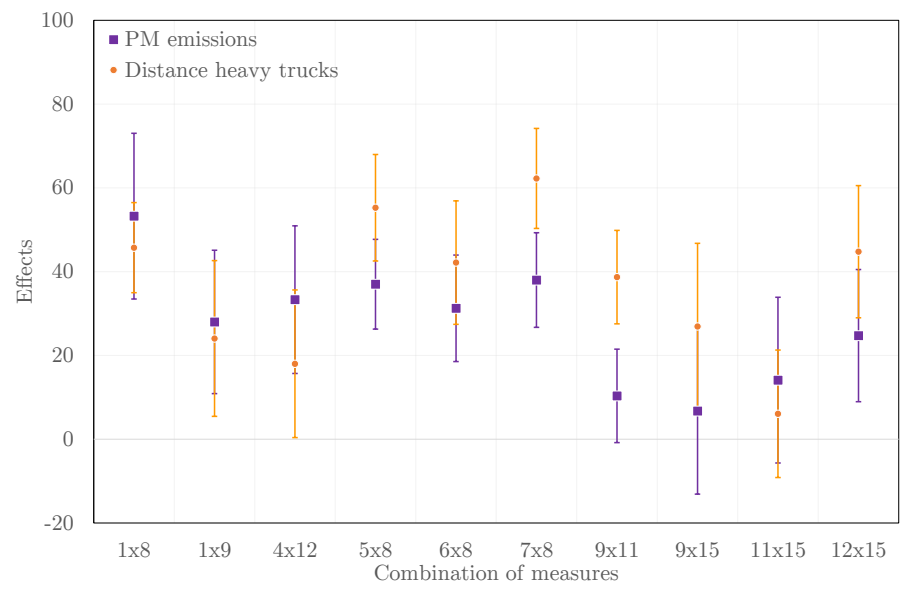

FIGURE 5.10. Interaction effects on the environmental performance indicators. 
The first two interactions that we discuss combine the access window (M1) and subsidization of either the carriers (M8) or the receivers (M9). Recall that the access window has a negative main effect on emissions, whereas the subsidies have positive main effects. We first discuss the combination M1xM8. This combination has a positive and significant interaction effect on the environmental KPIs, although the magnitude of the effect appears insufficient to compensate for the negative main effect of the access window. The combination has a significant positive effect on receivers, possibly due to receiving more bundled deliveries via the UCC. The effect on the profit of carriers is negative and significant; although carriers receive a subsidy for using the UCC, their monetary gains appear to be eclipsed by the transport inefficiency caused by the imposed access windows. We proceed to discuss the combination M1xM9, which consists of imposing the access window and subsidizing the receivers. The smaller magnitude of the environmental effects implies that the UCC is utilized less for combination M1xM9 than for M1xM8, nevertheless, the effect on both environmental KPIs is positive and significant. The interaction effect is also positive and significant for receivers, as under this combination of measures both carriers and receivers have an incentive to use the UCC, leading to more bundled deliveries. Although the carriers do not need to pay the UCC for orders that are outsourced by the receivers, they are still subject to the access windows and the consequent transport inefficiency, resulting in a negative effect on the carriers.

Next, we consider the interaction between imposing a minimum load factor and forming a carrier coalition (M4xM12). This combination has significant positive effects on both the carrier performance and the $\mathrm{PM}_{2.5}$ emission. Recall that the minimum load factor is effective only when the UCC is open. The combination appears to be effective as on the one hand we obtain the efficiency benefits of the coalition, whereas on the other hand any remaining trucks with low utilization must be outsourced to the UCC.

We continue by addressing three combinations that combine one of the three pricing policies with subsidizing the carriers; the combinations are denoted by M5xM8 (road pricing), M6xM8 (parking pricing), and M7xM8 (zone-access fee). All three combinations have comparable effects; they positively and significantly impact the $\mathrm{PM}_{2.5}$ emission and the heavy truck distance. Furthermore, the receivers significantly benefit with all three combinations. The combinations provide a double financial incentive for carriers to outsource their last-mile transport to the UCC; the combinations subsidize the use of the UCC and financially penalize carriers that perform the last-mile distribution themselves. In 
terms of environmental impact, the zone-access fee appears has the largest magnitude, although the differences between the combinations are relatively small.

Combinations M9xM11 and M9xM15 embed the following measures: subsidies allocated to the receiver (M9) and either the synchronization of delivery windows (M11) or the high shipping threshold policy (M15). Both combinations significantly reduce the heavy truck distance, whereas the combination M9xM15 also significantly improves the performance of carriers, shippers, and the UCC. In the combination M9xM11, the subsidies stimulate the receivers to use the UCC more frequently, whereas the synchronization of the delivery windows might make the last-mile distribution more efficient for the UCC. We explain the interaction effect of M9xM15 as follows: M9 stimulates the use of the UCC and thereby partially mitigates the effect of the threshold policy, yet on average the shippers still dispatch larger order batches. Therefore, the measures appear to work better in combination than separately.

The next interaction effect that we evaluate is M11xM15 and combines the synchronization of the delivery windows (M11) with the high shipping threshold (M15). The environmental effects of this interaction are insignificant; carriers are negatively and significantly impacted. Potential environmental benefits might stem from better consolidated freight flows, yet this presumption is not supported due the insignificance of the interaction effects.

The last interaction effect that we discuss combines the formation of the carrier coalition to the high shipping threshold (M12xM15). This combination has positive and significant effects on both environmental KPIs, but a negative (although minor) effect on the carriers. The combination of bundling orders at both the shipper level and the carrier level seems to contribute to the efficiency of last-mile transport.

We summarize the conclusions with respect to the interaction effects. Seven out of the ten measures with the highest impact on emissions contain a measure that subsidizes either carriers or receivers. In particular, the combination of subsidizing with pricing yields considerable environmental benefits, while also being financially acceptable combinations for the agents. Especially subsidies allocated to carriers appear to contribute to effective schemes. The rationale behind such schemes is that they combine two financial incentives for carriers to use the UCC. Furthermore, applying the high shipping threshold appears to support several measures, although the interaction effects are not in all cases significant. The best results are obtained when the high threshold is applied in 
combination with the carrier coalition. The high threshold results in more consolidated shipments; this seems to be beneficial for schemes in which carriers perform bundling upstream.

5.5.4. Scenario analysis. In this section, we perform a scenario analysis to obtain more insights into the structure and the performance of good urban logistics schemes. Section 5.5.2 established and quantified the sensitivity of the individual measures, whereas Section 5.5.3 quantified the main effect of each measure and the most important two-way interaction effects. Based on the obtained insights, we may deduce properties of good schemes. We use these properties to test a number of schemes. We evaluate their environmental impacts and how the agents perform compared to the default scheme. As our test instance, we use a uniform distribution of receivers and a pickup area of size $20 \mathrm{~km}^{2}$; the chosen distribution of receivers appears to be of minor influence, and we deem the small pickup area more realistic for a collaboration between carriers.

The schemes that we analyze are shown in Table 5.7. Scheme 1 represents the default scheme; the performance of all other schemes is expressed relative to this scheme. Based on the insights obtained from Section 5.5.2 and Section 5.5.3, we carefully construct a number of schemes. From the main effects, four measures positively stood out with respect to environmental performance: (i) forming a carrier coalition, (ii) opening the UCC, (iii) imposing a heavy truck ban, and (iv) subsidizing carriers to use the UCC. Schemes 2-5 represent these measures applied in isolation; schemes 3,4 , and 5 all require the UCC to be opened.

From the interaction effects, we gain insights into various combinations of measures that yield positive results on emissions, yet it is unclear whether implementing these measures in conjunction actually yields good schemes, particularly as many of the measures with positive interaction effects did not yield significant positive main effects. In Scheme 6-15, we test all the interaction effects that we discussed in Section 5.5.3.

We have not analyzed interactions between three or more measures in this chapter. Therefore, we conclude the scenario analysis with a number of schemes that contain three or more measures. The effects of simultaneously applying pricing policies and providing subsidies to carriers are additive; on the one hand such combinations increase the costs for the carrier if it conducts its last-mile transport itself, on the other hand they makes outsourcing less expensive. In schemes 16-18, we test the 3 different pricing policies (M5,M6,M7) in combination with subsidizing the carriers (M8), opening the UCC (M13), and imposing a minimum load factor (M4). Schemes 19-21 do the same, but instead of the minimum load factor set the high threshold policy (M15). Finally, we test four 
schemes (22-25) that combine the concepts of the carrier coalition and the UCC with various supporting measures.

Table 5.7 shows the results of the scenario analysis on a variety of KPIs. For the carrier and the UCC we report the profit margins; for the receivers and shippers we show the change in costs compared to the default scheme, with percentages higher than 0 indicating an improvement. The changes with respect to emissions are shown in the same way. The distances for both light and heavy trucks are represented by the average number of kilometers per day, measured only within the urban area. 


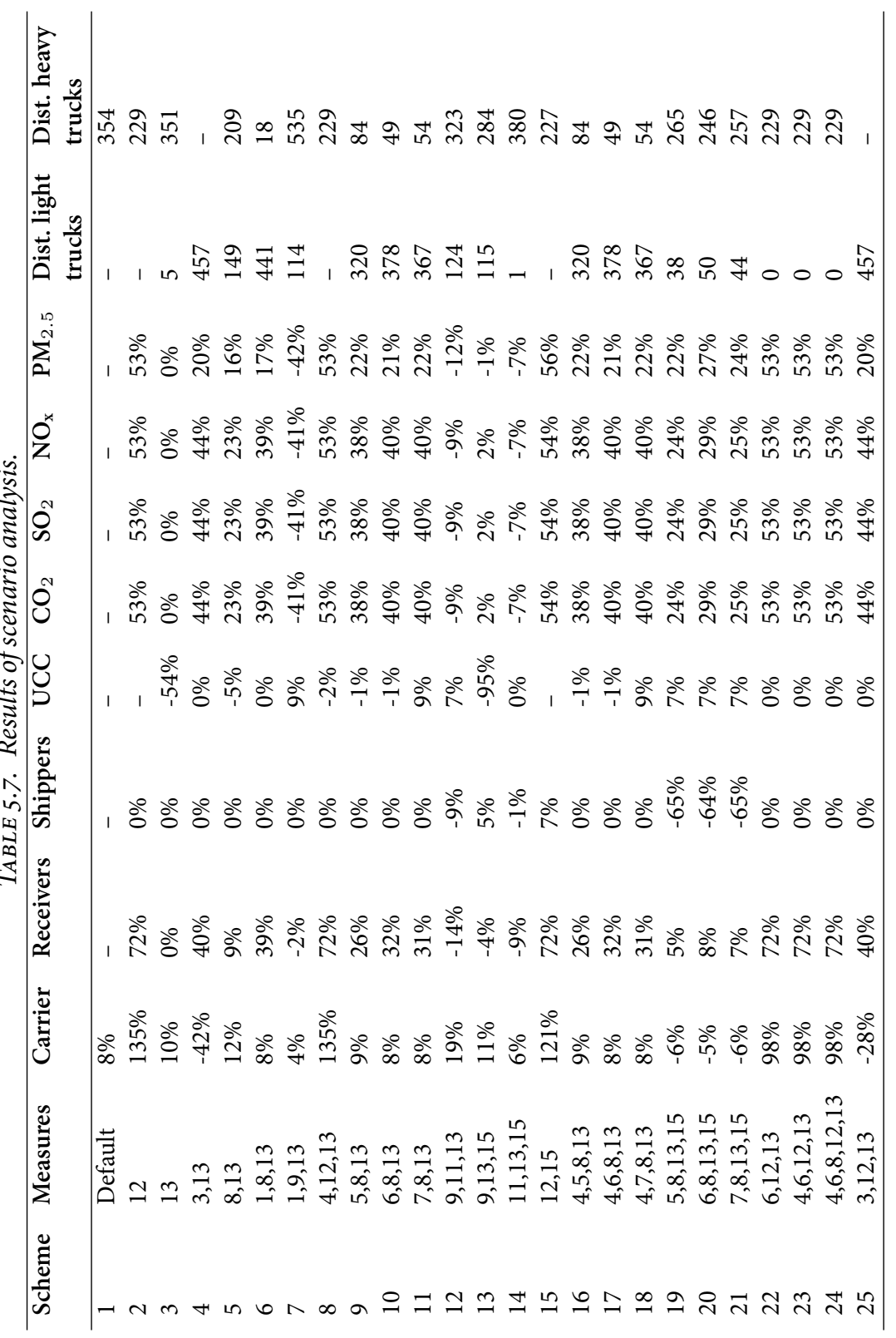


We reflect on the main insights obtained with the scenario analysis. First, we observe that a coalition of carriers (Scheme 2) is very effective in improving the environmental performance and does not require supporting measures. Second, we note that opening the UCC (M13) without any supporting measures (Scheme 3) is ineffective; the environmental impact is negligible and the UCC is far from being able to cover its expenses. Banning heavy trucks (M3) as in Scheme 4 or subsidizing the carriers (M8) as in Scheme 5 are effective measures to achieve significantly improved environmental performance by opening a UCC.

Next, we discuss the schemes relating to the two-way interaction effects that we have shown in Section 5.5.3, which are represented by Scheme 6-15. We evaluate whether the obtained results are in line with the effects computed based on the $2^{k-p}$ design. If effects cannot be reproduced by implementing the measures on a standalone basis, this might be an indication that undetected high-level interactions influence their performance. Also, a $2^{k-p}$ does not guarantee that the calculated effects are precise, as the design contains only a subset of the experiments that are performed in a full factorial design. We find that the effects of the combinations M1xM8 (access window and subsidizing the carriers) and M1xM9 (access window and subsidizing the receiver) are partially reproduced, with the main difference being that the impact of M1xM8 on the environmental performance is more positive than anticipated based on the computed effects. Interaction M4xM12 (Scheme 8) is not reproduced; imposing a minimum load factor does not appear to influence the behavior of the carrier coalition. The interactions between the pricing policies M5, M6, and M7 combined with subsidizing the carriers (M8) (Scheme 9-11) coincide with the calculated effects and all yield considerable environmental benefits. In Scheme 12 and Scheme 13 , combining the subsidization of receivers (M9) with either synchronizing delivery windows (M11) or a high threshold policy (M15) do not yield positive effects on the heavy truck distance of the predicted magnitude, however, the financial effects are in line with the computed effects. The effects predicted by interaction M11xM15 (Scheme 14), although of small magnitudes, are confirmed by the scenario analysis: both the decline in carrier performance and the slight improvements in the environmental performance (relative to the main effects of the separate measures, compared to the default scheme the impact is negative) are in agreement with the computed effects. The positive environmental effects of M12xM15 (Scheme 15) are confirmed by the scenario analysis; the combination of the high shipping threshold and the carrier coalition yields the best environmental performance of all tested schemes. 
We proceed to discuss Scheme 16 to 18 , in which we combine one of the pricing policies (M5,M6,M7), subsidization of the carriers (M8), opening the UCC (M13), and a minimum load factor (M4). We see that substantial environmental gains are made compared to Scheme 5 , in which we only test the combination of subsidizing and opening the UCC. Thus, adding a pricing policy appears to be a good practice; all three pricing policies produce roughly the same results.

In Scheme 19 to 21, we test schemes that are equivalent to schemes 16-18, but instead of adding a minimum load factor we set the high shipping threshold (M15). Although yielding positive environmental effects, many other schemes yield better results. Furthermore, the profit margins of the carriers and in particular the UCC are considerable affected under these schemes. Again, all three schemes yield comparable results.

The last schemes that we evaluate (22-25) aim to combine the carrier coalition (M12), opening the UCC (M13), and a number of measures that aim to stimulate the use of the UCC. For schemes 22, 23, and 24, the measures reduce the profit of the carriers, but are ineffective in stimulating the use of the UCC. In Scheme 25, we enforce the use of the UCC by imposing the heavy truck ban (M3); this yields results that are worse than when the carrier coalition conducts the last-mile distribution itself. These results imply that from an environmental perspective, the UCC should not be used for loads that already are efficiently consolidated.

We summarize the key findings of this section. The carrier coalition yields the best results both in terms of environmental performance; combining the coalition with a high threshold policy at the shipper level somewhat enhances the performance. The UCC also yields considerable environmental benefits, but requires supporting measures to be effective and thrives best when supported by multiple measures. Especially subsidizing the carriers in combination with implementing a pricing policy proves to be effective. A complete ban on heavy trucks aids in achieving good environmental performance as well, but compares unfavorably to the formation of the carrier coalition.

5.5.5. Discussion. In this section, we discuss the main conclusions that are obtained by the numerical experiments. We identify four measures that have the potential to yield considerable environmental benefits: (i) banning heavy trucks from the city center completely, (ii) opening a UCC, (iii) subsidizing carriers to use the UCC, and (iv) forming a coalition of carriers. Within the model, the first three measures are complementary, as in the model banning heavy trucks implies that carriers must use the UCC. Thus, we may state that the largest environmental gains are essentially obtained by consolidating and using truck capacity more efficiently, either upstream by the carriers or downstream by the UCC. 
Of the two alternatives, upstream consolidation by the carriers appears more promising, as it does not require an expensive reload procedure. Therefore, unlike the use of the UCC, in the simulation model it is a self-sustained solution that does not require subsidies or supporting regulations. However, collaboration by carriers has been treated as a best-case scenario in this study, and two major challenges have been omitted. First, we only model the transport flows related to a single city. In reality, trucks dispatched by carriers may well visit more than one city during a single trip; this makes successful planning and synchronizing logistics operations more challenging. Second, the model assumes that all carriers are willing to share their orders and trucks to achieve a common goal; in practice, competitive concerns often prevent carriers to cooperate with one another. Despite these shortcomings, the results imply that collaboration between carriers has significant benefits both for the environment and for the financial performance of carriers.

We proceed to discuss the impact of supporting measures on the use of the UCC. Although we show that such measures are generally ineffective when implemented on a stand-alone basis, they may aid in convincing carriers or receivers in using the UCC more frequently. Particularly the combination of subsidies allocated to carriers and imposing a pricing policy has a considerable effect on the utilization of the UCC. Besides providing a double incentive for the carriers to use the UCC, the income stemming from the pricing policies might also be used by the administrator to (partially) cover the subsidy expenses. In our test setting, however, the subsidy expenses are much higher than the policy income, with subsidy expenses exceeding the costs by a factor 30 . This financial gap might be addressed by monetizing external costs, but that is beyond the scope of this chapter. Furthermore, under certain conditions the use of a high dispatch threshold by shippers has a significant positive effect. Although the main effect of this measure is negative, it yields positive effects when combined with either a carrier coalition or when the UCC is used primarily by receivers. In the first case,the upstream bundling allows the carriers to operate more efficiently, whereas the second combination results in better bundled incoming goods at the UCC.

Finally, we reflect on the effects of a heavy truck ban. Although the environmental benefits of this measure are considerable, it strongly reduces the profit of the carriers and will likely be strongly opposed in practice. Furthermore, when the load capacity of carriers is effectively used, heavy trucks are in fact able to perform the last-mile distribution in a more environment-friendly way than the light trucks of the UCC. Thus, the UCC should preferably be used to bundle the 
loads of poorly utilized trucks, rather than aiming to take over the complete lastmile distribution process.

\subsection{Conclusions}

In this chapter, we have applied the simulation framework that we developed in Chapter 4. We have modeled the roles of five agent types: receivers, carriers, shippers, a UCC, and the local administrator. For each agent, we defined decisions both as one-time decisions and as operational decisions.

We collected urban transport data from a variety of sources to establish representative parameter ranges to define the properties of urban supply chains in Western European cities. Based on the collected data, we have defined 10 receiver profiles that reflect a variety of ordering systems. By assigning fixed sets of shippers to the receivers and fixed carriers to the shippers in our experiments, we created diversity in the modeled transport system. Furthermore, based on the collected data, we have developed six abstract networks that represent the characteristics of Western European cities; we distinguish three distinct distributions for the receiver locations in the urban area and two sizes for the pickup area.

As successful urban logistics schemes typically combine various measures from multiple categories, this chapter focused on revealing both the main effects of a variety of common urban logistics measures and the interaction effects between these measures. We defined 15 test measures in the following categories: (i) access restrictions, (ii) pricing policies, (iii) subsidy measures, and (iv) company-driven initiatives. To efficiently extract information on the main effects and the two-way interaction effects of the measures, we applied a fractional factorial experimental design. First, we performed sensitivity analysis to determine the 'low' and 'high' values that correspond to the $2^{k-p}$ design. Second, we have run the design to obtain insights in main effects and interaction effects. Third, we conducted a scenario analysis to further evaluate the design of good urban logistics schemes, which according to our criteria are schemes that yield a substantial improvement in environmental performance while being sustainable from a financial perspective as well. We compared the performance per agent type and on various environmental KPIs to the performance under a default scheme without any urban logistics measures being in effect.

Based on the numerical experiments, we have obtained a number of insights. We identified two main solution concepts that might be both financially viable and yield substantial environmental benefits: (i) collaboration between carriers allows to consolidate orders already upstream and (ii) outsourcing last-mile 
distribution to the UCC allows to consolidate orders downstream. Collaboration between carriers is preferable as it does not require supporting measures and yields better environmental effects. However, this chapter assumes a favorable collaboration structure that ignores competitive concerns and possible consequences of carriers visiting multiple cities. A 4PL that centrally plans and distributes orders, such as studied in Chapter 2, might negate such concerns. With respect to the UCC, we find that it only functions well when being substantially supported by regulation. A heavy truck ban is the most effective measure from the perspective of the UCC, but negatively affects the profits of carriers, while also yielding less environmental benefits than the collaboration between carriers does. A combination of a pricing policy (e.g., road pricing) and subsidizing the carriers appears to be a more fruitful solution, although requiring considerable subsidy expenses by the local administrator. The income from the pricing policies is insufficient to cover the subsidy expenses; in our experiments, the subsidy expenses are about 30 times higher than the policy income. Therefore, the administrator should be able to monetize external costs and subsequently determine whether these costs exceed the required subsidy amounts. The monetization of external costs is not addressed in this thesis.

This chapter has studied initiatives in urban logistics in a broad sense. To evaluate steady-state behavior and to test a large number of schemes on multiple instances, we have omitted the layer of tactical decision making. Furthermore, we addressed the system purely from a freight consolidation perspective, thereby ignoring the role of value-adding services. In Chapter 6 , we will focus specifically on the role of the UCC, also taking into account decisions that are made on the tactical level. This allows us to apply the simulation framework in more depth. 

CHAPTER 6

\section{Consolidation in the city of Copenhagen: a simulation study}

In the previous chapter, we used the framework that we described in Chapter 4 to evaluate a variety of measures. In this chapter , we apply the framework in a more demarcated setting, focusing on a single instance and the specific role of the UCC. As UCCs play an important role in many urban logistics initiatives, but still suffer from a low success rate and a lack of well-defined business models, we perform a simulation study to reduce this knowledge deficit. We create a test instance based on the city of Copenhagen, using actual retailer locations and a real UCC location. We use OpenStreetMap to generate a network with realistic travel times. Based on data gathered from both literature and expert interviews, we design multiple demand profiles for retailers. Each simulation run starts with a UCC that does not have a user base (users may be receivers carriers); we test various administrative measures that can be taken, with the objective being to reach a steady state in which it can operate without external funding after a given period of time. As UCCs do typically not reach such a steady state without initial support, we aim to identify the combinations of local regulations and temporary subsidies that are most effective to build a solid user base for the UCC.

The chapter is structured as follows. Section 6.1 provides a general introduction to the chapter. In Section 6.2, we present an overview of literature on business models for the UCC. The solution method, i.e., the specific application of the simulation framework, is described in Section 6.3. Section 6.4 provides the experimental setup, and Section 6.5 discusses the numerical results. Section 6.6 concludes the chapter.

\footnotetext{
${ }^{1}$ This chapter is based on the working paper: W.J.A. van Heeswijk, R. Larsen, \& A. Larsen (2017). An urban consolidation center in the city of Copenhagen: a simulation study. Van Heeswijk et al. (2017a)
} 


\subsection{Introduction}

It is well known that UCCs lack established business models (Allen et al., 2012b). Typically, the financial gains stemming from last-mile consolidation alone are insufficient to compensate for the additional transshipment costs, as we also illustrated in Chapter 5. Thus, a UCC requires additional sources of revenue. Although a few examples exist, the vast majority of UCCs does not receive perpetual subsidies, as this is typically politically untenable (although the city may ultimately need to spend more money to mitigate the negative impacts of freight transport). Therefore, business models based on subsidies are generally not successful in the long run. Value-adding services are a more promising source of income. Due to economies of scale, a UCC is often able to perform these services against considerably lower costs than the receivers themselves. Also, a sizable proportion of retailers is willing to pay for such services (Dablanc, 2011). Finally, rather than providing subsidies, the administrator might introduce regulation that monetizes external costs, such that end-users are willing to pay a higher fee to the UCC than they would otherwise.

The goal of this chapter is to identify sustainable business models for UCCs. To this end, we apply simulation to test a variety of subsidy schemes and regulations that may aid the UCC in building a sufficiently large user base. The starting point of each simulation run is a UCC without any users. When attracting more users and handling larger volumes, the operational costs per $\mathrm{m}^{3}$ of the UCC are reduced due to economies of scales. As a result, the UCC is able to offer more competitive prices and thereby attract more users. Based on this mechanism, we aim to find a situation in which the UCC can operate without external funding. We consider a subsidy period of two years, after which the UCC should be self-sustained. Supportive regulation is permanently implemented. However, as such regulations affect more parties than just the target group of the UCC, they should be as least intrusive as possible.

The study performed in this chapter is motivated by practice. Until recently Binnenstadservice, a Dutch UCC operator and one of the industry partners in the CONCOORD project (see Section 1.2), used a business model that focused on committing receivers. As the UCC became the formal delivery address for the carriers, this approach proved to be effective in taking control over last-mile deliveries. Receivers are typically easier to convince than carriers, as for them the costs of outsourcing to the UCC are relatively low compared to their overall costs. Furthermore, the offered value-adding services are often useful to them, as outsourcing these services allow receivers to dedicate more time to their core business. Although this business model is successful from an environmental 
point of view, Binnenstadservice struggles to make it financially sustainable. As receivers already pay the UCC for the last-mile distribution, the carriers essentially are able to outsource their last-mile distribution for free, while the income stemming from receivers alone is far too little to cover the operating costs of the UCC. However, if the carriers themselves decide to outsource their complete last-mile distribution - even if the carriers need to pay a moderate charge for this - the UCC is still able to sell value-adding services to the receivers. Thus, it may be sensible to focus primarily on committing carriers. In the simulation study, we test the effects of measures that provide incentives to both the receivers and the carriers to use the UCC. We evaluate how these measures impact the development of the user base over time and whether we eventually achieve a state in which the UCC is able to operate without external funding.

In this chapter, we test the performance of a UCC in a realistic setting, in which we evaluate the impact of various measures. We perform a simulation study on an instance based on the city of Copenhagen, with a geographical network that is as close as possible to the real street network. To model a setting that reflects economies of scale, we introduce a mechanism that periodically updates prices and costs based on the volume that is handled by the UCC. By collecting data from expert interviews and literature, we establish representative ranges for these prizes and costs. We incorporate the tactical decision layer of the simulation framework, with carriers and receivers opting in or opting out for the UCC for longer periods of time. The combination of varying costs and prices and tactical decisions reflects a system that dynamically evolves over time. This allows us to analyze changes in the system and to identify steady states, rather than using a trial-and-error approach on static instances.

\subsection{Literature on business models for UCCs}

Chapter 1 already briefly discussed the challenges associated with UCCs. We elaborate on their business models here. On a high level, two business models for UCCs can be distinguished (Van Rooijen and Quak, 2010). In the first one, it is the carrier that outsources the deliveries in the city to the UCC. The costs for last-mile distribution are disproportionally high for carriers, as travel speeds are low and unloading at the receivers is time-consuming. Restrictive local regulations may be an additional reason for carriers to outsource delivery to the UCC. Despite these incentives, the price charged by the UCC may still be unfeasibly high for carriers (Van Rooijen and Quak, 2010; Kin et al., 2016). In the second business model, the receiver in the urban area selects the UCC as its delivery address. The UCC can bundle goods stemming from multiple origins 
into a single delivery, such that the receiver needs to spend less time on receiving goods, thus requiring less personnel hours. However, as the shipping costs are generally embedded in the order price that the receiver pays, selecting the UCC as its delivery address introduces additional costs. The efficiency gains are unlikely to compensate for these costs (Verlinde et al., 2012), particularly if carriers are not willing to share their efficiency gains with the receivers. For the receiver, the key merits of the UCC are therefore the value-adding services that are offered. Van Rooijen and Quak (2010) and Allen et al. (2012b) describe various value-adding services. First, temporary storage at the UCC allows retailers to hold goods in a nearby position, without having to dedicate valuable shop floor space. Second, waste collection (e.g., cardboard waste) can be performed by the UCC; this service is typically not offered by carriers. Third, the UCC can collect goods that the retailer has sold online (e-tailing). From the UCC onwards, these goods are transported by an external carrier; due to the larger volumes handled, the UCC can negotiate lower transport rates than the individual retailers could. Fourth, home deliveries in the same city can also be performed by the UCC, being responsible for both collection and delivery. Finally, the UCC may offer specialized services tailored to the needs of individual retailers, such as splitting pallets into smaller loads, or putting clothes on hangers and labeling them before delivery to a fashion retailer.

In an elaborate review, Browne et al. (2005) analyze 67 UCC schemes (considering operational schemes, trials, and feasibility studies). They report that the vast majority of UCCs is unable to survive in the long term. For example, out of 200 known operational schemes in Germany, only 15 were still active at the time of the study (Browne et al., 2005). The main reasons for failure are (i) the high costs of the extra transshipment and (ii) a lack of added value from the perspective of both carriers and receivers (Browne et al., 2005; Van Duin et al., 2010; Verlinde et al., 2012). As a result, UCCs are often unable to generate a high throughput, failing to reach the break-even level required to operate in a financially sustainable manner. In the start-up phase of a UCC, local administrators often provide subsidies. When this financial support is ended, the UCC is generally unable to survive (Browne et al., 2005; Kin et al., 2016).

As the low success rate of UCCs might suggest, little analysis has been done on their long-term success factors (Van Rooijen and Quak, 2010). First of all, there is often an absence of a clearly defined target group of potential UCC users. Many logistics streams are already efficiently organized, and the transshipment may actually make them less efficient (Van Rooijen and Quak, 2010). A second challenge is that retailers, carriers, and administrators may all benefit in some 
way from a UCC, yet strive to accomplish divergent objectives (Bektaş et al., 2015). In particular, the objective to reduce environmental costs is often difficult to combine with cost efficiency for the actors involved. Therefore, systemwide optimization often does not generate solutions to which autonomous actors would commit in practice. Van Duin et al. (2016) state that carriers in particular are reluctant to outsource their last-mile distribution to a UCC.

We highlight a number of case studies that reflect on the performances and business models of various UCCs. Van Duin et al. (2010) perform a feasibility study for a UCC in the Hague (the Netherlands). Based on their cost-benefit analysis, they conclude that the UCC would only be financially sustainable when almost full participation of the target group of retailers is ensured. Furthermore, they suggest that the UCC might be set up as a collaboration between multiple carriers, such that they have control on the information structures that are required. The study by Van Rooijen and Quak (2010) addresses the case of a UCC in Nijmegen, the Netherlands, one of the UCCs that has been opened by Binnenstadservice. The authors state that this UCC differentiates itself in its business model by focusing on convincing receivers to join the UCC, rather than carriers. The approach proves successful in attracting a considerable number of UCC users and also shows that a UCC can significantly reduce environmental costs. However, the authors also acknowledge that the Nijmegen UCC remains financially unsustainable and depends on local subsidies for its existence. In a more recent study, Van Duin et al. (2016) state that Binnenstadservice now focuses more on attracting carriers as well. Gonzalez-Feliu and Morana (2010) describe a UCC initiative that has been implemented in the Italian city Padua. In the city, the local administrator has set a time access window from 10:00 to 12:00am for trucks heavier than 3.5 tonnes. The business model of the UCC depends on attracting carriers; prices are based on volume and negotiated individually with the carriers. Subsidies were reserved for its first four years of operations, but the UCC was claimed to be financially sustainable after the second year, having attracted 50 carriers as users. However, its buildings and vehicles were heavily subsidized during its start up phase. Ville et al. (2013) study the case of Vicenza; this historic Italian city has a UCC that is funded by the municipality. The municipality imposes very strict regulations on carriers, namely a combination of (i) an access time restriction that allows carriers within the city only between 7.00 and $9.00 \mathrm{am}$, (ii) a mandatory permit for every carrier, and (iii) access bans for trucks that do not meet the environmental requirements. Due to these restrictions, carriers utilize the UCC, although they heavily oppose the manner in 
which regulation is used to give a strong competitive advantage to the UCC. Similar to Vicenza, the UCC of La Rochelle (France) is also based on restrictive regulations. The municipality sets access time restrictions for vehicles heavier than 3.5 tonnes; these vehicles are only allowed in the city between 6.00 and 7.30am (Browne et al., 2005; Van Duin et al., 2010). As stores generally open later, extra personnel needs to be dedicated to receive the goods at these times. The administrator financially supports the UCC, covering a substantial amount of the costs. Due to the restrictive regulation and the subsidies, the UCC succeeds in its environmental objectives. However, it is not financially sustainable. The final UCC that we discuss is Citylogistik-kbh in Copenhagen, Denmark (Gammelgaard, 2015). The city has an access time windows from 9.00-11.00am for trucks, but the vehicles of the UCC must also abide this time restriction. Thus, unlike the aforementioned initiatives, from a regulatory perspective the Copenhagen UCC has no competitive advantage over carriers regarding the last-mile distribution phase. Financial support was pledged to the UCC for three years, after which it is supposed to operate independently. However, the UCC already decided to go private after two years. Gammelgaard (2015) states that the UCC struggles to attract sufficient UCC users, as both receivers and carriers do not perceive sufficient added value.

As pointed out in this section, no consensus exists regarding successful business models for UCCs. The first literature contribution of this chapter is the identification of sustainable business models for UCCs. Our second contribution is the construction of receiver profiles based on real data. As there is a high variation between receivers in terms of order frequency, order volumes and demand for value-adding services, it is challenging to create realistic test instances. Based on a combination of data collection and expert interviews, we create multiple profiles with accompanying parameters to address this challenge.

\subsection{Solution method}

To evaluate a variety of urban logistics schemes, we make use of the agentbased simulation framework introduced in Chapter 4 . The objective of the simulation study in this chapter is to identify urban logistics schemes that (i) reduce the environmental impact of freight transport in the city center, (ii) are based on a financially sustainable business model, and (iii) incentivize commitment of the actors involved. The agent types included in the model are receivers, carriers, the UCC, and the local administrator. To reflect the divergent goals of these agents, every agent pursues its own objectives within the constraints of the system. 
At the heart of the model is a discrete-event simulation over a finite decision horizon, with $\mathcal{T}=\{0,1, \ldots, T\}$ representing the set of decision moments. The decision moments are separated by equidistant time intervals that each represent one day. We distinguish between three levels of decision making (strategic, tactical, and operational); we discuss the different levels of decision making in more detail in Section 6.3.2. Strategic decisions are fixed at the start of the simulation, i.e., at $t=0$. Tactical decisions can only be made at a limited set of decision moments $\mathcal{T}^{t a c} \subset \mathcal{T}$ - for this study we set the interval between adjacent tactical decision moments equal to two months - and represent a commitment for a time period of medium length. Thus, decisions made at a given tactical decision moment are fixed until the subsequent tactical decision moment. Finally, orders (i.e., goods demanded by the receivers) are randomly generated at every decision moment $t \in \mathcal{T}$, upon which all agents make their operational decisions. In Section 6.3.3, we describe the cost functions and KPIs of the agents.

We denote the set of receivers by $\mathcal{R}$, the set of carriers by $\mathcal{C}$, and the UCC by $h$. To individual agents we refer as $r \in \mathcal{R}$ and $c \in \mathcal{C}$, respectively. The city of Copenhagen is represented by the graph $\mathcal{G}=\{\mathcal{V}, \mathcal{A}\}$, with the vertex set $\mathcal{V}$ containing both the UCC location and the retailer locations, and the arc set $\mathcal{A}$ connecting the vertices. The travel time between any pair of vertices is obtained with OpenStreetMap (OSM Foundation, 2017).

In our model, we make a number of key assumptions. We assume that (i) all orders that are generated at a given decision moment are delivered before the subsequent decision moment, (ii) all agents update their tactical decisions at the same decision moments, (iii) order frequencies and order volumes of receivers cannot be influenced during the simulation, (iv) receivers and carriers make their decisions whether to join the UCC independent of each other, and (v) UCC prices and costs decrease when the amount of volume that is handled by the UCC increases. Assumptions (i) and (ii) are made for the sake of computational speed. In addition, assumption (ii) ensures that agents are not able to react on the commitment decisions of other agents, i.e., the assumption assures a level playing field. Furthermore, assumption (iii) is based on the notion that receivers are subject to inventory constraints and the demand by end-consumers, (iv) reflects an absence of cooperation efforts and a lack of knowledge regarding the actions of competitors, and (v) implies that economies of scale improve the efficiency of the UCC. We note that the assumptions imply a non-cooperative problem structure, in which agents do not cooperate and gains are non-transferable to other agents. The remainder of the chapter should be viewed in this light. 
6.3.1. Outline of the simulation model. In this section, we describe the general outline of the simulation model. We start by introducing the notation required to define the problem state. Let $l \in \mathcal{L}=\left\{\frac{1}{y}, \ldots, 1\right\}$ be the volume of an order, with $y \in \mathbb{N}$. The element $l$ represents volume in terms of the vehicle capacity of the smallest vehicle type that is defined in the simulation, such that $l=1$ equals a full truckload of this vehicle type. It follows that every order can be transported by any vehicle. A unique combination of carrier, receiver, and volume represents the order type $[c, r, l]$; only these properties of the order are required for the simulation model. The number of orders of a given type is denoted by $I_{t, c, r, l} \in \mathbb{N}$. Now, let $I_{t}=\left[I_{t, c, r, l}\right]_{\forall[c, r, l] \in \mathcal{C} \times \mathcal{R} \times \mathcal{L}}$ be a vector that provides the number of orders per order type demanded at time $t$. All orders placed at decision moment $t$ are delivered before $t+1$, e.g., within one day. Every combination of numbers per order type demanded represents a unique order arrival; let $\Omega_{t}$ be the set of all possible order arrivals at decision moment $t$. We repres-

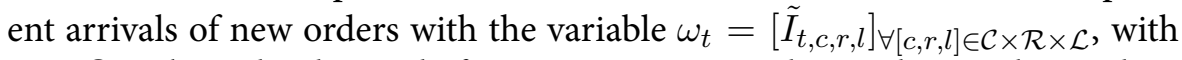
$\omega_{t} \in \Omega_{t}$. The order demand of receivers is generated according to the random variable $W_{t}$, with $\omega_{t}$ representing a simulated realization of $W_{t}$. As all orders in the system at $t$ are delivered before the next decision moment $t+1$, orders from previous decision moments have no impact on the system. Thus, at every decision moment $t \in \mathcal{T}$, we update the orders in the system as follows:

$$
I_{t, c, r, l}=\tilde{I}_{t, c, r, l} \quad \forall[c, r, l] \in \mathcal{C} \times \mathcal{R} \times \mathcal{L},
$$

Based on the order arrivals, both the UCC and the carriers determine their delivery routes. To determine which orders should be shipped via the UCC, we keep track of the agents that have committed themselves to use the UCC. If a carrier or receiver commits to the UCC, this means that all its shipments are handled by the UCC. The binary variable $\gamma_{t, r}^{r e c, t r} \in\{0,1\}$ represents whether receiver $r$ makes use of the base service of the UCC at time $t$; the vector $\gamma_{t}^{r e c, t r}=$ $\left[\gamma_{t, r}^{r e c, t r}\right]_{\forall r \in \mathcal{R}}$ stores this information for all receivers. The variable $\gamma_{t, r}^{r e c, v a l} \in$ $\{0,1\}$ and the vector $\gamma_{t}^{r e c, v a l}=\left[\gamma_{t, r}^{r e c, v a l}\right]_{\forall r \in \mathcal{R}}$ have a similar purpose, but instead describe whether the receiver outsources its value-adding services to the UCC. For a receiver to outsource its value-adding services, it must be pay the fee for the base service as well, i.e., $\gamma_{t, r}^{r e c, v a l}$ can have a value of 1 if and only if $\gamma_{t, r}^{r e c, t r}=1$. Finally, the variable $\gamma_{t, c}^{c a r} \in\{0,1\}$ and the corresponding vector $\gamma_{t}^{c a r}=\left[\gamma_{t, c}^{c a r}\right]_{\forall c \in \mathcal{C}}$ describe whether the carrier outsources its last-mile transport to the UCC. 
To reflect economies of scale that can be achieved by the UCC, various price functions and cost functions of the UCC are updated based on the ratio between the volume that passes through the UCC and the total volume that enters the city. We discuss this updating procedure in Section 6.3.2; for the definition of the problem state it suffices to introduce the notation for the volume ratio. This ratio is required for the tactical decisions of the UCC, and is therefore an element of the state. Let $l_{t^{\prime}, t^{\prime \prime}}^{u c c} \in[0,1]-$ with $t^{\prime}, t^{\prime \prime} \in \mathcal{T}^{t a c}$ and $t^{\prime}<t^{\prime \prime}$ - be the volume handled by the UCC in the period between the most recent tactical decision moment $t^{\prime \prime}$ and the second most-recent tactical decision moment $t^{\prime}$, divided by the total order volume entering the city during the same time period.

We have now introduced all elements necessary to define the problem state. The problem state is comprised of five elements: the vector of orders $I_{t}$, the vector of receivers that use the base transport service of the UCC $\gamma_{t}^{r e c, t r}=$ $\left[\gamma_{t, r}^{r e c, t r}\right]_{\forall r \in \mathcal{R}}$, the vector of receivers that outsource their value-adding services to the UCC $\gamma_{t}^{r e c, v a r}=\left[\gamma_{t, r}^{r e c, v a r}\right]_{\forall r \in \mathcal{R}}$, the vector of carriers that use the UCC $\gamma_{t}^{c a r}=\left[\gamma_{t, c}^{c a r}\right]_{\forall c \in \mathcal{C}}$, and the volume ratio $l_{t^{\prime}, t^{\prime \prime}}^{u c c}$, the price function for the receivers. We denote the problem state at time $t$ as

$$
S_{t}=\left[I_{t}, \gamma_{t}^{r e c, t r}, \gamma_{t}^{r e c, v a l}, \gamma_{t}^{c a r}, l_{t^{\prime}, t^{\prime \prime}}^{u c c}\right]
$$

6.3.2. Agent intelligence. In this subsection, we describe the agent intelligence embedded in the model. Small and independent actors will typically not use state-of-the-art algorithms; we reflect this practice by representing the decision processes of actors with relatively simple heuristics. As explained in the previous section, we distinguish between decisions made on the strategic, tactical and operational level, which we separately discuss here. Figure 6.1 provides a flowchart of the simulation model, describing the sequence of the decisions that are made by the various agent types.

In the model, strategic decisions are made only by the administrator. These long-term decisions are fixed at $t=0$ and involve fixing the subsidy levels, setting the length of the subsidy period, determining the accessibility measures to the city, and setting the policy costs. We test one accessibility measure, namely an access time window; large trucks may only drive in the environmental zone within this window. Furthermore, we set one cost measure, which is the zone access fees, i.e., a fixed fee per large truck entering the environmental zone of the city. As the UCC uses small trucks, it is exempted from both measures. 


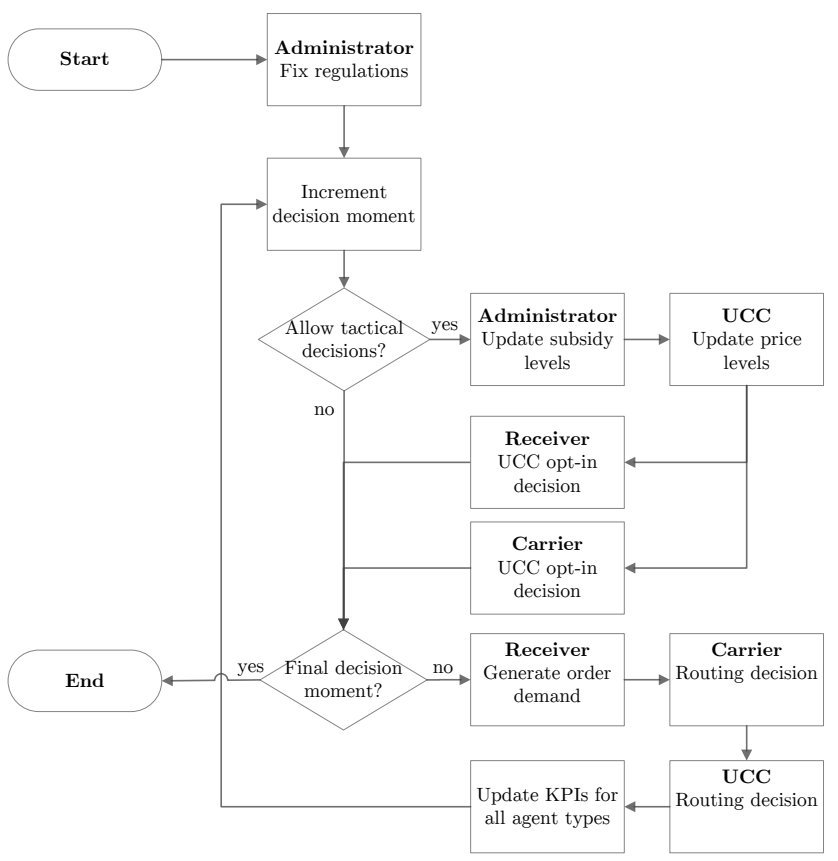

FIGURE 6.1. Flowchart of the simulation model.

The tactical decisions are made at the decision moments $t \in \mathcal{T}^{t a c}$; these decisions are made sequentially by the administrator, the UCC, and then (in parallel) by the receivers and carriers. First, the administrator may alter its subsidy levels. Subsidy levels are expressed as a percentage of the price charged by the UCC and function as a discount on the prices charged to the carriers and receivers. For example, when the carrier receives a $20 \%$ subsidy, this reduces the price it must pay to the UCC by $20 \%$. Subsidies allocated to the UCC are reflected as a discount in the prices charged to both the receivers and the carriers. Furthermore, when the administrator allocates subsidies to multiple agent types, their effect is cumulative, e.g., when both the UCC and carrier are subsidized for $20 \%$, the price reduction is $40 \%$ for the carriers and $20 \%$ for the receivers. The subsidy percentages are fixed at the start of the simulation, and are always set to $0 \%$ after the subsidy period has ended. 
Second, the cost levels and price levels of the UCC are altered. We adjust these levels based on the volume ratio $l_{t^{\prime}, t^{\prime \prime}}$, which was defined in Section 6.3.1. By varying the costs and prices for the UCC based on the volume it handles, we reflect economies of scale that are achieved by handling larger volumes. Here we focus on the updating procedures; the cost and price variables (or functions) themselves are explained in more detail in Section 6.3.3. At each tactical decision moment, we update three price variables (i) $P_{t}^{u c c, c a r, t r}$ (the price charged to the carrier for outsourcing its last-mile distribution, a fixed fee per outsourced delivery stop that is identical for each carrier), (ii) $P_{t}^{u c c, r e c, t r}$ (the fixed fee for the base service of bundled deliveries as charged to the receiver, identical for each receiver), (iii) $P_{t}^{u c c, v a l}$ (a receiver-dependent fee for performing value-adding services), and two cost variables, (iv) $C_{t}^{u c c, h d}$ (the volume-based costs for handling goods at the UCC), and (iv) $C_{t}^{u c c, v a l}$ (a receiver-specific cost to perform valueadding services). For each of the aforementioned price and cost variables, we define a range that contains the values that the variable can take. We express the volume handled by the UCC as the ratio of the total volume that enters the city (i.e., the cumulative volume of the target group). A ratio of 0 corresponds to the highest cost and pricelevels, a ratio of 1 to the lowest levels. Within the ranges, we assume a linear relation between costs/prices and the volume ratio. We provide an example of the updating procedure for the price variable $P_{t}^{u c c, r e c, t r}$; the other cost and price variables are updated in a similar manner. Let $P^{u c c, r e c, t r, \max }$ be the upper price bound and $P^{u c c, r e c, t r, m i n}$ be the lower price bound. The volume ratio $l_{t^{\prime}, t^{\prime \prime}}$ determines the price level within this range. We update receiver prices as follows:

$$
P_{t}^{u c c, r e c, t r}=\left(1-l_{t^{\prime}, t^{\prime \prime}}\right) \cdot P^{u c c, r e c, t r, \max }+l_{t^{\prime}, t^{\prime \prime}} \cdot P^{u c c, r e c, t r, \min } .
$$

After adjusting the subsidies, cost levels, and price levels, the receivers and carriers independently and in parallel decide whether or not to commit to the UCC. For any agent that chooses to use the UCC, the UCC becomes responsible for the last-mile distribution of all the agent's goods, until at least the next tactical decision moment. The decision to opt-in or opt-out is based on the expected future costs of both options, given the updated subsidy levels, and assuming that the agent unilaterally changes its decision. To compute the expected future costs at a given tactical decision moment, we first generate $N$ sample paths of order arrivals that stretch $\tau^{\text {sample }}$ decision moments into the future, with $n \in\{1, \ldots, N\}$ being the index for the sample path and $t_{n} \in\left\{1, \ldots, \tau^{\text {sample }}\right\}$ being the time 
index for the sample states of path $n$. For every $n \in\{1, \ldots, N\}$, we obtain a set of sample states $\left\{\tilde{S}_{t+1_{n}}, \ldots, \tilde{S}_{t+\tau_{n}}^{\text {sample }}\right\}$.

In each sample state $\tilde{S}_{t+t_{n}}$, we keep all but one binary variable at the same level as in $S_{t}$, i.e., for each agent we base the forecasts on the UCC commitments as they are before the update. We introduce the help variables $\tilde{\gamma}_{r}^{r e c, t r} \in$ $\{0,1\}, \tilde{\gamma}_{r}^{r e c, v a l} \in\{0,1\}$, and $\tilde{\gamma}_{c}^{c a r} \in\{0,1\}$. Adjusting the value of these variables allows us to compute cost forecasts for both the case in which it uses the UCC and the case in which it does not commit to the UCC. Based on the generated sample states, we compute the expected costs with the cost functions $\tilde{C}^{r e c}\left(\tilde{S}_{t+t_{n}}, \tilde{\gamma}_{r}^{r e c, t r}, \tilde{\gamma}_{r}^{r e c, v a l}\right)$ and $\tilde{C}^{c a r}\left(\tilde{S}_{t+t_{n}}, \tilde{\gamma}_{c}^{c a r}\right)$; these functions are similar to the functions that we define in Section 6.3.3. Computing the costs for the sets of sample states yields the expected future costs for both the case in which the agent unilaterally decides to use the UCC and the case in which the agent opts for direct transport. Minimizing the expected future costs yields the updated tactical decision. Equation (6.3.3) shows how we update the tactical decision for each receiver $r \in \mathcal{R}$, Equation (6.3.4) shows the same for each carrier $c \in \mathcal{C}$.

$$
\begin{array}{r}
{\left[\gamma_{t, r}^{\text {rec }, \text { tr }}, \gamma_{t, r}^{\text {rec }, v a l}\right]=} \\
\underset{\tilde{\gamma}_{r}^{r e c, t r} \in\{0,1\}, \tilde{\gamma}_{r}^{r e c, v a l} \in\{0,1\}}{\operatorname{argmin}} \frac{1}{N} \sum_{n=1}^{N} \sum_{t_{n}=1}^{\tau^{\text {sample }}} \tilde{C}^{r e c}\left(\tilde{S}_{t+t_{n}}, \tilde{\gamma}_{r}^{\text {rec }, t r}, \tilde{\gamma}_{r}^{\text {rec }, v a l}\right) \\
\gamma_{t, c}^{\text {car }}=\underset{\tilde{\gamma}_{c}^{\text {car }} \in\{0,1\}}{\operatorname{argmin}} \frac{1}{N} \sum_{n=1}^{N} \sum_{t_{n}=1}^{\tau^{\text {sample }}} \tilde{C}^{\text {car }}\left(\tilde{S}_{t+t_{n}}, \tilde{\gamma}_{c}^{\text {car }}\right) .
\end{array}
$$

We now discuss the operational decisions, which are made at every decision moment $t \in \mathcal{T}$. Based on the realization of the random variable $W_{t}-$ which translates into receivers placing orders - shipments are assigned to carriers. Both carriers and the UCC make routing decisions for the last-mile distribution. We use the Clarke-Wright savings algorithm to construct routes, followed by a 2 -opt improvement heuristic. Such an approach is similar to the routing algorithms that are often applied in practice (Quak and de Koster, 2009). We represent the resulting routes as follows. Let $\mathcal{Q}^{u c c}$ be the set of vehicles operated by the UCC, with $q \in \mathcal{Q}^{u c c}$ referring to an individual vehicle. The vehicle notation for carriers is similar. The delivery route of vehicle $q$ within the city is an ordered set of arcs, denoted by $\delta_{t, q}^{u c c}$ ( $\delta_{t, c, q}^{c a r}$ for carriers). When generating routes, we take into account the capacity of the vehicle and possible access time 
restrictions. To satisfy these restrictions, an agent may need to dispatch multiple vehicles at a single decision moment, which results in multiple routes performed by a single agent; sets of routes are denoted by $\Delta_{t}^{u c c}$ and $\Delta_{t, c}^{c a r}$ respectively. Finally, we use $\Delta_{t}=\Delta_{t}^{u c c} \cup \bigcup_{c \in \mathcal{C}} \Delta_{t, c}^{c a r}$ to denote the set of all routes executed at $t$.

6.3.3. Cost functions and KPIs. To quantify the results of the study, we monitor both environmental performance and financial performance. We measure environmental performance by three sets of indicators. First, we measure global emissions $\left(\mathrm{CO}_{2}\right)$. These emissions have a negative environmental impact, yet their effects are not restricted to the city boundaries. Second, we measure local emissions $\left(\mathrm{SO}_{2}, \mathrm{NO}_{\mathrm{x}}, \mathrm{PM}_{2.5}\right)$; these emissions directly affect health and environment in urban areas. Third, we measure two vehicle-related performance indicators, namely the number of vehicles in the urban area (itemized per vehicle type) and the distance covered per vehicle type. These indicators serve as a proxy for external costs that cannot be accurately measured in the model, such as noise hindrance, traffic safety, and influence on congestion. 


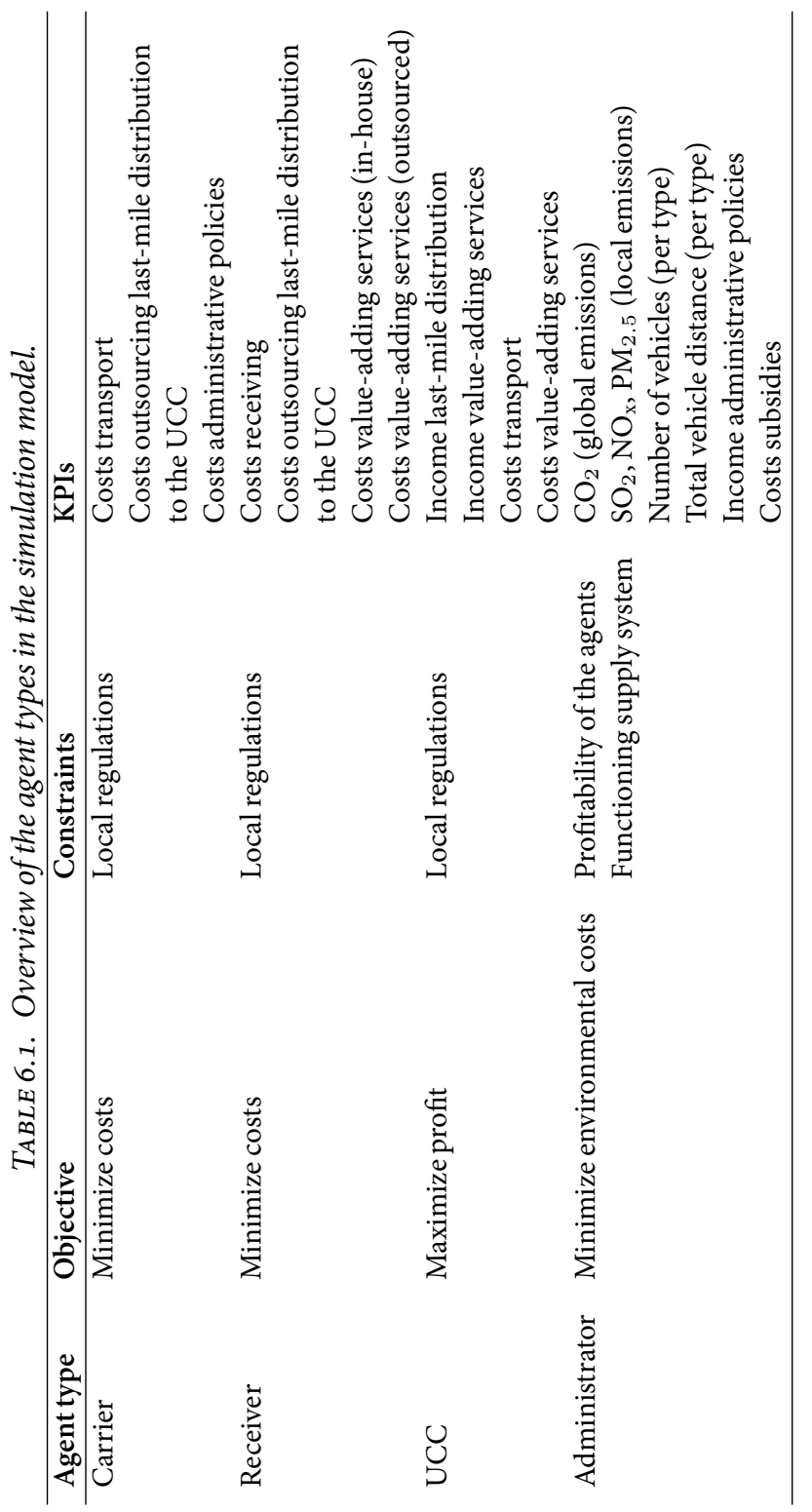


The four different agent types, along with their objectives, constraints and KPIs, are listed in Table 6.1. We now proceed to formalize the cost functions of the agents. We only introduce the notation required for a general understanding of the model; for a more detailed representation we refer to Chapter 4 .

We start by defining the cost function for the carriers. To distinguish between routes that visit all delivery addresses and routes that only visit the UCC, we again use the help variable $\tilde{\gamma}_{c}^{c a r}$. Let $\Delta_{t, c}^{c a r} \mid \tilde{\gamma}_{c}^{c a r}=0$ be the route set corresponding to the case in which the carrier visits all its delivery addresses (possibly including the UCC) itself, and let $\Delta_{t, c}^{c a r} \mid \tilde{\gamma}_{c}^{c a r}=1$ correspond to the case in which the carrier only visits the UCC. The function $C^{c a r, t r}(\cdot)$ returns the transport costs for a given route set, including the unloading costs at the receivers. With $C^{c a r, t r}\left(\Delta_{t, c}^{c a r} \mid \tilde{\gamma}_{c}^{c a r}=0\right)$ we obtain the transport cost that correspond to the carrier visiting all its delivery addresses itself; $C^{c a r, t r}\left(\Delta_{t, c}^{c a r} \mid \tilde{\gamma}_{c}^{c a r}=1\right)$ yields the transport costs if the carrier only visits the UCC. If the carrier outsources its lastmile distribution, the only destination of the route is the UCC. The information embedded in the route set suffices to compute the total travel time, the number of receivers visited, and the zone access fees paid. If the carrier outsources its last-mile distribution, a fixed amount per stop must be paid to the UCC. These costs are represented by $P_{t}^{u c c, c a r, t r}\left(\Delta_{t, c}^{c a r} \mid \tilde{\gamma}_{c}^{c a r}=0\right)$ - the route notation contains the number of stops that are outsourced - and depend on the price charged by the UCC at time $t$. Finally, $P_{t}^{c a r, u c c, s b}\left(\Delta_{t, c}^{c a r} \mid \tilde{\gamma}_{c}^{c a r}=0\right)$ denotes the subsidy that the carrier receives when using the UCC, which is a fixed percentage of the price per stop it pays to the UCC. The cost function of carrier $c \in \mathcal{C}$ at time $t$ is given by

$$
\begin{array}{r}
C_{t}^{c a r}\left(\gamma_{t, c}^{c a r}, \Delta_{t, c}^{c a r}\left|\tilde{\gamma}_{c}^{c a r}=0, \Delta_{t, c}^{c a r}\right| \tilde{\gamma}_{c}^{c a r}=1\right)= \\
\left(1-\gamma_{t, c}^{c a r}\right) C^{c a r, t r}\left(\Delta_{t, c}^{c a r} \mid \tilde{\gamma}_{c}^{c a r}=0\right)+\gamma_{t, c}^{c a r} C^{c a r, t r}\left(\Delta_{t, c}^{c a r} \mid \tilde{\gamma}_{c}^{c a r}=1\right)+ \\
\gamma_{t, c}^{c a r} P_{t}^{u c c, c a r, t r}\left(\Delta_{t, c}^{c a r} \mid \tilde{\gamma}_{c}^{c a r}=0\right)-\gamma_{t, c}^{c a r} P_{t}^{c a r, u c c, s b}\left(\Delta_{t, c}^{c a r} \mid \tilde{\gamma}_{c}^{c a r}=0\right) .
\end{array}
$$

The costs incurred by the receiver are comprised of five elements: (i) the receiving costs $C^{r e c, r c}\left(r, \Delta_{t}^{u c c}, \bigcup_{c \in \mathcal{C}} \Delta_{t, c}^{c a r}\right)$, which depend on the number of vehicles that visit its premises and - in case of visiting carrier trucks only whether shifted access windows are imposed, (ii) the receiver-specific costs for performing value-adding services in-house $C^{r e c, v a l}(r)$, (iii) the costs for outsourcing last-mile transport to the UCC $P_{t}^{u c c, r e c, t r}$ (the base service of bundled deliveries, for which the UCC charges the same fixed fee to every receiver), (iv) the receiver-specific costs for outsourcing value-adding services to the UCC 
$P_{t}^{u c c, v a l}(r)$, and (v) the subsidy income when using the UCC $P_{t}^{r e c, u c c, s b}$, which is a fixed percentage of the price that is paid to the UCC for the base service. The cost function of receiver $r \in \mathcal{R}$ at time $t$ is

$$
\begin{array}{r}
C_{t}^{r e c}\left(r, \gamma_{t, r}^{r e c, t r}, \gamma_{t, r}^{r e c, v a l}, \Delta_{t}\right)= \\
C^{r e c, r c}\left(r, \Delta_{t}^{u c c}, \bigcup_{c \in \mathcal{C}} \Delta_{t, c}^{c a r}\right)+\left(1-\gamma_{t, r}^{r e c, v a l}\right) C^{r e c, v a l}(r) \\
+\gamma_{t, r}^{r e c, t r} P_{t}^{u c c, r e c, t r}+\gamma_{t, r}^{r e c, v a l} P_{t}^{u c c, v a l}(r)-\gamma_{t, r}^{r e c, t r} P_{t}^{r e c, u c c, s b} .
\end{array}
$$

For the UCC, transport costs are calculated similarly to those of the carriers and are denoted by $C^{u c c, t r}\left(\Delta_{t}^{u c c}\right)$. The remainder of the costs and prices of the UCC are time-varying. The handling costs of incoming orders for the UCC are denoted by $C_{t}^{u c c, h d}\left(\bigcup_{c \in \mathcal{C}} \Delta_{t, c}^{c a r}\right)$ and the costs for performing value-adding services are given by $C_{t}^{u c c, v a l}(r)$. The prices charged by the UCC are the price charged per outsourced stop to the carrier $P_{t}^{u c c, c a r, t r}\left(\Delta_{t, c}^{c a r} \mid \tilde{\gamma}_{c}^{c a r}=0\right)$, the price for the base service charged to the receiver $P_{t}^{u c c, r e c, t r}$, and the price to perform the value-adding services for a receiver $P_{t}^{u c c, v a l}(r)$. Finally, the UCC may receive a subsidy $P_{t}^{u c c, s b}$, which is a percentage of the total prices charged to the carriers and the receivers (only for the base service, not the value-adding services). The cost function of the UCC is as follows:

$$
\begin{array}{r}
C_{t}^{u c c}\left(\gamma_{t}^{r e c, t r}, \gamma_{t}^{r e c, v a l}, \gamma_{t}^{c a r}, \Delta_{t}\right)= \\
C^{u c c, t r}\left(\Delta_{t}^{u c c}\right)+C_{t}^{u c c, h d}\left(\bigcup_{c \in \mathcal{C}} \Delta_{t, c}^{c a r}\right)+ \\
\sum_{r=1}^{|\mathcal{R}|} \gamma_{t, r}^{r e c, v a l} C_{t}^{u c c, v a l}(r)-\sum_{c=1}^{|\mathcal{C}|} \gamma_{t, c}^{c a r} P_{t}^{u c c, c a r, t r}\left(\Delta_{t, c}^{c a r} \mid \tilde{\gamma}_{c}^{c a r}=0\right)- \\
\sum_{r=1}^{|\mathcal{R}|} \gamma_{t, r}^{r e c, t r} P_{t}^{u c c, r e c, t r}-\sum_{r=1}^{|\mathcal{R}|} \gamma_{t, r}^{r e c, v a l} P_{t}^{u c c, v a l}(r)-P_{t}^{u c c, s b} .
\end{array}
$$

The final agent type is the administrator. For this agent, we do not define an explicit cost function; this would require either monetizing the environmental 
costs, or assigning weights to the different objectives. Instead, from the simulation output we can interpret how the administrator performs on its various KPIs. Although we monitor the financial performance of the administrator as we want to allocate subsidies as efficiently as possible - we assume that cost minimization is not a target in itself. Instead, financial expenses are a means to improve the environmental performance.

\subsection{Experimental setup}

In this section, we describe the setup of the simulation study. The goal of the study is to identify schemes that significantly reduce the environmental impact of freight transport by using the UCC. As high costs are the main barrier for a starting UCC, we consider the use of subsidies and regulations to encourage the use of the UCC in the startup phase. To this end, we test a variety of measures to support the UCC. We enforce that subsidies are only temporary; after two years the UCC must be able to operate independently. This implies that the UCC has limited time to create a sufficiently large user base. The UCC should reach a critical mass such that the operational costs are low enough to be able to offer competitive prices to its users. Each simulation runs represents a period of five years; the performance in the last two years is used to evaluate whether the UCC achieves the desired performance. Thus, unless stated otherwise, the results shown in this chapter relate to the final two years of the simulation.

Of particular interest is the sequence in which users are attracted. If the receiver selects the UCC, the carrier supplying this receiver essentially outsources its last-mile distribution without costs. From this perspective, it makes sense to first generate commitment from the carriers and obtain an income from them; this still leaves open the opportunity for receivers to pay for value-adding services. Receivers, on the other hand, may be easier to convince to use the UCC, as their perceived benefits (including value-adding services) are usually greater than for the carrier. We test various subsidy allocations to observe whether this affects the sequence in which users commit to the UCC.

6.4.1. Validation. The aim of this study is to provide insights into good business models for a UCC. To achieve this goal, the setup of the study should be closely related to practice. In this setup, we discuss the steps that we have taken to validate the match between the simulation model and the real world.

The problems that we study are motivated by practice and affirmed by the propositions posed in literature. Also, the measures that we evaluate are existing in the real-world. For the default setting, we consider the measures that are currently in effect in the city of Copenhagen. The other measures that we test 
are implemented or have been implemented in other Western-European cities; it is conceivable that these measures are implemented in Copenhagen as well. To select appropriate levels for the parameters and variables in the simulation model, we collect data both from a variety of literature sources and directly from industry; we provide a detailed description of the data collection in Section 6.4.3.

To validate both the data and the experimental setup, we conducted expert interviews with two parties. The first expert represents Binnenstadservice (Dutch for 'Inner City Service'), which operates 15UCCs in the Netherlands. The second expert is from the municipality of Copenhagen, who is involved with local regulations and logistics initiatives.

To present the virtual UCC in a realistic manner, we draw upon some properties of Citylogistik-kbh, the real UCC operating in Copenhagen. Despite similarities such as the physical location, the UCC that we study in this chapter is a fictive one; Citylogistik-kbh was not involved in this research.

6.4.2. Test instance. In this section, we provide the context of urban freight transport in Copenhagen. The current measures applied in Copenhagen are used to define the default scheme; the performance under this scheme is used as a benchmark to evaluate the effects of alternative measures.

Copenhagen is the capital of Denmark and is located on the island of Zealand. The city itself has about 600,000 inhabitants, whereas over 2 million people live in the metropolitan area. Copenhagen has a medieval city center with an area of $1 \mathrm{~km}^{2}$ (Geroliminis and Daganzo, 2005). About 500 retailers are located in this area; on a daily basis 6,000 trucks enter the center. Trucks may only visit stores in this area between 9.00 and 11.00am. The larger low-emission zone harbors approximately 2,000 retailers; trucks require a certificate to enter this zone. To be eligible to obtain the certificate, a truck must either be equipped with an effective particle filter or meet Euro 4 standards or higher. Currently, the city of Copenhagen charges $€ 12.5$ for the certificate, which is valid during the entire lifetime of the vehicle. The administrator is actively involved in reducing the impact of urban freight transport and has implemented or considered various interventions in the past. In 2002, Copenhagen implemented a zone access fee scheme, based on vehicle properties and average capacity utilization (Geroliminis and Daganzo, 2005). The city was also involved in starting Citylogistik-kbh, which like many other UCCs struggles to generate a sufficiently high throughput. The vast majority of goods destined for the city center (i.e., goods originating from other regions in Denmark or from elsewhere in Europe) arrives via the E20, a highway located south of Copenhagen. Citylogistik-kbh is located close to this highway; we assume the same location for the fictive UCC. In Figure 6.2, we 
highlight the key characteristics of the case instance. Using the properties of the city of Copenhagen helps to construct a realistic test instance, which we use to test various urban logistics schemes.

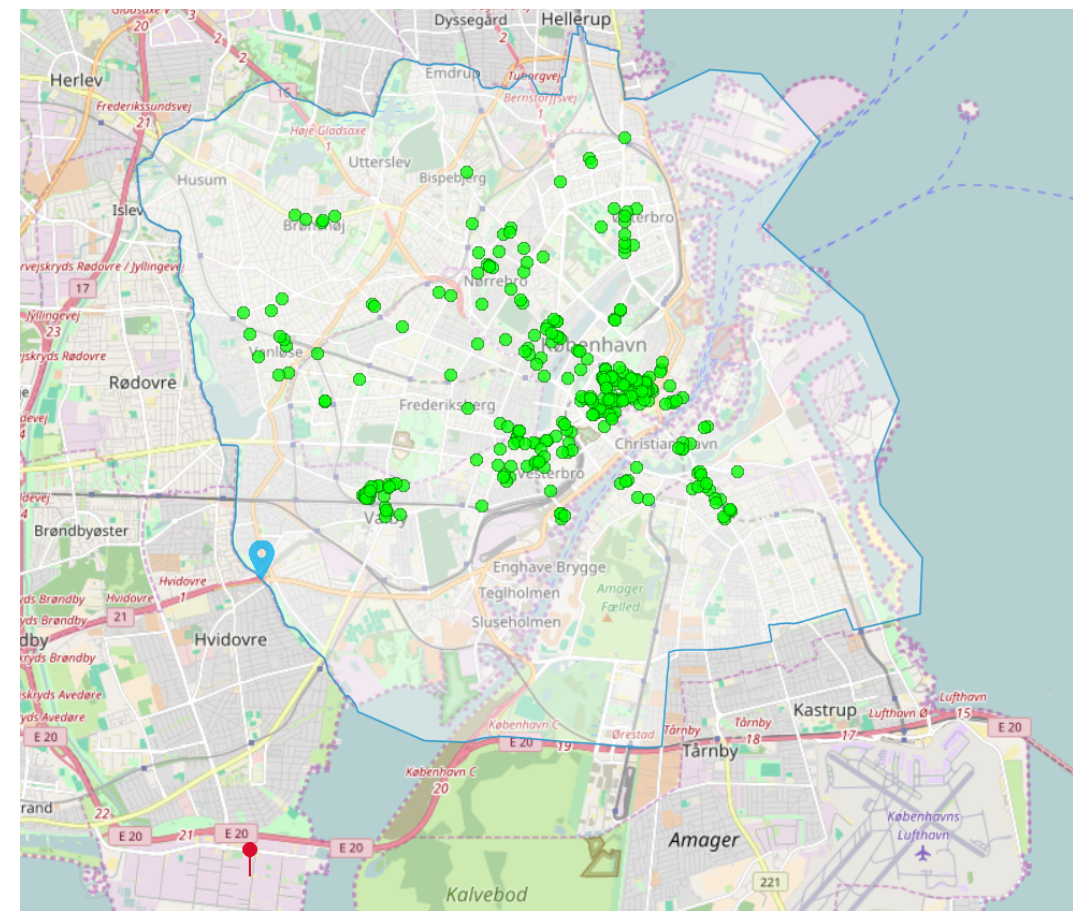

FIGURE 6.2. Map of Copenhagen, indicating the low-emission zone (shaded area), the UCC location (red pin marker), the entrance point for carriers arriving via the E20 (large blue marker), and the receiver locations (green dots).

6.4.3. Data collection. Our primary data sources are documents resulting from two large European projects on urban freight transport, namely the Green Logistics project and the BESTUFS project (Browne et al., 2005; Schoemaker et al., 2006; Allen et al., 2008). Both projects aggregated real-life data from many different cities and sources. We complement our data set with a number of smaller studies, as well as secondary sources of publicly available data. To obtain data that best represents the case of Copenhagen, we restrict ourselves to the data available for Western European cities. Furthermore, we exclude data 
corresponding to freight flows that do not fit the scope of this study, such as full truckload transport, construction logistics, and transport of perishable goods.

The data sources reveal great variations in urban logistics metrics, while some parameters lack proper documentation. This makes it challenging to select representative parameters for the simulation study. For certain parameters, we must therefore make justified assumptions on what our representation of reality looks like. The data set that we constructed has been validated during the expert interviews; various parameters were altered to obtain a closer fit with practice. We proceed to discuss the data set in the remainder of this section.

6.4.3.1. Network design. We obtain retailer locations in the low-emission zone from OpenStreetMap. To create a list of retailer locations, we looked for all addresses within the area labeled as 'shop'. Many shops do not fall into the target group of a typical UCC, which focuses on non-perishable goods that do not require any special sort of care or handling. For example, transporting food would require cooling installations both in the UCC and in the trucks, whereas the transport of jewelry brings a high risk of theft. To distill a representative target group for the UCC, we removed stores falling into the following categories: (i) food (e.g., seafood), (ii) high-value goods (e.g., jewelers), (iii) breakable goods (e.g., glaziers), (iv) fresh goods (e.g., florists), and (v) services (e.g., hairdressers). After this selection, we are left with a set of 1071 retailers. This set consists primarily of (i) fashion (clothes and shoes), (ii) bicycle stores, (iii) convenience stores and kiosks, and (iv) specialized stores (e.g., consumer electronics, sports stores). It is unrealistic to assume that freight flows related to all these retailers might be handled by the UCC. For example, Van Duin et al. (2010) state that a participation of about $10 \%$ is a realistic figure for UCCs. A successful example, such as the UCC in La Rochelle - which is heavily supported by regulations and subsidies - handles $30 \%$ of freight transport to the city (Browne et al., 2005). We adopt the figure of $30 \%$ as an upper bound for the size of the target group in the simulation study. Hence, to generate the test instance, we randomly select $30 \%$ of the retailers from the set of shops, leaving us with 321 retailer locations. We use the OpenStreetMap routing implementation of Luxen and Vetter (2011) to compute the travel times between all origin-destination pairs, adopting a driver profile that reflects a vehicle driving close to the maximum allowed speed. The generated travel times take into account factors such as the configuration of the street network, speed limits, and restrictions such as one-way streets. Variations in travel time during the day are not accounted for.

6.4.3.2. Receiver properties. The properties of the individual retailers are not directly available; both literature and expert interviews emphasize that the 
order patterns of retailers are subject to much variability. To model the receivers in a representative way, we collect aggregate data from literature. The order patterns and demands for value-adding services are highly unique. Rather than focusing on specific retail branches, we instead introduce various retailer profiles based on order patterns and demands for value-adding services. From the observed data, we distill the following properties that define a receiver profile: (i) average order volume, (ii) average order frequency, (iii) number of suppliers, and (iv) demand for value-added services.

The first three properties are related to each other, and they depend for a significant part on whether the receiver has a centralized or a decentralized supply system (Cherrett et al., 2012). In centralized supply systems, the receiver usually has a single supplier or logistics service provider that is responsible for all deliveries. Such supply chains tend to be characterized by relatively few deliveries and higher volumes, as consolidation already takes place upstream. To account for the correlation between these properties, we distinguish between receivers based on their supply system. Based on Cherrett et al. (2012), for centralized systems we estimate the average number of deliveries per week at 4.05 , whereas receivers with decentralized supply systems receive an average of 11.65 deliveries per week. We establish ranges from which we draw the order frequency and number of distinct shippers that the receiver orders at, these ranges are shown in Table 6.2. We assume that the ratio between receivers with centralized and decentralized supply chains is 50/50. In practice, the target group of the UCC may contain relatively more receivers with decentralized supply chains, which likely increases the profitability of the UCC due to the higher number of delivery stops made by the carriers. Thus, our assumption of a 50/50 ratio implies a safety margin for the results. Furthermore, we assume that every receiver has 3-5 ordering moments per week, but the centralized profiles (i-iii) place orders with one supplier at a time (averaging to $4 \approx 4.05$ deliveries per week), whereas decentralized profiles (iv-vi) place orders with 2-4 distinct suppliers at a single decision moment (averaging to $12 \approx 11.65$ deliveries per week). The order volumes are drawn from two empirical distributions (one for centralized and one for decentralized supply chains) that are based on the data of one of the Dutch UCC facilities.

Next, we consider the demand for value-adding services. To the best of our knowledge, there is no literature that quantifies the demand, cost levels and price levels for value-adding services. Our interviews reveal that demand is highly receiver-specific, both in terms of the required services and willingness to pay. Based on price data from the Dutch UCC, we categorize three levels of demands 
in terms of willingness to pay. Again, we distinguish between receivers with centralized and decentralized supply systems; both may be combined with one of three demand levels for value-adding services. This yields a total of six receiver profiles. In Table 6.2, we show the properties of the receiver profiles. Receiverspecific values are drawn randomly from the indicated ranges, assuming a uniform distribution.

Finally, based on Van Duin et al.(2010), we set the personnel costs for a retailer at $€ 15.3$ /hour. These costs are relevant to monetize the time a retailer spends on receiving goods, as well as the time a staff member must be present before the opening time of the shop in case of early deliveries. The average unloading time lies in the range of 7-34 minutes (Schoemaker et al., 2006; Allen et al., 2008). Contrary to what might be expected, deliveries of larger volumes do not translate into longer unloading times (Cherrett et al., 2012). As many factors (e.g., accessibility, handling equipment, quality checks) may influence both the total unloading time and the time the receiver itself is actually involved, we (i) randomly assign an unloading time to receivers from the indicated range (as experienced by the carrier) and (ii) randomly select a value between 2 minutes and the generated total unloading time to indicate how much time the receiver spends on unloading.

TABLE 6.2. Summary of the receiver profiles.

\begin{tabular}{lcccc}
\hline Profile & $\begin{array}{c}\text { Order frequency } \\
\text { per week }\end{array}$ & $\begin{array}{c}\text { \# orders placed per } \\
\text { ordering moment }\end{array}$ & $\begin{array}{c}\text { Demand value-adding } \\
\text { services per week }\end{array}$ & $\begin{array}{c}\% \text { of total } \\
\text { receivers }\end{array}$ \\
\hline i & {$[3-5]$} & {$[1]$} & {$[€ 0]$} & $10 \%$ \\
ii & {$[3-5]$} & {$[1]$} & {$[€ 6-20]$} & $37.5 \%$ \\
iii & {$[3-5]$} & {$[1]$} & {$[€ 60-150]$} & $2.5 \%$ \\
iv & {$[3-5]$} & {$[2-4]$} & {$[€ 0]$} & $10 \%$ \\
v & {$[3-5]$} & {$[2-4]$} & {$[€ 6-20]$} & $37.5 \%$ \\
vi & {$[3-5]$} & {$[2-4]$} & {$[€ 60-150]$} & $2.5 \%$ \\
\hline
\end{tabular}

6.4.3.3. Carrier properties. We proceed to describe the properties of carriers. First, we need to establish the number of carriers in the simulation model. With the used demand settings and distribution of receiver profiles, about 2500 deliveries per working week take place for the target group. Based on Browne et al. (2005), Allen et al. (2008), and Roca-Riu and Estrada (2012), we find that the average number of stops per carrier visiting a city is approximately 10 . As we are primarily interested in small, independent carriers, we select the number of carriers such that every carrier uses one truck on average for a delivery route. To achieve this average, we set the number of carriers in the simulation to 50 . The number of trucks actually deployed depends on the realization of order 
demand; a carrier may simultaneously deploy multiple trucks. As mentioned before, the total number of trucks entering Copenhagen is much higher; indeed most carriers and receivers would not fall in the target group of a UCC.

As the transport market is characterized by both high competition and high substitutability, we assume that all carriers are homogenous. We suppose that all carriers use medium-sized vehicles with a capacity of $28 \mathrm{~m}^{3}$. Emission data is obtained from Boer et al. (2011), based on engine standards that are set for the year 2020. The cost parameters are itemized in hourly costs (mainly driver's wage, including unloading time) and costs per $\mathrm{km}$ (diesel and depreciation). The corresponding values are obtained from Quak and de Koster (2009) and Roca-Riu et al. (2016), and can be found in Table 6.3. Recall that the average unloading time lies in the range of 7-34 minutes. Unlike the receiver, the driver is involved during the complete unloading process, hence the hourly costs are incurred for the duration of the process.

6.4.3.4. UCC properties. The operational costs of the UCC can be divided into two components, handling costs and transport costs. In the handling costs, we include the costs made at the facility in a broad sense, e.g., rent, insurance, equipment for material handling, and personnel hours. As these cost components are highly dependent on the setup of the UCC, it is difficult to accurately estimate handling costs. In the simulation model, we represent orders by means of volume. Therefore, when sources state handling costs per item rather than per volume unit (e.g., costs per parcel), assumptions on our part are required for the conversion from costs per item to costs per $\mathrm{m}^{3}$. Furthermore, the available figures may include a transport cost component that is not quantified; based on preliminary experiments, we estimate the transport costs for last-mile deliveries performed by the UCC at $€ 8$ per $\mathrm{m}^{3}$ (this figure is substantially higher than the costs estimated in Chapter 5 due to the smaller order volumes used in this chapter). We stress that we only use this figure to adjust the handling cost estimates of literature sources; in the simulation itself we compute the transport costs by solving its routing problems. The price levels and cost levels that are time-varying are updated as described in Section 6.3.2.

In a feasibility study, Van Duin et al. (2010) provide a detailed breakdown of the costs of a UCC, with the costs being related to the volumes handled by the UCC. Browne et al. (2005) provide several cost figures from UCCs in practice as well. By triangulating the estimates obtained from these sources with the expert estimates, we estimate the costs of goods handled at the hub. As indicated by Van Duin et al. (2010), handling costs strongly depend on the volumes handled and the corresponding economies of scale; we estimate handling costs 
at $€ 20 / \mathrm{m}^{3}$ for a UCC without any agents committed (i.e., the initialization of the handling costs) and $€ 7 / \mathrm{m}^{3}$ if all orders are delivered via the UCC. The updating procedure of the handling costs over time has been described in Section 6.3.2. Since data regarding the operational costs of UCCs is both scarce and subject to high variance, we test two additional cost ranges in our experiments, which are based on various literature sources.

We propose that the UCC uses small trucks for the last-mile distribution. Although smaller transport modes - such as bicycles and delivery vans - are often used for urban logistics initiatives, their inability to handle pallets and rolling containers has been identified as a hampering factor in binding UCC users (Van Duin et al., 2010). An additional effect is that due to the small load capacities, the number of vehicles required vastly increases. Heavy trucks are also unsuitable for last-mile distribution, given their negative impact on traffic and the environment. Hence, in line with Van Duin et al. (2010), we assume that the UCC operates a fleet of light trucks with a loading capacity of $18 \mathrm{~m}^{3}$. We use the same data sources as for the carrier; the vehicle parameters for light trucks can also be found in Table 6.3. Finally, we estimate that the upper bounds for the costs of the UCC to perform the value-adding services fall in the range of $70 \%$ to $95 \%$ of the costs the receiver makes to perform these services in-house, these upper bounds are generated randomly for each receiver. The lower bounds are equivalent to 0.8 times the upper bounds. Thus, in the best case, the UCC can perform the value-adding services at $0.8 \cdot 70 \%=56 \%$ of the in-house costs.

We set the prices imposed by the UCC based on data provided by the UCC and the expert interviews. The exact price levels and pricing methods cannot be disclosed for confidentiality reasons, yet the indicated ranges are representative for real life. Receivers always pay a monthly fee for the base service of the UCC (i.e., bundled deliveries); the fee is independent of location and the volumes delivered. We set the price range for the base service for receivers at $€ 60-70$ per month. Carriers that make use of the UCC must pay per outsourced delivery stop. The corresponding price range is set at $€ 12-18$ per stop. Finally, valueadding services are a significant source of income, the UCC makes a profit of $25 \%$ on these services. Thus, to compute the prices for these services, we set them at 1.25 times the costs that the UCC incurs to perform them.

6.4.3.5. Administrator properties. Aside from the implementation of regulations, the main design choice for the administrator is how to distribute subsidies. One might think of various distribution keys that encourage a certain behavior, e.g., subsidy based on the forwarded volume, the number of trucks, or time-varying subsidies. A good subsidy scheme should be in accordance with 
TABLE 6.3. Vehicle properties for the UCC (light truck) and carriers (medium-sized truck).

\begin{tabular}{lcc}
\hline Vehicle type & Light truck & Medium-sized truck \\
\hline Load capacity $\left(\mathrm{m}^{3}\right)$ & 18 & 28 \\
Driver's wage $(€ /$ hour $)$ & 21 & 21 \\
Costs urban transport $(€ / \mathrm{km})$ & 0.72 & 0.86 \\
(excluding driver's wage) & & \\
$\mathrm{CO}_{2}(\mathrm{~g} / \mathrm{km})$ & $455-553$ & $821-1,065$ \\
$\mathrm{SO}_{2}(\mathrm{mg} / \mathrm{km})$ & $3.5-4.2$ & $6.3-8.1$ \\
$\mathrm{NO}_{\mathrm{x}}(\mathrm{g} / \mathrm{km})$ & $1.5-1.8$ & $2.7-3.5$ \\
$\mathrm{PM}_{2.5}(\mathrm{mg} / \mathrm{km})$ & $35-37$ & $53-59$ \\
\hline
\end{tabular}

three principles: (i) it should be simple and predictable to create valid business models, (ii) it must not favor or discriminate individual actors (as this is politically prohibited), and (iii) it should be feasible to implement and to verify (e.g., the administrator should be able to check if allocation criteria are satisfied). In the simulation model, subsidies are provided to agents as a fixed percentage of the UCC price charged to the agents using the UCC, thereby essentially serving as a price discount to the end-users. For receivers, the subsidy is based only on the price for the basic last-mile delivery service, not the prices for value-adding services. This price-based distribution key is simple, does not discriminate among agents, and its allocation can be verified by the administrator. In the simulation, we assume that the subsidy scheme is terminated after two years.

6.4.4. Scenarios. We conclude this section by outlining the scenarios. We introduce seven test variables (indicated by the capital letters A-G), for which we evaluate three different levels corresponding to 'low' (I), 'medium' (II), and 'high' (III) estimates of the variable. An exception is variable B, which only has two levels. The variable levels are shown in Table 6.4; every unique combination of variables represents a scheme. We apply a full factorial design, which gives us $2^{1} \cdot 3^{6}=1458$ schemes to evaluate. If possible, we select the variable levels based on the collected data and the expert interviews. However, preliminary experiments indicate that several variables are particularly prone to changes, such that small changes in their values may lead to different outcomes. To provide insight in the impact of setting alternative levels for these variables, we perform sensitivity analysis in Section 6.5.3. 
TABLE 6.4. Variable levels.

\begin{tabular}{|c|c|c|c|c|}
\hline Indicator & Variable & Level I & Level II & Level III \\
\hline $\mathrm{A}$ & Access times & 7.00-9.00am & 9.00-11.00am & No restrictions \\
\hline B & Zone access fee & $€ 0$ & & $€ 7$ \\
\hline $\mathrm{C}$ & Subsidies carriers & $\begin{array}{l}0 \% \text { of cost } \\
\text { per stop }\end{array}$ & $\begin{array}{l}10 \% \text { of cost } \\
\text { per stop }\end{array}$ & $\begin{array}{l}20 \% \text { of cost } \\
\text { per stop }\end{array}$ \\
\hline $\mathrm{D}$ & Subsidies receivers & $\begin{array}{l}0 \% \text { of cost } \\
\text { for base service }\end{array}$ & $\begin{array}{l}10 \% \text { of price } \\
\text { for base service }\end{array}$ & $\begin{array}{l}20 \% \text { of price } \\
\text { for base service }\end{array}$ \\
\hline $\mathrm{E}$ & Subsidies UCC & $\begin{array}{l}0 \% \text { of prices per } \\
\text { stop and } \\
\text { base service }\end{array}$ & $\begin{array}{l}10 \% \text { of price } \\
\text { stop and } \\
\text { base service }\end{array}$ & $\begin{array}{l}20 \% \text { of price } \\
\text { stop and } \\
\text { base service }\end{array}$ \\
\hline $\mathrm{F}$ & UCC handling costs & $2-11 € / \mathrm{m}^{3}$ & $7-20 € / \mathrm{m}^{3}$ & $26-56 € / \mathrm{m}^{3}$ \\
\hline G & $\begin{array}{l}\text { Margin value-adding } \\
\text { services }\end{array}$ & $0 \%$ & $25 \%$ & $50 \%$ \\
\hline
\end{tabular}

The expert interviews revealed that the municipality has limited power to implement regulations, as they are bound by government laws. This view is confirmed by the study of Gammelgaard (2015). Measures such as, e.g., city access exclusively for electric trucks, are not viable in the foreseeable future. In this study, we therefore restrict ourselves to measures that are perceivable in the current context of urban freight transport within Denmark.

We start by describing the administrative measures. As mentioned, Copenhagen currently allows vehicles to deliver in the medieval center only between 9.00 and $11.00 \mathrm{am}$. Although this restriction keeps the city free of trucks for most of the day, it may also cause inefficiencies when a carrier has to visit multiple receivers, possibly requiring additional vehicles. The first test variable (A) therefore relates to the adjustment of this access time restriction. Van Duin et al. (2010) study a variant of access time restrictions, in which they set the latest allowed access time before the opening times of the shops. This requires shop personnel to be in early to receive the goods, thus requiring extra salary payments. This approach is adopted in La Rochelle as well, with the UCC being exempted from the time restriction (Browne et al., 2005). We test this measure to evaluate how it compares to the current access time restrictions, requiring receivers to assign a staff member for two additional hours on delivery days. Another measure that we test is to completely abandon access time restrictions; the potential efficiency losses may outweigh the intended benefits. The second restriction corresponding to test variable (B) - is the access fee for trucks, which in the current situation in Copenhagen is valid for the entire low-emission zone. As stated before, a certificate to gain access to the low-emission zone costs $€ 12.5$ and is valid for the lifetime of the truck. For trucks that regularly visit the city, the costs 
per visit are negligible; we therefore set the current access costs equal to 0 . In the simulation, we test the impact of raising the fee. In 2002, the city of Copenhagen charged $€ 7$ for a one-day access certificate $(\overline{\mathrm{OECD}}, 2003)$; this fee was intended for trucks that did not meet certain vehicle criteria. We use this value as the high variable level for the access fee, with the smaller UCC vehicles being exempted from paying the fee.

Next, we discuss the subsidy measures that we test. Although the municipality expert indicated that the willingness to subsidize UCC initiatives is currently low, subsidy schemes are common in many comparable initiatives (Browne et al., 2005). We therefore consider subsidies as a realistic measure. Also, the Danish Transportation Authority was prepared to fund Citylogistik-kbh for three years, after which it was supposed to be financially sustainable (Gammelgaard, 2015). Ultimately, Citylogistik-kbh went private after two years. As stressed in our literature review, subsidies should indeed be of a temporary nature. In the experiments, we assume a two year subsidy period. Traditionally, it is the UCC that receives subsidies, yet these could also be awarded to receivers or carriers for utilizing the UCC. Subsidizing carriers (variable C) or receivers (variable D) could generate initial commitment from these parties, which may aid to reach the critical mass of users and sufficiently lower the cost structure of the UCC to be sustainable when subsidies are halted. Both agent types receive a monthly subsidy when using the UCC; all subsidies stop after two years. Variable E represents subsidies awarded directly to the UCC.

The operational costs of the UCC have a strong impact on its performance; as noted before, the obtained estimates for these costs vary widely. With variable F, we set three cost ranges that represent an upper and lower bound for the handling costs, with the handling costs per $\mathrm{m}^{3}$ decreasing linearly with the increase in volume handled. This variable helps us to determine what the costs for a UCC should be to perform in a sustainable manner. Finally, variable G tests the impact of the profit margin that the UCC makes on value-adding services; we test profit margins of $0 \%, 25 \%$, and $50 \%$. Although the latter may seem high, in practice the economies of scales for services such as storage and parcel shipping is often substantial.

\subsection{Numerical experiments}

In this section, we present the results of the simulation experiments. First, we address the financial performance of the individual agents in Section 6.5.1. 
Section 6.5.2 discusses the impact that the various schemes have on the environment. We perform sensitivity analysis on several variables in Section 6.5.3. In Section 6.5.4, we discuss the key findings and pose a number of propositions.

Each simulation run represents a time period of five years. Subsidies may be awarded in the first two years, the third year is simulated for the system to stabilize and to reach a steady state. To compute the KPIs, we use the final two years of the simulation. We compare the KPIs obtained for all tested schemes to the performance under the default scheme, in which the city can be accessed by carriers between 9.00 and 11.00am $\left(\mathrm{A}_{I I}\right)$, there is no zone access fee $\left(\mathrm{B}_{I}\right)$, there are no subsidies $\left(\mathrm{C}_{I}, \mathrm{D}_{I}, \mathrm{E}_{I}\right)$, and the UCC has handling costs between 7 and $20 € / \mathrm{m}^{3}$ $\left(\mathrm{F}_{I I}\right)$ and a profit margin on value-adding services of $25 \%\left(\mathrm{G}_{I I}\right)$. Comparing KPIs to this default scheme provides insights into the financial performance of the agents. We take the average performance of all agents for a given agent type to compute the performance indicators.

6.5.1. Financial performance. In this section, we discuss how the financial performance of carriers, receivers, and the UCC is affected by adjusting the variable levels. First, we summarize the performance per agent type for all simulate schemes. Second, we show the effects of changing variable levels, both in isolation and in combination with other measures. Third, we illustrate the performance difference for the UCC between carrier-oriented schemes and receiveroriented schemes. Fourth, we discuss the properties of the scheme under which the UCC performs best financially, assuming default cost settings.

Figure 6.3 shows the financial performance per agent type (excluding the administrator) for each scheme; a positive percentage implies an improvement for the financial KPIs for carriers and receivers (i.e., cost reductions) and for the UCC (net cost reduction, with a cost reduction greater than $100 \%$ implying that the UCC makes a profit). A performance below the $0 \%$ line implies that the agent loses money compared to the default scheme and would likely oppose the scheme in real life (with the exception of the administrator). The solid horizontal line at 100\% indicates the break-even point for the UCC, e.g., the point at which its income (excluding subsidies) equals its costs. To show how carriers and receivers perform under schemes that are good for the UCC, the scenarios are sorted from high to low based on the performance of the UCC. Based on our analysis, the main findings with respect to financial performance are that (i) it is challenging to find schemes that result in a profitable UCC (most performances are below the break-even line), (ii) receivers are very inclined to use the UCC when shifted access time windows are introduced, (iii) carriers strongly benefit from the UCC under receiver-oriented schemes, as they can 
freely outsource their last-mile distribution, and (iv) the schemes under which the UCC performs best are schemes under which carriers considerably improve their performance and receivers improve marginally. Various correlations can be observed in Figure 6.3. On the far left side, we see the results for the schemes under which the UCC performs best. We see that for these schemes, carriers considerable reduce their costs, whereas receivers are not worse off than under the default scheme. In the remainder of the graph, we observe that when receivers bear all the costs of the UCC due to opting in early (performance indicators are about $12 \%$ below the $0 \%$ line), the carriers perform very well as they can freely outsource their distribution, while the UCC performs poorly as it obtains its income only for the receivers.

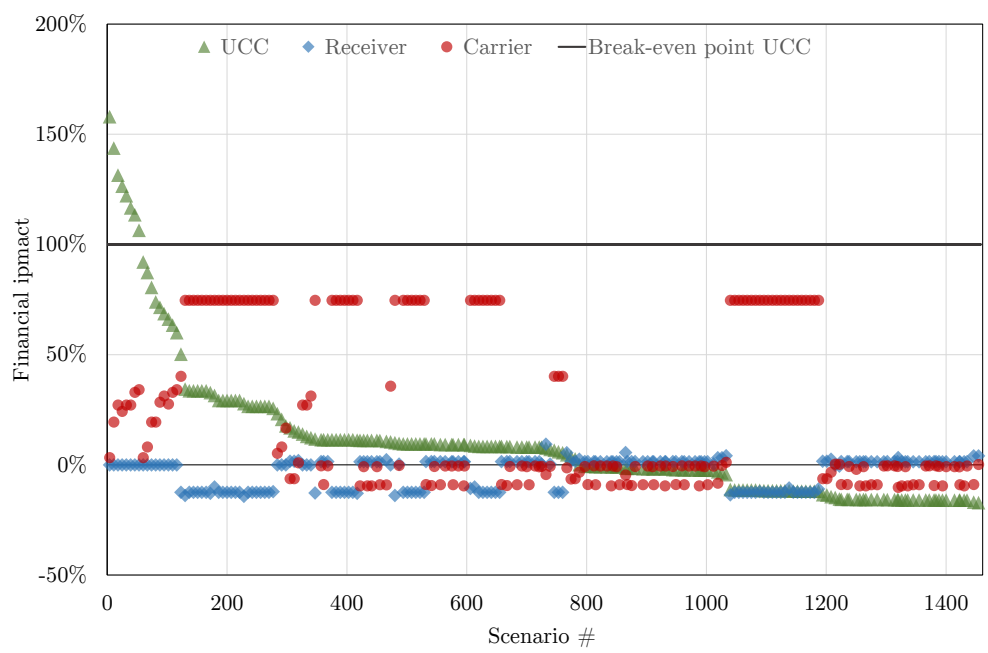

FIGURE 6.3. Financial performance, segregated per agent type for all scenarios. Performance is relative to the default scenario, which is indicated by the 0\% line; percentages higher than $0 \%$ indicate an improvement. The break-even point for the UCC is indicated by the line at $100 \%$.

In Table 6.5, we show the impact of changing each variable on the financial KPIs. For the carriers, receivers, and the UCC, we show four measures for every 
low and high variable level. First, we show the isolated effect, which we compute by only changing the level of a single variable, with all other variable levels being kept as under the default scheme. Second, we compute the main effect, which is the average difference between all pairs of equivalent schemes; each pair contains one scheme in which the variable level of interest is adjusted, whereas in the other scheme of the pair it is kept at the default level. As an example, suppose we compute the main effect for variable level $\mathrm{A}_{I I I}$. A pair of schemes may then consist, e.g., of $\left[\mathrm{A}_{I I I}, \mathrm{~B}_{I}, \mathrm{C}_{I I}, \mathrm{D}_{I I I}, \mathrm{E}_{I}, \mathrm{~F}_{I}, \mathrm{G}_{I I I}\right]$ and $\left[\mathrm{A}_{I I}, \mathrm{~B}_{I}, \mathrm{C}_{I I}, \mathrm{D}_{I I I}, \mathrm{E}_{I}, \mathrm{~F}_{I}, \mathrm{G}_{I I I}\right]$; we compute the average performance difference between all such pairs. Third, we show the worst-case effect. As for the main effect, we compute the differences between pairs, but rather than taking the average, we show only the worst result. Fourth, we compute the best-case effect similarly to the worst-case effect, now only showing the best result. A positive sign indicates a performance improvement. To complement Table 6.5, Figure 6.4 graphically represents the main effects per variable level for the carriers, receivers, and the UCC. 


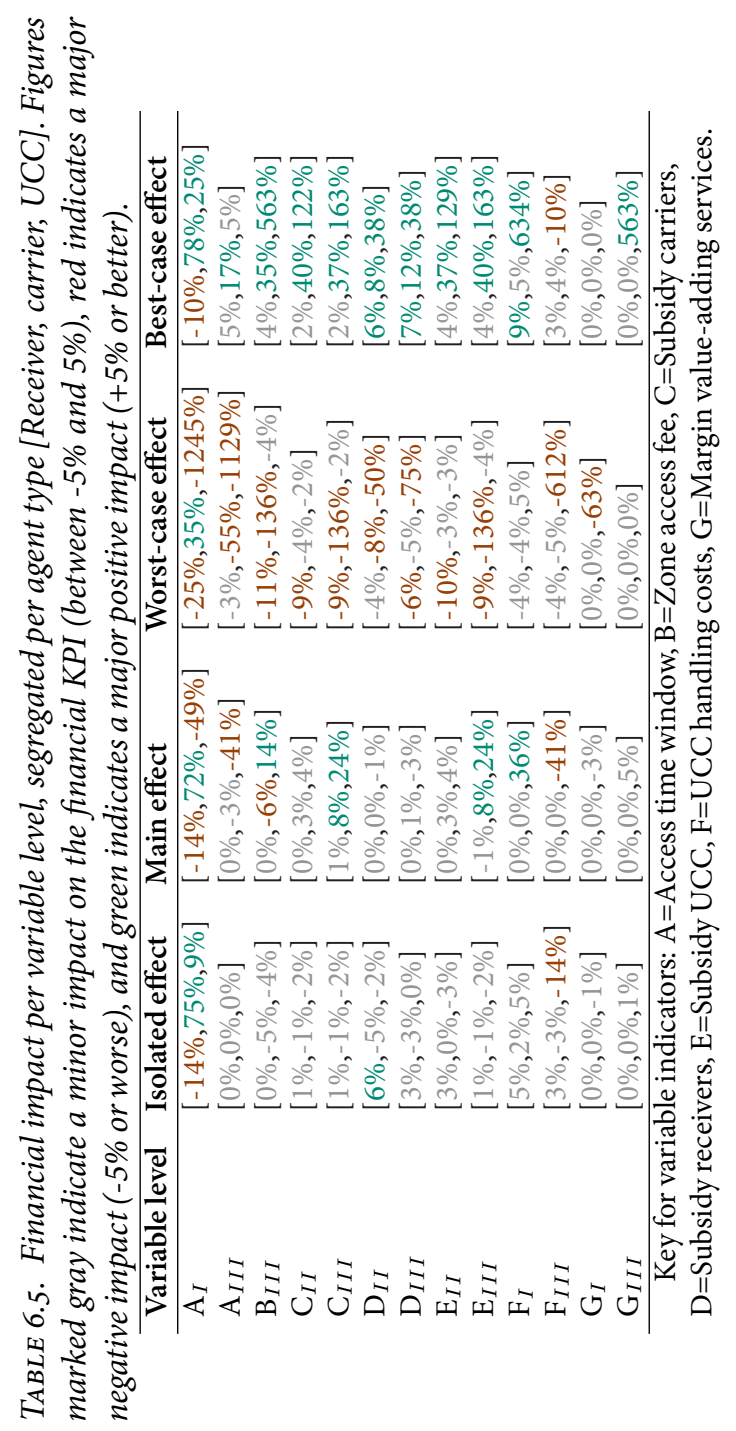




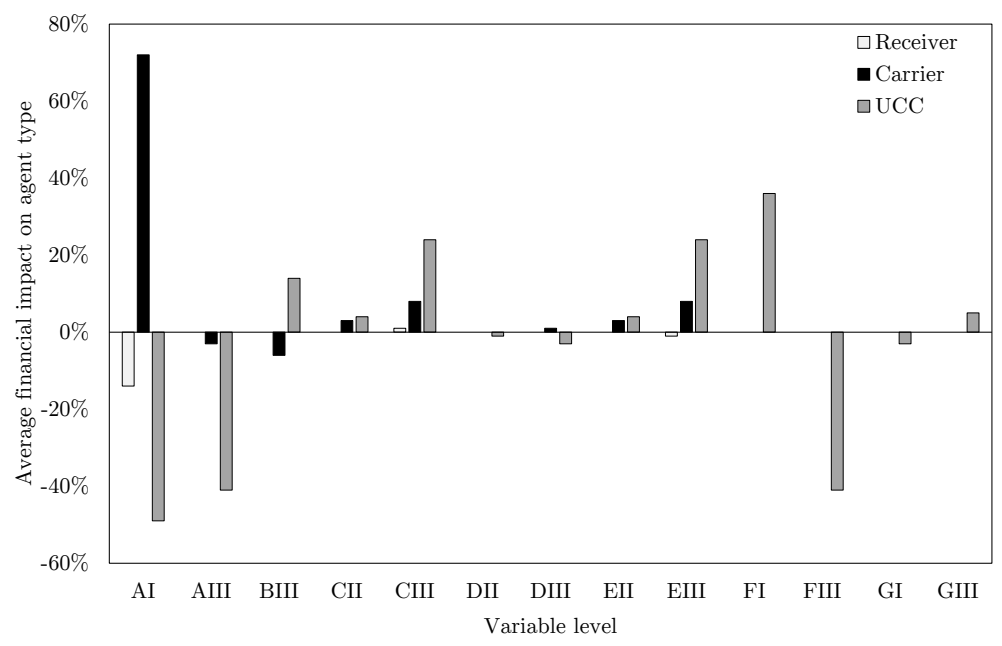

FIGURE 6.4. Barchart, visualizing the financial main effects as listed in Table 6.5 per variable level for the carriers, receivers, and the UCC.

We reflect on the influence of each variable. Shifting time windows $\left(\mathrm{A}_{I}\right)$ is a very effective measure to commit receivers to the UCC; as the trucks operated by the UCC are exempted from the access time windows, the receiver does not need to dedicate additional personnel hours when outsourcing. The consequence, however, is that carriers can outsource their last-mile distribution for free. As a result, UCCs are typically not able to generate sufficient income in schemes that contain this measure. Removing time access restrictions $\left(\mathrm{A}_{I I I}\right)$ on average results in 6 less carriers selecting the UCC, which ultimately translates into higher losses for the UCC. Imposing a zone-access fee $\left(\mathrm{B}_{I I I}\right)$ results in a higher use of the UCC when combined with other measures; as a stand-alone measure it does not suffice to make carriers adjust their behavior significantly. Although the isolated measure results in a somewhat higher utilization of the UCC, only carriers that typically transport low numbers of orders are inclined to outsource (as for them the fee is relatively high), such that the UCC itself must deal with orders that are difficult to deliver efficiently and subsequently incurs higher operational costs. We have tested six settings related to subsidies 
$\left(\mathrm{C}_{I I}, \mathrm{C}_{I I I}, \mathrm{D}_{I I}, \mathrm{D}_{I I I}, \mathrm{E}_{I I}, \mathrm{E}_{I I I}\right)$. Both subsidizing carriers and subsidizing the UCC seems to have a positive effect on the financial performance of carriers, receivers and the UCC. However, it appears that subsidizing the UCC is less efficient than subsidizing carriers; a higher expenditure is required to obtain the same net effect. Subsidizing receivers does not yield a positive effect; the resulting cost reduction on the base service is only a small component of their overall costs, and value-adding services are not subsidized. The effect of receiver subsidies on the number of receivers joining is therefore negligible. For all subsidy measures, we see that they have a limited impact as a stand-alone measure; they must be combined with other measures to achieve the desired outcomes, otherwise agents simply revert to their former behavior after the subsidy period ends. Adjusting the estimated handling costs at the $\mathrm{UCC}\left(\mathrm{F}_{I}\right.$ and $\left.\mathrm{F}_{I I I}\right)$ has a considerable impact on the financial performance of the UCC, with an average improvement of $36 \%$ in the net result for the low-cost setting and a $41 \%$ reduction of the net result for the high-cost setting. The experimental results imply that sustainable UCC schemes do not exist for the high-cost setting. The final variable that we consider is the profit margin of value-adding services $\left(\mathrm{G}_{I}\right.$ and $\left.\mathrm{G}_{I I I}\right)$. Varying the profit margin has an impact on the net income of the UCC. However, as in profitable schemes only about $15 \%$ of the revenue stems from value-adding services, the overall impact of varying the profit margin remains relatively small. To summarize, most measures have a limited effect when implemented on a standalone basis (only the shift of time windows has a considerable impact on all agent types), but in combination with other measures particularly subsidies (to UCC and carrier) and zone-access fees generally have a positive impact. Later in this section, we list the parameter settings that correspond to the best performing scheme under average cost settings. Furthermore, in Section 6.5.3, we will test how adjusting the parameter settings of these measures affects performance.

Our analysis of the numerical results indicates that the sequence in which UCC users are attracted is decisive for the eventual profitability of a scheme. Figure 6.5 shows the income and costs for the UCC over time for a scheme that focuses on attracting carriers before receivers, Figure 6.6 shows the same information for a scheme that aims to attract receivers first. Both schemes assume low costs for the UCC $\left(\mathrm{F}_{I}\right)$ and a medium profit margin on value-adding services $\left(\mathrm{G}_{I I}\right)$. It can be seen that the scheme that aims to first commit carriers performs considerably better than the other scheme. Although the latter attracts more UCC users overall, the costs for the UCC are consistently higher than its income, as it generates its income only from the receivers. In the first scheme 
we observe a drop in the number of committed receivers when the subsidies are ended, yet the UCC remains profitable in the years that follow.

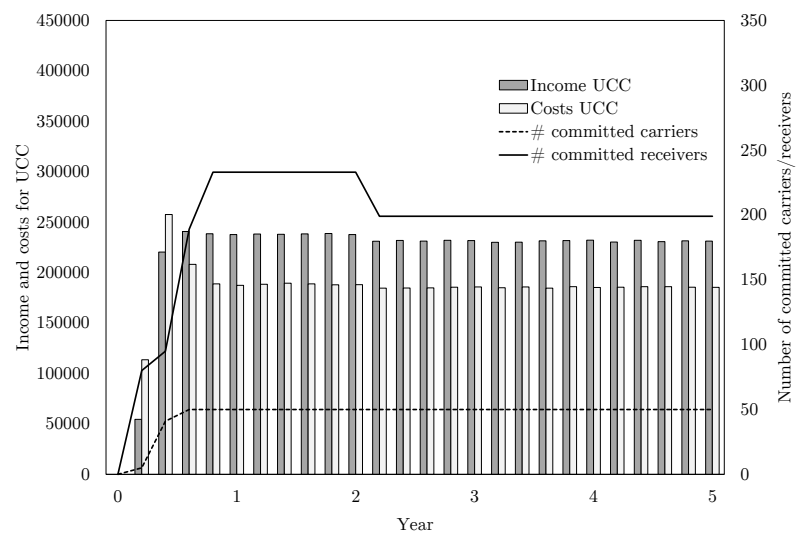

FIGURE 6.5. Financial performance for the UCC under a scheme that primarily aims to attract carriers. Assumed are low handling costs $\left(F_{I}\right)$ and a medium profit margin $\left(G_{I I}\right)$. 


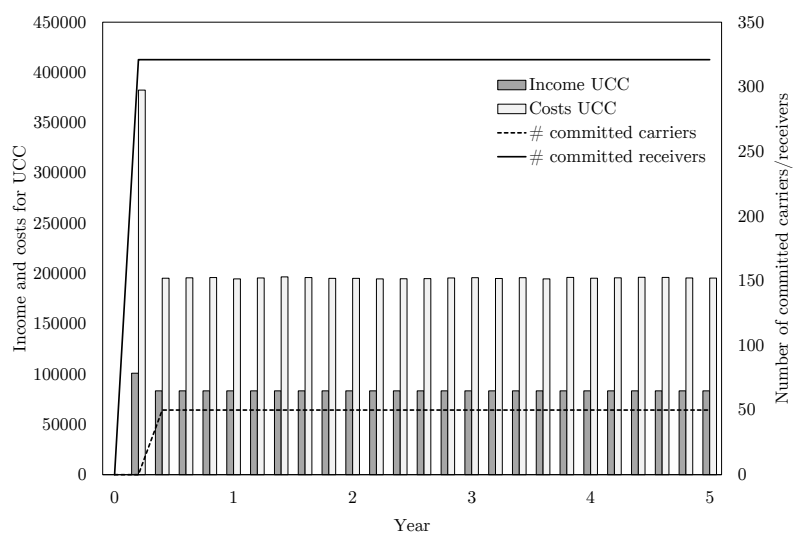

FIGURE 6.6. Financial performance for the UCC under a scheme that primarily aims to attract receivers. Assumed are low handling costs $\left(F_{I}\right)$ and a medium profit margin $\left(G_{I I}\right)$.

The objective of the simulation study is to identify schemes that yield a positive net result for the UCC, while not decreasing the financial performance of the other agents. We are primarily interested in whether such schemes exist for medium cost levels for the UCC, i.e., handling costs of $7-20 € / \mathrm{m}^{3}$ and a profit margin of $25 \%$ on value-adding services. In Figure 6.7, we show the financial KPIs for the best scheme - in terms of UCC profitability - compared to the performance under the default scheme. Despite being the best performing scheme under default cost settings, it still yields a loss of $8.5 \%$ to the UCC. The scheme has the following properties: an access time window from 9.00 to $11.00,20 \%$ subsidies to both carriers and the UCC, and a zone-access fee of $€ 7$. We observe a cost reduction for the carriers, virtually no cost change for the receivers, and a major reduction in net costs for the UCC. Under the best scheme, the UCC generates considerable more revenue than under the default scheme, while the revenue is also proportionally larger compared to the costs. To see whether slight adjustments of the best scheme yield a profitable situation for the UCC, in Section 6.5 .3 we finetune several variables to verify whether profits are attainable under average cost settings for the UCC. 


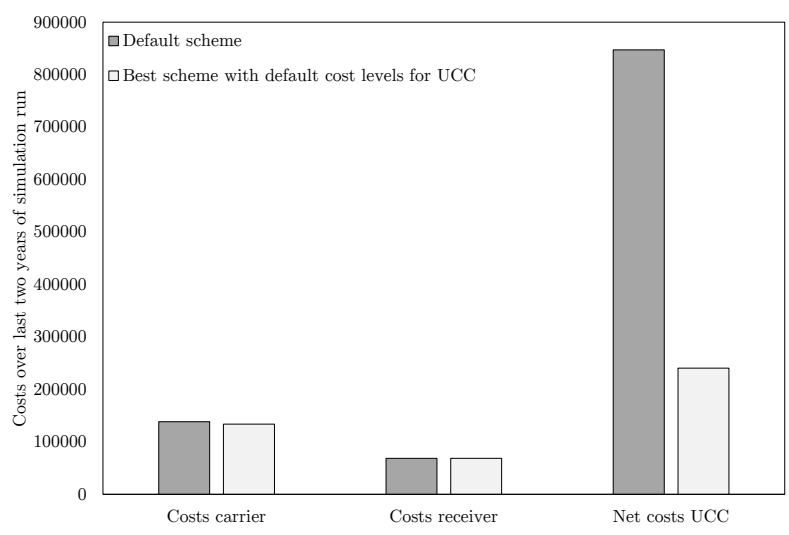

FIGURE 6.7. Financial performance of carriers, receivers, and UCC under both the default scheme and the financially best-performing scheme, both under default handling costs $\left(F_{I I}\right)$.

6.5.2. Environmental performance. In this section, we discuss the environmental performance of the schemes that we tested. First, we discuss the environmental impact of all simulated schemes. Second, as we have seen that most schemes perform poorly from a financial point of view, we reflect on the relation between financial and environmental performance, and show the environmental impact of the scheme under which the UCC performs best financially.

As the emission levels are measured based on distance and the emission ratios between the two truck types for $\mathrm{CO}_{2}, \mathrm{SO}_{2}, \mathrm{NO}_{\mathrm{x}}$, and $\mathrm{PM}_{2.5}$ are almost equivalent, the individual emission levels would be hard to graphically distinguish. Therefore, we aggregate them for the sake of our virtual representation. Figure 6.8 shows the average emission levels that correspond to each scheme. The emission levels are normalized with respect to those of the default scheme, which are set equal to $100 \%$. Furthermore, the average emission level for the scenario without UCC is indicated by the horizontal lines. The scenarios are sorted based on their resulting emission reduction. It can be seen that considerable reductions of emissions are achievable; the left side of the graph shows emission reductions for schemes with high utilization of the UCC. From an environmental perspective, all schemes perform better than in the scenario without a UCC. The best schemes reduce emissions by approximately $70 \%$ compared to the default 
scenario. Furthermore, such schemes reduce the total number of trucks in the city - i.e., both from carriers and the UCC - by up to $60 \%$ and the total distance driven by up to $65 \%$ (not shown in the figure). These considerable benefits indicate that the concept of a UCC makes sense from an environmental perspective. In practice, these effects might even justify permanent subsidies of the UCC.

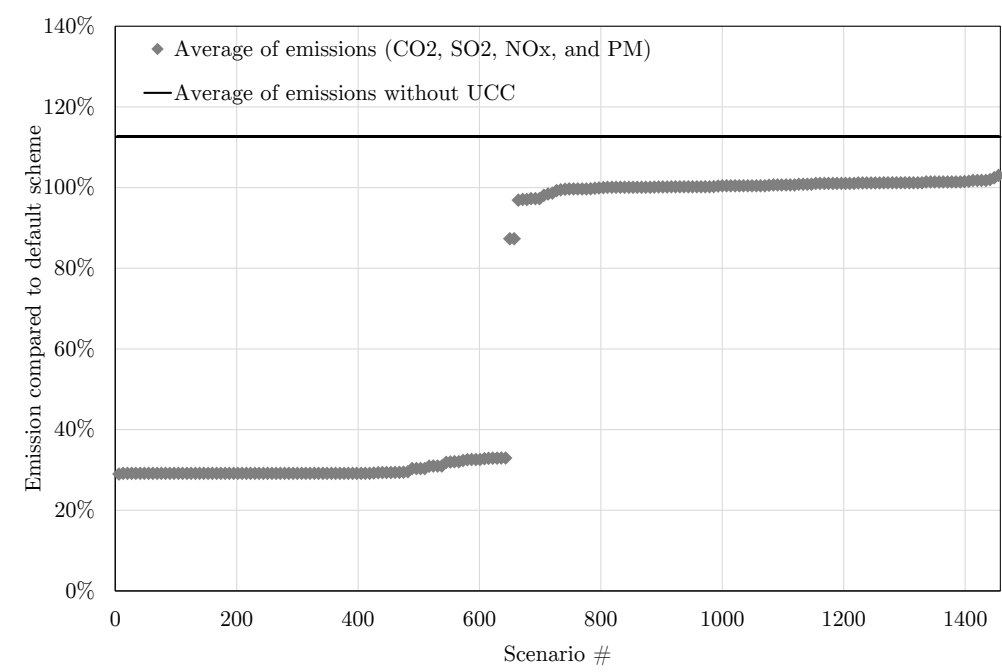

FIGURE 6.8. Average emission levels compared to the default scheme for all tested schemes. The 100\% line indicates the emission levels under the default scheme, values below $100 \%$ indicate an improvement. The average emission levels without a UCC are shown by the solid line.

Although many schemes strongly improve the environmental performance, it is challenging to find a scheme that is also financially viable. However, when a scheme performs well financially, this also implies a good environmental performance. Financial results depend on attracting a sufficiently high number of UCC users, such that a considerable number of stops is outsourced to the UCC. The inverse relation between environmental and financial performance is not necessarily true: a scheme may perform well from an environmental perspective, but be unsustainable financially, e.g., when committing all receivers before the carriers join. 
In Table 6.6, we compare the environmental KPIs for the financially bestperforming scheme (see Figure 6.7) to the KPIs under the default scheme. The difference between both schemes shows a considerable improvement on all KPIs. Compared to the default scheme, the best scheme reduces emission levels by $68 \%$ to $72 \%$. Although the number of small trucks in the city increases due to the higher use of the UCC, the total number of trucks reduces by $61 \%$, whereas the total distance driven decreases by $67 \%$.

TABLE 6.6. Performance on environmental KPIs compared between the default scheme and the financially best-performing scheme. All outcomes correspond to the final two years of the simulation.

\begin{tabular}{llll}
\hline KPI & Default scheme & Best scheme & Change \\
\hline $\mathrm{CO}_{2}$ (ton) & 383.51 & 70.42 & $72 \%$ \\
$\mathrm{SO}_{2}$ (kilogram) & 2.92 & 0.53 & $72 \%$ \\
$\mathrm{NO}_{\mathrm{x}}$ (ton) & 1.26 & 0.23 & $72 \%$ \\
$\mathrm{PM}_{2.5}$ (kilogram) & 22.97 & 4.99 & $68 \%$ \\
\# small trucks & 2,937 & 11,493 & $-291 \%$ \\
\# large trucks & 26,244 & 0 & $100 \%$ \\
Total \# trucks & 29,181 & 11,493 & $61 \%$ \\
Distance small trucks $(\times 1000 \mathrm{~km})$ & 38 & 139 & $-261 \%$ \\
Distance large trucks $(\times 1000 \mathrm{~km})$ & 385 & 0 & $100 \%$ \\
Total distance trucks $(\times 1000 \mathrm{~km})$ & 423 & 139 & $67 \%$ \\
\hline
\end{tabular}

6.5.3. Sensitivity analysis. Although we strive to use data that reflects the real world as accurately as possible, the nature of the simulation study inherently requires simplifications and assumptions on the real-world variablelevels. A full factorial design for a large number of values per variable would be computationally intractable. In this section, we therefore test the impact of variables that are both subject to considerable variability and are expected (based on preliminary tests) to have a significant impact on the results. For each variable that we test, we simulate with multiple numerical values for the variable of interest, while keeping all other variables at their default levels. We perform sensitivity analysis on the following variables: (i) the width of the access time windows, (ii) the subsidy levels to the carrier, and (iii) the price that the UCC charges to the carriers. Furthermore, we finetune several variables in the financially best-performing scheme, as this scheme - under average cost settings - yields financial losses for the UCC.

The access time restriction of two hours that is currently applied in the city of Copenhagen appears to be ineffective to persuade carriers to use the UCC. We test the impact of various widths of the time access window on the number of 
carriers that commit to the UCC. The results are shown in Figure 6.9. We see that windows with a width up until one hour have the intended effect; for larger windows the number of committed carriers becomes lower. When access time restrictions are used as a standalone measure, windows wider than two hours do not aid in attracting carriers to utilize the UCC. As only very small windows of an hour or less have a strong impact on UCC usage, one may argue that access windows only effectively support the UCC if the window essentially serves as a ban on large trucks.

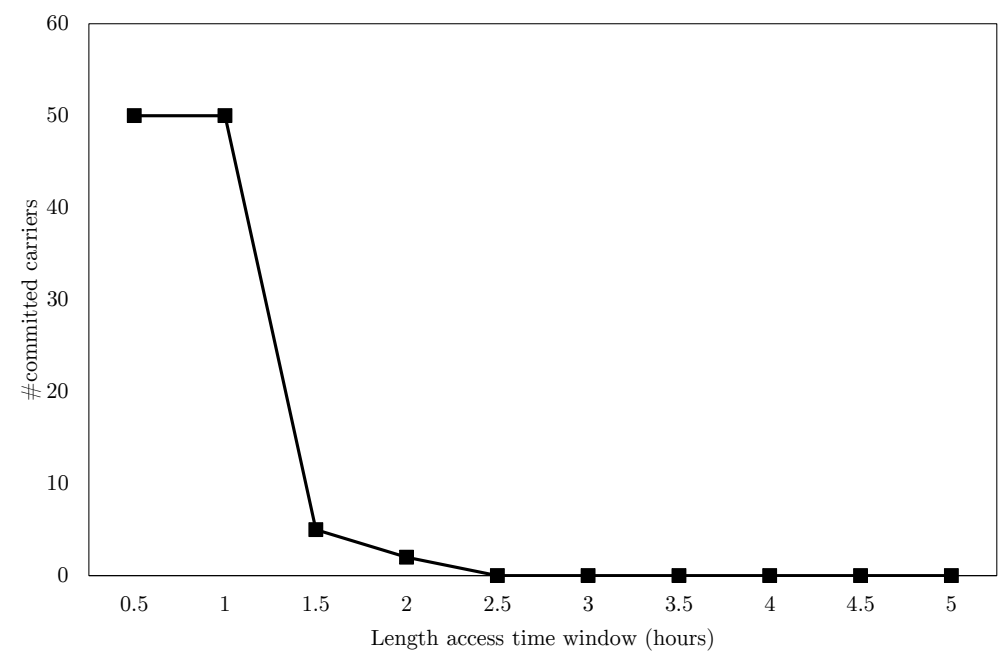

FIGURE 6.9. Impact on the number of committed carriers for a variety of access time window widths.

We have already established that subsidies as an independent measure are not sufficient to permanently attract carriers; they need to be combined with other measures to yield a sustainable solution. More specifically, to reach a steady state in which the UCC makes a profit, the carriers should be attracted before the receivers, and subsidy levels should be set in accordance with this goal. Nevertheless, it remains useful to know the smallest subsidy amount that the administrator needs to spend in order to commit carriers within a certain subsidy period. 
In Figure 6.10, we show the impact of 10 different subsidy levels on the commitment of carriers over time, measured during the two-year subsidy period. We see that for levels over $12 \%$, half a year of subsidies suffices to attract all carriers. For a level of $12 \%$ it takes one year; levels lower than $12 \%$ fail to attract all carriers within two years. To attract higher numbers of carriers directly at the start, subsidies higher than $20 \%$ are required; these levels are not shown in the figure;

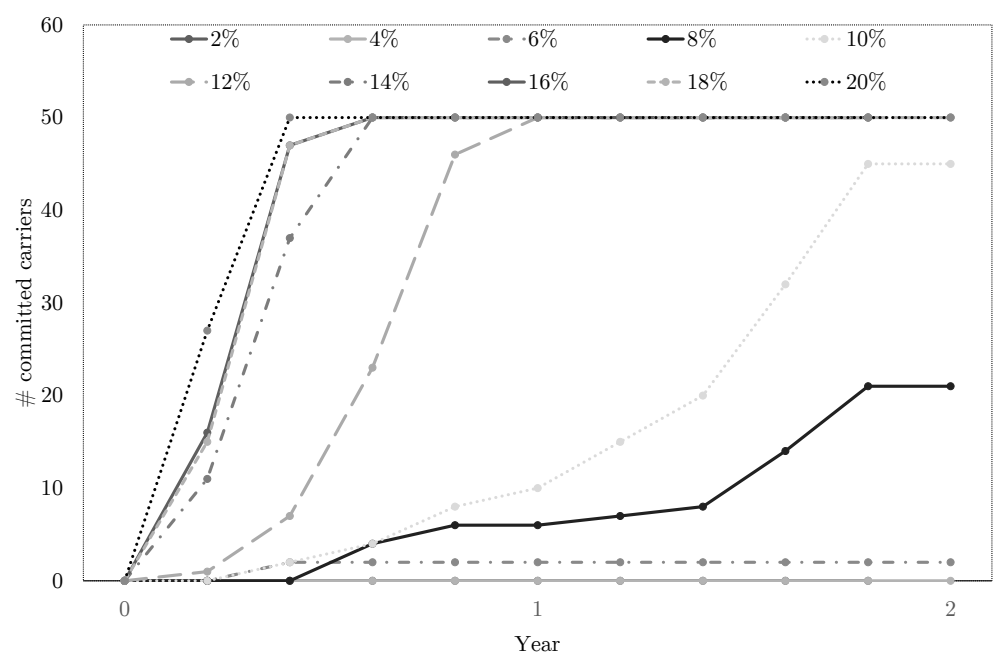

FIGURE 6.10. Impact on the number of committed carriers for a variety of subsidy levels.

Analysis of our results indicates that the carriers are very price-sensitive. Figure 6.11 shows the effects of various price levels on the commitment of carriers to the UCC under the base scenario. In contrast to the price bounds used in the main experiments, for this sensitivity analysis we assume a fixed price that is not altered over time. For price levels higher than $€ 9.5$ per stop, the number of carriers that use the UCC rapidly declines. However, at price levels of $€ 9.5$ and below, the UCC is not financially sustainable; a higher price in combination with supporting measures is required to ensure the required income for the UCC.

We conclude this section with an evaluation of the impact of finetuning the best performing scheme (see Figure 6.7), as the achieved net profit of $-8.5 \%$ is 


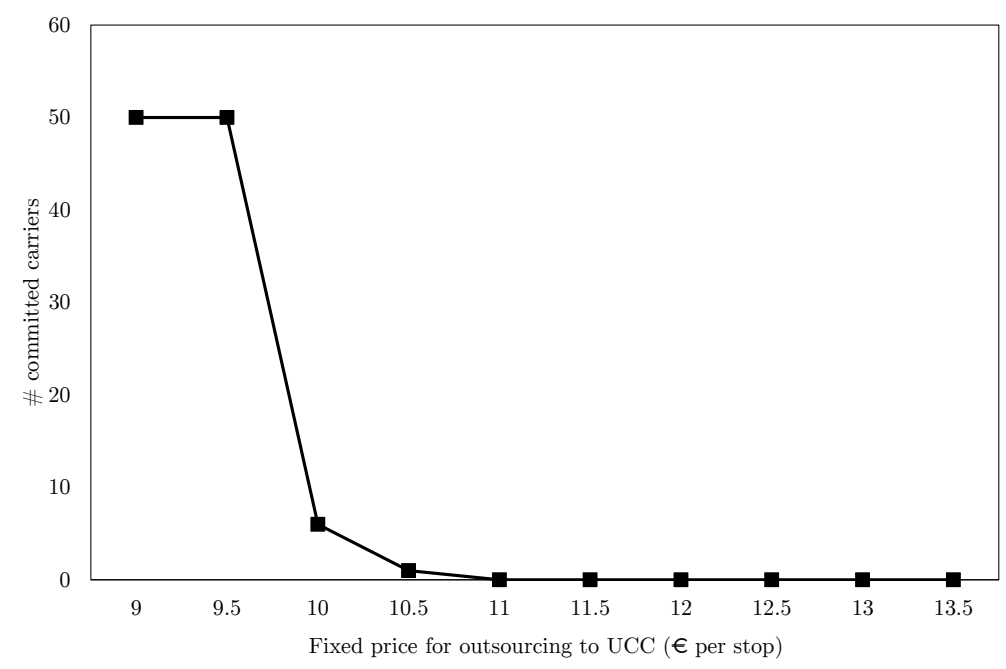

FIGURE 6.11. Impact on the number of committed carriers for a variety of price levels.

insufficient for the UCC to survive in the long term. Recall that this scheme has an access time window from 9.00 to $11.00,20 \%$ subsidies to both carriers and the UCC, and an access fee of $€ 7$. We finetune various cost and subsidy variables, and highlight the adjustments that resulted in a profit. In terms of profit, increasing subsidy levels for either the UCC or the carriers from $20 \%$ to $30 \%$ yields the best results. This change instantly commits almost all carriers from the start - such that the profit from this group is maximized - and results in a profit margin for the UCC of $12.1 \%$. Another successful measure is to lower the price of the base service from $€ 60-70$ to $€ 40-50$. These lower costs result in $15 \%$ more receivers in the steady state and push the profit margin of the UCC to $2.2 \%$. Finally, raising the zone access fee for from $€ 7$ to $€ 9$ yields a profit margin of $0.3 \%$ for the UCC, due to committing several extra carriers in the early stages. The impact of these measures show that relatively small price changes may impact the profitability of the UCC considerably. 
6.5.4. Discussion. In this section, we reflect on the key insights obtained from the numerical experiments, provide a number of propositions with respect to good business models for UCCs, and discuss the impact of discrepancies between reality and the simulation model. We stress that a solid business model requires both good financial performance and a good environmental performance. As mentioned before, a scheme that attracts high numbers of UCC users implies good environmental performance, but is not necessarily financially viable.

Proposition 1: The commitment of carriers to the UCC should be ensured before targeting the receivers.

The numerical results show that in the most successful schemes, the bulk of the carriers commit to the UCC before the receivers do. As carriers only pay for outsourcing stops at receivers that have not committed to the UCC at the time of the tactical decision, first committing the carriers maximizes the revenues stemming from this group. Subsequently, value-adding services can still be sold to the receivers. At that point in time, the UCC already handles larger volumes due to the committed carriers, enabling to offer lower prices to the receivers. After the startup years, the UCC should be able to offer sufficiently competitive prices such that carriers remain users of the UCC.

Proposition 2: Subsidies are most effectively allocated to the carriers.

As stated in Proposition 1, carriers should be the primary target for a UCC that aims to attract a base of users. Allocating subsidies to the carriers appears to be the most effective measure to achieve this goal. The subsidies should be sufficiently large to commit many carriers within a relatively short period of time. The numerical results show that relatively high subsidies are necessary for this purpose. Subsidies that are allocated to the UCC have similar effects as subsidizing the carriers, but in a less efficient manner. Subsidies allocated to receivers are less effective, as attracting receivers before carriers negatively affects total revenues.

Proposition 3: Access time restrictions only aid the UCC if the access window is set sufficiently narrow.

When an access time window is used as a stand-alone measure, the current width of two hours is ineffective for the purpose of committing carriers to the UCC. Particularly when part of the receivers outsource their last-mile distribution to 
the UCC, the access restrictions are not stringent for carriers. However, in combination with other measures, access time windows appear to have a positive effect on the usage of the UCC.

Proposition 4: Setting access time restrictions before the opening times of stores is an effective measure to generate commitment from receivers.

Setting the time access restrictions before the opening times of stores requires receivers to dedicate additional personnel for receiving goods, unless it outsources the last-mile delivery to the UCC; the small vehicles operated by the UCC are exempted from the access time windows. This makes it a very effective measure to commit receivers to the UCC. Despite its success in attracting receivers, this measure tends not to create viable schemes. When receivers commit to the UCC before carriers do, revenues can no longer be obtained from carriers, as for them the UCC has already become the final delivery address.

Proposition 5: Zone access fees can have a positive effect in combination with other measures.

As a stand-alone measure, zone access fees at their current level are ineffective in realizing a change in the behavior of carriers. However, in combination with other measures, zone access fees may have a modest positive effect on the utilization of the UCC, as they increase the costs for carriers and therefore make the UCC prices more favorable. For carriers that need to make only few stops in the city, the zone access fee is a relatively large component of their costs, which makes such carriers more inclined to outsource their shipments to the UCC.

We acknowledge that our simulation model deviates from practice on various aspects; we discuss the impact of the two most important deviations. First, for the performance of the UCC in the simulation model, the sequence in which carriers and receivers commit to the UCC is very important. Due to instantaneous decision making by the agents combined with varying price levels, decisions made in the early stages of the simulation greatly impact the steady-state performance of the UCC. Although it is obvious that decisions made in practice will be more gradual and involve negotiations with the UCC, the main takeaway remains that the focus should be on attracting carriers first as they generate the bulk of the revenue for the UCC, and that measures should be in support of this approach. A second deviation from practice is that we assume price ranges that 
vary based on the volumes that are handled by the UCC. Although the underlying argument is to reflect economies of scale, in practice the continuous price changes would likely cause confusion for the UCC users and require frequent contract renegotiation. The main reason for using ranges rather than fixed price levels is that they make it easier to identify steady states, rather than finding them by means of trial-and-error. As fixed price levels may only work for a specific scheme, it is difficult to make generic statements regarding single price levels.

\subsection{Conclusions}

In this chapter, we have presented a simulation study on the feasibility of implementing a UCC under a variety of urban logistics schemes. Based on the framework presented in Chapter 4, we designed an agent-based simulation model, which represents receivers, carriers, the UCC, and the local administrator as autonomous entities. Decisions within the simulation were divided into three levels: strategic, tactical, and operational. The strategic level represents the subsidy schemes and regulations implemented by the administrator. On the tactical level, subsidies and UCC price levels are adjusted. Subsequently, receivers and carriers decide whether or not to join the UCC, computing estimated downstream costs. Finally, on the operational level routing decisions are made and handling cost are computed, allowing to calculate the operational costs of the agents. The goal of this chapter was to identify schemes that - after an initial subsidy period - enable all agents to operate in a financially sustainable manner, while simultaneously yielding substantial environmental improvements.

The simulation study has been applied to a case inspired by the city of Copenhagen. Based on an OpenStreetMap implementation, we generated a realistic network to represent the city. We have gathered data to accurately portray the agents via various meta-studies, individual case studies, and publicly available information. Both the setup of the case study and the reliability of the data are validated by means of interviews with two experts. We have created receiver profiles to reflect the large variance between receivers in practice.

We have tested 1458 different schemes, for which we measured both financial and environmental KPIs. We have shown that considerable environmental improvements can be achieved through the use of a UCC, reducing the number of trucks in the city by up to $60 \%$ and reducing emissions by about $70 \%$. However, it is challenging to find schemes that are also financially sustainable. We have shown that the UCC obtains the highest revenues by first convincing carriers to outsource their last-mile deliveries, and then selling value-adding services 
to the receivers in the city. The concept of the UCC appears to be unsuccessful without supporting measures; temporary subsidies to the carriers and imposing a zone access fee appear to be the most effective measures in achieving a steady state in which the UCC is profitable and can eventually operate without external funding, once having received a sufficient scale of operations.

This chapter concludes Part III of the thesis. In this part, we have introduced an agent-based simulation framework to evaluate urban logistics schemes. The framework has been applied in two distinct simulation studies. Part IV, Chapter 7, concludes this thesis. 



\section{Part IV}

\section{Conclusions}





\section{CHAPTER 7}

\section{Conclusions and further research}

This chapter concludes the thesis. In Section 7.1, we reflect on the research objective and corresponding research questions that we introduced in Chapter 1. Section 7.2 reviews the key managerial insights of this thesis. Finally, we discuss some implementation challenges and suggest directions for future research in Section 7.3.

\subsection{Reflection on research objective and research questions}

We repeat our main research objective:

To develop mathematical models that support coordinating actors in consolidated freight planning, and to obtain quantitative insights into the challenges and requirements to establish integrated logistics systems, in which independent actors coordinate their decisions to improve the efficiency of urban transport.

We first list the methodological contributions of this thesis. In Chapter 2, we have developed a planning algorithm for a $4 \mathrm{PL}$, which coordinates the allocation of freight over a network of contracted carriers, with transshipments and reloads being allowed at the transfer hubs. Chapter 3 provides an algorithm that yields a consolidation policy for UCCs. An agent-based simulation framework to analyze urban logistics schemes has been introduced in Chapter 4. Additional insights are obtained in Chapter 5 and Chapter 6 , where we test a variety of urban logistics schemes. Chapter 5 provides a broad overview of the impact of urban logistic schemes in abstract representations of European cities, whereas Chapter 6 has a more demarcated scope with its analysis of UCC performance in a setting that embeds an additional degree of realism.

We proceed to reflect on the individual research questions. 
1. How can we efficiently allocate dynamically arriving orders of fractional truckload size to transport resources that operate in a network with transfer hubs?

We address this research question in Chapter 2. We have defined a problem in which orders arrive over time and need to be planned by a 4PL over a network of contracted carriers. The orders are subject to a pickup time and a delivery time; the receiver does not impose constraints on how the order is transported. The network consists of arcs that represent line-haul services, which may be subject to timetables. Furthermore, the network contains transfer hubs, in which transport units (e.g., trailers, containers) can switch transport modes, but loads can also be bundled and split on the level of individual orders.

In the proposed solution method, orders are tentatively planned the moment they become known, but their physical route and their dispatch times per node may be adjusted in response to future consolidation opportunities. As routes may consist of multiple arcs, we must take into account how consolidation actions on a specific arc impact the rest of the system. Changing dispatch times and routes affects other orders as well; we enforce that solutions must be feasible at all times. To efficiently construct solutions and check their feasibility, we make use of a decision tree structure, in which we evaluate the consolidation opportunities per arc. By sorting the solutions based on savings, we only need to check solutions until we identify the first one that is feasible.

We make three key design choices in the development of the algorithm, namely (i) we only store a limited number of routes per order, (ii) we allow to adjust the physical route of an order only once, and (iii) orders are planned dynamically rather than periodically. The main motivation for these design choices is to keep computational effort limited and to allow providing an immediate reply to customers when an order request arrives. We tested the impact of each design choice with numerical experiments. Regarding the first assumption, we find that storing more than 30 routes per order virtually never yield further improvements. Thus, storing a relatively small number of routes already suffices to capture most of the consolidation opportunities. For the second assumption, the results indicate that continuous replanning yield performance improvements of less than $3 \%$, while requiring a much higher computational effort. To test the impact of the third assumption, we solve various small instances to optimality, resulting in an average performance gap of less than $5 \%$.

The experimental results indicate that evaluating multiple routes significantly improve performance. By improving the efficiency of transport, considerable 
gains in both financial and environmental performance are achieved. The performance of the algorithm was validated on a variety of virtual networks, as well as on a real instance provided by a Dutch $4 \mathrm{PL}$. Compared to direct road transport, we improve fill rates by $57 \%$ on average, resulting in a cost reduction of $34 \%$ and a reduction of $\mathrm{CO}_{2}$ emission of $30 \%$. Furthermore, comparing to a benchmark in which consolidation takes place on the best routes of orders, fill rates improve by $21 \%$, while achieving significant reductions of both costs and emissions.

\section{How can we efficiently plan the last-mile distribution of dynamically arriving} orders at an urban consolidation center?

In Chapter 3, we formulate a Markov decision model that describes the stochastic arrival process of orders and the corresponding dispatching problem faced by the UCC operator. The state of the problem is defined by the number of orders per order type that the UCC has in inventory and the earliest dispatch availability per vehicle. The UCC has no perfect insight into the future order arrivals, but we assume that the stochastic arrival process is known to the operator. Based on the corresponding arrival probabilities, the operator is able to make informed decisions regarding the dispatch of orders. The operator must decide to either dispatch an order at the current decision moment or keep it in inventory for a later decision moment. Newly arriving orders may allow to achieve better utilization of vehicle capacity or constructing more efficient routes. However, as the orders are subject to dispatch time windows and there is no guarantee that better consolidation opportunities arise, the operator must make intelligent decisions on both the subset of orders to dispatch and the timing of dispatch.

Only toy-sized instances of the dispatching problem can be solved to optimality; computational problems quickly arise for larger instances. We develop an algorithm based on approximate dynamic programming to tackle three computational challenges. First, as every combination of orders represents a unique arrival scenario, there may be too many outcomes for which we need to compute the downstream costs. Therefore, we replace the full outcome space with a single estimated outcome, corresponding to the state of the system directly after the dispatch action. We determine this estimated outcome by means of repeated sampling in a simulation. Second, the number of order combinations increases exponentially with the number of orders in the system, which implies that the number of states quickly becomes too large to enumerate. We solve the problems related to the size of the state space by introducing a relatively small number of explanatory variables that explains the cost structure of the problem, 
and learn the associated weights through the observation of simulated outcomes during an offline learning phase. Third, to handle large action spaces, we define an integer linear program to solve the embedded decision problems with limited computational effort, instead of enumerating the full action space. This program is typically able to solve the decision problems in less than 5 seconds.

After testing a variety of explanatory variables, we select a set of variables that works well to capture the cost structure of the problem. Experiments on small instances show a gap of less than $3 \%$ between the learned policy and the optimal policy. Numerical experiments confirm that the proposed method is able to overcome the computational challenges associated with the problem. The resulting policies outperform a variety of myopic benchmark policies by $10-15 \%$ on average. Furthermore, we compare the performance to a benchmark policy that estimates lookahead costs based on a scenario sampling procedure. This benchmark policy is typically outperformed by $2-8 \%$; the outperformance is generally stronger for instances that offer more flexibility. We validate the algorithm on a number of virtual instances, as well as a realistic instance based on the Copenhagen street network.

3. How can we test the behavior and interaction of autonomous actors in urban supply chains under a variety of circumstances?

To answer this research question, we develop an agent-based simulation framework, which is described in Chapter 4. Agent-based simulation is particularly suitable for applications in the context of urban freight transport, as we deal with multiple stakeholders who have a high degree of autonomy and often divergent objectives. By modeling autonomous actors that make their decisions based on a set of rules, we can evaluate what decisions rational actors would make, and how they interact with other actors. In this way, we observe what the impacts of urban logistics schemes are on the various actor types, and if they yield the desired environmental performance. Based on how the performances per agents change, we can gauge whether a scheme might be implemented in a practical setting, or if certain actors would oppose such a scheme. As the method is simulation-based, the framework can be flexibly adjusted to incorporate alternative measures to test. A large variety of scenarios can be analyzed within reasonable computational time.

The framework introduces five agent types in the context of urban logistics, along with their KPIs, objective functions, action space, and system constraints. The framework considers both the financial and environmental impact of urban logistics schemes, with the financial performance being itemized 
per actor. We describe the interactions between actors, their information exchanges, and their monetary flows. In defining the actions of agents, we distinguish between three levels of decision-making: strategic, tactical, and operational. Strategic decisions are decisions that are fixed for a longer period of time (e.g., multiple years). These decisions are fixed at the beginning of the simulation, and are essentially used as input that define a scenario. Tactical decisions commit actors for a time period of medium length (e.g., on a yearly or monthly base). We propose a lookahead procedure that samples the costs associated with the various tactical decision, such that the actor can rationally assess the impact of these decisions. Operational decisions are made at every decision moment in the simulation. The operational decisions facilitate an accurate calculation of, for example, vehicle distances, monetary flows, and the throughput of the UCC.

The working of the framework is briefly illustrated to assert the functionality of the model and to show how to interpret the outcomes. The ideal solution is one in which every actor benefits, but we show this is difficult to realize. Instead, what we propose is to identify schemes that have a positive environmental impact, benefit the actors that we require to alter their behavior, and where necessary support or steer behavior by means of restrictions, regulations, and (temporary) subsidies.

4. What are abstract representations of urban supply chains for small retailers in Western European cities, which can be used to test the impact of interventions in urban freight transport?

In Chapter 5, we conduct a literature study to gather data on the properties of supply chains in European cities. We used the data provided in several metastudies, and complemented our data set with figures obtained from various case studies and publicly available sources. The resulting data set was verified - and where necessary altered - in a number of expert interviews. For the carriers, we have defined realistic ranges for the number of stops per trip, the line-haul distance, and the costs. For receivers, we have provided unloading times, the timing of delivery, delivery frequencies, the percentage of scheduled orders, and the volume of orders. For UCCs, we consider the size of the target area, the distance from the target area, and various parameters that help to determine the costs. Finally, for the administrator we have collected data regarding zone access fees, access time restrictions, and weight restrictions.

Based on the data set we created, we created instances that represent supply chains in Western European cities in an abstract manner. We developed a number of receiver profiles to reflect the real-life variety of the order patterns 
of retailers; particularly the distinction between centralized and decentralized supply chains significantly influences order patterns.

5. How is the urban freight system affected when combining various companydriven initiatives and government regulations?

Chapter 5 applies the simulation framework that has been designed in Chapter 4 on a variety of abstract networks. We test 15 distinct measures that may be either administrative or company-driven, and analyze both their main effects and twoway interaction effects. Ultimately, we find that the most significant environmental benefits are obtained by either consolidating freight upstream by means of order sharing between carriers, or by consolidating downstream at a UCC. The collaboration between carriers that we study yields good performances from an environmental perspective as well as from a financial perspective. However, for a real-life implementation, such a collaboration needs to overcome various practical and competitive concerns. Combining the carrier coalition with a consolidation policy at the shipper level appears to yield the best environmental results, achieving emission reductions up to $56 \%$. Downstream consolidation at the UCC level results in emission reductions up to $44 \%$. However, without supporting measures, the UCC is too expensive to be a financially viable alternative for both carriers and receivers. A ban on heavy trucks is effective in increasing the utilization of the UCC, yet it is a very harsh measure from a practical point of view. Subsidization of carriers also proves to be effective, particularly when applied in combination with a pricing policy such as road pricing. However, the income from pricing policies is insufficient to cover the subsidy expenses; permanent subsidization can only be successful if the administrator is committed to monetize and redistribute external costs in the long term. Although in practice many subsidy streams are halted at some point in time, the administrator does have some legitimate incentives to provide permanent subsidies. The hazardous effects of urban freight transport (e.g., damage to structures, poor air quality) eventually have negative financial consequences for the administrator as well. Thus, the administrator may decide to prevent these indirect costs by subsidization, potentially against lower costs over the long term. In Chapter 6, we perform a more detailed study on the role of the UCC; the results from this study suggest that temporary subsidies may suffice to achieve a state in which a UCC is able to function in a self-sustained manner. We further discuss these findings in our answer to Research Question 6. Our final conclusion obtained from the simulation study performed in Chapter 5 is that the UCC should ideally be used to 
bundle fractionalloads; there are no environmental benefits to outsourcing if the carrier already dispatches trucks that efficiently utilize their transport capacity.

6. What are the prospects for an urban consolidation center in a realistic setting? Again applying the agent-based simulation framework, we have performed a simulation study based on the city of Copenhagen. This simulation study is described in Chapter 6. We adopt the location of the real Copenhagen UCC for the virtual consolidation center. Furthermore, we make a selection of shops in the city that could potentially use the UCC, and use their real locations. Using an OpenStreetMap implementation, we generate a network with realistic travel times, taking into account the configuration of the real street network.

The main problem for UCCs is that they introduce extra costs in the supply chain due to the required transshipment, whereas efficiency gains in transport can only be made on a short distance. To evaluate whether these gains can exceed the costs for various amounts of throughput, we study a setting in which a UCC starts without a customer base. Customers (both receivers and carriers) can periodically decide to join or leave the UCC. When attracting more customers, the UCC is able to benefit from economies of scale. Consequently, it can lower its price, which may convince more customers to join. For a limited period of time, the administrator may allocate subsidies to the UCC, or to receivers and carriers that commit themselves to the UCC. Furthermore, the administrator can implement access time restrictions or access fees to encourage the use of the UCC. The goal is to identify business models that are sustainable in the long run, meaning that they should not require lasting external funding. Value-adding services play an important role in the model; real-life UCCs obtain a sizable portion of their revenues from these services.

Our numerical experiments indicate that the UCC is able to significantly improve the environmental performance for freight transport of the target group, with $60 \%$ less trucks in the city and $70 \%$ reductions of emissions. However, it is challenging to identify schemes that are also financially sustainable. We found that the sequence in which customers are attracted is essential; carriers should be attracted before the receivers to generate a sufficiently high income. For good schemes, almost $80 \%$ of the UCC revenue stems from carriers; the majority from the remaining income stems from providing value-adding services. We did not encounter schemes that generate positive revenues without supporting administrative measures. Temporary subsidies to the carrier appear to be the most effective measure to eventually reach financial stability, whereas 
zone access fees - in combination with other measures - also help to improve the financial performance of the UCC.

\subsection{Managerial insights}

In this section, we summarize the key managerial insights of this thesis.

By preserving flexibility in both the dispatch times of orders and the construction of routes composed of multiple segments, the efficiency offractional truckload line-haul operations in networks with transfer hubs may be improved significantly. We have developed an algorithm to schedule the transport of LTL orders via a network with transfer hubs that allow for reloads. The numerical experiments imply that both in terms of financial performance and environmental performance, preserving the flexibility to alter physical routes as well as the time of departure yields significantly better results, with increases in fill rates over $20 \%$. In a setting in which orders arrive dynamically over time, we therefore propose not to fix routes in advance, but rather allow to adjust schedules when new consolidation opportunities arise.

Using explanatory variables for expected downstream costs based on the available vehicle capacity, the volumes per latest dispatch time, and the number of locations that might be visited, lookahead policies may significantly improve the efficiency of order dispatching decisions for urban consolidation centers.

To address the dispatching problem that UCCs face, we have developed an algorithm that yields a consolidation policy. In this algorithm, three types of explanatory variables - available vehicle capacity per future decision moment, volumes per latest dispatch time, and the number of locations that might currently be visited - are used to estimate the downstream costs associated with the decisions that are made based on the current inventory and vehicle availability. By observations of future costs, we learn about the downstream costs associated to these explanatory variables. The resulting estimates of downstream costs help to significantly improve the quality of decisions. The proposed solution method consistently outperforms the benchmark policies, including a policy based on sampling downstream costs that notably outperforms the myopic benchmark policies. If we do not have a perfect estimate of downstream costs, but are able to rank actions well, it still pays off to consider the future impact of dispatching orders now against holding them until a future decision moment. With our numerical experiments, we show an average outperformance of myopic benchmarks between $10 \%$ and $15 \%$.

An analysis of urban logistics schemes should address urban supply chains and all its stakeholder as a coherent entity, rather than treating a city as an isolated 
system unaffected by upstream logistics decisions.

The majority of studies in urban logistics consider the city as an isolated system, and do not take into account decisions that are made outside the city boundaries. However, important decisions are already made upstream. Shippers usually located at distant locations - make decisions that impact the transport efficiency within the city. Carriers make the bulk of both their revenues and costs with their transport over longer distances; last-mile distribution is often a minor component of their transport process. The decisions that shippers and carriers make may be efficient from their own perspective (e.g., visiting five cities with a single truck), but may simultaneously be deemed inefficient from the perspective of the city. It is therefore imperative that when analyzing urban supply chains, one takes into account the logistics decisions of all stakeholders involved, including those made upstream. To exemplify this statement: our numerical experiments show attainable emission reductions up to $56 \%$, solely achieved by altering upstream decisions by shippers and carriers.

When evaluating urban logistics measures, all involved stakeholders must be taken into account in order to find solutions that both yield considerable environmental improvements and provide a financial incentive to the stakeholders that are required to alter their behavior.

Urban logistics takes place in a complex environment, in which multiple stakeholders are involved. These stakeholders often have divergent objectives. Many initiatives in urban logistics fail, because they have a negative impact on (or at least insufficient benefits for) certain stakeholders. Without a proper incentive, actors are generally not willing to commit to solutions. A quantitative analysis of the impact of solutions on the level of individual actors should be performed. If a solution requires some actors to alter their behavior, the solution ideally provides a positive incentive to these agents. If a solution does not satisfy this property, the administrator may re-allocate costs and gains over the actors by means of pricing and subsidizing. This re-allocation might sufficiently alter the system, such that actors have an incentive to adjust their decisions. However, the impact of such measures on the financial performance of the administrator should also be addressed. Another way to enforce a change of behavior is by means of restrictions. When imposing restrictions, the solution should ensure that the affected actors have reasonable alternatives, in order to safeguard the economic viability of the city. The numerical results from Chapter 5 and Chapter 6 demonstrate that particularly the financial performances of carriers and the UCC are heavily influenced by subsidies and restrictions. 


\subsection{Discussion and further research}

In Section 7.3.1, we discuss various possible extensions of the work that has been presented in this thesis. In Section 7.3 .2 , we present a variety of implementation challenges in the implementation of urban freight initiatives, and points out corresponding directions for future research.

7.3.1. Model extensions. We discuss a number of possible extensions for the work presented in this thesis.

7.3.1.1. Extending the solution method for the dynamic line-haul planning problem. We consider four possible extensions for the 4PL decision support system that we developed in Chapter 2. So far, we have assumed that travel times and handling times are deterministic. As a consequence, the $4 \mathrm{PL}$ is able to postpone dispatching until the last possible moment. In a practical setting, decision makers often anticipate factors such as delays and disruptions by allocating some slack in their plans. The first extension we consider to address this point is within our deterministic setting. We might take into account possible delays and disruptions by, e.g., putting a constraint on the minimum amount of slack, or by penalizing solutions that contain insufficient slack. A second extension would be to model stochastic travel times instead. This would allow to evaluate the robustness of solutions, which may have an impact on the amount of slack and the number of transshipments. Third, in response to disturbances, the 4PL might decide to re-plan schedules during the execution of routes. This can be done both in a deterministic and a stochastic setting. Although we touch upon this subject, it is worth to be studied in more detail. Fourth, the model can be extended by incorporating a lookahead policy similar to the one described in Chapter 3.

7.3.1.2. Extending the solution method for the DDP-TW. Various extensions can be considered for our algorithm to solve the Delivery Dispatching Problem; we discuss two of them. The focus in Chapter 3 is on the dispatching problem itself, rather than the embedded routing problem. In the bulk of the experiments, we have applied a relatively simple analytical expression to estimate the properties of the routing component. We also have tested the impact of solving the routing problem embedded in the dispatching problem with a VRP heuristic, but this solution is not scalable to larger instances. In the context of urban logistics, VRPs may embed properties such as access time restrictions, zone access fees, and delivery windows. The first extension we discuss is therefore to take into account these richer VRPs; some references to alternative analytical expressions are provided in the chapter. Embedding such expressions 
may yield additional insights into the performance of the solution method. Altering the VRP also gives rise to re-evaluate the set of explanatory variables that we use to capture the cost structure; new variables may be required to obtain a better fit with more complex VRPs. A second topic that is worthy of additional research is that of alternative methods to solve instances with large action spaces. Although we can solve fairly large instances with the ILP that we formulated, this approach has its computational limits, given that the ILP must be solved many times when learning about the consolidation policy. The efficiency of solving the decision problem might be improved by heuristically reducing the action space, which requires removing bad actions a priori. This requires an additional study regarding the common properties of good actions, such that we do not discard good actions without evaluating them.

7.3.1.3. Optimization within the agent-based simulation framework. In the agent-based simulation studies (Chapter 4 to Chapter 6), we have deliberately adopted relatively simple planning algorithms for the decision processes of actors, to accurately reflect the common practices of smaller actors in logistics. This corresponds with our aim to evaluate urban logistics schemes in the prevailing context of urban logistics. However, the agent-based simulation framework may also be used to optimize system-wide performance. Efficiency gains can be achieved by adopting more advanced solution methods. A natural extension of our work might therefore be to implement these smarter solution methods in the simulation framework and study the potential gains that can be achieved on the planning level.

7.3.1.4. Integration of line-haul and last-mile planning systems. As already discussed in the introduction of this thesis, the focus of this work is on the consolidation and coordination of freight within flexible transport networks, in the spirit of the Physical Internet. If this concept becomes reality, it will be commonplace for goods to be transported from origin to destination via multiple transport modes, ensuring an efficient use of the transport network. For urban logistics, this implies that planning on the line-haul distances and on the last-mile distribution level should be tightly integrated. Consequently, a single planning system will be responsible for both the line-haul network and for a large number of cities (e.g., on the national or European level). Practitioners involved in the CONCOORD project also stress the importance of systems that integrate the line-haul transport network and the last-mile distribution networks for a multitude of cities, each with its own UCC.

With our work in this thesis on both planning in line-haul networks and the last-mile dispatch problem, we have provided building blocks for such systems. 
Actual integration of the algorithms may pose (computational) challenges that require additional design steps. The complete integration of planning systems for the line-haul and the last-mile distribution would be a valuable contribution to literature, both methodologically and for evaluation purposes. A full integration allows us to study network-wide effects of interventions. We may measure the environmental impact on a higher level, and address possible performance tradeoffs between line-haul and last-mile. An integrated system can aid in analyzing the impacts of local administrators implementing their own regulations, which on a system-wide level may have detrimental effects. Simulations that simultaneously measure performance on the line-haul and within the cities might aid in the synergy of regulations.

7.3.2. Implementation challenges. Our main research objective has stated that we aim to obtain quantitative insights into the effects of urban logistics schemes. As the vast majority of urban freight transport literature is of a qualitative nature - often lacking a quantitative analysis to support claims regarding the alleged benefits - this is one of the key contributions of our thesis. Specifically, we have illustrated the difficulty in finding feasible solutions that satisfy all stakeholders. Despite the importance of quantifying the impact of a solution, simply showing that a solution is feasible and beneficial is not sufficient for a practical implementation.

Based on an empirical study, Cruijssen et al. (2007) list various objections that actors have against cooperation in logistics. We provide the following categorization of these objections: (i) lack of quantification of costs and gains, (ii) unclear how gains and workloads can be fairly allocated, (iii) reasons of competitiveness, (iv) lack of resources and knowledge, and (v) lack of support from government and transport sector.

In this thesis, we have addressed the first concern in detail with our optimization method and our agent-based simulation studies. To various extents, we have addressed or touched upon the remaining concerns. This section elaborates on directions for future research that are required to address these concerns in more detail and aim to overcome them in a practical setting.

7.3.2.1. Allocation of costs and gains. In our agent-based simulations, we have quantified the financial and environmental effects of various urban logistics schemes. The nature of our simulations was explanatory. We have measured how the system-wide performance and the performance of agent types are influenced. However, showing that system-wide gains can be made does not guarantee that rational agents would commit to a solution. For example, carriers may perform better overall when collaborating, but the gains should be 
fairly divided over the individual carriers in order to ensure their participation. Furthermore, so far we have ignored the possibility that agent types are able to compensate other agent types, i.e., the option to re-allocate system-wide gains by means other than subsidies.

A model based on game theory is expected to help modeling such business cases in more detail. The field of game theory is concerned with the strategic interactions between rational and autonomous actors. On a high level, we can distinguish between two forms of game theory: non-cooperative game theory and cooperative game theory.

In non-cooperative game theory, the interaction between independent actors is studied. It aims to predict the actions and payoffs of rational actors, given their beliefs of what other actors will do. The solution to a non-cooperative game is usually a Nash equilibrium, which is the set of actions for which no actor can improve its payoff by unilaterally changing its action. The Nash equilibrium does not guarantee that actors maximize their payoff, as illustrated by the classical 'prisoner's dilemma'. In cooperative game theory, players can form lasting alliances (coalitions), which in practice can be reflected by legally binding contracts between the actors. A necessary assumption to apply cooperative game theory is that the payoff of the coalition exceeds the sum of payoffs for individually operating actors. More specifically, games are typically designed such that their payoff function is superadditive (i.e., the union of disjoint coalitions has a value that is at least equal to coalitions' separate values) and monotonic (i.e., larger coalitions obtain at least the same payoff as their subcoalitions). Given that such a payoff function exists, the main question is how to distribute the coalition gains, allocating the gains according to some fairness properties. Common properties for fairness are individual rationality (every actor obtains at least the same payoff than he would individually), symmetry (identical actors receive exactly the same payoff), and zero allocation to null players (players that do not contribute to the coalitional gains should not receive any gains). In addition, the allocation is preferably efficient (distributing exactly the gains of coalitions), additive (the allocated payoffs of a sum of games is equal to the sum of payoffs of the individual games), existing for every coalition, unique for every coalition, and easy to compute. A coalition is stable if no actor would be better off by leaving the coalition. Common solution concepts for a cooperative game are the computation of Shapley values, the core, the nucleolus, and the kernel. (Harsanyi et al., 1988; Osborne and Rubinstein, 1994; Brandenburger, 2014)

Without attempting to provide an exhaustive overview, we refer to some game theoretical applications in a logistics setting that relates to the topic of this thesis. 
Reyes (2005) studies a flow game in the context of freight transport networks with transshipments, with the arcs in the network being operated by independent carriers. He solves the problem by computing the Shapley value. HafezaIkotob and Makui (2015) study a more complex variant of the flow game that considers the stability of the throughput of the network, and apply various solution concepts to solve the problem. Yang and Odani (2007) study the collaboration between carriers and a UCC, formulating solution concepts based on the core of the game. Finally, Dahlberg (2015) studies a cooperative game that contains carriers, a UCC, and a financing municipality, comparing various solution concepts.

We suggest that simulations of an explorative nature are used to identify good schemes, and subsequently analyze the best schemes into more detail with the use of game-theoretical methods. Such a game-theoretical analysis not necessarily needs to take place on a stand-alone basis. Shoham and Leyton-Brown (2008) discuss the integration of game-theoretical elements within agent-based systems. Such an integration would allow to retain the favorable properties of agent-based analysis, while simultaneously implementing behavioral rules for agents that are consistent with the mechanisms of game theory.

7.3.2.2. Resources and knowledge. Solutions in the field of logistics are frequently based on some form of collaboration with other parties, and as such often require sizable investments and specialist knowledge (Cruijssen et al., 2007; Mason et al., 2007). One requirement that is at the heart of many initiatives is the presence of an information system that enables the exchange of detailed real-time information. Such a system is essential to flexibly plan operations, and couple orders to transport resources in an efficient manner. Aside from the system itself, companies may also need to invest in sensors, tags, and scanners. For many companies, adopting such an information system is a significant investment. Other possible resource investments include standardized loading units that are shared by multiple partners, such that orders can seamlessly be reloaded from one vehicle to another.

Although significant resource investments are required, the efficiency gains are ultimately expected to reduce operational costs. For logistics companies to remain competitive, they must strive for supply chain efficiency; ignoring new developments likely results in losing their competitive position. Dalmolen et al. (2015) propose a four-layer model for inter-organizational information systems in supply chains, which integrates the supply chain-, organizational, semantic, and technical levels. The model provides some guidelines for establishing information systems that facilitate collaboration within supply chains. 
Cruijssen et al. (2007) mention various objections of logistics actors against collaboration that correspond to a lack of knowledge. Collaboration initiatives pose various challenges; logistics companies are often not equipped with the right knowledge to handle those challenges. As observed by Aghion et al.(2009), successful innovation requires that the development of human skills is in line with the applied technologies, policies, and degree of business support. Zijm and Klumpp (2017) state that logistics education is an essential factor for the success of future innovations. They furthermore stress that the adoption of new technologies and their implementation has become a parallel process, in which the role of human intelligence is partially taken over by artificial intelligence.

We identify two main research directions that require additional attention. First, the design of the information systems required for integrated logistics systems needs to be crystallized. Compared to existing systems that facilitate cooperation in supply chains, the context of urban logistics differs in the sense that the environment is comprised of many small, independent actors. Information systems should be implemented in a way that requires minimal investments and knowledge, while still enabling far-reaching flexibility in urban supply chains. Second, although abstract assessments exist to overcome the lack of resources and knowledge, there are no concrete applications that tell us how to handle the specific shortcomings in the field of urban logistics. In an effort to close the gap between resources and knowledge on the one hand and implementation requirements on the other hand, it is necessary to have a detailed cost-benefit analysis that includes the specific resource- and knowledge deficits that need to be overcome, the required (capital) investments, and the long-term benefits.

7.3.2.3. Competitive concerns. Cruijssen et al.(2007) indicate that small logistics actors in particular oppose collaboration opportunities. They fear that large parties will disproportionally benefit from collaboration. In addition, they may have concerns to lose clients to competitors or to lose their competitive advantages. Their concerns can partially be addressed by applying allocation mechanisms as described previously in this section. Contractual obligations should sharply define the distributions of gains, costs, and workloads to safeguard the position of small actors within the coalition.

The previously mentioned objections regarding a lack of knowledge and resources are especially relevant for small actors. They often do not have in-house specialist knowledge, and lack the financial means to make large investments in, e.g., standardized load units, information infrastructures, and logistics training. The reluctance of small actors to collaborate with competitors is problematic from a system point of view. As indicated in this thesis, these parties have the 
most problems to work efficiently, due to the increasing fragmentation of freight flows. Therefore, the most gains are expected to be achieved by grouping these actors into a coalitional structure. Eventually, small companies may have no choice but to collaborate, as fragmentation in freight transport will continue to hinder their operations.

Various works suggest that discussion, stakeholder analysis, and a methodological implementation plan are key in overcoming competitive concerns. The paper of Macharis and Bernardini (2015) highlights the importance of explicitly including the involved stakeholders in the logistics decision-making process, proposing a multi-actor, multi-criteria analysis to illuminate discrepancies in stakeholder objectives and to encourage discussions. Verlinde and Macharis (2016) stress that discussion between government, industry and academia is essential to successfully implement solutions. Furthermore, they mention the supporting roles of banks, insurance companies, real estate companies and the media in the realization of urban freight initiatives. Verstrepen et al. (2009) provide a four-stage framework for managing horizontal collaboration in logistics, with a focus on the role of logistics service providers. In this model, the collaborating actors move through the stages of strategic positioning (e.g., defining motives and objectives), design (e.g., selecting partners, negotiating), implementation (e.g., of information systems and contractual agreements), and moderation (e.g., control and evaluation). During the last phase, feedback may give rise to reconsider the design of the cooperation. The final collaboration framework that we discuss is that of Gonzalez-Feliu et al. (2013). They develop a decision support methodology that contains the following phases: strategy design (define decision makers, objectives, and strategies), strategy simulation (quantify the effects on each decision maker for all strategies), individual clustering phase (rank strategies individually per decision maker), system clustering phase (rank strategies from a system perspective), dominance phase (identify dominant strategies), and finally decision group identification (reach a consensus between the decision makers).

We have discussed various frameworks that provide a methodological approach to organize collaboration in a structured manner, but thorough applications in the field of urban logistics are lacking. Most assessments of collaboration in urban freight networks are on a qualitative level only. In contrast, we believe that a solid quantitative analysis should be firmly embedded in the process of forming coalitions; the framework of Gonzalez-Feliu et al. (2013) illustrates this idea. 
7.3.2.4. Lack of support. Many actors in urban supply chains experience a lack of support from either the government or the transport sector in their efforts to collaborate or work more efficiently. We separately discuss the roles that both government and the transport sector might have in the support of urban freight initiatives.

On the level of the government, the use of subsidies and regulations has been extensively discussed in this thesis. In addition to these measures, an opportunity for governments lies in the legislative area. In the transport sector, the legislative framework is of essential importance. Traditionally, the transport of an order is formalized by two contracts: a contract between the shipper and the receiver, and a contract between the shipper and the carrier (Verlinde et al., 2012). With the introduction of additional actors such as the 4PL, the UCC, and multiple carriers, the distribution of obligations and responsibilities becomes increasingly complex. Contracts are often based on so-called 'incoterms', which are standardized international commercial terms for the transport of goods, developed by the International Chamber of Commerce (Szymonik, 2014). These standards define, for example, the place of delivery, and the burden of risk insurance during transport. The current incoterms are not well equipped to deal with innovative supply chain concepts. For example, if a receiver specifies the UCC as its delivery address, there is no standard for the responsibilities on the last mile. Typically, this requires additional contracts between the UCC and the receiver. Clearly, the higher the number of actors involved in the supply chain, the more complicated the contractual aspects become. New incoterms will appear in 2020; when addressing the specific requirements of modern urban supply chains, they have the potential to significantly reduce the administrative burden in urban logistics initiatives.

We proceed with an assessment of the support opportunities within the transport sector. The solutions that we propose rely on the presence of coordinating parties such as a 4PL and a UCC. As discussed earlier in this section, the importance of information systems is essential for the success of collaborative solutions in urban freight transport. These solutions require real-time exchange of accurate information on the level of individual orders. Mason et al. (2007) stress that standardization of information systems is essential to facilitate cooperation in logistics. Therefore, the transport sector should make an effort into moving towards industry standards for information systems. The same holds for other resources (e.g., loading units), such that the logistics systems of a multitude of actors can be integrated seamlessly (Quak, 2011). In a study that addresses both horizontal and vertical collaboration in logistics, Mason et al. (2007) state that 
there are many barriers to overcome, and that certain logistic actors should take the lead in setting up collaboration structures. The authors refer to various actors with a coordinating role, such as 4PLs and UCCs in an urban logistics environment. It is unlikely that small actors - such as independent carriers and receivers themselves - take the initiative to adjust their practices. Support from actors with a central role in the supply chain is necessary for collaborative effort to succeed, taking away as many barriers as possible in order to convince logistics actors to alter their behavior.

With respect to supporting collaboration efforts, we identify two literature gaps that require attention. On the governmental side, it is essential to fit legislation to the requirements of the logistics parties. This requires a study on how to distribute responsibilities and liabilities in a fair manner, while simultaneously facilitating transport contracts that are simple to adopt. On the side of the transport sector, the key contributions that are required are the definition of clear supply chain strategies, the introduction of industry standards, and the dissemination of knowledge. In this process, coordinating actors should adopt active leadership roles. Future research could delve further into their facilitating roles, rather than only focusing on the coordination aspect. 


\section{Summary}

Recent developments in the field of urban freight transport necessitate a move towards integrated logistics systems, which facilitate the efficient planning of fragmented freight flows. In this thesis, we study the role of consolidation and coordination to improve the efficiency of urban freight transport. We summarize the contributions of the thesis per chapter.

Part I of the thesis is comprised of Chapter 1 and provides an introduction to the problem setting. We present a summary of trends and developments in urban logistics. Among the key trends are increased urbanization, a revival of small-format stores, and an adoption of Just-in-Time ordering principles, resulting in fragmented freight flows and low utilization of transport capacity. This in turn has negative impacts on the economy, the environment, and social wellbeing. A variety of solution concepts has been proposed in theliterature to tackle these problems, which can be divided into four categories: (i) infrastructural changes, (ii) transport system reorganization, (iii) company-driven initiatives, and (iv) administrative measures. Although many measures have the potential to improve the efficiency of urban transport and reduce hazardous impacts, many solutions fail in practice. An important reason for this is that urban logistics takes place in a complex field, in which multiple actors with divergent objectives operate. With this thesis, we therefore address the following research objective:

To develop mathematical models that support coordination actors in consolidated freight planning, and to obtain quantitative insights into the challenges and requirements to establish integrated logistics systems, in which independent actors coordinate their decisions to improve the efficiency of urban transport.

Part II is composed of two chapters. These chapters focus primarily on the methodological contributions to the field of consolidated freight planning. 
Chapter 2 presents an algorithm to facilitate the dynamic planning of fractional truckloads in a network with transfer hubs. The algorithm is designed from the perspective of a 4th Party Logistics Service Provider (4PL) that plans the transport of orders in an intermodal network comprised of contracted carriers, but has the opportunity to replan later in response to arising consolidation opportunities. Transport prices in the network are volume-based, thus giving the $4 \mathrm{PL}$ an incentive to use the capacity of transport units as efficiently as possible. At the transfer hubs in the network, transport units may be transshipped between transport modes. Furthermore, the reload capabilities of the transfer hubs are used to transfer orders from one transportation unit to another. Orders are subject to time windows and the line-haul services may be subject to timetables. Therefore, the evaluation of consolidation opportunities must take into account the time feasibility of solutions. Our algorithm constructs and evaluates decision trees that take into account both the cost savings and the feasibility of solutions in which orders are transported by multiple transport means. We test the performance of the algorithm on a variety of virtual networks, as well as on a real data set provided by a leading Dutch 4PL. Our findings indicate that both the financial and environmental performance improve significantly by retaining flexibility in both the structure of routes and the dispatch times; compared to direct transport we are able to improve container fill rates by $57 \%$, resulting in cost savings of $34 \%$ and reductions of $\mathrm{CO}_{2}$ emissions of $30 \%$. When we compare to the case in which we only consolidate on the best routes for each individual order, still average increases in fill rates of $28 \%$ are achieved.

In Chapter 3, we develop an algorithm that provides a consolidation policy for the operator of an Urban Consolidation Center (UCC). To estimate the future costs associated to current decisions, the operator makes use of its knowledge of the stochastic order arrival process. The goal of the operator is to minimize delivery costs over time. At each decision moment, the operator of the UCC faces the decision which subset of orders to dispatch. Holding orders for some time may result in better consolidation opportunities at a later point in time, but there is no guarantee that such opportunities will arise. We model this decision problem as a Markov Decision Model. In order to solve large instances of this problem, we propose a solution method based on Approximate Dynamic Programming (ADP) with a linear value function approximation. We study a variety of explanatory variables that may be used to estimate the future costs corresponding to dispatching decisions and identify a set of explanatory variables that can be used to accurately capture these costs. To solve the dispatching problem that is embedded into the larger optimization problem, we develop an Integer Linear 
Program; the applicability of this method is validated for action spaces that contain up to $2^{120}$ decisions in the numerical experiments. We test the algorithm on a variety of network configurations; the results imply that the method works best when there is sufficient flexibility with respect to the times that orders are dispatched. Both myopic benchmark policies and lookahead benchmark policies are consistently outperformed. On average, our ADP algorithm outperforms the myopic benchmark policies by $10 \%$ to $15 \%$ and the lookahead benchmark policies by $2 \%$ to $8 \%$.

Part III contains three chapters that focus on agent-based simulation of urban freight transport.

Chapter 4 introduces an agent-based simulation framework to evaluate urban logistics schemes, with a scheme being composed of one or more companydriven initiatives and/or (local) government policies. We define five different types of agents: receivers, carriers, shippers, the UCC, and the local administrator. By defining these agent types and their corresponding actions, interactions, and objective functions, we are able to model and analyze a wide range of measures in urban logistics. The framework is explicitly designed to test combinations of company-driven initiatives and administrative policies; practice indicates that combinations of solution concepts from both classes typically yield the best results. Furthermore, we consider line-haul transport as an integral part of routes and decisions, rather than focusing only on the processes within the city boundaries. This design choice is better aligned with the profit model of carriers and the dispatching decisions made by shippers, thus allowing to more accurately evaluate the impact of urban measures on the upstream process and vice versa. We distinguish between three levels of decision making (strategic, tactical, and operational) and propose solution methods for each level. The working of the framework and the interpretation of results are illustrated with some small numerical experiments. In line with practice, the results of these experiments show that most measures are able to achieve environmental improvements, but that it is challenging to find schemes that are also financially sustainable for the involved stakeholders. Typically, multiple measures need to be combined to achieve both objectives; schemes that only contain companydriven initiatives or only administrative measures appear to be ineffective.

In Chapter 5, we apply our agent-based simulation framework to evaluate a large number of scenarios. To be able to generalize our conclusions, we create a data set that represents a large number of Western European cities. To this 
end, we collect data from a multitude of overview studies, case studies, and publicly available data sources. We use the collected data to assign realistic values for properties to the agents in urban supply chains. To reflect the large differences that exist between such supply chains in practice, we create a number of receiver profiles that represent the ordering systems of receivers. These profiles differ in the number of distinct shippers that deliver to one receiver, delivery frequencies, and delivery volumes. By randomly assigning profiles to receivers, we reflect the real-life variety of urban supply chains. Furthermore, we test several geographically distinct instances. On these instances, we test 15 distinct measures that are either company-driven or imposed by the local administrator. First, we test the measures individually to obtain insights into their impact in isolation for various parameter settings. Then, we apply a fractional factorial design to quantify the main effects and two-way interaction effects. This way, we evaluate the symbiosis between measures, in particular between administrative policies and company-driven initiatives. Third, we perform scenario analysis for the most promising measures to evaluate the effects of various combinations of measures on both environmental KPIs and the financial performance of the agents. We conclude that bundling of orders is essential to achieve significant environmental benefits; this bundling may either take place upstream at the carrier level or downstream at the UCC level. When carriers cooperate with each other and centrally plan their order transport, emission reductions of $56 \%$ are attainable; by consolidating at the UCC, emission reductions up to $44 \%$ are achievable. Collaboration between carriers appears to be sustainable from a financial point of view, although for such a collaboration to materialize in practice, significant hurdles should be overcome. The concept of the UCC is also challenging to successfully implement, particularly from a financial point of view. Stringent regulations or substantial subsidies allocated to carriers are effective in altering the behavior of decision makers, but require a long-term commitment from the administrator.

Another application of the agent-based simulation framework is described in Chapter 6. This chapter focuses explicitly on the feasibility of UCCs. The test instance is based on the city of Copenhagen. Using an OpenStreetMap implementation, we generate a realistic network based on the street network of Copenhagen. By making use of geo-tags, retailer locations are also obtained from OpenStreetMap. Furthermore, we use the location of an existing UCC in Copenhagen. To accurately describe the supply chains and measures, we use the data collected in Chapter 5, specific data for the city of Copenhagen, and data obtained in expert interviews. The initial state of each simulation run is a UCC 
without a user base. To support the UCC, subsidies may be allocated to receivers, carriers and the UCC, but only for a limited period of time. After that period, the UCC must have attracted sufficient users to operate without external funding. Furthermore, regulations may be implemented to support the UCC. We numerically test 1,458 distinct urban logistics schemes. In line with both literature and practice, we find that a UCC without any support has very slim chances for long-term survival; both external funding and supporting regulation appear to be necessary. For less than $5 \%$ of the scenarios we find a profitable long-term situation for the UCC (when ignoring potential environmental benefits, which are not translated in financial terms); these scenarios mainly assume favorable cost settings for the UCC. We find that the sequence in which users commit to the UCC is essential for its long-term viability. The bulk of the revenues can be generated from carriers, yet when receivers have already set the UCC as their delivery address, the carrier can essentially outsource its last-mile distribution for free. We therefore propose measures that focus on attracting carriers; zoneaccess fees and in particular (temporary) subsidies are effective for this purpose. Despite the financial challenges, the numerical results show that the environmental benefits of a UCC may be substantial; we achieve emission reductions of about $70 \%$ and are able to reduce the number of trucks in the city by up to $60 \%$.

Part IV concludes the thesis. Chapter 7 reflects on both our main research objective and the individual research questions. We summarize the key managerial insights that were obtained with our research:

- By preserving flexibility in both the dispatch times of orders and the construction of routes composed of multiple segments, the efficiency of fractional truckload line-haul operations in networks with transfer hubs may be improved significantly.

- Using explanatory variables for expected downstream costs based on the available vehicle capacity, the volumes per latest dispatch time, and the number of locations that might be visited, lookahead policies may significantly improve the efficiency of order dispatching decisions for urban consolidation centers.

- An analysis of urban logistics schemes should address urban supply chains and all its stakeholder as a coherent entity, rather than treating a city as an isolated system unaffected by upstream logistics decisions. 
- When evaluating urban logistics measures, all involved stakeholders must be taken into account in order to find solutions that both yield considerable environmental improvements and provide a financial incentive to the stakeholders that are required to alter their behavior.

Finally, we provide a number of directions for further research. First, we suggest extensions of the work presented in this thesis. Our solution method for line-haul planning might be enriched by incorporating stochastic properties such as time-dependent travel times and uncertain handling times, attaining a closer fit to reality. For our DDP-TW solution method, we propose the design of algorithms to intelligently reduce the action space. Furthermore, analytical expressions for richer VRPs might be incorporated to better reflect the practice of urban logistics. A new use can be found for our agent-based simulation framework by incorporating more intelligent planning methods, so that it becomes suitable for optimization rather than evaluating within the current context. The last model extension that we discuss is the integration of our line-haul and lastmile planning methods, signifying another step towards an integrated logistics system in the spirit of the Physical Internet.

The second category of further research topics is comprised of a number of possible research directions that are related to the implementation challenges. A major challenge relates to the allocation of gains and costs; we believe that game theory offers a suitable approach to solve this problem. Other implementation challenges are overcoming gaps in resources and knowledge, addressing competitive concerns of stakeholders, and improving the facilitating roles of both the government and transport sector agencies. 


\section{Samenvatting}

Recente ontwikkelingen op het gebied van stadsdistributie voor goederen scheppen de noodzaak voor een geïntegreerd logistiek systeem dat een efficiënte planning van gefragmenteerde goederenstromen mogelijk maakt. In dit proefschrift bestuderen we de rol van consolidatie en coördinatie in het verbeteren van de efficiëntie van stedelijk goederentransport. Het proefschrift is opgebouwd uit diverse delen en daaronder vallende hoofdstukken, we geven een samenvatting van de bijdrage per hoofdstuk.

Deel I van het proefschrift omvat hoofdstuk 1; in dit hoofdstuk introduceren we de probleemstelling. We vatten de trends en ontwikkelingen die spelen in stadsdistributie kort samen. De voornaamste trends zijn de toenemende verstedelijking, een heropleving van kleine, specialistische winkels en het verwezenlijken van Just-In-Time bestelprincipes. Tezamen resulteren deze trends in gefragmenteerde goederenstromen en een lage bezettingsgraad van vrachtwagens. Dit heeft een negatieve invloed op de economie, de omgeving en de sociale welvaart. In de literatuur is een variëteit aan oplossingen aangedragen om deze problemen aan te pakken; deze oplossingen kunnen in vier categorieën verdeeld worden: (i) veranderingen in de infrastructuur, (ii) reorganisatie van transportsystemen, (iii) door bedrijven geïnitieerde innovaties, en (iv) maatregelen vanuit de (lokale) overheid. Hoewel veel oplossingen de potentie hebben om de efficiëntie van stedelijk goederentransport te verbeteren en daarmee de schadelijke effecten te verminderen, blijkt dat in de praktijk veel oplossingen niet het gewenste resultaat opleveren. Een belangrijke reden hiervoor is dat stadsdistributie plaatsvindt in een complexe omgeving, waarin meerdere actoren met uiteenlopende doelen opereren. Aansluitend op deze problematiek formuleren we de onderzoeksdoelstelling van dit proefschrift als volgt:

Het ontwikkelen van wiskundige modellen die coördinerende actoren ondersteunen in de geconsolideerde planning van goederentransport en het verkrijgen van kwantitatieve inzichten in de uitdagingen en vereisten om een geïntegreerd logistiek 
systeem op te zetten, waarin onafhankelijke actoren hun beslissingen afstemmen om de efficiëntie van stedelijke goederendistributie te verbeteren.

Deel II van dit proefschrift bestaat uit twee hoofdstukken. Dezehoofdstukken richten zich primair op methodologische bijdragen op het gebied van geconsolideerde planningen van goederentransport.

Hoofdstuk 2 beschrijft een algoritme dat de dynamische planning van deelladingen van transporteenheden (zoals bijv. containers) ondersteuning in netwerken waarin overslag op de tussenliggende goederenstations mogelijk is. Het algoritme is ontwikkeld vanuit het perspectief van een 4th Party Logistics Service Provider (4PL) die de planning van het transport verzorgt in een intermodaal netwerk waarin meerdere gecontracteerde vervoerders opereren. De 4PL plant orders direct wanneer ze bekend worden, maar houdt de mogelijkheid open de planning later aan te passen wanneer mogelijkheden voor consolidatie (het samenvoegen van meerdere orders in een transporteenheid) ontstaan. De transportprijzen die gehanteerd worden in het netwerk zijn gebaseerd op volume; deze structuur geeft de 4PL een stimulans om de transportcapaciteit in het netwerk zo efficiënt mogelijk te gebruiken. De overslagpunten in het netwerk kunnen gebruikt worden om transporteenheden van de ene naar de andere modaliteit over te dragen. Daarnaast kunnen orders op de overslagpunten naar andere transporteenheden verplaatst worden. Orders zijn gebonden aan tijdsvensters en de transportdiensten opereren mogelijk op basis van een vaste dienstregeling. Om deze redenen moet bij de evaluatie van consolidatiemogelijkheden rekening gehouden worden met haalbaarheid van oplossingen. Het algoritme construeert en evalueert beslisbomen die rekening houden met zowel de kostenbesparingen als de haalbaarheid van oplossingen die bestaan uit meerdere verbindingen. Om inzicht te krijgen in prestaties van het algoritme, passen we deze toe op verschillende virtuele netwerken. Daarnaast passen we het algoritme ook toe op een echte dataset die is aangedragen door een prominente Nederlandse 4PL. De numerieke resultaten tonen aan dat zowel de financiële als de milieugerelateerde prestaties aanzienlijk verbeterd kunnen worden door flexibiliteit te behouden in zowel de structuur van routes als de verzendtijden. Vergeleken met transport zonder overslag is het algoritme in staat de gemiddelde beladingsgraad van transporteenheden met $57 \%$ te verbeteren; dit resulteert in kostenbesparingen van $34 \%$ en reduceert de $\mathrm{CO}_{2}$-uitstoot met $30 \%$. Wanneer we vergelijken met het geval waarin we alleen kunnen consolideren op de beste routes voor individuele orders zijn verbeteringen in de gemiddelde beladingsgraad van $28 \%$ haalbaar. 
In hoofdstuk 3 ontwikkelen we een algoritme dat een stelsel van beslisregels geeft voor de consolidatie van goederen door een stadsdistributiecentrum. Om de toekomstige kosten gerelateerd aan huidige beslissingen te schatten maakt de operator gebruik van stochastische informatie betreffende het tijdsaankomstproces van orders. Het doel van het stadsdistributiecentrum is om de kosten over een vooraf vastgestelde tijdshorizon te minimaliseren. Op ieder beslismoment bepaalt het stadsdistributiecentrum welke orders verscheept worden naar de klanten. Door orders gedurende een bepaalde tijd vast te houden kan het stadsdistributiecentrum anticiperen op consolidatiemogelijkheden die ontstaan wanneer nieuwe orders arriveren, maar er is geen garantie dat zulke mogelijkheden zich daadwerkelijk voordoen. We modelleren dit planningsprobleem als een Markov-beslismodel. Om grote instanties van dit probleem op te lossen ontwikkelen we een methode die gebaseerd is op Approximate Dynamic Programming (ADP) met een lineaire approximatie van de waardefunctie. We evalueren een uiteenlopende verzameling van verklarende variabelen die gebruikt kunnen worden om de verwachte toekomstige kosten behorende bij specifieke beslissingen te berekenen. Op basis van deze evaluatie bepalen we de meest geschikte verzameling van verklarende variabelen. Om de operationele beslissingen binnen het overkoepelende optimalisatieprobleem te bepalen voor grote instanties ontwikkelen we een Integer Linear Program; op basis van numerieke experimenten wordt de doeltreffendheid van deze methode gevalideerd voor beslisruimtes die tot $2^{120}$ unieke beslissingen bevatten. We testen het ADP-algoritme op verscheidene netwerkconfiguraties; de resultaten tonen aan dat de methode het best werkt wanneer er voldoende flexibiliteit aanwezig is in de mogelijke verzendtijd van orders. We vergelijken de prestaties van het algoritme met diverse benchmarks; gemiddeld presteert ons ADP-algoritme 10\% tot $15 \%$ beter dan kortzichtige beslisregels en $2 \%$ tot $8 \%$ beter dan alternatieve beslisregels die ook de verwachte toekomstige kosten meenemen.

Deel III bestaat uit drie hoofdstukken die gericht zijn op agentgebaseerde simulaties van stadsdistributie.

In hoofdstuk 4 introduceren we een agentgebaseerd simulatieframework om oplossingsconcepten in stadsdistributie te evalueren; een oplossingsconcept bestaat uit een of meerdere bedrijfsinitiatieven en/of beleidsmaatregelen van (lokale) overheden. We definiëren vijf verschillende rollen voor de agenten: ontvangers, vervoerders, verladers, het UCC en de lokale overheid. Door het definiëren van deze rollen en de bijbehorende beslissingen, interacties tussen agenten 
en doelfuncties voor de agenten zijn we in staat een grote variëteit aan maatregelen in stadsdistributie te modelleren en te analyseren. Het simulatieframework is expliciet ontwikkeld om combinaties van bedrijfsinitiatieven en beleidsmaatregelen te testen; in de praktijk blijkt dat dergelijke combinaties vaak de beste resultaten opleveren. Verder beschouwen we langeafstandtransport als een integraal onderdeel van routes en beslissingen, in plaats van ons alleen te richten op de processen die plaatsvinden binnen de stedelijke grenzen. Deze ontwerpkeuze sluit beter aan bij het verdienmodel van vervoerders en de verzendbeslissingen van verladers. Hierdoor kunnen we de stroomopwaartse effecten van maatregelen die binnen de stad van toepassing zijn nauwkeuriger analyseren, en vice versa. We maken een onderscheid tussen drie niveaus van besluitvorming (strategisch, tactisch, en operationeel) en definiëren beslissingen voor ieder van deze niveaus. De werking van het framework wordt geilllustreerd aan de hand van een aantal numerieke experimenten. In lijn met bevindingen uit de praktijk zien we dat de meeste maatregelen een positief effect hebben op de omgeving, maar dat het een uitdaging is om oplossingsconcepten te vinden die ook financieel acceptabel zijn voor de betrokken actoren. Meestal zijn combinaties van meerdere maatregelen noodzakelijk om beide doelstellingen te behalen; oplossingsconcepten die alleen initiatieven van bedrijven of alleen administratieve maatregelen bevatten zijn in ons model niet effectief.

In hoofstuk 5 passen we ons agentgebaseerde simulatieframework toe om de effecten van een groot aantal scenario's op zowel omgevingsgerelateerde KPIs als de financiële prestaties van de agenten te evalueren. Om onze conclusies te kunnen generaliseren, creëren we een representatieve dataverzameling voor West-Europese steden. Hiervoor verzamelen we data van een aantal overzichtsstudies, case-studies en openbaar beschikbare databronnen. We gebruiken de verzamelde data om de agenten in het simulatiemodel realistische karakteristieken te geven. Om de grote diversiteit die in de praktijk in bevoorradingsketens bestaat na te bootsen, definiëren we een aantal profielen voor ontvangers. Deze profielen verschillen in het aantal verladers per ontvanger, de leverfrequenties en de ordervolumes. Door willekeurig profielen aan ontvangers toe te wijzen, houden we rekening met de diversiteit in bevoorradingsketens zoals die in de praktijk speelt. Verder testen we een diversiteit aan geografische instanties. Op deze instanties passen we 15 verschillende interventies toe, die ofwel door bedrijven geïnitieerd zijn ofwel zijn ingevoerd door de lokale overheid. Eerst testen we iedere interventie afzonderlijk om inzicht te verkrijgen in de effecten voor verschillende parameterwaarden. Vervolgens passen we een 'fractional factorial design' toe om de primaire effecten van en interactie-effecten 
tussen interventies te kwantificeren. Op deze wijze beoordelen we de synergie tussen interventies, in het bijzonder tussen overheidsbeleid en initiatieven van bedrijven. Tot slot passen we scenario-analyse toe om de invloed van diverse oplossingsconcepten te bepalen. We concluderen dat het bundelen van orders essentieel is om significante verbeteringen te realiseren met betrekking tot de uitstoot van schadelijke stoffen en een reductie van het aantal vrachtwagens in de stad; deze bundeling kan stroomopwaarts plaatsvinden op het niveau van de vervoerders of stroomafwaarts op het niveau van het distributiecentrum. Wanneer vervoerders met elkaar samenwerken en een centrale planning voor hun orders opstellen, zijn emissiereducties tot $56 \%$ haalbaar. Wanneer consolidatie bij het UCC plaatsvindt worden emissiereducties tot $44 \%$ gemeten. Samenwerking tussen vervoerders lijkt een financieel houdbare oplossing op de lange termijn, hoewel voor een implementatie van de voorgestelde oplossing in de praktijk diverse knelpunten zullen moeten worden opgelost. Het is eveneens een uitdaging het concept van een UCC op succesvolle wijze te implementeren; de voornaamste bezwaren zijn van financiële aard. Strikte regulering of het toekennen van substantiële subsidies aan vervoerders zijn effectieve maatregelen om de beslissingen van de agenten te beïnvloeden, maar vereisen betrokkenheid van de overheid op de lange termijn.

Een andere toepassing van het agentgebaseerde simulatieframework is beschreven in hoofdstuk 6. In dit hoofdstuk richten we ons expliciet op de levensvatbaarheid van stadsdistributiecentra. Onze testinstantie is gebaseerd op de Deense hoofdstad Kopenhagen. Met behulp van een implementatie in OpenStreetMap genereren we een realistisch netwerk dat is gebaseerd op het stratennetwerk van Kopenhagen. Door gebruik te maken van geografische labels in OpenStreetMap bepalen we ook winkellocaties in Kopenhagen. Verder gebruiken we een bestaande locatie van een stadsdistributiecentrum in de stad. Om de goederenstromen en maatregelen nauwkeurig te beschrijven maken we gebruik van de verzamelde data in hoofdstuk 5; specifieke data voor de stad Kopenhagen en data is verkregen door middel van interviews met experts. Iedere simulatierun start met een distributiecentrum zonder gebruikers. Om het distributiecentrum te ondersteunen kan de gemeente voor een bepaalde periode subsidies toekennen aan ontvangers, vervoerders, en het distributiecentrum zelf. Na deze periode moet het distributiecentrum voldoende gebruikers hebben aangetrokken om zonder externe financiering te kunnen opereren. Ter verdere ondersteuning kan regelgeving worden geïmplementeerd om het distributiecentrum te ondersteunen. We testen 1.458 verschillende oplossingsconcepten door middel van numerieke experimenten. In lijn met bevindingen uit zowel de literatuur 
als de praktijk concluderen we dat de levensvatbaarheid van een distributiecentrum zonder enige externe ondersteuning zeer beperkt is; zowel financiering als toegangsrestricties lijken noodzakelijk. Voor minder dan $5 \%$ van de geteste scenario's is het distributiecentrum op de lange termijn winstgevend (waarbij overigens gunstige milieu-effecten niet vertaald worden in monetaire termen); deze scenario's nemen meestal gunstige schattingen voor de kosten van het UCC aan. De resultaten wijzen uit dat de volgorde waarin gebruikers aangetrokken worden essentieel is voor het uiteindelijke succes. De meeste inkomsten kunnen behaald worden bij de vervoerders, maar wanneer ontvangers het distributiecentrum als hun afleveradres aanwijzen, kunnen transporteurs in essentie gratis hun stedelijk transport uitbesteden. Om deze reden adviseren we het invoeren van maatregelen die gericht zijn op het aantrekken van vervoerders als gebruikers van het distributiecentrum; toegangsprijzen voor de stad en vooral (tijdelijke) subsidies zijn effectief om dit doel te bereiken. Ondanks de financiële uitdagingen wijzen de simulatieresultaten uit dat de voordelen van een distributiecentrum voor de omgeving substantieel kunnen zijn; we behalen emissiereducties van rond de $70 \%$ en zijn in staat het aantal vrachtwagens in de stad met $60 \%$ terug te brengen.

Met deel IV sluiten we het proefschrift af. In Hoofdstuk 7 blikken we terug op zowel ons primaire onderzoeksdoel als de individuele onderzoeksvragen. We vatten de belangrijkste managementinzichten verkregen met ons onderzoek samen:

- Door flexibiliteit in routeringsbeslissingen te behouden - zowel in verzendtijden als in de structuur van routes bestaande uit meerdere segmenten - kan de efficiëntie van transport van deelladingen over netwerken met overslagmogelijkheden significant verbeterd worden.

- Met behulp van verklarende variabelen voor verwachte toekomstige kosten, gebaseerd op (i) beschikbare voertuigcapaciteit, (ii) volumes voor de laatst mogelijke verzendtijd en (iii) het aantal locaties dat bezocht kan worden, kan de efficiëntie van verzendbeslissingen door het stadsdistributiecentrum significant verbeteren.

- De analyse van oplossingsconcepten in stadsdistributie moet de goederenstromen en de betrokken belanghebbenden als een geheel beschouwen in 
plaats van de stad als een geïsoleerd systeem te beschouwen dat niet beïnvloed wordt door stroomopwaarts genomen logistieke beslissingen.

- Wanneer we interventies in stadsdistributie evalueren, moet rekening gehouden worden met alle betrokkenen om oplossingen aan te dragen die significante verbeteringen in de leefomgeving bewerkstelligen en daarnaast financiële drijfveren aanreiken aan de betrokkenen die hun gedrag dienen aan te passen.

Aansluitend op het gedane werk in dit proefschrift beschrijven we een aantal mogelijke richtingen voor toekomstig onderzoek. De eerste categorie van toekomstig onderzoek bestaat uit uitbreidingen op de in dit proefschrift gepresenteerde modellen. Onze oplossingsmethode voor het plannen van ordertransport via een netwerk met overslagpunten kan verrijkt worden met stochastische elementen zoals tijdsafhankelijke reistijden en onzekere handelingstijden, hierdoor kan een betere aansluiting op de realiteit verkregen worden. Voor onze oplosmethode voor de DDP-TW is het ontwikkelen van algoritmes die op intelligente wijze de beslissingsruimte verkleinen een zinvolle uitbreiding. Verder kunnen analytische uitdrukkingen voor meer gedetailleerde routeringsproblemen geïntegreerd worden om de praktijk van stadsdistributie beter te modelleren. Een mogelijke nieuwe toepassing van ons simulatieframework zou intelligentere planningsmethoden kunnen bevatten, zodat het framework ook toepasbaar is voor optimalisatie. De laatste uitbreiding die we hier noemen is de integratie van onze oplossingsmethoden voor netwerken met overslagpunten en het planningsprobleem voor distributiecentra; dit zou een stap kunnen zijn richting geïntegreerde logistieke systemen naar analogie van het concept van het Physical Internet.

De tweede categorie van toekomstig onderzoek omvat een aantal mogelijke richtingen die gerelateerd zijn aan uitdagingen in de implementatie van oplossingen in stadsdistributie. Een grote uitdaging is de allocatie van opbrengsten en kosten; hiervoor zien we de toepassing van speltheorie als een geschikte oplossingsmethode. Andere uitdagingen zijn het adresseren van tekortkomingen in middelen en kennis, het overbruggen van concurrentie-tegenstellingen tussen besluitvormers en het verbeteren van de ondersteunende rollen van zowel de overheid als de transportsector in het opzetten van nieuwe initiatieven. 



\section{Bibliography}

Agatz, N., Erera, A., Savelsbergh, M. W. P., and Wang, X. (2012). Optimization for dynamic ride-sharing: A review. European Journal of Operational Research, 223(2):295-303.

Aghion, P., David, P. A., and Foray, D. (2009). Science, technology and innovation for economic growth: linking policy research and practice in 'stig systems.' Research policy, 38(4):681-693.

Allen, J., Browne, M., and Cherrett, T. (2012a). Investigating relationships between road freight transport, facility location, logistics management and urban form. Journal of Transport Geography, 24:45-57.

Allen, J., Browne, M., Cherrett, T., and McLeod, F. (2008). Review of UK urban freight studies. Green Logistics project, Universities of Westminster an Southampton.

Allen, J., Browne, M., Woodburn, A., and Leonardi, J. (2012b). The role of urban consolidation centres in sustainable freight transport. Transport Reviews, 32(4):473-490.

Ambrosini, C. and Routhier, J.-1. (2004). Objectives, methods and results of surveys carried out in the field of urban freight transport: an international comparison. Transport Reviews, 24(1):57-77.

Ambrosino, G., Boero, M., Di Bugno, M., Guerra, S., and Librato, A. (2007). A centre for eco friendly city freight distribution: Urban logistics innovation in a mid-size historical city in Italy. ICL 2007.

Ambrosino, G., Boero, M., Nelson, J. D., and Romanazzo, M. (2005). Systems and advanced solutions for eLogistics in the sustainable city. ENEA.

Anand, N., Quak, H. J., Van Duin, J. H. R., and Tavasszy, L. A. (2012). City logistics modeling efforts: Trends and gaps - a review. Procedia-Social and Behavioral Sciences, 39:101-115.

Anand, N., Van Duin, J. H. R., and Tavasszy, L. A. (2016). Framework for modelling multi-stakeholder city logistics domain using the agent based modelling approach. Transportation Research Procedia, 16:4-15. 
Anderson, S., Allen, J., and Browne, M. (2005). Urban logistics - how can it meet policy makers' sustainability objectives? Journal of Transport Geography, 13(1):71-81.

Arslan, A., Agatz, N., Kroon, L. G., and Zuidwijk, R. A. (2016). Crowdsourced delivery-a pickup and delivery problem with ad-hoc drivers. Working paper, Erasmus University Rotterdam.

Baldacci, R., Mingozzi, A., Roberti, R., and Calvo, R. W. (2013). An exact algorithm for the two-echelon capacitated vehicle routing problem. Operations Research, 61(2):298-314.

Bektaş, T. and Crainic, T. G. (2008). A brief overview of intermodal transportation. In Taylor, G., editor, Logistics Engineering Handbook, chapter 28, pages 1-16. Taylor and Francis Group, Boca Raton, Florida.

Bektaş, T., Crainic, T. G., and Van Woensel, T. (2015). From managing urban freight to smart city logistics networks. CIRRELT 2015-17.

Bektaş, T., Demir, E., and Laporte, G. (2016). Green vehicle routing. In Green Transportation Logistics, pages 243-265. Springer.

Bertazzi, L., Bosco, A., Guerriero, F., and Lagana, D. (2013). A stochastic inventory routing problem with stock-out. Transportation Research Part C: Emerging Technologies, 27:89-107.

Bertsekas, D. P. (2005). Dynamic programming and optimal control, volume 1. Athena Scientific Belmont, MA.

Berwick, M. D. and Dooley, F. (1997). Truck costs for owner/operators. Technical report, Upper Great Plains Transportation Institute, North Dakota State University.

Boardman, B. S., Malstrom, E. M., Butler, D. P., and Cole, M. H. (1997). Computer assisted routing of intermodal shipments. Computers \& Industrial Engineering, 33(1):311-314.

Bock, S. (2010). Real-time control of freight forwarder transportation networks by integrating multimodal transport chains. European Journal of Operational Research, 200(3):733-746.

Boer, E., Otten, M. B. J., and Essen, H. (2011). Comparison of various transport modes on a EU scale with the STREAM database. CE Delft.

Boerkamps, J. and van Binsbergen, A. (1999). Goodtrip - a new approach for modelling and evaluating urban goods distribution. In Taniguchi, E. and G, T. R., editors, City Logistics I, pages 175-186. ARRB Group Limited, Melbourne.

Bohne, S., Ruesch, M., and Barrera, G. (2015). Best practice handbook 2. Technical report, BESTFACT. 
Bookbinder, J. H., Cai, Q., and He, Q.-M. (2011). Shipment consolidation by private carrier: the discrete time and discrete quantity case. Stochastic Models, 27(4):664-686.

Boussier, J.-M., Cucu, T., Ion, L., and Breuil, D. (2011). Simulation of goods delivery process. International Journal of Physical Distribution \& Logistics Management, 41(9):913-930.

Brandenburger, A. (2014). The language of game theory: putting epistemics into the mathematics of games, volume 5 . World scientific.

Bräysy, O. and Gendreau, M. (2005). Vehicle routing problem with time windows, Part i: Route construction and local search algorithms. Transportation Science, 39(1):104-118.

Browne, M., Sweet, M., Woodburn, A., and Allen, J. (2005). Urban freight consolidation centres. Transport Studies Group, 10.

Buhrkal, K., Larsen, A., and Ropke, S. (2012). The waste collection vehicle routing problem with time windows in a city logistics context. Procedia-Social and Behavioral Sciences, 39:241-254.

Burger, M. and Repiskỳ, J. (2012). Problems of linear least square regression. Proceedings in ARSA-Advanced Research in Scientific Areas, 1(1):257-262.

Cai, Q., He, Q.-M., and Bookbinder, J. H. (2014). A tree-structured markovian model of the shipment consolidation process. Stochastic Models, 30(4):521553.

Campbell, A. M. and Savelsbergh, M. W. P. (2004). Efficient insertion heuristics for vehicle routing and scheduling problems. Transportation Science, 38(3):369-378.

Caris, A., Macharis, C., and Janssens, G. K. (2008). Planning problems in intermodal freight transport: accomplishments and prospects. Transportation Planning and Technology, 31(3):277-302.

Cattaruzza, D., Absi, N., Feillet, D., and González-Feliu, J. (2015). Vehicle routing problems for city logistics. EURO Journal on Transportation and Logistics, pages 1-29.

Centre for Retail Research (2016). World's population increasingly urban with more than half living in urban areas. http://www.retailresearch. org/onlineretailing.php. Accessed: 2016-08-22.

Çetiner, S., Sepil, C., and Süral, H. (2010). Hubbing and routing in postal delivery systems. Annals of Operations Research, 181(1):109-124.

Çetinkaya, S. (2005). Coordination of inventory and shipment consolidation decisions: A review of premises, models, and justification. In Applications of supply chain management and e-commerce research, pages 3-51. Springer. 
Çetinkaya, S. and Bookbinder, J. H. (2003). Stochastic models for the dispatch of consolidated shipments. Transportation Research Part B: Methodological, 37(8):747-768.

Chang, H. S., Hu, J., Fu, M. C., and Marcus, S. I. (2013). Simulation-based algorithms for Markov decision processes. Springer Science \& Business Media.

Chang, T.-S. (2008). Best routes selection in international intermodal networks. Computers \& operations research, 35(9):2877-2891.

Cheng, C., Qi, M., Wang, X., and Zhang, Y. (2016). Multi-period inventory routing problem under carbon emission regulations. International Journal of Production Economics, 182:263-275.

Cherrett, T., Allen, J., McLeod, F., Maynard, S., Hickford, A., and Browne, M. (2012). Understanding urban freight activity-key issues for freight planning. Journal of Transport Geography, 24:22-32.

Coelho, L. C., Cordeau, J.-F., and Laporte, G. (2014). Thirty years of inventory routing. Transportation Science, 48(1):1-19.

Coelho, L. C., Laporte, G., and Cordeau, J.-F. (2012). Dynamic and stochastic inventory-routing. Technical Report CIRRELT 2012-37. CIRRELT.

Crainic, T. G. and Kim, K. H. (2006). Intermodal transportation. Transportation, 14:467-537.

Crainic, T. G. and Laporte, G. (1997). Planning models for freight transportation. European Journal of Operational Research, 97(3):409-438.

Crainic, T. G., Ricciardi, N., and Storchi, G. (2009). Models for evaluating and planning city logistics systems. Transportation Science, 43(4):432-454.

Cruijssen, F., Cools, M., and Dullaert, W. (2007). Horizontal cooperation in logistics: opportunities and impediments. Transportation Research Part E: Logistics and Transportation Review, 43(2):129-142.

Cuda, R., Guastaroba, G., and Speranza, M. G. (2015). A survey on two-echelon routing problems. Computers \& Operations Research, 55:185-199.

Dablanc, L. (2007). Goods transport in large European cities: Difficult to organize, difficult to modernize. Transportation Research Part A: Policy and Practice, 41(3):280-285.

Dablanc, L. (2011). City distribution, a key element of the urban economy: guidelines for practitioners. In Macharis, C. and Melo, S., editors, City distribution and urban freight transport: Multiple perspectives, pages 37-56. Edward Elger, Cheltenham, UK.

Daganzo, C. F. (1984). The distance traveled to visit $\mathrm{n}$ points with a maximum of c stops per vehicle: An analytic model and an application. Transportation Science, 18(4):331-350. 
Dahlberg, J. (2015). Cooperative transportation planning and cost allocation. Licentiate thesis, Linköping University.

Dalmolen, S., Moonen, H., and Van Hillegersberg, J. (2015). Building a supply chain ecosystem: How the enterprise connectivity interface (eci) will enable and support interorganisational collaboration. In International Workshop on Global Sourcing of Information Technology and Business Processes, pages 228-239. Springer.

Dantzig, G. B. (1998). Linearprogrammingand extensions. Princeton University Press.

Dasburg, N. and Schoemaker, J. (2006). Quantification of urban freight transport effects ii. BESTUFS Consortium, www. bestufs. net.

De Transporters (2016). De transporters - tarieven. http: //detransporters.nl/tarieven/. Accessed: 2016-10-15.

Dellaert, N., Saridarq, F. D., Van Woensel, T., and Crainic, T. G. (2016). Branch \& price based algorithms for the two-echelon vehicle routing problem with time windows. CIRRELT Working Paper series 2016, 45.

Delling, D. and Wagner, D. (2009). Time-dependent route planning. In Robust and Online Large-Scale Optimization, pages 207-230. Springer.

Dewitt, W. and Clinger, J. (2000). Intermodal freight transportation. Transportation in the New Millennium, pages 1-6.

Ding, B., Yu, J. X., and Qin, L. (2008). Finding time-dependent shortest paths over large graphs. In Proceedings of the 11th international conference on extending database technology: Advances in database technology, pages 205-216. ACM.

Ehmke, J. F., Campbell, A. M., and Thomas, B. W. (2016). Vehicle routing to minimize time-dependent emissions in urban areas. European Journal of Operational Research, 251(2):478-494.

Eurostat (2016). Eurostat: your key to European statistics. http: //ec . europa. eu/eurostat/. Accessed: 2016-08-19.

Ferrucci, F. and Bock, S. (2015). A general approach for controlling vehicle en-route diversions in dynamic vehicle routing problems. Transportation Research Part B: Methodological, 77:76-87.

Figliozzi, M. A. (2009). Planning approximations to the average length of vehicle routing problems with time window constraints. Transportation Research Part B: Methodological, 43(4):438-447.

Ford Jr, L. R. and Fulkerson, D. R. (1958). Constructing maximal dynamic flows from static flows. Operations Research, 6(3):419-433. 
Gammelgaard, B. (2015). The emergence of city logistics: the case of Copenhagen's Citylogistik-kbh. International Journal of Physical Distribution \& Logistics Management, 45(4):333-351.

Geroliminis, N. and Daganzo, C. F. (2005). A review of green logistics schemes used in cities around the world. Technical report, University of California, Berkeley.

Gevaers, R., Van de Voorde, E., and Vanelslander, T. (2011). Characteristics and typology of last-mile logistics from an innovation perspective in an urban context. In Macharis, C. and Melo, S., editors, City distribution and urban freight transport: Multiple perspectives, pages 56-71. Edward Elger, Cheltenham, UK.

Gilbert, G. N. (2008). Agent-based models. Number 153 in Quantitative applications in the social sciences. Sage.

Goel, A. (2010). The value of in-transit visibility for supply chains with multiple modes of transport. International Journal of Logistics: Research and Applications, 13(6):475-492.

Golden, B. L., Bodin, L., Doyle, T., and Stewart Jr, W. (1980). Approximate traveling salesman algorithms. Operations research, 28(3-part-ii):694-711.

Golden, B. L., Raghavan, S., and Wasil, E. A. (2008). The vehicle routing problem: latest advances and new challenges, volume 43 . Springer Science \& Business Media.

Gonzalez-Feliu, J. (2012). Freight distribution systems with cross-docking: a multidisciplinary analysis. In Journal of the Transportation Research Forum, volume 51, pages 93-109.

Gonzalez-Feliu, J. and Morana, J. (2010). Are city logistics solutions sustainable? the cityporto case. TeMA-Trimestrale del Laboratorio Territorio Mobilità Ambiente, 3(2):55-64.

Gonzalez-Feliu, J., Morana, J., Grau, J.-M. S., and Ma, T.-Y. (2013). Design and scenario assessment for collaborative logistics and freight transport systems. International Journal of Transport Economics, pages 207-240.

Goodson, J. C., Ohlmann, J. W., and Thomas, B. W. (2013). Rollout policies for dynamic solutions to the multivehicle routing problem with stochastic demand and duration limits. Operations Research, 61(1):138-154.

Goodson, J. C., Thomas, B. W., and Ohlmann, J. W. (2017). A rollout algorithm framework for heuristic solutions to finite-horizon stochastic dynamic programs. European Journal of Operational Research, 258(1):216-229.

Groothedde, B., Ruijgrok, C., and Tavasszy, L. A. (2005). Towards collaborative, intermodal hub networks: A case study in the fast moving consumer goods 
market. Transportation Research Part E: Logistics and Transportation Review, 41(6):567-583.

Hafezalkotob, A. and Makui, A. (2015). Cooperative maximum-flow problem under uncertainty in logistic networks. Applied Mathematics and Computation, 250:593-604.

Harsanyi, J. C., Selten, R., et al. (1988). A general theory of equilibrium selection in games. MIT Press Books, 1.

Hayashi, K., Nemoto, T., and Visser, J. G. S. N. (2014). E-commerce and city logistics solution. In Taniguchi, E. and Thompson, R. G., editors, City Logistics: Mapping The Future, pages 55-78. CRC Press.

Hemmelmayr, V. C., Cordeau, J.-F., and Crainic, T. G. (2012). An adaptive large neighborhood search heuristic for two-echelon vehicle routing problems arising in city logistics. Computers \& operations research, 39(12):3215-3228.

Higginson, J. K. and Bookbinder, J. H. (1995). Markovian decision processes in shipment consolidation. Transportation Science, 29(3):242-255.

Horn, M. E. T. (2004). Procedures for planning multi-leg journeys with fixedroute and demand-responsive passenger transport services. Transportation Research Part C: Emerging Technologies, 12(1):33-55.

Janic, M. (2007). Modelling the full costs of an intermodal and road freight transport network. Transportation Research Part D: Transport and Environment, 12(1):33-44.

Janjevic, M., Kaminsky, P., and Ballé Ndiaye, A. (2013). Downscaling the consolidation of goods-state of the art and transferability of micro-consolidation initiatives. European Transport/Trasporti Europei, 54(4).

Jerry, B., Carson, J., Nelson, B., and Nicol, D. (2005). Discrete-Event System Simulation. Pearson/Prentice Hall.

Kanungo, T., Mount, D. M., Netanyahu, N. S., Piatko, C. D., Silverman, R., and $\mathrm{Wu}, \mathrm{A}$. Y. (2002). An efficient k-means clustering algorithm: Analysis and implementation. IEEE transactions on pattern analysis and machine intelligence, 24(7):881-892.

Kim, G., Ong, Y.-S., Heng, C. K., Tan, P. S., and Zhang, N. A. (2015). City vehicle routing problem (city VRP): A review. Intelligent Transportation Systems, IEEE Transactions, 16(4):1654-1666.

Kin, B., Verlinde, S., Van Lier, T., and Macharis, C. (2016). City vehicle routing problem (city VRP): A review. Transportation Research Procedia, 12:357-369.

Klapp, M. A., Erera, A. L., and Toriello, A. (2016). The one-dimensional dynamic dispatch waves problem. Transportation Science, Articles in Advance. 
Klausner, M. and Hendrickson, C. T. (2000). Reverse-logistics strategy for product take-back. Interfaces, 30(3):156-165.

Köhler, E., Langkau, K., and Skutella, M. (2002). Time-expanded graphs for flow-dependent transit times. In Algorithms-ESA 2002, pages 599-611. Springer.

Kok, A. L., Hans, E. W., Schutten, J. M. J., and Zijm, W. H. M. (2010). A dynamic programming heuristic for vehicle routing with time-dependent travel times and required breaks. Flexible Services and Manufacturing Journal, 22(1-2):83-108.

Koller, D. and Pfeffer, A. (1997). Representations and solutions for gametheoretic problems. Artificial intelligence, 94(1):167-215.

Konings, J. W. (1996). Integrated centres for the transshipment, storage, collection and distribution of goods: A survey of the possibilities for a high-quality intermodal transport concept. Transport Policy, 3(1):3-11.

Krajewska, M. A. and Kopfer, H. (2006). Collaborating freight forwarding enterprises. OR Spectrum, 28(3):301-317.

Laporte, G. (1992). The vehicle routing problem: An overview of exact and approximate algorithms. European Journal of Operational Research, 59(3):345-358.

Larsen, A., Madsen, O. B. G., and Solomon, M. (2002). Partially dynamic vehicle routing - models and algorithms. Journal of the operational research society, 53(6):637-646.

Law, A. M. (2007). Simulation Modeling \& Analysis. McGraw-Hill.

Law, A. M. and Carson, J. S. (1979). A sequential procedure for determining the length of a steady-state simulation. Operations Research, 27(5):1011-1025.

Lenstra, J. K. (1997). Local search in combinatorial optimization. Princeton University Press, Princeton, NJ, USA.

Lium, A.-G., Crainic, T. G., and Wallace, S. W. (2009). A study of demand stochasticity in service network design. Transportation Science, 43(2):144-157.

Luxen, D. and Vetter, C. (2011). Real-time routing with OpenStreetMap data. In Proceedings of the 19th ACM SIGSPATIAL International Conference on Advances in Geographic Information Systems, GIS '11, pages 513-516, New York, NY, USA. ACM.

Macharis, C. (2007). Multi-criteria analysis as a tool to include stakeholders in project evaluation: the mamca method. Transport Project Evaluation. Extending the Social Cost-Benefit Approach, pages 115-131. 
Macharis, C. and Bernardini, A. (2015). Reviewing the use of multi-criteria decision analysis for the evaluation of transport projects: Time for a multi-actor approach. Transport Policy, 37:177-186.

Macharis, C. and Bontekoning, Y. M. (2004). Opportunities for or in intermodal freight transport research: A review. European Journal of Operational Research, 153(2):400-416.

Macharis, C., Milan, L., and Verlinde, S. (2014). A stakeholder-based multicriteria evaluation framework for city distribution. Research in Transportation Business \& Management, 11:75-84.

Macharis, C., Turcksin, L., and Lebeau, K. (2012). Multi actor multi criteria analysis (mamca) as a tool to support sustainable decisions: State of use. Decision Support Systems, 54(1):610-620.

Maibach, M., Schreyer, C., Sutter, D., Van Essen, H. P., Boon, B. H., Smokers, R., Schroten, A., Doll, C., Pawlowska, B., and Bak, M. (2008). Handbook on estimation of external costs in the transport sector. CE Delft.

Mason, R., Lalwani, C., and Boughton, R. (2007). Combining vertical and horizontal collaboration for transport optimisation. Supply Chain Management: An International Journal, 12(3):187-199.

Masson, R., Lehuédé, F., and Péton, O. (2013). An adaptive large neighborhood search for the pickup and delivery problem with transfers. Transportation Science, 47(3):344-355.

Meng, Q., Wang, T., and Wang, S. (2012). Short-term liner ship fleet planning with container transshipment and uncertain container shipment demand. European Journal of Operational Research, 223(1):96-105.

Mes, M. R. K. and Iacob, M.-E. (2016). Synchromodal transport planning at a logistics service provider. In Logistics and Supply Chain Innovation, pages 23-36. Springer.

Minkoff, A. S. (1993). A Markov decision model and decomposition heuristic for dynamic vehicle dispatching. Operations Research, 41(1):77-90.

Montgomery, D. C. (2008). Design and analysis of experiments. John Wiley \& Sons.

Montreuil, B. (2011). Toward a Physical Internet: meeting the global logistics sustainability grand challenge. Logistics Research, 3(2-3):71-87.

Muñuzuri, J., Cortés, P., Guadix, J., and Onieva, L. (2012). City logistics in Spain: Why it might never work. Cities, 29(2):133-141.

Muñuzuri, J., Larrañeta, J., Onieva, L., and Cortés, P. (2005). Solutions applicable bylocal administrations for urban logistics improvement. Cities, 22(1):15-28. 
Mutlu, F., Çetinkaya, S., and Bookbinder, J. H. (2010). An analytical model for computing the optimal time-and-quantity-based policy for consolidated shipments. IIE Transactions, 42(5):367-377.

Nesterova, N. and Quak, H. J. (2015). Validating freight electric vehicles in urban Europe. Technical report, TNO.

Niazi, M. and Hussain, A. (2011). Agent-based computing from multi-agent systems to agent-based models: a visual survey. Scientometrics, 89(2):479-499.

Nuzzolo, A., Crisalli, U., and Comi, A. (2012). A delivery approach modeling for urban freight restocking. Journal of Civil Engineering and Architecture, 6(3):251.

OECD (2003). Delivering the Goods: 21st Century Challenges to Urban Goods Transport. OECD Publishing, Paris.

Oertel, P. (2000). Routing with reloads. PhD thesis, Universität zu Köln.

Osborne, M. J. and Rubinstein, A. (1994). A course in game theory. MIT Press.

OSM Foundation (2017). OpenStreetMap. https://www . openstreetmap. org/about. Accessed: 2017-01-04.

Pérez Rivera, A. E. and Mes, M. R. K. (2016). Anticipatory freight selection in intermodal long-haul round-trips. Transportation Research Part E: Logistics and Transportation Review.

Pillac, V., Gendreau, M., Guéret, C., and Medaglia, A. L. (2013). A review of dynamic vehicle routing problems. European Journal of Operational Research, 225(1):1-11.

Ploos Van Amstel, W. (2015). City Logistics: Working on livable cities through sustainable city logistics. Amsterdam University of Applied Sciences.

Powell, W. B. (1987). An operational planning model for the dynamic vehicle allocation problem with uncertain demands. Transportation Research Part B: Methodological, 21(3):217-232.

Powell, W. B. (2009). What you should know about approximate dynamic programming. Naval Research Logistics, 56(3):239-249.

Powell, W. B. (2011). Approximate Dynamic Programming: Solving the Curses of Dimensionality, volume 842. John Wiley \& Sons.

Powell, W. B., Simão, H. P., and Bouzaiene-Ayari, B. (2012). Approximate dynamic programming in transportation and logistics: a unified framework. EURO Journal on Transportation and Logistics, 1(3):237-284.

Psaraftis, H. N., Wen, M., and Kontovas, C. A. (2016). Dynamic vehicle routing problems: Three decades and counting. Networks, 67(1):3-31.

Puterman, M. L. (2014). Markov decision processes: discrete stochastic dynamic programming. John Wiley \& Sons. 
Quak, H. J. (2011). Urban freight transport: the challenge of sustainability. In Macharis, C. and Melo, S., editors, City distribution and urban freight transport: Multiple perspectives, pages 37-56. Edward Elger, Cheltenham, UK.

Quak, H.J. and De Koster, M. B. M. (2007). Exploring retailers' sensitivity to local sustainability policies. Journal of Operations Management, 25(6):1103-1122.

Quak, H. J. and de Koster, M. B. M. (2009). Delivering goods in urban areas: how to deal with urban policy restrictions and the environment. Transportation Science, 43(2):211-227.

Reyes, P. M. (2005). Logistics networks: A game theory application for solving the transshipment problem. Applied Mathematics and Computation, 168(2):1419-1431.

Ritzinger, U., Puchinger, J., and Hartl, R. F. (2016). A survey on dynamic and stochastic vehicle routing problems. International Journal of Production Research, 54(1):215-231.

Robusté Antón, F., Estrada, M., and López-Pita, A. (2004). Formulas for estimating average distance traveled in vehicle routing problems in elliptic zones. Transportation Research Record: Journal of the Transportation Research Board, 1873(1):64-69.

Roca-Riu, M. and Estrada, M. (2012). An evaluation of urban consolidation centers through logistics systems analysis in circumstances where companies have equal market shares. Procedia-Social and Behavioral Sciences, 39:796-806.

Roca-Riu, M., Estrada, M., and Fernández, E. (2016). An evaluation of urban consolidation centers through continuous analysis with non-equal market share companies. Transportation Research Procedia, 12:370-382.

Rossetti, M. D. (2015). Simulation modeling and Arena. John Wiley \& Sons.

Russo, F. and Comi, A. (2010). A classification of city logistics measures and connected impacts. Procedia-Social and Behavioral Sciences, 2(3):6355-6365.

Savelsbergh, M. W. P. and Van Woensel, T. (2016). 50th anniversary invited article - City logistics: Challenges and opportunities. Transportation Science, 50(2):579-590.

Schoemaker, J., Allen, J., Huschebek, M., and Monigl, J. (2006). Quantification of urban freight transport effects i. BESTUFS Consortium, www. bestufs. net.

Shoham, Y. and Leyton-Brown, K. (2008). Multiagent systems: Algorithmic, game-theoretic, and logical foundations. Cambridge University Press.

$\mathrm{Si}$, J. (2004). Handbook of learning and approximate dynamic programming, volume 2. John Wiley \& Sons. 
Simão, H. P., Day, J., George, A. P., Gifford, T., Nienow, J., and Powell, W. B. (2009). An approximate dynamic programming algorithm for large-scale fleet management: A case application. Transportation Science, 43(2):178-197.

Spady, R. H. and Friedlaender, A. F. (1978). Hedonic cost functions for the regulated trucking industry. The Bell Journal of Economics, pages 159-179.

Stathopoulos, A., Valeri, E., and Marcucci, E. (2012). Stakeholder reactions to urban freight policy innovation. Journal of Transport Geography, 22:34-45.

SteadieSeifi, M., Dellaert, N. P., Nuijten, W. P. M., Van Woensel, T., and Raoufi, R. (2014). Multimodal freight transportation planning: A literature review. European Journal of Operational Research, 233(1):1-15.

Steiger, N. M. and Wilson, J. R. (2001). Convergence properties of the batch means method for simulation output analysis. INFORMS Journal on Computing, 13(4):277-293.

Sutton, R. S. and Barto, A. G. (1998). Reinforcement learning: An introduction, volume 1. MIT press Cambridge.

Suzuki, Y. (2011). A new truck-routing approach for reducing fuel consumption and pollutants emission. Transportation Research Part D: Transport and Environment, 16(1):73-77.

Szymonik, A. (2014). International Logistics. Lodz University of Technology Press.

Tamagawa, D., Taniguchi, E., and Yamada, T. (2010). Evaluating city logistics measures using a multi-agent model. Procedia-Social and Behavioral Sciences, 2(3):6002-6012.

Taniguchi, E., Thompson, R. G., and Yamada, T. (2012). Emerging techniques for enhancing the practical application of city logistics models. Procedia-Social and Behavioral Sciences, 39:3-18.

Taniguchi, E., Thompson, R. G., and Yamada, T. (2014). Concepts and visions for urban transport and logistics relating to human security. In Taniguchi, E., Fwa, T. F., and Thompson, R. G., editors, Urban transportation and logistics: Health, safety, and security concerns, pages 1-30. CRC Press, Boca Raton, USA.

Taniguchi, E. and Van Der Heijden, R. E. C. M. (2000). An evaluation methodology for city logistics. Transport Reviews, 20(1):65-90.

Taniguchi, E., Yamada, T., and Okamoto, M. (2007). Multi-agent modelling for evaluating dynamic vehicle routing and scheduling systems. Journal of the Eastern Asia Society for Transportation Studies, 7:933-948. 
Teo, J. S. E., Taniguchi, E., and Qureshi, A. G. (2014). Evaluation of load factor control and urban freight road pricing joint schemes with multi-agent systems learning models. Procedia-Social and Behavioral Sciences, 125:62-74.

Topaloglu, H. and Powell, W. B. (2006). Dynamic-programming approximations for stochastic time-staged integer multicommodity-flow problems. INFORMS Journal on Computing, 18(1):31-42.

Transmodal, M. (2012). DG MOVE European Commission: Study on urban freight transport.

Trip, J. J. and Bontekoning, Y. (2002). Integration of small freight flows in the intermodal transport system. Journal of Transport Geography, 10(3):221-229.

Ulmer, M. W., Brinkmann, J., and Mattfeld, D. C. (2015). Anticipatory planning for courier, express and parcel services. In Logistics Management, pages 313-324. Springer.

United Nations (2014). World's population increasingly urban with more than half living in urban areas. https://www.un.org/development/desa/ en/news/population/world-urbanization-prospects.html. Accessed: 2016-03-22.

Van Duin, J. H. R., Quak, H. J., and Muñuzuri, J. (2010). New challenges for urban consolidation centres: A case study in The Hague. Procedia-Social and Behavioral Sciences, 2(3):6177-6188.

Van Duin, J. H. R., van Dam, T., Wiegmans, B., and Tavasszy, L. A. (2016). Understanding financial viability of urban consolidation centres: Regent street (London), Bristol/Bath \& Nijmegen. Transportation Research Procedia, 16:61-80.

Van Duin, R. J. H., Van Kolck, A., Anand, N., and Taniguchi, E. (2012). Towards an agent-based modelling approach for the evaluation of dynamic usage of urban distribution centres. Procedia-Social and Behavioral Sciences, 39:333-348.

Van Essen, H., Schroten, A., Otten, M., Sutten, D., Schreyer, C., Zandonella, R., Maibach, M., and Doll, C. (2011). External costs of transport in Europe.

Van Heeswijk, W. J., Larsen, R., and Larsen, A. (2017a). An urban consolidation center in the city of Copenhagen: a simulation study. Beta Working Paper Series, 523:1-34.

Van Heeswijk, W. J. A., Mes, M. R. K., and Schutten, J. M. J. (2015). An approximate dynamic programming approach to urban freight distribution with batch arrivals. Lecture Notes in Computer Science, 9335:61-75.

Van Heeswijk, W. J. A., Mes, M. R. K., and Schutten, J. M. J. (2016a). An agent-based simulation framework to evaluate urban logistics schemes. 
Lecture Notes in Computer Science, 9855:369-383.

Van Heeswijk, W. J. A., Mes, M. R. K., and Schutten, J. M. J. (2017b). The delivery dispatching problem with time windows for urban consolidation centers. Transportation Science, pages 1-37.

Van Heeswijk, W. J. A., Mes, M. R. K., Schutten, M. J. M. J., and Zijm, W. H. M. (2016b). Freight consolidation in intermodal networks with reloads. Flexible Services and Manufacturing Journal, pages 1-34.

Van Rooijen, T. and Quak, H. J. (2010). Local impacts of a new urban consolidation centre-the case of Binnenstadservice.nl. Procedia-Social and Behavioral Sciences, 2(3):5967-5979.

Van Woensel, T., Kerbache, L., Peremans, H., and Vandaele, N. (2008). Vehicle routing with dynamic travel times: A queueing approach. European journal of operational research, 186(3):990-1007.

Veenstra, A. and Zuidwijk, R. (2010). The future of seaport hinterland networks. Liber Amicorum Jo van Nunen, Dinalog and RSM-Erasmus University of Rotterdam, pages 205-215.

Verlinde, S. and Macharis, C. (2016). Innovation in urban freight transport: The triple helix model. Transportation Research Procedia, 14:1250-1259.

Verlinde, S., Macharis, C., Milan, L., and Kin, B. (2014). Does a mobile depot make urban deliveries faster, more sustainable and more economically viable: results of a pilot test in Brussels. Transportation Research Procedia, 4:361-373.

Verlinde, S., Macharis, C., and Witlox, F. (2012). How to consolidate urban flows of goods without setting up an urban consolidation centre? Procedia-Social and Behavioral Sciences, 39:687-701.

Verlinden, T., Van de Voorde, E., and Dewulf, W. (2016). Ho. re.ca. logistics and medieval structured cities: A market analysis and typology. In Commercial Transport, pages 369-384. Springer.

Verstrepen, S., Cools, M., Cruijssen, F., and Dullaert, W. (2009). A dynamic framework for managing horizontal cooperation in logistics. International Journal of Logistics Systems and Management, 5(3-4):228-248.

Ville, S., Gonzalez-Feliu, J., and Dablanc, L. (2013). The limits of public policy intervention in urban logistics: Lessons from Vicenza (Italy). European Planning Studies, 21(10):1528-1541.

Voccia, S. A., Campbell, A. M., and Thomas, B. W. (2015). The same-day delivery problem for online purchases. Working paper, University of Iowa.

Wangapisit, O., Taniguchi, E., Teo, J. S. E., and Qureshi, A. G. (2014). Multi-agent systems modelling for evaluating joint delivery systems. Procedia-Social and Behavioral Sciences, 125:472-483. 
West, D. B. et al. (2001). Introduction to graph theory, volume 2. Prentice hall Upper Saddle River.

Wiesenthal, T., Leduc, G., Cazzola, P., Schade, W., and Köhler, J. (2011). Mapping innovation in the european transport sector. Technical report, Institute for Prospective Technological Studies.

Williams, H. P. (2013). Model building in mathematical programming. John Wiley \& Sons.

Winston, W. L. and Goldberg, J. B. (2004). Operations research: applications and algorithms, volume 3. Duxbury Press, Belmont, CA, USA.

Yan, S., Bernstein, D., and Sheffi, Y. (1995). Intermodal pricing using network flow techniques. Transportation Research Part B: Methodological, 29(3):171-180.

Yang, D. and Odani, M. (2007). An analysis on city freight cooperative transportation system using game theory. Journal of the Eastern Asia Society for Transportation Studies, 7:989-1001.

Zijm, W. H. M. and Klumpp, M. (2017). Future logistics: What to expect, how to adapt. In Freitag, M., Kotzab, H., and Pannek, J., editors, Dynamics in Logistics, Lecture Notes in Logistics, pages 365-379. Springer International Publishing.

Ziliaskopoulos, A. and Wardell, W. (2000). An intermodal optimum path algorithm for multimodal networks with dynamic arc travel times and switching delays. European Journal of Operational Research, 125(3):486-502. 



\section{List of abbreviations}

$\begin{array}{ll}\text { ADP } & \text { Approximate Dynamic Programming } \\ \text { DP } & \text { Dynamic Programming } \\ \text { DDP } & \text { Delivery Dispatching Problem } \\ \text { DDP-TW } & \text { Delivery Dispatching Problem with Time Windows } \\ \text { IRP } & \text { Inventory Routing Problem } \\ \text { ILP } & \text { Integer Linear Program } \\ \text { KPI } & \text { Key Performance Indicator } \\ \text { LP } & \text { Linear Program } \\ \text { LSP } & \text { Logistics Service Provider } \\ \text { LTL } & \text { Less than TruckLoad } \\ \text { SND } & \text { Service Network Design } \\ \text { TSP } & \text { Traveling Salesman Problem } \\ \text { UCC } & \text { Urban Consolidation Center } \\ \text { VFA } & \text { Value Function Approximation } \\ \text { VRP } & \text { Vehicle Routing Problem } \\ \text { 4PL } & \text { 4th Party Logistics Service Provider }\end{array}$





\section{List of mathematical symbols}

We provide an overview of the mathematical symbols that are used in Chapter 2 to Chapter 6 this thesis. To aid the reader, the overview is itemized per chapter. Although the thesis contains a variety of subjects and solution methods, we try to be consistent in our notation throughout the thesis. This list is meant to aid the reader in understanding the main text; symbols that are used only in appendices are not included in this overview.

We use a few standard notations and apply various subscripts, superscripts, and accents to distinguish their roles. With respect to time indices, we use $t$ to denote a decision moment, $T$ to denote the total number of decision moments, $\tau$ to describe a time interval, and $\mathcal{T}$ to define a set of times. The symbol $C$ indicates a cost function, whereas $c$ is a cost parameter. We use $I_{t}$ to describe an inventory at time $t$. Graphs are represented by $\mathcal{G}=\{\mathcal{V}, \mathcal{A}\}$. Here, $\mathcal{V}$ is the set of vertices and $\mathcal{A}$ is the set of arcs connecting the vertices. The symbol $v \in \mathcal{V}$ denotes a vertex, $a \in$ $\mathcal{A}$ denotes an arc. Finally, $\pi$ is used to denote a policy, i.e., a set of decision rules.

\section{Chapter 2}

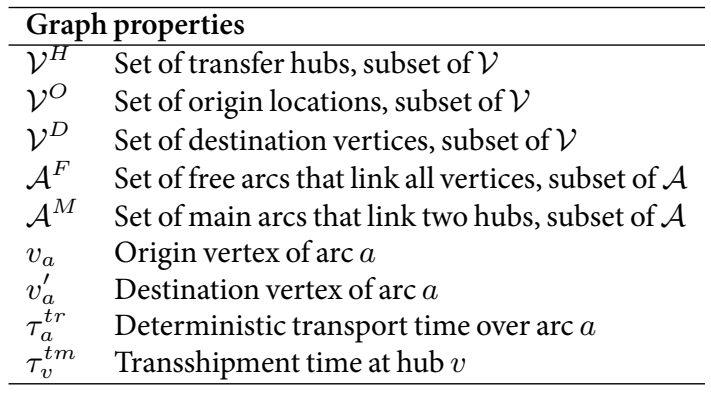




\begin{tabular}{ll}
\hline \multicolumn{2}{l}{ Cost functions and parameters } \\
\hline$C_{a}^{t r}$ & Volume-dependent cost function for arc $a$ \\
$C^{t r}(i, n)$ & Function for transport costs of route $r_{i, n}$ \\
$C^{t m}(i, n)$ & Function for transshipment costs of route $r_{i, n}$ \\
$C^{r l}(i, n)$ & Function for reload costs of route $r_{i, n}$ \\
$c_{v}^{t m}$ & Costs for placing a transport unit on a mode at hub $v$ \\
$c_{v^{\prime}}^{t m}$ & Costs for lifting a transport unit off a mode at hub $v^{\prime}$ \\
$c_{v^{\prime}}^{r l}$ & Costs for a reload at hub $v^{\prime}$ \\
\hline
\end{tabular}

\begin{tabular}{ll}
\hline \multicolumn{2}{l}{ Order properties } \\
\hline$l$ & Volume of an order \\
$i$ & Indicator for current order \\
$j$ & Indicator for open order \\
$v_{i}^{O}$ & Origin of order $i$ \\
$v_{i}^{D}$ & Destination for order $i$ \\
$t_{i}^{\text {min }}$ & Earliest pickup time of order $i$ \\
$t_{i}^{\text {max }}$ & Latest delivery time of order $i$ \\
$l_{i}$ & Volume of order $i$ \\
$\mathcal{L}$ & Set of order volumes \\
\hline
\end{tabular}

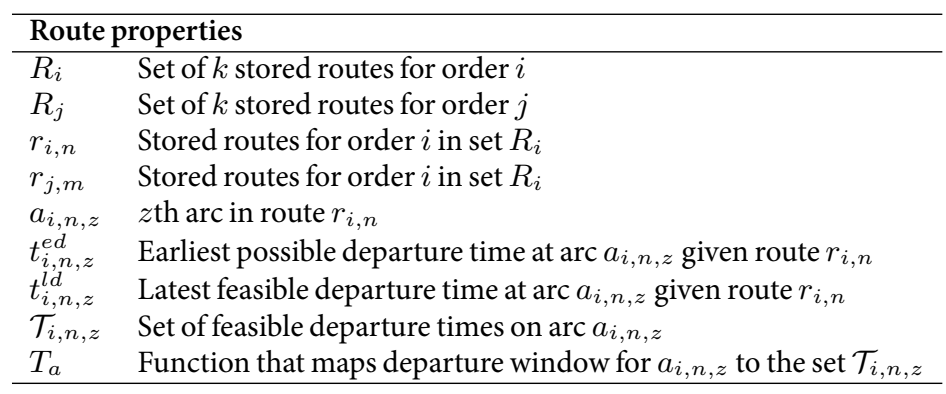

\begin{tabular}{ll}
\hline \multicolumn{2}{l}{ Algorithmic input } \\
\hline$k$ & Number of routes stored per order \\
$\beta$ & Threshold value, upper bound for route costs \\
$M$ & Number of main arcs in a route \\
$M^{\text {max }}$ & Maximum allowed number of main $\operatorname{arcs}$ in a route \\
$\#$ Route $s_{M}$ & Number of routes generated with $M$ main arcs \\
$\#$ Route $s_{M}^{\text {max }}$ & Maximum number of routes generated with $M$ main arcs \\
$\mathcal{J}$ & Set of open orders that have not yet reached their destination \\
\hline
\end{tabular}




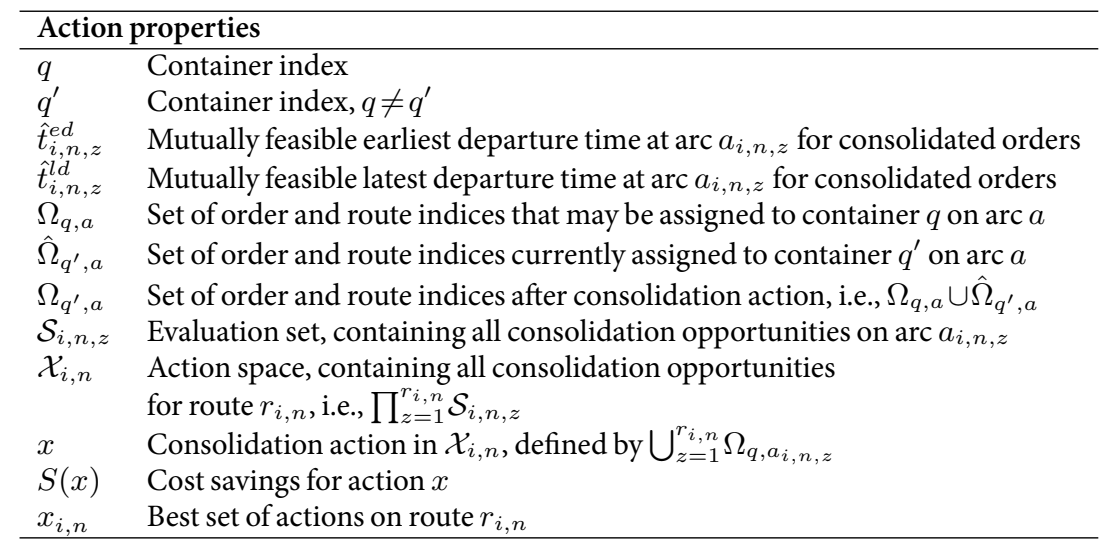

\section{Chapter 3}

\begin{tabular}{ll}
\hline \multicolumn{2}{l}{ Vehicle properties } \\
\hline $\mathcal{Q}^{p r}$ & Set of primary vehicles \\
$\mathcal{Q}^{s e}$ & Set of secondary vehicles \\
$q$ & Indicator for a vehicle in $\mathcal{Q}^{p r} \cup \mathcal{Q}^{s e}$ \\
$r_{t, q}$ & Route duration for vehicle $q$ dispatched at decision moment $t$ \\
$\tau^{\text {Max Route }}$ & Maximum route duration for any vehicle \\
$\mathcal{V}_{t, q}$ & Subset of locations visited by vehicle $q$ dispatched at $t$ \\
$\Delta$ & Routing function, returning the route duration given $\mathcal{V}_{t, q}$ \\
$\tau_{t, q}$ & Earliest dispatch availability of vehicle $q$ relative to $t$ \\
$\tau_{t, q}^{x}$ & Earliest dispatch availability of vehicle $q$ relative to $t$, after applying action $x_{t}$ \\
$Q_{t}$ & Dispatch availability of all primary vehicles, $\left(\tau_{t, 1}, \ldots, \tau_{t,\left|\mathcal{Q}^{p r}\right|}\right)$ \\
\hline
\end{tabular}




\begin{tabular}{ll}
\hline Order properties \\
\hline$v$ & Destination vertex of an order \\
$l$ & Load size of an order, expressed in fractional vehicle capacity \\
$t^{e}$ & Earliest dispatch time of an order \\
$t^{l}$ & Latest dispatch time \\
$\mathcal{L}$ & Set of possible load sizes \\
$\mathcal{T}^{e}$ & Set of possible earliest dispatch times \\
$\mathcal{T}^{l}$ & Set of possible latest dispatch times \\
$\tau^{\text {Max Ahead }}$ & Maximum time interval an order is announced in advance \\
$\tau^{\text {MaxWindow }}$ & Maximum width of an order dispatch window \\
$I_{t, v, l, t^{e}, t^{l}}$ & Number of orders of an order type, i.e., a unique \\
& combination of destination, volume, and dispatch window \\
$I_{t}$ & Number of orders in system per order type \\
$I_{t, v, l, t^{e}, t^{l}}^{x}$ & Number of orders of an order type in post-decision state $S_{t}^{x}$ \\
$I_{t}^{x}$ & Number of orders in system per order type in post-decision state $S_{t}^{x}$ \\
\hline
\end{tabular}
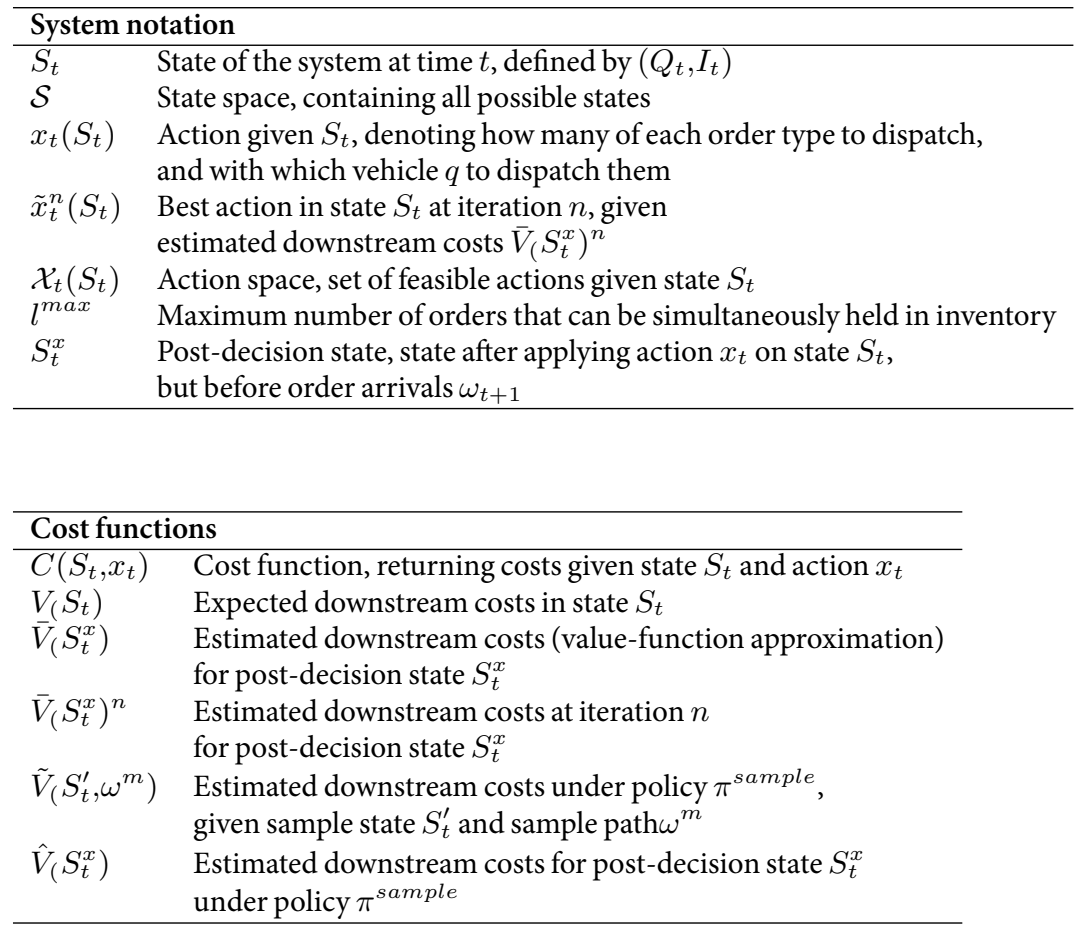


\begin{tabular}{|c|c|}
\hline Policies & \\
\hline$\pi_{t}$ & $\begin{array}{l}\text { Consolidation policy, set of decision rules that returns } \\
\text { an action } x_{t} \text { given state } S_{t}\end{array}$ \\
\hline$\Pi_{t}$ & Set of all consolidation policies \\
\hline$\pi^{A D P}$ & Consolidation policy obtained with approximate dynamic programming \\
\hline$\pi^{\text {direct }}$ & $\begin{array}{l}\text { Consolidation policy that directly ships orders in inventory, } \\
\text { provided that primary vehicle capacity is available }\end{array}$ \\
\hline$\pi^{\text {post }}$ & $\begin{array}{l}\text { Consolidation policy that postpones the shipment of orders, } \\
\text { unless dispatched vehicles have idle capacity }\end{array}$ \\
\hline$\pi^{\text {min }}$ & $\begin{array}{l}\text { Consolidation policy that minimizes the direct } \\
\text { costs at decision moment } t\end{array}$ \\
\hline$\pi^{\text {sample }}$ & $\begin{array}{l}\text { Consolidation policy that uses sampling to estimate } \\
\text { lookahead costs for each action in } \mathcal{X}_{t}\end{array}$ \\
\hline$\tau^{\text {sample }}$ & Length of sample paths corresponding to policy $\pi^{\text {sample }}$ \\
\hline$\omega^{m}$ & Sample path corresponding to policy $\pi^{\text {sample }}$ \\
\hline$\Omega^{m}$ & Set of sample path corresponding to policy $\pi^{\text {sample }}$ \\
\hline$m$ & $\begin{array}{l}\text { Number of sample paths generated per decision moment } \\
\text { under policy } \pi^{\text {sample }}\end{array}$ \\
\hline
\end{tabular}

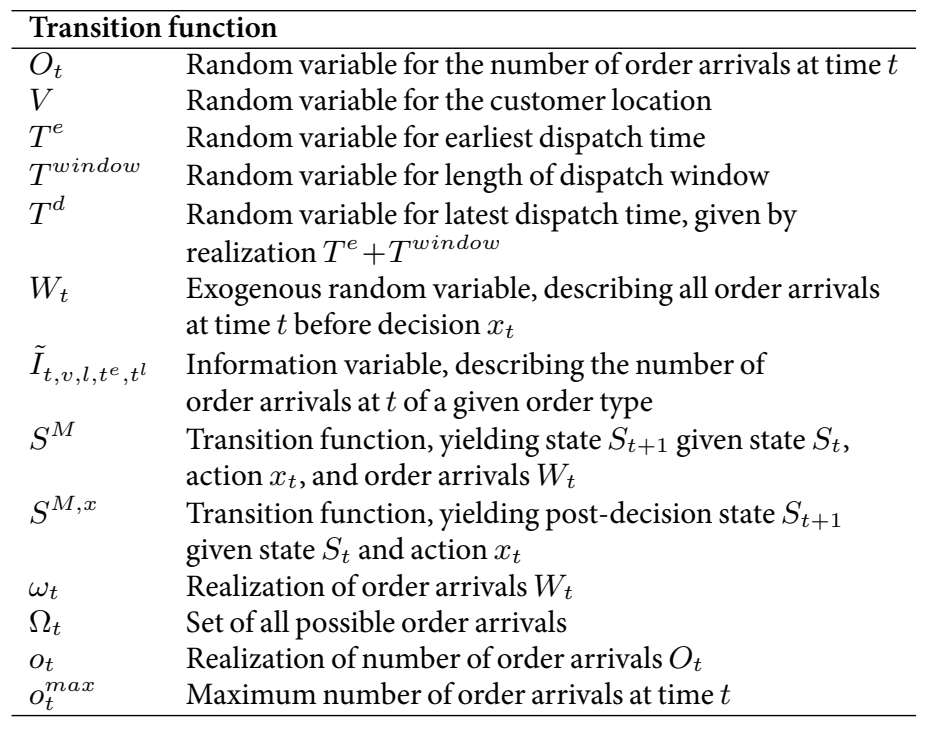




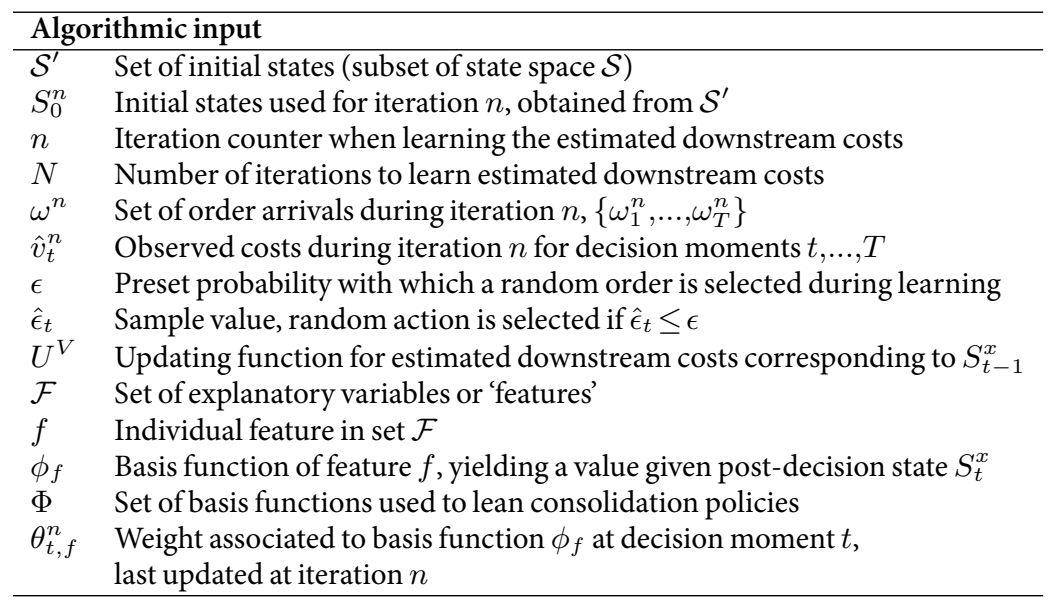

\section{Chapter 4}

\begin{tabular}{ll}
\hline \multicolumn{2}{l}{ Graph properties } \\
\hline $\mathcal{C}$ & Set of carriers \\
$\mathcal{R}$ & Set of receivers \\
$\mathcal{S}$ & Set of shippers \\
$\mathcal{H}$ & Set of UCCs \\
$\mathcal{V}^{R}$ & Set of receiver vertices \\
$\mathcal{V}^{S}$ & Set of shipper vertices \\
$\mathcal{V}^{C}$ & Set of carrier vertices \\
$\mathcal{V}^{H}$ & Set of UCC vertices \\
\hline
\end{tabular}

\begin{tabular}{ll}
\hline Order properties & \\
\hline$c$ & Carrier in $\mathcal{C}$ \\
$r$ & Receiver in $\mathcal{R}$ \\
$s$ & Shipper in $\mathcal{S}$ \\
$\gamma$ & Indicates whether order is delivered via UCC \\
$l$ & Volume of an order \\
$t^{e}$ & Earliest delivery time of an order \\
$t^{l}$ & Latest delivery time of an order \\
$I_{t, t^{e}, t^{l}, v, r, c, s, l, \gamma}$ & Number of orders of a specific order type \\
$I_{t}$ & Vector of number of orders per type in the system at $t$ \\
\hline
\end{tabular}



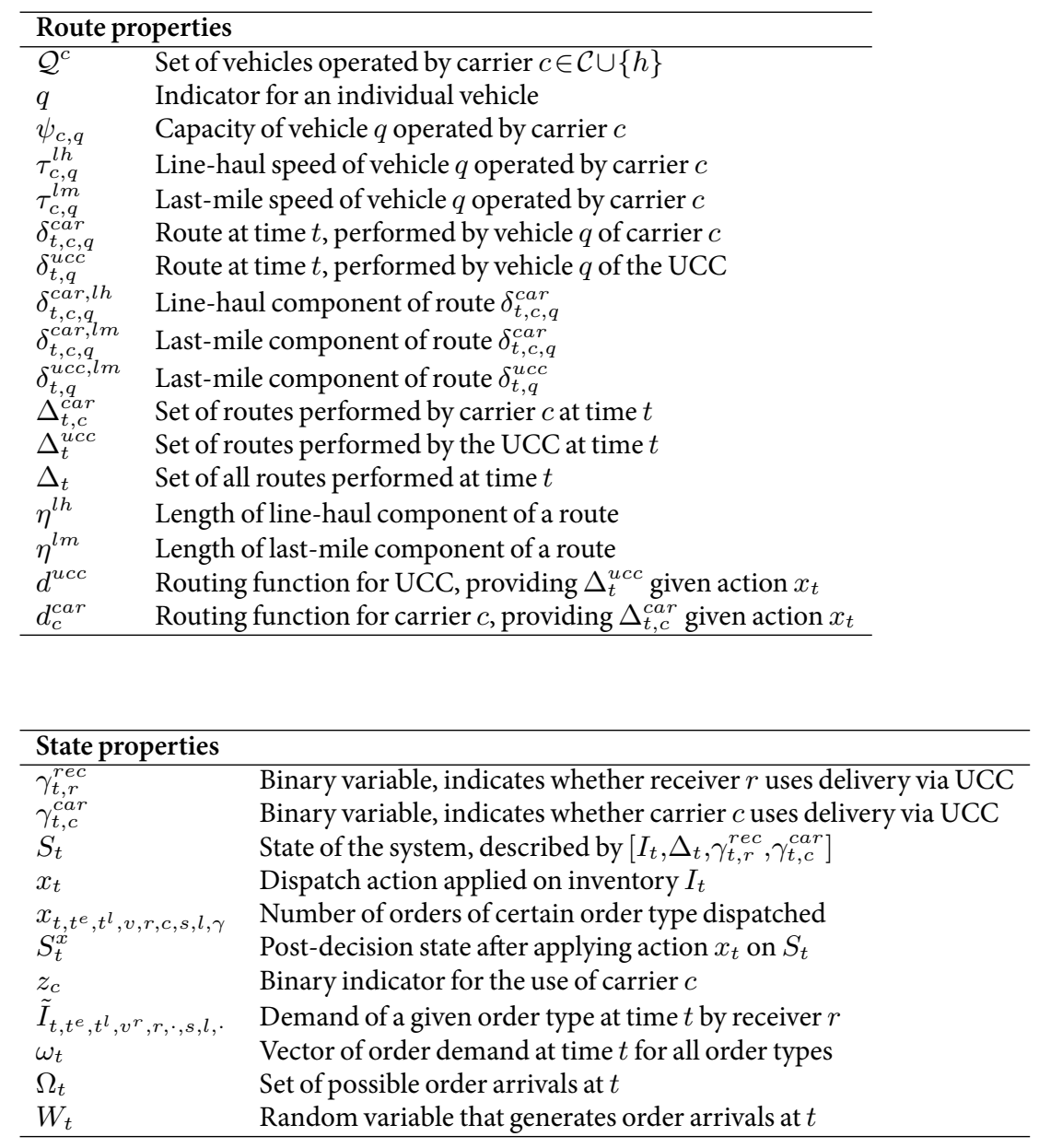


\begin{tabular}{|c|c|}
\hline \multicolumn{2}{|l|}{ Cost and price functions } \\
\hline$\alpha$ & Fixed price/cost component \\
\hline$\beta$ & Variable price/cost component \\
\hline$C_{t, r}^{r e c}$ & Cost function of receiver $r$ at time $t$ \\
\hline$C_{t, s}^{s h p}$ & Cost function of shipper $s$ at time $t$ \\
\hline$C_{t, c}^{c a r}$ & Cost function of carrier $c$ at time $t$ \\
\hline$C_{t, h}^{u, c c}$ & Cost function of UCC $h$ at time $t$ \\
\hline$P_{t, r}^{r e c, s b}\left(l_{t, v^{r}, r, \gamma \mid \gamma=1}\right)$ & Income subsidies if $r$ selects UCC \\
\hline$C_{t, r}^{r, r} c, r c\left(l_{t, v^{r}, r, c}\right)$ & Costs for receiver $r$ when receiving shipments \\
\hline$C_{t, r}^{r e c, l m}\left(l_{t, v^{r}, r, \gamma \mid \gamma=1}\right)$ & $\begin{array}{l}\text { Costs for receiver } r \text { when } \\
\text { outsourcing last-mile distribution }\end{array}$ \\
\hline$C_{t, r}^{r e c, v a l}$ & $\begin{array}{l}\text { Costs for receiver } r \text { when performing } \\
\text { in-house value-adding services }\end{array}$ \\
\hline$C_{t, r}^{r e c, v a l, u c c}$ & $\begin{array}{l}\text { Costs for receiver } r \text { when } \\
\text { outsourcing value-adding services }\end{array}$ \\
\hline$C_{t, s}^{s h p, t r}\left(l_{t, v^{s}, c, s}, \eta^{l h}\right)$ & Costs for transportation incurred by shipper $s$ \\
\hline$C_{t, s}^{s h p, s p}\left(l_{t, v^{s}, c, s}\right)$ & Costs for shipping incurred by shipper $s$ \\
\hline$P_{t, c}^{c a r, s b}\left(l_{t, v^{c}, c, \gamma \mid \gamma=1}\right)$ & Income from subsidies if $c$ selects UCC \\
\hline$P_{t, c}^{c a r, t r}\left(l_{t, v^{c}, c}, \eta^{l h}\right)$ & Shipping price by carrier $c$ \\
\hline$C_{t, c}^{c a r, t r}\left(\eta^{l h}, \eta^{l m}, \mathcal{S}^{\prime}, \mathcal{R}^{\prime}\right)$ & Costs full transport for carrier $c$ \\
\hline$C_{t, c}^{c a r, l m}\left(l_{t, v^{c}, c}, \eta^{l h}, \mathcal{S}^{\prime}\right)$ & Costs for carrier $c$ when outsourcing to UCC \\
\hline$P_{t}^{u c c, s b}\left(l_{t, v u c c}\right)$ & Income from subsidies for UCC \\
\hline$P_{t}^{u c c, v a l}(r)$ & Price value-adding services for receiver $r$ \\
\hline$P_{t}^{u c c, r e c, t r}\left(l_{t, v^{r}, r, \gamma \mid \gamma t, r^{r e c}=1}\right)$ & Price for distribution if $r$ selects UCC \\
\hline$P_{t}^{u c c, c a r, t r}\left(l_{t, v^{c}, c, \gamma \mid \gamma t, c^{c a r}=1}\right)$ & Price for distribution if $c$ selects UCC \\
\hline$C_{t}^{u c c, r c}\left(l_{t, v^{u c c}}\right)$ & Costs receiving for UCC \\
\hline$C_{t}^{u c c, t r}\left(l_{t, v} u c c, \eta^{l m}, \mathcal{R}^{\prime}\right)$ & Costs distribution for UCC \\
\hline & Shapley value allocated to player $c$ \\
\hline $\mathcal{C}^{\prime}$ & Coalition of carriers, $\mathcal{C}^{\prime} \subseteq \mathcal{C}$ \\
\hline
\end{tabular}

\begin{tabular}{ll}
\hline \multicolumn{2}{l}{ Sampling procedure } \\
\hline $\mathcal{T}^{\text {tac }}$ & Set of tactical decision moments, subset of $\mathcal{T}$ \\
$n$ & Indicator for sample path \\
$N$ & Number of sampling paths \\
$\tau^{\text {sample }}$ & Length of sample path \\
$\omega_{t}^{n}$ & Sample arrival at time $t$ of sample path $n$ \\
$\tilde{S}_{t}^{n}$ & Sample state at time $t$ of sample path $n$ \\
$\tilde{\gamma}_{r}^{\text {rec }}$ & Sample indicator that mandates use of UCC for receiver $r$ \\
\hline
\end{tabular}




\section{Chapter 5}

\begin{tabular}{ll}
\hline \multicolumn{2}{c}{ Graph properties } \\
\hline $\mathcal{C}$ & Set of carriers \\
$\mathcal{R}$ & Set of receivers \\
$\mathcal{S}$ & Set of shippers \\
$\mathcal{H}$ & Set of UCCs \\
$\mathcal{V}^{R}$ & Set of receiver vertices \\
$\mathcal{V}^{S}$ & Set of shipper vertices \\
$\mathcal{V}^{C}$ & Set of carrier vertices \\
$\mathcal{V}^{H}$ & Set of UCC vertices \\
\hline
\end{tabular}

\begin{tabular}{ll}
\hline Order properties & \\
\hline$c$ & Carrier in $\mathcal{C}$ \\
$r$ & Receiver in $\mathcal{R}$ \\
$s$ & Shipper in $\mathcal{S}$ \\
$\gamma$ & Indicates whether order is delivered via UCC \\
$l$ & Volume of an order \\
$t^{e}$ & Earliest delivery time of an order \\
$t^{l}$ & Latest delivery time of an order \\
$I_{t, t^{e}, t^{l}, v, r, c, s, l, \gamma}$ & Number of orders of a specific order type \\
$I_{t}$ & Vector of number of orders per type in the system at $t$ \\
\hline
\end{tabular}

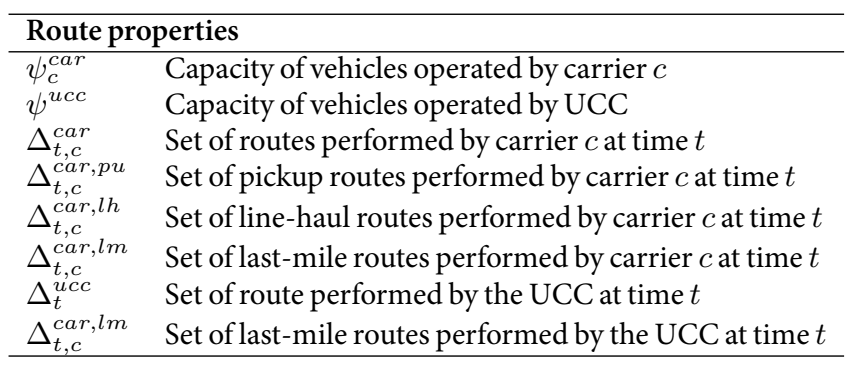




\begin{tabular}{ll}
\hline State properties & \\
\hline$\gamma_{t, r}^{r e c}$ & Binary variable, indicates whether receiver $r$ uses delivery via UCC \\
$\gamma_{t, c}^{c a r}$ & Binary variable, indicates whether carrier $c$ uses delivery via UCC \\
$x_{t}$ & Dispatch action applied on inventory $I_{t}$ \\
$x_{t, t^{e}, t^{l}, v, r, c, s, l, \gamma}$ & Number of orders of certain order type dispatched \\
$\tilde{I}_{t, t^{e}, t^{l}, v^{r}, r, \cdot, s, l, \cdot}$ & Demand of a given order type at time $t$ by receiver $r$ \\
$\omega_{t}$ & Vector of order demand at time $t$ for all order types \\
$\Omega_{t}$ & Set of possible order arrivals at $t$ \\
$W_{t}$ & Random variable that generates order arrivals at $t$ \\
\hline
\end{tabular}

\begin{tabular}{|c|c|}
\hline \multicolumn{2}{|l|}{ Cost functions } \\
\hline$C_{r}^{r e c}\left(\Delta_{t}^{u c c} \cup \bigcup_{c \in \mathcal{C}} \Delta_{t, c}^{c a r}\right)$ & Cost function for receiver $r$ \\
\hline$C_{c}^{c a r}\left(\Delta_{t, c}^{c a r},\left[l_{t, \cdot, \cdot, v_{s}^{s h p}, \cdot, c, s, \cdot, \cdot}\right]_{s \in \mathcal{S}}\right)$ & Cost function for carrier $c$ \\
\hline$C_{s}^{s h p}\left(l_{t, \cdot, \cdot, v_{s}^{s h p}, \cdot, c, s, .,}\right)$ & Cost function for shipper $s$ \\
\hline$C^{u c c}\left(\Delta_{t}^{u c c}, l_{t, \cdot, \cdot, v}^{u c c}, \cdot, \cdot, \cdot, \cdot, 1\right)$ & Cost function for UCC \\
\hline$P_{c}^{c a r, t r}\left(l_{t, \cdot, \cdot, v_{s}^{s h p}, \cdot, c, s, \cdot, \cdot}\right)$ & $\begin{array}{l}\text { Volume-dependent transport price charged } \\
\text { by carrier } c \text { to shipper } s\end{array}$ \\
\hline$C_{c}^{c a r, t r}\left(\Delta_{t, c}^{c a r}\right)$ & $\begin{array}{l}\text { Transport costs for carrier } c \text { for } \\
\text { performing route set } \Delta_{t, c}^{c a r}\end{array}$ \\
\hline$C_{s}^{s h p, s p}$ & Fixed shipping fee per shipment for shipper $s$ \\
\hline$C^{u c c, h d}\left(l_{t, \cdot, \cdot, v^{u c c}, \cdot, \cdot, \cdot, \cdot, 1}\right)$ & $\begin{array}{l}\text { Handling costs for UCC for order } \\
\text { volume } l_{t, \cdot, \cdot, v} u c c,,, \cdot, \cdot,, 1\end{array}$ \\
\hline$C^{u c c, t r}\left(\Delta_{t}^{u c c}\right)$ & $\begin{array}{l}\text { Transport costs for UCC for } \\
\text { performing route set } \Delta_{t}^{u c c}\end{array}$ \\
\hline$P^{u c c, t r}\left(l_{t, \cdot, \cdot, v}^{u c c}, \cdot, \cdot, \cdot, \cdot, 1\right)$ & $\begin{array}{l}\text { Price charged by UCC for last-mile } \\
\text { distribution volume } l_{t} \ldots v u c c \ldots .\end{array}$ \\
\hline$C_{r}^{r e c, r c}\left(\Delta_{t}^{u c c} \cup \bigcup_{c \in \mathcal{C}} \Delta_{t, c}^{c a r}\right)$ & $\begin{array}{l}\text { Receiving costs for receiver } r \\
\text { for routes } \Delta_{t}^{u c c} \cup \bigcup_{c \in \mathcal{C}} \Delta_{t, c}^{c a r}\end{array}$ \\
\hline
\end{tabular}




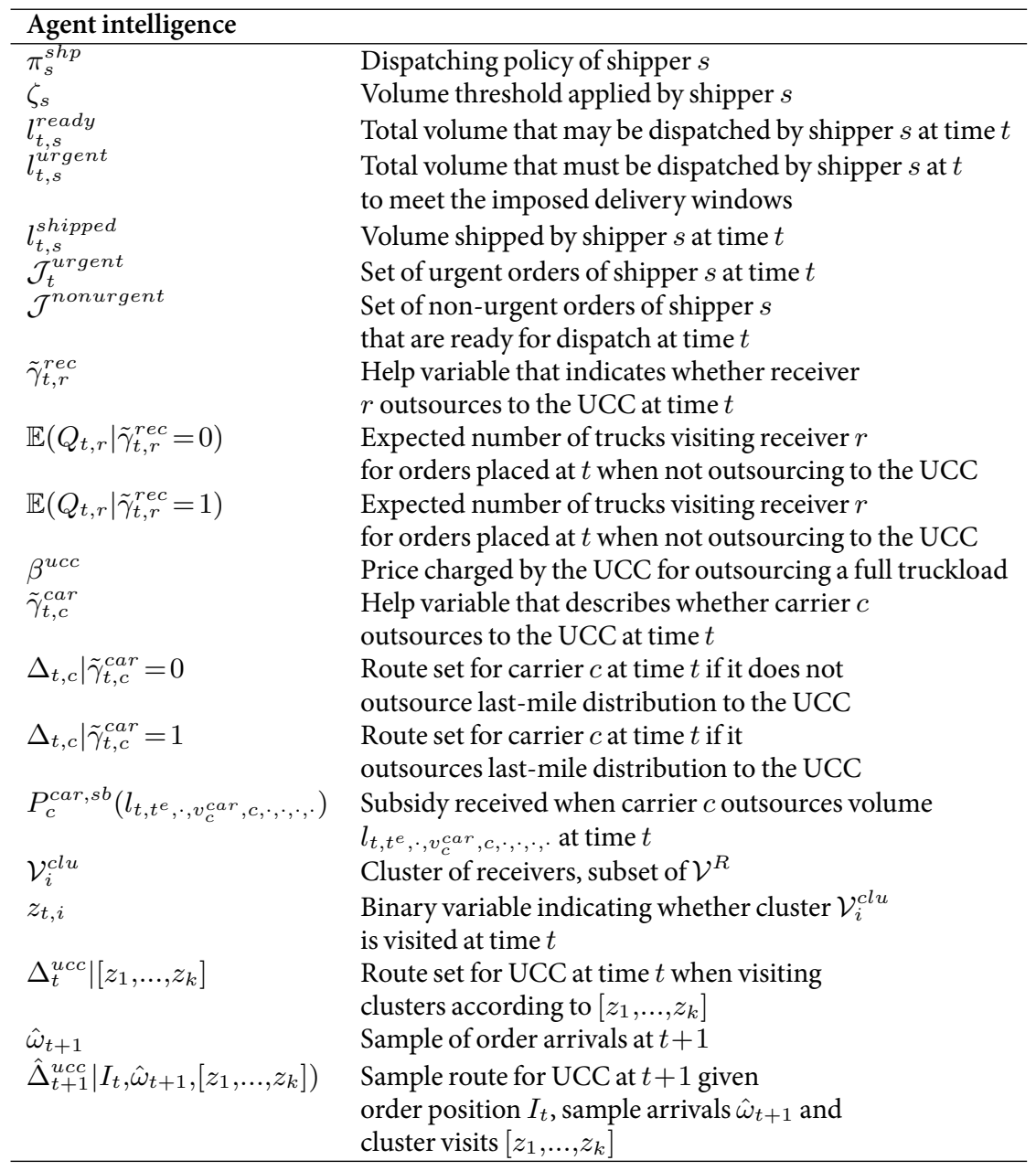




\section{Chapter 6}

\begin{tabular}{ll}
\hline \multicolumn{2}{l}{ State properties } \\
\hline $\mathcal{T}^{\text {tac }}$ & Set of tactical decision moments, subset of $\mathcal{T}$ \\
$\mathcal{R}$ & Set of receivers \\
$\mathcal{C}$ & Set of carriers \\
$\mathcal{L}$ & Set of order volumes \\
$h$ & Indicator for UCC \\
$r$ & Indicator for receiver in $\mathcal{R}$ \\
$c$ & Indicator for carrier in $\mathcal{C}$ \\
$l$ & Order volume in $\mathcal{L}$ \\
$I_{t, c, r, l}$ & Number of orders at time $t$ of order type $(c, r, l)$ \\
$I_{t}$ & Vector of number of orders per order type \\
$\gamma_{t, r}^{r e c t r}$ & Binary variable, indicates whether receiver $r$ uses delivery via UCC \\
$\gamma_{t, r}^{r e c, v a l}$ & Binary variable, indicates whether receiver $r$ outsources \\
$\gamma_{t, c}^{c a r}$ & value-adding services to UCC \\
$S_{t}$ & Binary variable, indicates whether carrier $c$ uses delivery via UCC \\
\hline
\end{tabular}

\begin{tabular}{ll}
\hline State properties \\
\hline$\tilde{I}_{t, c, r, l}$ & Number of order arrivals at time $t$ of order type $(c, r, l)$ \\
$\omega_{t}$ & Vector of order demand at time $t$ for all order types \\
$\Omega_{t}$ & Set of possible order arrivals at $t$ \\
$W_{t}$ & Random variable that generates order arrivals at $t$ \\
$S^{M}$ & Transition function to move from $S_{t}$ to $S_{t+1}$, based on $W_{t+1}$ \\
\hline
\end{tabular}

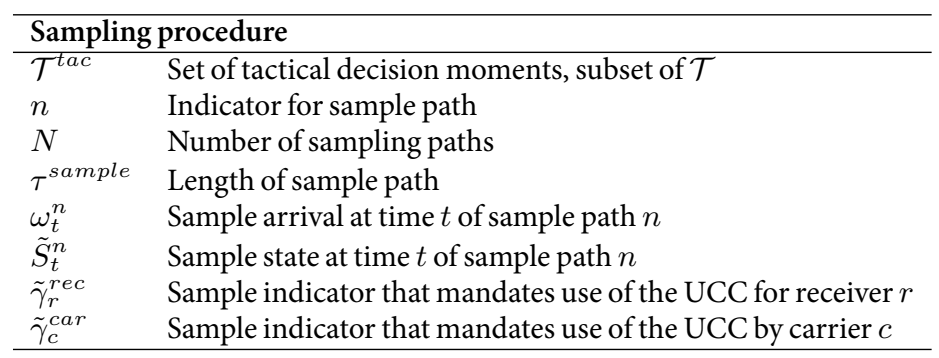




\begin{tabular}{ll}
\hline \multicolumn{2}{l}{ Route properties } \\
\hline$q$ & Vehicle indicator \\
$\mathcal{Q}^{u c c}$ & Set of vehicles operated by the UCC \\
$\delta_{t, q}^{u c c}$ & Route of vehicle $q$ dispatched by the UCC at time $t$ \\
$\delta_{t, c, q}^{c a r}$ & Route of vehicle $q$ dispatched by carrier $c$ at time $t$ \\
$\Delta_{t}^{u c c}$ & Route set for vehicles dispatched by the UCC at time $t$ \\
$\Delta_{t, c}^{c a r}$ & Route set for vehicles dispatched by carrier $c$ at time $t$ \\
$d^{u c c}$ & Route function for the UCC, providing $\Delta_{t}^{u c c}$ given $S_{t}$ \\
$d_{c}^{c a r}$ & Route function for carrier $c$, providing $\Delta_{t, c}^{c a r}$ given $S_{t}$ \\
\hline
\end{tabular}

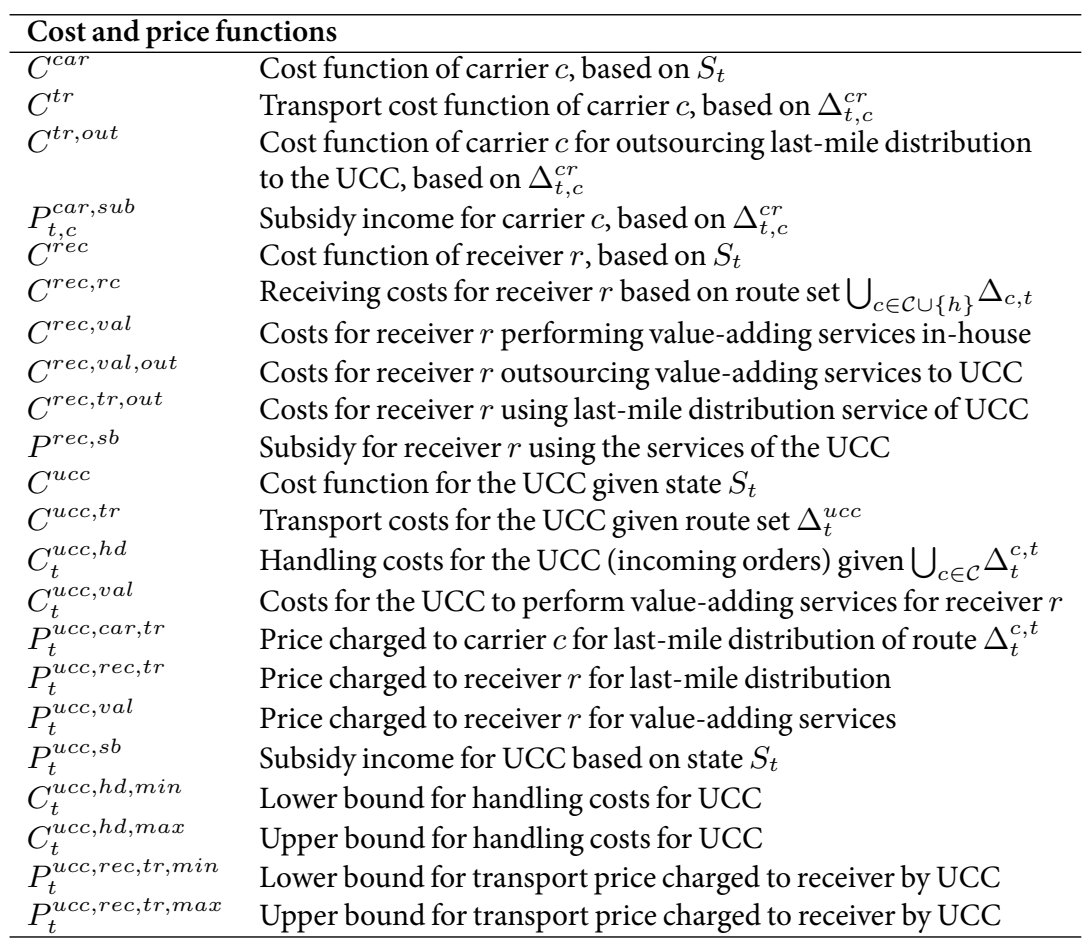





\title{
List of academic work
}

\author{
Journal papers
}

Heeswijk, W.J.A. van, Mes, M.R.K. \& Schutten, J.M.J. (2017). The delivery dispatching problem with time windows for urban consolidation centers. Transportation Science.

Heeswijk, W.J.A. van, Mes, M.R.K., Schutten, J.M.J. \& Zijm, W.H.M. (2016). Freight consolidation in intermodal networks with reloads. Flexible Services and Manufacturing.

\section{Conference papers}

Heeswijk, W.J.A van, Mes, M.R.K \& Schutten, J.M.J. (2016). An agent-based simulation framework to evaluate urban logistics schemes. Lecture Notes in Computer Science 9855, 369-383.

Heeswijk, W.J.A van, Mes, M.R.K \& Schutten, J.M.J. (2015). An approximate dynamic programming approach to urban freight distribution with batch arrivals. Lecture Notes in Computer Science, 9335, 61-75.

\section{Working papers}

Heeswijk, W.J.A. van, Larsen, R. \& Larsen, A. (2017). An urban consolidation center in the city of Copenhagen: a simulation study. Beta Working Paper series 523. Under review.

Heeswijk, W.J.A van, Joosten, R.A.M.G., Huisman, K. \& Bos, C. (2013). Real option analysis in a replicating portfolio perspective. University of Twente. 


\section{Peer-reviewed conference proceedings}

Heeswijk, W.J.A. van, Mes, M.R.K., \& Schutten, J.M.J. (2016). An agentbased simulation study on the effectiveness of urban consolidation initiatives. Proceedings of the Triennial Symposium on Transportation Analysis IX.

Heeswijk, W.J.A. van, Mes, M.R.K., \& Schutten, J.M.J. (2015). Freight consolidation in urban networks with transshipments. Proceedings of the 5th INFORMS Transportation Science and Logistics Society Workshop.

\section{Academic presentations at international conferences}

Heeswijk, W.J.A. van (2016). Evaluating urban logistics schemes using agentbased simulation. 7th International Conference on Computational Logistics. Presented on 8-9-2016, Lisbon, Portugal.

Heeswijk, W.J.A. van (2016). An-agent based simulation framework to evaluate urban logistics initiatives. Triennial Symposium on Transportation Analysis IX. Presented on 15-6-2016. Oranjestad, Aruba.

Heeswijk, W.J.A. van (2015). An ADP algorithm for the delivery dispatching problem with time windows. 6th International Conference on Computational Logistics. Presented on 25-9-2015, Delft, The Netherlands.

Heeswijk, W.J.A. van (2015). An ADP approach towards the delivery dispatching problem with time windows. 5th INFORMS Transportation Science and Logistics Society Workshop. Presented on 8-7-2015, Berlin, Germany.

Heeswijk, W.J.A. van (2014). Urban freight delivery under uncertain batch arrivals. 2014 INFORMS Annual Meeting. Presented on 12-11-2014, San Francisco, United States.

Heeswijk, W.J.A. van (2013). Consolidation of intermodal freight transport: k-shortest path matching. 2013 INFORMS Annual Meeting. Presented on 810-2013, Minneapolis, United States. 


\section{Theses}

Heeswijk, W.J.A. van (2017). Consolidation \& coordination in urban freight transport. $\mathrm{PhD}$ thesis, University of Twente.

Heeswijk, W.J.A. van (2012). The application of real option analysis on a gasto-wire investment scenario. MSc thesis, University of Twente.

Heeswijk, W.J.A.van (2011). Analyse van de rechtsgeldigheid van een elektronisch geldsysteem binnen de financiële wetgeving. BSc thesis, University of Twente. 



\section{Layout and design}

\section{Layout}

This thesis has been designed with the open-source document system ${ }_{\mathrm{AT}} \mathrm{T}$ X, using the distribution $\mathrm{TEXNicCenter} \mathrm{and} \mathrm{the} \mathrm{document} \mathrm{class} \mathrm{'amsbook'.} \mathrm{We}$ have used the typesetting engine $\mathrm{X}_{\mathrm{H}} \mathrm{AT}_{\mathrm{E}} \mathrm{X}$ to generate the document.

\section{Font}

The Adobe Original typeface Minion $\mathrm{Pro}^{\circledR}$ serves as the font for the main text, using font size 11. For the figures, the font Computer Modern Serif was used. This font belongs to Computer Modern, the original family of typefaces used by $\mathrm{T}_{\mathrm{E}} \mathrm{X}$.

\section{Figures}

Several figures depicted in this thesis are based on OpenStreetMap, which is a project with the purpose to create freely available and freely adaptable geographical maps. The maps of OpenStreetMap may be modified and re-used under the Creative Commons license; the copyright of the maps lies with the contributors to OpenStreetMap. The remainder of the figures and plots have been designed in Microsoft Visio and Microsoft Excel ${ }^{\circ}$.

\section{Cover}

The cover design shows the urban landscape of the city of Dubai. The picture was obtained from Pixabay and released under the Creative Commons CC0 license, allowing for redistribution and modification without restriction.

URL: https://pixabay.com/en/dubai-cityscape-emirates-travel-1767540/ 



\begin{abstract}
About the author
Wouter van Heeswijk was born in Etten, the Netherlands on September 14th, 1988. In 2012, he obtained his master degree in Industrial Engineering and Management, with specialization Financial Engineering, at the University of Twente. He performed his graduation assignment at research institute TNO in Utrecht. He studied the application of real option valuation in the natural gas industry and developed a simulation model to value natural gas fields with the potential to generate electricity.

In 2013, Wouter started his $\mathrm{PhD}$ research as part of the CONCOORD project on urban freight transport. The objective of the project was to investigate methods to improve the efficiency of urban freight transport by consolidating freight and coordinating between actors in the supply chain. Wouter executed his research in the department of Industrial Engineering \& Business Information Systems, under the supervision of prof. dr. W.H.M. Zijm, dr. ir. M.R.K. Mes, and dr. ir. J.M.J. Schutten. The results of his studies are presented in this thesis.
\end{abstract}


